\title{
MICRO- AND NANOPLASTIC IN THE AQUATIC ENVIRONMENT FROM RIVERS TO WHALES
}




\section{Thesis committee}

\section{Promotor}

Prof. Dr A.A. Koelmans

Personal chair, Aquatic Ecology and Water Quality Management Wageningen University \& Research

\section{Co-promotor}

Dr E.M. Foekema

Researcher, Wageningen Marine Research

Wageningen University \& Research

\section{Other members}

Prof. Dr C. Kroeze, Wageningen University \& Research

Prof. Dr A.P. Van Wezel, Utrecht University

Dr A.J. Verschoor, National Institute for Health and Environment, Bilthoven

Prof. Dr A.D. Vethaak, VU Amsterdam

This research was conducted under the auspices of the Graduate School for Socio- Economic and Natural Sciences of the Environment (SENSE) 


\section{MICRO-AND NANOPLASTIC IN THE AQUATIC ENVIRONMENT FROM RIVERS TO WHALES}

\section{THESIS}

submitted in fulfilment of the requirements for the degree of doctor at Wageningen University by the authority of the Rector Magnificus Prof. Dr. A.P.J. Mol in the presence of the

Thesis Committee appointed by the Academic Board to be defended in public on Monday $16^{\text {th }}$ of April 2018 at 4 in the Aula 


\section{Ellen Besseling}

Micro- and nanoplastic in the aquatic environment - From rivers to whales

312 pages

$\mathrm{PhD}$ thesis, Wageningen University, Wageningen, NL (2018)

With references, with summaries in English and Dutch

ISBN: 978-94-6343-259-7

DOI: https://doi.org/10.18174/441167 


\section{Table of contents}

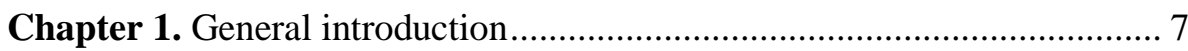

Chapter 2. Fate of nano- and microplastic in freshwater systems: a modeling study....

Chapter 3. Nanoplastic affects growth of S. obliquus and reproduction of $D$. magna

Chapter 4. Effects of microplastic on fitness and PCB bioaccumulation by the lugworm Arenicola marine (L.)

Chapter 5. Plastic as a carrier of POPs to aquatic organisms:

A model analysis

Chapter 6. The effect of microplastic on the uptake of chemicals by the lugworm Arenicola marina (L.) under environmentally relevant exposure conditions 91

Chapter 7. Microplastic in a macro filter feeder: humpback whale Megaptera novaeangliae.

Chapter 8. Synthesis 121

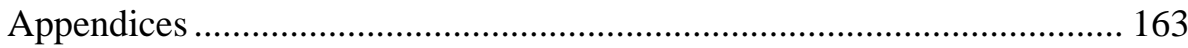

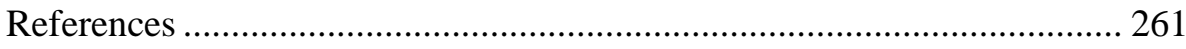

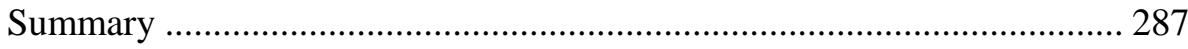

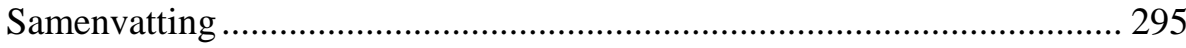

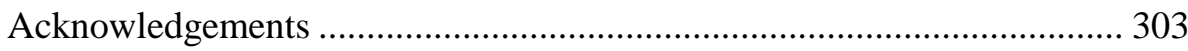

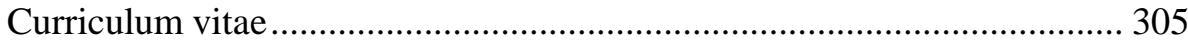

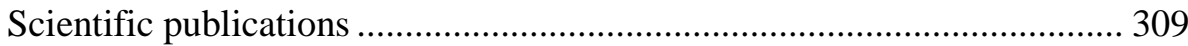

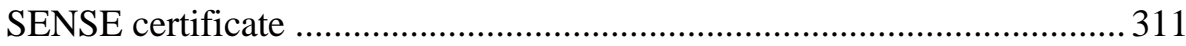




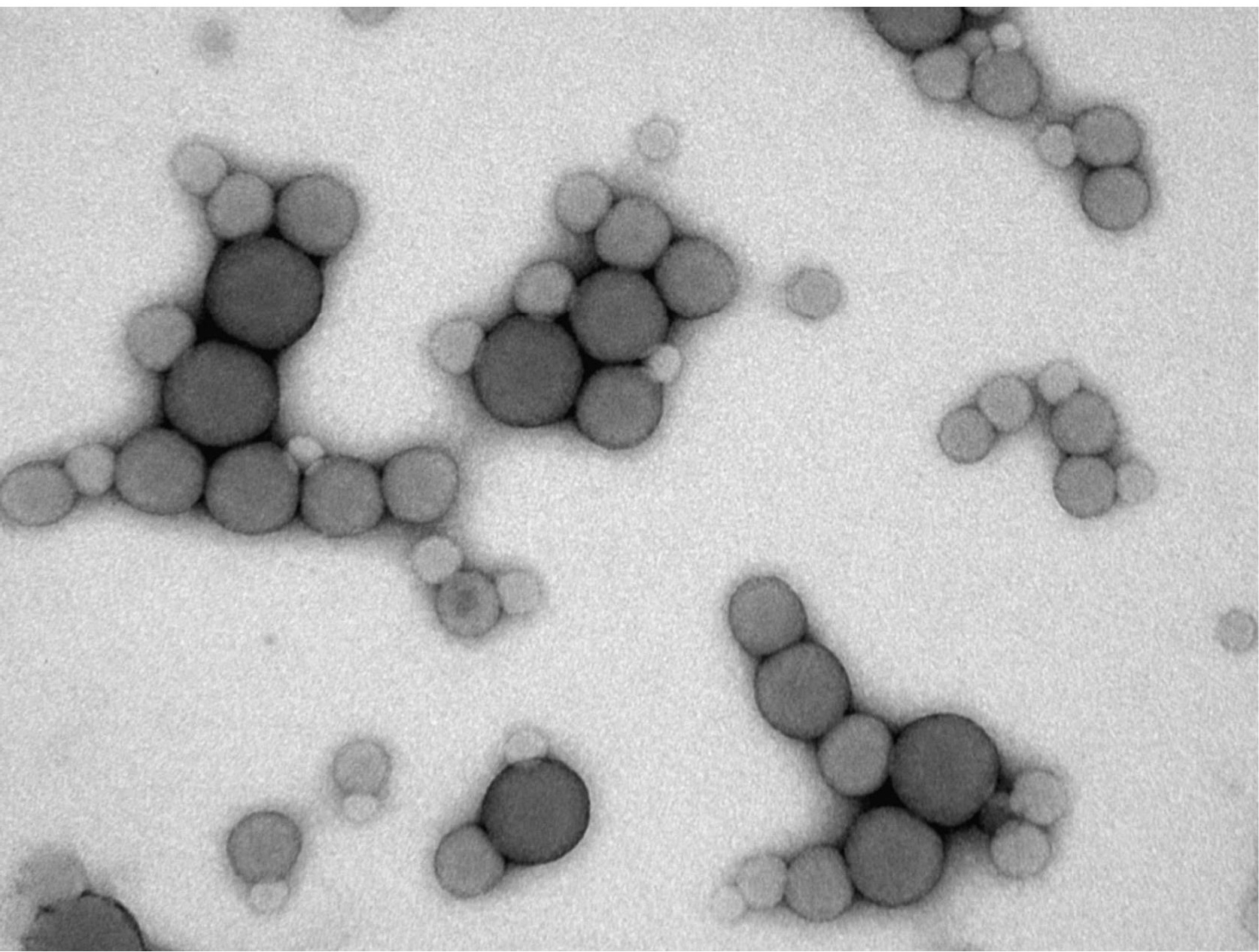




\section{General introduction}

\subsection{Plastic pollution}

Plastic has unquestionable benefits to modern society, ${ }^{1}$ however, its current use leads to pollution of the environment. In 2015 a total of 322 million tonnes was produced globally, and this yearly production is still increasing. ${ }^{2}$ Although being a very versatile material which also is used in durable products, the present use of plastic is for a large part in single-use products. About $40 \%$ of the plastic production consists of packaging material, ${ }^{2}$ therewith making up the majority of the single use plastic products. For an important part of these single used products, emission of pollutants at the production site is prevented, consumers take care of proper discard and plastic waste gets a new life via down-cycling. In that way, about a fifth of the single use plastic reaches the official waste stream, of which about two-third is recovered by recycling or energy production and about one-third goes to landfills. ${ }^{2}$ However, the other fraction, the fraction of plastic that initiated the necessity of this thesis, is the fraction that escapes the pathway from production towards recovery. During production, transport, consumption and discard, plastic is released to air, soil and water systems. This occurs due to accidents, nonoptimal treatment of waste streams and indifferent user behaviour, and happens to plastic of all sizes. The result of the growing plastic production and use is that the estimated worldwide emission of 45 thousand metric tonnes three decades $\mathrm{ago}^{3}$ has risen by at least two orders of magnitude, as $4.8-12.7$ million metric tonnes of plastic entered the ocean in 2010. If no measures are taken, this emission will even be increased by another order of magnitude by the year $2025 .{ }^{4}$ The emission of plastic leads to socio-economic and ecological harm, and is because of these negative consequences considered pollution. Socio-economic harm due to plastic comprises reduction of recreational and aesthetic attractiveness, possible human health risks and income loss in, amongst others, fisheries, tourism and shipping. Ecological harm includes potential negative effects of plastic on species and habitats. ${ }^{5}$ 


\subsection{Plastic size classes}

Most visible are the large plastic items in which we still recognize their intended use, like bottles, plastic bags and fishing-nets. First concerns about this macroplastic being found on the ocean surface were raised in the scientific literature already four decades ago. ${ }^{6,7}$ However, the public debate about plastic in the environment became large during the last decade, when repeated discovery of garbage patches, also referred to as plastic islands, and suspended tiny plastic particles, in the Netherlands referred to as plastic soup, in respectively the ocean gyres and subsequently many seas took place. ${ }^{8,9}$ Les visible than this macroplastic are microplastic particles. ${ }^{10}$ This second size class consists of plastic particles smaller than 1 or than $5 \mathrm{~mm} \cdot{ }^{8,11}$ Although a definition $\leq 1 \mathrm{~mm}$ might be more intuitive as it really considers the micro size range, a definition of $\leq 5 \mathrm{~mm}$ presently is the most commonly accepted definition and will therefore be used in this thesis. Plastic is often transported in $\leq 5 \mathrm{~mm}$ pellet form. These microplastic particles are to be melted down and formed into products elsewhere. ${ }^{8}$ Microplastic pellets are known to be omnipresent on beaches, due to accidental spillage from ships. Smaller microplastic is present in a range of applications, from personal care products (PCPs) like scrubs and toothpaste to medicine and air-blasting media. ${ }^{11,12}$ When wastewater treatment plants are unable to retain microplastic particles from PCPs, they reach surface water via effluent. ${ }^{13}$ Subsequently, retention in sewage sludge can via application on agricultural land cause presence in soils and via run-off in surface water. ${ }^{14}$ These forms of microplastic mentioned so far are intentionally fabricated small sized plastic particles, often called primary microplastic particles. Another group of microplastic particles is formed by unintended degradation from larger plastic items, usually referred to as secondary microplastic. ${ }^{11}$ As plastic is a resistant material, degradation is slow. Nevertheless, under harsh user conditions or prolonged exposure to sun, waves, wind or (micro)organisms, plastic wears out, becomes brittle and disintegrates from the outside. Examples are synthetic fibres released when washing clothes, and sheets, threads and fragments from degrading bags, ropes and other plastic items. ${ }^{8,13}$ Apart from macro- and microplastic, a third size class of plastic is made up by nanoplastic. A common definition of nanoplastic is lacking, from $\leq 100 \mathrm{~nm}$ to also $\leq 20 \mu \mathrm{m}$ have been suggested. ${ }^{15,16}$ In this thesis the definition of nanoplastic being $\leq 100 \mathrm{~nm}$ in at least one of the particle's dimensions is used, as this corresponds with the size definition of nanoparticles of other types of materials. ${ }^{17} \mathrm{With}$ the latest techniques it is still not possible to detect these $\leq 100 \mathrm{~nm}$ particles in environmental samples. However, their intended use in consumer products, release by processing 
plastic by f.e. 3D printing and cutting of styrene foam and expected creation by weathering, makes their presence in the environment highly likely. ${ }^{8,15,18}$ The small size of micro- and nanoplastic has given rise to concerns about special characteristics and therewith size-specific behaviour and effects of these particles. This is why the focus of this thesis is on these size classes.

\subsection{Plastic composition}

Environmental plastic consists of different polymer types. Most found polymer types in the marine environment represent the globally most produced types: polyethylene (PE) in low- and high-density form (LDPE, HDPE), polypropylene (PP), polystyrene (PS), polyamide nylon (PA), polyethylene terephthalate (PET), polyvinyl chloride (PVC) and cellulose acetate (CA) ${ }^{8}$ All these polymer types are found as macroplastic items, f.e. bags are often made of LDPE, jugs of HDPE, rope of PP, foam of PS, netting of PA, PP or LDPE, bottles of PET or PVC and cigarette filters of CA. Degradation of these macroplastic items, together with intentionally produced microplastic, makes that also smaller plastic particles of all these polymer types are found in the environment. The different polymer types have different molecular and crystal structures and therewith different characteristics. The share of relatively open, amorphous versus dens, crystalline structures affects the polymer density: PE, PP, PS and PA have densities $\leq 1.05 \mathrm{~kg} / \mathrm{L}$ while PET and PVC have densities $\geq 1.37 \mathrm{~kg} / \mathrm{L} .{ }^{8}$ These different densities might influence the fate of the particles that are made of them, as it affects their tendency to either float, suspend or settle in water. Furthermore, plastic can contain additives to give it specific properties. Plasticizers, stabilizers and flame-retardants are well-known examples of additives. Whether environmental plastic still contains those additives depends on the diffusivity of the additives and on the time-span of the plastic in the environment. Apart from additives present in the plastic since manufacturing, plastic can also pick up chemicals during its use and route through the environment. As especially hydrophobic chemicals are likely to sorb to plastic, the transport of persistent organic pollutants (POPs) is a large concern related to plastic pollution. ${ }^{19-21}$ Ongoing debate on whether or not plastic particles will transport contaminants from the environment into organisms (vector effect) or out of organisms (cleaning effect) reveals that more research on this particular sub-topic is needed.

\subsection{Plastic fate and effects}

Relatively little was known about where in the environment plastic occurred, how it behaved and what effects it caused on organisms, until research on this topic boomed within the last decade. Macroplastic was found on the ocean 
surface, seafloor or washed ashore, ${ }^{22}$ depending on the environment, polymer density and on whether it gained extra weight by coverage with marine organisms or not. Apparent effects of macroplastic were suffocation of organisms that tried to swallow plastic, and entanglement of organisms in ropes and nets. ${ }^{23}$ Also, decaying carcasses of birds unmasked stomachs full of plastic items. ${ }^{24,25}$ Therewith raising the question whether these birds died of starvation due to non-nutritious fill or blockage of their intestines. ${ }^{3}$ The same items as found in bird stomachs, and among them microplastic pellets, were found when taking a detailed look at beaches. Together with first examined fine nets scooped through the ocean gyres, ${ }^{26}$ this contributed to public debate about where the smaller size fraction, the microplastic particles ended up. Next to the fate of these particles, it was wondered what effects they caused. Suggested effects of microplastic were alteration of sediment porosity, shading, diluting the overall nutritious value of food by being a non-nutritious fraction in the diet of organisms, blockage of intestines, serving as a vector for invasion of alien species and transfer of chemicals into the food chain. . $^{27,28}$ For nanoplastic and the smaller size range of microplastic in particular, hypothesised effects were translocation from the intestines into other tissues, oxidative stress, immune response and particle toxicity. ${ }^{11,27-30} \mathrm{~A}$ new field of research emerged, with environmental scientists, oceanographers and most of all marine biologists involved.

\subsection{Emerging field of research}

With a main focus on the marine environment, plastic pollution studies started on the entanglement of organisms by large plastic items ${ }^{31}$ and on the occurrence of macro- and microplastic in water and biota. More specifically, a lot of research was carried out on the occurrence of plastic on the ocean surface, ${ }^{8,26}$ beaches $^{8}$ and on which species of organisms contained plastic. ${ }^{8,25,32-36}$ These were very sensible first steps to get insight in the magnitude of the pollution of the environment with plastic. Subsequent steps for the abiotic environment were to expand our knowledge on occurrence on the ocean surface and beaches ${ }^{37}$ to seas, freshwater ${ }^{38}$ and include the water bottom sediment. ${ }^{39}$ Further steps for the biotic environment were to analyse which species would take up plastic under which circumstances and what the effects of this uptake were. ${ }^{29,40}$ These subsequent steps would provide insight in the behaviour of plastic in the abiotic environment and the effects on organisms. This is where this thesis started. 


\subsection{Knowledge gaps}

At the time this thesis started it was unclear to what extent micro- and nanoplastic particles pose a risk to aquatic life. This unclearness about risk entailed both lack of understanding with respect to the 1) fate of micro- and nanoplastic in the abiotic and 2) biotic environment, and the 3) physical and 4) chemical effects on the biotic environment. Gap 1: Transport patterns of floating plastic on the ocean surface were fairly clear and macro- and microplastic on beaches encountered. Inland emitted plastic was hypothesised to be transported down the drain, adding upon the encountered marine plastic islands. ${ }^{13}$ However, uncertain was whether retention in freshwater systems would occur and only very first measurements were done there. ${ }^{38}$ Also, no differentiation between the fate of different plastic sizes and types was included. Gap 2: Around the fate of plastic in biota, several questions were unanswered. It was unknown whether ingestion of plastic by organism could lead to uptake beyond the gut system into the tissues of organism. ${ }^{41}$ Ingestion by organisms was expected to result in accumulation of plastic in these organisms and subsequent transfer along the food chain, from algae to zooplankton to higher organisms, but this was not confirmed. Gap 3: Ingestion of plastic was hypothesised to have a likely negative effect on organisms. Suffocation and entanglement were apparent effects of macroplastic but whether the suggested types of effects of micro- and nanoplastic on organisms indeed occurred was uncertain, as well as their effect thresholds. Gap 4: In particular one hypothesised effect of micro- and nanoplastic received a lot of attention, namely the possibility of plastic functioning as a vector, ${ }^{19-21}$ transporting other pollutants from the surrounding environment into organisms. However, whether this indeed entailed an important consequence of exposure to micro- or nanoplastic, was unknown.

\subsection{A need for quantification of mechanisms}

When this thesis started, research on plastic debris in the aquatic environment was mostly descriptive. For instance for the Pacific Ocean and Mediterranean Sea appearance of floating plastic was described and outcomes were given in numbers of particles per surface area. ${ }^{26,42}$ Furthermore, occurrence of plastic in many species was described, often reporting numbers of particles per organism and \% of organisms containing plastic. ${ }^{34-36,43-47}$ Therewith, the main focus was at raising attention for plastic pollution itself and to a lesser extent at getting insight in the mechanisms underlying the observations. A systems analysis approach aiming at quantitatively understanding mechanisms underlying what was observed in the field, seemed to lack. Nonetheless, a 


\section{Chapter 1}

mechanistic understanding and quantification of occurrence, fate and effects of micro- and nanoplastic is needed to assess risk, to decide on risk mitigation and to mitigate the risk of micro- and nanoplastic to the environment and human health. Plastic in the environment might be a relatively recent type of pollution and therewith a relatively young field of research. However, all the aforementioned knowledge gaps relate to issues addressed by and methods developed in other research fields. For example, sediment transport models, analytical techniques for analyses of (fluorescent) algae and nanoparticle behaviour, organism mass balances, food web models, ecotoxicological exposure assays and passive samplers have been used before and might beneficial in understanding mechanisms behind plastic fate and effects. As using this existing knowledge and methods could accelerate insight in risks of plastic in the environment, this thesis specifically aimed to quantitatively address the micro- and nanoplastic knowledge gaps at stake, thereby integrating knowledge and tools from adjacent disciplines and lessons learned from assessment of more traditional pollutants.

\subsection{Aim of this thesis}

This thesis aims to quantitatively address the following four major general knowledge gaps on micro- and nanoplastic:

- To what extent do biotic and abiotic processes influence the transport and fate of micro- and nanoplastic, therewith affecting their aquatic fate?

- To what extent does exposure to micro- and nanoplastic lead to uptake in organisms?

- What are the particle effects of micro- and nanoplastic on organisms, and at what thresholds?

- What are the effects of ingestion of micro- and nanoplastic by organisms on bioaccumulation of plastic associated chemicals, and at what thresholds?

- To what extent are micro- and nanoplastic a risk, when environmental exposure concentrations and effects on organisms are compared to one another? 


\subsection{Outline}

After this general introduction and outline of the thesis in Chapter 1, the above mentioned knowledge gaps are addressed. Chapter $2-7$ touch upon those knowledge gaps by six studies that relate to these gaps. These studies follow the route that a plastic particle might follow through the environment, from freshwater on to the marine system (Figure 1.1). In Chapter 8 these studies are combined to provide a provisional assessment of the ecological risks of microplastic. In short what is outlined in each chapter:

Chapter 2 addresses the mechanisms of fate and transport of micro- and nanoplastic. This is done for a river, as rivers are considered important carriers of microplastic to the oceans. By developing a transport model for plastic and performing scenario studies, the effect of several abiotic and biotic processes in rivers on the transport and retention of plastic particles is assessed. Plastic particles ranging from the nanoscale $(100 \mathrm{~nm})$ to microplastic $(<5 \mathrm{~mm})-$ up to small macroplastic scale $(10 \mathrm{~mm})$ are included. Because plastic particles come in different sizes, but are also composed of different polymer types and can occur as either pristine or fouled particles, the effect of these varying characteristics on their aquatic behaviour was investigated. Therewith, this modeling study reveals under which conditions, which particles are likely to be found in either riverine water or sediment. Furthermore, it points out whether and where hotspot locations are to be expected and whether riverine transport of micro- and nanoplastic is likely to contribute to pollution of the marine environment.

From Chapter 3 onwards we assess effects of micro- and nanoplastic, starting with physical effects of pristine versus fouled nanoplastic. The first two trophic levels of the freshwater food web are exposed, namely algae and zooplankton species. Endpoints chlorophyll- $a$ concentration, survival, growth and reproduction quantity and quality are assessed. High concentrations are included to provoke effects, such that effect thresholds can be quantified. Effect thresholds are subsequently compared with environmentally relevant concentrations. We consider the resilience of aquatic organisms both with plastic as a single stressor, as well as in a multiple stressor environment, by adding treatments with predator hormones. If plastic is a stressor to aquatic life, this stressor is expected to be one amongst multiple stressors such as toxicants, food scarcity, oxygen depletion and predators. As such, exposure to multiple stressors increases environmental realism and allows indication of interaction effects between stressors. 
In Chapter 4 we address both particle effects and chemical effects by exposure to different microplastic concentrations. A benthic marine deposit feeder from the base of the North Sea food web is exposed to polystyrene microplastic in presence of polychlorinated biphenyls (PCBs). Differences between ingestion and egestion are used to infer the likeliness of accumulation in the subjected species and transfer along the food web. Fitness indicators survival, feeding activity and bodyweight are assessed, as well as transfer of PCB congeners from plastic and sediment to the worms. For the first time, the relation between exposure to different microplastic concentrations and changes in body burden concentrations of persistent organic pollutants (POPs) like PCBs is investigated with a bioassay. Additionally, biota to sediment accumulation factors (BSAFs) on a biota dry weight basis are assessed. A comparison of the findings with environmentally realistic concentrations is made, as well as a reflection on differences in chemical partitioning processes in a bioassay versus the marine environment.

In Chapter 5 we continue with the chemical transfer between organisms and their surroundings when exposed to microplastic, by developing a bioaccumulation model that is capable of simulating chemical transfer from ingested plastic. Within this quantitative framework, the exposure assay of the previous chapter is simulated. Subsequently an extrapolation to the open ocean scenario is made, where due to the relative excess of pollutants microplastic only marginally affects sediment and aqueous phase concentrations of POPs. Different possible processes of plastic affecting concentrations of POPs are distinguished: 'diluting' POP exposure concentrations, 'carrying' POPs from the environment towards an organism and 'cleaning' POPs from an organism. The importance of these processes in laboratory versus environmental setting is distinguished for different polymer types and particle sizes. Thereafter, the outcomes are put in the perspective of a risk assessment.

Chapter 6 uses the insights from the previous chapters to optimize a bioassay for revealing effects of microplastic on transfer of chemicals between organisms and the environment. Again a benthic marine deposit feeder is exposed, this time to the polymer type polyethylene, which has a higher affinity for POPs than the polystyrene used in Chapter 4. Novel in microplastic exposure assays, the following further technical improvements are implemented: quantification of exposure through all media using passive samplers, and assessment of uptake fluxes through all natural exposure pathways. PCB concentrations in the worms are normalised on lipid content of the organisms, in order to derive biota sediment accumulation factors 
(BSAFs), bioaccumulation factors (BAFs) and a new metric to assess bioaccumulation from media containing plastic: the biota plastic accumulation factor (BPAF). The plastic inclusive bioaccumulation model of Chapter $\mathbf{5}$ is used to support the interpretation of the presented bioassay. Subsequently, a reflection is provided on the importance of chemical transfer in the risk assessment of microplastic.

In Chapter $\mathbf{7}$ microplastic is studied in the intestines of a stranded humpback whale. Until now no data was available on microplastic ingestion in whales. By showing the presence of microplastic in whales, this study adds to the database of species in which microplastic is detected. The non-natural content of the humpback intestines is analysed by FTIR (Fourier transform infrared) to confirm the polymer composition of the particles. The detected polymer types are compared to globally produced quantities of polymers and polymers mostly used in marine activities. Furthermore, based on the microplastic findings in the humpback whale we lay the foundation of a quantitative approach to assess bioaccumulation of microplastic particles, by linking microplastic counts in the whale intestines to known microplastic concentrations in the ocean. As such, we get an indication of whether humpback whales are likely to accumulate microplastic from their prey and the water, or that their intestines contain concentrations that just correspond with the concentrations in the water.

In the final Chapter 8 we reflect on the current status of the research field. The content of this thesis is combined with findings of others to state to what extent the mentioned knowledge gaps continue to exist. We assess exposure in the environment by reflecting on what concentrations are found in different media. Additionally, we nuance how fate is thought to be influenced by particle characteristics, biotic and abiotic processes based on models. We evaluate the (thresholds of) particle as well as chemical effects of micro- and nanoplastic on organisms and put this in perspective of ecological consequences by providing a provisional species sensitivity distribution. By comparing the fate and uptake with effects levels of aquatic micro- and nanoplastic, we provide a provisional quantification of their risk. 
Chapter 1

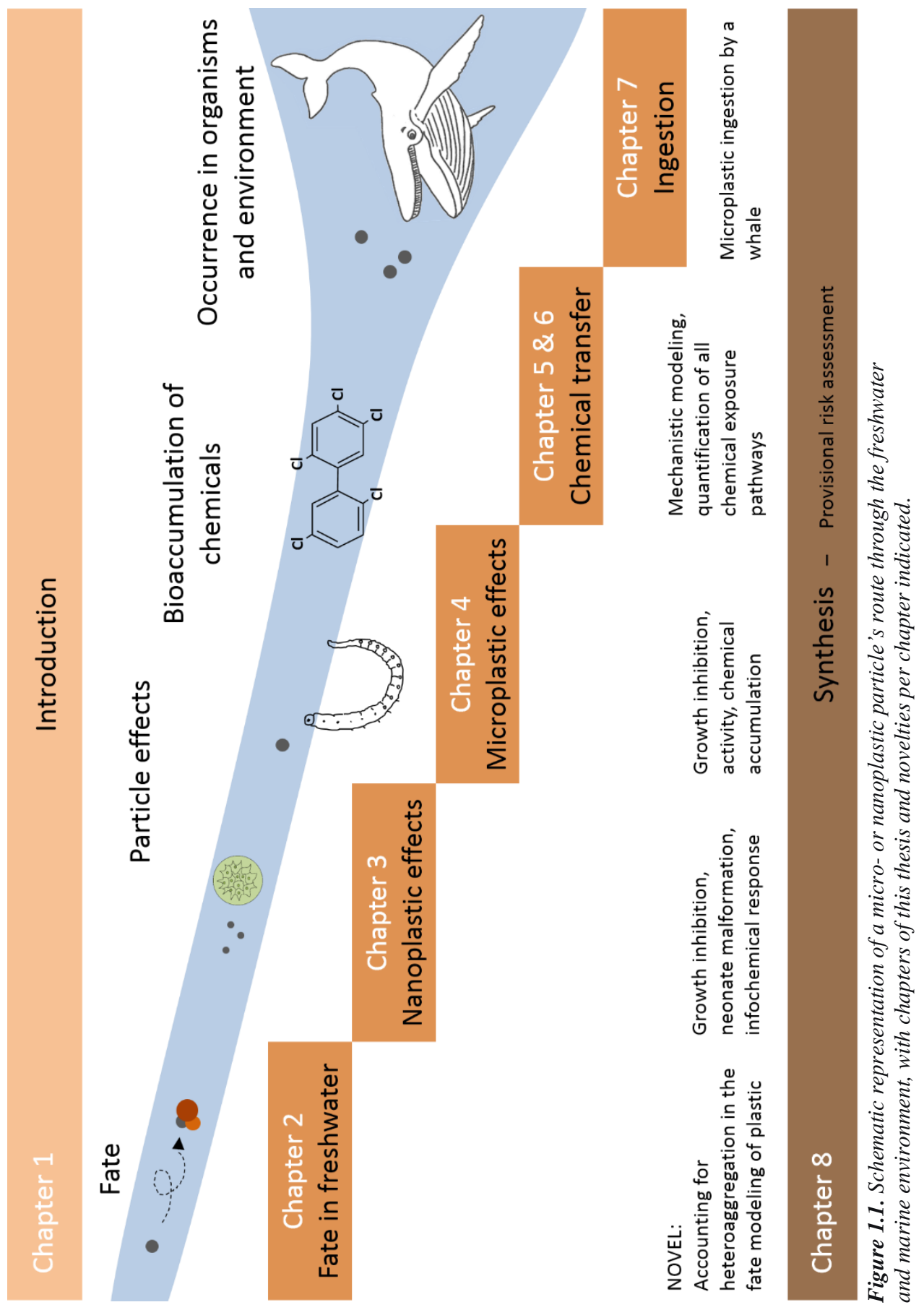


Introduction 

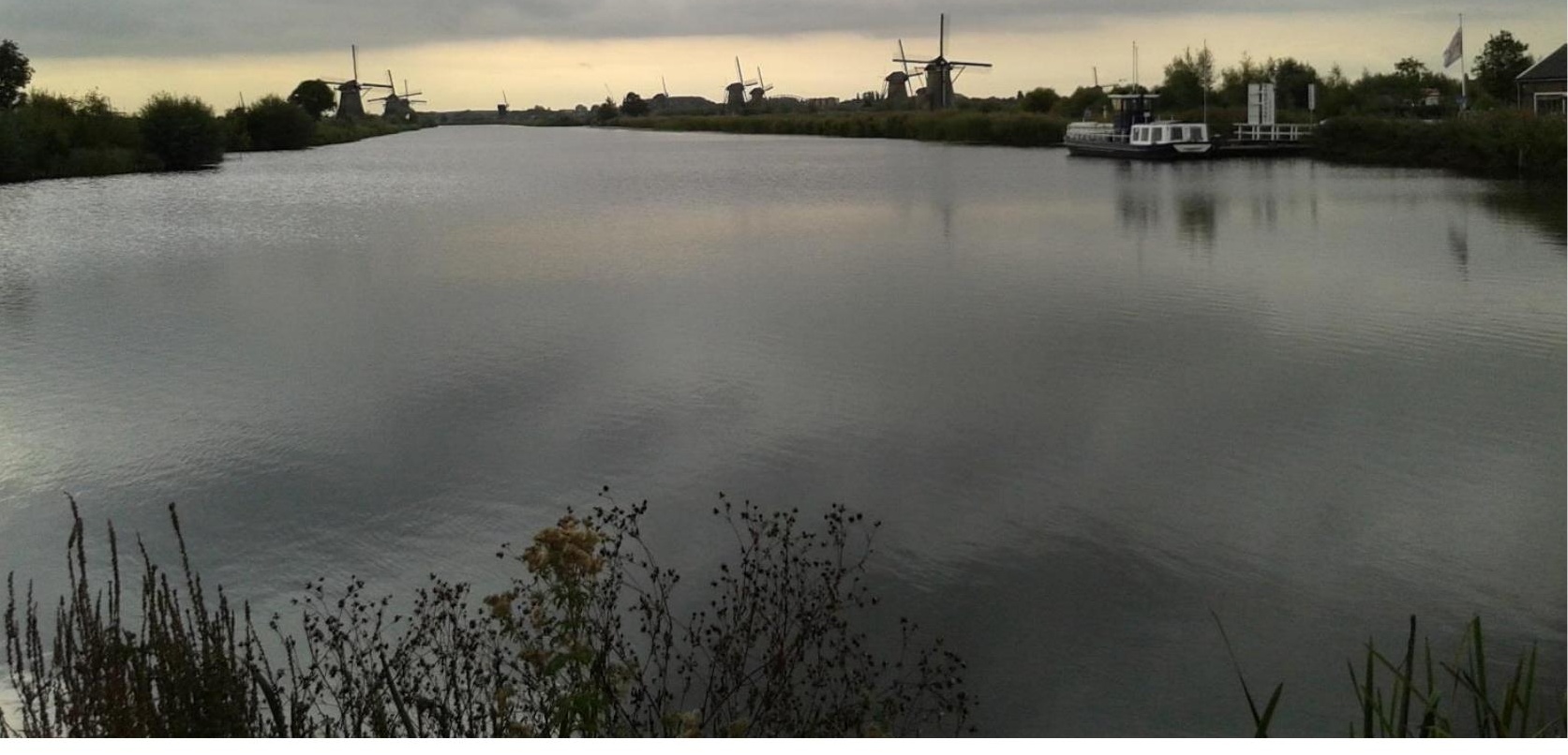


\title{
Fate of nano- and microplastic in freshwater systems: a modeling study
}

\author{
Published as: Besseling, E., Quik, J.T.K., Sun, M., Koelmans, A.A., 2017. \\ Fate of nano- and microplastic in freshwater systems: a modeling study. \\ Environmental Pollution 220, 540-548
}

\begin{abstract}
Riverine transport to the marine environment is an important pathway for microplastic. However, information on fate and transport of nano- and microplastic in freshwater systems is lacking. Here we present scenario studies on the fate and transport of nano- to millimetre sized spherical particles like microbeads $(100 \mathrm{~nm}-10 \mathrm{~mm})$ with a state of the art spatiotemporally resolved hydrological model. The model accounts for advective transport, homo- and heteroaggregation, sedimentation-resuspension, polymer degradation, presence of biofilm and burial. Literature data were used to parameterize the model and additionally the attachment efficiency for heteroaggregation was determined experimentally. The attachment efficiency ranged from 0.004 to 0.2 for $70 \mathrm{~nm}$ and $1050 \mathrm{~nm}$ polystyrene particles aggregating with kaolin or bentonite clays in natural freshwater. Modeled effects of polymer density $(1-$ $1.5 \mathrm{~kg} / \mathrm{L}$ ) and biofilm formation were not large, due to the fact that variations in polymer density are largely overwhelmed by excess mass of suspended solids that form heteroaggregates with microplastic. Particle size had a dramatic effect on the modeled fate and retention of microplastic and on the positioning of the accumulation hot spots in the sediment along the river. Remarkably, retention was lowest (18-25\%) for intermediate sized particles of about $5 \mu \mathrm{m}$, which implies that the smaller submicron particles as well as larger micro- and millimetre sized plastic are preferentially retained. Our results suggest that river hydrodynamics affect microplastic size distributions with profound implications for emissions to marine systems.
\end{abstract}




\subsection{Introduction}

Land-based sources are thought to make a large contribution to plastic debris in the oceans. ${ }^{4,48}$ Plastic items come in a wide variety of sizes and types, with microplastic $(<5 \mathrm{~mm})$ being a size class of growing concern due to possible impacts on marine life. Indeed, microplastic can negatively affect organisms in freshwater and marine environments (Chapter 3, Chapter 4). ${ }^{49-54}$ Microplastic has been detected in the marine environment ${ }^{39,55,56}$ and, more recently, also in freshwater. ${ }^{38,57-61}$ One major route for the occurrence of small plastic particles in the environment is expected to be wastewater, containing plastic particles from cosmetics and fibres from washing synthetic clothes. ${ }^{13}$ The other route is thought to be degradation of larger plastic items, which has the potential to release large numbers of microplastic particles. ${ }^{62}$

To date, techniques for detecting microplastic particles in natural samples are still in their infancy. Although important progress is being made in the development of detection methods, ${ }^{60,63,64}$ present methods still are insufficiently distinguishing for some particle sizes and completely lacking for others. ${ }^{65}$ The occurrence of nanoplastic is very plausible,${ }^{66}$ however, current techniques are not yet capable of demonstrating their presence in the environment. ${ }^{15,67}$ When microplastic from domestic sources passes wastewater treatment plants, or is formed by degradation of larger items, this leads to contamination of aquatic systems. It is generally hypothesised that once reaching surface water, plastic particles will be transported with the water, along rivers and into the sea. However, because of the limitations in standardized detection methods, the wide variability of plastic types and sizes, and insufficient knowledge on the mechanisms driving the fate of nanoplastic in rivers, support for this hypothesis is lacking. Therefore, there is a high need for the parallel development of tools that provide mechanism-based hypothesis on system behaviour that can be experimentally validated later on. This is similar to the development of nanoparticle models where first simple mass flow models evolved into validated spatiotemporal explicit models. ${ }^{68}$ Fate models might also provide information on which particle sizes are retained in rivers, which in turn defines which size classes freshwater species are exposed to, and which size classes reach the marine environment. For instance, a relative lack of millimetre sized plastic was found in the plastic size distribution of the marine environment, which was hypothesized to be caused by size-selective oceanic sinks. ${ }^{69}$ However, unexpected particle size distributions in marine ecosystems may be attributed to differential retention of plastic size categories in rivers. 
We are not aware of earlier studies that use a fate model for plastic in rivers. Here, we simulate the transport of nano-, micro-, and millimetre sized spherical or near-spherical plastic particles in freshwater with a hydrodynamic model. The model simulates spatially and temporally explicit hydrodynamic particle behaviour, accounting for advective transport, particle aggregation, sedimentation, resuspension, polymer degradation and burial. We model polymer particles from $100 \mathrm{~nm}$ to $10 \mathrm{~mm}$, and account for biofilm formation. For engineered nanomaterials, previous model studies showed that aggregation with suspended solids and subsequent sedimentation is the most likely removal process for these particles in water. ${ }^{70-72}$ The rates of these processes are important for microplastic as well, as they will determine the extent to which transport or transformation occurs.

The aim of this study is to analyse the theoretical fate and retention of plastic in a river using modeling, and to analyse the dependence of these endpoints on upstream initial microplastic concentration, particle size, polymer density and presence of biofilm. Following earlier nanoparticle study approaches ${ }^{70-72}$ our aim was to model plastic fate mechanistically within uncertainty limits, but even more so to launch a concept that can trigger further development of models. Prospective modeling based on first principles can provide guidance for monitoring network design and for identifying priorities for the mitigation of plastic contaminated sites. Parameters were taken from the literature. Heteroaggregation, the aggregation of nano- and microplastic with suspended solids, is highly affected by the efficiency of attachment between these particles, $\alpha_{\text {het }}$, a parameter which however is unknown for nano- and microplastic. This uncertainty was accounted for by scenario studies that used different values for $\alpha_{\text {het }}$. To assess which of these scenarios for $\alpha_{\text {het }}$ may be more likely, values for $\alpha_{\text {het }}$ in natural water were experimentally determined and used as input in the model. The scenario studies were performed using hydrodynamic data of the Dommel river, which represents a realistic case study showing the spatiotemporal distribution of microplastic particles of different size, and which allows for the detection of accumulation hot spots. To cover the variety of (spherical) plastic particles, different scenarios are studied with a range of particle sizes, attachment efficiencies, with and without biofilm formation, several polymer densities and varying upstream concentrations. 


\subsection{Materials and methods}

\subsubsection{Experimental determination of the attachment efficiency $(\alpha$ het $)$ between nanoplastic, microplastic and clays}

Heteroaggregation was studied in three experiments for two plastic particle sizes and two clay types representing suspended solids. These aggregationsedimentation experiments of plastic with suspended solids were performed in $0.9 \mathrm{~L}$ glass columns (diameter $5 \mathrm{~cm}$, height $43 \mathrm{~cm}$ ) with natural lake water (Wageningen, Droevendaalsesteeg) filtered through $0.7 \mu \mathrm{m}$ (Whatman, GF/F). The suspended solids kaolin clay (Fluka 60609) or bentonite clay (Sigma 285234) were dispersed at a concentration of $5 \mathrm{mg} / \mathrm{L}$, which is a representative value for rivers with low discharge. ${ }^{73,74}$ Either $70 \mathrm{~nm}$ or 1050 $\mathrm{nm}$ polystyrene (Chapter 3$)^{49,75}$ was mixed with the lake water at a concentration of $50 \mathrm{mg} / \mathrm{L}$. These plastic concentrations were chosen to be higher than in the environment for accurate detection. This agrees to recent approaches to determine $\alpha_{\text {het }}$ for engineered nanomaterials. ${ }^{73,76}$ Supernatants were sampled after settling times of 20 and 40 minutes, 1, 4 and 6 hours and 1 - 3 days and directly measured by Dynamic Light Scattering (DLS), using a Cobolt Samba-300 DPSS laser at a wavelength of $532 \mathrm{~nm}$, an ALV7002-USB as correlator and an ALV PM-15 as detector, ${ }^{52}$ with linear calibration lines between 0 and $50 \mathrm{mg} / \mathrm{L}\left(\mathrm{R}^{2}>0.93\right)$. Representative subsamples of the same supernatants were stored for 1 day in the refrigerator and analysed by spectrophotometry (DU® 730 Life Science UV/Vis Spectrophotometer A23616) at a wavelength of 310, 470 and $600 \mathrm{~nm}$, with linear calibration lines between 20 and $100 \mathrm{mg} / \mathrm{L}\left(\mathrm{R}^{2}>0.98\right)$. Values for $\alpha_{\text {het }}$ were calculated following the method of Barton et al. ${ }^{74}$ using the data obtained by both detection methods (calculation provided in the Appendix).

\subsubsection{Modeling the fate of spherical nanoplastic and microplastic}

The fate of microplastic was modeled with the NanoDUFLOW model, ${ }^{71}$ parameterised for the investigation of plastic particle behaviour in riverine systems, including for the first time micro- and millimetre sized plastic particles. Using traditional particle model concepts that were, amongst others, used for suspended solids, colloids and algae ${ }^{77-79}$ and more recently in (nano)particle transport models, ${ }^{70-72,80,81}$ we described plastic particle behaviour as a function of (plastic) particle related processes homo- and heteroaggregation, sedimentation based on Stokes settling theory, and degradation, dissolution, resuspension and burial. Homoaggregation, the mutual aggregation of particles, was included following a simplification as described by Quik et al. ${ }^{71}$ 
To be able to study the effect of microplastic particle size, scenarios were calculated for monodisperse plastic particles, so no mixture interactions between plastic particles of different sizes were included. Accordingly, heteroaggregation, the aggregation of plastic particles with suspended solids, was included by assuming five size classes of suspended solids interacting with one size class of plastic particles and four size classes of plastic homoaggregates. While theoretically more than one microplastic particle can attach to one suspended solid, it has been demonstrated for nanoparticles that the chance this happens is low. ${ }^{72,73,82}$ This is caused by the excess number concentration of natural colloids and suspended solids in rivers, compared to that of nanoparticles, which also holds for micro- and nanoplastic (Table A2.2, Eerkes-Medrano et al., ${ }^{61}$ Lenz et al. $\left.{ }^{83}\right)$. Therefore, only primary heteroaggregates, that is, aggregates of a natural colloid with one plastic particle or one homoaggregate, were considered. This approach might be less suitable for shapes like fibres, that may aggregate through knotting and/or may exhibit non-Stokes settling behaviour.

Heteroaggregation rates were calculated as the product of (a) the collision frequency in which orthokinetic, perikinetic and differential settling were accounted for (Eq. A2.5), and (b) the attachment efficiency ${ }^{71}$ (Eq. A2.4). The modeled river system was a $40 \mathrm{~km}$ stretch of the river Dommel, a Dutch lowland river, with a flow velocity averaging $0.199 \mathrm{~m} \mathrm{~s}^{-1}$ (Table A2.1). Water works present in the Dommel are a sediment settling area at $14.4 \mathrm{~km}$ flow distance and multiple weirs. A wastewater treatment plant (WWTP) in the city of Eindhoven discharges at the river Dommel.$^{84} \mathrm{~A}$ simulation time of 9 days was used. No storm, rain or other weather influences were investigated in this scenario study. The DUFLOW hydrological component for the Dommel has previously been validated.$^{85}$ The NanoDUFLOW particle model has recently been validated for metal-based submicron $(<0.45 \mu \mathrm{m})$ particles. ${ }^{84}$ A detailed description of the present implementation is provided in the Appendix. $\mathrm{R}$ Studio software v0.98.976 ${ }^{86}$ was used to control DUFLOW calculations and to process model output. Interpretation of NanoDUFLOW simulation results was assisted by modeling several particle specific sub-processes independent of the hydrological model, like modeling the collision rate and settling rates as a function of particle size according to Eqs. A2.5 and A2.8. NanoDUFLOW, including the equations, is described in detail in the Appendix. 


\subsubsection{Parameterization}

Particle diameter. The modeled plastic particles were assumed to be spherical. The model was run for twenty-five microplastic diameters in the range from $100 \mathrm{~nm}$ to $10 \mathrm{~mm}$. For all these particle diameter scenarios, the upstream microplastic input concentration was kept equal in mass, resulting in decreasing particle number concentrations with increasing diameter (Table A2.4C).

Collision frequencies. The collision frequencies were calculated according to Quik et $a l^{71}$ (Eq. A2.5), who followed traditional approaches for nonnanoparticles. ${ }^{77,78}$ This required inputs of particle radius and density, which could be taken directly from the defined scenarios.

Attachment efficiency. For the attachment efficiencies, $\alpha$, in freshwater for both homo- and heteroaggregation, a default value of 0.01 was used. ${ }^{74,87} \mathrm{We}$ used literature values for nanoparticles as a proxy to motivate our default values as they comprise a broad range of suspended solid types. Due to the relative low concentration of the spherical plastic particles compared to suspended solids, homoaggregation plays a negligible role compared to heteroaggregation, ${ }^{71,73,88}$ which renders the model output insensitive to uncertainty in the attachment efficiency for homoaggregation. The importance and the uncertainty of the attachment efficiency for heteroaggregation $\left(\alpha_{\text {het }}\right)$ may be higher..$^{72,74,78,82,89,90}$ Therefore, following Praetorius et al. ${ }^{72}$ the model's sensitivity to the magnitude of $\alpha_{\text {het }}$ was investigated by a scenario approach in which $\alpha_{\text {het }}$ was ranged from no $\left(\alpha_{\text {het }}=0\right)$ to complete $\left(\alpha_{\text {het }}=1\right)$ attachment. Additionally, $\alpha_{\text {het }}$ for nano- and microplastic was estimated from aggregationsedimentation experiments as described above.

Biofilm. While part of the particles may have no biofilm because they are freshly emitted or deposited from the atmosphere, ${ }^{91,92}$ another fraction of plastic particles entering aquatic systems like river Dommel can be assumed to be already colonized by biofilms ${ }^{87,93-97}$ and it is likely that this affects the hydrodynamics of the particle behaviour. We therefore provide simulations without as well as with biofilm. Biofilm formation is likely to alter the fate of microplastic by affecting diameter, overall density and attachment efficiency. Biofilm formation was included by adding on the particles a $0.4 \mu \mathrm{m}$ thick biofilm layer, representing average bacteria width, ${ }^{98}$ with a density of 1250 $\mathrm{kg} / \mathrm{m}^{3}$, representing the density of organic matter. ${ }^{99}$ Bacterial cell density can be lower (e.g. Godin et al. ${ }^{100}$ ), which implies that the modeled effect of biofilm can be seen as a maximum boundary. Attachment efficiencies are 
heterogeneous in nature, but previous work has shown that on the system level, average values can be used. ${ }^{71,72,80,84}$ Although being subject to uncertainty, biofilms have been found to increase the attachment efficiency of nanoparticles $2-3$ times. ${ }^{70,87}$ Therefore, we explored the effect of increased attachment efficiencies of 0.02 and 0.03 for the biofilm scenarios.

Polymer density. By default, microplastic was assigned a density of 1040 $\mathrm{kg} / \mathrm{m}^{3}$, which is the average of polymer densities found in the marine environment. ${ }^{8}$ Furthermore, polymer density was varied from $1000-1500$ $\mathrm{kg} / \mathrm{m}^{3}$, representing a wide range of polymer types and with $1000 \mathrm{~kg} / \mathrm{m}^{3}$ also representing non-settling plastic. For the scenarios with different polymer densities, the upstream plastic input concentration was kept constant on a volume basis.

Upstream concentration. A default upstream plastic mass concentration of 1 $\mathrm{ng} / \mathrm{L}$ was used. This concentration represents the average order of magnitude of published concentrations of microplastic in freshwater (Table A2.2) ${ }^{38,57,58,101}$ Besides this 'realistic' simulation, we performed simulations that studied the proportionality of predicted concentrations to the initial concentration, because initial concentration might as such affect the fate of particles. ${ }^{71}$ Hence, additional scenarios were analysed with upstream input concentrations varying by a factor of $10^{6}$, i.e. a factor $10^{2}$ lower and a factor $10^{4}$ higher than the default upstream concentration.

Burial and degradation. Burial to lower sediment layers was included with a rate of $3.17 \times 10^{-9} \mathrm{~s}^{-1}{ }^{102}$ Plastic degradation and abrasion in water and sediment were modeled as first order removal processes. The first order removal rate constant for biodegradation was set at $6.81 \times 10^{-9} \mathrm{~s}^{-1}$ based on a maximum biodegradation of $1.75 \%$ per month observed by Harshvardhan and Jha (2013). Whereas degradation and abrasion processes may play a role at very long time scales, the estimated kinetic constants are too low to affect transport and retention given the present flowtimes for the river Dommel. This also renders the model output insensitive to uncertainty in the parameters for degradation. Further parameterization was as specified in the Appendix (Table A2.1, A2.5). 


\subsection{Results and discussion}

\subsubsection{Attachment efficiencies ( $\alpha$ het $)$ for heteroaggregation of nanoplastic and microplastic with suspended solids}

There are several published methods to estimate $\alpha_{\text {het }}$ values from experimental aggregation data, ${ }^{73,74,76,104}$ the discussion of which is beyond the scope of this hydrological modeling chapter. Here, we pragmatically estimated $\alpha_{\text {het }}$ values for two near-spherical plastic particle sizes with two clay types in three combinations in natural freshwater following a procedure previously applied to nanoparticles. ${ }^{74}$ For $70 \mathrm{~nm}$ polystyrene with kaolin clay the average $\alpha_{\text {het }}$ for triplicates each measured by two detection methods (see below) was 0.04 (range 0.004-0.07) and with bentonite clay 0.1 (range 0.09-0.1). For $1050 \mathrm{~nm}$ polystyrene with bentonite clay $\alpha_{\text {het }}$ was on average 0.09 (range 0.06-0.2) (Table A2.6). As far as we know, these are the first aggregation data reported for nano- and microplastic in natural lake water. The type of suspended solids (i.e. kaolin vs. bentonite) slightly affected $\alpha_{\text {het, }}$ which confirms earlier findings that attachment efficiencies in the environment partly depend on the characteristics of suspended solids. ${ }^{73}$ The two detection methods DLS and spectrophotometry gave very comparable outcomes in particle numbers. The experimentally determined values ranged between 0.004 and 0.2 and thus are close to the values that were taken from the literature to motivate our default values $^{74,87}$ and therefore support using these values in the modeling, as discussed further in the next sections. At the same time, we emphasise that our experiments are the first that use nano- and microplastic particles for heteroaggregation experiments and that they will not apply equally to plastic particles of all shapes, like for instance fibres. To fully understand the aggregation characteristics of the wide variety of nano- and microplastic particles in the aquatic environment, more research is recommended.

\subsubsection{Modeling the fate of nano- and microplastic in water and sediment of the Dommel river}

Simulated concentrations of nano- and microplastic: To study the effect of microplastic particle size on spatial distribution, transport in the river Dommel was modeled for 25 microplastic size classes separately. Within 5 days of simulation time, microplastic concentrations in the water reached steady-state over the entire $40 \mathrm{~km}$ river stretch, thus confirming the sufficiency of a 9 days simulation period for all size classes (Fig. A2.5A). For the sediment, however, steady-state is not reached within the 9 days simulation period (Fig. A2.5BD). The locations of peak concentrations in sediment are mainly related to the emission scenario and spatial properties of the river Dommel. The height of 
these peak concentrations largely depended on particle size of either plastic $(P l)$ or natural $(S S)$ particles (Fig. 2.1). Particles $<5 \mu \mathrm{m}$ reached their highest concentrations in the sedimentation area $14 \mathrm{~km}$ downstream, whereas bigger particles settled earlier upstream (Fig. 2.1D, F, H, Fig. 2.2). Within the 9 days simulation period, peak concentrations in sediment of (a) up to $13 \mu \mathrm{g} / \mathrm{kg}$ were reached within $1 \mathrm{~km}$ from the source (Fig. 2.1J, plastic particle diameter $>500$ $\mu \mathrm{m}$ ), (b) up to $0.1 \mu \mathrm{g} / \mathrm{kg}$ in the sedimentation area $14 \mathrm{~km}$ downstream (Fig. A2.1R, plastic particle diameter $50 \mu \mathrm{m}$ ) and (c) up to $0.01 \mu \mathrm{g} / \mathrm{kg}$ at the end of the $40 \mathrm{~km}$ river stretch (Fig. A2.1 A, C, E, N, P, plastic particle diameter $<500$ $\mathrm{nm}$ and $10-20 \mu \mathrm{m})$. The settling of plastic particles to the sediment phase resulted in a linear increase in sediment concentrations with a maximum rate of $1.5 \mu \mathrm{g} \mathrm{kg}^{-1} \mathrm{day}^{-1}$ for the $>500 \mu \mathrm{m}$ particles within $1 \mathrm{~km}$ from the source, a maximum of $1.2 \times 10^{-2} \mu \mathrm{g} \mathrm{kg}^{-1} \mathrm{day}^{-1}$ for the $\sim 50 \mu \mathrm{m}$ sized particles in the sedimentation area and a maximum of $1.5 \mathrm{ng} \mathrm{kg}^{-1} \mathrm{day}^{-1}$ for the nano and 10$20 \mu \mathrm{m}$ sized particles at the end of the river system (Table A2.3, Fig. A2.5BD). By combining these accumulation rates with the loss rates from the sediment by burial, steady-state concentrations can be calculated. ${ }^{82,102}$ The maximum steady-state concentration in the river stretch was $5 \mathrm{mg} / \mathrm{kg}$, which was reached for $>1 \mathrm{~mm}$ particles at $<1 \mathrm{~km}$ from the source (Fig. 2.2, Table A2.3). However, for most other sizes and locations the steady-state concentrations were a factor 2 to 15 lower. The calculated steady-state concentrations within $1 \mathrm{~km}$ from the source (Fig. 2.2) correspond with concentrations of microplastic found by Wagner et al..$^{16}$ in sediment in Europe of 34-64 particles $/ \mathrm{kg}$, which, with a size of $10^{2}-10^{3} \mu \mathrm{m}$ based on their figures and a particle weight of $5 \mu \mathrm{g}$ per particle, ${ }^{105}$ would be around $0.25 \mathrm{mg} / \mathrm{kg}$.

Spatial distribution of nano- and microplastic: The concentration profiles of nano- and microplastic in the water and sediment were clearly related to the spatial characteristics of the river section and the size of plastic particles (Fig. 2.1). Here, spatial characteristics relate to differences in river width, depth and flow rate (Table A2.1) resulting in net sedimentation and resuspension areas. ${ }^{71}$ The $100 \mathrm{~nm}$ singular nanoplastic particles $\left(P l_{1}\right)$ show a gradual decrease in concentration in the water phase, coupled to an increase in concentration of heteroaggregates (Fig. 2.1A). There is no sharp decrease in $100 \mathrm{~nm}$ nanoplastic concentration at the sedimentation area after $14.4 \mathrm{~km}$, which implies that their removal from the water column is by aggregation with suspended solids and barely by direct settling. With increasing size of the plastic particles (Fig. 2.1, Fig. A2.1), removal of singular particles from the water phase occurs earlier, i.e. within a shorter flow distance. This can be 


\section{Chapter 2}

explained from the dependence of the various sub-processes with particle size. First, the rates of direct Stokes settling increase substantially with size (Fig. A2.6A, Table A2.4B). Whereas settling of smaller plastic particles is dependent on their aggregation with suspended solids (governed by $\alpha_{\text {het }}$ in Fig. 2.3A), larger plastic particles settle at a rate which is independent of the heteroaggregation rate. Second, the frequencies of the collisions between
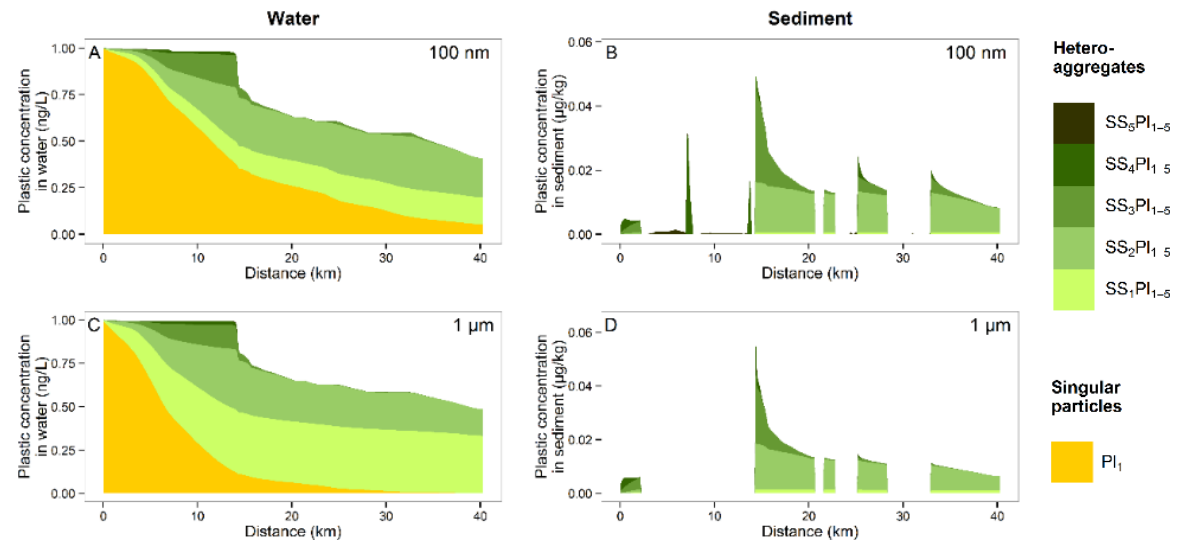

Singular particles
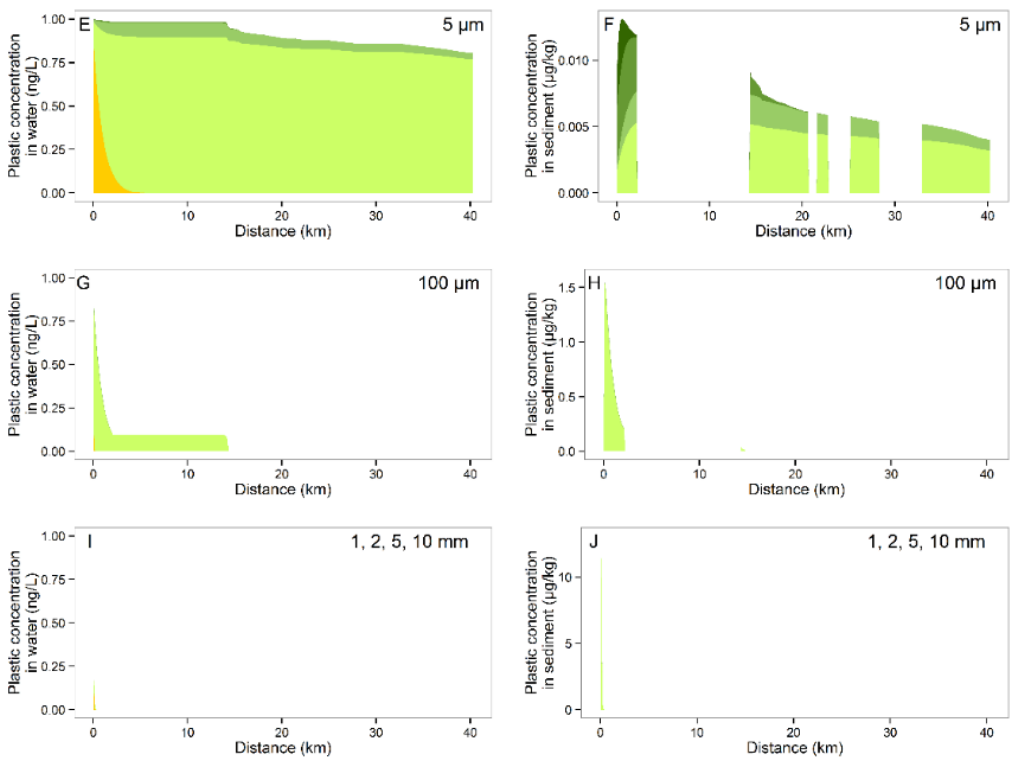

Figure 2.1. Spatial distribution of plastic over a $40 \mathrm{~km}$ river stretch. Plastic concentrations are given for the water column (left-sided panels) and for sediment (right-sided panels). These concentrations are reached after nine days of plastic input into the river, representing steadystate concentrations for the water column and intermediate state for the sediment. Different sections along the river are characterised by either net sedimentation or net resuspension, sections with net resuspension show no accumulation of plastic in the sediment. From top to 
bottom panels are ordered based on the order of increasing initial plastic particle size. The simulations used default settings with an average density of $1040 \mathrm{~kg} / \mathrm{m}^{3}$, which is similar to that of polystyrene. The upper curves in the panels indicate the total concentration of microplastic, whereas the coloured areas indicate how plastic particles in singular form $\left(\mathrm{Pl}_{I}\right)$ and plastic in heteroaggregates $\left(\mathrm{SS}_{1-5} \mathrm{Pl}_{1-5}\right)$ with suspended solids of different size classes $\left(\mathrm{SS}_{1-5}\right)$, contribute to the total concentration. Homoaggregate $\left(\mathrm{Pl}_{2-5}\right)$ concentrations were negligible and therefore not visible in the figure. Heteroaggregate concentrations are plotted as sum for $\mathrm{SS}_{x} \mathrm{Pl}_{1-5}$, though are mainly composed of $\mathrm{SS}_{x} \mathrm{Pl}_{1}$.

plastic particles and suspended solids depend on the relative sizes of plastic particles and suspended solids due to altered orthokinetic aggregation and differential settling resulting in formation of heteroaggregates (Fig. A2.6E-F, Table A2.4D). Heteroaggregation rates depend on particle number concentrations, which are highest for the smallest size class (Eq. A2.4, Fig. A2.6B, Table A2.4C). Initially, heteroaggregate concentrations in the water phase increased for all particle sizes and subsequently decreased due to settling of the heteroaggregates, which occurred predominantly within $1 \mathrm{~km}$ from the input source for particles $\geq 5 \mu \mathrm{m}$, and at the sedimentation area at 14 $\mathrm{km}$ for particles $<5 \mu \mathrm{m}$ (Fig. 2.1, Fig. A2.1). This corresponds well with peak concentrations in the sediment, as mentioned above. Homoaggregation played a minor role compared to heteroaggregation due to lower collision frequencies (Fig. A2.7, Table A2.4B), ${ }^{71}$ causing that homoaggregate concentrations are not visible in Fig. 2.1.

Retention of microplastic with implications for transport to sea: Using the data from the default scenario presented in Figure 2.1, the retention of microplastic in the $40 \mathrm{~km}$ river stretch was calculated as the percentage of the upstream microplastic input concentration that remained within the sediment of the 40 $\mathrm{km}$ river stretch. Remarkably, the relationship of retention with microplastic particle size was not monotonous but showed two maxima, up to $60 \%$ for plastic particles $\leq 1 \mu \mathrm{m}$ and up to $100 \%$ for plastic particles $\geq 50 \mu \mathrm{m}$, with a clear minimum in between where retention was only $18 \%$ for particles of $\sim 4$ $\mu \mathrm{m}$ (Fig. 2.3A). This typical pattern in retention for particles with increasing diameter was caused by a trade-off between sedimentation of heteroaggregates (driven by the larger size and higher density of the suspended solids), and sedimentation of the plastic particles. For plastic particles between $100 \mathrm{~nm}$ and $2 \mu \mathrm{m}$, increasing the diameter resulted in a reduced sedimentation rate of the heteroaggregates and for bigger plastic particles a larger diameter resulted in an increased sedimentation rate of the heteroaggregates (Table A2.4B).

If we know the percentage of microplastic retained in the $40 \mathrm{~km}$ river stretch, we can calculate how many stretches i.e. what distance is needed to retain $99 \%$ 
of the microplastic in the sediment of a river (RD99). This assumes that on average, retention percentages will be roughly similar across river stretches of this length, an assumption the validity of which of course is highly dependent on river morphology. The rationale for extrapolating to such a percentage is that discharges further from sea than the RD99, can be assumed to mainly contribute to plastic pollution in freshwater, whereas discharges within the RD99 will contribute to plastic pollution in both the fresh and marine environment. The RD99 was calculated by linear interpolation where this distance lied within $40 \mathrm{~km}$ and by exponential extrapolation where this distance lied further away than $40 \mathrm{~km}$. Highly depending on particle size, the RD99 was calculated to be around $200 \mathrm{~km}$ for nanoplastic and reaches up to $>900 \mathrm{~km}$ for microplastic, whereas millimetre sized plastic retained within a few kilometres (Fig. 2.3B). These calculations thus imply that for river morphologies like that of the Dommel, the intermediate size class of microplastic is preferentially transported downstream.

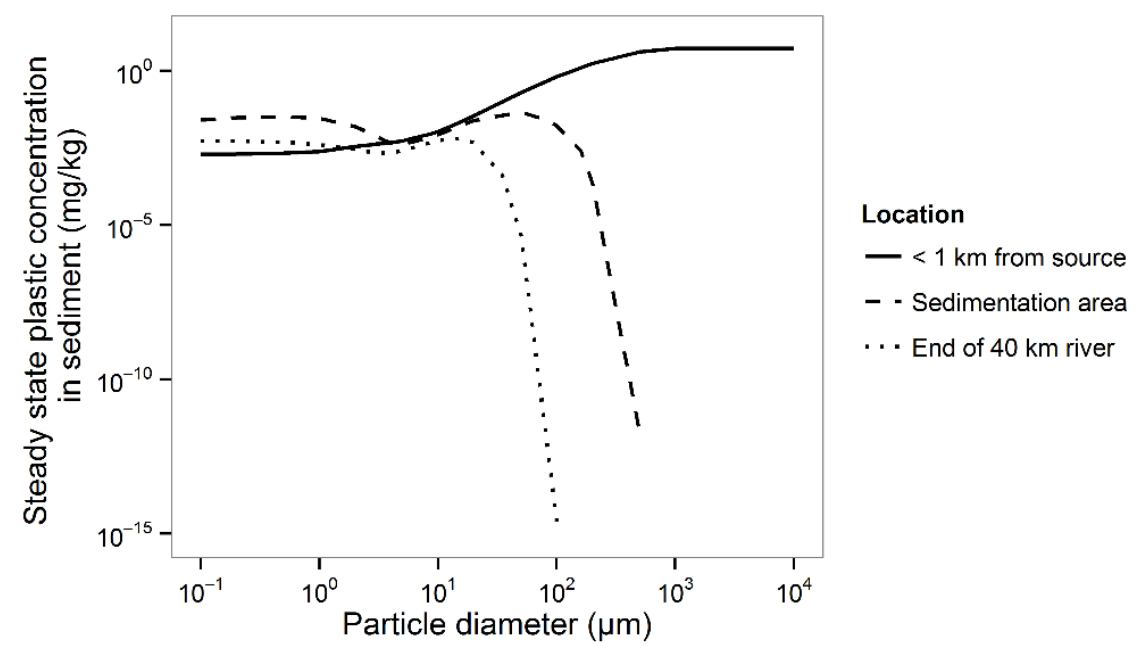

Figure 2.2. Steady-state plastic concentrations in sediment at a distance within $1 \mathrm{~km}$ from the input source, at the main sedimentation area $14.4 \mathrm{~km}$ downstream and at the end of the $40 \mathrm{~km}$ river stretch. Steady-state concentrations based on linear increase rates by aggregation and sedimentation, and loss due to burial (Table A2.3).

Effect of attachment efficiency, biofilm formation, polymer density and input concentration on retention: Attachment efficiency. We used a default attachment efficiency for heteroaggregation $\alpha_{\text {het }}$ of 0.01 , whereas we showed experimentally that the attachment efficiency is very similar within error 
limits, i.e. $0.004-0.2$ (Table A2.6). To be able to evaluate the relevance of such variations, we calculated all spatial profiles for a range of lower and higher attachment efficiencies (Fig. 2.3A). Increasing the attachment efficiency above 0.01 affected the overall retention by a factor $0.72-1.03$. Of this change in overall retention, a decrease by a factor $0.72-0.99$ is seen in the lower size range, whereas the retention is not affected by more than $1 \%$ for particles $\geq 4 \mu \mathrm{m}$ (factor $1-1.03$ ). Consequently, the range and variability observed between the default literature value of 0.01 and the experimentally determined average $\alpha_{\text {het }}$ values of about $0.04-0.1$ (range 0.004-0.2, Table A2.6) also had negligible impact on the observed profiles and retention, which shows the robustness of the modeling results and the adequacy of the default value. However, reducing the attachment efficiencies to 0 (no aggregation at all) or 0.001 (very low aggregation) resulted in a reduction of retention for small particles down till $0 \%$, whereas for the middle and bigger sized particles this increased retention by up to a factor 1.6 ( $4 \mu \mathrm{m}$ sized particles) (Fig. 2.3A). Given the literature values and experimentally determined values it is not likely that $\alpha_{\text {het }}$ was in fact smaller than 0.01 .

Biofilm formation. The previous simulations did not consider fouling or presence of biofilm, as explained above. Simulating the presence of a biofilm by changes in particle density and size did not change the overall qualitative trends and patterns in the behaviour of the particles, as can be seen by comparing Fig. 2.1 and Fig. A2.3. More quantitatively, this presence of biofilm on the plastic particles resulted in some size dependent changes in retention (Fig. 2.3). For particles $\geq 50 \mu \mathrm{m}$, which represent a major fraction of the microplastic particles in the environment, no effect of biofilm on retention was calculated. For particles $\leq 2 \mu \mathrm{m}$ retention decreased from around 60-50\% down to $50-40 \%$, which was caused by a faster formation of heteroaggregates through reduced settling of these heteroaggregates. In the middle part of Fig. 2.3 increases in retention from around $40 \%$ up to $70 \%$ were calculated, due to an increase in the settling of heteroaggregates (Fig. 2.3B, Fig. A2.6-7). When also accounting for the effect of biofilm on the attachment efficiency (i.e. an increase of $\alpha_{\text {het }}$ by a factor 2 to 3 ), the retention of particles $\leq 2 \mu \mathrm{m}$ is further reduced to about $50-30 \%$ (Fig. 2.3B).

Polymer density. For plastic particles $\geq 200 \mu \mathrm{m}$ retention in the $40 \mathrm{~km}$ river stretch was calculated to be high, approaching $100 \%$. For particles $100 \mathrm{~nm}-$ $1 \mu \mathrm{m}$ retention was low; about $50 \%$, which was nearly independent of polymer density. However, for the size classes in-between $(1-200 \mu \mathrm{m})$, retention is highly determined by the polymer type at hand, namely strongly increasing 


\section{Chapter 2}
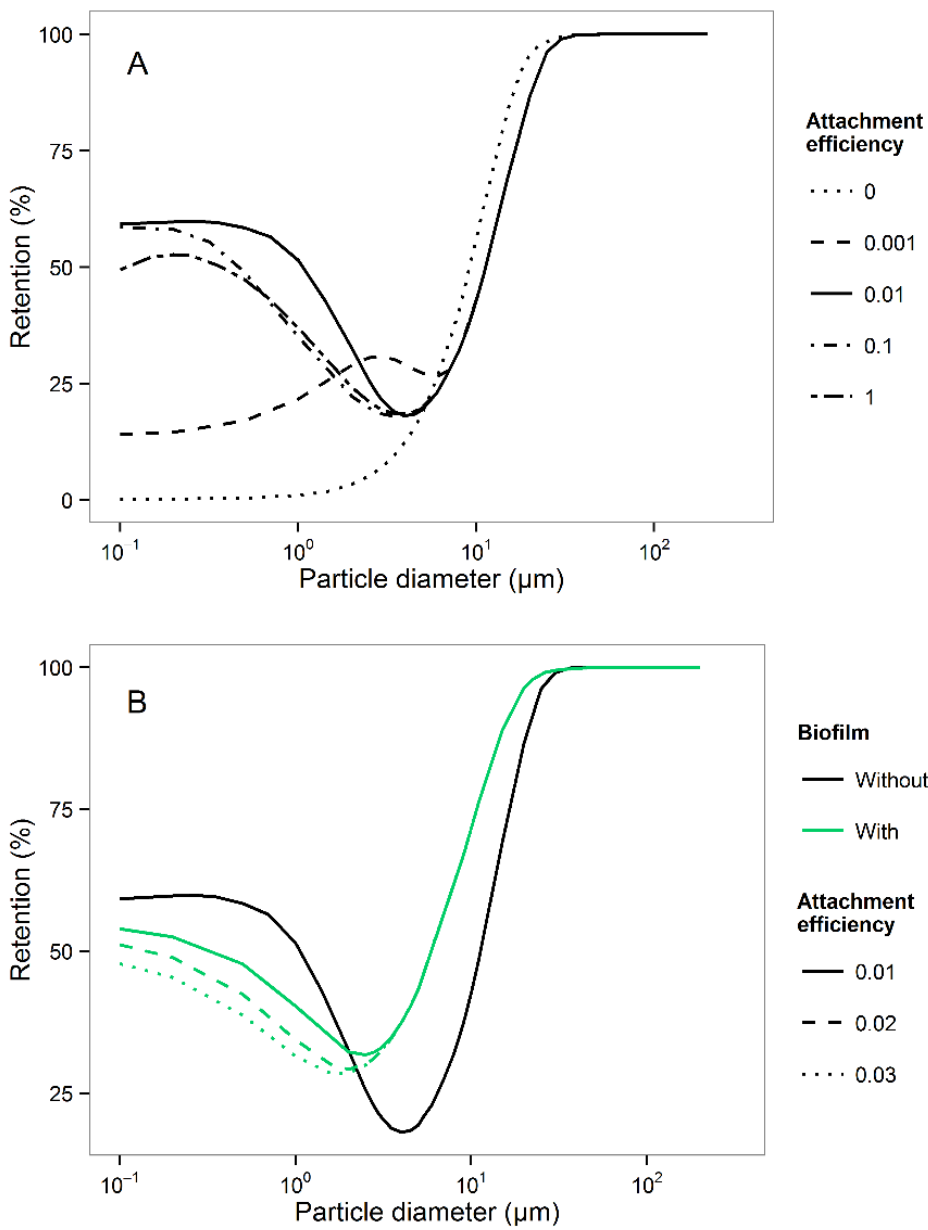

Biofilm

- Without

- With

Attachment

efficiency

$-0.01$

- 0.02

$\cdots 0.03$

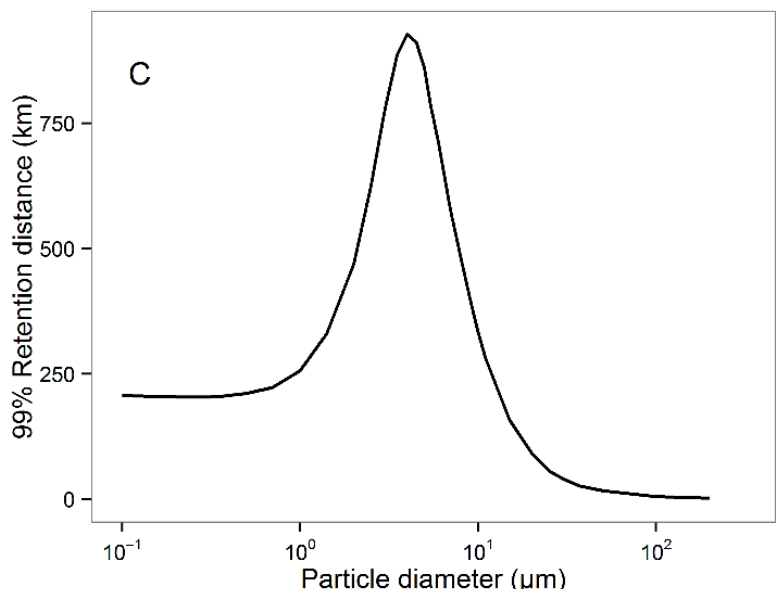

Attachment efficiency

$-0.01$ 
Figure 2.3. Retention and $99 \%$ retention distance of microplastic with a density of $1040 \mathrm{~kg} / \mathrm{m}^{3}$ as a function of particle size in a $40 \mathrm{~km}$ river stretch. Panel A: effects of attachment efficiency $\left(\alpha_{h e t}\right)$ on retention. Panel B: effects of biofilm and $\alpha_{\text {het }}$ on retention. Panel C: 99\% retention distance for the default value of ahet (0.01).

with increasing polymer density (Fig. 2.4A, Fig. A2.2). A special case exists for nano- and micrometre sized polymers that have a density equal or slightly lower than that of water, like for instance polyethylene and polypropylene. It is often assumed that such polymer particles will float and show low retention in water systems. However, for such plastic particles, dispersion into the water column will still occur due to common mixing and shear or due to wind induced mixing of surface water, ${ }^{106}$ after which they will rapidly heteroaggregate with suspended solids. Alternatively, the low density polymers $\left(<1000 \mathrm{~kg} / \mathrm{m}^{3}\right)$ are likely to aggregate with natural or anthropogenic non-polymer particles in the surface microlayer (SML), where particle concentrations are high. ${ }^{107}$ For low density microplastic $\leq 1 \mu \mathrm{m}$, the density and numbers of suspended solids overwhelm that of the microplastic, such that the settling of heteroaggregates virtually is determined by that of the suspended solids, leading to a retention of about 50\% (Fig. 2.4A). However, for low density microplastic particles larger than a few $\mu \mathrm{m}$, retention of heteroaggregates is largely dependent on polymer density, leading to zero retention (Fig. 2.4A). Consequently, these low density microplastic particles, those remaining in singular form or those newly settling on the water surface, are expected to be transported further downstream and have longer RD99.

Input concentration. In our default scenarios, the particle number concentration was highest for the smallest particles, as input concentration was kept constant for all particle sizes. Because in our environmentally realistic scenarios the particle number concentration of microplastic was relatively low compared to the suspended solids, homoaggregation played a negligible role compared to heteroaggregation. Although peak concentrations in water and sediment varied with input concentration, the overall retention $(\%)$ remained unaffected by input concentration at input concentrations $<1 \mathrm{ng} / \mathrm{L}$ (Fig. 2.4B). 
Chapter 2
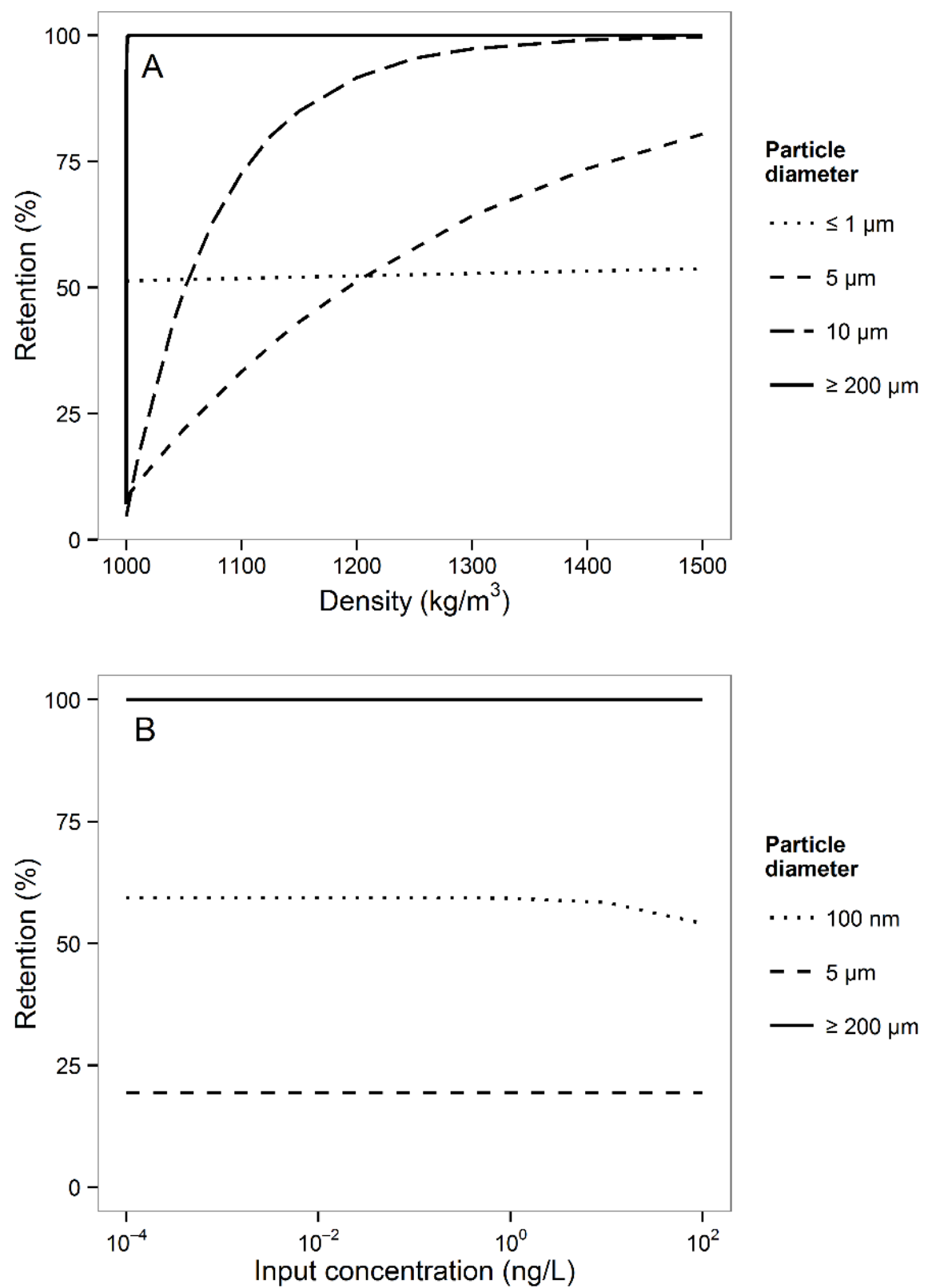

Figure 2.4. Panel A: Influence of polymer density on retention for different particle diameters with a default value of $\alpha_{\text {het }}(0.01)$ and input concentration $(1 \mathrm{ng} / \mathrm{L})$. Panel B: Influence of input concentration on retention for different particle diameters with a default value of $\alpha_{\text {het }}(0.01)$ and polymer density $\left(1040 \mathrm{~kg} / \mathrm{m}^{3}\right)$. 


\subsection{General discussion and Implications}

Rivers are often viewed simply as sources of plastic from terrestrial to marine ecosystems, but our modeling results indicate that part of the nano- and microplastic fraction can be efficiently retained in river systems. Therefore, emissions of microplastic to freshwater will not just result in 'down the drain' transport to coastal areas and the oceans. The strong dependence of retention on size in the $1-50 \mu \mathrm{m}$ range (Fig. 2.3A) can be evaluated with respect to know sizes of microplastic being emitted to freshwater. Tyre dust, abrasion and shedding from polymer-based textiles, ${ }^{108}$ or personal care products (PCPs) ${ }^{109}$ are often mentioned with respect to emissions to sea. The size of tyre dust is reported to range $60-80 \mu \mathrm{m}$, which may imply substantial retention in freshwater, based on the present simulations. Concentrations of black carbon in freshwater sediments indeed have been shown to be high, i.e. median black carbon contents as a fraction of total organic carbon are $9 \%$ for sediments ${ }^{110}$. Microplastic particles in personal care products are reported to range between $1-800 \mu \mathrm{m} .{ }^{108}$ Whereas the lower end of this range thus would be rather mobile in freshwater, particles at the higher end of this range would be retained efficiently in aquatic sediments, implying a potential risk to sediment dwelling organisms. We showed that especially nanoplastic and millimetre sized plastic are likely to be retained in rivers to a relatively high extent, which may define implications for species with specific feeding traits and sensitivities to these size fractions. The other side of the coin is that it suggests that especially micrometre sized particles are transported by rivers, resulting in exposure of marine and coastal areas to micrometre sized particles. Furthermore, it can be speculated that the preferential river retention of millimetre sized plastic particles may contribute to the lack of millimetre sized plastic as observed recently in the marine environment, ${ }^{69}$ compared to the expected particle size distribution.

Our model shows that based on initial waste sources and downstream distance, exposure concentrations can be predicted. Recently, the occurrence of microplastic in freshwater sediments has been experimentally confirmed. ${ }^{16,111}$ This calls for a thorough model validation and risk assessment for microplastic in freshwater systems. As mentioned, we did not aim to provide a fully validated model that can simulate all types of plastic particles with certainty. Still, the model is valid with respect to its conformance to known theory and parameter constraints, whereas its adequacy to simulate measured concentrations for other types of nanoparticles has been demonstrated recently. ${ }^{84}$ The present implementation was designed for (near-)spherical 
particles, and effects of particle shape need to be assessed before the model can be applied to more diverging types of plastic particles such as single fibres or thin sheets. For fibres, knotting may be more important than attachment, leading to effectively higher attachment efficiencies. Aggregates of knotted fibres would eventually approach sphericity implying they could be accommodated by the model once size and density would be known, or otherwise by including shape correction factors for sedimentation. Furthermore, uncertainty with respect to fouling, aggregation and sedimentation would affect absolute concentrations, but probably not the general trends with respect to location of hot spots. After all, model analysis showed that biofilm formation would not have large effects on particle fate. In that sense, the model provides a generalised tool that can be implemented for other catchments as well, and that can help in the design of optimum sampling grids and frequencies, based on a priori prospective simulations.

\section{Acknowledgements}

We thank Edwin Foekema for his valuable comments on earlier versions of the manuscript and Sil Nieuwhof for his great support in the use of R. J.Q. and A.A.K. acknowledge financial support from NanoNextNL, a micro and nanotechnology consortium of the Government of the Netherlands and 130 partners. 
Freshwater fate modeling 


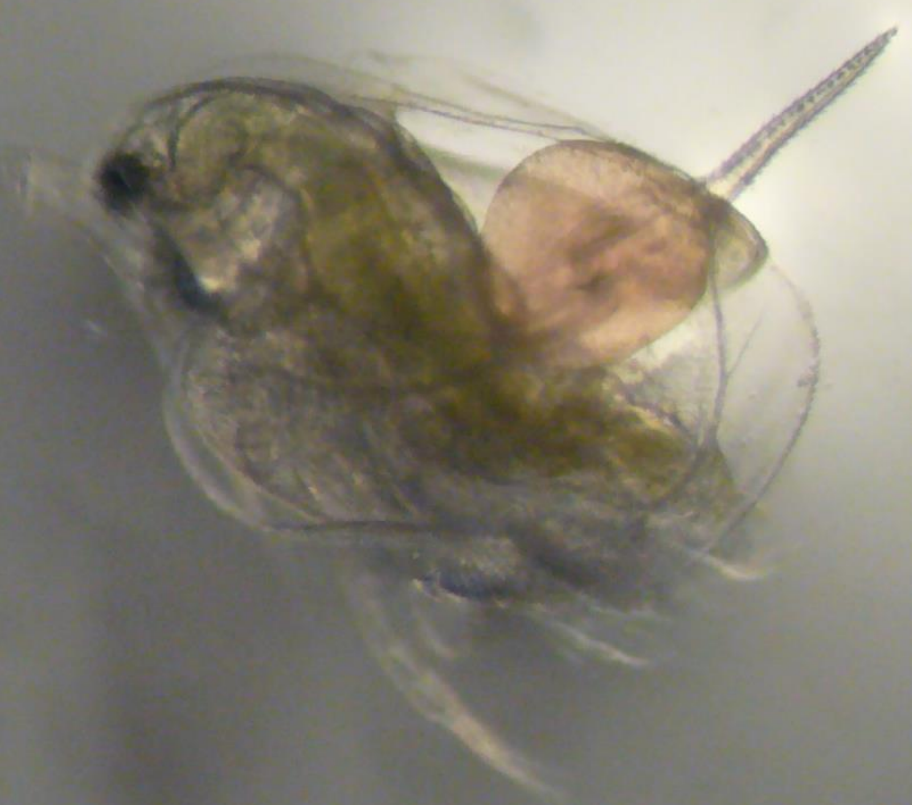




\title{
Nanoplastic affects growth of $S$. obliquus and reproduction of $D$. magna
}

Based on: Besseling, E., Wang, B., Lürling, M., Koelmans, A.A., 2014. Nanoplastic affects growth of $S$. obliquus and reproduction of D. magna. Environmental Science \& Technology 48, 12336-12343

\begin{abstract}
The amount of nano- and microplastic in the aquatic environment rises due to the industrial production of plastic and the degradation of plastic into smaller particles. Concerns have been raised about their incorporation into food webs. Little is known about the fate and effects of nanoplastic, especially for the freshwater environment. In this study, effects of nano polystyrene (Nano-PS) on the growth and photosynthesis of the green alga Scenedesmus obliquus and the growth, mortality, neonate production and malformations of the zooplankter Daphnia magna were assessed. Nano-PS reduced population growth and reduced chlorophyll concentrations in the algae. Exposed Daphnia showed a reduced body size and severe alterations in reproduction. Numbers and body size of neonates were lower, while the number of neonate malformations among neonates rose to $68 \%$ of the individuals. These effects of Nano-PS were observed between 0.22 and $103 \mathrm{mg}$ Nano-PS/L. Malformations occurred from $30 \mathrm{mg}$ Nano-PS/L onwards. Such plastic concentrations are much higher than presently reported for marine as well as freshwater, but may eventually occur in sediment pore waters. As far as we know, these results are the first to show that direct life history shifts in algae and Daphnia populations may occur as a result of exposure to nanoplastic.
\end{abstract}




\subsection{Introduction}

Pollution with plastic is a growing concern in the marine environment. ${ }^{8}$ However, emissions from land-based sources reach rivers first and freshwaters provide an important source of marine plastic pollution through riverine transport. ${ }^{12}$ Therefore, the occurrence of plastic in the freshwater environment receives increasing attention. ${ }^{16,113-115}$ Special concerns exist with respect to nanoplastic particles because of their large surface area and hypothesized ability to penetrate cells. ${ }^{116-120}$ Both primary particles from personal care and cosmetic products and secondary particles from degradation of larger plastic items are expected to contribute to pollution of the environment with nanoplastic. ${ }^{121}$ Recent reports showed the importance of physical abrasion as a source of secondary micro- and nanoplastic. ${ }^{122,123}$ Yet there are hardly any proven life history effects of micro- and nanoplastic on marine organisms and effect data for freshwater organisms are lacking. For microplastic, the first reported data on effects on invertebrates relate to survival, feeding, oxidative status and PCB uptake in lugworms (Arenicola marina) (Chapter 4). ${ }^{51,53,124}$ In marine zooplankton, decreased feeding ${ }^{125}$ as well as reduced survival and fecundity have been observed. ${ }^{50}$ Even less is known about the effects of nanoplastic. For mussels (Mytilus edulis), an increased pseudofeces production and reduced filtering activity have been reported. ${ }^{52}$ For algae, nanoplastic has been shown to reduce $\mathrm{CO}_{2}$ uptake and increase the production of reactive oxygen species (ROS). ${ }^{28}$

As the interaction of organisms with pollutants in particulate form is completely different from that with conventional dissolved chemicals, there is a potential high risk associated with particles. ${ }^{16,126}$ Given the limited data, there is an urgent need to quantify the effects of nanoplastic on freshwater organisms. Effects of nanoplastic may be related to particle toxicity, toxicity of plastic-associated chemicals or both and will depend on the characteristics of the nanoplastic, like particle size, polymer type and age. However, previous research on nanoparticle behaviour and effects was often conducted using pristine particles, ${ }^{127}$ whereas aged and naturally altered particles are of higher importance considering environmental relevance, which will therefore be addressed in the present study.

Plastic interacts with man-made organic compounds (Chapter 5), ${ }^{128}$ as studied for several kinds of pollutants as well as additives (Chapter 5). ${ }^{19,124,128,129}$ Recently, an exceptionally strong sorption of PCBs to nanoplastic was observed, which might imply a strong transport capacity including increased exposure upon penetration of cells or tissues. ${ }^{75}$ Effects of 
nanoplastic might also be caused by direct particle toxicity, attachment to algae, reduction of light penetration, reduced food quality, release of additives, or interference with chemical communication. Here we hypothesize that nanoplastic might also interact with natural organic molecules like kairomones, which may yield unforeseen effects on the interactions among species. Daphnia are known to express life history traits such as altered adult and/or neonate body size and altered neonate quantity in response to the presence of predator kairomones. ${ }^{130,131}$ Sorption of kairomones to nanoplastic might disturb these life history traits. ${ }^{132}$

The aim of the present study was to investigate effects of nanoplastic at the first two trophic levels of the freshwater aquatic food chain; algae, represented by Scenedesmus obliquus, and zooplankton, represented by Daphnia magna. Both species are widely used for ecotoxicity tests. Nano-sized polystyrene (Nano-PS) spheres were used as the test substance, as polystyrene is one of the most widely used commercial polymer types in the world and was used in earlier toxicity tests (Chapter 4). ${ }^{28,51,52} \mathrm{We}$ investigated direct and indirect effects of a broad range of expected environmentally relevant as well as elevated concentrations of Nano-PS in freshwater bioassays. The bioassay we present here is the first interaction bioassay of Nano-PS combined with an interspecific organic molecule: fish kairomone. We took the interaction time between plastic particles and algae into account by using both pristine and aged dispersions of Nano-PS, thereby providing novel information about the potential role of particle aging.

\subsection{Experimental procedures}

Bioassays were performed with algae (S. obliquus), as well as with water flea (D. magna) fed with these algae.

\subsubsection{Organisms}

Scenedesmus obliquus SAG 276/3A was obtained from the University of Göttingen, Germany and was maintained in modified algal growth medium (WC-medium). ${ }^{133}$ Stock cultures and the Scenedesmus bioassay were maintained similar to previous procedures at $20{ }^{\circ} \mathrm{C}$ in a climate chamber with 24 hours continuous light $\left(\sim 100 \mu \mathrm{mol}\right.$ quanta $\left.\mathrm{m}^{-2} \mathrm{~s}^{-1}\right)$ and $100 \mathrm{rpm}$ rotational shaking. ${ }^{134}$ Algae inoculum was prepared three days ahead of the Scenedesmus bioassay, to obtain exponential growth at the start of the test. Daphnia magna originated from lake Zwemlust, Nieuwesluis, ${ }^{135}$ the Netherlands and were 
cultured in artificial growth medium (RT medium ${ }^{135}$ ) with a $\mathrm{pH}$ of 7.7-8.1. The Daphnia cultures and bioassay were kept at a temperature of $21 \pm 1{ }^{\circ} \mathrm{C}$ with the natural spring daylight regime (low beam day conditions $<20 \mu \mathrm{mol}$ quanta $\left.\mathrm{m}^{-2} \mathrm{~s}^{-1}\right)$. In the Daphnia bioassay two generations were used: (1) Daphnia of age $<24$ hours at the start, maturing during the bioassay and (2) their offspring, i.e. neonates hatched while being in the bioassay. In all dispersions used in the Daphnia bioassay, S. obliquus served as food at approximately $0.36 \mathrm{mg}$ carbon/Daphnia.

\subsubsection{Nano-PS beads}

Polystyrene nanoparticle stocks were supplied as $20 \%$ Nano-PS dispersion by AVT-PCC, Wageningen UR. The particles were synthesized from styrene monomers with sodium dodecyl sulphate (SDS) as surfactant and potassium persulphate as initiator. ${ }^{136}$ SDS concentrations were kept far below toxicity thresholds of Daphnia ${ }^{137,138}$ and Scenedesmus. Absence of toxicity to Scenedesmus was confirmed in separate pilot tests with SDS, which are provided in the Appendix. Similarly, because of its hydrophobicity and high volatility with reported half-lives of 1-3 hours in lake water, ${ }^{139}$ presence of styrene monomers in the aqueous phase can be assumed negligible. The polystyrene beads had a primary nominal size of $\sim 70 \mathrm{~nm}$ (confirmed by Transmission Electron Microscopy) and contained $0.01 \%$ on mass basis of the hydrophobic fluorescent dye (Nile Red), which was immobilised by the polymer matrix. Consequently, presence of Nile Red in the aqueous phase can also be assumed negligible, which is consistent with the use of Nile Red as a tracer in numerous studies of biological systems. ${ }^{11,140,141}$ Furthermore, even if all Nile Red in the polystyrene would have been bioavailable, the concentration would still have been a factor $1.5 \times 10^{4}$ below the effect concentration reported by Wu et al. ${ }^{142}$ (Calculation provided in the Appendix). To better represent Nano-PS occurring in products and in the environment, ${ }^{143}$ the spheres were functionalised with carboxylic acid groups. As the glassliquid transition temperature of polystyrene ${ }^{144}$ is much higher than the maximum temperature in our bioassay $\left(21^{\circ} \mathrm{C}\right)$, leaching of chemicals from the polymer matrix and therewith their occurrence in the exposure dispersions is negligible. The form of Nano-PS in aqueous suspension was extensively characterised before (See Fig. A3.1) ${ }^{75}$

\subsubsection{Scenedesmus bioassay}

Scenedesmus obliquus were exposed to $44-1100 \mathrm{mg}$ Nano-PS/L in $80 \mathrm{~mL}$ WC medium in a $72 \mathrm{~h}$ bioassay. Details about the used concentration range are provided in the Appendix. Algae populations with an initial density of 
approximately $3 \times 10^{6}$ cell/mL were used. A growth inhibition test was performed three times with controls in 6-fold and Nano-PS treatments in triplicate. ${ }^{137,145}$ Cell densities were determined using a CASY counter (CASY® Model TT, INNOVATIS) at the start and after every $24 \pm 1$ hour. At the end of two of the bioassays, Chlorophyll- $a$ (Chl- $a$ ) was extracted and determined by spectrophotometry (Beckman Coulter, DU® 730 Life Science UV/Vis) to assess photosynthetic capacity and biomass following a hot ethanol extraction method with phaeopigment correction. ${ }^{146}$

\subsubsection{Daphnia bioassay}

Daphnia magna were exposed individually to $80 \mathrm{~mL}$ Nano-PS test dispersion in a 21 day bioassay, according to OECD guidelines. ${ }^{147,148}$ Four types of NanoPS test dispersions were tested, that are referred to as (1) 'Pristine', (2) 'Pristine-kairomone', (3) ‘Aged' and (4) 'Aged-filtered' (Fig. 3.1). (1) Pristine refers to the treatment where the exposure of the Daphnia started immediately after mixing algae and Nano-PS. Nano-PS dispersions were dilutions of NanoPS stock in RT medium to which algae were added, just before use in the bioassay. Pristine exposures were applied at ten nanoplastic concentrations in the range of $0.22-150 \mathrm{mg}$ Nano-PS/L. Details about the used concentration ranges are provided in the Appendix. (2) For the Pristine-kairomone dispersions, the only difference with the Pristine dispersions was the presence of fish kairomones in the initial RT medium. Fish kairomones were kindly obtained from a parallel study at our university, where three individuals of Perca fluviatilis (total overall length $\pm 12 \mathrm{~cm}$ ) were inhabited in $20 \mathrm{~L}$ aerated RT medium for a week. Perca fluviatilis is a predator known to induce life history responses in Daphnia. ${ }^{149,150}$ Three times a week, the fish were fed with Daphnia. Before use in the Daphnia bioassay, the RT medium with fish kairomones was filtered over a $0.45 \mu \mathrm{m}$ membrane filter (Whatman ${ }^{\mathrm{TM}}$ Cellulose Nitrate Membrane - Grade NC45). The Pristine-kairomone dispersions were applied at concentrations of 0.88 and $1.8 \mathrm{mg}$ Nano-PS/L. (3) The Aged dispersion was prepared in the same way as the Pristine dispersions, the only difference was that the Aged dispersions were not used immediately after addition of the algae, but instead aged at the conditions used for stock cultures (see paragraph Organisms) for 5 days. The Aged treatment was applied at one concentration; $32 \mathrm{mg}$ Nano-PS/L. (4) The Aged-filtered dispersion was made in the same way as the Aged dispersion at the same nanoplastic concentration. Thereafter, it was further processed as follows: the algae were separated from the water phase by filtering over a $1.2 \mu \mathrm{m}$ glass fibre filter (Whatman GF/C). The residue was rinsed from the filter with new 
RT medium and the new RT medium applied in the bioassay. As controls original RT medium and RT medium with fish kairomones were included, both with algae, but without Nano-PS. The control treatment with original RT medium without Nano-PS was replicated 16-fold, all other treatments were replicated 12-fold. The four dispersion types enabled us to make various mechanistic comparisons. For instance, comparison of Pristine with Pristinekairomone enabled to reveal interaction between plastic and kairomones. Comparison of Pristine with Aged allowed to show the consequences of aging of the Nano-PS dispersions on the Daphnia. The difference in preparation between Aged and Aged-filtered reduced the relative importance of aquatic exposure to plastic of the Daphnia. Although it can be assumed that the functionalised Nano-PS stays dispersed, ${ }^{75}$ the replacement of the aged aquatic phase by fresh RT medium in the Aged-filtered dispersion allows a check on the relative importance of the (Nano-PS absorbed to) aged algae being an exposure route. Daphnia were transferred to glass tubes with $80 \mathrm{ml}$ new medium three times a week. S. obliquus is known as good food source for Daphnia. ${ }^{151}$ Survival of Daphnia was checked and reproduction was counted on a daily basis. Body size ${ }^{152}$ of both adult and neonate Daphnia were measured and number of malformed neonates were counted using a stereobinocular (Nikon SMZ-10, magnification 0-40). During the bioassay, three times a week, water quality was measured in a randomly chosen replicate of each treatment. On average the $\mathrm{pH}$ was $7.80 \pm \mathrm{SE} 0.015$, oxygen concentration was $8.80 \pm \mathrm{SE} 0.012 \mathrm{mg} / \mathrm{L}$ and conductivity was $296.92 \pm \mathrm{SE} 0.71 \mu \mathrm{S} / \mathrm{cm}$, thereby being within the range of the guideline.

\subsubsection{Data analysis}

Algae growth inhibition rates were derived from cell density over time according to ISO guidelines, ${ }^{153}$ by using nominal initial cell densities. Daphnia population growth rates $(r)$ were estimated from Euler-Lotka's equation. ${ }^{152}$ Statistical analyses were performed with ' $\mathrm{R}$ ' statistical software (R Development Core Team) by 2-way ANOVA, (Multiple) Linear regression, Kruskal-Wallis and Nemenyi-Damico-Wolfe-Dunn (NDWD) tests with $\alpha=0.05$. 


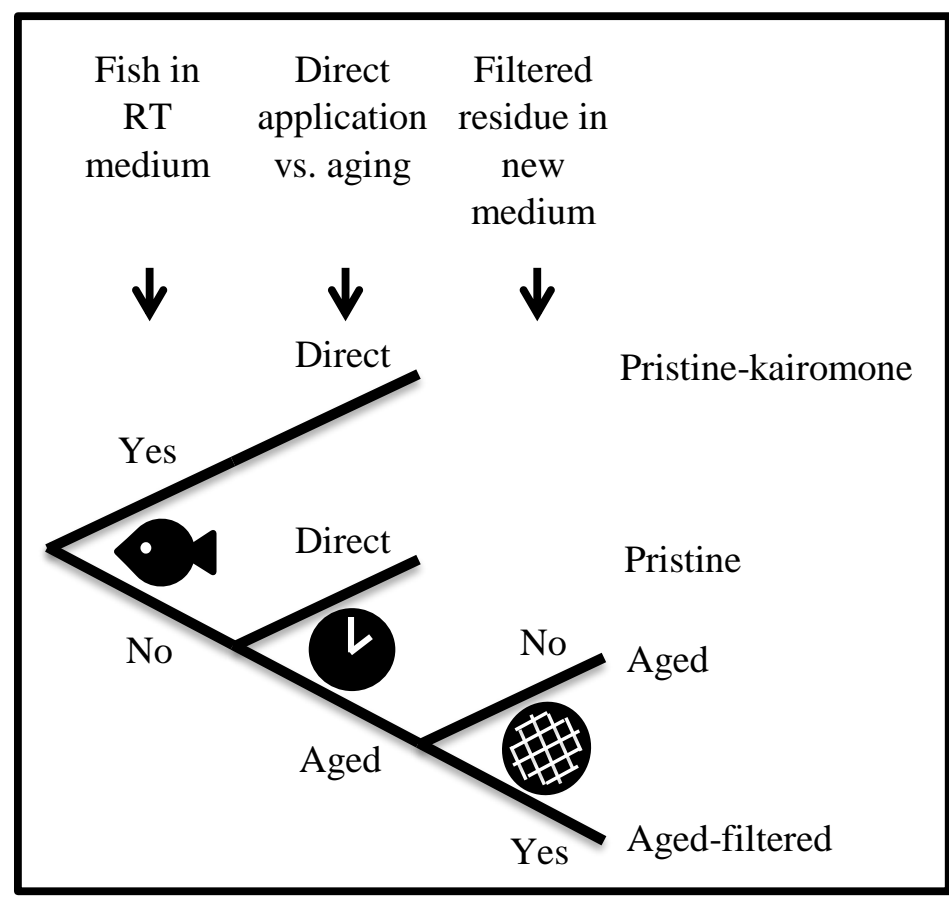

Figure 3.1. Visualization of the four different types of test dispersions. All dispersions contain RT medium with Nano-PS and algae.

\subsection{Results and discussion}

\subsubsection{Effect of Nano-PS on growth and Chlorophyll-a of Scenedesmus}

We performed three bioassays with the green alga Scenedesmus obliquus and show that exposure to Nano-PS leads to inhibition of growth (Fig. 3.2A) and to reduced Chl- $a$ levels in the cells (Fig. 3.2B). As far as we know, these are the first direct negative effects of nanoplastic on algae populations established. The growth inhibition had limited magnitude, yet the increased growth inhibition with increasing Nano-PS concentration was statistically significant and did not differ among the three tests (2-way ANOVA, plastic treatment significant, $p$-value $=0.013$ ). At a high Nano-PS concentration of $1 \mathrm{~g} / \mathrm{L}$ there was approximately $2.5 \%$ growth inhibition of $S$. obliquus. The negative relationship between Nano-PS concentration and Chl- $a$ concentration is similar for both tests and statistically significant, although the variability within controls and Nano-PS treatments is high and below $100 \mathrm{mg}$ Nano-PS/L no reduced Chl- $a$ concentration is expected to occur. The negative 
relationships of growth and Chl- $a$ with Nano-PS concentration are independent, as after correction for cell density, Chl- $a$ concentration remains significantly negatively related to Nano-PS concentration (2-way ANOVA, plastic treatment significant, $p$-value $=5.1 \times 10^{-5}$ ). Previous research showed absorption of nanoplastic by algae and indications of reduced algal health i.e. reduced $\mathrm{CO}_{2}$ uptake observed at concentrations higher than $1.8 \mathrm{mg} / \mathrm{L}$ and promoted production of ROS. ${ }^{28}$ Our present results add that also direct effects of nanoplastic on algae growth and Chl- $a$ levels may occur. Before, it was suggested that shading by plastic might cause the observed effects on $\mathrm{CO}_{2}$ uptake and production of oxygen species. ${ }^{28}$ However, as shading is known to cause an upward correction of the Chl- $a$ level in cells, ${ }^{154}$ our findings of a Chl$a$ reduction with increasing Nano-PS concentration contradict this suggestion. Thereby, although at relatively high concentrations, our novel observed reduction in Chl- $a$ implies that another mechanism is at work, which may help to direct further mechanistic effect research. Note that we do not fully distinguish here between the possible mechanisms explaining the toxicity of Nano-PS, to which direct nanoparticle toxicity as well as effects of Nano-PS associated-chemicals like styrene, may contribute.
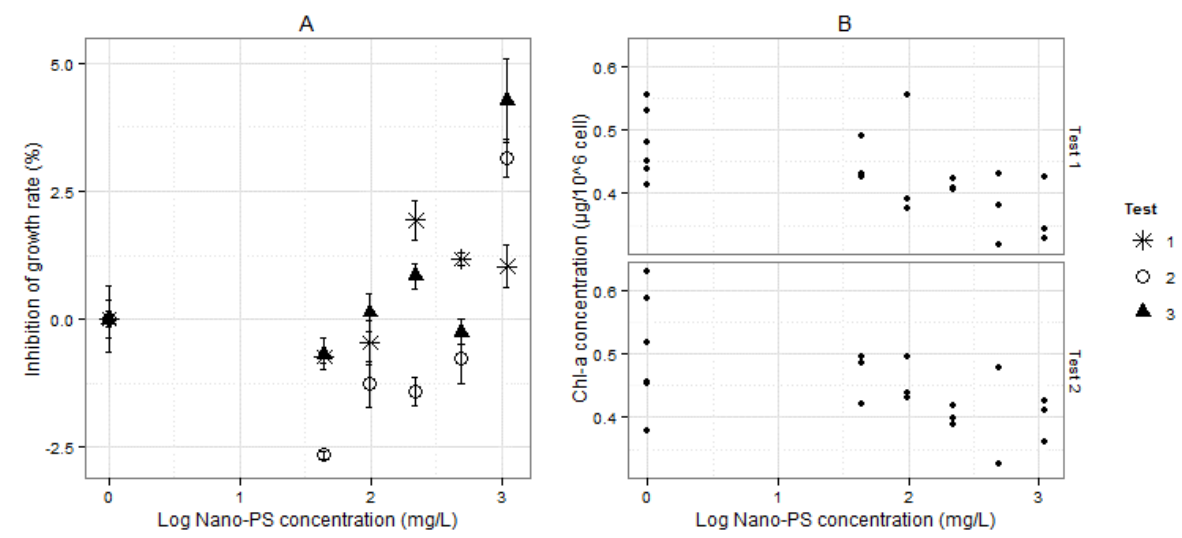

Figure 3.2. Nano-PS effects on Scenedesmus obliquus. Panel A: Inhibition of the growth rate (\%) as a function of Nano-PS concentration after 72 hours of exposure. Panel B: Upper part: test 1, lower part: test 2. Chl-a concentration $/ 10^{6}$ cell as a function of the 72 hours Nano-PS exposure. 


\subsubsection{Effects of Nano-PS on survival and body size of Daphnia} Effects of pristine and aged Nano-PS dispersions. Across the treatments, the Daphnia mortality ranged from $0-100 \%$, with an average of $27 \%$. The mortality of $18.8 \%$ in the control groups was within the limit set by the OECD guidelines 2008. ${ }^{148}$ Pristine suspensions of Nano-PS were not lethal to $D$. magna, but the aged dispersions were (Kruskal-Wallis; NDWD test: aging sign. $p$-values $\leq 0.014$ ). Aging of algae with plastic caused a $4.4-6$ times higher mortality in Daphnia as compared to a diet without pre-exposure of the algae. Several explanations for this difference in mortality can be considered. First, in the Aged-filtered treatment, after the exposure of the algae the plastic was removed from the water phase using a glass fibre filter. Some release of glass fibres into the Aged-filtered treatment was observed and it may be speculated that this contributed to the mortality in this treatment. However, in the Aged treatment no glass fibre filter was used and a similar mortality was observed, which renders the speculation less likely. Second, the higher mortality could relate to a plastic treatment effect implying that the preexposed algae adsorbed Nano-PS, thereby being the route for exposure of the Daphnia resulting in an elevated mortality. An increase of the uptake via food might be the explanation for the six times higher mortality compared to pristine exposure when the Aged dispersion was used. While in pristine dispersions Nano-PS mainly resided in the water medium, Nano-PS might be absorbed to the $S$. obliquus in aged dispersions, thereby changing the main uptake route or degree of exposure. A third explanation could be that although the presence of aqueous phase styrene is unlikely, aging may increase the transfer of styrene monomers from the Nano-PS ${ }^{155}$ into algae, thus increasing the bioavailability of styrene. It is very important to take the effect of aging and plastic associated chemicals into account in the risk identification of nanoplastic, as this affects the outcomes of bioassays as well as the comparability with environmental conditions.

\subsection{Interacting effects of kairomones and Nano-PS on Daphnia}

A $10.7 \%$ reduction in Daphnia body size due to kairomones was observed in our bioassay (Fig. 3.3A). The reduction in Daphnia body size due to kairomones only, was also observed by Hanazato and Dodson (1995) and Riessen (1999) and was explained by differences in survival strategy with/without predator presence. From the Daphnia that were treated with aged dispersions, not enough individuals survived to consider body size as a representative endpoint. The presence of plastic also had a negative effect on 
Chapter 3
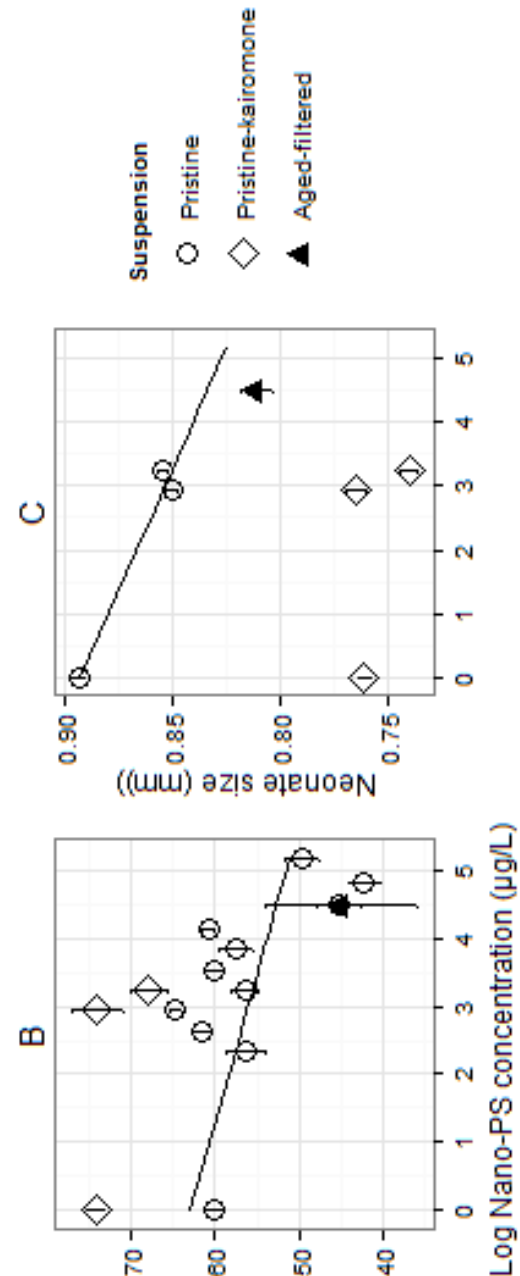

((u) Jəqunu әџеuоәN

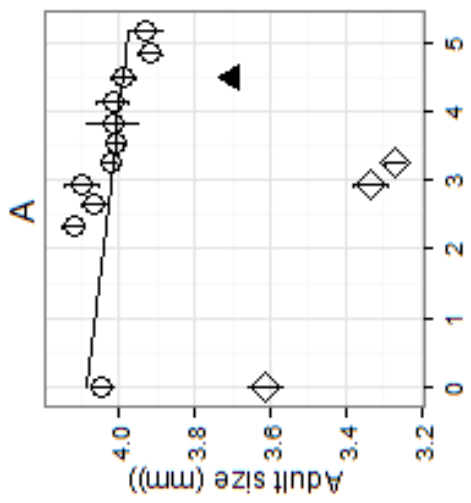

है

s.

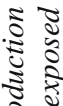

ลั

$\ddot{0}$

रे

咅 $\frac{1}{5}$

23

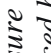

0

3.

政。

8 ะ

స

ذิ ะี

s.

辛

दे दे

0

记

棺

का

出告告

고워

ก ร

这客

ฐ)

$5 \div 2$

ริ)

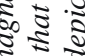

3
3.3
2

इ है है

201

ㅇำ

ปั

S.

a

ว.

$z$ \&

m:

ปे

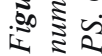


body size with up to $3.1 \%$ reduction in length. The significance of the term accounting for interaction between Nano-PS and kairomones implies that with kairomones present, the body size reduction with Nano-PS concentration is stronger than without kairomones (Fig. 3.3A) (Multiple linear regression: $\log$ (Nano-PS), kairomones as well as the interaction between them were significant, $R_{\text {adj }}^{2}=0.80, p$-values $\left.<1.7 \times 10^{-3}\right)$. At a concentration of $1.8 \mathrm{mg}$ Nano-PS/L, interaction with kairomones reduced the body size by up to $18.9 \%$. This might constitute an additive negative effect of both kairomones and Nano-PS or an interaction between Nano-PS and kairomones. For example, the presence of Nano-PS could change the exposure concentration of kairomones in water, the uptake route for kairomones or the susceptibility of Daphnia, resulting in an altered growth reduction. This possible Nano-PS interference with kairomones is the first report of an effect of plastic on chemical communication among organisms. This hypothesis of an increased kairomone effect might be less relevant for other, for instance more hydrophobic kairomones, which implies that more studies on these interactions are recommended.

\subsubsection{Effects on reproduction and neonate malformations of Daphnia}

Effects on life history traits of aquatic organisms often provide sensitive metrics for ecological stress or chemical toxicity. To determine the effect of Nano-PS on reproduction, we investigated the neonates produced by the exposed adult Daphnia. Only those replicates where the adult Daphnia survived the bioassay were included in the analyses of neonate number and size. The total number of neonates produced in the first three broods was in the control without kairomones $53.4 \pm$ SE 18.9 and in the control with kairomones 55.7 \pm SE 33.7. Exposure to Nano-PS in the Pristine treatments reduced the cumulative number of neonates in the first three broods (Figure 3.3B). A slightly lower decrease in Daphnia neonate quantity was observed in the presence of kairomones (Multiple linear regression, Nano-PS, kairomones and interaction significant, $R^{2}$ adj $=0.52, p$-value $<10^{-16}$ ). The overall neonate number per surviving adult was $19.4 \%$ higher with kairomones present, which is consistent with previous findings. ${ }^{131,149}$ Neonate number was also significantly related to adult body size, although it had a lower significance than Nano-PS and kairomones. Multiple linear regression performed with the explanatory variables Nano-PS, kairomone (interaction) and adult body size had an adjusted $R^{2}$ of 0.53 ( $p$-value $<10^{-16}$ ). Population growth rates $(r)$ were in the range of $0.23-0.55$ day $^{-1}$, with $r=0.23-0.42$ day $^{-1}$ for aged dispersions, 
0.44-0.45 day ${ }^{-1}$ for dispersions with kairomones and 0.46-0.55 day ${ }^{-1}$ for pristine dispersions. Replication of the bioassay would allow calculations of significant differences in population growth rates between treatments.

From the first three broods, a random selection of 16 neonates per treatment was subjected to body size measurements. The body size of the neonates was negatively affected by the Nano-PS concentration, and aging of dispersions increased this effect. Neonates of Daphnia exposed to kairomones were much smaller, but further reduction in body size caused by Nano-PS was smaller than in the pristine treatment (Fig. 3.3C) (Multiple linear regression, $\log$ (plastic), kairomones and interaction significant $\left(R_{\text {adj }}^{2}=0.93, p\right.$-value $=$ 0.035). Former research on Daphnia showed that a trade-off between clutch size and neonate body size exists. Low food availability results in fewer but larger neonates, whereas small mature Daphnia or the presence of fish kairomone causes a greater number of smaller neonates, ${ }^{149}$ the latter mechanism being confirmed in our bioassay. It has been demonstrated that the overall maternal investment often decreases by exposure to pollutants. ${ }^{156}$ Here we show that Nano-PS reduces both clutch and neonate body size, thereby acting as a stressor similar to other contaminants.

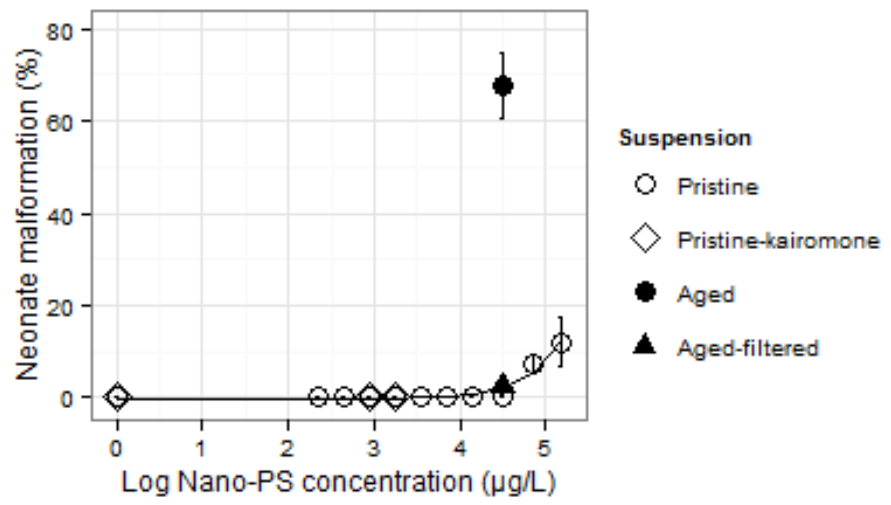

Figure 3.4. Percentage occurrence of malformations in neonates, that were produced by Daphnia during 21 days exposure to Nano-PS. 
While low numbers $(<2.5 \%)$ of malformations were observed in the control treatment and the lower range of Pristine treatments, from $32 \mathrm{mg}$ Nano-PS/L onwards elevated numbers of malformed neonates were observed (Fig. 3.4) and a factor 2.2-4.9 increase in plastic caused a 7-12\% increase in the occurrence of malformations. When exposed to Aged dispersions the occurrence of malformations increased radically, by $67 \%$ compared to the Pristine treatment at $32 \mathrm{mg}$ Nano-PS/L (Multiple linear regression plastic, aging and interaction significant $\left(R^{2}\right.$ adj $=0.81, p$-value $\left.<10^{-16}\right)$. This increase also implies once more that the malformations are not due to any initially present co-contaminant (e.g. styrene, SDS or Nile Red) because these were used in the Pristine treatment too. For the analyses of malformation occurrence all neonates were taken along, including those of adult Daphnia that did not survive the exposure. Nano-PS affected several developmental stages of Daphnia neonates, as different malformation types were observed (Fig. 3.5). In order of decreasing occurrence: internal vacuoles, shortened antenna and lump in carapace, altered tail spine. The normal embryonic development of Daphnia consists of six stages, i.e. 'Cleavage', 'Gastrulation', 'Early embryonic maturation', 'Mid-embryonic maturation', 'Late embryonic maturation' and 'Fully developed neonate'. ${ }^{157}$ In our study we observed a considerable number of neonates with incomplete developed second antennae setae and curved tail spines (Fig. 3.5). These malformed neonates were mainly observed in the high Nano-PS exposures, especially in the Aged treatment. This indicates disruption of one or more embryonic development stages from the stage of mid-embryonic maturation onwards, as the second antenna (including setae) and tail spine are developed and extended in these stages. ${ }^{157}$ Malformed tail spines and incomplete developed antennae setae have been reported to occur in D. magna exposed to cyanobacterial toxins, mercury and a mixture of Clofibric acid and fluoxetine. ${ }^{158-160} \mathrm{We}$ did not find any report of styrene or related compounds causing such malformations. Also, to exceed the $1.9 \mathrm{mg} / \mathrm{L}$ NOEC of styrene for Daphnia, ${ }^{139}$ in the treatment with highest malformation occurrence, $6 \%$ of the Nano-PS in that Aged treatment would need to be monomer styrene in suspension. That is an unlikely high percentage. As mentioned before, given the rapid volatilization of styrene, ${ }^{139}$ it is even less likely that such concentrations occurred during our bioassay. In contrast to the Aged treatment, effects of the Aged-filtered suspension did not significantly differ from the Pristine and Pristine-kairomone treatments. Thereby, it validates our assumption that the Nano-PS did not aggregate with the algae to such an extent that only exposure via algae (Aged-filtered) would cause effects equal to combined aquatic and algal exposure (Aged). Although 
not irreversibly bound to algae, a change in allocation of the Nano-PS in the dispersion with algae might however have changed due to aging and thereby influenced the extent of exposure. The occurrence of a lump in neonates' carapaces (Fig. 3.5, top-middle), has not been observed in our laboratory before and we are not aware of any other publications on this type of malformation. The colour of the observed lumps was similar to the Nano-PS colour and might indicate accumulation of Nano-PS in neonates. Solubilization of carbon nanotubes with polymers as well as by fouling is thought to increase the uptake in biological systems, ${ }^{161,162}$ implying that polymer nanoparticles are likely to reach these systems too. The observed malformations might relate to the alteration of membrane properties found by Rossi et al. ${ }^{163}$ We recommend further, detailed histological study for direct evidence of uptake and transfer of nanoplastic from Daphnia adults to offspring.

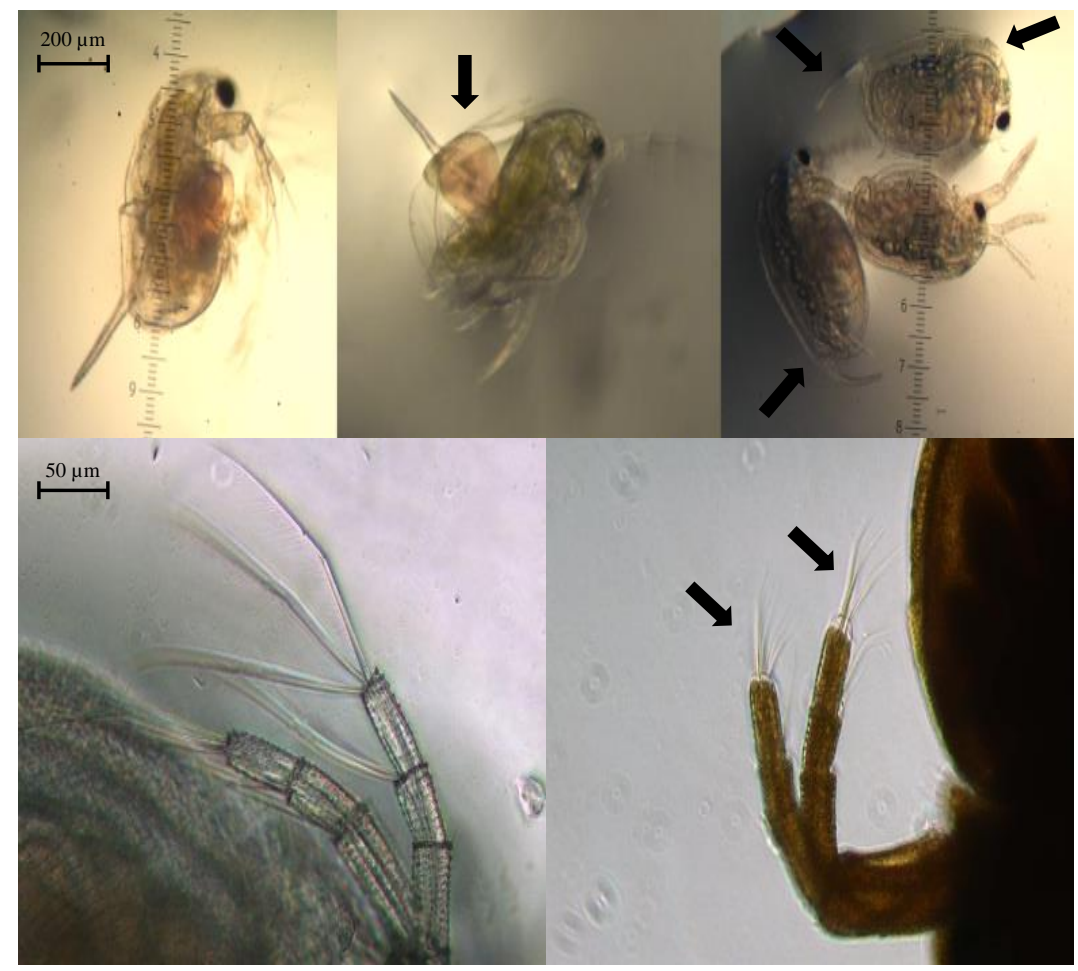

Figure 3.5. Malformations in different developmental stages of Daphnia neonates. Top-right: incomplete developed antenna setae, curved shell spine and vacuoles around ovary, top-middle: lump in the carapace, top-left: normal developed neonate, bottom-right: short antenna setae, bottom-left: normal developed antenna setae. The arrows depict malformed body parts. 


\subsection{Implications}

These bioassays are the first to show that direct life history responses in algae and Daphnia populations can occur as a result of exposure to nanoplastic. We observed $67.7 \%$ malformed offspring at exposure to $32 \mathrm{mg}$ Nano-PS/L aged Nano-PS. For pristine Nano-PS, $0.3 \%$ of the offspring malformed at a concentration of $32 \mathrm{mg}$ Nano-PS/L and $12.1 \%$ malformed at $155 \mathrm{mg}$ Nano$\mathrm{PS} / \mathrm{L}$. These thresholds are a factor $10^{6}-10^{8}$ higher than the 0.4-34 ng/L microplastic concentrations found in freshwater in Europe and USA, and about two orders of magnitude higher than the highest reported microplastic concentration in marine water, ${ }^{11,38,58,101,113}$ based on reported densities of $7.9 \times$ $10^{-5} \mathrm{n} / \mathrm{L}-6.8 \times 10^{5} \mathrm{n} / \mathrm{km}^{2}$, an estimated trawling depth of $0.1 \mathrm{~m}$ and an average particle weight of $5 \mu \mathrm{g} /$ particle. $^{164}$ Environmental concentrations of microplastic in sediment reach up to $81 \mathrm{mg} / \mathrm{kg}$ dry weight, ${ }^{165}$ which with a sediment density of $2 \mathrm{~kg} / \mathrm{L}$ and a water content of $50 \%$ on mass basis would equate to a concentration in pore water of $162 \mathrm{mg} / \mathrm{L}$. Assuming that microplastic degrades into nanosized plastic particles in the environment, for organisms inhabiting porewater or the sediment-water interface, ${ }^{166}$ these environmental concentrations exceed the observed effect thresholds for NanoPS. Furthermore, effects of plastic should not be considered in isolation. Other anthropogenic stressors are known to cause similar effects on reproduction, including malformations. ${ }^{158-160,167}$ The relevance of the present findings therefore does not only follow from the environmentally relevant plastic concentrations or those anticipated in the near future, but merely from the joint effects of multiple stressors per category of responses. Plastic simply adds to the stress already existing from tradiational contaminants and therefore make organisms less tolerant and more vulnerable to additional stressors. This implies that the effects identified in this study may in general reduce the resilience of aquatic ecosystems.

\section{Acknowledgements}

The authors thank Joris Sprakel (AVT-PCC, Wageningen UR, the Netherlands) for supplying the Nano-PS and Edwin Foekema (WMR, Wageningen UR, the Netherlands) and three anonymous reviewers for their useful comments on an earlier version of the manuscript. 


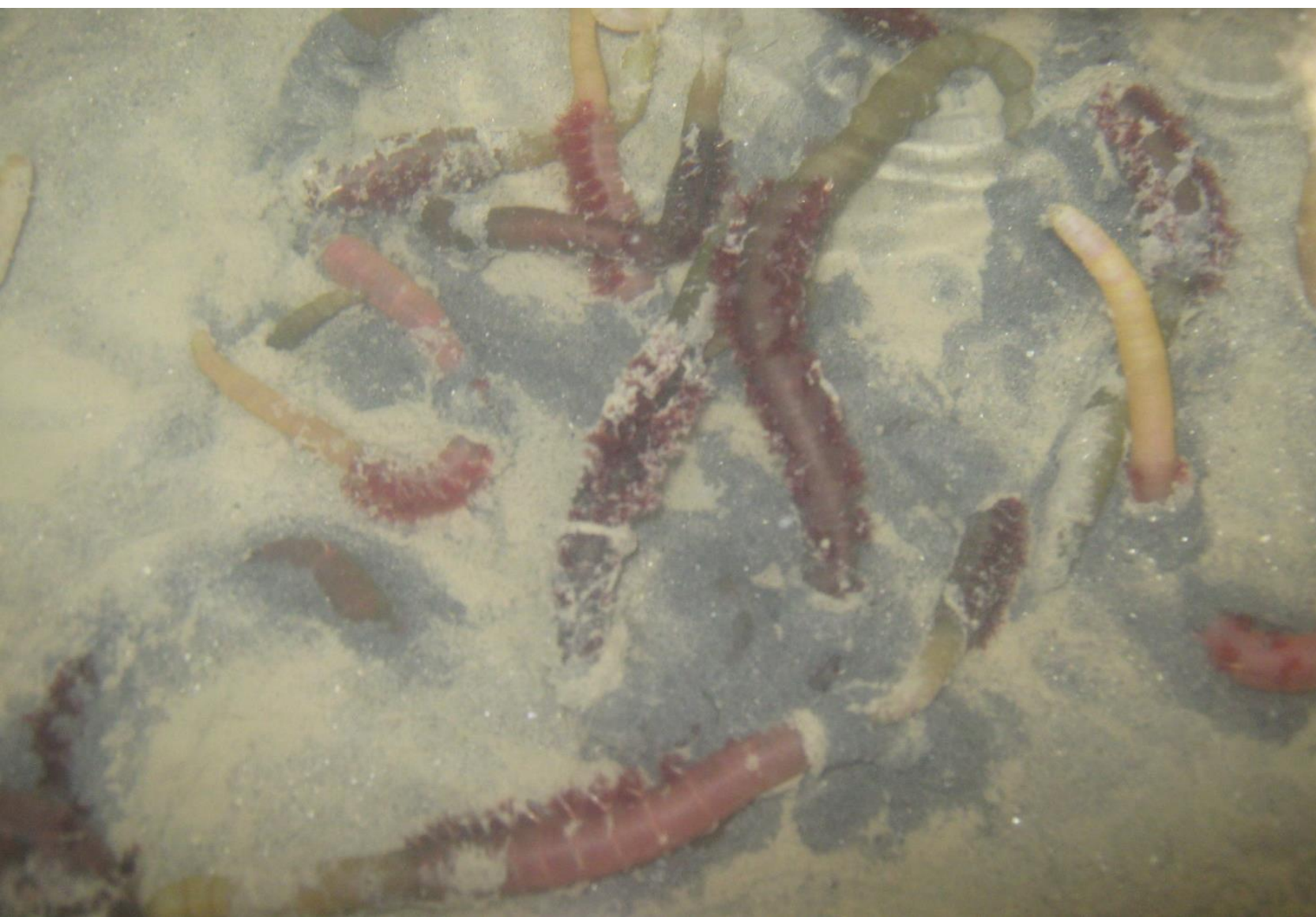




\title{
Effects of microplastic on fitness and PCB bioaccumulation by the lugworm Arenicola marine (L.)
}

\author{
Published as: Besseling, E., Wegner, A., Foekema, E.M., Van den Heuvel- \\ Greve, M.J., Koelmans, A.A., 2013. Effects of microplastic on fitness and \\ PCB bioaccumulation by the lugworm Arenicola marina (L.). Environmental \\ Science \& Technology 47, 593-6
}

\begin{abstract}
It has been speculated that marine microplastic particles may cause negative effects on benthic marine organisms and increase bioaccumulation of persistent organic pollutants (POPs). Here, we provide the first controlled study of plastic effects on benthic organisms including transfer of POPs. The effects of polystyrene (PS) microplastic on survival, activity, and bodyweight as well as the transfer of 19 polychlorinated biphenyls (PCBs), were assessed in bioassays with Arenicola marina (L.). PS was pre-equilibrated in natively contaminated sediment. A positive relation was observed between microplastic concentration in the sediment and both uptake of plastic particles and weight loss by A. marina. Furthermore, a reduction in feeding activity was observed at a PS dose of 7.4\% dry weight (DW). A low PS dose of $0.074 \%$ increased bioaccumulation of PCBs by a factor $1.1-3.6$, an effect that was significant for $\Sigma \mathrm{PCBs}$ and several individual congeners. At higher doses, bioaccumulation decreased compared to the low dose, which however, was only significant for PCB105. PS has statistically significant effects on the organisms' fitness and bioaccumulation, but the magnitude of the effects was not high. This may be different for sites with different plastic concentrations, or polymer types with a higher affinity for POPs.
\end{abstract}




\subsection{Introduction}

Plastic makes up the largest part of marine litter and the amount of marine plastic is still increasing due to both maritime activities and land-based sources. ${ }^{55}$ The buoyant plastic debris disperses along the globe due to its floating behaviour and is known to be ingested and to have negative effects on marine organisms including birds, mammals and turtles. ${ }^{55}$ Transfer of plastic particles along food chains is observed, but it is unknown for which plastic particle size transfer and effects occur. ${ }^{29}$

In addition to large plastic litter, also small plastic particles are disposed to the marine environment. An important part of the microplastic particles $\left(<5 \mathrm{~mm}^{(55)}\right)$ consists of fibres washed out of synthetic clothes, which is likely to increase with increasing human population. ${ }^{13}$ In addition to direct disposal, the decay of larger debris will contribute to increasing microplastic concentrations. ${ }^{55}$ Mechanical stress, UV radiation and biofouling play a key role in the decay process. ${ }^{22,168}$ The durability of large marine plastic items is estimated to be 5 to 50 years, but the fragmentation into micro particles might take longer as the thermal stability of plastic increases with decreasing particle size. ${ }^{169}$ Information on microplastic concentrations in the water column is scarce and also little is known about concentrations in marine sediments. Reported microplastic concentrations in sediments range up to 124 fibres/L in the United Kingdom and Portugal, ${ }^{13} 7.2 \mathrm{mg} / \mathrm{kg}$ in Belgium ${ }^{39}$ and $81 \mathrm{mg} / \mathrm{kg}$ in India. ${ }^{165}$ The uptake of microplastic by lugworms, mussels, amphipods, barnacles, sea cucumbers and fish has been described, but negative biological effects of microplastic have not been determined yet. ${ }^{22,29,170}$

Aside from causing adverse biological effects, microplastic particles are hypothesised to act as a carrier for persistent organic pollutants (POPs) in marine food webs. ${ }^{171}$ Microplastic is an effective sorbent for POPs ${ }^{172,173}$ and may transport POPs from the surface water to the sediment, thereby increasing the exposure of benthic organisms to POPs. ${ }^{171}$ Reported concentrations of POPs in marine plastic pellets range from 1 - 10,000 ng/g plastic pellet worldwide. ${ }^{37,174}$ For polychlorinated biphenyls (PCBs), concentrations of 4 $980 \mathrm{ng} / \mathrm{g}$ plastic pellet were found worldwide ${ }^{171,175}$ and $169-324 \mathrm{ng} / \mathrm{g}$ plastic pellet in the North Sea. ${ }^{174,176}$ A positive relation between internal macroplastic and PCB concentrations in seabirds was found in a feeding experiment ${ }^{21}$ and a field survey. ${ }^{177,178}$ However, this relation has not been quantified yet for microplastic ${ }^{8}$ and not for species other than birds. Microplastic may also reduce the body concentration of POPs. The hypothesized mechanism is that relatively 'clean' plastic adsorbs POPs from the organism, resulting in 
removal of POPs by egestion of the plastic. ${ }^{19}$ To date, the POP carrier feature of microplastic has been addressed by modeling ${ }^{19,20}$ and for birds by feeding experiments and field surveys. ${ }^{21,177,178}$ To our knowledge, no reports are yet available for fish or benthic organisms.

The aim of this study is to investigate the uptake of microplastic by marine (epi)benthic organisms and the effects of uptake of microplastic on their survival, growth, activity and internal PCB concentrations. We chose Arenicola marina (L.) (lugworm) as test organism, because it is a robust and quantitatively important deposit feeder at the base of the North Sea food web and is commonly used in marine sediment toxicity tests. ${ }^{22,179,180}$ Moreover, microplastic particles have recently been detected in A. marina collected from the field. ${ }^{181}$ The uptake of plastic by the lugworm has been shown before, ${ }^{22}$ but only after short exposure and only for one microplastic concentration. Longer exposure is important for a realistic assessment of potential chronic impacts of microplastic uptake on the fitness of A. marina. ${ }^{27}$ We are not aware of earlier studies that address the effects of microplastic on bioaccumulation of POPs to marine benthic organisms. Polystyrene (PS) was selected as a representative model plastic, since $\mathrm{PS}$ is one of the five main high production volume polymer types that make up about $90 \%$ of the total plastic demand, and is therefore commonly found in the marine environment. ${ }^{182}$ Furthermore, because the density of PS is higher than that of seawater, it may settle faster than equally sized microplastic particles with a lower density, thus contributing to increased concentrations in sediment. ${ }^{39}$

\subsection{Materials and Methods}

\subsubsection{Experimental design}

A. marina was exposed for 28 days to natively PCB contaminated sediment pre-equilibrated with PS (0 - 7.4\% based on dry weight; DW). Direct toxic effects caused by PCBs were prevented by applying sufficiently low PCB concentrations. ${ }^{183}$ We selected a narrow PS size range of $400-1300 \mu \mathrm{m}$, which is within the feeding range of $A$. marina.${ }^{179}$ The bioassay was carried out in two versions. A pilot experiment followed previously described procedures ${ }^{184}$ and aimed at the development of the main bioassay, which was optimized to detect effects of plastic particles. Details about the pilot experiment are provided in the Appendix (SI). The main bioassay was optimized to isolate effects of PS by keeping water quality variables constant. Bioaccumulation of PCB is only reported for the main bioassay. 


\subsubsection{Materials}

PS (crystal 1160, diameter $400-1300 \mu \mathrm{m}$ ) was purchased from Ter Hell Plastic GMBH (Herne, Germany). For PCB analysis, PCB18, 20, 28, 29, 31, 44, 52, 101, 105, 118, 138, 143, 149, 153, 155, 170, 180, 194, 204 and 209 were obtained from Dr. Ehrenstorfer GmbH (Augsburg, Germany). Diatomaceous earth was obtained from Dionex (Camberly, UK). Acetone and n-hexane (picograde) were obtained from Promochem (Wesel, Germany). Isooctane was obtained from Acros (Geel, Belgium). Silicia gel 63 - 200 mesh was obtained from Merck KGaA (Darmstadt, Germany) and was activated overnight at $180{ }^{\circ} \mathrm{C}$. Aluminium oxide super was obtained from ICN Biomedicals (Eschwege, Germany) and was deactivated with 10 mass\% Barnstead $^{\mathrm{TM}}$ nanopure water. Copper powder, $99.7 \%$, from Merck KGaA (Darmstadt, Germany) was Soxhlet-extracted with hexane for $4 \mathrm{~h}$ before use.

\subsubsection{Sediment sampling and pre-treatment}

The sediment for the bioassays was a 18:1 mixture of clean marine sediment and a sediment that was natively contaminated with PCBs. The clean sediment ${ }^{185}$ was sampled from the Oesterput in the Eastern Scheldt (the Netherlands) in autumn 2011 and had a density of $1.8 \mathrm{~kg} / \mathrm{L}$ (wet weight; WW) and an organic matter $(\mathrm{OM})$ content of $1.0 \%$. The PCB contaminated sediment was dredged in Diemen (the Netherlands) in spring 2010, had a density of $1.1 \mathrm{~kg} / \mathrm{L}$ (WW), an OM content of $15.6 \%$, and was sieved in order to remove objects $>2 \mathrm{~mm}$. Representative subsamples of the two sediment types were mixed with pre-defined PS quantities, to achieve homogeneous concentrations of $0,1,10$ and $100 \mathrm{~g}$ PS/L sediment. The resultant mixture was used for the upper layer in the test aquaria. Similarly, sediment without PS was mixed, which was used for the lower layer in the aquaria. The PS concentrations agree to $0,0.074,0.74$ and $7.4 \%$ DW PS in the sediment. The mixture had an OM content of $1.7 \%$. Mixing occurred on a roller apparatus (Willemsen Proefinstallaties, Spijk, the Netherlands) during six weeks before the bioassay. This procedure ascertained (a) a thorough mixing and homogenisation of particles, (b) re-equilibration of fast and slowly desorbing fractions from the PCB contaminated sediment to the marine sediment, which have typical desorption half-lives of $0.4-4 \mathrm{~d}^{-1}(186,187)$, and (c) equilibration of PCBs in $0.4-1.3 \mathrm{~mm}$ microplastic particles, for which equilibration times of 1 to 35 days can be estimated based on the PS particle sizes and reported diffusivities of $2 \times 10^{-10}-4 \times 10^{-10} \mathrm{~cm}^{2} / \mathrm{s} .{ }^{172,188}$ Due to the high mixing ratio, sediment characteristics were close to that of the original marine sediment, except for the PCB concentrations. PCB concentrations in the mixture are 
listed in the Appendix (Table A4.1). In addition to the treatments with 0 - 7.4\% PS on PCB contaminated sediment, a treatment without plastic containing only clean sediment (treatment Oesterput) was included as a control without PCB contamination.

\subsubsection{Test organisms}

A. marina were collected in the southern Wadden Sea by a professional bait collector (Lugworm wholesale business Rotgans, Hippolytushoef, the Netherlands). Before the start of the pilot experiment, the organisms were allowed to clear their gut. This step was omitted for the bioassay, in order to prevent weakening of their condition. Before the start of the bioassay, the 'digging-in' speed of the organisms in clean sediment was tested. In this way, healthy, fast digging organisms were selected for this assay.

\subsubsection{Bioassays}

Two L test beakers filled with $\pm 2.6 \mathrm{~kg} \mathrm{WW}$ sediment ( $6 \mathrm{~cm}$ thick layer) were placed in a glass aquarium containing $\pm 110 \mathrm{~L}$ sea water $(39 \times 78 \times 38 \mathrm{~cm})$. Each aquarium contained four test beakers with the same treatment, such that no exchange of plastic via the water column could occur between different treatments. Aquaria were placed in random order. Apart from the control (no PS), PS was present in the upper one-third of the sediment $(2 \mathrm{~cm}, 0.86 \mathrm{~kg})$, because the lugworm feeds on the upper sediment layer. ${ }^{189}$ Following previous procedures, ${ }^{190}$ no extra food source was provided. The aquaria were stabilised for five days before adding five individuals of $A$. marina per test beaker, which started the bioassay. The $\mathrm{n}=5$ group weights averaged $20.2 \pm 2.6 \mathrm{~g}$ per beaker. Because transport of sediment, PS and sediment associated PCBs between replicates was negligible, the beakers were independent units even though they shared the overlying seawater. PCB transfer among the beakers was irrelevant for two reasons. First, exchange between pore water in one beaker to another would involve passage through two stagnant benthic boundary layers and transport across $15 \mathrm{~cm}$ of stagnant water between the beakers, which can be calculated to be negligible in 28 days (not shown). Second, even if PCBs would exchange between beakers, their aqueous phase concentrations would be too low to cause additional detectable exposure to the worms. A schematic representation of both bioassays is provided in the Appendix (Fig. A4.3). Aeration was applied and the water was renewed every two-days. Dissolved oxygen (DO) saturation, temperature, $\mathrm{pH}$ and salinity were measured twice a week with a $\mathrm{HACH} \mathrm{HQd}$ Field Case. $\mathrm{NH}_{4}{ }^{+}$and $\mathrm{NO}_{2}{ }^{-}$were monitored with reagent kits from Aquamerck (Darmstadt, Germany), having a range of 0.5 $10 \mathrm{mg} / \mathrm{L}$ and $0.025-0.5 \mathrm{mg} / \mathrm{L}$, respectively. Average values were $10.1 \mathrm{mg} / \mathrm{L}$ 
(94\% saturation) for DO, $12.3{ }^{\circ} \mathrm{C}$ for temperature, 8.2 for $\mathrm{pH}, 32 \%$ for salinity, $0.2 \mathrm{mg} / \mathrm{L}$ for $\mathrm{NH}_{4}{ }^{+}$and $0.03 \mathrm{mg} / \mathrm{L} \mathrm{NO}_{2}{ }^{-}$.

\subsubsection{Endpoints}

Mortality and feeding activity were assessed daily. Following previously reported methods for heap analysis, ${ }^{180,191,192}$ feeding activity was defined as the amount of faeces heaps produced per organism per day. In the early stage of the bioassay, some organisms were able to escape from the beakers. For the subsequent part of the assay, this was prevented with nets (mesh size about 3 $\mathrm{mm}$ ). The activity analysis was however hindered by the nets and therefore stopped at day 10. After $28 \mathrm{~d}$ exposure, the lugworms were collected and allowed to clear their guts in beakers with clean seawater overnight. ${ }^{190} \mathrm{Gut}$ content was harvested and the whole sample was analysed for PS particle numbers using a microscope (Nikon, Binocular 200786). The lugworm tissue was stored at $-18{ }^{\circ} \mathrm{C}$ prior to homogenization. After homogenization by scalpel, determination of the internal amount of plastic particles was done by microscopy. Furthermore, WW, DW (after heating $\pm 1 \mathrm{~g}$ tissue per group at 60 ${ }^{\circ} \mathrm{C}$ during $24 \mathrm{~h}$ ), and AFDW (ash free dry weight, after heating at $600{ }^{\circ} \mathrm{C}$ during $2 \mathrm{~h}$ ) were determined.

\subsubsection{PCB analysis}

PCB analysis followed previously published procedures. ${ }^{186,193}$ The lugworm tissue was homogenized by scalpel, pulverised and dehydrated ( $\pm 2 \mathrm{~g}$ tissue with $\pm 10 \mathrm{~g}$ diatomaceous earth) in a mortar. For the sediment, the same procedure was used. Glassware was rinsed with acetone prior to use. PCBs were extracted with an accelerated solvent extractor (ASE 350, Dionex, see Table A4.2), using n-hexane, and cleaned over a combined column filled with a dot of quartz wool, $1.5 \pm 0.1 \mathrm{~g}$ silica gel and $3 \pm 0.1 \mathrm{~g}$ aluminiumoxide. The solution was concentrated on a Kuderna-Danish apparatus followed by evaporation to $1 \mathrm{~mL}$ under a gentle stream of nitrogen. Sulphur was removed using copper. PCBs were detected with standard gas chromatograph procedures (GC Hewlett-Packard 5890 II from Agilent equipped with an HP7673A autosampler, two ${ }^{63} \mathrm{Ni}$ electron capture detectors and two 50-m capillary fused silica columns: CP Sil-8 CB and CP Sil-5/C18 CB from Varian), and PCB143 as an internal standard. Procedural blanks contained generally $\Sigma \mathrm{PCBs}$ of $0 \mu \mathrm{g} / \mathrm{kg}$ (SD 0.04). PCB recoveries averaged $85,9 \%$ (SD 12.7). If not stated otherwise, $\mathrm{PCB}$ concentrations are reported on a WW basis. Detection limit (DL) was $0.01 \mu \mathrm{g} / \mathrm{kg}$. Biota sediment accumulation factors $\left(\mathrm{BSAF}=\mathrm{C}_{\mathrm{worm}} / \mathrm{C}_{\text {sediment }}\right)$ are based on tissue and sediment concentrations on a DW basis. 


\subsubsection{Data analysis}

Prior to further analysis, normality of the data and equality of variances were tested with a Q-Q plot and Levene's test, respectively. Linear regression analysis, one-way-ANOVA, Kruskal-Wallis and Mann-Whitney U tests were performed using SPSS versions 17 and 19, with significance level $\alpha=0.05$ as a criterion for effect.

\subsection{Results and Discussion}

\subsubsection{Effects of water quality variables}

Water quality variables were rather constant in the bioassay (Table A4.1) and conform to the ICES guidelines. ${ }^{184}$ Consequently, there was no significant relation between the investigated endpoints and average oxygen concentration, temperature, $\mathrm{pH}$, salinity, $\mathrm{NH}_{4}{ }^{+}$or $\mathrm{NO}_{2}{ }^{-}$. A clear difference in effects of water quality variables was observed between the two bioassays. Because of the positive relation between oxygen concentration and average number of days of survival (Fig. A4.9.A) in the pilot experiment, we conclude that oxygen depletion rather than PS treatment has been the main stressor there.

\subsubsection{Effects of microplastic on fitness and performance of $A$. marina.}

Survival. Lugworm survival was $94 \%$. In total, $12 \%$ of the worms was removed during the bioassay, of which $50 \%$ was because of incidental escapes from the test beakers into the large aquaria and 50\% because of mortality. There was no significant relation between treatment and the amount of worms that remained in the test beakers (ANOVA, $\mathrm{p}=0.817$ ) (Fig. A4.5.D). Furthermore, no effect of treatment on survival was detected $(\mathrm{p}=0.276)$. The absence of an effect on survival for contaminated sediment without PS implies that the observed mortality in the pilot experiment probably was not caused by sediment contamination. As mentioned above, mortality in the pilot experiment is ascribed to oxygen depletion.

Ingestion of plastic. In some of the organisms that died during the bioassay, plastic was encountered after dissection. No plastic remained in the organisms that survived the entire 28 days exposure period after allowing them to clear their guts. We only found internal plastic particles in worms that were, because of escape or mortality, preliminary removed from the $0.74 \%$ treatment, which was the highest plastic concentration from which we preliminary removed worms (two removed worms with respectively 1 and 2 internal PS particles). There was a significant higher amount of internal plastic particles in organisms 
Chapter 4
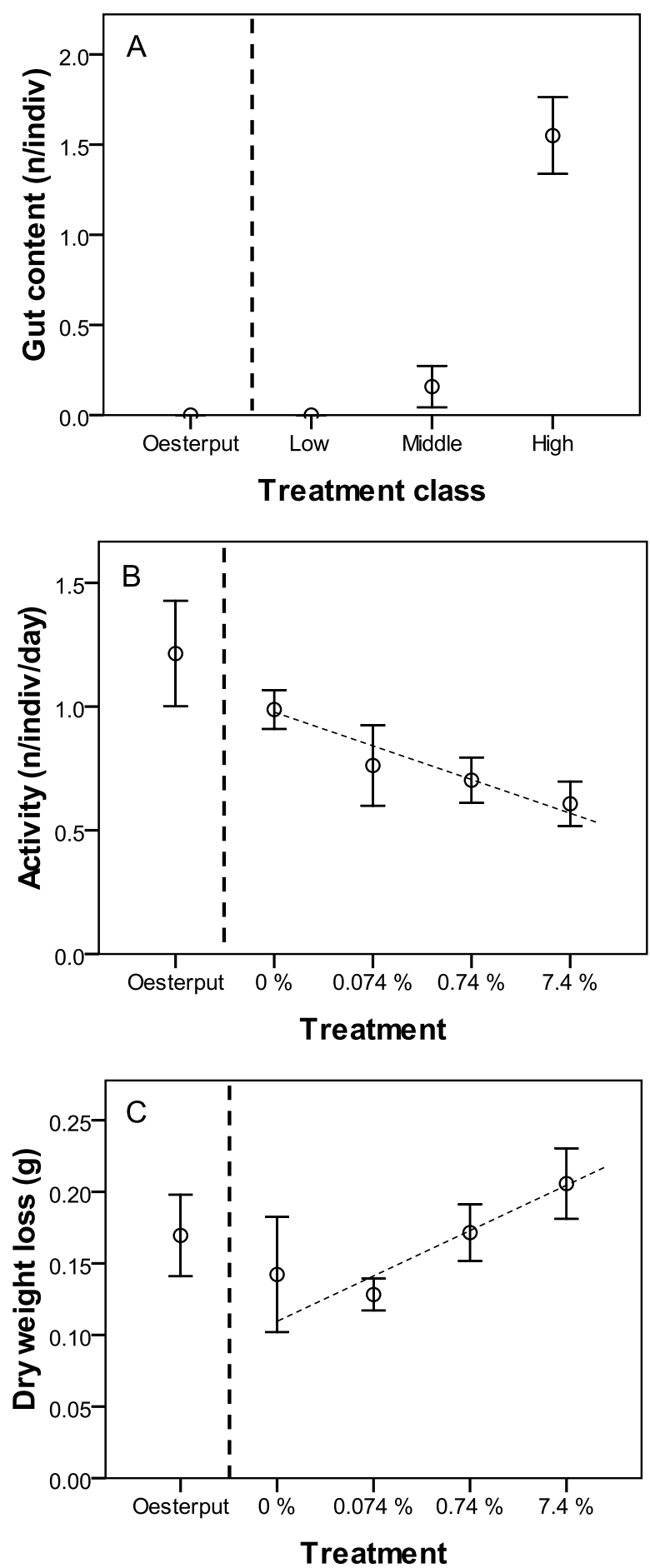
Figure 4.1. Effects of polystyrene dose $(0,0.074,0.74$ and $7.4 \%)$ in the bioassay on the endpoints: Number of PS particles detected in gut content, expressed in treatment classes Low (0 and $0.074 \%)$, Middle $(0.74 \%)$ and High (7.4\%) (panel A); Activity observed as average heap production (panel $B$ ) and DW loss after 28 days (panel $C$ ). Oesterput $=$ treatment with clean Oesterput sediment; The thin dashed lines in panels $B$ and $C$ visually indicate the relation between $\log (P S$ dose) and activity and DW loss, respectively. Error bars relate to $\pm 1 S E$.

that did not clear their guts (due to preliminary removal from the bioassay, no gut clearance applied in this stage) compared to organisms that did clear their guts (at the end of the assay) (Mann-Whitney $U$ test: $\mathrm{p}=6.96 \times 10^{-6}$ ), which indicates that $A$. marina ingested PS particles $\geq 400 \mu \mathrm{m}$, but that the particles did not accumulate in this organism. The higher amount of plastic particles in organisms that died could also imply that the ingested plastic negatively affected survival or that weak organisms were less capable to egest microplastic particles. However, we consider it more likely that the higher amount is related to the lacking of gut clearance.

Gut content. Organisms that were exposed for the whole 28-day exposure period, egested faeces during gut clearance overnight. Plastic was only found in these faeces of organisms exposed to the plastic concentration of $7.4 \%$ (average of 6.8 PS particles per group of 5 worms, SD 4.7, on average 1.36 particle/worm). In the previous section we concluded that dying worms were unable to excrete their gut content. Therefore, for the definition of 'gut content', no differentiation is made between internally detected plastic, i.e. 'gut content' of worms that died during the bioassay, and plastic egested during gut clearance, i.e. 'gut content' of worms that survived the bioassay. Because of the non-normality of the data, we investigated the relation between plastic exposure and amount of plastic particles in the gut content with the KruskalWallis test, which gave significant differences between treatments $\left(\mathrm{p}=1.90 \times 10^{-18}\right)$. As a post hoc test, pair-wise comparisons of the treatments were done with the Mann-Whitney U test, which revealed three significantly different treatment classes: low (0 and $0.074 \%)$, middle $(0.74 \%)$, high (7.4 \%) $\left(1\right.$-sided $\left.\mathrm{p}=0.019, \mathrm{p}=3.43 \times 10^{-14}, \mathrm{p}=1.31 \times 10^{-7}\right)$. As expected, we found no plastic particles in the gut content of the organisms in treatment Oesterput. The positive relation between the amount of plastic particles in the gut content and plastic exposure concentration (Fig. 4.1.A) is consistent with our expectation that $A$. marina feeds unselective ${ }^{179}$ with respect to particle type. This is also supported by the results from the pilot experiment (Fig. A4.4A).

Activity. Feeding activity was observed during the whole bioassay, but recorded only till day 10 (Fig. A4.7.B). In that time span, the mean activity 
was 0.53 heap/individual/day (SD 0.46). We observed a negative relation between plastic concentration and activity in the bioassay (Fig. 4.1.B, ANOVA, $\mathrm{p}=0.045$ ). The activity in clean sediment (Oesterput) turned out to be significantly higher than in all treatments with plastic $(0.074,0.74$ and 7.4 $\%$ ) (1-sided p-values $0.018,0.010$ and 0.004 respectively). Theoretically, the effect could have been caused by the plastic as well as by the PCB contamination in the sediment containing plastic. However, considering only the treatments with PCB contaminated sediment, the activity in the treatment without plastic (0\% PS) was significantly higher than in the $7.4 \%$ treatment (1-sided p-value 0.035). This identifies the effect as an effect of plastic, which is consistent with our experimental design criterion that sediment PCB concentrations were at least a factor 100 lower than reported effect thresholds for benthic invertebrates. ${ }^{183}$ That the PCB concentrations were not toxic to $A$. marina also followed from the fact that no difference in feeding activity was detected for the PCB contaminated versus the clean sediment. As far as we know, such a negative effect of plastic on the activity of A. marina has not been detected before.

Weight loss. In the bioassay all groups lost weight. The bioassays were accomplished in autumn, a season in which individual weight loss of $A$. marina is common ${ }^{192}$ and might (partially) be caused by spawning, ${ }^{179,194}$ as was observed in various test beakers during the pilot experiment. Spawning was not recorded in the main experiment, but might have occurred there too. The mean WW loss was $1.20 \mathrm{~g} /$ individual $(29.7 \%$, SD 0.41$)$, the mean DW loss was $0.16 \mathrm{~g} /$ individual $(28.4 \%$, SD 0.06) and the mean AFDW loss was $0.06 \mathrm{~g}$ /individual (16.6\%, SD 0.04). We observed an increasing weight loss with increasing plastic concentration, but this was not for all weight indicators significant. There was a positive significant relation between plastic concentration and absolute DW loss (Regression, 1-sided p-value=0.028) (Fig. 4.1.C). To our knowledge, this is the first time that a negative effect of plastic on the bodyweight of lugworms is quantified. We found no significant difference in DW loss between the contaminated and clean sediment in absence of plastic (treatment $0 \%$ and Oesterput) (ANOVA, $\mathrm{p}=0.994$ ). This again confirms the similarity of sediment quality and the absence of PCB toxicity that was aimed for by mixing unpolluted and polluted sediment in a 1:18 mixing ratio. AFDW loss and WW loss had no significant relation with plastic concentration ( 1 -sided $\mathrm{p}=0.058$ and $\mathrm{p}=0.205$, respectively). One could expect that weight loss is an indirect effect of reduced feeding activity, but there was no significant link between activity and WW, DW, AFDW loss 
(Regression, $\mathrm{p}=0.721, \mathrm{p}=0.894, \mathrm{p}=0.656$ ). That the water quality variables were constant and favourable for A. marina in this assay, might be the reason why an effect of plastic exposure concentration on activity and weight loss was visible here, and not in the pilot experiment.

\subsubsection{Effects of microplastic on bioaccumulation of PCBs.}

Detection of PCBs. $\Sigma$ PCB in the sediment was $1.84 \pm 0.22 \mu \mathrm{g} / \mathrm{kg}$ (Table A4.4), which is a factor 350 lower than the probable effect $\Sigma \mathrm{PCB}$ concentration of $676 \mu \mathrm{g} / \mathrm{kg}$ reported by MacDonald et al. ${ }^{183}$ This further supports the conclusion that effects discussed in the previous section are effects of plastic and do not relate to PCB toxicity. Because PCB concentrations were designed to be low, several individual congener concentrations were below the detection limit (overview in Table A4.3). However, a complete dataset is available for $11 \mathrm{PCBs}$ over a large hydrophobic range $(\operatorname{LogK} \mathrm{Ow}=5.58-7.21)$ (Table A4.3), such that treatment effects can be tested for this range. Relative errors (\%SD) among replicates generally were $10-30 \%$ (85\% of the cases), with $6 \%$ of the cases $>40 \%$, of which 2 cases $>100 \%$. These 2 cases had suspected outliers (Dixons Q-test $\mathrm{p}<0.01$ ). However, excluding them did not change the number of detected significant treatment effects so outliers were kept in the dataset. Amongst others, spawning might be an explanation for the observed variation.

Treatment effects on bioaccumulation. Per PCB congener, an ANOVA was applied to detect differences between treatments. There were significant differences in PCB tissue concentration between treatments for all PCBs and $\Sigma$ PCBs (p-values $\leq 0.001$ ), except for PCB29, 194 and 204, for which too many values were below DL (Table A4.3). For testing which treatments significantly differed from each other, a post-hoc test was done for $\mathrm{PCCBs}$ and for PCBs with all concentrations $>$ DL, i.e. PCB31, 44, 52, 101, 105, 118, 138, 149, 153, 170 and 180. The discussion below focuses on these congeners. One PCB concentration pattern occurred most frequently among treatments (Fig. 4.2). The tissue PCB concentrations of lugworms exposed to clean Oesterput sediment, did not differ from the tissue concentrations of non-exposed lugworms (treatment 'start') (ANOVA, for individual PCBs $\mathrm{p} \geq 0.620$ ). For all $(\Sigma)$ PCBs subjected to further analysis, the worms exposed to contaminated sediment (i.e., treatments 0, 0.074, 0.74 and $7.4 \%$ ) had a significantly higher PCB concentration than the clean Oesterput sediment, non-exposed worms ('Start') and worms exposed to clean Oesterput sediment (p-values $\leq 0.017$ ). Generally, the worms exposed to contaminated sediment contained 2 - 7 times the concentration found in worms exposed to clean sediment (Table A4.6). 
PCB concentrations in worms exposed to $0.074 \%$ PS were generally a factor 1.1 - 1.5 higher than those exposed to contaminated sediment without plastic (0\% PS) (Table A4.7). This was statistically significant for PCB31, 52 and 105 (p-values $0.022,0.016$ and $8.2 \times 10^{-6}$ respectively). This means that at our lowest PS dose of $0.074 \%$, bioaccumulation of these PCBs increased. For most PCBs, a decrease in PCB concentration was observed for plastic concentrations $>0.074 \%$ (generally a factor 1.1-1.3 difference with $0 \%$ PS) (Fig. 4.2). However, this decrease was significant only for PCB105, that is, PS treatments 0.074 and $0.74 \%$ resulted in significantly higher PCB105 tissue concentrations than the $7.4 \%$ PS treatment (p-values $\leq 0.001$ ).

The less hydrophobic PCBs 28, 31 and 52 showed an accumulation pattern deviating from the most frequent pattern described above, with the PCB concentration in the $0.74 \%$ PS treatment being lower than in the treatments with 0.074 and $7.4 \%$. This is significant for PCB28 (p-values $\leq 0.002$ ). For PCB20 and 44 the PCB concentration increased with plastic concentration till the treatment with $0.74 \%$, after which it decreased. However, the only statistically significant difference here is that the concentration of PCB44 was higher in worms from the treatment with $0.74 \%$ than from the treatment without plastic $(\mathrm{p}=0.015)$. So, the maximum in observed bioaccumulation as a function of PS dose is seen here as well, but the maximum appears at a higher plastic concentration.

Biota sediment accumulation factors. PCB congener concentrations in the contaminated sediment correlated well with congener concentrations in the exposed worms (correlations not shown), which suggests constant partitioning between sediment and organism and calls for a consideration of BSAF values. BSAFs ranged from about 10 to 40, except for PCB29 and 209 (BSAFs $<7$ ) and PCB155 (BSAFs>80) (Table A4.5). These BSAFs are $1-2$ orders of magnitude higher than previously found in lugworms ${ }^{190,195}$ and an order of magnitude higher than equilibrium partitioning theory (EPT) would predict if BSAFs would have been normalised on biota lipid and sediment organic matter fractions. ${ }^{196}$ However, BSAFs were not normalised as lipid content was not measured in this study due to lack of sample material. A possible explanation for the high BSAFs may be that the worms were actively feeding on sediment, which increases their steady-state BSAF to higher levels than what would be predicted by EPT. ${ }^{197}$ 


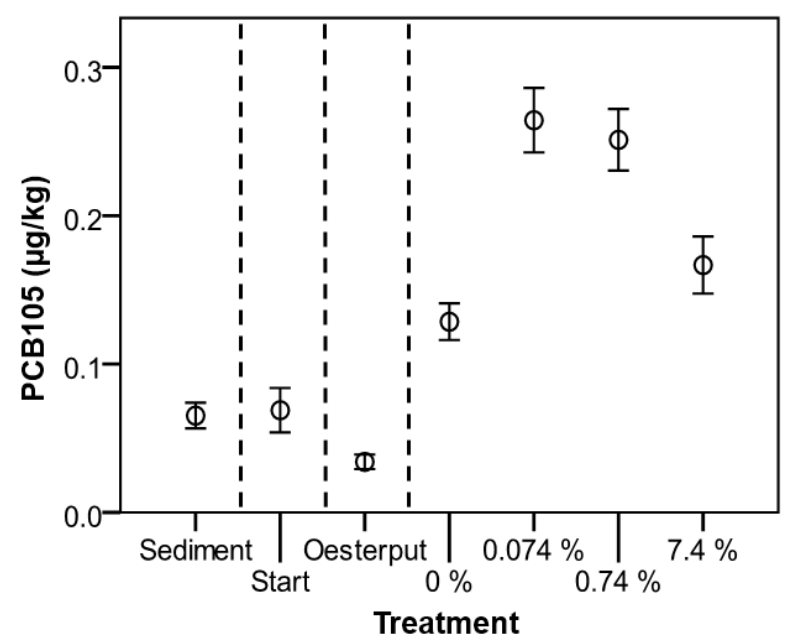

Figure 4.2. PCB105 concentration in contaminated sediment ('sediment'), non-exposed start worms ('start'), worms exposed to clean Oesterput sediment ('Oesterput') and worms exposed to polystyrene concentrations of 0, 0.074, 0.74 and $7.4 \%$ in contaminated sediment, expressed on dry weight basis. Error bars relate to $\pm 1 S E$.

\subsubsection{General discussion and implications}

The bioassay showed that A. marina ingested PS spheres of $400-1300 \mu \mathrm{m}$. As plastic is ingested by A. marina, its predators will be exposed to plastic as well. The difference in amount of plastic particles in lugworms that did or did not clear their guts indicates that $A$. marina ingests PS particles $\geq 400 \mu \mathrm{m}$, but that these particles do not accumulate in this organism. Therefore, obstruction of the digestive tract, as proposed for marine birds, ${ }^{198}$ is not likely for the particles that were used. In our study an effect concentration for feeding activity of $7.4 \%$ was found, implying that high environmental PS concentrations negatively affect feeding activity. This is in accordance with the positive relation between sediment nutritional value and feeding activity found by Cadée et al. ${ }^{180}$ To the authors knowledge, birds are the only marine organism for which a reduced feeding activity related to high internal plastic concentrations is found. ${ }^{199}$ The suggested concern about the ability of these birds to lay down fat deposits might apply to lugworms as well. So far, microplastic concentrations up to $81 \mathrm{mg} / \mathrm{kg}$ have been found in marine sediment, ${ }^{165}$ which are three orders of magnitude lower than our effect concentration of $7.4 \%$. However, to date sediment microplastic concentrations have been investigated for only a limited number of sites. ${ }^{13,22,39,165}$ Higher concentrations might exist in harbour sediment or in coastal areas in densely populated regions, or arise due to future disposal or 
breakdown of plastic. Like activity reduction, a negative effect of plastic on weight loss has, so far, only been quantified for marine birds. ${ }^{200}$ For birds, the proposed explanatory mechanism is a lower feeding efficiency, as a result of food dilution. ${ }^{200,201}$ In our experiment, the OM content of the sediment was diluted from $1.73 \%$ down to $1.64 \%$ due to the addition of plastic, which constitutes a relative decrease of OM content up to $5.3 \%$. This means a larger volume of sediment needs to be processed to obtain the same nutritional value. Furthermore, PS ingestion may have caused physical stress because the PS particles were larger than sediment particles. Consequently, the reductions of the OM content and presence of PS might have been large enough to reduce the energy assimilation efficiency of $A$. marina and thereby explain its weight loss. As food availability appears to be the crucial factor for the growth of $A$. marina ${ }^{202}$ plastic pollution might negatively affect the viability of lugworm populations if the pollution causes a reduction of the OM content.

To our knowledge, this is the first bioassay that reports on the relation between exposure to different microplastic concentrations in sediment and internal PCB concentrations of marine benthic organisms. Increased PCB accumulation was observed at our lowest PS dose of $0.074 \%$. An increase in bioaccumulation by a factor 1.1-1.5 is scientifically interesting, but probably not that relevant for the risk assessment of POPs nor that of marine microplastic particles. Despite the limited magnitude of the effect, it was significant for several PCBs, whereas the general pattern was observed for 68 $\%$ of the congeners studied. Several explanations for the observations may be given. It has been speculated that an increase in bioaccumulation upon addition of plastic may be explained by plastic facilitated transfer of PCBs from the sediment to the organisms. ${ }^{19-21,203}$ However, PS has a reported range of PCB partition coefficients of $10^{2}-10^{3} \mathrm{~L} / \mathrm{kg},{ }^{188}$ which is much lower than $\mathrm{OM}$ or lipid water partition coefficients for the same PCBs. Therefore, it is not likely that PS acted as a vector for PCBs. PS also might affect bioaccumulation by diluting pore water PCB concentrations. This, however, also is not likely for our lowest PS dose as it can be calculated (not shown) that the change would be marginal, which agrees to an earlier analysis of the same mechanism. ${ }^{19}$ Two other mechanisms may play a role. First, the decreased (diluted) OM content and presence of PS may have resulted in increased sediment feeding, ${ }^{204}$ more than compensating the aforementioned small dilution effect and thus in a higher net accumulation. However, our measurement of feeding was based on faeces count and not on faeces weight, such that our data can neither support nor contradict this. Second, the 
experienced stress in the experiments may have resulted in reduced lipid contents, which is supported by the observed DW loss of $28.4 \%$. Similarly, it has been reported that activated carbon in sediments reduced lipid contents in aquatic worms. ${ }^{205} \mathrm{~A}$ reducing lipid volume might in particular concentrate hydrophobic PCBs that have the lowest elimination rates. The dilution mechanism however, might partly explain the decrease in PCB accumulation at the doses of 0.74 and $7.4 \%$. These high plastic concentrations might have diluted the directly available free pore water PCB concentrations in the sediment to an extent that overwhelms the other mechanisms, resulting in lower net PCB transfer to the organism.

To date, there are no reports of plastic concentrations as high as our range of $0.074-7.4 \%$ in marine sediment. Consequently, we cannot unambiguously conclude that currently reported plastic concentrations will cause an increase in organisms PCB concentrations. Also, in the marine environment, the plastic will already contain PCBs when entering the sediment, such that any speculated dilution effect in this assay plays a minor role. ${ }^{20} \mathrm{PS}$ appeared to increase accumulation by a factor of $1.1-1.5$, with peculiarities up to 3.6. Given the low magnitude and uncertainty of these factors, the impact of PS on PCB transfer to A. marina can be considered small. This may, though, be different for polymer types for which POPs have an equal or higher affinity than for biota tissue, like polyethylene. ${ }^{206}$ However, the interplay of simultaneous processes is complex, which calls for the development of validated models for plastic facilitated uptake of POPs.

\section{Acknowledgements}

We thank Frits Gillissen, Gerrit Hoornsman, Erika Koelemij and Darya Kupryianchyk for their valuable contributions to this work. 


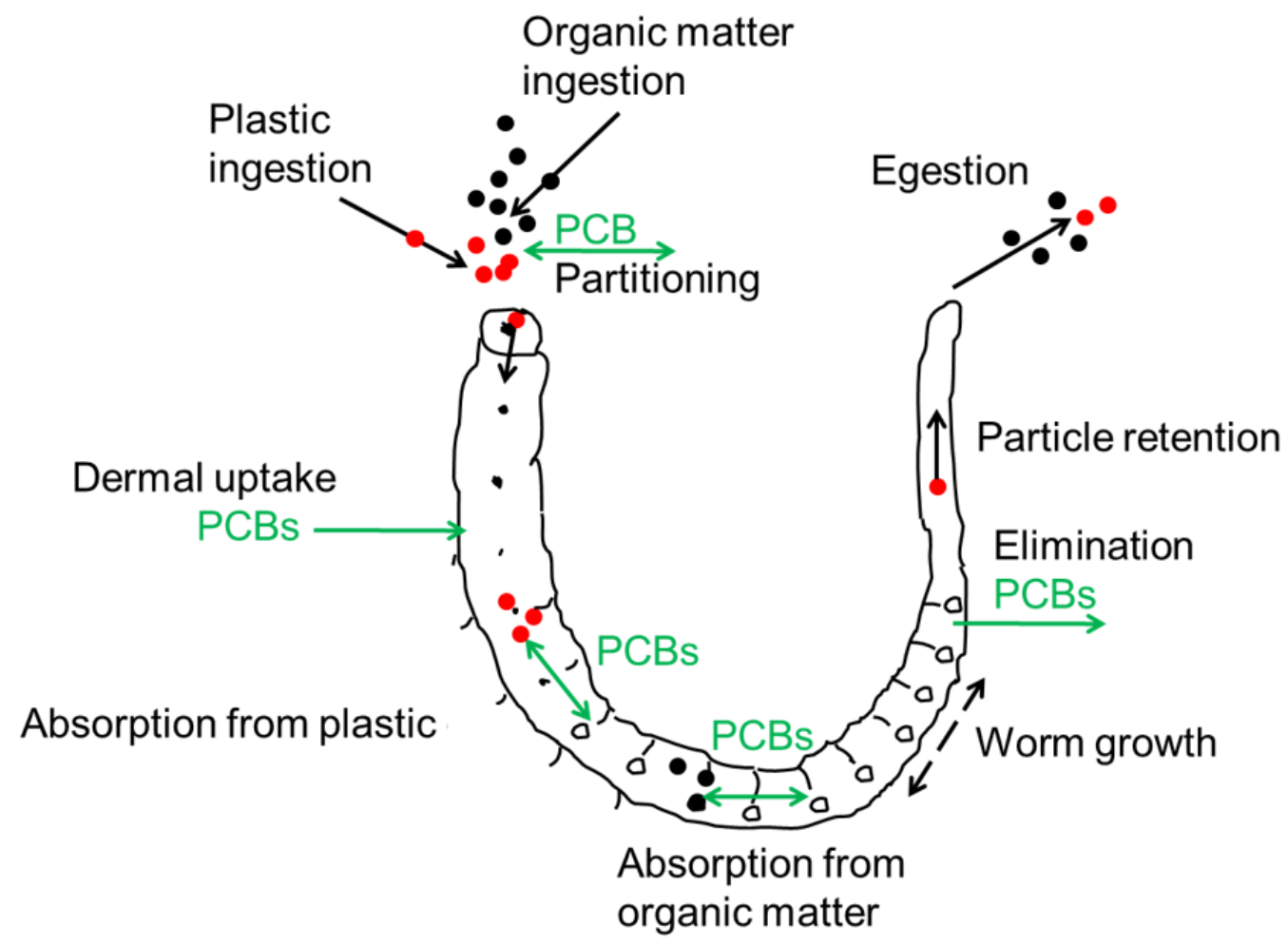




\title{
Plastic as a carrier of POPs to aquatic organisms: A model analysis
}

\author{
Based on: Koelmans, A.A., Besseling, E., Wegner, A., Foekema, E.M., 2013. \\ Plastic as a carrier of POPs to aquatic organisms: A model analysis. \\ Environmental Science \& Technology 47, 7812-7820
}

\begin{abstract}
It has been hypothesised that persistent organic pollutants (POPs) in microplastic may pose a risk to aquatic organisms. Here, we develop and analyse a conceptual model that simulates the effects of plastic on bioaccumulation of POPs. The model accounts for dilution of exposure concentration by sorption of POPs to plastic (POP 'dilution'), increased bioaccumulation by ingestion of plastic containing POPs ('carrier'), and decreased bioaccumulation by ingestion of clean plastic ('cleaning'). The model is parameterised for the lugworm Arenicola marina and evaluated against recently published bioaccumulation data for this species from laboratory bioassays with polystyrene microplastic. Further scenarios include polyethylene microplastic, nano-sized plastic and open marine systems. Model analysis shows that plastic with low affinity for POPs, like polystyrene will have a marginal decreasing effect on bioaccumulation, governed by dilution. For stronger sorbents like polyethylene, the dilution, carrier and cleaning mechanism are more substantial. In closed laboratory bioassay systems, dilution and cleaning dominate, leading to decreased bioaccumulation. Also in open marine systems a decrease is predicted due to a cleaning mechanism that counteracts biomagnification. However, the differences are considered too small to be relevant from a risk assessment perspective.
\end{abstract}




\subsection{Introduction}

Accumulation of plastic is recognized as one of today's major marine water quality problems ${ }^{23,207}$ Plastic is very persistent, ${ }^{208}$ yet breaks down gradually by abrasion and by UV-B radiation ${ }^{22,209}$ to form smaller fragments, i.e. microand nanoplastic particles defined as $<5 \mathrm{~mm}$ and $<100 \mathrm{~nm}$ sized particles, respectively. ${ }^{19,182,203}$ Microplastic particles have been shown to be the most common size fractions in the oceanic gyres ${ }^{210}$ and microplastic densities in the North Pacific have increased by two orders of magnitude over the past 40 years. ${ }^{211}$ One of the observed effects relates to ingestion, which negatively affects benthic invertebrates, birds, mammals and turtles (Chapter 4). ${ }^{22,29,51,52,212,213}$ Microplastic particles are also hypothesised to act as a carrier for persistent organic pollutants (POPs) in marine ecosystems ${ }^{20,177}$ which is the focus of this chapter. Marine plastic particles are known to absorb POPs like for instance polychlorobiphenyls (PCBs)..$^{37,171,174,175,214,215}$ High plasticwater partitioning coefficients ${ }^{172,188,216,217}$ make plastic particles potential candidates as carriers of POPs to marine organisms. Concentrations of POPs absorbed to plastic in the marine environment range from 1 to $10,000 \mathrm{ng} / \mathrm{g}$ plastic pellet worldwide. ${ }^{37,174}$ After settling, microplastic particles may increase the exposure of benthic organisms to POPs. ${ }^{20}$ It has also been suggested that plastic may reduce bioaccumulation of POPs. One proposed mechanism is that equilibrium partitioning between plastic and POPs dilutes free aqueous concentrations, ${ }^{19,20}$ i.e. decreases bioavailability and bioaccumulation of POPs. Another mechanism might occur if the plastic is relatively clean compared to the organisms' body burden. Then, plastic would absorb POPs from the organism's tissue, resulting in removal of POPs by egestion of the plastic. ${ }^{19}$ Recently, the dilution hypothesis has been investigated by model and mass flux analysis, which showed that the dilution effect will be small in diluted systems such as the oceans. ${ }^{19,20,182}$ However, this may be different in 'hot spots' of plastic pollution near the coast or in harbour areas, or if microplastic concentrations keep rising. Then, plastic ingestion by benthic organisms might be substantial, leading to either increased or decreased accumulation compared to a situation without plastic, dependent on initial POP concentrations. The importance of plastic ingestion as an exposure pathway has not been quantified yet. Following Gouin et al. ${ }^{19}$ we conclude there is a need for dynamic models that are able to simulate these simultaneous and possibly counteracting processes for prognostic risk assessment of microplastic and future scenario studies. 
Here, we present a biodynamic model for uptake of POPs in a sediment - water system, in the presence of microplastic. The model accounts for dilution of pore and overlying water by microplastic, uptake from plastic loaded with POPs as well as depuration of POPs from the tissue in case of ingestion of clean microplastic. Although the core model is general for aquatic organisms, it is first parameterised and validated for the lugworm Arenicola marina (L.). This benthic organism feeds on sediment material and will thus ingest microplastic that is present in the sediment (Chapter 4). ${ }^{51}$ Furthermore, $A$. marina is the only species for which a dataset on uptake of a series of PCB congeners at different polystyrene (PS) microplastic-sediment mixing ratios is available (Chapter 4) ${ }^{51}$ Second, the parameterised model is used for scenario studies simulating closed systems, where microplastic dilutes chemical concentrations. Such systems mimic laboratory test systems, field enclosures or 'hot spots' where microplastic concentrations are exceptionally high. Third, the model is used for scenario studies simulating open systems, where aqueous phase and sediment PCB concentrations are only marginally affected by the presence of microplastic. These scenarios mimic oceanic systems where microplastic is much more diluted. ${ }^{19}$ Aim of the presented model is to outline the processes, then quantify them in the form of a tentative mass balance model that is consistent with available empirical data to capture the key processes and dependencies.

\subsection{Modeling approach}

\subsubsection{General biodynamic model for POP uptake by aquatic organisms in an environment containing plastic}

Following traditional bioaccumulation models, ${ }^{218-221}$ bioaccumulation of PCBs $\left(d C_{B, t} / d t\right)$ from an environment containing plastic is modeled as a mass balance of uptake and loss processes (Fig. A5.1), to which a novel term for PCB transfer to and from plastic is added (units are provided in Table 5.1):

$$
\frac{d C_{B, t}}{d t}=k_{d e r m} C_{w}+I R_{t}\left(S_{S E D} a_{S E D} C_{S E D}+S_{P L} C_{P L R, t}\right)-k_{l o s s} C_{B, t}
$$

In Eq. 5.1 and below, time dependent variables are indicated with subscript ' $t$ '. The first term in Eq. 5.1, quantifies dermal uptake from ambient water by passive partitioning. The second term quantifies uptake from ingested sediment and exchange with plastic particles. The third term quantifies overall loss due to elimination and egestion. The first and third term are parameterised following traditional approaches, ${ }^{219,221}$ where $C_{w}$ is the concentration in the ambient water and $k_{\text {derm }}$ and $k_{\text {loss }}$ are first order rate constants for dermal uptake 
and overall loss. In the second term, $I R_{t}$ represents total particle mass ingested per unit of time, $a_{S E D}$ is the absorption efficiency from sediment, $S_{S E D}$ and $S_{P L}$ are the mass fractions of sediment and plastic in ingested material respectively $\left(S_{S E D}+S_{P L}=1\right)$ and $C_{S E D}$ is the PCB concentration in sediment. The product $a_{S E D} \times C_{S E D}$ quantifies the contaminant concentration that is transferred from sediment to organism during gut passage. The transferred concentration from plastic, $C_{P L R, t}$, is a novel plastic-specific term in this type of models. Absorption from plastic particles is calculated in another way than absorption from sediment, which is why the product $S_{P L} C_{P L R, t}$ in Eq. 5.1 is separated from that for sediment particles. Plastic particles are assumed not to degrade inside the organism, but pass through the gut after which they are defecated. This allows for reversible exchange of POPs between plastic particle and biota lipids during gut passage. Consequently, the absorption efficiency for plastic particles is dependent on the concentration gradient between ingested plastic and biota lipids, plastic-lipid forward and backward exchange transport rate constants and duration of exchange, which can be defined as the gut residence time $\left(G R T_{t}\right)$. The concentrations in the biota $\left(C_{B, t}\right)$ change over time, whereas the gut residence time $\left(G R T_{t}\right)$ may increase with growth and thus with time. Consequently, absorption from plastic has to account for both effects and is supposed to change over time, unless a steady-state is reached. For calculation of $C_{P L R, t}$ we approximate exchange of POPs between plastic and lipids as a first order reversible process $\mathrm{C}_{\mathrm{PL}, \mathrm{t}} \leftrightarrow \mathrm{C}_{\mathrm{L}, \mathrm{t}}$ with forward and backward rate constants $k_{1}$ and $k_{2}$ :

$\frac{d C_{P L, t_{G P}}}{d t_{G P}}=k_{2} C_{L, t_{G P}}-k_{1} C_{P L, t_{G P}}$

in which $C_{P L, t_{G P}}$ and $C_{L, t_{G P}}$ are concentrations in plastic and lipids during gut passage (GP), respectively. Time $t$ in Eq. 5.2 is elapsed time during gut passage $\left(0<\mathrm{t}_{\mathrm{GP}}<G R T_{t}\right.$. $)$ and therefore carries a subscript 'GP' to distinguish this variable from bioaccumulation time in Eq. 5.1. With $C_{P L}^{I n g}$ and $C_{L}^{I n g}$ are the concentrations of the POP in the ingested plastic particle and the biota lipids at the moment of ingestion (i.e. at $\mathrm{t}_{\mathrm{GP}}=0$ and $C_{L, t}^{I n g}=C_{B, t} / f_{\text {lip }}$ ) and $C_{P L R, t_{G P}}$ is the net concentration originally in the plastic that has been removed (absorbed) from the plastic (i.e. $C_{P L, t_{G P}}=C_{P L}^{I n g}-C_{P L R, t_{G P}}$ and $C_{L, t_{G P}}=$ $\left.C_{L}^{I n g}+\frac{M_{P L}}{M_{L}} C_{P L R, t_{G P}}\right)$, Eq. 5.2 can be rewritten as: ${ }^{222}$

$\frac{d C_{P L R, t_{G P}}}{d t_{G P}}=k_{1}\left(C_{P L}^{I n g}-C_{P L R, t_{G P}}\right)-k_{2}\left(C_{L}^{I n g}+\frac{M_{P L}}{M_{L}} C_{P L R, t_{G P}}\right)$ 
which can be solved to yield

$C_{P L R, t}=\frac{k_{1} C_{P L}^{I n g}-k_{2} C_{L, t}^{I n g}}{k_{1}+\frac{M_{P L}}{M_{L}} k_{2}}\left(1-e^{-\left(k_{1}+\frac{M_{P L}}{M_{L}} k_{2}\right) G R T_{t}}\right)$

in which $\mathrm{M}_{\mathrm{PL}} / \mathrm{M}_{\mathrm{L}}$ is the ratio of plastic and lipid mass in the organism. For a detailed explanation of Equations 5.2-5.4, see the Appendix. In Eq. 4, gut passage time $\left(\mathrm{t}_{\mathrm{GP}}\right)$ is replaced by the fixed value $G R T_{t}$, the actual gut residence time of the plastic particles in the organism at bioaccumulation time t. The variables $C_{P L R, t}, C_{L, t}^{I n g}$ and $G R T_{t}$ now carry the subscript ' $\mathrm{t}$ ' because they depend on bioaccumulation time ' $\mathrm{t}$ '. The variable $C_{P L R, t}$ for plastic is analogous to the product $a_{S E D} \times C_{S E D}$ for sediment and can be used directly in Eq. 5.1 (i.e. without a separate absorption efficiency for plastic). However, to enable comparison with $a_{S E D}$ an absorption efficiency $a_{P L, t}$ for plastic can be calculated as the net concentration that has been removed from the plastic by gut passage $\left(C_{P L R, t}\right)$ divided by the concentration at ingestion $\left(C_{P L}^{I n g}\right)$ :

$a_{P L, t}=\frac{C_{P L R, t}}{C_{P L}^{I n g}}$

This assumes that the concentrations in Eq. 5.5 are larger than zero and that $C_{P L R, t} \leq C_{P L}^{I n g}$. Equations 5.4 and 5.5 quantify how the absorption efficiency from plastic depends on concentrations in plastic and organism lipids, kinetic constants and gut residence time. As long as $k_{1} C_{P L}^{I n g}>k_{2} C_{L, t}^{I n g}$ (Eq. 5.4), the absorption efficiency is positive and the organism acquires POPs from the plastic. It follows from Eq. 5.2 that the ratio $k_{1} / k_{2}\left(C_{L} / C_{P L}\right.$ at equilibrium) is a lipid-plastic partition coefficient, $K_{P L I P}$. If for instance, $K_{P L I P}$ is equal to 1 , transport from plastic to lipids occurs as long as the concentration in plastic is higher than that in the lipids (carrier mechanism). However, if $k_{1} C_{P L}^{I n g}<$ $k_{2} C_{L, t}^{I n g}$ (Eq. 5.4), mass transfer is reversed and plastic ingestion effectively leads to depuration of POPs from the organism (cleaning mechanism). Note that Eq. 5.4 is general for $C_{P L}^{I n g}$ and $C_{L, t}^{I n g}$ regardless of initial conditions. This way, Eq. 5.1-5.4 can simulate increased bioaccumulation by plastic as well as cleaning by plastic. Reversible mass transfer between plastic and biota lipids (plastic $\leftrightarrow$ lipids, Eq. 5.2-5.4) simplifies a number of transport steps that can be assumed to occur in series. Consequently, the simplification requires one of the internal transport resistances to dominate, which will be motivated below. 
Chapter 5

Table 5.1. Symbols and units. ${ }^{a)}$

\begin{tabular}{|c|c|}
\hline$\delta$ & $=$ gut content's density $\left(\mathrm{g} / \mathrm{mm}^{3}\right)$ \\
\hline asED & $=$ absorption efficiency from sediment (-) \\
\hline asED,L & $=$ absorption efficiency per mm worm length $\left(\mathrm{mm}^{-1}\right)$ \\
\hline aPL & $=$ absorption efficiency from plastic $(-)$ \\
\hline $\mathrm{C}_{\mathrm{B}, \mathrm{t}}$ & $=$ Concentration in biota $(\mu \mathrm{g} / \mathrm{g} \mathrm{DW})$ \\
\hline $\mathrm{C}_{\mathrm{L}, \mathrm{t}}$ & $\begin{array}{l}=\text { Lipid normalised concentration in biota }(\mu \mathrm{g} / \mathrm{g} \mathrm{DW}) . \mathrm{C}_{\mathrm{L}}^{0} \text { is } \\
\text { the concentration at } t=0 . \mathrm{C}_{\mathrm{L}, \mathrm{t}}^{\text {Ing }} \text { is the concentration at the } \\
\text { time of plastic ingestion. }\end{array}$ \\
\hline $\mathrm{C}_{\mathrm{PL}}$ Ing & $\begin{array}{l}=\text { concentration in plastic (outside worm and at time of } \\
\text { ingestion by worm) }(\mu \mathrm{g} / \mathrm{g})\end{array}$ \\
\hline $\mathrm{C}_{\mathrm{PLR}, \mathrm{t}}$ & $\begin{array}{l}=\text { concentration in plastic that has been absorbed by the } \\
\text { worm, referenced to plastic mass }(\mu \mathrm{g} / \mathrm{g})\end{array}$ \\
\hline $\mathrm{C}_{\mathrm{SED}}$ & $=$ concentration in sediment $(\mu \mathrm{g} / \mathrm{g}$ sediment $)$ \\
\hline $\mathrm{C}_{\mathrm{w}}$ & $=$ concentration in water $(\mu \mathrm{g} / \mathrm{L})$ \\
\hline $\mathrm{D}$ & $=$ POP polymer diffusivity $\left(\mathrm{m}^{2} / \mathrm{d}\right)$ \\
\hline$f_{O C}$ & $=$ sediment organic carbon fraction $(-)$ \\
\hline$f_{\text {OM }}$ & $=$ sediment organic matter fraction $(-)$ \\
\hline $\mathrm{GRT}_{\mathrm{t}}$ & $=$ gut residence time $(\mathrm{d})$ \\
\hline $\mathrm{IR}_{\mathrm{t}}$ & $=$ ingestion rate (g/g DW per day) \\
\hline $\mathrm{k}_{1}$ & $\begin{array}{l}=\text { apparent first order rate constant for plastic to lipid } \\
\text { transport }\left(\mathrm{d}^{-1}\right)\end{array}$ \\
\hline $\mathrm{k}_{2}$ & $\begin{array}{l}=\text { apparent first order rate constant for lipid to plastic } \\
\text { transport }\left(\mathrm{d}^{-1}\right)\end{array}$ \\
\hline $\mathrm{k}_{\mathrm{derm}}$ & $=$ rate constant for uptake from water $\left(\mathrm{L} / \mathrm{g} \mathrm{DW} \mathrm{d}^{-1}\right)$ \\
\hline $\mathrm{k}_{\text {loss }}$ & $=$ loss rate constant $\left(\mathrm{g} / \mathrm{g} \mathrm{DW} \mathrm{d}^{-1}\right)$ \\
\hline
\end{tabular}


Table 5.1. Continued.

\begin{tabular}{|c|c|}
\hline $\mathrm{K}_{\mathrm{OC}}$ & $=$ sediment organic carbon - water partition coefficient $(\mathrm{L} / \mathrm{kg})$ \\
\hline Kow & $=$ octanol water partition coefficient (-) \\
\hline $\mathrm{K}_{\mathrm{P}}$ & $=$ sediment-water partition coefficient $(\mathrm{L} / \mathrm{kg})$ \\
\hline $\mathrm{K}_{\text {PLIP }}$ & $\begin{array}{l}\text { = ratio of lipid-water and plastic-water equilibrium constant } \\
(-)\end{array}$ \\
\hline $\mathrm{L}_{\mathrm{t}}$ & $=$ length of the worm $(\mathrm{mm})$ \\
\hline $\mathrm{M}_{\mathrm{L}}$ & $=$ mass of lipids in worm (g DW) \\
\hline $\mathrm{M}_{\mathrm{PL}}$ & $=$ mass of plastic in worm (g DW). \\
\hline $\mathrm{M}_{\mathrm{PS}}$ & $=$ mass of plastic in bioassay system (kg DW) \\
\hline $\mathrm{M}_{\mathrm{SED}}$ & $=$ mass of sediment in bioassay system $(\mathrm{kg} \mathrm{DW})$ \\
\hline Q & $=$ volumetric flow rate ingested particles $\left(\mathrm{mm}^{3} / \mathrm{d}\right)$ \\
\hline $\mathrm{r}_{\mathrm{P}}$ & $=$ radius of plastic particle $(\mathrm{m})$ \\
\hline $\mathrm{r}_{\mathrm{t}}$ & $=$ radius of the worm $(\mathrm{mm})$ \\
\hline $\mathrm{S}_{\mathrm{SED}}$ & $=$ mass fraction of sediment particles ingested $(-)$ \\
\hline $\mathrm{S}_{\mathrm{PL}}$ & $=$ mass fraction of plastic particles ingested $(-)$ \\
\hline $\mathrm{V}_{\text {gut }}$ & $=$ lugworm gut volume $\left(\mathrm{mm}^{3}\right)$ \\
\hline $\mathrm{W}_{\mathrm{t}}$ & $=$ dry weight of organism $(\mathrm{g})$ \\
\hline
\end{tabular}

${ }^{a}$ Source of parameter values and additional equations are defined in Table A5.1.

\subsubsection{Modeling PCB transfer to $A$. marina in closed bioassays} with polystyrene

Parameterization. We first parameterised the model to simulate results from previously published bioassays with the lugworm A. marina (Chapter 4). ${ }^{51}$ In these assays natural PCB contaminated sediment was pre-equilibrated with 0.4 - 1.3 mm PS microplastic particles for 6 weeks on a roller bank (Chapter 4), ${ }^{51}$ to obtain PS sediment concentrations of $0,0.074,0.74$ and $7.4 \%$ dry weight, and PS in sorption equilibrium with the sediment. A. marina individuals were added to replicated treatments and exposed for $28 \mathrm{~d}$. Lugworm PCB 
concentrations and weights were known at start and after exposure (Chapter 4). ${ }^{51}$ Six weeks is long enough to equilibrate PCBs in $\sim 1 \mathrm{~mm}$ microplastic particles, either based on reported PCB diffusivities of $2-4 \times 10^{-10} \mathrm{~cm}^{2} / \mathrm{s}$ in $\mathrm{PS}^{188}$ or on undisturbed boundary layer (UBL) transfer rates in the vigorously agitated dense sediment slurry. ${ }^{223,224}$ This means that for $C_{w}$ in the first term in Eq. 5.1 we can write:

$$
C_{w}=\frac{M_{S E D} C_{S E D}}{V_{w}+M_{P S} K_{P L}+M_{S E D} K_{P}}
$$

with $\mathrm{C}_{\mathrm{SED}}$ is the measured $\mathrm{PCB}$ concentration in sediment, $\mathrm{M}_{\mathrm{SED}}$ and $\mathrm{M}_{\mathrm{PS}}$ known (added) masses of sediment and PS in the experimental system, $V_{w}$ is known water volume and $\mathrm{K}_{\mathrm{P}}$ and $\mathrm{K}_{\mathrm{PL}}$ are sediment-water and plastic-water partition coefficients. $K_{\mathrm{P}}$ values were estimated from measured organic carbon content and $\log K_{O W}$ using the well-established relationship $\log K_{O C}=\log K_{O W^{-}}$ 0.48 by Seth et al. ${ }^{225} \mathrm{~K}_{\mathrm{PL}}$ values for PS $(\sim 100-1000 \mathrm{~L} / \mathrm{kg})$ were taken from the literature. ${ }^{188}$ Eq. 5.6 shows how in a closed system, addition of plastic may decrease the initial exposure concentration $C_{w}$, an effect that is larger if more plastic is added and if the PCBs have a higher affinity for plastic than for sediment ${ }^{20}$ (dilution mechanism). Because of the excess of PCB contaminated sediment compared to mass of worms and plastic, uptake by the worms can be assumed not to affect sediment PCB concentrations in these bioassays. Furthermore, reported fast- and slow desorption half-lives for PCBs are short $\left(0.4 \text { to } 4 \mathrm{~d}^{-1}\right)^{(186,187)}$ so that we can assume pore water concentration $\left(C_{w}\right)$ not to vary over time after pre-equilibration. Similarly, we can assume that PCB concentrations in PS particles $\left(C_{P L}\right)$ stayed constant in time due to the excess of contaminated sediment. The dermal uptake rate constant $k_{\text {derm }}$ was estimated using allometric relationships ${ }^{221}$ (detailed in the Appendix, Table A5.1).

In the second term of Eq. 5.1, the ingestion rate $I R_{t}\left(\mathrm{~g} / \mathrm{g} \mathrm{DW} \mathrm{d}^{-1}\right)$ quantified following Cammen, ${ }^{204}$ increases with growth of the organism but decreases with sediment organic matter (food) content $f_{\text {OM }}$ as can be approached by: ${ }^{204}$

$$
I R_{t}=0.001 \times 0.435 \times\left(1000 \times W_{t}\right)^{-0.771} \times f_{O M}^{-0.92} / W
$$

with $W_{t}$ the dry weight of the organism as a function of time. The factors 1000 and 0.001 relate to the fact that Cammen defined IR as mg sediment ingested per day, and organism weight in $\mathrm{mg}$, whereas we used organism weight in $\mathrm{g}$. The dependency on $f_{O M}$ allows organisms to increase their ingestion rate if the nutritional status of the sediment is lower, for instance caused by low organic 
matter content or by dilution of sediment with plastic particles. The absorption efficiency from sediment $a_{S E D, t}\left(0 \leq a_{S E D, t} \leq 1\right)$ is supposed to increase with length of the worm: ${ }^{218}$

$$
a_{S E D, t}=a_{S E D, L} \times L_{t}
$$

This assumes that sediment particles in worms have a fixed absorption efficiency per unit of length, $a_{S E D, L} . L_{t}$ is the length of the worms which is supposed to increase over time, or to be constant if growth is limited. Length of the worms were not observed to change during the modeled bioassays, so a default value $a_{S E D, t}=0.15$ was used. ${ }^{218,221} S_{S E D}$ is calculated as $M_{S E D} /\left(M_{S E D}+M_{P L}\right)$, and $S_{P L}$ is $1-S_{S E D}$. For the calculation of $C_{P L R, t}$ using Eq. 5.4, estimates for the rate constants $k_{1}$ and $k_{2}$, for chemical concentration in the ingested plastic, $C_{P L}$ and for the gut residence time $G R T_{t}$ are required. There are, to our knowledge, no published rate constants for the release of PCBs from plastic particles in the lugworm. Here, we follow earlier reasoning that polymer diffusion most probably will be rate limiting in the exchange of POPs between plastic in the gut and biota lipids. ${ }^{20,21}$ For POPs with $\log \mathrm{K}_{\mathrm{Ow}}>4$ such as PCBs, polymer-water exchange by planar passive samplers deployed in surface water is usually rate limited by transfer across the UBL. ${ }^{217,226}$ However, for plastic spheres in the gut of A. marina this transfer will be much faster, due to (a) the spherical shape of the UBL leading to a steeper concentration gradient across the UBL, ${ }^{227}$ (b) solubility increase in the gut, ${ }^{228}$ (c) DOC facilitated PCB transport across the UBL, ${ }^{229}$ and (d) frequent direct contact between plastic and sediment particles in the gut, leading to decreased thickness of the UBL. ${ }^{224}$ A quantitative analysis of these processes with additional references is provided in the Appendix. The calculations show that UBL transfer for micro- and nanoplastic is so fast that polymer diffusion is expected to be rate limiting (Table A5.2, A5.3, Fig. A5.2). In case of polymer diffusion, first order rate constants can be estimated using the established approximation $k_{1} \approx 23 D / r_{P}^{2}$, (227) in which $r_{P}$ is particle radius and $D$ is polymer diffusivity. Using reported PCB diffusivities in pristine PS at room temperature of $(1.7-3.5) \times 10^{-9} \mathrm{~m}^{2} / \mathrm{d},{ }^{(188)}$ this yields $k_{l}$ values of $0.1-2 \mathrm{~d}^{-1}$ for 0.4-1.3 mm plastic particles and $>100 \mathrm{~d}^{-1}$ for $0.1-1 \mu \mathrm{m}$ micro- and nanoplastic particles. Such estimates however, can be considered conservative. Weathered microplastic will be brittle ${ }^{215}$ and can be expected to have higher release rates in the gut than the pristine polymer. ${ }^{21}$ At higher temperatures as in warm blooded animals, transfer rates may even be higher. ${ }^{221}$ Recent data showed release rates of phenanthrene from microplastic in artificial gut fluid, ranging 
between 2-20 d $\mathrm{d}^{-1} \cdot{ }^{(20,230)}$ Therefore, by evaluating a range of $k_{1}$ values $\left(1-10 \mathrm{~d}^{-}\right.$ ${ }^{1}$ ) in different model runs, we cover the effect of a wide range of parameters on the diffusion rates from microplastic and are able to elucidate the influence and importance of this parameter on the overall uptake of PCBs from plastic by A. marina. Based on Eq. 5.2, reversed rate constants $k_{2}$ were calculated as $k_{1} / K_{P L I P}$, with $K_{P L I P}$ is the ratio between the lipid water partition coefficient ( $K_{L I P}$, Table A5.5) and $K_{P L}$. For $\mathrm{C}_{\mathrm{PL}}$, we used the equilibrium condition $C_{P L}=K_{P L} C_{w}$, in which $C_{w}$ follows from Eq. 5.6 and the $K_{P L}$ value for PS was taken from the literature. ${ }^{188}$ It has been described that the gut of A. marina is segmented, which may lead to different exchange kinetics of sediment bound chemicals in different parts of the gut. ${ }^{231}$ Here, an average gut residence time $G R T_{t}$ is defined, which depends on the volume of the gut $V_{\text {gut }}$ and the volumetric flow rate of ingested particles $(Q)$. If the organism grows, $G R T_{t}$ changes over time according to: 220

$$
G R T_{t}=\frac{V_{g u t}}{Q}=\frac{\pi \times r_{t}^{2} \times L_{t} \times 0.3}{\frac{I R_{t}}{\delta} W_{t}}
$$

with $r_{t}, L_{t}$ and $W_{t}$ measured radius, length and dry weight of the worm (g DW), which may vary over time. In the denominator of Eq. 5.9, $W_{t}$ in $\mathrm{g} \mathrm{DW}$, multiplied with $I R_{t}$ in $\mathrm{g} / \mathrm{g}$ DW per day, gives the ingested mass per individual worm per day. Divided by the density of the ingested particles $\delta$, calculated as the weighted average of sediment and plastic particle density, this gives the volume ingested per worm per day (volumetric flow rate, Q). Because density of plastic is lower than that of sediment, $\delta$ is calculated to decrease with increasing plastic content of sediment. The overall loss rate constant $k_{\text {loss }}$ is estimated using previously published data with respect to its dependence on $\log K_{o w}{ }^{(221)}$ (Table A5.1). $\log K_{\text {ow }}$ values for the estimation of $K_{P}, k_{\text {derm }}$ and $k_{\text {loss }}$ were taken from van Noort et al. ${ }^{232}$ Lipid content of $A$. marina for the sampling site was taken from a data compilation by Hauck et al., which averaged $5.2 \pm$ $1.2(\mathrm{n}=25){ }^{233}$

Model evaluation. The model was evaluated using data previously published (Chapter 4). ${ }^{51}$ An overview of the raw data and parameter values is provided in the Appendix (Table A5.4, A5.5). All parameters were inferred from the experimental set up or were independently measured or estimated as described above. Modeled tissue concentrations were compared to measured tissue concentrations. Because PCB congener concentrations in the sediment were different, modeled and measured tissue concentrations were scaled to PCB 
concentration in the sediment (i.e. as biota sediment accumulation factors, BSAF).

\subsubsection{Scenario studies for PCB transfer to $A$. marina in closed bioassays with polyethylene}

To evaluate scenarios for a plastic type for which PCBs have a high affinity, the above implementation for PS was adapted for low density polyethylene (PE). This means that all parameters and variables remained the same except those for the plastic, that is, $K_{P L}$ and the ad- and desorption rate constants ( $k_{l}$, $k_{2}$ ) in the gut. PE - water partition coefficients were calculated as $\log K_{P E}=1.18 \times \log K_{O W^{-}}-1.26 .^{(216,217)}$ Reported PCB diffusivities in PE range from $(1.7-34) \times 10^{-9} \mathrm{~m}^{2} / \mathrm{d},{ }^{(217,234)}$ which yields $k_{l}$ values of $0.1-20 \mathrm{~d}^{-1}$ for particles of $0.4-1.3 \mathrm{~mm}$. Similar ranges up to $4-12 \mathrm{~d}^{-1}$ were reported for desorption of phenanthrene from PE in seawater and seawater to which surfactant was added to mimic conditions in the gut. ${ }^{20,230}$ Based on these data we selected a range of $k_{l}=1$ and $10 \mathrm{~d}^{-1}$ for our scenarios.

\subsubsection{Scenario studies for PCB transfer to $A$. marina in open marine systems for micro- and nanoplastic}

PCB concentrations after $28 \mathrm{~d}$ bioaccumulation were calculated for scenarios with and without plastic present in the sediment, with PE as a model for high affinity plastic. Gouin et al. ${ }^{19}$ have shown that plastic will not dilute POP concentrations in sediment and seawater, due to the large excess of sediment and water compared to plastic. This implies that $C_{W}$ in Eq. $5.1 \mathrm{can}$ be entered as a constant instead of being dependent on the plastic concentration (Eq. 5.6). Assuming partitioning equilibrium at field relevant time scales, ${ }^{215} C_{S E D}$ and $C_{P L}$ are calculated from $C_{S E D}=K_{P} C_{W}$ and $C_{P L}=K_{P L} C_{W}$ respectively. Sedimentwater partitioning $\left(K_{P}\right)$ was based on the regression with $\log K_{O W}$ provided by Seth et al. ${ }^{225}$ For time zero, lugworm lipids are also assumed to be at equilibrium with pore water concentrations following $C_{L, t=0} \approx K_{L I P} C_{W},{ }^{(221)}$ after which the model calculates a new steady-state taking into account ingestion of sediment and PE. This follows the logic that larvae are already in partitioning equilibrium with their environment after hatching and at some point start ingesting sediment with plastic. Nano- and micro sized particles $(100 \mathrm{~nm}, 1$ $\mu \mathrm{m}$ and $1 \mathrm{~mm}$ ) of the same plastic polymer type were modeled assuming uptake in the gut, with equal partitioning constants but size dependent first order rate constant $k_{1} \approx 23 D / r_{P}^{2}$. Effects of $0.01,0.1,1$ and $10 \mathrm{wt} \% \mathrm{PE}$ were calculated as increase of steady-state bioaccumulation due to plastic addition, i.e. by dividing the modeled tissue concentrations in presence of $\mathrm{PE}$, by the 
modeled tissue concentrations without PE present. Simulations covered POPs with $\log K_{O W}$ between 4 and 8 .

\subsection{Results and Discussion}

\subsubsection{Modeling PCB transfer to $A$. marina in closed bioassays with polystyrene}

The model with all parameters at their chemically or biologically realistic default value was consistent with measured BSAFs within a factor of three (Fig. A5.3). A factor of three is satisfactory given reported ranges of agreement for traditional bioaccumulation models of about an order of magnitude. For PCB105 as an example, initial lugworm tissue concentrations increase till a plateau was reached at about $1.2 \mu \mathrm{g} / \mathrm{kg}$ dry weight (Fig. A5.4). Calculated tissue concentrations after $28 \mathrm{~d}$ show a marginal increase of bioaccumulation due to presence of PS in the order of $7.4 \%>0.074 \%>0.74 \%$ PS in the sediment (Fig. A5.4). There is no consistent trend with PS concentration due to small differences in initial organism weight at start of the bioassays, which affect the ingestion rates through Eq. 5.7. If in the model one (average) weight for all individuals is used, the order in bioaccumulation is $7.4 \%>0.74 \%>0.074 \%$ PS (Fig. 5.1A). In terms of prediction, the calculated differences are too small to be meaningful, also considering parameter uncertainty and propagation of error in models with this number of parameters. ${ }^{197,219}$ The modeled increase in bioaccumulation, however, does qualitatively agree with the small increase in bioaccumulation observed (Chapter 4). ${ }^{51}$ (raw data provided in Table A5.4). Their BSAF values for PCB105, decreased slightly with increasing PS concentration. Besseling et al. (Chapter 4) $)^{51}$ observed treatment-specific changes in weight during the bioassays and argued that this may also have affected the fitness and/or lipid content of the lugworms thus leading to variations in PCB bioaccumulation not accounted for by the model.

The parameterised model now can be used to evaluate to what extent each of the postulated mechanisms contributes to bioaccumulation in the presence of PS. For PCB105 and the default exchange rate $k_{l}=10 \mathrm{~d}^{-1}$ as an example, modeled absorption efficiencies from PS (Eq. 5.5) decrease from $\sim 8 \%$ to less than zero in five days, which is different from the absorption efficiency from sediment, which is $15 \%$ by default ${ }^{221}$ (Fig. 5.1B). The initially positive PCB absorption efficiency from PS $\left(a_{P L}>0\right)$ implies a 'carrier' mechanism, which however turned into a 'cleaning' mechanism after 5 days. The decrease in absorption efficiency over time is caused by the bioaccumulation, that is, the 
increase in calculated $\mathrm{C}_{\mathrm{L}, \mathrm{t}}$ with time (Fig. 5.1A). An increase in $\mathrm{C}_{\mathrm{L}, \mathrm{t}}$ decreases the gradient between biota lipids and plastic $k_{1} C_{P L}^{I n g}-k_{2} C_{L, t}^{I n g}$, leading to a decrease in $\mathrm{C}_{\mathrm{PLR}, \mathrm{t}}$ and apL (Eq. 5.4). Calculated $G R T_{t}$ (Eq. 5.9) was 43 minutes, which fairly agrees to a similarly modeled range of 30-200 minutes during growth of the polychaete species $N$. arenaceodentata reported by Janssen $e t$ $a l .{ }^{218}$
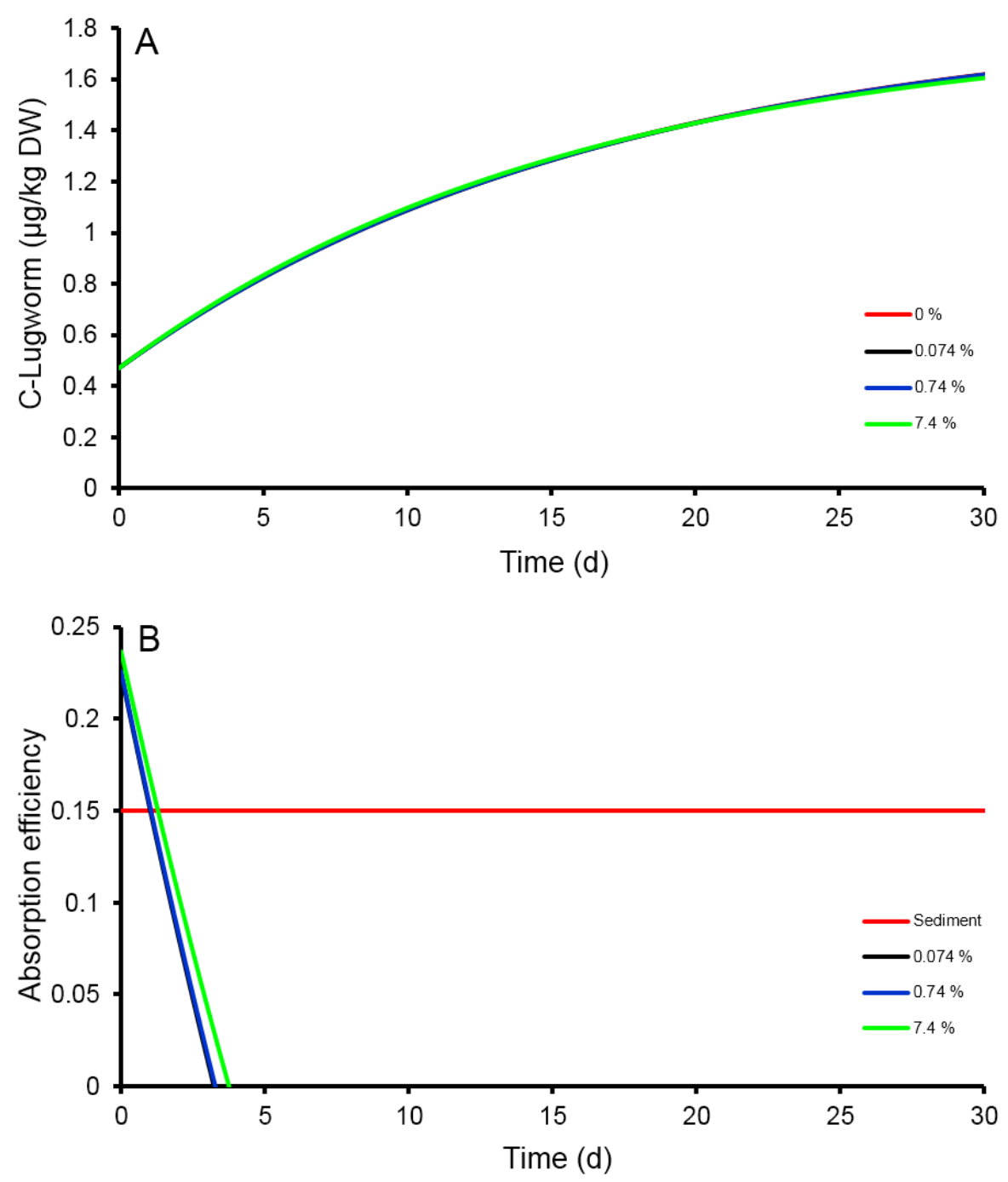

Figure 5.1. Simulated PCB105 concentration in lugworm tissue as a function of time in closed bioassay systems with polystyrene $(A)$, and absorption efficiencies from sediment and polystyrene $(B)$, for three polystyrene concentrations. Default exchange rate constant $k_{1}$ is 10 $d^{-1}$. 
The initially positive absorption efficiency for PS does not imply that PS was an important route for uptake of PCBs. If we express the contribution of the plastic term in Eq. 5.1 as a percentage of all uptake terms, it follows that even at a very high concentration of $7.4 \%$ PS, uptake by PS contributes for only $0.2 \%$ to total uptake (Fig. A5.5A). Main reason is not that PCB absorption from PS during the first days of gut passage is insignificant, but that the PCB concentrations in this low affinity polymer were too low to constitute a substantial pathway even if absorption would have been $100 \%$. This low relative contribution of PS in PCB transfer implies that the model outcomes are not sensitive to uncertainty in $K_{P L}$ or $k_{l}$. Further, this implies that for PS, also the 'carrier' mechanism contributed only to a minor extent to the bioaccumulation of PCB in the lugworm. Instead, the dominant terms in Eq. 5.1 were the terms for dermal uptake and uptake from sediment with approximately equal shares (Fig. A5.5A).

The model calculations as well as the experimental data (Chapter 4$)^{51}$ showed a small increase in bioaccumulation in the presence of PS. We inspected the individual terms in the model to identify the main driver for the increased bioaccumulation. It appears that addition of PS increased the modeled aqueous phase concentration $C_{w}$ and consequently the importance of dermal uptake. The increase in $C_{w}$ follows from the fact that PS replaces sediment in the mixture, whereas PS is a lower affinity sorbent for PCBs (i.e. lower $K_{P}$ ) than the sediment. Adding PS causes an overall decrease in the partition coefficient of the mixture, leading to a higher dermal uptake. A higher $C_{w}$ also causes a higher $C_{P L}^{I n g}\left(C_{P L}=K_{P L} C_{w}\right)$ and thus a higher initial gradient $k_{1} C_{P L}^{I n g}-k_{2} C_{L, t}^{I n g}$ in Eq. 5.4. Addition of PS, however, also leads to an increase in the time constant $k_{1}+\frac{M_{P L}}{M_{L}} k_{2}$, leading to a net decrease in absorption efficiency. Decreased partitioning to the mixture, however, decreases the ingestion term in Eq. 5.1, so there also is a counteracting mechanism. At equal ingestion rate IR, uptake by ingestion decreases with PS addition because the PCB concentration per unit of weight is lower for PS than for sediment. Theoretically this might be (partly) compensated by higher absorption efficiency from the plastic compared to sediment. However, the calculated efficiency apL is calculated to be much lower than asED during 28 days of bioaccumulation.

We argue that the theoretical model analysis provides a plausible explanation for the small increase in bioaccumulation observed by Besseling et al. $(\text { Chapter 4 })^{51}$ For all PS concentrations studied, two mechanisms play a role, 
with counteracting effects on overall uptake: an initial carrier mechanism through the plastic ingestion term in Eq. 5.1 that nevertheless decreased bioaccumulation by replacing the more efficient uptake from sediment ingestion, and a 'reversed dilution' mechanism, increasing dermal uptake due to increased concentrations in water. Given the biological variation in the bioassays, the small differences calculated for different PS concentrations could not be experimentally detected.

\subsubsection{PCB transfer to $A$. marina in closed bioassays with polyethylene}

For evaluation of bioaccumulation of POPs in closed system bioassays with PE, no experimental data are available. However, by introducing the $K_{P L}$ and $k_{l}$ estimates derived for PE (see also Table A5.5) a plausible scenario can be provided for PE. All organism conditions were kept the same, which assumes that chemical transport changes, but that biological effects of plastic particles as such remain the same as for PS. This also enables a direct comparison with the PS scenarios. The simulation for the control without PE is identical to that for the control without PS (compare 0\% Fig. 5.1A and 5.2A). For the PE scenarios, however, simulated bioaccumulation decreases substantially (Fig. 5.2A) and is much lower compared to the simulations for PS. Without PE, dermal transfer and uptake from sediment dominates uptake (Fig. A5.5B). With $0.074 \%$ PE, absorption is positive for the first 2 days of the simulation (Fig. 5.2B), which indicates that during this stage PE acts as a carrier for PCB105. However, the absorption efficiency rapidly decreases due to the decrease in the gradient between plastic and biota lipids. Despite the initial carrier mechanism, overall bioaccumulation is slightly lower, which is explained from a 'cleaning' mechanism acting after 2 days and a dilution of aqueous phase and sediment concentrations by the high affinity plastic PE (Eq. 5.6), which more than compensates for the initial carrier effect. Interestingly, at $0.74 \%$ the initial absorption efficiency already is negative, which is caused by a much stronger dilution at this higher PE dose (Fig. 5.2). This implies that PE effectively 'cleans' the organism for the entire simulation. Consequently, steady-state bioaccumulation at $0.74 \%$ is reduced by roughly a factor of three compared to the treatment without PE (Fig. 5.2A). For 7.4\% PE, initial pore water concentrations $C_{w}$ are even lower, such that the plastic term in Eq. 5.1 (i.e. $\left.C_{P L R, t}\right)$ also is negative already at start of bioaccumulation. This transport from biota lipids to PE more than compensates dermal uptake and uptake from ingested sediment, leading to net depuration of PCB from the organism and a 


\section{Chapter 5}

25 times lower steady-state concentration compared to the system without PE (Fig. 5.2A).
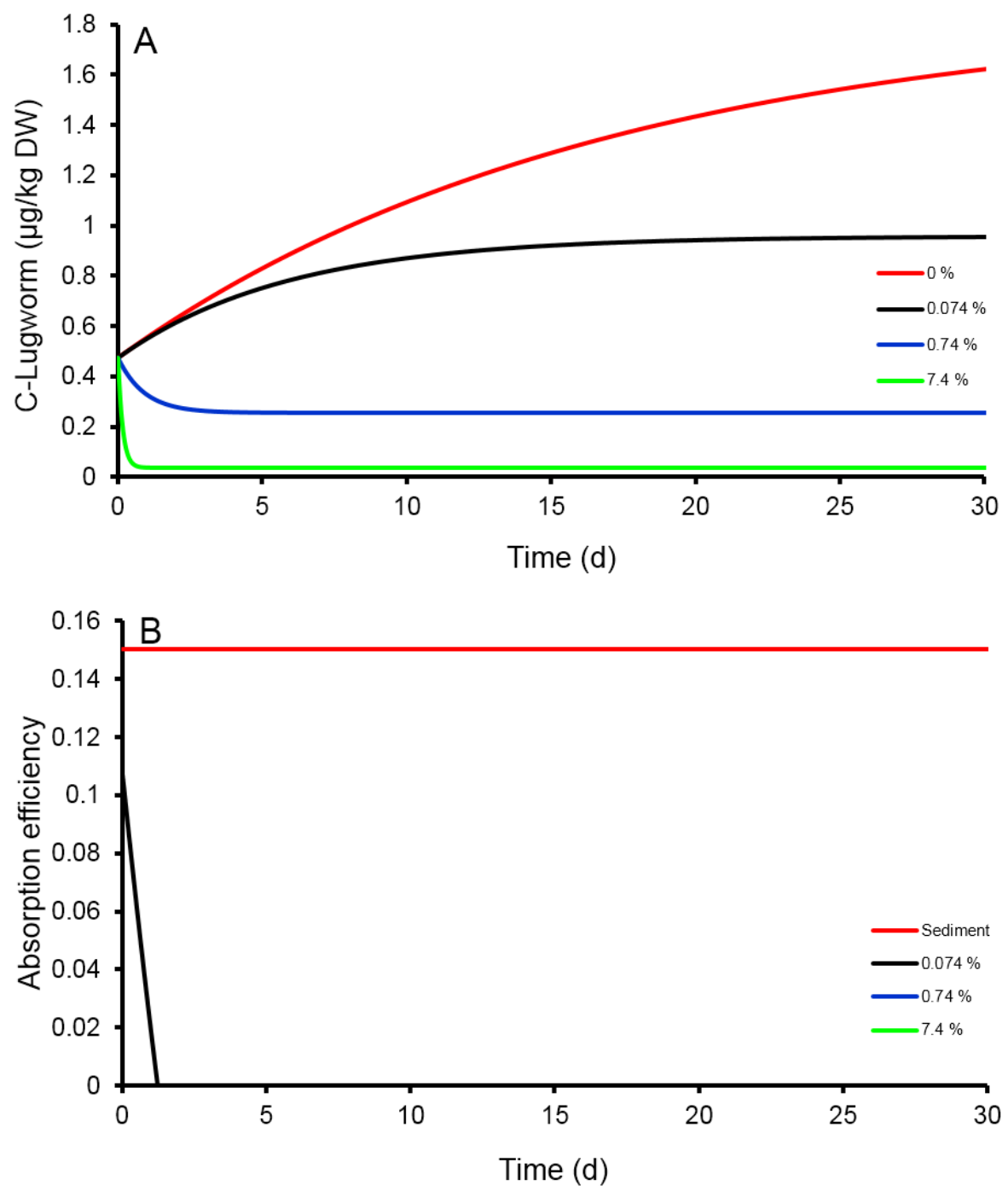

Figure 5.2. Simulated PCB105 concentration in lugworm tissue as a function of time in closed bioassay systems with polyethylene (A), and absorption efficiencies from sediment and polyethylene $(B)$, for three polystyrene concentrations. Default exchange rate constant $k_{1}$ is 10 $d^{-1}$. 
These features illustrate the trade-off between (a) strong dilution of initial PCB pore water concentrations leading to decreased exposure through dermal uptake, which however changes with sediment-plastic mass ratio (Eq. 5.6), and (b) a case specific role of PE as either carrier or cleaning phase dependent on the gradient between plastic and organism lipids that changes over time (Eq. 5.4). This PE case based on realistic laboratory bioassay conditions, illustrates that although the sub processes are well understood, the net impact of their simultaneous effects can be difficult to predict. This also illustrates the importance of models as condensed in Equations 5.1 to 5.6, which allow for a quantitative analysis of the sub processes.

\subsubsection{Modeling effects of plastic on bioaccumulation of PCBs in open systems}

Microplastic. Simulations for $\sim 1 \mathrm{~mm}$ PS showed negligible effects, that is, no more than a $1 \%$ decrease in bioaccumulation compared to sediment without PS, even with $k_{l}$ set to $10 \mathrm{~d}^{-1}$ and an unrealistically high concentration of $10 \%$ PS (data not shown). The decrease is consistent with a cleaning or sediment OM dilution mechanism because of the lower affinity of PS compared to sediment OM, as was discussed in the previous bioassay scenarios with PS. For $\sim 1 \mathrm{~mm}$ PE, however, steady-state bioaccumulation is predicted to decrease for $\log K_{O W}$ values higher than 5 or 6 (Fig. 5.3). The predicted relative decrease compared to sediment without $\mathrm{PE}$ is a factor of two and five for sediment PE concentrations of 0.1 , and 1 to $10 \%$, respectively.

The relative decrease is independent of $C_{w}$ and is fully caused by the negative plastic ingestion term, which reduces the relative importance of dermal uptake and uptake through sediment ingestion (Fig. A5.5C). This simulated negative effect of plastic ingestion on bioaccumulation can be explained as follows. Initially, biota lipids, pore water and plastic are in partitioning equilibrium, such that the gradient between plastic and biota lipids (Eq. 5.4) is zero. When feeding however, absorption of PCBs from the sediment causes a slowly increasing concentration in the biota lipids, leading to a negative gradient between plastic and lipids, i.e. $k_{1} C_{P L}^{I n g}-k_{2} C_{L, t}^{I n g}<0$. Plastic ingestion thus counteracts the biomagnification mechanism by attenuating the gradient, a mechanism recognized earlier by Gouin et al. ${ }^{19}$ If $K_{O W}$ is higher, the gradient is more negative because the initial concentration in biota lipids is higher. This explains that the effect is larger at higher $\log K_{O W}$. For comparison with Fig. 5.1 and 5.2, a simulation for PCB105 is provided in the Appendix (Fig. A5.6). Without chemical dilution by $\mathrm{PE}$ as in the closed bioassay scenario, PE no 
longer leads to steady-state bioaccumulation being less than in the control (Fig. A5.6). However, increasing PE concentrations attenuate biomagnification, apparently resorbing all PCBs originating from the sediment so that the concentration stays at the initial level.

For the scenario where $k_{l}$ is $1 \mathrm{~d}^{-1}$ the pattern is comparable but the predicted decrease in bioaccumulation is less $(10 \%$ decrease for $0.1 \% \mathrm{PE}$, about a factor of two decrease for 1 and 10\% PE, Fig. A5.7A). This is explained from the lower exchange rates at the same $G R T$, which results in a lower $a_{P L}$.

Nanoplastic. Plastic nanoparticles have been shown to be taken up by various marine organisms..$^{29,52}$ For instance, for the mussel, Wegner et al. ${ }^{52}$ showed that $30 \mathrm{~nm}$ nanoplastic particles in seawater were taken up as $\sim 1 \mu \mathrm{m}$ aggregates. At entrance of the gut, the aggregates may have slower release kinetics than the primary nanoparticles, yet still with $k_{l}>100 \mathrm{~d}^{-1}$ based on radial diffusion. For PE particles in the nano- and submicron particle size range of $<100$ to $1000 \mathrm{~nm}$, exchange of POPs like PCBs will be faster than for mm sized microplastic particles due to the high surface area and short diffusion path lengths $\left(k_{l}>>10 \mathrm{~d}^{-1}\right)$, theoretically leading to higher differences compared to sediment without PE. The extra decrease, however, was calculated to be marginal (0.5\%; Fig. A5.7B) compared to the PE effect that was calculated already for the PE microparticles (Fig. 5.3).

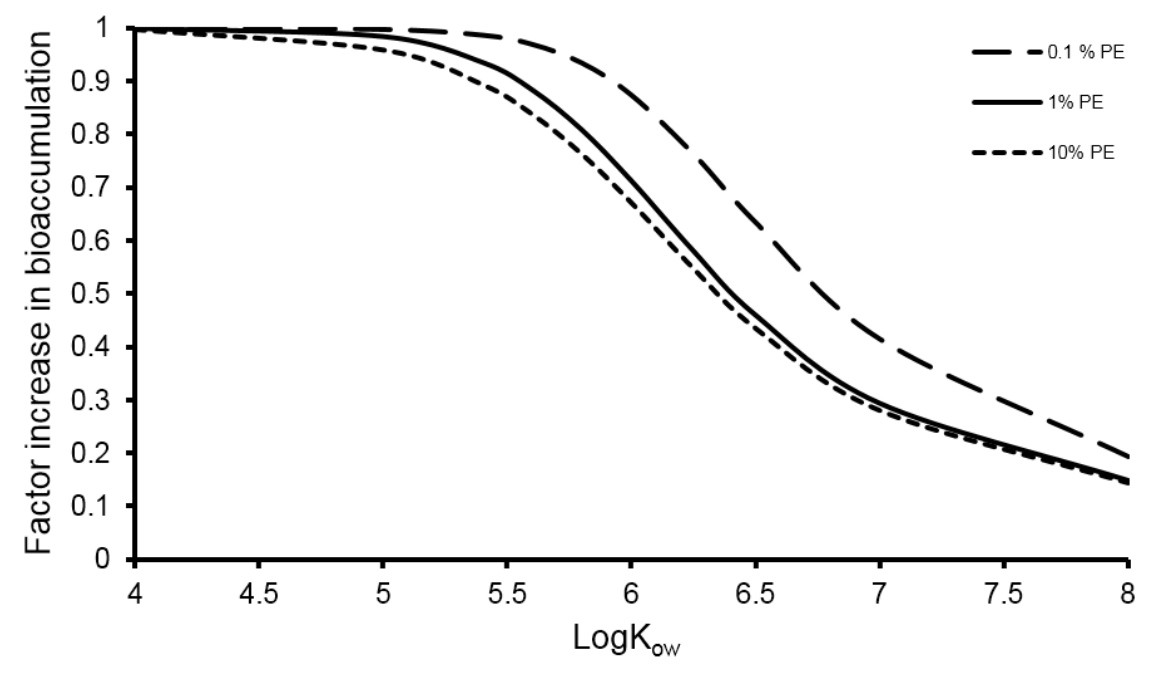

Figure 5.3. Steady-state bioaccumulation in the lugworm in sediment with plastic relative to a scenario without plastic for open marine systems, for 0.1, 1 and 10\% polyethylene (PE). Simulations are for microplastic particles with diameter $\sim 1 \mathrm{~mm}, k_{1}=10 \mathrm{~d}^{-1}$. 


\subsubsection{Implications for risk assessment}

The above sections show how process descriptions for sorption, bioaccumulation and polymer diffusion can be combined in a quantitative framework to estimate uptake of POPs through plastic ingestion. The framework is general and can be implemented for different combinations of plastic type, species and chemicals. As an example, we presented an implementation for the lugworm and showed that plastic ingestion may affect bioaccumulation for this species in marine systems. Scenario studies showed that the effects of plastic can be manifold, dependent on chemical and polymer properties, and species traits. Error propagation due to parameter uncertainty can be expected to be considerable. Using probabilistic modeling, Selck et $a l .{ }^{197}$ showed that the uncertainty in modeled bioaccumulation for benthic invertebrates was an order of magnitude. This implies that the effects calculated for the open ocean scenario are not to be considered statistically significant. Biological variability among species and individuals in the field also can be expected to be large. Given this variability, the differences in bioaccumulation will be insignificant or undetectable if the abundance of plastic is limited. The prognostic assessment in this chapter showed that small effects might occur at concentrations of 1 to $10 \%$ PE in sediment (Fig. 5.3, A5.6, A5.7B), which is still a factor $100-1000$ higher than the highest plastic concentrations reported in marine sediments today $(81 \mathrm{mg} / \mathrm{kg})^{(165)}$. Given this difference and the small magnitude of the calculated effects, we conclude that the role of plastic in bioaccumulation of POPs is scientifically interesting but probably not very relevant from a risk assessment perspective. This, however, may be different for chemicals for which plastic is the main source of bioaccumulation like additives leaching from microplastic.

\section{Acknowledgements}

We thank three anonymous reviewers for their valuable comments on the manuscript. 


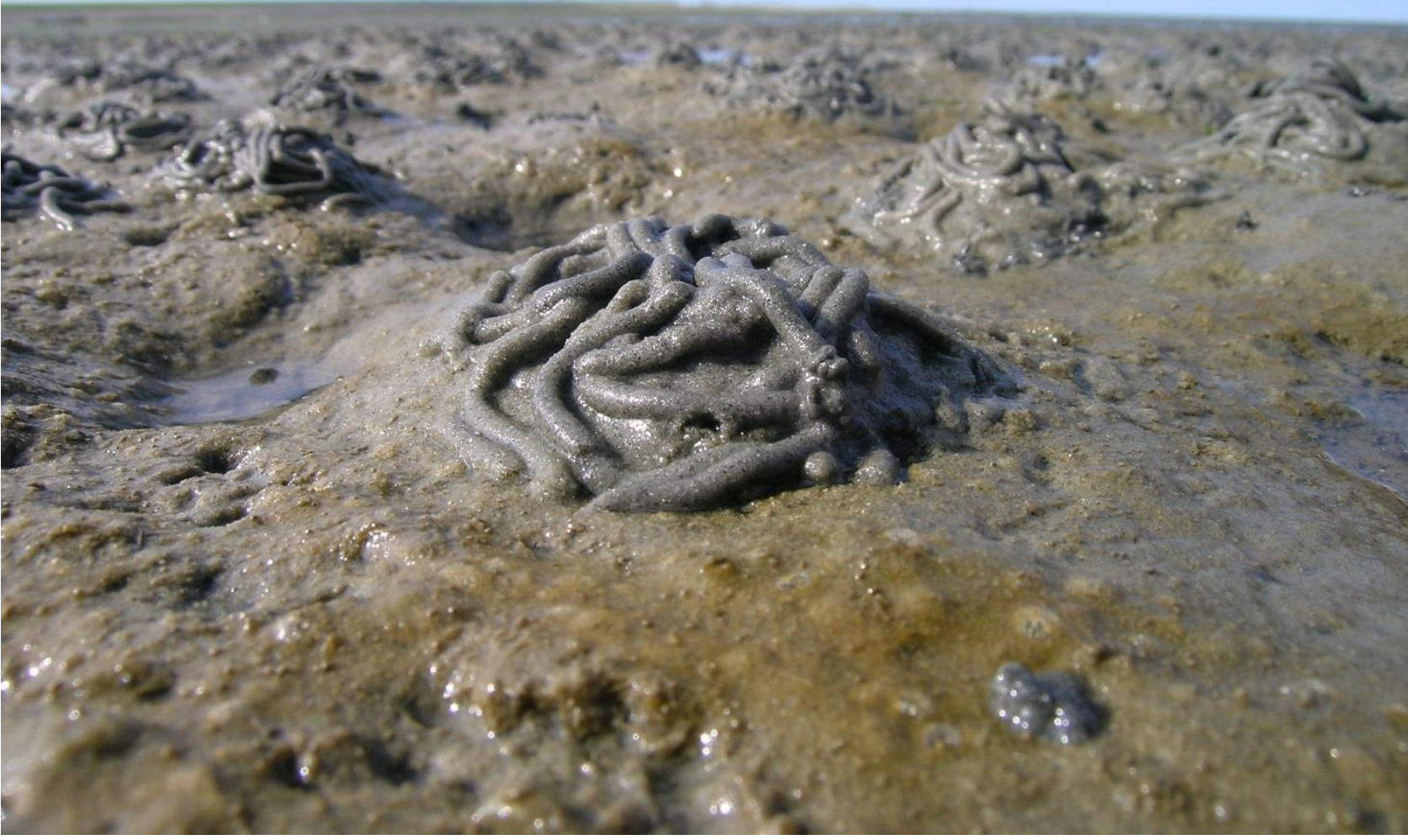




\title{
The effect of microplastic on the uptake of chemicals by the lugworm Arenicola marina (L.) under environmentally relevant exposure conditions
}

\author{
Published as: Besseling, E., Foekema, E.M., van den Heuvel-Greve, M.J., \\ Koelmans, A.A., 2017. The effect of microplastic on chemical uptake by the \\ lugworm Arenicola marina (L.) under environmentally relevant conditions. \\ Environmental Science \& Technology 51, 8795-8804
}

\begin{abstract}
It has been hypothesized that ingestion of microplastic increases exposure of aquatic organisms to hydrophobic contaminants. To date, most laboratory studies investigated chemical transfer from ingested microplastic without taking other exposure pathways into account. Therefore we studied the effect of polyethylene (PE) microplastic in sediment on PCB uptake by Arenicola marina as a model species, quantifying uptake fluxes from all natural exposure pathways. PCB concentrations in sediment, biota lipids $\left(\mathrm{C}_{\text {lip }}\right)$ and porewater measured with passive samplers were used to derive lipid-normalised bioaccumulation metrics $\mathrm{C}_{\text {lip }}$, Biota sediment accumulation factor (BSAF), Bioaccumulation factor (BAF) and the Biota plastic accumulation factor (BPAF). Small effects of PE addition were detected suggesting slightly increased or decreased bioaccumulation. However, the differences decreased in magnitude dependent on the metric used to assess bioaccumulation, in the order: $\mathrm{C}_{\text {lip }}>\mathrm{BSAF}>\mathrm{BPAF}>\mathrm{BAF}$, and were non-significant for BAF. The fact that $\mathrm{BAF}$, i.e. normalization of $\mathrm{C}_{\text {lip }}$ on porewater concentration, largely removed all effects of PE, shows that PE did not act as a measurable vector of PCBs. Biodynamic model analysis confirmed that PE ingestion contributed marginally to bioaccumulation. This work confirmed model-based predictions on the limited relevance of microplastic for bioaccumulation under environmentally realistic conditions, and illustrated the importance of assessing exposure through all media in microplastic bioaccumulation studies.
\end{abstract}




\subsection{Introduction}

It has been hypothesized that ingestion of microplastic increases exposure of aquatic organisms to hazardous contaminants. ${ }^{20,171,177}$ This increased chemical exposure is often perceived as a major concern. Because chemical sorption to polymers is reversible, the transport of chemicals via plastic is possible in two directions: both transporting chemicals from plastic into organisms, and transporting chemicals from the organisms lipids into the plastic ('cleaning' Chapter 5). ${ }^{19,128}$ Some laboratory studies have shown an elevating effect of plastic in food on the uptake of chemicals (Chapter 4), ${ }^{51,124,235-239}$ whereas others found no effect, for all or some of the chemicals studied (Chapter 4). ${ }^{51,239-242}$ These studies often used an experimental design where parallel uptake from water or food/sediment was not explicitly considered, rendering them less ecologically relevant for conditions in nature, where these parallel uptake pathways do occur. ${ }^{243,244}$ Instead, often plastic loaded with persistent organic pollutants (POPs) was considered as the only route for chemical uptake. Furthermore, usually (relatively) clean organisms were used, which forces chemical transfer from the plastic to the organism. Modeling studies have attempted to asses more environmentally relevant conditions systematically, by (a) including the environmental uptake pathways water, sediment and food, and (b) accounting for the fact that organisms in the environment already would be chemically contaminated, which reduces the fugacity gradient driving chemical transfer. These studies indicated that the uptake of plastic-associated chemicals in organisms would be a minor contribution to total bioaccumulation in more environmentally realistic scenarios (Chapter 5). ${ }^{19,128,129,240,243-246}$ Therewith, there tends to be growing consensus among recent studies by various groups that plastic in the environment will have minor effects on bioaccumulation in organisms (Chapter 4, Chapter 5). ${ }^{19,51,128,129,240-246}$ Although first principles on chemical partitioning and kinetics suggest this, there is a lack of empirical environmentally realistic studies confirming these modeling outcomes. The few experimental studies that included uptake routes other than plastic so far indicated no measureable vector effect of plastic on bioaccumulation (Chapter 4)..$^{51,241,242,247}$ Quantification of chemical uptake from all environmental pathways jointly is however still lacking, as well as normalization of the bioaccumulation on lipid content of the organisms. Additionally, in the relatively small volume of the bioassays in previous studies the addition of an extra absorbing pool, namely the plastic, diluted porewater POP concentrations (Chapter 4, Chapter 5). ${ }^{51,128}$ This hampered the comparability of outcomes with environmentally relevant settings, where 
the excess availability of POPs from the surrounding media is virtually infinite. Therefore, empirically, the lack of effect of plastic on bioaccumulation under environmentally realistic exposure conditions with all uptake pathways quantified and accounted for yet has to be proven. ${ }^{248}$

The aim of the current study was therefore to assess the role of microplastic as a vector of plastic associated chemicals for Arenicola marina (L.) (lugworms) under environmentally relevant exposure conditions and with full quantification of all exposure pathways. Sediment, porewater and two realistic plastic doses were PCB spiked and equilibrated for 6 weeks after which PCB concentrations were assessed in sediment and in the porewater with the aid of polyoxymethylene (POM) passive samplers. ${ }^{75,249}$ This enabled determination of the in situ partitioning of PCBs among biota lipids, plastic, porewater and sediment. There were two exposure scenarios. Since the addition of clean plastic was expected to slightly dilute the chemical concentrations, the first scenario was referred to as the 'chemical dilution' (CD) scenario. Another set of PCB congeners was used to represent a second scenario. The spiked quantity of these PCB congeners was slightly increased to roughly a priori compensate for the anticipated PCB dilution by plastic addition. This second scenario aimed to represent open seafloor conditions where PCBs can be considered present from a virtually infinite source (Chapter 5), ${ }^{128}$ which is why the second scenario is referred to as the 'Infinite Source' (IS) scenario. Lugworms were chronically exposed for 28 days with endpoints survival, feeding activity, growth, lipid content and PCB bioaccumulation. As such, we focused on relevant scenarios where (a) as in nature, chemicals spread out over the environmental compartments and parallel uptake pathways exist, (b) environmentally relevant low plastic and chemical doses were used. We used the polymer high density polyethylene, because of its relatively high affinity for POPs and large global production. A mixture of environmentally relevant particle sizes in the smaller microplastic size range was used (10-180 $\mu \mathrm{m}) .{ }^{250,251}$ Ten PCB congeners were used as a proxy for environmentally sorbing and native plastic associated chemicals, spanning a wide hydrophobicity range. The $\sum$ PCB concentrations in the sediment were a factor $>24$ lower than the reported NOEC, and thus were too low to cause any toxic effect. ${ }^{183}$ In bioaccumulation assessment for benthic invertebrates, several bioaccumulation metrics are usually applied. Biota sediment accumulation factors (BSAFs) correct for variations in lipid content $\left(\mathrm{f}_{\text {lip }}\right)$ and organic matter content ( $f_{\mathrm{OM}}$ ) of organisms and sediment respectively. ${ }^{252}$ Hence, for bioassays with microplastic, this metric can reveal whether significant differences in 
bioaccumulation exist between organisms that were or were not exposed to plastic, as they are supposed to eliminate the above mentioned differences between organism and sediment characteristics. However, BSAF is composed of four measured variables $\left(\left(\mathrm{C}_{\text {organism }} / \mathrm{f}_{\text {lip }}\right) /\left(\mathrm{C}_{\text {sediment }} / \mathrm{f}_{\text {OM }}\right)\right)$, which makes this type of metric inherently sensitive to error propagation and may limit its rigor in detecting subtle differences in bioaccumulation. ${ }^{252}$ Instead, Bioaccumulation factors (BAFs) correct observed bioaccumulation for porewater concentrations $\left(\left(\mathrm{C}_{\text {organism }} / \mathrm{f}_{\text {lip }}\right) /\left(\mathrm{C}_{\mathrm{PW}}\right)\right)$ and thus more directly eliminate differences in bioaccumulation caused by differences in chemical concentrations in the porewater of the sediment. Here we report for the first time effects of microplastic on lipid normalised bioaccumulation in lugworms, lipid and organic matter (OM) normalised BSAFs and BAFs, and use and evaluate these metrics with respect to the vector effect of plastic on bioaccumulation. Furthermore, we introduce and evaluate the Biota Plastic Accumulation Factors $\left(\mathrm{BPAF}=\left(\mathrm{C}_{\text {organism }} / \mathrm{f}_{\text {lip }}\right) / \mathrm{C}_{\text {plastic }}\right)$ as a new metric relevant for the assessment of bioaccumulation from microplastic. Furthermore, the relative importance of PCB uptake pathways for the various scenarios was assessed using a plastic-inclusive biodynamic model (Chapter 5). ${ }^{128,129,245}$

\subsection{Materials and Methods}

\subsubsection{Materials}

Polyethylene (PE, green fluorescent UVPMS-BG, spherical, diameter 10 $180 \mu \mathrm{m}$, density $0.94 \mathrm{~kg} / \mathrm{L})^{75}$ was used in the bioassay. PE polymer identity was confirmed by FTIR (ThermoFisher, iN10 MX). For microscope images and particle size distributions of the PE the reader is referred to the publication by Velzeboer et al. ${ }^{75}$ Polyoxymethylene sheets (POM, $76 \mu \mathrm{m}$ thickness) were employed as passive samplers. ${ }^{75,249,253}$ The selected PCB congeners were 28 , $31,44,52,101,118,138,153,170$ and 180 . Further details are provided in the SI.

\subsubsection{Sediment sampling and pre-treatment}

The sediment was sampled from the Eastern Scheldt (the Netherlands) (Chapter 4). ${ }^{51,185} \mathrm{PE}$ was added to the sediment, accomplishing plastic concentrations of $0,0.05$ and $0.5 \% \mathrm{DW}$, which are within and above the range found in the marine environment, respectively (Chapter 4). ${ }^{39,51,165,254}$ Subsequently, the sediment-plastic mixture was spiked with the PCB congeners and mixed for six weeks. During the last four weeks of mixing, three POM passive samplers $(\approx 0.3 \mathrm{~g} \text { each })^{75,172,249,253}$ were added to each PEsediment mixture for determination of porewater PCB concentrations. Six and 
four weeks have been shown sufficient to reach chemical equilibrium between sediment porewater, and 10-180 $\mu \mathrm{m}$ PE particles and POM passive samplers, respectively (Chapter 5), ${ }^{75,128,172,173,216,223,224,249,255-258}$ for PE also is confirmed by the linearity of the $\log \mathrm{K}_{\mathrm{PE}}-\log \mathrm{K}_{\mathrm{Ow}}$ plot (Fig. A6.7). Further details are provided in the Appendix.

\subsubsection{Experimental set up}

Glass aquaria with dimensions $16 \times 16 \times 16 \mathrm{~cm}$ were filled with $4 \mathrm{~kg}$ wet PEcontaining PCB equilibrated sediment $(3.2 \mathrm{~kg}$ DW, $\pm 8.7 \mathrm{~cm}$ thick layer) and covered with stainless steel gauze with a mesh size of $2 \mathrm{~mm}$ to prevent exchange of lugworms. These aquaria were placed per five replicates in large $(80 \times 40 \times 40 \mathrm{~cm})$ aquaria, following previously published procedures (Chapter 4) ${ }^{51}$ Subsequently, $\pm 90 \mathrm{~L}$ of sea water from the Eastern Scheldt (the Netherlands) was added. After a two week stabilization period, the bioassay was started by adding pools of 5 individuals of $A$. marina to each small aquarium. Following our previous bioassays, no extra food source was provided (Chapter 4). ${ }^{51,190}$ Three times a week, water quality was measured, and about $30 \mathrm{~L}$ of overlying water was refreshed. Further details on the maintenance of the systems and the test organisms can be found in the SI.

\subsubsection{Treatments}

In this study we aimed at scenarios with and without a diluting effect of PCBs in the environment by plastic, referred to as the chemical dilution (CD) scenario and the infinite source (IS) scenario. These two chemical exposure scenarios were combined within the same experimental units to eliminate any influence of biological variability in the comparison of the two exposure scenarios. We achieved this by spiking the sediment plastic mixtures with pairs of chemically comparable PCB congeners. PCB pairs were 28 and $31^{*}$, 52 and $44^{*}, 101$ and $118^{*}, 153$ and $138^{*}$, and 180 and 170*. Within each of these pairs of chemically comparable PCB congeners, one of the congeners was spiked equally among all treatments (CD scenario, $\sum \mathrm{PCBs} \approx 5 \mu \mathrm{g} / \mathrm{kg}$ DW sediment mixture). The other congeners per pair, the IS scenario PCB congeners (congeners marked $*$ ), were spiked in higher quantity in the treatments with PE to compensate for the anticipated dilution effect of the added PE $\left(0.05 \%\right.$ PE treatment: $\sum$ PCBs* $\approx 7 \mu \mathrm{g} / \mathrm{kg}$ DW sediment mixture; $0.5 \%$ PE treatment: $\sum \mathrm{PCBs}^{*} \approx 23 \mu \mathrm{g} / \mathrm{kg}$ DW sediment mixture). Two plastic free treatments were included. The first one, referred to as ' $0 \% \mathrm{PE} \mathrm{A',}$ consisted of the CD scenario PCB congeners plus an equal spike of IS scenario PCB congeners ( $\sum \mathrm{PCBs}^{*} \approx 5 \mu \mathrm{g} / \mathrm{kg}$ DW sediment mixture). This $0 \% \mathrm{PE} \mathrm{A}$ treatment did not receive an increased spike of the IS scenario PCBs, because 
no dilution by added PE would occur at $0 \%$ PE. The second plastic free treatment (' $0 \%$ PE B') contained the same CD scenario PCB congener spike concentrations as the ' $0 \%$ PE A', but now with the IS scenario PCBs at the higher spiked quantity of the $0.5 \% \mathrm{PE}$ treatment $\left(\sum \mathrm{PCBs} * \approx 23 \mu \mathrm{g} / \mathrm{kg} \mathrm{DW}\right)$ (Fig. A6.1). The extra spiked quantities of the IS scenario PCB congeners were designed in such a way that the porewater concentrations in the sediment were expected to be similar in the PE free and PE containing treatments. The latter was based on a priori estimates of the organic matter-water and PE-water partitioning coefficients $\mathrm{K}_{\mathrm{OM}}{ }^{(225)}$ and $\mathrm{K}_{\mathrm{PE}}{ }^{(217)}$ respectively (Table A6.2). In this experimental design, the effect of $\mathrm{PE}$ on bioaccumulation in the $\mathrm{CD}$ scenario can be seen from the difference between concentrations of the $\mathrm{CD}$ scenario PCB congeners among all four treatments. The effect of PE on bioaccumulation in the IS scenario can be seen from the difference between concentrations of the IS scenario PCB congeners in the 0\% PE B and the $0.5 \%$ $\mathrm{PE}$ treatment. Comparison of the IS scenario PCB congeners in the $0 \% \mathrm{PE} \mathrm{A}$, $0.05 \% \mathrm{PE}$ and $0.5 \% \mathrm{PE}$ treatment, is a comparison of systems with a designed similar porewater concentration. Note that as $\mathrm{K}_{\mathrm{OM}}$ and $\mathrm{K}_{\mathrm{PE}}$ in the design phase were estimated using literature values, ${ }^{217,225}$ an exact compensation for the dilution effect leading to identical porewater concentration was not anticipated. PCB concentrations added to the sediment mixtures are listed in the Appendix (Table A6.1).

\subsubsection{Endpoints}

During the exposure assay, mortality was assessed daily and dead lugworms were removed. Feeding activity was assessed following previous procedures, as the number of faeces heaps produced per organism per day (Chapter 4), ${ }^{51,180,191,192}$ and additionally as mass of faeces heaps produced per organism per day. The latter was done by flattening all sediment surfaces with a spatula at the $27^{\text {th }}$ day, and subsequently collecting all faeces heaps at the $28^{\text {th }}$ day. Of these faeces, wet weight (WW), dry weight (DW, $60{ }^{\circ} \mathrm{C}$ during $24 \mathrm{~h}$ ) and ash free dry weight (AFDW, $600{ }^{\circ} \mathrm{C}$ during $2 \mathrm{~h}$ ) were determined and corrected for the number of surviving organisms to calculate faeces weight produced per organism per day. Results were corrected for the initial polyethylene fraction of the sediment $\left(\mathrm{f}_{\mathrm{PE}}\right)$ to estimate the $\mathrm{OM}$ content of the faeces. Thereby the assumption was made that all PE burned during the AFDW determination. After the exposure period of $28 \mathrm{~d}$, the lugworms were transferred to clean seawater to clear their guts overnight (Chapter 4) ${ }^{51,190}$ Lugworms were rinsed with demineralised water, air dried on tissue paper for 15 minutes and subsequently stored at $-18{ }^{\circ} \mathrm{C}$. After defrosting and homogenization of the 
tissue, WW, DW ( $\approx 1$ g tissue per pool), AFDW, lipid content ${ }^{259}$ and PCB concentrations were analysed.

\subsubsection{PCB analysis and QA}

PCB concentrations of initial (i.e. $t=0$ d) porewater, initial and final (i.e. $t=28$ d) sediment and initial and final lugworm tissue were determined. Sample preparation and PCB analysis followed previously published procedures (Chapter 4). ${ }^{51,186,193}$ For details the reader is referred to Chapter $4 .^{51}$ Porewater concentrations $\left(\mathrm{C}_{\mathrm{PW}}\right)$ were determined by analysing the POM passive samplers. ${ }^{172}$ After the four weeks equilibration, POM strips were rinsed with demineralised water, air dried for 15 minutes on tissue paper and stored at $7{ }^{\circ} \mathrm{C}$ till analysis. Recoveries of PCB congeners were determined in triplicate and averaged for the individual congeners $83.6 \pm$ SE $1.5 \%$. PCB concentrations were corrected for procedural blanks. The concentration of PCB congener 52 was only incidentally identified in initial lipids as well as in the sediment at the start and end of the bioassay, and therefore left out of further analysis of the these metrics.

\subsubsection{Data analysis}

Normality of the data and equality of variances were tested with a ShapiroWilk Normality test and Levene's test, respectively. Linear regression (LM), ANCOVA, Kruskal-Wallis, Tukey HSD and Nemenyi-Damico-WolfeDunn (NDWD) tests were performed with $\mathrm{R}$ statistical software (R Development Core Team), with a significance level of $\alpha=0.05$. Unless stated otherwise, results are reported \pm standard error. $\mathrm{C}_{\mathrm{PW}, \mathrm{t}=0}$ was derived from the passive sampler data using measured PCB partitioning coefficients to $76 \mu \mathrm{m}$ POM provided by Hawthorne et al. ${ }^{249}$ (K $\mathrm{K}_{\mathrm{POM}}$, Table A6.2, Eq. A6.1). Calculation methods for $\mathrm{C}_{\mathrm{PW}, \mathrm{t}=28}, \mathrm{~K}_{\mathrm{OM}}, \mathrm{K}_{\mathrm{PE}}, \mathrm{C}_{\mathrm{PE}}, \mathrm{C}_{\mathrm{OM}}, \mathrm{BSAF}, \mathrm{BPAF}$ and $\mathrm{BAF}$ are provided in the Appendix (Eq. A6.2-A6.9). We included a figure that illustrates, theoretically, the various scenarios associated with each of the bioaccumulation metrics BSAF, BPAF and BAF (Fig. A6.2).

\subsubsection{Bioaccumulation modeling}

Bioaccumulation was modeled according to Chapter 5. ${ }^{128}$ For details, the reader is referred to the Appendices of Chapter 5 \& Chapter 6. 


\subsection{Results and discussion}

\subsubsection{Lugworm survival, feeding activity, weight and lipid content}

Overall lugworm survival was $81 \%$, with no significant differences between the treatments (Fig. A6.3A). This implies that the PCBs nor the PE additions had physical or chemical effect on survival. Feeding activity started in all treatments within the first week of exposure. The feeding activity expressed as number of heaps per individual worm was significantly highest in the treatment with $0.05 \% \mathrm{PE}$ and lowest in the treatment with $0.5 \% \mathrm{PE}$ (Fig. A6.3B, ANOVA, $p$-value $=0.010$, Tukey HSD, $p$-value $=0.006)$. However, the size of the faeces heaps was observed to be rather variable. Furthermore, individual heap count seemed to be affected by the number of alive lugworms (linear regression, treatment and survival both explanatory for activity (heaps/individual/day), $\mathrm{R}^{2}=0.91$ ). This can be explained by a high feeding activity causing the heaps to be less well distinguished from one another. Heap production in mass might therefore be a better indicator of lugworm condition than number of heaps and is more relevant as a relative measure of egestion rate. In the $0 \% \mathrm{PE}$ treatments the heap mass production per individual varied from $9.4-19.9 \mathrm{~g} \mathrm{DW}$, whereas in the treatments with PE this was significantly lower by a factor of two, i.e. only $4.1-11.6 \mathrm{~g} \mathrm{DW}$ (Fig. A6.3C, ANOVA, $p$ value $=7.20 \times 10^{-3}$, Tukey HSD, $p$-values $\leq 0.049$ ). After correction by $f_{\mathrm{PE}}$, the only significant difference in $\mathrm{f}_{\mathrm{OM}}$ in the heaps was the $\mathrm{f}_{\mathrm{OM}}$ of the $0 \% \mathrm{PE} \mathrm{A}$ treatment being higher than that of the $0 \%$ PE B treatment (Fig. A6.3D, black markers, Kruskal-Wallis, $p$-value $=0.012$, NDWD, $p$-value $=0.022$ ). The concurrence of lowest feeding activity with highest PE concentration confirms the negative effect of plastic on feeding activity that was previously observed for 7.4\% PS (Chapter 4) ${ }^{51}$ and 5\% UPVC. ${ }^{53}$ Weight loss was calculated as average weight of the surviving lugworms divided by average initial weight in that pool. The initial pooled average weight varied between 3.0 and $4.1 \mathrm{~g}$ WW/individual. After 28 days of exposure this was for the surviving lugworms reduced to $2.5-3.5 \mathrm{~g}$. This weight loss of $3.7-28.8 \%$ did not significantly differ among the treatments (Fig. A6.3E). On average the lipid fraction was $1.8 \pm \mathrm{SE} 0.03 \%$. No significant difference in lipid fraction of the lugworms exposed to the different treatments was found (Fig. A6.3F).

\subsubsection{PCB concentrations in porewater and sediment}

$\mathrm{PCB}$ concentrations in porewater at the start of the bioassay $\left(\mathrm{C}_{\mathrm{PW}, \mathrm{t}=0}\right.$, Fig. 6.1) can be used to check whether PE indeed diluted concentrations of CD scenario $\mathrm{PCB}$ congeners in the porewater and whether concentrations of IS scenario 
PCB congeners in the porewater remained more similar among treatments. Overall, $\mathrm{C}_{\mathrm{PW}}$ values ranged between 0.001 and $10 \mathrm{ng} / \mathrm{L}$, therewith being environmentally relevant. ${ }^{260,261} \mathrm{PE}$ treatment, chemical exposure scenario and $\mathrm{K}_{\mathrm{Ow}}$ were significant explanatory variables of $\mathrm{C}_{\mathrm{PW}}$ (ANCOVA, $\mathrm{R}^{2}$ adj $=0.91$, $p$-values $\leq 0.021)$. The negative trend of $\mathrm{C}_{\mathrm{PW}}$ with $\log \mathrm{K}_{\mathrm{Ow}}$ in Fig. 6.1 was explained by spiking in similar quantities (Table A6.1), which resulted in lower PCB concentrations in the porewater with increasing hydrophobicity of the congeners. The $\mathrm{C}_{\mathrm{PW}}$ of the IS scenario PCB congeners was somewhat higher and showed a higher variability among treatments than that of the $\mathrm{CD}$ scenario PCB congeners, which was explained by the higher spiking concentrations of the IS scenario PCB congeners (Table A6.1). As such, the extra spiking of the IS scenario PCB congeners turned out to be an overcompensation for the sorption by PE. This difference from exact compensation could be expected, since literature $\mathrm{K}_{\mathrm{PE}}$ values were used for the experimental design, ${ }^{217}$ which were in line with, though not exactly equal to, measured $\mathrm{K}_{\mathrm{PE}}$ values (Fig. A6.7) ${ }^{75}$ Per scenario, the order of $\mathrm{C}_{\mathrm{PW}}$ among the different treatments was generally equal for all congeners (Fig. 6.1). Although designed to have a similar $\mathrm{C}_{\mathrm{PW}}, \mathrm{C}_{\mathrm{PW}}$ of the $\mathrm{CD}$ scenario $\mathrm{PCB}$ congeners was a factor 1.9 lower in the $0 \% \mathrm{PE}$ A than in the $0 \% \mathrm{PE} \mathrm{B}$ sediment at the start of the experiment $\left(0.19-0.41 \log\right.$ unit, Fig. A6.6, ANCOVA, $\mathrm{R}^{2}$ adj $=0.97, p$ values $\leq 3.35 \times 10^{-4}$ ). IS scenario $\mathrm{PCB}$ congeners had a relatively low $\mathrm{C}_{\mathrm{PW}}$ in the $0 \% \mathrm{PE}$ A sediment too. We explain the lower than expected $\mathrm{C}_{\mathrm{PW}}$ of all congeners in the $0 \%$ PE A sediment by sediment heterogeneity during the preparation phase or random variability. For instance, spiking the $\mathrm{CD}$ scenario PCB congeners caused already an average factor 1.1 lower concentration of PCBs in the $0 \%$ PE A sediment compared to the $0.05 \%$ PE sediment. After the preparation phase of six weeks sediment mixing, porewater PCB concentrations were a factor $1.1-1.8$ lower in the $0 \%$ PE A sediment compared to the $0.05 \% \mathrm{PE}$ sediment, even before exposure started. A plausible explanation for these lower $\mathrm{C}_{\mathrm{PW}}$ values is a higher $\mathrm{f}_{\mathrm{OM}}$, or a different $\mathrm{OM}$ quality, in the initial $0 \% \mathrm{PE} \mathrm{A}$ sediment compared to the other treatments, which also is consistent with the aforementioned faeces heaps OM content. In the sediment containing $0.05 \% \mathrm{PE}, \mathrm{C}_{\mathrm{PW}}$ of the $\mathrm{CD}$ scenario $\mathrm{PCB}$ congeners was higher compared to the $0 \% \mathrm{PE}(\mathrm{A})$ sediment (a factor 1.8 increase, range $1.2-2.6$, Tukey HSD, significant for 3 out of 5 congeners, $p$-values $\leq 0.015$ ), and statistically identical compared to the $0 \% \mathrm{PE}$ (B) sediment (factor 0.94 , Tukey HSD, $p$-values $\geq 0.198$ ). At the ten times higher PE dose of $0.5 \% \mathrm{PE}$, $\mathrm{C}_{\mathrm{PW}}$ of the CD scenario $\mathrm{PCB}$ congeners was insignificantly different compared to the $0 \%$ PE A sediment (Tukey HSD, $p$-values $\geq 0.057$ ) and was reduced 
compared to the 0\% PE B sediment by a factor 3.5 (1.9 - 5.6, Tukey HSD, $p$ values $\leq 0.011)$. The latter phenomenon has previously been referred to as the 'dilution effect of plastic' (Chapter 4, Chapter 5)). ${ }^{51,128} \mathrm{C}_{\mathrm{PW}}$ of the IS scenario PCB congeners were in the $0.05 \%$ PE sediment equal (Tukey HSD, $p$-values $\geq 0.231)$ and in the $0.5 \% \mathrm{PE}$ sediment elevated compared to the $0 \%$ PE A sediment (Fig. 6.1, dark grey colour, factor $1.4-2.3$ increase, Tukey HSD, significant for 3 out of 5 congeners, $p$-values $\leq 0.022$ ). This was caused by the extra spike but also implied that the PCBs sorbed less to PE and OM than a priori assumed. After all, the extra spike of the IS scenario PCB congeners was designed to keep the $\mathrm{C}_{\mathrm{PW}}$ in the treatments with $\mathrm{PE}$ more or less constant compared to the 0\% PE A treatment. The difference between these sediments was also increased by the random variability that lowered the $\mathrm{C}_{\mathrm{PW}}$ of all PCB congeners in the $0 \%$ PE A treatment. Nevertheless, a substantial part of the extra spike was indeed sorbed to the PE, as one can see by comparison with the $0 \%$ PE B treatment. In the latter sediment, as expected, the extra spike of IS scenario PCB congeners in absence of PE resulted in higher porewater concentrations (factor $8.2-22.5$ ). The extra spike of IS scenario PCB congeners thus compensated for the dilution effect as planned, although at the same time it was not fully representative of an open seafloor scenario where porewater concentrations would have been the same among environments with and without PE (0\% PE A, $0.05 \% \mathrm{PE}$ and $0.5 \% \mathrm{PE})$. This however, does not interfere with interpretation of treatment effects, as will be discussed later on.
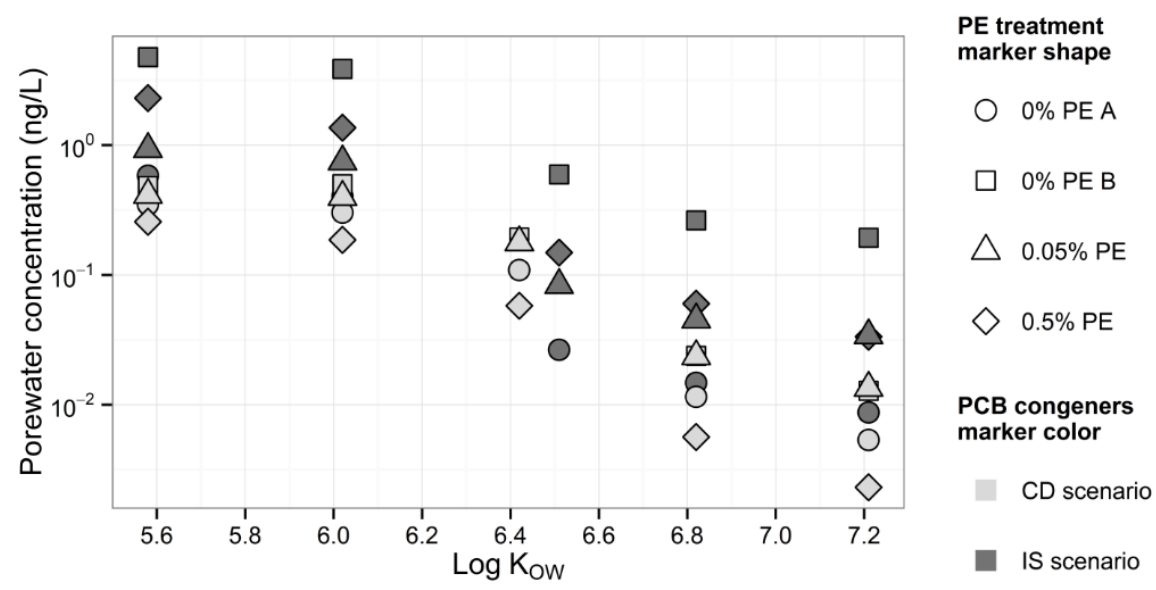

Figure 6.1. Average $C P W$ values $\pm S E 0.011-0.075$ (not shown) at $t=0$ days measured with passive samplers. $C D$ scenario $P C B$ congeners: $28,52,101,153,180$. IS scenario PCB congeners: 31 , 44, 118, 138, 170. Log KOW values from Van Noort et al., ${ }^{232}$ Table A6.2. 
Concentrations in $\mathrm{OM}$ and PE at the start of the experiment $(t=0 \mathrm{~d})$, and in porewater, OM and PE after exposure at $t=28 \mathrm{~d}$ were calculated (Fig. A6.4, A6.7), using measured total (sediment plus PE) concentrations in sediment at $t=0$ and $28 \mathrm{~d}$, porewater concentrations at $t=0$ and assuming OM-porewater and PE-porewater partition coefficients to remain constant during $28 \mathrm{~d}$ of exposure (Appendix, equations A6.2-6.6). PCB concentrations in porewater did not differ substantially between 0 and $28 \mathrm{~d}$ (Fig. A6.5), hence constant exposure was concluded and $\mathrm{C}_{\mathrm{PW}, \mathrm{t}=28}$ was used to derive the accumulation factors discussed hereafter.

\subsubsection{OM-porewater and PE-porewater partitioning coefficients} Partitioning coefficients to $\mathrm{OM}\left(\mathrm{K}_{\mathrm{OM}}\right)$ were calculated from $\mathrm{C}_{\mathrm{PW}, \mathrm{t}=0}$ and the PCB concentrations in the sediment $\left(\mathrm{C}_{\mathrm{SED}, \mathrm{t}=0)}\right)$ and are close to literature values $^{225}$ (Fig. A6.6). Because of the measured, as designed, statistically identical porewater concentrations of the $\mathrm{CD}$ scenario $\mathrm{PCB}$ congeners in the $0 \%$ PE B and $0.05 \%$ PE sediment, $\mathrm{K}_{\mathrm{OM}}$ values of the $0 \%$ PE B sediment were used to calculate $\mathrm{K}_{\mathrm{PE}}$ values in the sediments with PE. The following formula was used:

$K_{P E}=\frac{\left(K_{p-t o t a l}-f_{O M} \times K_{O M}\right)}{f_{P E}}$

with $\mathrm{K}_{\mathrm{p} \text {-total }}$ being the PCB partitioning to the total mixture of sediment including $\mathrm{OM}$ and $\mathrm{PE}$, and $\mathrm{f}_{\mathrm{OM}}$ and $\mathrm{f}_{\mathrm{PE}}$ being the fraction $\mathrm{OM}$ and polyethylene in that sediment mixture, respectively. This follows the same procedure as Rakowska et al. ${ }^{262,263}$ who derived activated carbon partitioning coefficients in mixtures of activated or black carbon and sediment. Log $\mathrm{K}_{\mathrm{PE}}$ showed a linear increase with $\log \mathrm{K}_{\mathrm{Ow}}$ (Fig. A6.7, LM, $p$-value $=2.17 \times 10^{-12}$ ). $\log \mathrm{K}_{\mathrm{PE}}$ values did not differ significantly between the treatment with 0.05 and $0.5 \%$ PE. Furthermore, they were in line with previous findings by Lohmann et al. ${ }^{217}$ and Velzeboer et al. ${ }^{75}$ This conformance in $\mathrm{K}_{\mathrm{PE}}$ among PE treatments and literature studies that used pure PE, confirms the reliability of the presently used procedures. The PCB concentrations in $\mathrm{PE}\left(\mathrm{C}_{\mathrm{PE}}\right)$ were calculated from $\mathrm{K}_{\mathrm{PE}}$ and $\mathrm{C}_{\mathrm{PW}}$ according to Eq. A6.5 and ranged up to about one $\mu \mathrm{g} / \mathrm{g}$ (Fig. A6.8). The above analysis provided a clear view on the chemical concentrations in all relevant media, water, sediment and PE, and how PE additions in the different treatments changed these concentrations. This facilitates the interpretation of the data on the effects of PE additions on bioaccumulation. 


\subsubsection{Effects of PE on bioaccumulation}

Effects of PE treatments were assessed first by evaluating lipid normalised PCB concentrations $\left(\mathrm{C}_{\text {lip }}\right)$ in the lugworms, representing a direct measure of bioaccumulation (Fig. 6.2, Fig. 6.3A). After 28 days of exposure to $0.05 \%$ PE, concentrations of CD scenario PCB congeners in lugworm lipids were increased by on average a factor $2(1.6-2.5$, significant for four out of five congeners (not for PCB 180) compared to the 0\% PE A treatment (Tukey HSD, $p$-values $\leq 4.33 \times 10^{-4}$ ). This factor difference is similar to that found in earlier studies that assessed parallel pathways (Chapter 4), ${ }^{51,239}$ and complies with the general conclusion by Koelmans et al..$^{244}$ that a factor two increase or decrease may occur due to complex counteracting mechanisms affecting accumulation. However, compared to the $0 \%$ PE B treatment, $\mathrm{C}_{\text {lip }}$ remained equal or was lower after exposure to $0.05 \% \mathrm{PE}$ by on average a factor $1.3(1.1$ - 1.7, Fig. 6.2, Fig. 6.3A, Fig. A6.9, significant for one out of five congeners, Tukey HSD, $p$-value $=0.047$ ). Exposure to sediment containing $0.5 \% \mathrm{PE}$ caused bioaccumulation of the CD scenario PCB congeners to be on average a factor $1.6(1.1-2.2)$ lower for all five congeners compared to the $0 \%$ PE A treatment, and even a factor $4.2(3.2$ - 6.1) compared to the $0 \% \mathrm{PE} \mathrm{B}$ treatment. This suggests that the factor two increase in apparent bioaccumulation observed at the $0.05 \%$ compared to the $0 \%$ PE A treatment is more than compensated for by chemical dilution at a PE dose of $0.5 \%$. One could explain the increased bioaccumulation after exposure to the $0.05 \% \mathrm{PE}$ compared to the $0 \%$ PE A treatment by release of PCBs from the ingested PE (vector effect). However, the lack of this increase when comparing the $0.05 \%$ $\mathrm{PE}$ to the $0 \%$ PE B treatment, in which $\mathrm{C}_{\mathrm{PW}}$ was similar, makes it more likely that the low $\mathrm{C}_{\mathrm{PW}}$ observed in the 0\% PE A treatment (Fig. 6.1) explains the low $\mathrm{C}_{\text {lip }}$ in that treatment. For the five IS scenario $\mathrm{PCB}^{*}$ congeners, bioaccumulation was strongly elevated after exposure to the 0\% PE B treatment compared to the $0 \%$ PE A treatment (Fig. 6.2, Fig. 6.3A, Fig. A6.9, ANOVA, $p$-values $\leq 1.70 \times 10^{-5}$, Tukey HSD, $p$-values $\leq 3.74 \times 10^{-5}$ ), which is explained by the higher spike quantity used. For the IS scenario PCB* congeners, the presence of 0.05 or $0.5 \%$ PE resulted as expected in decreased bioaccumulation compared to the $0 \%$ PE B treatment (Tukey HSD, $p$-values $\leq 1.80 \times 10^{-4}$ ) and no significant differences with the $0 \%$ PE A treatment. The elevation of bioaccumulation in the $0 \%$ PE B treatment was up to a factor 10 compared to the treatments where these PCBs were added in the presence of plastic (Fig. 6.2). As there was extra spiking of PCBs in the PE treatments too, the lack of this elevated bioaccumulation in the treatments with PE compared to the IS control thus can be attributed to sorption to PE. 
In summary, a small increase in bioaccumulation was detected for the $\mathrm{CD}$ scenario PCB congeners after exposure to the $0.05 \%$ PE compared to the $0 \%$ PE A treatment. However, when comparing to reference systems with statistically identical porewater concentration ( $0 \%$ PE B) as would occur in nature, no difference in bioaccumulation was detected. Dilution due to PE addition was detected at higher PE dose and for the IS scenario PCB congeners. Dosing extra PCBs to compensate for dilution in the IS scenario confirmed but did not provide a clear additional mechanistic view on these processes when looking directly at bioaccumulation in lipids of lugworms. Hereafter, we tease out dilution effects due to presence of plastic by normalizing bioaccumulation to concentrations in respectively sediment organic matter (BSAF), PE (BPAF), or porewater (BAF) below.
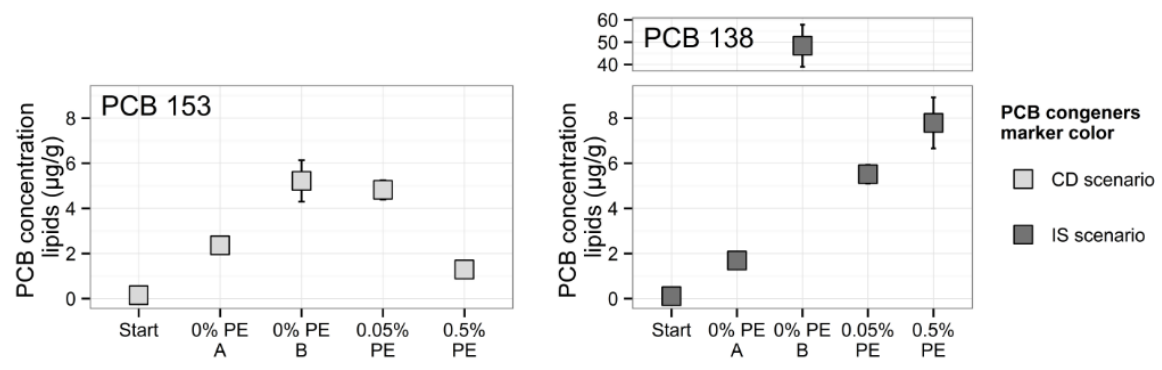

Figure 6.2. Average PCB concentrations \pm SE (lipid normalised) in lugworms after exposure to the different treatments and their background $P C B$ concentrations before start of the exposure assay for the representative PCB congeners 153 and 138. Left panel: PCB congener 153 spiked equally in all treatments representing the $C D$ (chemical dilution) scenario. Right panel: PCB congener 138 extra spiked in the treatments with PE and the 0\% PE B to correct for the dilution mechanism representing the IS (infinite source) scenario. Where error bars are invisible they are small and thus lie behind the markers. The (similar) results for eight more $P C B$ congeners can be found in the Appendix (Fig. A6.9) and the results of all congeners combined in Fig. 6.3A.

\subsubsection{Biota to sediment accumulation factors (BSAFs)}

Biota to sediment accumulation factors (BSAFs) were calculated from PCB concentrations in the lipids $\left(\mathrm{C}_{\text {lip }}\right)$ and $\mathrm{OM}\left(\mathrm{C}_{\mathrm{OM}}\right)($ Eq. A6.7, Fig. 6.3B). Overall, for BSAF, variability among treatments was less than when $\mathrm{C}_{\text {lip }}$ was used as a metric for bioaccumulation (compare Fig. 6.3A and 6.B). In case increase of bioaccumulation would occur due to extra spiking or chemical dilution effects would occur due to addition of PE, these would respectively increase or decrease $\mathrm{C}_{\text {lip }}$ as well as $\mathrm{C}_{\text {OM. }}$. Accordingly, BSAFs can be expected to provide a clearer view on the role of PCB uptake from ingested PE than $\mathrm{C}_{\text {lip. }}$. Average 
BSAFs ranged $30-272$. This implies that bioaccumulation did not comply to Equilibrium Partitioning Theory (EPT), which would suggest normalised BSAF values of $1-2 .{ }^{252}$ Similarly high BSAFs for PCBs were reported for lugworms by Diepens et al. ${ }^{264}$ and Chapter 4. ${ }^{51}$, and for several other species and chemicals. ${ }^{265,266}$ One reason for higher BSAFs is that binding to Oesterput $\mathrm{OM}$ was relatively low, that is, $\mathrm{K}_{\mathrm{OM}}$ values were an order of magnitude lower than $\mathrm{K}_{\text {ow }}$ values (Fig. A6.6), where $\mathrm{K}_{\mathrm{ow}}$ is taken as a proxy for the binding affinity to lipids. BSAFs can also be increased due to biomagnification from ingested sediment OM. This would imply a higher BSAF with higher LogKow, which however was not observed. This in turn can be explained by not having reached steady-state for the more hydrophobic congeners in lipids. ${ }^{264}$ For the CD scenario PCB congeners, BSAFs were elevated after exposure to $0.05 \%$ PE compared to the 0\% PE A treatment by a factor 4.4 (1.9-10.6), which was significant for one congener (PCB 28). After exposure to 0.5\% PE, BSAFs were elevated by a factor $3.6(2.5-4.6)$ which was significant for three congeners (PCB 28, 153, 180, Fig. 6.3A, NDWD, $p$-values $\leq 0.030$ ). However, compared to the $0 \%$ PE B treatment, the treatment with similar porewater concentration, BSAFs were not elevated after exposure to PE. Also for the IS scenario PCB congeners compared to the $0 \%$ PE B treatment, BSAFs did not increase after exposure to PE (factor $1.2(0.9-1.7)$, statistically insignificant). For both the CD and IS scenario PCB congeners, differences in BSAFs after exposure to either 0.05 or $0.5 \% \mathrm{PE}$ were not significant (average ratio $\mathrm{BSAF}_{0.5 \% \mathrm{PE}} / \mathrm{BSAF}_{0.05 \% \mathrm{PE}}=1.1$, range $0.4-2.0$ ). This implies that a vector effect of PE is not likely, because a ten times higher PE dose then would have resulted in a higher BSAF. The latter corresponds with the lack of difference in BSAFs of the PE treatments compared to the $0 \% \mathrm{PE} \mathrm{B}$ treatment as mentioned above. We conclude that by using BSAF as a metric for bioaccumulation, magnitude and statistical significance of differences among treatments were smaller compared to $\mathrm{C}_{\mathrm{lip}}$, as expected.

\subsubsection{Biota-to-plastic accumulation factors (BPAFs)}

Biota to plastic accumulation factors (BPAFs) were calculated from PCB concentrations in the lipids $\left(\mathrm{C}_{\text {lip }}\right)$ and $\mathrm{PE}\left(\mathrm{C}_{\mathrm{PE}}\right)$ (Eq. A6.9, Fig. 6.3C). Overall, for BPAF, variability among the $0.05 \%$ and $0.5 \% \mathrm{PE}$ treatments was even less than when BSAF was used as a metric for bioaccumulation (Fig. 6.3B, C). BPAFs ranged $7.6-44.8$, suggesting a contribution of OM bound PCBs to $\mathrm{C}_{\text {lip. }}$ A decreasing trend of BPAF with increasing Kow was observed, which might be explained by (a) slow kinetics towards the worm lipids of PCBs with a higher hydrophobicity, ${ }^{264}$ and (b) sorption to PE increasing more than 
proportionally with $\log \mathrm{K}_{\mathrm{OW}}$ (Fig. A6.7). BPAFs were lower than BSAFs, as $\mathrm{K}_{\mathrm{PE}}$ values were higher than $\mathrm{K}_{\mathrm{OM}}$ values. There were no significant differences (average ratio $\mathrm{BPAF}_{0.5 \% \mathrm{PE}} / \mathrm{BPAF}_{0.05 \% \mathrm{PE}}=1.0$, range $0.5-1.2$ ) between these plastic normalised BPAFs after exposure to 0.05 and $0.5 \%$ PE for all tested PCB congeners (Kruskal-Wallis, $p$-values $\geq 0.22$ ). This again implies that no vector effect of PE was found. After all, when PE would act as a vector for PCBs, a ten times higher PE dose would have resulted in a higher BPAF. The lower variability among PE doses when using BPAF instead of BSAF indicates that normalizing on $\mathrm{PE}$ is a useful, more representative way to interpret bioaccumulation in sediments containing plastic.

\subsubsection{Bioaccumulation factors}

Lipid normalised bioaccumulation factors (BAF, Eq. A6.9) were calculated as the ratio of $\mathrm{C}_{\text {lip }}$ and $\mathrm{C}_{\mathrm{PW}}$ (Eq. A6.5, Fig. 6.1). Overall, for BAF, the variability among treatments and among treatment replicates was far less than that for all other metrics. LogBAF increased linearly with increasing hydrophobicity of the PCB congeners (Fig. 6.3D), with a hydrophobicity cut-off visible at LogK $\mathrm{K}_{\mathrm{OW}}>6.8 .{ }^{267}$ Interestingly, BAF showed no significant differences among treatments in both the $\mathrm{CD}$ and IS scenario, except for the least hydrophobic PCB congener. Only for PCB 28, the BAF at $0.05 \%$ and $0.5 \%$ PE was increased by a factor of 5.3 and 2.3 respectively, compared to the $0 \%$ PE A treatment (NDWD, $p$-values $\leq 0.045$ ). This also implies that the BAF normalization removed the aforementioned deviation for the $0 \%$ PE A treatment for all but one PCB. Differences in BAF between the 0.05 and $0.5 \%$ $\mathrm{PE}$ treatments were not significant either, and the ratio between them again was one, equalling that of the aforementioned BSAF and BPAF.

In summary, differences among treatments appeared to decrease in terms of magnitude and statistical significance in the order: $\mathrm{C}_{\text {lip }}>\mathrm{BSAF}>\mathrm{BPAF}>\mathrm{BAF}$, to become non-significant when BAF was used as a metric for treatment effect. This implies that the main driver of treatment effects was the difference in porewater concentration (Fig. 6.1), which in turn was driven by partitioning phenomena among worm lipids, PE, water and sediment OM. Because $\mathrm{C}_{\text {lip }}$ closely followed the concentrations in the porewater for each of the PCBs, we conclude that bioaccumulation was not affected by extra exposure due to $\mathrm{PE}$ ingestion. 
Chapter 6

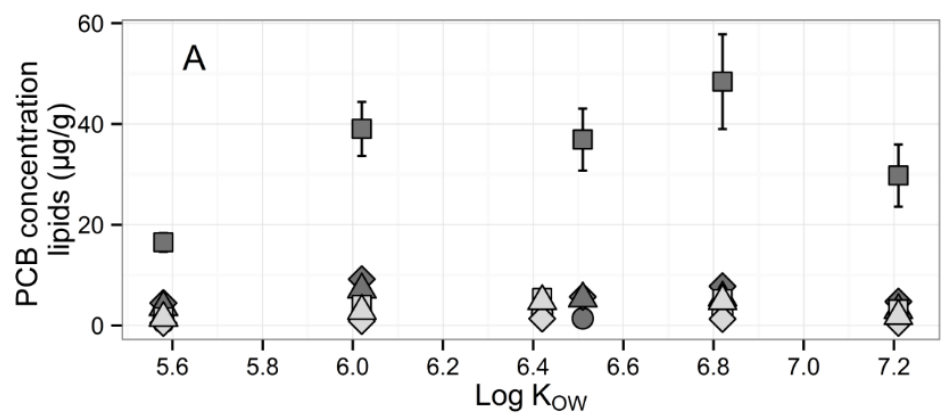

PE treatment marker shape

○ $0 \%$ PE A

$\square \quad 0 \%$ PE B

$\triangle 0.05 \% \mathrm{PE}$

$\diamond 0.5 \% \mathrm{PE}$

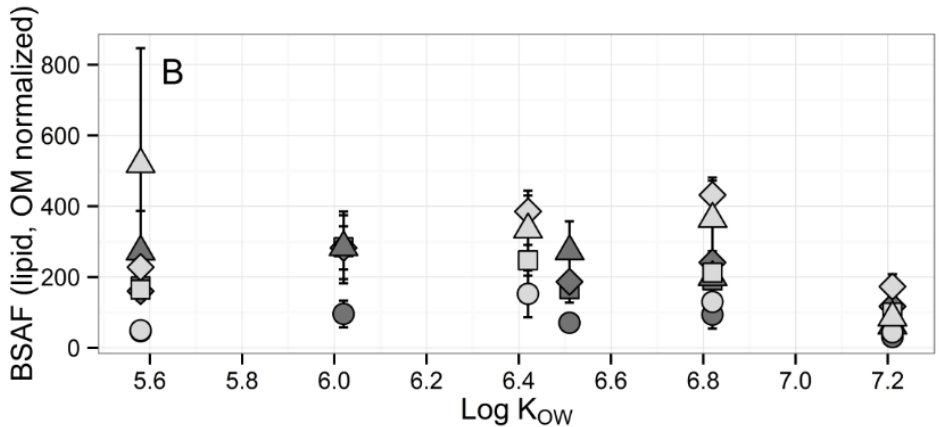

PCB congeners marker color
CD scenario
IS scenario
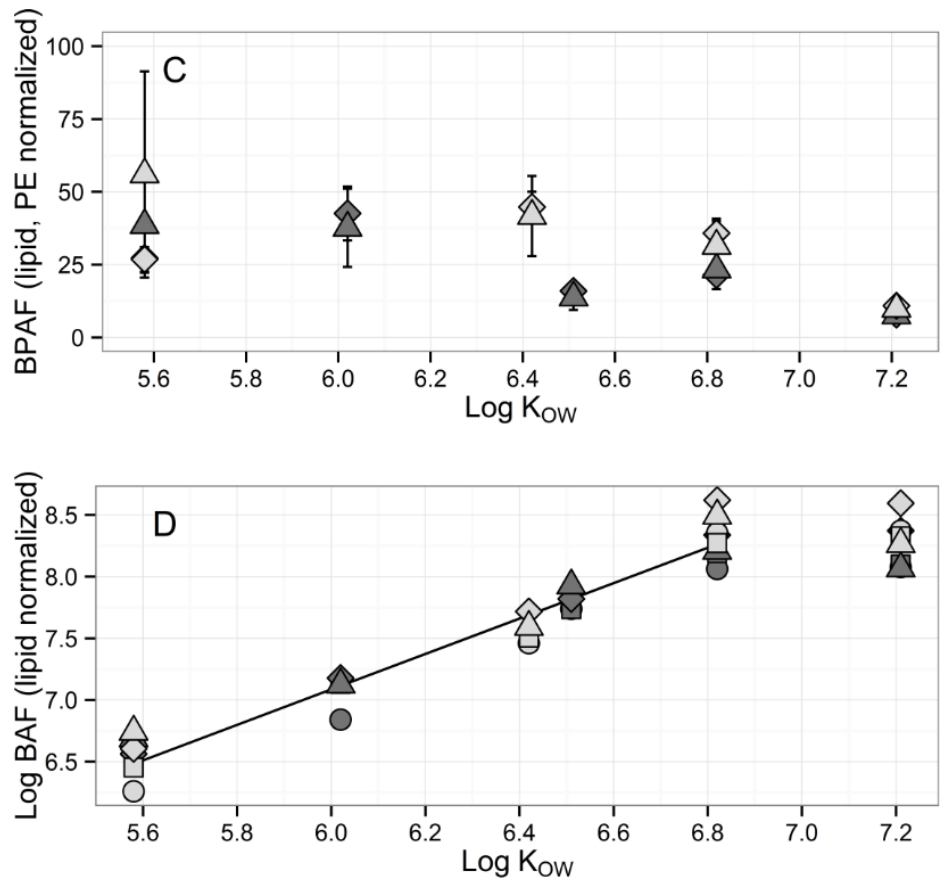
Figure 6.3. Average bioaccumulation and biota to sediment, $P E$ and porewater accumulation factors per PCB congener. Panel A: Lipid normalised PCB concentrations $\pm S E$ in lugworms after exposure to the different treatments. Concentrations of ten congeners as a function of their hydrophobicity (Log KOW). Panel B: BSAFs normalised on concentrations of PCBs in lipids in lugworm tissue and $O M$ in sediment. Panel C: BPAFs normalised on concentrations of PCBs in lipids in lugworm tissue and PE in sediment. Panel D: Bioaccumulation factors (Log BAFs) normalised on concentrations of PCBs in lipids in lugworm tissue $( \pm S E 0.011-0.198$, not shown). Linear regression line with cut off at $\log K O W>6.8: \log B A F=1.44 \times \operatorname{LogKOW}-$ $1.53, R 2=0.95$.

\subsubsection{Model supported assessment of relative importance of uptake pathways}

We modeled bioaccumulation of the PCBs $\left(\mathrm{dC}_{\mathrm{B}, \mathrm{t}} / \mathrm{dt} ; \mu \mathrm{g} \times \mathrm{g}^{-1} \mathrm{~d}^{-1}\right)$ as a mass balance of uptake and loss processes (Chapter 5): 128,129,240,244,246

$\frac{\mathrm{dC}_{\mathrm{B}, \mathrm{t}}}{\mathrm{dt}}=\mathrm{k}_{\mathrm{derm}} \mathrm{C}_{\mathrm{PW}}+\mathrm{IR} \times \mathrm{S}_{\mathrm{SED}} \mathrm{a}_{\mathrm{SED}} \mathrm{C}_{\mathrm{SED}}+\mathrm{IR} \times \mathrm{S}_{\mathrm{PL}} \mathrm{C}_{\mathrm{PLR}, \mathrm{t}}-\mathrm{k}_{\mathrm{loss}} \mathrm{C}_{\mathrm{B}, \mathrm{t}}$

A detailed description of the modeling is provided in the Appendix. The first term in Eq. 6.2 quantifies uptake from the porewater. The second and third term quantify uptake from ingested sediment and ingested PE, respectively. The fourth term quantifies loss due to elimination and egestion. For the CD scenario PCB congeners, bioaccumulation after $28 \mathrm{~d}$ was modeled using Eq. A6.17, after which the relative shares of the uptake pathways on accumulation were assessed. A tiered parameter estimation was applied. First, parameters were set at default values except the sediment ingestion rate (IR), which was fitted using the bioaccumulation data of the $0 \%$ PE B treatment. This resulted in $\mathrm{IR}=9.98 \mathrm{~g} / \mathrm{g} \mathrm{DW} \times \mathrm{d}^{-1}$, a value which is close to the value estimated following the equation provided by Cammen ${ }^{204}$ of $7.1 \mathrm{~g} / \mathrm{g} \mathrm{DW} \times \mathrm{d}^{-1}$. For the $0.05 \%$ and $0.5 \%$ PE scenario calculations, IR was set at $55 \%$ and $33 \%$ of this IR value in the control, derived from the measured heap mass production, here taken as a relative measure of ingestion. Second, the uptake rate constant in the gut (plastic-gut fluid exchange coefficient $\mathrm{k}_{1}$, Chapter 5) ${ }^{128}$ was optimized, which resulted in values of 0.080 and $0.27 \mathrm{~d}^{-1}$ for the $0.05 \%$ and $0.5 \% \mathrm{PE}$ treatments, respectively. Using this parameterization, the terms in Eq. 6.2 were evaluated for both PE scenarios (Table A6.3). It appears that at $0.05 \%$, PE contributed less than 3\% to PCB uptake and also the loss rate changed marginally (not shown), indicating no substantial effect of $\mathrm{PE}$ at this environmentally realistic dose. At the high concentration of $0.5 \%$, PE contributed more to uptake, but still to a minor extent for most PCBs with up to $62 \%$ of uptake for the most hydrophobic congener. This percentage should not be interpreted as extra bioaccumulation but as the contribution of plastic to the total uptake term in 
Eq. A6.1, meaning that the overall uptake term does not necessarily increase. For the latter PCBs, loss rates are predicted however to increase, leading to an overall predicted factor two decrease in bioaccumulation, as was suggested before (Chapter 5). ${ }^{19,128,243,245}$ These flux estimates, based on measured concentrations in all exposure media confirm the lack of a vector effect deduced from the bioaccumulation metrics discussed in the previous sections, thus mechanistically explaining the empirical observations.

Our results further illustrate that measuring bioavailability using passive samplers is crucial to understand exposure at the extremely low aqueous phase concentrations typical for hydrophobic chemicals in environmentally realistic exposure studies. To date, most studies that tested the effects of plastic ingestion on bioaccumulation did not assess aqueous phase concentrations with the detection limits that can be achieved with passive samplers, and they usually neglected the possibility of aqueous exposure. With the aid of metrics that normalize bioaccumulation to concentrations in the various exposure media and biodynamic modeling, we showed that these extremely low $\mathrm{pg} / \mathrm{L}$ concentrations in porewater still drive exposure and can explain bioaccumulation, a phenomenon that is widely recognized in the bioaccumulation literature. This work confirms model-based predictions on the limited relevance of microplastic for bioaccumulation under environmentally realistic exposure conditions, and illustrates the importance of assessing exposure through all media in microplastic bioaccumulation studies.

\section{Acknowledgements}

We thank Frits Gillissen, Gerrit Hoornsman, Yu Ren, Noël Diepens, Marie Trijau and Oscar Bos for their valuable contributions to this work. 
Chemical uptake under environmentally relevant conditions 


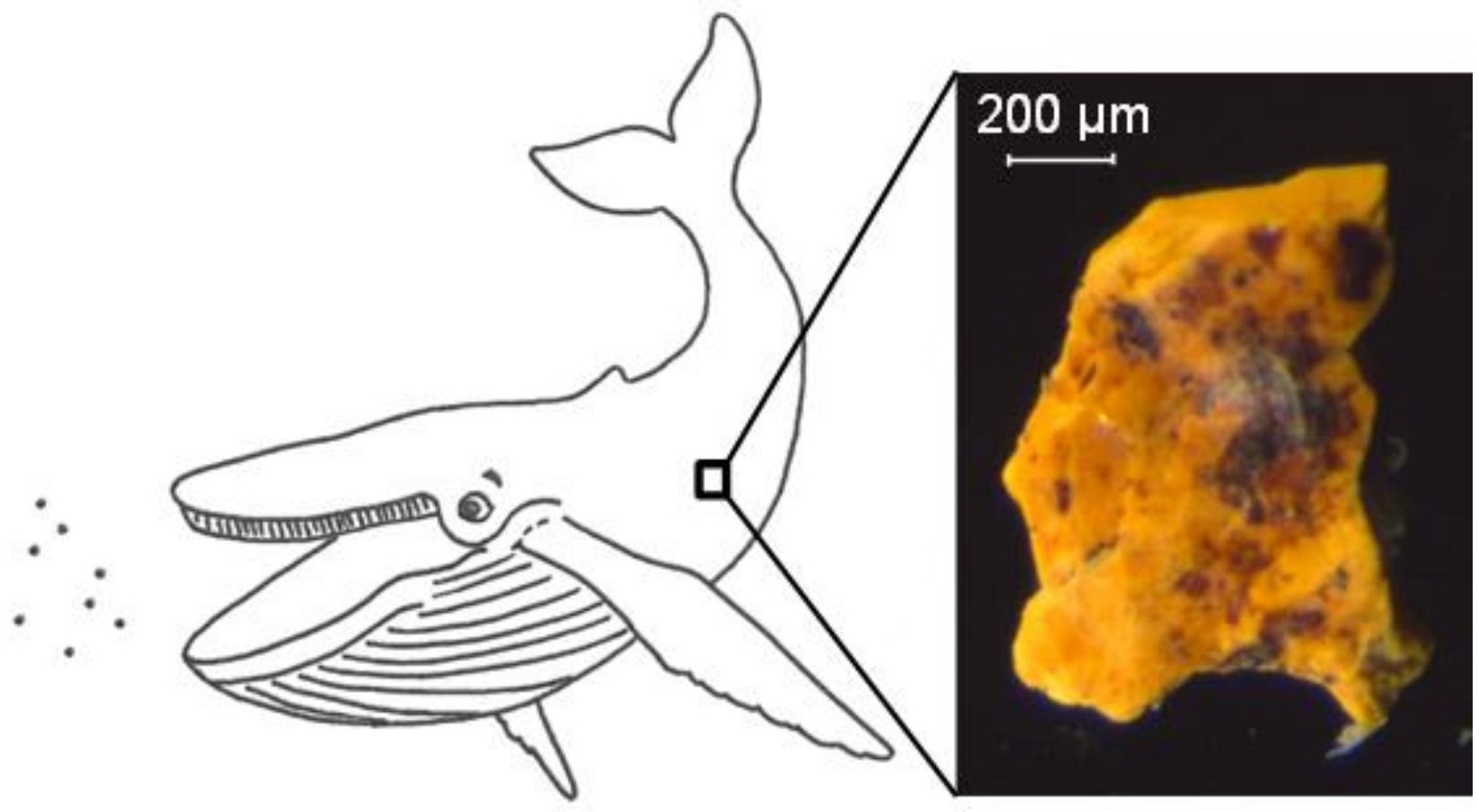




\title{
Microplastic in a macro filter feeder: humpback whale Megaptera novaeangliae
}

\author{
Published as: Besseling, E., Foekema, E.M., Van Franeker, J. A., Leopold, \\ M.F., Kühn, S., Bravo Rebolledo, E.L., Heße, E., Mielke, L., IJzer, J., \\ Kamminga, P., Koelmans, A.A., 2015. Microplastic in a macro filter feeder: \\ Humpback whale Megaptera novaeangliae. Marine Pollution Bulletin 95, \\ $248-252$
}

\begin{abstract}
Marine filter feeders are exposed to microplastic because of their selection of small particles as food source. Baleen whales feed by filtering small particles from large water volumes. Macroplastic was found in baleen whales before. This study is the first to show the presence of microplastic in intestines of a baleen whale (Megaptera novaeangliae). Contents of its gastrointestinal tract were sieved, dissolved in $10 \%$ potassium hydroxide and washed. From the remaining dried material, potential synthetic polymer particles were selected based on density and appearance, and analysed by Fourier transform infrared (FTIR) spectroscopy. Several polymer types (polyethylene, polypropylene, polyvinylchloride, polyethylene terephthalate, nylon) were found, in varying particle shapes: sheets, fragments and threads with a size of $1 \mathrm{~mm}$ to $17 \mathrm{~cm}$. This diversity in polymer types and particle shapes, can be interpreted as a representation of the varying characteristics of marine plastic and the unselective way of ingestion by Megaptera novaeangliae.
\end{abstract}




\subsection{Introduction}

Microplastic (i.e. particles with a synthetic origin $<5 \mathrm{~mm}$ ) ${ }^{(55)}$ is present in the marine environment due to direct disposal and degradation of larger plastic items ${ }^{55}$ and was first emphasized in the 1970's. ${ }^{10}$ Because of its small size and wide spread occurrence, microplastic is now thought to be available to species throughout the marine food web. ${ }^{11}$ Only a few studies about possible negative effects of microplastic on organisms have been published. ${ }^{50,51,53,124,268}$ So far reported possible negative effects of microplastic are on survival, feeding, oxidative status and uptake of persistent organic pollutants (Chapter 3, Chapter 4). ${ }^{49,51}$

Due to their feeding behaviour, filter feeders are thought to collect microplastic particles from the water column. Microplastic has indeed been encountered in bivalves ${ }^{269,270}$ and in planktivorous fish. ${ }^{46,213}$ By filtering a size range from plankton up to small fish, ${ }^{271,272}$ baleen whales can potentially ingest microplastic directly from the water column as well as via prey species. Exposure of baleen whales to microplastic has therefore been hypothesised recently. ${ }^{273,274}$ Phthalates in the blubber tissue as indirect indication of microplastic in a fin whale have been suggested by Fossi et al., ${ }^{274}$ although this does not differentiate between phthalate uptake from food items (zooplankton, small fish) and microplastic. Baleen whales are suggested to be useful as a monitoring species in the implementation of Descriptor 10 (Marine litter) of the Marine Strategy Framework Directive (MSFD), ${ }^{273,274275}$ even though direct measurement of microplastic in baleen whales has not yet been reported.

Mesoplastic (i.e. items with a synthetic origin of 5-20 mm) is often included in the macroplastic size category (i.e. items with a synthetic origin $>20 \mathrm{~mm}$ ) ${ }^{55}$ This includes plastic lids, bags and fishing lines and has been found in 31 marine mammal species, including baleen whales. ${ }^{276}$ Records of macroplastic in Cetacean species are increasing. While being reported in at least 26 Cetacean species before, ${ }^{44}$ macroplastic is reported in 48 (56\% of) Cetacean species by $2014^{(277)}$ and in $61.5 \%$ in the review by Kühn et al. ${ }^{278}$ Examples are $28 \%$ of examined Franciscana dolphins (Pontoporia blainvillei) having plastic in their stomach, including microplastic, ${ }^{44}$ micro- and mesoplastic in True's beaked whales (Mesoplodon mirus), ${ }^{279}$ severe incidences of large macroplastic quantities causing starvation and death in a beaked whale and several sperm

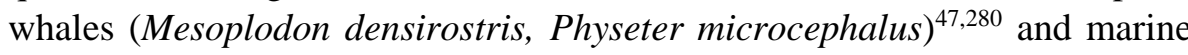
debris in two baleen whale species, Minke and Sei whale (Balaenoptera acutorostrata, B. borealis). ${ }^{277}$ Raised hypotheses based on these incidences 
are that 1) chances of micro- and macroplastic ingestion are higher for relatively passive feeders, as compared to active predators, ${ }^{281}$ 2) even small amounts of macroplastic can cause obstruction of the digestive tract, ${ }^{280,282}$ and 3) microplastic might be of special concern as it may clog the filtering apparatus of organisms. ${ }^{276}$ Theoretically, all of these hypotheses apply to baleen whales.

The non-selective feeding mode of many baleen whale species by ingesting material surrounding the intended prey in the water with a size large enough to be retained by their baleens, ${ }^{283}$ might result in exposure to microplastic. The ratio between microplastic and zooplankton ${ }^{42}$ indicates a possible daily intake of 3.7 thousand microplastic particles in fin whales in the Mediterranean. ${ }^{273}$ Negative effects of microplastic uptake on organisms in the marine environment might occur, though the information about effects is still limited. Meanwhile, microplastic is already present in the marine environment. ${ }^{11}$ This is why we studied the occurrence of microplastic in a stranded baleen whale, a humpback whale. Our study describes the first reported case of microplastic ingested by a humpback whale, and discusses it within the context of microplastic uptake related to ecological traits.

\subsection{Materials and methods}

\subsubsection{Animal}

At December $12^{\text {th }} 2012$, a $10.34 \mathrm{~m}$ long, ca. 16 thousand $\mathrm{kg}$ juvenile female humpback whale (Megaptera novaeangliae) stranded on a sandbank between harbour city Den Helder and the island Texel in the Netherlands, and was publically called 'Johanna'. Four days later, it died.

\subsubsection{Sampling}

Two days post-mortem, necropsy was performed on the severely autolytic carcass. Wood shreds were used around the humpback carcass for absorption of body fluids. Multiple tissue samples were preserved, including part of the gastrointestinal tract for content analysis. Gastrointestinal tract samples were stored at $-18{ }^{\circ} \mathrm{C}$ till further processing. After thawing, samples were sequentially sieved over two sieves with a mesh size of $1 \mathrm{~mm}$ and $0.5 \mathrm{~mm}$. Subsequently, the residues were dissolved in $10 \%$ potassium hydroxide $(\mathrm{KOH})$ solution. The remainder was washed according to previous methods in a washing machine in double washing bags, the inner bag having a mesh size of $300 \mu \mathrm{m}$ and the outer bag $120 \mu \mathrm{m} .{ }^{284}$ After washing, the samples were dried for three hours at $70{ }^{\circ} \mathrm{C}$. From the remaining material, possible synthetic polymer particles were selected based on density (floating/sinking in saturated 
$\mathrm{NaCl}$ dispersion) and appearance (Zeiss Stereo Discovery V8 microscope) according to previous procedures, ${ }^{285}$ measured by marking gauge (for subsequent volume calculation) and subjected to Fourier transform infrared (FTIR) analyses. FTIR spectra of the samples were gained with a Varian Scimitar 1000 FT-IR spectrometer equipped with a DTSG-detector. Sample and reference spectra were obtained using a measurement resolution of $4 \mathrm{~cm}^{-}$ ${ }^{1}$, following Gonzalez-Contreras et al. ${ }^{286}$

\subsubsection{Data analysis}

FTIR spectra of the particles were compared with reference polymer spectra (Thompson et al. 2004; Ng and Obbard 2006) of the seven most produced polymers polyethylene (PE), polypropylene (PP), polyvinylchloride (PVC), polyethylene terephthalate (PET), polystyrene (PS) and nylon (PA). ${ }^{8}$ Additionally, comparisons with reference spectra of natural rubber and cellulose were made. Statistical analyses were performed with linear regression in 'RStudio' statistical software (Version 0.98.976, R Development Core Team). Particles were the quality index i.e. the correlation coefficient $\left(\mathrm{R}^{2}\right)$ of the comparison with reference spectra was $>0.7$ were classified as synthetic polymers.

\subsection{Results}

\subsubsection{Post-mortem examination}

According to the well-developed musculature and blubber thickness, the humpback whale was in good nutritional condition. Severe post-mortal decomposition of all internal organs prevented detailed macroscopic and microscopic evaluation. About a fifth to tenth of the total length of the gastrointestinal tract was sampled for content analysis. There were few contents in the gastrointestinal tract. Continued digestion of the contents of the gastrointestinal tract during the four days of stranding, might have resulted in fluid contents that were partly deflated from the gastrointestinal tract during sampling. The primary cause of the stranding could not be identified. However, prolonged stranding in itself caused deterioration and death of the animal.

\subsubsection{Plastic}

A total of 45 particles of possible synthetic origin was found in the gastrointestinal tract samples. Of these, $77.7 \%$ was large enough $\left(>1 \mathrm{~mm}^{2}\right)$ to be analysed by FTIR. Of these particles, $45.7 \%$ had a synthetic origin (Figure 7.1), $25.7 \%$ had a natural origin and for $28.6 \%$ no matching spectra were obtained. The identified polymer types were PE, PP, PVC, PET and PA (Table 
7.1). For size categories, we follow the classification of Barnes et al ${ }^{55}$ Of the synthetic particles, $12.5 \%$ had a maximum dimension of 2.5 to $17 \mathrm{~cm}$ (macroplastic). These were threads, with a diameter of $0.1-0.23 \mathrm{~mm} .50 \%$ had a size of $5.8-12.0 \mathrm{~mm}$ by $0.3-8.2 \mathrm{~mm}$ (mesoplastic). Those were all sheets. The remaining $37.5 \%$ consisted of sheets and fragments with a size of 1.1 to $4.7 \mathrm{~mm}$ by $0.4-2.4 \mathrm{~mm}$ (microplastic). The found plastic particles all made up less than $3.5 \mathrm{~mm}^{3}$ per particle. As particles might fall apart during passage of the gastrointestinal tract, sampling or processing, we discuss particle volumes instead of particle numbers. A total plastic volume of $13.7 \mathrm{~mm}^{3}$ was encountered, of which most was PE and PA (Table 7.1), which might represent the worldwide most produced polymer (PE) as well as polymers used in the marine environment in fishing gear. ${ }^{8}$

There were few remainders of fish found in the gastrointestinal tract samples, being small fish bones, vertebra and otoliths of herring and sprat. Wood shreds were found in the gastrointestinal tract samples, most likely originating from the necropsy site. Twenty-five gram of comparable wood shreds was investigated by microscope. No plastic particles were found among these wood shreds.

The high variation in particle appearance and polymer type of the plastic found in the gastrointestinal tract samples is an indication that the particles originate from the marine environment. Studying synthetic fibres in the gastrointestinal tract samples was omitted, because of the high risk of fibres being caused by methodological contamination during sampling and analyses, i.e. clothes, washing bags. ${ }^{213,289}$ As fragments, threads and sheets are less than fibres prone to contaminate samples during the used methods and additionally because of the eroded condition of the found particles, we render it likely that they were ingested by the humpback whale at sea.

Humpback whales have an intestine length of about 5.5 times their body length ${ }^{290}$ such that the sampled humpback whale was estimated to have an intestine length of about $57 \mathrm{~m}$. As a fifth to a tenth of this length was sampled, it is likely that five to ten times as much plastic was present on a whole organism basis if we neglect gastrointestinal tract section type, than encountered in the subsample. That would result in an estimate of up to 160 small plastic particles or a volume of up to $137 \mathrm{~mm}^{3}$ of plastic in the whole humpback whale. 


\section{Chapter 7}

Table 7.1. Particle shape, number and size of particles and percentage of the total plastic volume found in the studied humpback whale, per polymer type with average $R^{2}$ values and ranges, together with the percentage of the total global production of these polymer types, $P E$ (polyethylene), PA (nylon), PP (polypropylene), PVC (polyvinylchloride) and PET (polyethylene terephthalate). ${ }^{8}$

\begin{tabular}{|c|c|c|c|c|c|c|}
\hline 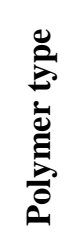 & 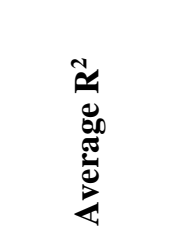 & 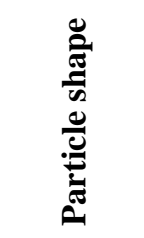 & 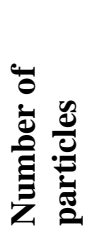 & 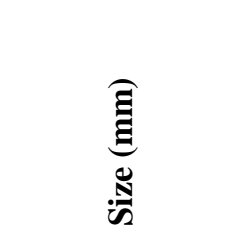 & 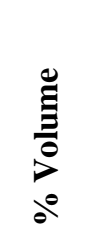 & 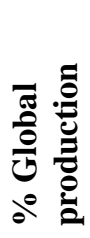 \\
\hline PE & $\begin{array}{c}0.90 \\
(0.88-0.98)\end{array}$ & Sheet & 9 & $\begin{array}{c}3.3-12 \times 0.3-8.2 \\
\times 0.04-0.2\end{array}$ & 55.01 & 38 \\
\hline PA & $\begin{array}{c}0.80 \\
(0.70-0.96)\end{array}$ & $\begin{array}{l}\text { Thread, } \\
\text { fragment }\end{array}$ & 4 & $\begin{array}{c}2.3-170 \times 0.1- \\
1.5 \times 0.1-0.4\end{array}$ & 37.64 & $<3$ \\
\hline $\mathrm{PP}$ & 0.82 & Sheet & 1 & $3.6 \times 2.4 \times 0.1$ & 5.61 & 24 \\
\hline $\mathrm{PVC}$ & 0.82 & Sheet & 1 & $5.8 \times 3.3 \times 0.01$ & 0.97 & 19 \\
\hline PET & 0.82 & Fragment & 1 & $1.1 \times 0.8 \times 0.2$ & 0.77 & 7 \\
\hline
\end{tabular}

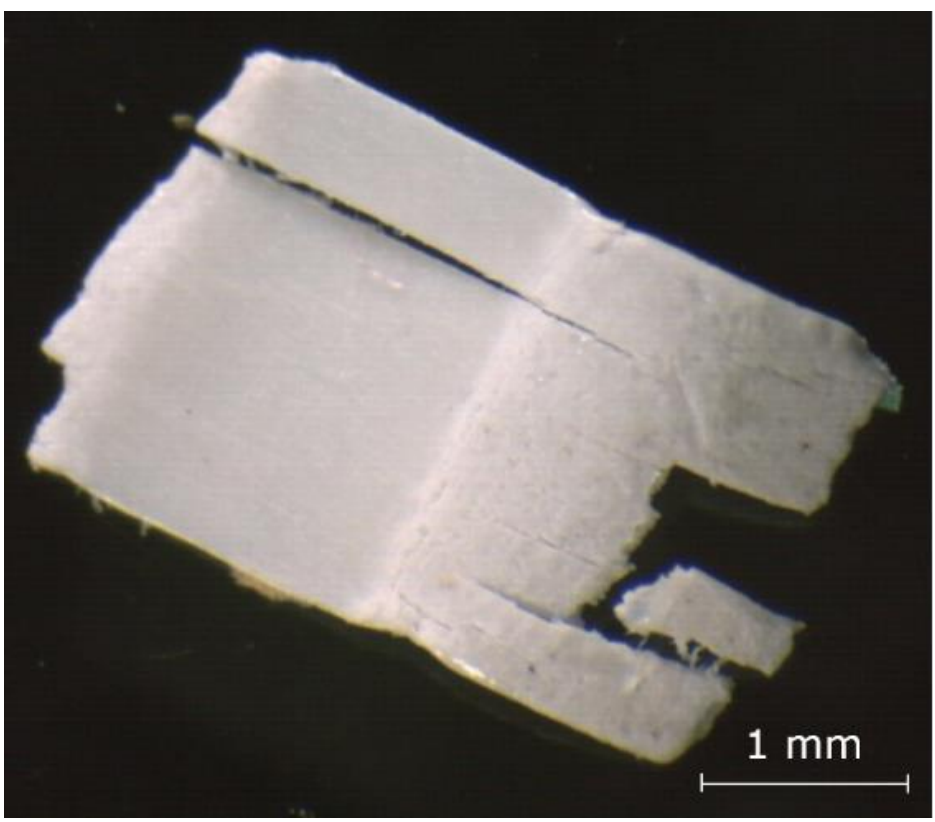

Figure 7.1. Polypropylene (PP) particle found in the gastrointestinal tract samples of the studied humpback whale, $R^{2}=0.82$. Additional photos of other particles found in the gastrointestinal tract of the studied humpback whale are given in the Appendix of this chapter. 


\subsection{Discussion}

By combining information on daily filtered water volume, gut passage time and plastic particle numbers in an organism, it is possible to calculate a plastic concentration in an organisms' foraging area from biological traits. The estimated concentration then can be compared with data on measured concentrations. Here we performed such a calculation by using plastic numbers of the humpback whale we studied. The daily filtered water volume of humpback whales has to our knowledge not been reported yet. For baleen whale species with similar feeding type, ${ }^{271,272}$ we here assume that daily filtered water volume is constant per unit surface of the baleen plate rows. We use the formula:

$V_{W, y}=V_{W, x}\left(\frac{B P R_{y}}{B P R_{x}}\right)$

with $\mathrm{V}_{\mathrm{W}}\left(\mathrm{m}^{3}\right)$ the average daily filtered water volume and BPR $\left(\mathrm{m}^{2}\right)$ the average surface of the baleen plate rows for species $\mathrm{Y}$ and species X. Fossi et $a l .{ }^{273}$ report a daily filtered water volume of 5.9 thousand $\mathrm{m}^{3}$ for fin whales. With the BPR of $4 \mathrm{~m}^{2}$ of fin whales and $2.6 \mathrm{~m}^{2}$ of humpback whales, ${ }^{271}$ we calculate a daily filtered water volume of 3.8 thousand $\mathrm{m}^{3}$ for humpback whales. The gut passage time (GPT) of baleen whales is to our knowledge unknown. We here make the assumption that the GPT can be assumed to be constant per unit length of the gastrointestinal tract, across Cetacean species. We use the formula:

$G P T_{y}=G P T_{x}\left(\frac{B G_{y}}{B G_{x}}\right)\left(\frac{B L_{y}}{B L_{x}}\right)$

with GPT (h) the average gut passage time, BG the body to gastrointestinal tract length ratio and $\mathrm{BL}$ the body length for species $\mathrm{Y}$ and species $\mathrm{X}$. A GPT $\mathrm{x}$ of $4.2 \mathrm{~h}$ has been reported for (Cetacean species) Amazon river dolphins (Inia geoffrensi) with a $\mathrm{BL}_{\mathrm{x}}$ of $2.22 \mathrm{~m} .{ }^{291} \mathrm{By}$ lacking the $\mathrm{BG}_{\mathrm{x}}$ for the Amazon river dolphin species we use $\mathrm{BG}_{\mathrm{x}}$ of another river dolphin (Gangetic dolphin, Platanista gangetica) of 7.3. ${ }^{290}$ We use a $\mathrm{BG}_{\mathrm{y}}$ of 5.5 for humpback whales ${ }^{290}$ and $\mathrm{BL}_{\mathrm{y}}$ of the studied humpback whale of $10.34 \mathrm{~m}$, and calculate the GPT of the studied humpback whale to be $\sim 14.6 \mathrm{~h}$. For inert particles, the particle content of the water volume ingested within the GPT, can be seen as the steady-state concentration of plastic in a filter feeding organism. We use the formula:

$$
C_{S S}=\frac{N P}{V_{W}\left(\frac{G P T}{24}\right)}
$$


with $\mathrm{C}_{\mathrm{SS}}$ (plastic particles $/ \mathrm{m}^{3}$ ) the steady-state concentration of plastic in the humpback whale, NP (n), $\mathrm{V}_{\mathrm{W}}\left(\mathrm{m}^{3}\right)$ and GPT $(\mathrm{h})$ respectively the number of particles, daily filtered water volume and gut passage time of the studied humpback whale. With this formula we calculate the filtered water volume of the humpback whale within the gut passage time $V_{W}\left(\frac{G P T}{24}\right)$ to be 2.3 thousand $\mathrm{m}^{3}$ of water and $C_{\mathrm{ss}}$ to be 0.07 plastic particles $/ \mathrm{m}^{3}$. We hypothesis that $\mathrm{C}_{\mathrm{Ss}}$ is directly related to the concentration of plastic in the foraging area of a filter feeding organism. We use the formula:

$C_{S S} \approx C_{f a}$

with $\mathrm{C}_{\mathrm{fa}}$ the concentration of plastic in the foraging area of the humpback whale. The average microplastic particle concentration in the Northeast Atlantic is 0.1 microplastic particles $/ \mathrm{m}^{3}$ excluding synthetic fibres. ${ }^{292}$ This measured concentration in water is thus very close to the calculated concentration based on the small plastic particles in the humpback whale, implying that formula 4 may hold. It must be noted that this calculation concerns only one humpback whale and includes several uncertain conversion factors. Further research is needed to confirm whether this relation between plastic concentration in organisms and foraging area applies more generically.

Microplastic has different characteristics based on the large surface to volume ratio compared to macroplastic. ${ }^{55,63,171}$ As first studies on physical as well as chemical effects of microplastic on organisms have only recently appeared (Chapter 4), ${ }^{50,51,53,124,268}$ there is still a large knowledge gap about possible negative effects of microplastic. Therefore, although not reported yet for most whale species, ingestion of microplastic might be of specific concern and is recommended to be studied alongside ingestion of macroplastic.

The estimated number of small plastic particles in the studied humpback whale may be lower than in other baleen whales. Humpback whales are mainly lunge feeders, that is, swallowing a mouth full of preferably concentrated masses of planktonic crustaceans or fish, subsequently retaining the prey by filtering the water through the baleens. ${ }^{271,272,290}$ This may result in lower plastic uptake compared to other preferably water or mud skimming baleen whales. Feeding by these strategies as well as filter feeding by making use of the water flow, which in general can be created by either hydrodynamics or organisms themselves, might result in a higher plastic intake compared to lunge feeding. Mortality at sea and decay of carcasses before necropsy diminishes the number of opportunities to study plastic occurrence in whales. ${ }^{276}$ Together with the 
likelihood of (micro)plastic ingestion, this indicates the importance of reporting also singular incidences of plastic in gastrointestinal tracts of examined baleen whales.

\section{Acknowledgements}

The authors like to thank Gerrit Hoornsman and André Meijboom (WMR, Wageningen UR, the Netherlands) for their help at the sampling site and Wouter Teunissen (Food \& Biobased Research, Wageningen UR, the Netherlands) for his help on the FTIR measurements. 


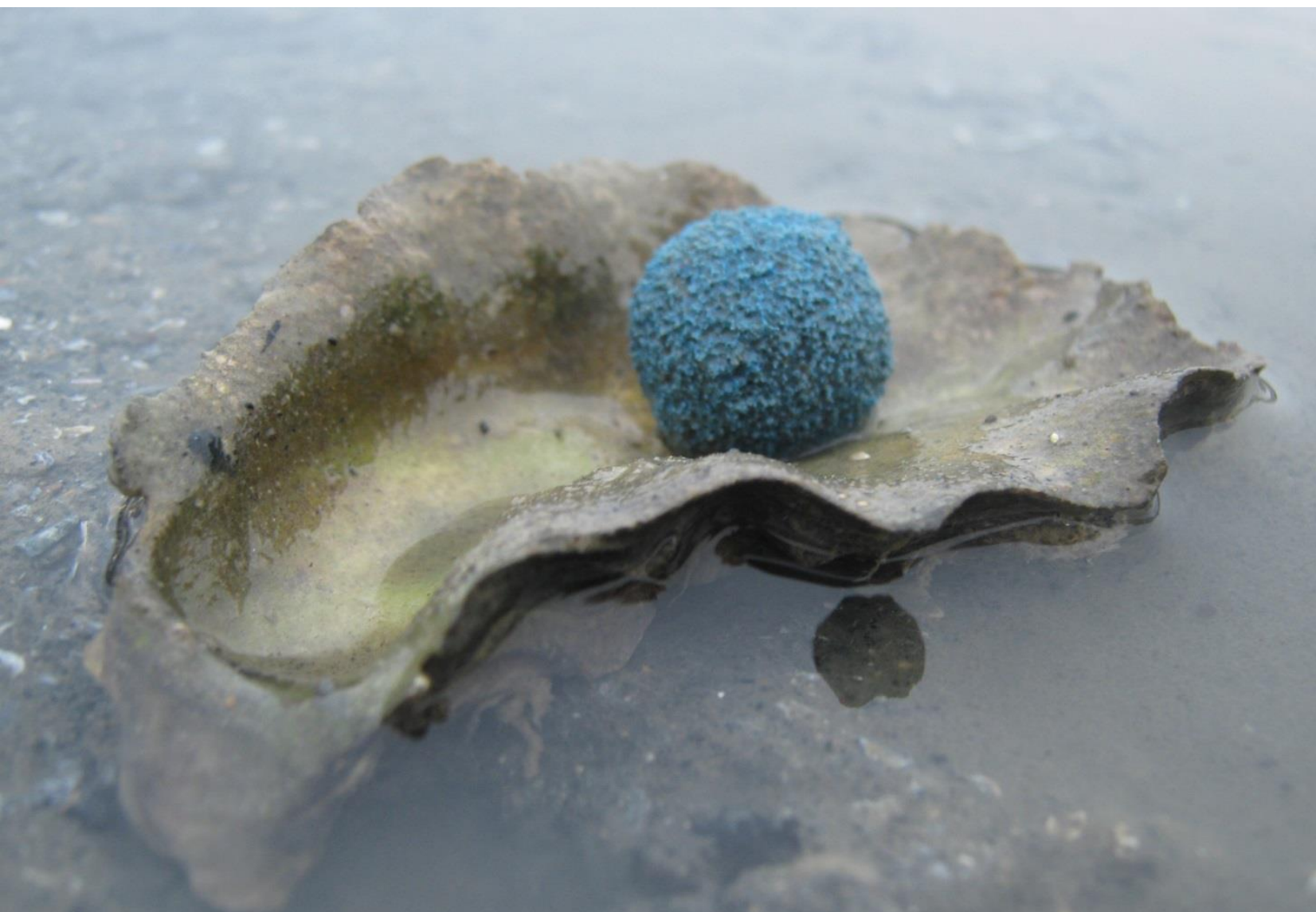




\section{Synthesis}

\section{Towards quantification of the risk of aquatic micro- and nanoplastic}

\section{Introduction}

Pollution with micro- and nanoplastic is thought to cause risks to aquatic ecosystems. ${ }^{15,293}$ Several effects of microplastic have been hypothesised or demonstrated, such as alteration of sediment porosity, shading, dilution of food, blockage of the gastrointestinal tract of animals, increased transport of invasive species and increased transfer of chemicals along the food chain (the 'vector effect').${ }^{5,27,28}$ Effects hypothesised or demonstrated for nanoplastic and the smaller size range of microplastic in particular include translocation from the intestines into other tissues, oxidative stress, immune response and particle toxicity. ${ }^{11,27-30}$ However, whether effects will occur in nature depends on the actual exposure. Environmental risk assessment (ERA) methodology generally requires exposure concentrations to exceed effect threshold concentrations in order to conclude that a risk exists. ${ }^{294}$ This chapter presents a provisional quantitative assessment of the risk of micro- and nanoplastic in the aquatic environment. The assessment is based on the data and results reported in this thesis, including unpublished data on two topics that are not reported in the previous chapters, supplemented with data from the literature. The assessment is based on seven steps: (1) assessing ranges of exposure concentrations that are currently found in the aquatic environment globally, (2) assessing ranges of exposure concentrations in Dutch river deltas as a case study for the Netherlands, (3) refining the expected exposure by use of exposure models, (4) assessing the nano- and microplastic effect thresholds reported to date, (5) assessing community level effect thresholds using a species sensitivity distribution (SSD) approach, (6) reflecting on the latest insights into the effect of micro- and nanoplastic on chemical transfer and risk, and (7) comparison of exposure and effect levels to characterise risk (Figure 8.1). Finally, the chapter reflects on public concerns, the current state of knowledge and the direction of further research into micro- and nanoplastic in the environment. 


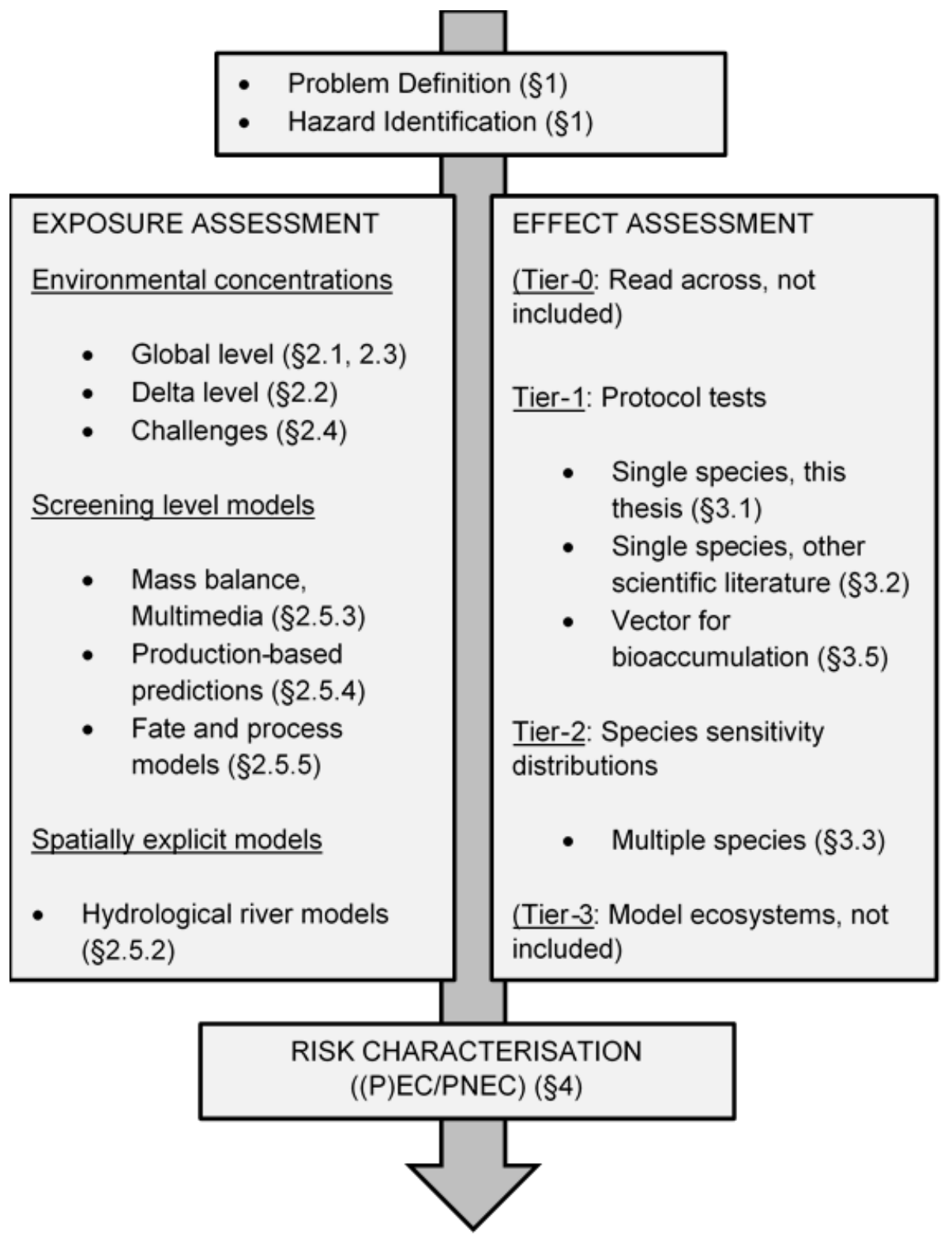

Figure 8.1. Tools for exposure and effect assessment as part of the general environmental risk assessment framework for micro- and nanoplastic. Based on Koelmans et al. ${ }^{295}$ The symbol $\S$ marks the section in this chapter in which each tool is discussed. 


\subsection{Exposure assessment}

Defining the actual risk of micro- and nanoplastic requires information on exposure concentrations. Below, these exposure concentrations are compared with effect concentrations in the risk characterisation of the actual risk assessment (Figure 8.1). This section provides an overview of measured and modeled ranges of micro- and nanoplastic concentrations.

\subsubsection{Microplastic concentrations from source to sea}

Are aquatic organisms being exposed to micro- and nanoplastic in the environment? And if so, at what concentrations? These questions underlie the so-called retrospective exposure assessment. The aquatic environment consists of various habitats, and microplastic concentrations have been found to differ among regions. Concentrations of microplastic (plastic with a $0.1 \mu \mathrm{m}$ - $5 \mathrm{~mm}$ particle size) found in the environment are summarised in the table below (Table 8.1), arranged from inland water locations towards the ocean: surface freshwater, freshwater sediment, near-shore or estuarine surface water, subtidal sediment, beach sediment, open sea or ocean surface water and seafloor sediment. The concentrations in freshwater and estuarine habitats are shown for each continent. Open sea or ocean concentrations are provided for each oceanic region for which microplastic concentrations could be found in the scientific literature. Data was gathered from about 120 studies mentioned in the reviews by Eerkes-Medrano et al. ${ }^{61}$ and Lusher, ${ }^{296}$ supplemented with studies on the occurrence of microplastic in the aquatic environment published in the years 2015-2016. The concentrations reported to date vary greatly among compartments and regions but also within studies. Here, only the highest limits of reported ranges (HLRR) are summarised, because (1) the lower limits of the reported ranges often exclude zero-encounters, which would thus imply bias, and (2) this represents the worst-case information with respect to exposure. Most concentrations in the water compartment rely on surface sampling. A recent study of samples taken from different depths down to $5 \mathrm{~m}$ below the ocean surface revealed that microplastic concentrations approach zero at a depth of $5 \mathrm{~m}$, but are considerably higher in the water column above. The data indicated that total buoyant microplastic amounts are underestimated by a factor of $1.04-30$ when based on surface sampling by instruments like manta trawls. ${ }^{297}$ 


\section{Chapter 8}

Table 8.1. Highest limits of reported ranges (HLRR) of particle concentrations going from inland towards the ocean, in the following compartments: freshwater surface water, freshwater sediment, near-shore or estuarine surface water, subtidal sediment, beach sediment, open sea or ocean surface water and seafloor sediment, per continent or oceanic region. Because of the orders of magnitude of variation, concentrations are presented on a logarithmic scale. As most studies have reported environmental concentrations in numbers of particles per mass or per volume, these are the units used in this table. The following conversion factors were used to convert to approximate particles per mass or volume where needed: microplastic particle weight $5 \mu \mathrm{g} /$ particle, trawling depth $0.1 \mathrm{~m}$, sediment density $1.8 \mathrm{~kg} / \mathrm{L}$ and sediment porewater fraction 20\%. These factors are explained in more detail in section 8.2.4 Studies up to and including the year 2016 were used.

\begin{tabular}{|c|c|c|c|c|c|c|c|}
\hline 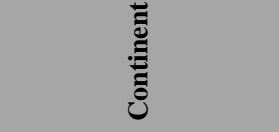 & 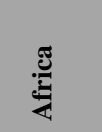 & $\frac{\pi}{2}$ & 产 & z & 苞 & 遏 & 巡 \\
\hline $\begin{array}{l}\text { Freshwater surface } \\
\text { water }\left(\log \text { particles } L^{-1}\right)\end{array}$ & & -5.36 & -0.85 & -3.92 & & & 61 \\
\hline $\begin{array}{l}\text { Freshwater sediment } \\
\left(\log \text { particles } \mathrm{kg}^{-1} \mathrm{DW}\right)\end{array}$ & & & 1.81 & 3.18 & & & 61 \\
\hline $\begin{array}{l}\text { Near-shore surface } \\
\text { water }\left(\log \text { particles } L^{-1}\right)\end{array}$ & -6.14 & 1.20 & 2.01 & -0.27 & -3.59 & -6.07 & 296 \\
\hline $\begin{array}{l}\text { Subtidal sediment (log } \\
\left.\text { particles } \mathrm{kg}^{-1} \mathrm{DW}\right)\end{array}$ & & & 2.32 & 2.43 & -0.55 & & 296 \\
\hline $\begin{array}{l}\text { Beach sediment (log } \\
\left.\text { particles } \mathrm{kg}^{-1} \mathrm{DW}\right)\end{array}$ & & 4.31 & 3.62 & 3.00 & 2.32 & 1.84 & 296 \\
\hline 蔍 & 吾 & 晜 & Е & E & & & \\
\hline $\begin{array}{l}\text { Open ocean surface } \\
\text { water }\left(\log \text { particles } L^{-1}\right)\end{array}$ & -4.40 & -4.52 & -2.66 & -5.27 & & & 296 \\
\hline $\begin{array}{l}\text { Seafloor sediment (log } \\
\left.\text { particles } \mathrm{kg}^{-1} \mathrm{DW}\right)\end{array}$ & -0.55 & & & & & & 296 \\
\hline
\end{tabular}

The HLRR of microplastic concentrations in the environment vary by more than ten orders of magnitude (Table 8.1). Even after correcting for the sediment density (approximated as $1.8 \mathrm{~kg} / \mathrm{L}$ ) concentrations in sediment on a volume basis are generally higher than in surface water, which is explained by the settling of particles. The particles settle either as singular particles, or in 
aggregated or fouled form, as long as their density is higher than that of the surrounding water. ${ }^{298}$ Consequently, although sediment-buried plastic may be less available, sediment-dwelling organisms are expected to be exposed to higher concentrations than pelagic organisms. Nevertheless, microplastic in sediment is expected to affect the pelagic foodweb, via transfer through the foodchain and resuspension. Due to increased water turbulence or defouling, originally settled plastic particles are expected to become resuspended in the water column and lead to exposure of pelagic organisms. ${ }^{299}$

Variations in HLRR of microplastic concentrations among continents seem to be lower in the open sea and ocean areas compared to that in freshwater systems and near-shore areas. For example, the HLRR of microplastic concentrations in surface water vary by three orders of magnitude in seas and oceans, compared to five and nine orders of magnitude in fresh and near-shore water, respectively. This difference in observed heterogeneity might reflect better mixing or a difference in representativeness of the sampling in the marine environment and less mixing and larger variability in magnitude of sources and flow conditions for fresh- and coastal waters. Sampling with a trawl over several hundreds of metres in open water might give a more representative sample than pointwise sampling with a bucket or flask from the shore. Furthermore, one could expect less mixing and a greater influence of location-specific hotspot characteristics for sediment compared to water. ${ }^{298}$ Although this is not yet reflected in a greater variation of microplastic concentrations in sediment compared to water among continents, such a large variation is seen among studies. For example, the HLRR in near-shore sediment in Europe are found at a plastic factory site and are generally a factor $10^{2}$ to $10^{8}$ higher than in the surrounding areas. The absence of observations of large variations in HLRR in sediment concentrations among continents might be due to the fact that sediment sampling has been done in fewer continents than water sampling. Intercontinental comparison is especially hampered for marine sediment, as the number of studies that have included marine sediment is very limited.

The HLRR of microplastic concentrations in near-shore or estuarine areas seem to be higher than concentrations in freshwater systems and open sea or ocean. Accumulation of microplastic in these areas might be high, due to input from rivers and beaches, and from wash-back by marine water currents. Nearshore hydrodynamics, combined with high biological activity, might trap, degrade and foul both microplastic and macroplastic, ${ }^{300-302}$ which could explain the high microplastic concentrations found here. Yet, the development 
of the research field may also have influenced these differences between areas: whereas awareness of marine microplastic pollution has increased through studies of the marine environment, ${ }^{8}$ the freshwater environment has remained relatively underexamined. ${ }^{16,61}$ Furthermore, logistics make remote open seas or oceans harder to study than near-shore locations. ${ }^{296}$ Since the chances of finding a high microplastic concentration are affected not only by the actual environmental concentrations but also by the sampling effort, this factor might partly explain the HLRR of microplastic concentrations found in near-shore areas.

The HLRR of concentrations in the near-shore regions are higher in sediments than in the overlying water, which also applies to freshwater and the open ocean. Concentrations in beach sediments are even higher than in subtidal sediments. This can most probably be explained by the relatively low density of plastic compared to seawater, causing floating and suspended plastic to be washed ashore, ${ }^{303-308}$ while beaches may act as a filter for plastic (and other) particles. The high exposure of microplastic to sunlight, wind and waves near and on beaches increases the degradation of larger plastic items to microplastic $(<5 \mathrm{~mm}){ }^{300-302}$ The chances of detecting these high concentrations are substantial, as beaches have by far the highest sampling effort of all habitats. ${ }^{296}$

Globally, the overall HLRR of microplastic concentrations in freshwater and near-shore surface water are to be found in Europe, those in freshwater sediment and subtidal sediment in North America, those on beaches in Asia, those in open ocean surface water in the North Pacific and those in marine sediment in the North Atlantic. However, there are remarkable data gaps regarding microplastic concentrations in several compartments for the continents of Africa and Oceania in particular, and to a lesser extent for Asia and South America. Macroplastic concentrations are known to be high on African beaches (Figure 8.2), which makes it very likely that high microplastic concentrations occur there too.

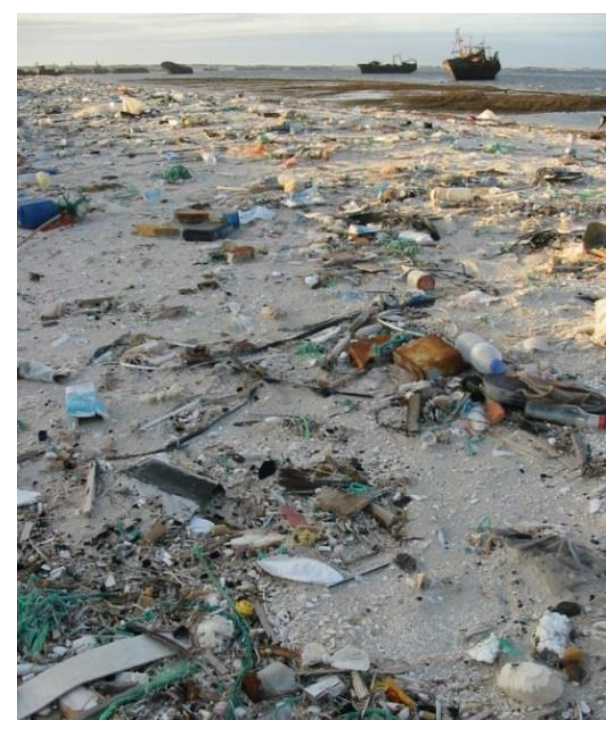

Figure 8.2. West-African coastline. Kees Goudswaard, Wageningen Marine Research. 


\subsubsection{Microplastic concentrations in freshwater. A case study on a river delta in the Netherlands}

Little is known about the distribution of microplastic along river deltas, in terms of concentrations, hotspots and shape and size distributions. Relatively high concentrations have been found in Europe (Table 8.1). Although data have been reported for many countries, no data were available for the Netherlands at the start of our research. This section zooms in on microplastic concentrations in the Dutch river delta as a case study. Below, the results are reported of the first surveys ${ }^{309,310}$ of the occurrence of microplastic that included both freshwater, estuarine and marine sediment and surface water locations of the main river delta in the Netherlands.

Methodology. During four sampling campaigns in 2013-2015, partly carried out in collaboration with the waterboard Rivierenland, sediment and surface water samples were collected. Sediment samples were taken from 34 locations along the Dutch river delta using a Van Veen grab or a spade. Sediment sampling was partly replicated: 1-, 3- and 5-fold for the freshwater, estuarine and marine parts of the delta, respectively. Water was sampled at 23 freshwater locations by filtering $10 \mathrm{~m}^{3}$ successively over $1 \mathrm{~mm}$ and $300 \mu \mathrm{m}$ sieves and a $50 \mu \mathrm{m}$ net (Figure 8.3). For five locations, this was done in triplicate. At 18 locations, an additional $1 \mathrm{~L}$ water sample was filtered over a $0.45 \mu \mathrm{m}$ filter (Whatman, cellulose nitrate membrane) to determine the $<0.45$ $\mu \mathrm{m}$ size fraction. Both urban and rural areas were sampled, including rural water outlets into rivers as well as inlets of river water into rural areas, and samples were taken both upstream and downstream from wastewater treatment plants

Furthermore, influent water, effluent water and sewage sludge at three WWTPs were sampled. Sediment samples were treated by density separation (saturated $\mathrm{NaCl}$

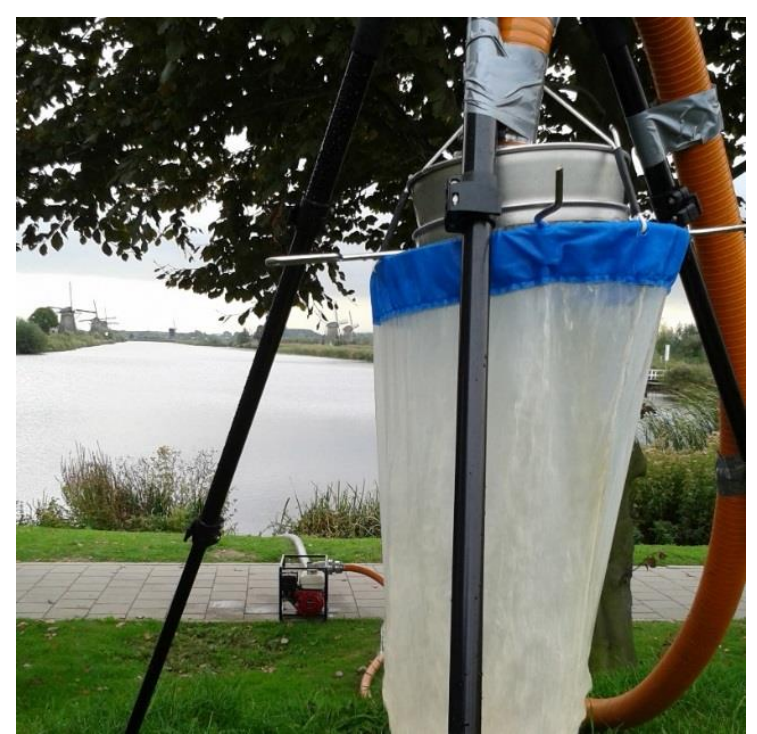

Figure 8.3. Sampling unit showing the $1 \mathrm{~mm}$ and $300 \mu \mathrm{m}$ sieves and $50 \mu \mathrm{m}$ net. 
solution) and filtered over a $50 \mu \mathrm{m}$ sieve. Both sediment and water samples were treated to degrade organic matter $(10 \% \mathrm{KOH}$ solution) as described for the treatment of tissue samples in Chapter 7.213,311,312 Subsequently, microplastic particles were selected from the filtrate by microscopy, based on visual appearance (Zeiss Stereo Discovery V8 microscope). Except for fibres (see section 8.2.4), all particle shapes were included (see for example Figure 8.4). All laboratory analyses were performed in a laminar flow cabinet, and no plastic materials or synthetic clothes were used during the analyses. Procedural blanks were included, by analysing demineralised water samples before and after running them through the whole procedure. The detection limit was defined as the number of particles that would have been found per standard volume if one particle had been detected in the analysed sample volume.

Results and discussion. For sediment, the highest concentration was 56.3 particles/kg dry weight (DW), with highest concentrations in the freshwater part of the delta, moderate in the estuarine part and lowest in de marine part (Figure 8.5). The microplastic concentrations in the sediment in this study are in the same range as those in the German part of the Rhine catchment (up to 64 particles $/ \mathrm{kg}$, Wagner et al. ${ }^{16}$ ). A concentration of 20.6 particles $/ \mathrm{kg}$ DW was found in the sediment of a ditch into which a WWTP discharges. For the sake of comparison, the effluent and sludge of this WWTP were analysed too, and contained on average $2.7 \pm 1.9(\mathrm{SD})$ particles $/ \mathrm{m}^{3}$ and $7.1 \times 10^{2} \pm 7.7 \times 10^{2}$ particles/kg DW, respectively. ${ }^{310}$

Concentrations of $50 \mu \mathrm{m}-5 \mathrm{~mm}$ microplastic in freshwater ranged from $<0.1$ to 6 particles $/ \mathrm{m}^{3}$. Of these particles, $12 \%$ were in the $50-300 \mu \mathrm{m}$ size class, $58 \%$ were in the $300 \mu \mathrm{m}-1 \mathrm{~mm}$ size class and $30 \%$ were in the $>1 \mathrm{~mm}$ size class. Concentrations of $>0.45 \mu \mathrm{m}$ microplastic in freshwater ranged from $<5$ to 40 particles/L. As the latter is at least three orders of magnitude higher than the concentration range for $>50 \mu \mathrm{m}$ particles, this implies that a major fraction of the microplastic in freshwater consists of the smaller size range of microplastic particles. A comparison with the $>50 \mu \mathrm{m}$ particle numbers found in sediment and water also shows a three orders of magnitude difference, with concentrations being much higher in sediment than in water. This indicates that microplastic is removed from the water phase by settling, as already predicted by the modeling described in Chapter $\mathbf{2}$ of this thesis. ${ }^{298}$ The same modeling predicts that this settling causes the smaller size fraction to accumulate in sediments too. This implies that the $>0.45 \mu \mathrm{m}$ particle concentrations of up to 40 particles/L found here in freshwater might even be 
three orders of magnitude higher for freshwater sediments. The microplastic concentrations found in freshwater systems in this study are at the lower end of the 0.3-0.5 particles $/ \mathrm{m}^{3}$ range in freshwater elsewhere in Europe and the US. ${ }^{38,57,58}$

Elsewhere in the Netherlands, Leslie et al $^{313}$ found much higher concentrations of microplastic in freshwater systems, especially in freshwater sediments. Nevertheless, a similar trend could be observed in their data, with highest concentrations in freshwater systems $(48-187$ particles/L in urban canal water; $1.4-4.9 \times 10^{3}$ particles $/ \mathrm{kg} \mathrm{DW}$ in riverine suspended matter; $<68$ $-1.05 \times 10^{4}$ particles $/ \mathrm{kg}$ DW in urban canal sediment) compared to coastal waters $\left(100-3.6 \times 10^{3}\right.$ particles $/ \mathrm{kg}$ DW in coastal or offshore sediment). Furthermore, the latter study included analyses of 7 WWTPs, whose influent, effluent and sewage sludge contained $68-910$ particles/L, $51-81$ particles/L and $510-760$ particles/kg WW, respectively. That these concentrations in sediment, surface water and WWTP influent and effluent are higher than the concentrations found elsewhere in the Netherlands and elsewhere in Europe and the US might relate to local differences or to methodological differences. Since some studies include fibre-shaped particles, and extrapolations are made based on relatively small samples, ${ }^{313}$ these outcomes might be prone to overor underestimation.
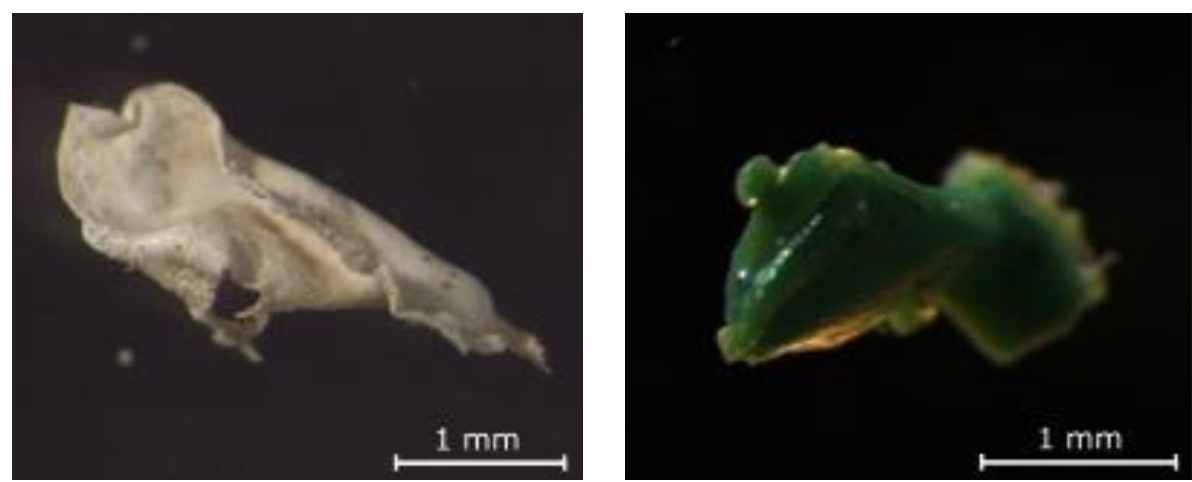

Figure 8.4. Microplastic particles with different shapes (left: sheet, right: fragment) found in sediment and water in the Rhine and Meuse delta. Adapted from Besseling et al. ${ }^{310}$

In the current study, differences between freshwater concentrations in rural versus urban areas were not large, and similarly no large differences were found between inlets and outlets from and towards international rivers. This either means that there are no large regional or international differences in microplastic pollution, or that water is not the appropriate medium to detect 


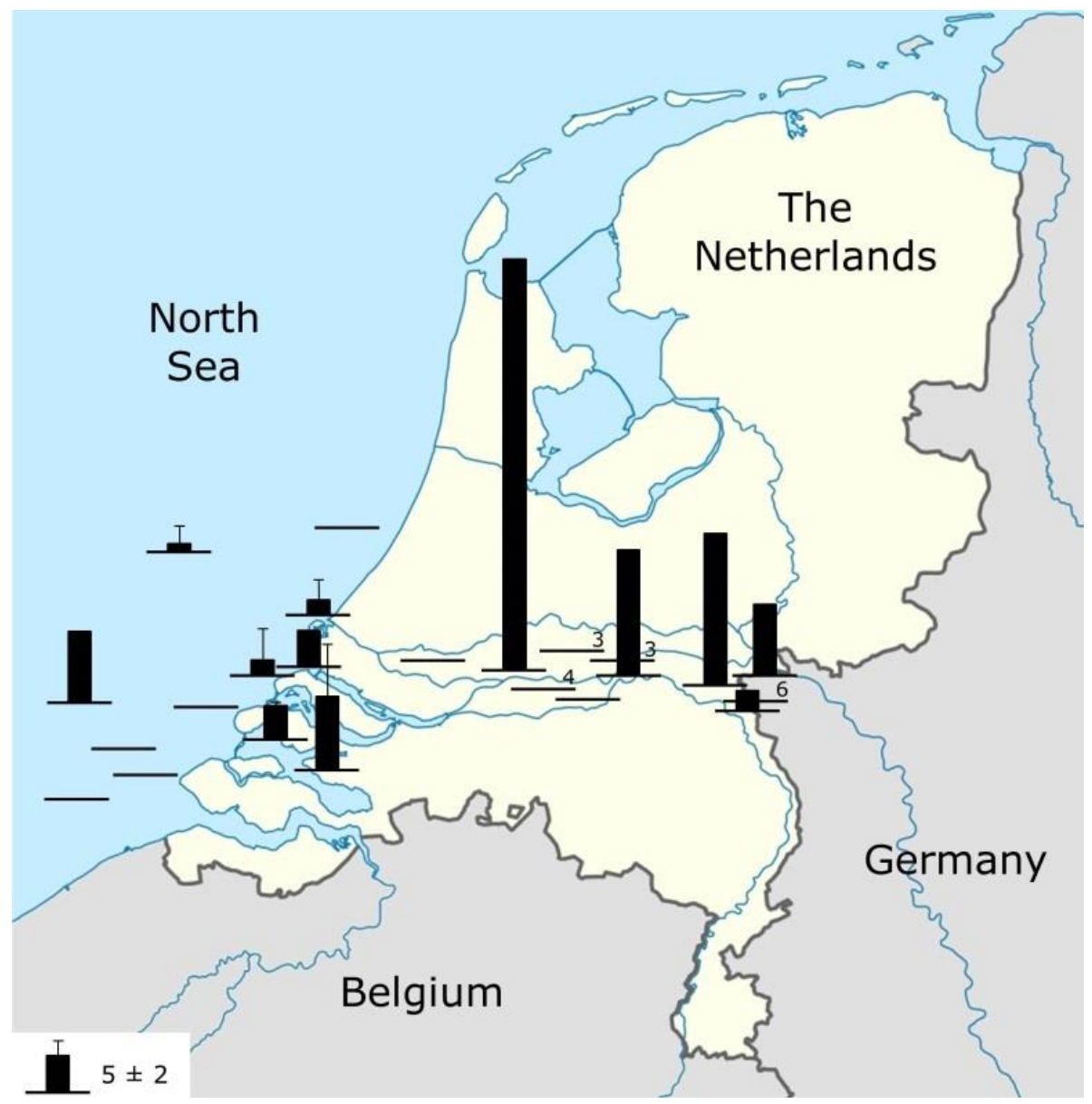

Figure 8.5. Number of plastic particles (particles $/ \mathrm{kg}$ DW sediment, \pm STD, size range $50 \mu m-$ $5 \mathrm{~mm}$ ) along the Dutch part of the Rhine/Meuse delta. Adapted from Besseling et al. ${ }^{310}$ and supplemented with data from Foekema et al. ${ }^{309}$ Horizontal lines indicate locations were no microplastic was detected. Numbers in the figure (3-6) indicate multiple nearby locations with zero-encounters of microplastic. Locations are approximate.

microplastic in, due to high removal rates from the water as indicated in Chapter 2 of this thesis. ${ }^{298}$ The large regional variations in shapes of particles that were found point to the latter explanation. The concentrations of microplastic in WWTP effluents were generally about a factor of $4.4 \pm 4.0$ (SD) lower than those in WWTP influents (Table 8.2, factor difference calculated by dividing the effluent by the influent concentration, using the detection limit as concentration when no particles were found), although this varied among the different plants. This corresponds with data by Leslie et al. ${ }^{313}$ on different WWTPs. Concentrations of microplastic in effluent were higher 
than those in the surrounding freshwater systems. Nevertheless, there was no large difference between upstream and downstream concentrations near WWTPs. This further supports the idea that surface water is not the ideal medium in which to quantify microplastic pollution sources, due to dilution and settling out of the water column, and due to the fact that the concentrations are greatly influenced by rain, wind and the flow rate of the water on the specific sampling date. The triplicate samples of sewage sludge we took at one WWTP still showed a range of $2 \times 10^{2}-1.6 \times 10^{3}$ particles $/ \mathrm{kg} \mathrm{DW}$. One year later, no particles were found in the sewage sludge from this WWTP and two others. Detection limits by then were $<400-500$ particles/kg DW, so that concentrations can be assumed to be below this limit. Despite these differences between time points and WWTPs, sewage sludge can be considered a potentially large source of microplastic when reused in the environment.

Table 8.2. Microplastic concentrations in freshwater in the Dutch delta. For samples in which no particles were found, the < sign indicates a detection limit $(D L)$. The $D L$ is the number of particles that would have been detected in a standard volume in case one particle had been present in the sample. Thus the DL is lower when a larger sample volume was analysed. ${ }^{309}$

\begin{tabular}{|c|c|c|c|}
\hline 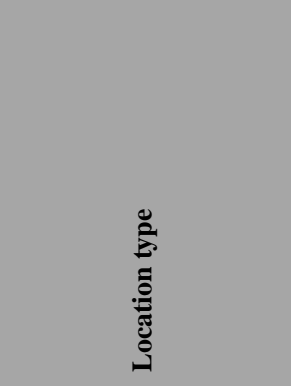 & 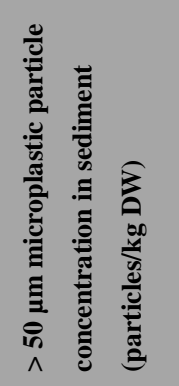 & 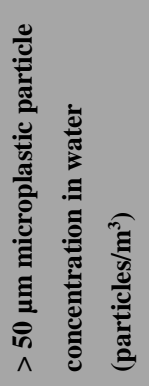 & $\begin{array}{l} \\
\\
\end{array}$ \\
\hline WWTP influent & & $<10-<25$ & $20-40$ \\
\hline WWTP effluent & & $2.2-9.6$ & $<6-21$ \\
\hline WWTP sewage sludge & $<408-706$ & & \\
\hline Upstream WWTP & $<2.5-<25.9$ & $<0.2-6.0$ & $<5-10$ \\
\hline Downstream WWTP & $<2.6-21$ & $0.1-3.2$ & $<5$ \\
\hline Urban area & $<2.6-56$ & $0.1-0.4$ & $<5$ \\
\hline Rural area & $<4.5-17$ & $0.1-0.4$ & $<5$ \\
\hline Outlet rural area & $<2.4-<30.8$ & $<0.1-0.3$ & $<5-<7$ \\
\hline Inlet river & $<2.7-<10.3$ & $<0.1-0.1$ & $<5-5$ \\
\hline Subtidal zone & $\leq 9.9$ & & \\
\hline Off-coast & $\leq 9.7$ & & \\
\hline
\end{tabular}




\subsubsection{Conclusion microplastic monitoring}

There is relatively low spatial variation in microplastic concentrations in water, but a large spatial variation in microplastic concentrations in sediment. This corresponds with global findings, as presented in the previous section, suggesting that:

(a) many diffuse sources contribute to microplastic pollution; ${ }^{314}$

(b) non-buoyant microplastic settles out of the water column within relatively short distances from their source (Chapter 2, this thesis); ${ }^{298}$

(c) model-guided sediment monitoring focusing on hotspots is the best way to reveal the extent of microplastic pollution (Chapter 2, this thesis), ${ }^{298}$ for example using sediment traps;

(d) sediment-dwelling organisms living at hotspot locations are exposed chronically to microplastic, ${ }^{16}$ whereas acute exposure of pelagic organisms may occur mainly during resuspension events;

(e) differences between sampling locations might not be detectable when selecting microplastic visually, which calls for the use of polymer identification techniques.

\subsubsection{Exposure to nanoplastic}

It is commonly assumed that nanoplastic, that is, plastic with a size of $\leq 100$ $\mathrm{nm}$ in at least one of the particles' dimensions, is emitted to ${ }^{18,315}$ and formed within the environment. ${ }^{50}$ The presence of nanoplastic has been proved under controlled laboratory conditions using nanoparticle tracking analysis ${ }^{66}$ and dynamic light scattering. ${ }^{67}$ To date, no technique is available that allows the detection of nanoplastic in environmental samples. Pyrolysis GC-MS might be used and has been proved to identify low concentrations of polymers in an environmental matrix, ${ }^{316}$ while UV-VIS spectroscopy and field flow fractionation (FFF) are techniques under development which might be promising in this respect. ${ }^{15}$ Since no technique is so far able to detect nanoplastic in environmental samples, it is not possible to provide actual measured environmental exposure concentrations for it. Due to the diffuse nature of sources and the lack of information on routes towards and removal mechanisms from the aquatic environment, even estimates of current concentrations are hard to make. The expected trend over time, however, is that environmental concentrations of nanoplastic will increase, because of (1) their increased application in a variety of products, ${ }^{317}(2)$ its production as byproduct during manufacturing ${ }^{18,318}$ and (3) the huge potential release by 
fragmentation and degradation of macro- and microplastic. ${ }^{15,50,319}$ Nanoplastic is used, inter alia, in paints, coatings, medicines, electronics and research. ${ }^{15}$ Manufacturing processes that are known to release nanoplastic as a by-product include thermal cutting of polystyrene foam and 3D printing. ${ }^{18,318}$

Under the influence of mechanical, thermal, chemical and biological stressors in the environment, microplastic is expected to degrade into smaller particles. ${ }^{15,50,319}$ The highest reported values on aquatic concentrations of microplastic in the Netherlands are, as mentioned above, 10 particles/ $\mathrm{L}$ in surface water and 56.3 particles $/ \mathrm{kg}$ DW in sediment until the year 2016, and as published by Leslie et al. in $2017,{ }^{313} 187$ particles/L in surface water and $1.05 \times 10^{4}$ particles $/ \mathrm{kg}$ DW in sediment. On a global scale, highest reported microplastic concentrations in the aquatic environment are 102 particles/L water and 1529 particles/kg DW sediment, with considerable differences between regions and between freshwater, estuarine and marine habitats. Values for beach sediments, which are not included in these global maxima, are even an order of magnitude higher. Depending on the size of the microplastic particles that have been found and the estimated size of the eventual nanoplastic particles, degradation of microplastic particles with a size of $>0.1 \mu \mathrm{m}-5 \mathrm{~mm}$ into $100 \mathrm{~nm}$ nanoplastic particles can lead, based on volumes of spherical particles, to particle concentrations that are ultimately $1.25 \times 10^{14}$ times higher than the currently found microplastic particle concentrations. Although non-spherical particles, such as sheets, degrade into smaller numbers of nanoplastic particles, macroplastic can potentially degrade to even larger numbers of nanoplastic particles. Thus, the estimate of $1.25 \times$ $10^{14}$ times higher nanoplastic particle concentrations compared to measured microplastic particle concentrations seems not to greatly overestimate the potential generation of nanoplastic in the environment. Model-based estimations of the time-scale at which such fragmentation and degradation into nanoplastic would occur are of the order of several hundreds of years. ${ }^{15}$ Nevertheless, the first indications that a fraction of the marine litter consists of nanoplastic have already been reported. ${ }^{67}$

\subsubsection{Challenges in defining micro- and nanoplastic concentrations}

The previous sections reported on assessments of the HLRR of microplastic concentrations in different habitats of different continents (Table 8.1). Mean concentrations from the same studies or study regions were generally a factor of two to a thousand lower. However, the differences between methodologies makes generalisation unreliable. ${ }^{294}$ Variations in methodology regard the 
included size range and particle shapes; reported units; calculation of maxima and the extraction and identification of plastic. These factors are briefly discussed below.

Size range and shape. The size cut-off for microplastic used in this thesis is $0.1 \mu \mathrm{m}-5 \mathrm{~mm}$. However, some studies include only particles in a narrower range as microplastic, often determined by their sampling methodology or the detection limit of devices they used. A commonly used lower limit due to mesh size lies between $300-800 \mu \mathrm{m}$, while the upper limit is often set between 2.16 and $4.75 \mathrm{~mm}$ or up to $5 \mathrm{~mm} .{ }^{58}$ The limits set in these studies result in microplastic numbers being underestimated compared to the definition used in this thesis. Furthermore, studies differ as to whether all particle shapes are included, distinguishing between fragments, spheres, sheets, pellets, ropes and fibres. The choice of particle shapes being included is affected by variations in the conditions under which samples in different studies are collected and analysed. In addition, microplastic literature in recent years has involved some discussion on the effect of contamination of samples during sampling and analyses. ${ }^{213}$ Nowadays, attempts are made to perform studies under fully clean air and plastic-free conditions, that is, completely eliminating exposure of samples to air, as the later could possibly contain fibres from the clothes of researchers or other dust, as well as the use of plastic materials for collection, storage and analysis. ${ }^{311}$ Consequently, reported microplastic concentrations vary due to:

(a) having fibres excluded due to their high risk of methodological contamination;

(b) no fibres being reported, without mention of exclusion;

(c) all particle shapes being included, irrespective of methodology;

(d) inclusion of fibres because of clean air and plastic-free methods. ${ }^{296}$

The work reported on in this thesis aimed to include the whole $0.1 \mu \mathrm{m}-5 \mathrm{~mm}$ microplastic size range and to avoid methodological contamination and exclude fibres. Nevertheless, the studies listed in Table 8.1 were included irrespective of whether they gave reasons for including or excluding certain particle shapes. Nor does the present chapter use any correction for the size ranges included in these studies, because insufficient information is often provided by the individual studies to allow conversions. 
Reported unit and unit conversions. Microplastic concentrations are variably reported as mass or as particle numbers per mass, per volume or per surface area of water or sediment, or even per study site. Some studies, even from recent years, only compare the results of their investigated sites qualitatively. These differences in units and lack of quantification make quantitative comparisons between studies very difficult. Several studies have used conversion factors to be able to make comparisons. ${ }^{294}$ However, this adds another source of uncertainty. Also, sampling depth, time of day and sampling technique were often not described in detail in the studies reviewed. Conversion factors used to calculate the values given in Table 8.1 are as follows. Concentrations reported as particle mass have been converted to numbers and vice versa ${ }^{49}$ by using a mass per particle factor of $5 \mu \mathrm{g} /$ particle, based on the weight of an average microplastic particle as detected on shores. ${ }^{105}$ When outcomes were given per surface area, an assumed manta trawl sampling depth of $0.1 \mathrm{~m}$ has been used to convert surface area-based concentrations (particles $/ \mathrm{km}^{2}$ ) to volume-based concentrations (particles $/ \mathrm{m}^{3}$ ). ${ }^{320}$ Volume concentrations or mass concentrations in wet weight have been transferred to dry weight concentrations by assuming a sediment density of $1.8 \mathrm{~kg} / \mathrm{L}^{51}$ and a porewater fraction of $20 \% .{ }^{321}$

Calculation of highest limits of reported ranges of microplastic concentrations. Comparing environmental concentrations of microplastic often requires medians or ranges of reported concentrations per water body, habitat type, ocean or compartment. However, an extra source of uncertainty in defining maximum, or average, concentrations is that the calculations underlying reported environmental concentrations are often unclear and differ between studies. ${ }^{296}$ The present literature appears to report highly variable metrics of concentration, such as averages, medians, maximum averages, average maxima and maxima. Even a description like 'average' can imply multiple calculation methods: it can imply that the average of all samples in the study is given, or that averages per subsample or sampling site were first calculated and then the study average. Remarkably, zero-encounters or nondetects are often excluded before calculation of the averages, ${ }^{61,296}$ which erroneously leads to higher predicted environmental concentrations. Furthermore, calculation of a meaningful average concentration for a certain region based on data from different studies should involve a weighted average, because of the varying numbers of sampling sites used in studies. In view of these obvious deficiencies and to provide an impression of exposure under worst-case conditions, Table 8.1 only shows the HLRR of microplastic 
concentrations. These may also have been affected by differences in calculations, e.g. because of differences between average maxima and real maxima, and may be highly dependent on the number of sampling sites because of spatial heterogeneity. However, the number of additional calculation steps used to combine studies is smaller than for averages. Furthermore, compared to effect thresholds, the high variability of concentrations among sampling sites means that if averages are below effect concentrations, they are not indicative of whether there could be a risk or not. By using the HLRR only, one cannot assess the risk for the entire region, but at least one can assess whether there is a risk. Furthermore, from a risk perspective, evaluating the HLRR of microplastic concentrations would comply with the 'worst case' approach in risk assessment. If, for instance, the HLRR were sufficiently below effect threshold concentrations, all other sites studied in the region can be assumed to be free of risk.

Extraction and identification of plastic. Environmental media that may contain nano- or microplastic will also contain a fraction of water, mineral constituents and/or organic matter. This is the case for abiotic as well as biotic samples. A variety of methods have been applied to isolate nano- or microplastic from the samples for plastic analyses. These include density separations with varying types and concentrations of salts, sieving, removal of organic matter with acids, bases and peroxide (or combinations thereof) and drying or decomposing samples at different temperatures. ${ }^{63,65,250,311,322-324}$ These methods differ in the extent to which particles other than plastic are removed, the likelihood of contamination of the samples with procedural plastic particles from materials used or the work space, ${ }^{213,325}$ but also in the extent to which plastic particles that are present in the environmental samples are retained in the samples. Losses of plastic particles occur during these procedures, depending on the number of extraction cycles involved and the aggressiveness of the chemicals used. Several chemicals used for sample preservation or for the digestion of tissue or organic matter, as well as high temperatures, are known to degrade specific polymer types, which thus will affect the analysis results. ${ }^{83,322,323,326}$ After clean-up of the samples, the subsequent detection of microplastic particles is usually done by visual inspection and increasingly by polymer identification techniques using reference spectra, like FTIR and Raman spectroscopy. The eventual identification, however, is highly dependent on the method used. For instance, of the particles visually judged as plastic, a percentage varying from 1.4 to $70 \%$ was identified as plastic by focal plane array (FPA), micro-Fourier 
transform infrared (micro-FTIR) spectroscopy or Raman spectroscopy. ${ }^{57,63,294,311,327}$

The above challenges illustrate that the analysis of plastic debris in environmental samples is not straightforward. Nevertheless, the number of studies underlying the data summarized in Table 8.1 is high. If the above restrictions are kept in mind, Table 8.1 thus gives us at least an impression of the HLRR of environmental microplastic concentrations known to date. Future studies should stick to standardised quality assurance criteria such as those provided by Hermsen et al., ${ }^{294,311,328}$ as there seems to exist a relation between reported particle counts and the degree of quality assurance. ${ }^{311}$

\subsubsection{Modeling the fate of and exposure to plastic}

Introduction. Models can be used to assess the exposure to micro- and nanoplastic. For microplastic, models can complement measurements and guide monitoring campaigns. ${ }^{212,298}$ And as the current techniques are not yet capable of detecting nanoplastic in environmental samples, prospective exposure assessment for nanoplastic is completely dependent on modeling. Since transport and fate models have been used before for other pollutants and other particle types like algae, micro-organisms, sediment and nanomaterials, models for micro- and nanoplastic can build upon this existing knowledge. ${ }^{212,298}$ Spatiotemporally explicit models are most helpful when it comes to transport and retention patterns within freshwater and marine systems. For freshwater systems, this type of model has been developed for micro- and nanoplastic at different scales, ranging from single river catchments ${ }^{298,329}$ to the global scale. ${ }^{314,330}$ So far, these models have been theoretical, and though validated with other particle types, they have not been fully validated for plastic particles yet. For marine systems, particle behaviour and ocean circulation models are used to model the fate of microplastic and to find optimal clean-up strategies. ${ }^{299,331}$ Below, currently available microplastic models are summarised going from their source towards and inside the oceans.

River transport modeling. Spatiotemporally explicit models have addressed catchment hydrology, soil erosion, sediment budgets, ${ }^{329}$ advective transport, homo- and heteroaggregation, sedimentation-resuspension, polymer degradation, presence of biofilm and sediment burial processes. ${ }^{298}$ In Chapter 2 of this thesis it was shown that for particles in the $100 \mathrm{~nm}$ to $10 \mu \mathrm{m}$ size range, concentrations in the water of a $40 \mathrm{~km}$ river stretch dropped from an input concentration of $1 \mathrm{ng} / \mathrm{L}$ to steady-state concentrations between 0.5 and $0.8 \mathrm{ng} / \mathrm{L}$ at the end of the stretch. ${ }^{298}$ Another model study indicated that leaving 
the aggregation of small plastic particles with other suspended solids out of consideration results in higher percentages being predicted to remain in the water column. ${ }^{329}$ However, several empirical studies, including the aggregation-sedimentation study described in Chapter 2, have shown that fouling followed by aggregation and settling is relevant for particle fate in aquatic systems. ${ }^{84,298,332}$ Therefore, leaving aggregation out of consideration might lead to overestimation of aquatic plastic particle concentrations. Both of these river catchment modeling studies agree that concentrations of particles in the upper part of the microplastic size range become reduced to 0 to $20 \%$ of the input concentration in the water column. ${ }^{298,329}$ High flow periods might, however, cause resuspension and remobilise this pool. ${ }^{329}$ The locations of hotspot concentrations in riverine sediment largely depend on the particle size of the plastic and on river morphology. In our study, the highest steadystate concentration in riverine sediment was predicted to be $5 \mathrm{mg} / \mathrm{kg}$ for $>1$ $\mathrm{mm}$ particles at $<1 \mathrm{~km}$ from the $1 \mathrm{ng} / \mathrm{L}$ input source. ${ }^{298}$ For most other sizes and locations, the steady-state concentrations were a factor 2 to 15 lower. The calculated steady-state concentrations within $1 \mathrm{~km}$ from the source are in line with concentrations of microplastic found by Wagner et al. (2014) in the sediment of freshwater systems in Europe. ${ }^{298}$ Apart from relatively close to the source, hotspot concentrations of microplastic are also expected in sedimentation areas characterised by a reduced flow velocity. Hence, monitoring campaigns and impact assessments should focus on these regions. ${ }^{298,329}$

Modeled effects of polymer density 298,329 and formation of biofilms ${ }^{298}$ on the fate of plastic particles were not large, although this formation of biofilms and its effects on fate processes has been empirically confirmed, and is receiving increasing attention in the recent literature. ${ }^{94,299,332-334}$ As the modeled plastic particles were spherical, particles of different shape might behave differently. ${ }^{298,314}$ A global modeling study incorporating microplastic of different shapes and point sources predicted that the majority of riverine transport of microplastic to sea would consist of synthetic polymers from tyre abrasion $(>40 \%)$. The other fractions are made up by plastic-based textiles abraded during laundry (29\%), synthetic polymers and plastic fibres in household dust (19.4\%) and microbeads in personal care products $(9.8 \%) .{ }^{330}$ Yearly, 1.15 to 2.41 million tonnes of plastic waste are estimated to enter the oceans from rivers, $67 \%$ of which is made up by the top 20 most polluting rivers. ${ }^{335}$ 
Emission-based mass flow modeling and multi-media modeling. Emissionbased mass flow modeling and multi-media modeling have been used before to estimate fluxes of pollutants and particles between compartments like the atmosphere, terrestrial soil, surface water and water-bottom sediment. ${ }^{80,295,314}$ For microplastic, the first provisional calculations of mass flows from WWTPs to water bodies predicted a retention for microplastic of 40 to $96 \%$ in WWTPs, depending on the type of plant. ${ }^{336}$ In comparison, the new data provided in section 8.2.2 indicate a retention of $53 \% \pm 46(\mathrm{SD})$ in three WWTPs, while Leslie et al. reported $72 \pm 61 \%$ (SD). Multi-media models like SimpleBox4Nano (SB4N) calculate average background concentrations in different compartments. ${ }^{314}$ Assuming a yearly emission of $20 \mathrm{kt},{ }^{337}$ of which $50 \%$ to the water and $50 \%$ to the soil, resulted in the average background predicted environmental steady-state concentrations (PECs) in the Rhine catchment (Europe) shown for each compartment in Table 8.3. ${ }^{314}$ These PECs are based on the yearly emission of $20 \mathrm{kt}$, such that PECs based on new estimated yearly predictions can be derived from the ratios between the $20 \mathrm{kt}$ used here and the respective emissions. Although the resulting output concentrations are highly dependent on the assumed yearly emissions in a catchment, SB4N clearly predicted different fates for microplastic particles of different sizes. ${ }^{314}$ Whereas PECs of nanometre and micrometre sized plastic particles are highest in the soil and water compartments, PECs of particles bigger than $0.1 \mathrm{~mm}$ are expected to be highest in aquatic sediments (Table 8.3). This largely corresponds with the distribution of micrometre and millimetre sized plastic particles over water and sediment calculated with the aforementioned spatiotemporally explicit models (Chapter 2) ${ }^{298,329}$ However, compared to the outcomes of SB4N, spatiotemporally explicit modeling predicted a higher retention of nanoplastic in sediments due to settling of aggregated nanoplastic. Another emission-based mass flow model was used by Siegfried et al. ${ }^{330}$ to calculated riverine transport of microplastic in Europe. The model revealed large spatial differences in transported plastic mass, largely as the result of differences in the technological status of WWTPs. For the transport of plastic from production and consumption sites towards the coastal and marine environment, Siegfried et al. used the output from the modeling simulations discussed in Chapter 2. An extrapolation towards the year 2050 showed that riverine transport of plastic will increase in some rivers while decreasing in others, the latter decreases being mainly explained by the expected improved wastewater treatment. ${ }^{330}$ 


\section{Chapter 8}

Table 8.3. Distribution of plastic particles of different sizes over the soil, water and aquatic sediment compartments, as predicted by the multi-media model SB4N. PECs are based on a yearly emission of $20 \mathrm{kt}^{314}$

\begin{tabular}{|l|c|c|c|c|c|}
\hline Particle size & $\mathbf{0 . 1} \boldsymbol{\mu m}$ & $\mathbf{1} \boldsymbol{\mu \mathbf { m }}$ & $\mathbf{1 0} \boldsymbol{\mu \mathbf { m }}$ & $\mathbf{1 0 0} \boldsymbol{\mu \mathbf { m }}$ & $\mathbf{1 ~} \mathbf{~ m}$ \\
\hline Soil $\left(\log \mu \mathrm{g} / \mathrm{m}^{3}\right)$ & 6.43 & 6.38 & 6.17 & 4.57 & 2.62 \\
\hline Water $\left(\log \mu \mathrm{g} / \mathrm{m}^{3}\right)$ & 5.45 & 5.44 & 5.39 & 4.89 & 3.08 \\
\hline Sediment $\left(\log \mu \mathrm{g} / \mathrm{m}^{3}\right)$ & 1.52 & 2.41 & 4.42 & 6.07 & 6.26 \\
\hline
\end{tabular}

Coastal and marine microplastic concentration estimates based on plastic production. Recently, Van Cauwenberghe predicted microplastic concentrations in the coastal and marine environment by using plastic production data. ${ }^{338}$ Van Cauwenberghe calculated the cumulative emission of plastic based on the world plastic production, assuming that 1.7 to $4.7 \%$ of this annual plastic production reaches the oceans. ${ }^{338}$ Using this percentage, she calculated current coastal and open ocean microplastic abundance as well as extrapolations of future concentrations (Table 8.4). ${ }^{338}$ She used two scenarios to forecast a range of future environmental plastic concentrations: a businessas-usual continuation of the current growth of the annual plastic production of $4.5 \%$ per year, and an immediate stop to plastic production (Table 8.4). ${ }^{338}$ The production-based estimates obtained this way appeared to exceed current measured concentrations of total floating plastic debris particles by only one order of magnitude. Given the uncertainties in data and calculation, this overestimation was considered acceptable, ${ }^{338}$ also because the calculation did not take into account the fact that part of the emitted plastic is missed during sampling, settles in deeper layers ${ }^{339}$ and/or resides at beaches.

Table 8.4. Coastal and open-ocean microplastic concentrations in surface water and sediment, estimated by Van Cauwenberghe. ${ }^{338}$

\begin{tabular}{|l|c|c|c|}
\hline \multirow{2}{*}{ Particle size } & 2015 & \multicolumn{2}{|c|}{2100} \\
\cline { 3 - 4 } & $4.7 \times 10^{-4}-2.1$ & $\begin{array}{c}\text { Immediate } \\
\text { production stop }\end{array}$ & Business-as-usual \\
\hline $\begin{array}{l}\text { Coastal surface water } \\
\text { (particles/L) }\end{array}$ & $9.7 \times 10^{-3}-11.9$ & $0.03-129.4$ \\
\hline $\begin{array}{l}\text { Coastal sediment } \\
\text { (particles/L) }\end{array}$ & $1.3 \times 10^{-4}-0.3$ & $7.5 \times 10^{-4}-2.0$ & $0.01-21.1$ \\
\hline $\begin{array}{l}\text { Open ocean surface } \\
\text { water (particles/L) }\end{array}$ & $0.7-15.7$ & $3.7-91.1$ & $40.5-987.2$ \\
\hline $\begin{array}{l}\text { Seafloor sediment } \\
\text { (particles/L) }\end{array}$ & $0.1 \times 10^{4}$ & $597-2.2 \times 10^{5}$ \\
\hline
\end{tabular}

(a) Based on an assumed average polymer density of $1100 \mathrm{~kg} / \mathrm{m}^{3}$ and a particle size distribution of 10\% $1-5 \mathrm{~mm}, 25 \% 0.3-1 \mathrm{~mm}$ and $65 \% 1-300 \mu \mathrm{m}$ sized particles. 


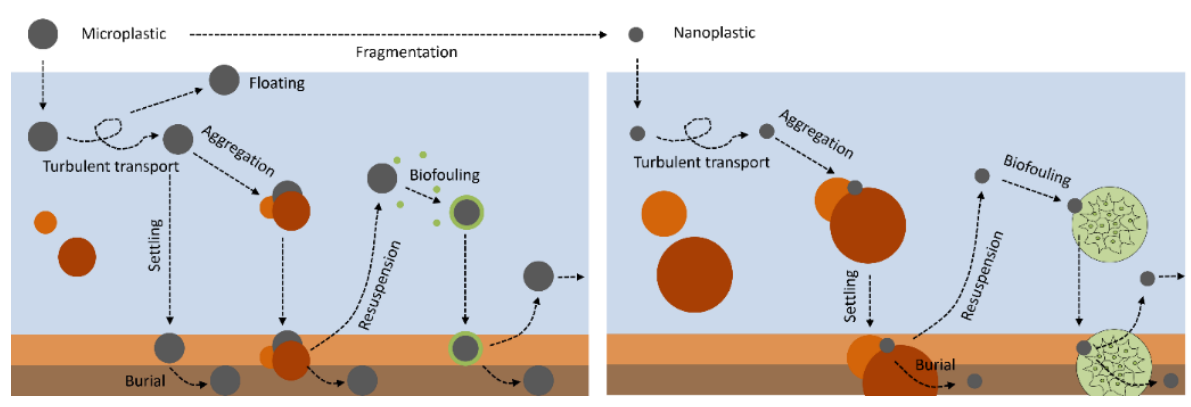

Figure 8.6. Processes that affect the fate of plastic particles in the aquatic environment, adapted from Kooi et al. ${ }^{340}$

Fate processes and models for the estuarine and marine environment. Overview of current models. As plastic particles reach the river mouth, the relative importance of the different processes that affect their fate in rivers is assumed to change (Figure 8.6). Here, the higher salinity causes a wider variety of polymer types to drift within or float upon the water column. The change in salinity can also destabilise dispersed submicron particles, causing aggregation of particles ${ }^{15}$ that might become non-buoyant, and may result in settling. Particles with intermediate polymer density would settle in freshwater but float in marine water. Polymer types that are on their way to settle, might change direction when they reach the estuarine environment. Their fate depends on the type of estuarine system. ${ }^{341,342}$ In a salt-wedge estuary, these polymer types will most probably drift above the halocline within the relatively fresh upper part of the water column, whereas in well-mixed estuaries they are likely to be pushed even further upward towards the water surface. The presence of a halocline ${ }^{343}$ as well as the strength of the tides ${ }^{344,345}$ will affect the time during which plastic particles remain within the water column. In the marine environment too, the vertical distribution might consist of a buoyant, a settling and an in-between fraction. The in-between fraction, at intermediate depth, is thought to remain there due to the opposing mechanisms of fouling and buoyancy. ${ }^{299}$ Whereas many previous studies predicted that the majority of microplastic in the marine environment would be present in the surface layer of the upper few metres, ${ }^{297}$ recent modeling scenario studies indicate that the fraction at a lower, intermediate, depth may well be larger. ${ }^{299}$ Aggregation of plastic particles with materials of higher density will accelerate settling, ${ }^{298,299}$ but the same process will slow down the settling rate of other mineral or organic particles, as inclusion of plastic in the aggregate reduces their overall density. ${ }^{332}$ Diurnal and seasonal fluctuations in photosynthesis, and hence in biofilm growth, might induce an oscillating 
movement of micrometre sized plastic over a depth range down to about $75 \mathrm{~m}$ below the ocean surface. ${ }^{299,301}$

The global spatial distribution of plastic is affected by several oceanographic processes. Global ocean circulation, tides and Ekman transport cause accumulation to occur in five to six main regions. ${ }^{301,346}$ Using ocean surface current models, combined with debris concentrations or spatial macroplastic beach clean-up data, resulted in surface concentration estimates for macroplastic which could be compared with spatial distributions or speciesspecific habitats of sea turtles and birds. ${ }^{347-349}$ These modeling efforts revealed where turtles and sea birds have the highest encounter rates with plastic and suggested where cleaning efforts might be most cost-effective. Interestingly, this is not so much in the main subtropical oceanic plastic accumulation regions, but instead in parts of the Southern Ocean where plastic concentrations are not extremely high but the number of seabird species is, ${ }^{347}$ namely off the coast of China and in the Indonesian archipelago near large sources of debris from land ${ }^{331,349}$ and near the typical entry point where debris enters the Gulf of Carpentaria. ${ }^{348}$

Some examples of processes currently missed by transport models. So far, plastic particle fate models have mainly included abiotic processes only, the exception being the influence of biofilm formation. However, it is not only the abiotic processes of beaching and incorporation into marine snow, but also more biotic processes, like ingestion and bioturbation, which are likely to affect the fate of plastic particles. As part of the experimental work described in Chapter 6 of this thesis, we studied the feedback between microplastic and bioturbation at the sediment-water interface. ${ }^{350}$ The unpublished data derived from parallel cosms with and without lugworms (Arenicola marina) were obtained as follows. Cosms with lugworms inhabiting polyethylenecontaminated sediment contained 200 individuals $/ \mathrm{m}^{2}$, which is within the range of environmentally realistic population densities. ${ }^{194}$ Microplastic concentrations in water were measured by microplate reader with Microplate Data Collection \& Analysis Software (BioTek, USA). Further description of methods and materials can be found in Chapter 6 .

Bioturbation by A. marina appeared to increase the concentration of microplastic in the overlying water (Figure 8.7). The production of faeces heaps and the movement of lugworms along the sediment-water interface facilitated the release of polyethylene particles from the sediment. The data thus reveal that microplastic can not only have effects on organisms, but 
organisms can also affect the distribution of microplastic in their surrounding compartments. This suggests a direct feedback between microplastic fate and effects. Bioturbating organisms can be hypothesised to affect the relative importance of sediments as a sink and the temporal bioavailability of microplastic. ${ }^{350}$ Other species of ecosystem engineers, including bivalves, ${ }^{52,351}$ can also be expected to alter micro- and nanoplastic concentrations in sediment and water compartments.

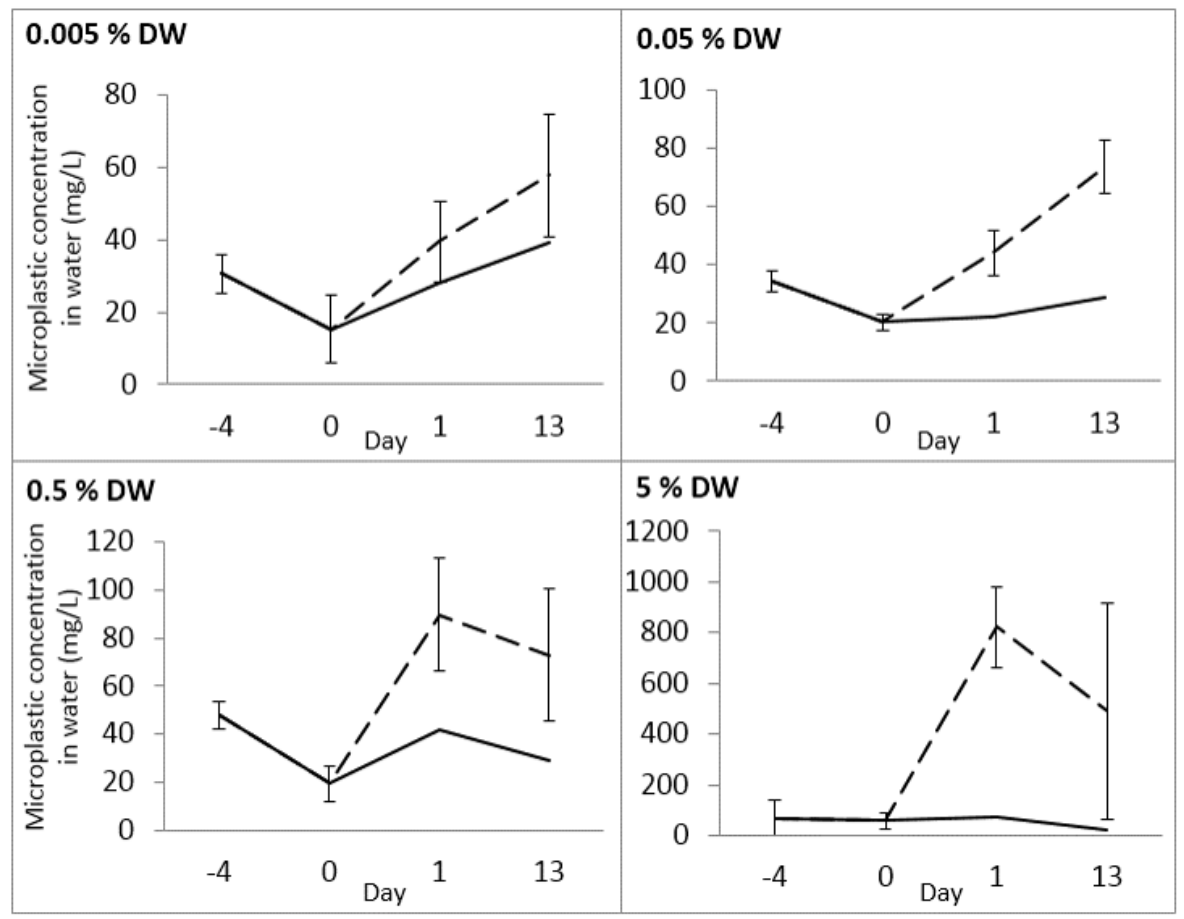

Figure 8.7. Microplastic concentrations $(g / L \pm S D)$ in overlying water as a result of bioturbation in sediment, with initial microplastic concentrations of (top - bottom) 0.005, 0.05, 0.5 and $5 \%$ dry weight $(D W)$. Lugworms were added to the cosms on day 0 (dotted lines). Solid lines represent cosms without lugworms. ${ }^{350}$

Apart from the environmental concentrations, internal exposure to plastic particles in the aquatic environment depends on the actual ingestion by organisms. Plastic uptake can be modeled as a mass balance of ingestion and loss processes, such as that calculated for a humpback whale in Chapter $\mathbf{7}$ of this thesis. This biodynamic modeling approach has also been used to model the ingestion of nano-, micro- and macroplastic by worms, fish and birds. ${ }^{129,240,312,352}$ Most of these studies, including the study reported on in 
Chapter 5, have modeled ingestion to determine bioaccumulation of hydrophobic contaminants ${ }^{129,240,352}$ and rarely solely in the interest of ingestion itself, as was done in Chapter 7. ${ }^{312}$ This calculation of steady-state microplastic concentration may apply more universally to other organisms too and can be used to model plastic concentrations in entire foodwebs. ${ }^{353}$ Tissue or organ concentrations of nanoplastic particles that are possibly transferred beyond the gut system might be modeled in a similar way.

Since plastic concentrations can vary along the vertical gradient of the water column, one should take care to use the appropriate average aqueous concentration over an appropriate depth interval to calculate steady-state concentrations in organisms. The presence or absence of a halocline in estuaries, as well as oscillatory movements in oceans, affect the concentrations of plastic particles and to some extent also those of organisms. Oscillatory movements of microplastic ${ }^{299}$ might reflect the diurnal movements of algae and zooplankton. Consequently, the use of average aquatic concentrations can mean that actual exposure concentrations are underestimated.

Furthermore, characteristics of different filter feeding species affect whether plastic ingestion is solely a matter of chance of encounters. Several copepoda and bivalves are known to be able to discriminate between edible and nonedible particles, leading to post-ingestive food selection or pausing of feeding when edible to non-edible particle ratios are insufficient. ${ }^{52,354-358}$ Other species are known to adjust ingestion rates depending on the nutritional value or the size of the food. ${ }^{352,359}$ Thus, species-specific characteristics affect whether lower or higher ingestion rates than determined by chance encounter can be anticipated. For species with different feeding types, such as scavengers and active predators, ingestion rates are differently related to the prevailing environmental concentrations. One way to model internal concentrations in these organisms could be by defining a plastic encounter rate, using their beak width, beak open-to-close ratio, beak open-to-feeding ratio, swimming distance and the aqueous plastic concentration. Large differences in stomach concentrations between individuals of the same species ${ }^{25,213,280,360}$ are partly explained by the regions they inhabit. However, when large differences between individuals of one species are found within the same region, it might suggest that individual food selection preferences greatly affect ingestion. Such large differences in individual food selection strategies are seen in many species and mean that plastic encounter rates can mainly be useful to predict average ingestion rates by a species in a region, not aiming to represent the possibly large individual differences. 


\subsection{Effect assessment}

To define the actual risks of micro- and nanoplastic, an assessment of exposure needs to be combined with an assessment of effect thresholds. Eventually, these can be combined with one another in a risk characterisation (Figure 8.1). The sections below present an overview of effect thresholds for physiological effects of micro- and nanoplastic and effects on bioaccumulation of chemicals in organisms.

\subsubsection{Effect thresholds reported in the previous chapters of this thesis}

There is considerable data available on the occurrence of macro- and microplastic in biota, mainly for the higher trophic levels, but also for invertebrates. ${ }^{34,41,47,213,280,311,361-363}$ Effects of plastic on organisms have been hypothesised or demonstrated to relate to entanglement, blocking of intestines, reduced nutritional value of food, increased exposure to plastic-associated chemicals and particle toxicity. Some of these effects have mostly been observed for macroplastic, such as entanglement and blocking of intestines of organisms in the wild (Figure 8.8). ${ }^{278}$ Other effects are more likely to be caused by smaller particles such as micro- and nanoplastic. Effect levels of micro- and nanoplastic on organisms as determined by the studies included in this thesis (Chapters 3, $\mathbf{4}$ and 6) are summarised in Table 8.5. In this chapter, the uptake into the gastrointestinal tract itself, without measured adverse effects on the organism's functioning, is not considered to be an effect.

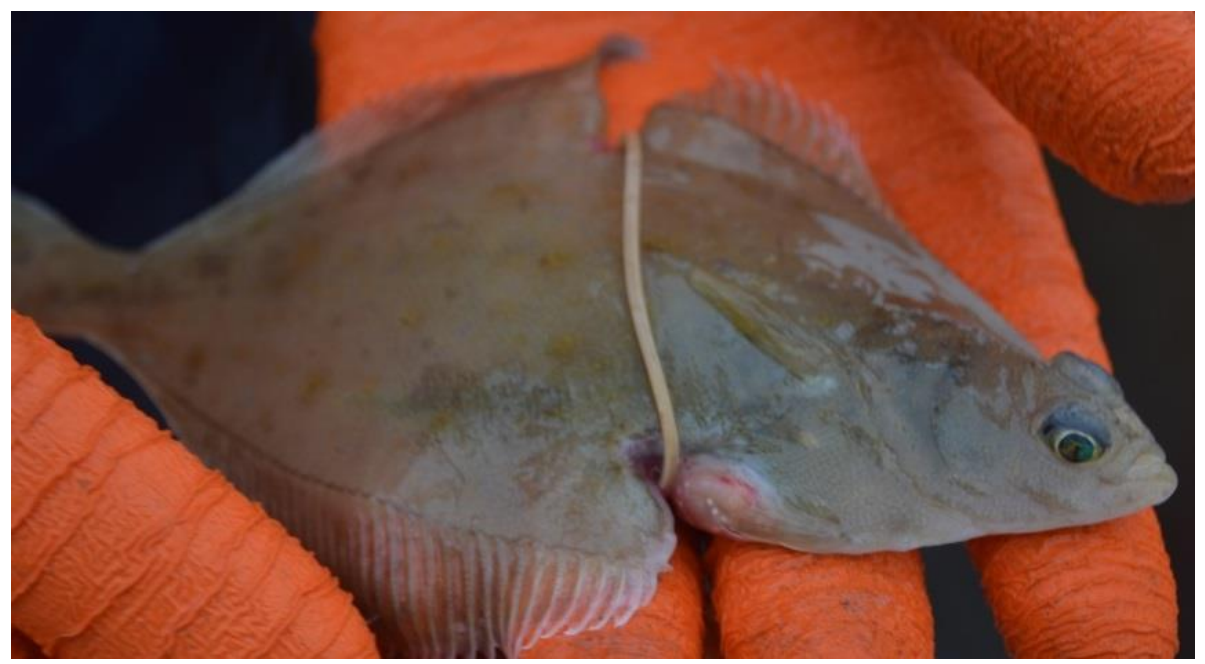

Figure 8.8. Common dab (Limanda limanda) malformed by marine litter. Kees Goudswaard, Wageningen Marine Research. 
Chapter 8

\begin{tabular}{|c|c|c|c|c|c|c|c|c|c|c|c|c|}
\hline \multirow{4}{*}{ 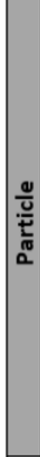 } & squezoefuns & $\begin{array}{l}\tilde{u} \\
\text { 今 }\end{array}$ & $\begin{array}{l}\tilde{0} \\
\text { I }\end{array}$ & $\begin{array}{l}\tilde{0} \\
\text { क } \\
\text { I }\end{array}$ & $\frac{\tilde{O}}{\stackrel{n}{\perp}}$ & $\begin{array}{l}\tilde{a} \\
\stackrel{0}{I}\end{array}$ & 咢 & $\begin{array}{l}\tilde{O} \\
\text { I }\end{array}$ & & & & \\
\hline & әdeys & $\begin{array}{l}\frac{0}{0} \\
\frac{5}{0} \\
0\end{array}$ & $\begin{array}{l}\frac{0}{\pi} \\
\frac{c}{0} \\
\text { n }\end{array}$ & $\begin{array}{l}\frac{0}{0} \\
\frac{5}{0} \\
\text { ஸे }\end{array}$ & $\begin{array}{l}\frac{0}{0} \\
\frac{0}{2} \\
0\end{array}$ & $\begin{array}{l}\frac{0}{2} \\
\frac{0}{0} \\
0\end{array}$ & $\begin{array}{l}\frac{0}{0} \\
\frac{c}{0} \\
\text { n }\end{array}$ & $\begin{array}{l}\frac{0}{0} \\
\frac{5}{0} \\
0\end{array}$ & $\begin{array}{l}\frac{0}{0} \\
\frac{0}{2} \\
0\end{array}$ & $\begin{array}{l}\frac{0}{0} \\
\frac{\tilde{c}}{0} \\
\text { n }\end{array}$ & $\begin{array}{l}\frac{0}{0} \\
\frac{0}{2} \\
0\end{array}$ & $\begin{array}{l}\frac{0}{0} \\
\frac{0}{2} \\
\text { in }\end{array}$ \\
\hline & 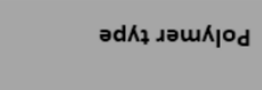 & $\cong$ & $\approx$ & 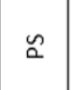 & $\check{\Sigma}$ & $\check{\Sigma}$ & 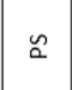 & $\approx$ & 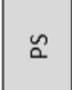 & $\cong$ & $\begin{array}{l}\text { 뜸 } \\
\text { oิ }\end{array}$ & $\begin{array}{l}\text { 믐 } \\
\text { 오 }\end{array}$ \\
\hline & әz!S & $\begin{array}{l}E \\
\check{\Sigma} \\
\stackrel{R}{R}\end{array}$ & $\begin{array}{l}\varepsilon \\
\Sigma \\
D\end{array}$ & $\begin{array}{l}\varepsilon \\
\qquad \\
尺\end{array}$ & 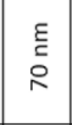 & $\begin{array}{l}\varepsilon \\
\qquad \\
尺\end{array}$ & $\begin{array}{l}\varepsilon \\
\Sigma \\
\circ \\
尺\end{array}$ & $\begin{array}{l}\varepsilon \\
\Sigma \\
尺 \\
尺\end{array}$ & 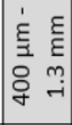 & $\mid$\begin{tabular}{cc}
1 & $\varepsilon$ \\
$\xi$ & $\varepsilon$ \\
$\vdots$ & $\varepsilon$ \\
\hdashline & $m$ \\
$\dot{q}$ & -1
\end{tabular} & 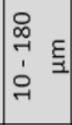 & 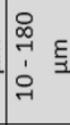 \\
\hline \multirow{3}{*}{ 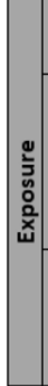 } & (p) uo!̣edna & $m$ & $m$ & $\vec{N}$ & $\vec{N}$ & $\vec{N}$ & $\vec{N}$ & $\vec{N}$ & $\stackrel{\infty}{\sim}$ & 악 & $\stackrel{\infty}{N}$ & $\stackrel{\infty}{\sim}$ \\
\hline & 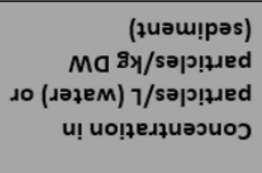 & $\begin{array}{l}\stackrel{n}{+} \\
\stackrel{+}{山} \\
\stackrel{m}{m} \\
\text { in }\end{array}$ & 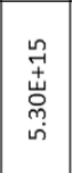 & 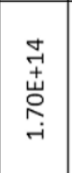 & 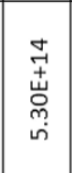 & 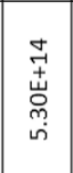 & 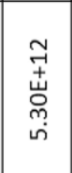 & 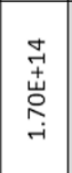 & $\begin{array}{l}\stackrel{n}{0} \\
\text { 岀 } \\
\stackrel{7}{\sim}\end{array}$ & 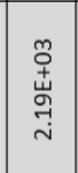 & $\begin{array}{l}\hat{O} \\
+ \\
\text { 㟧 } \\
\rightarrow \\
-1\end{array}$ & 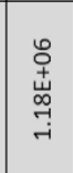 \\
\hline & un!pəw & 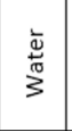 & 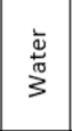 & 㐫 & 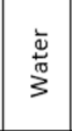 & 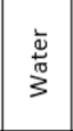 & $\begin{array}{l}\bar{\Phi} \\
w^{\infty} \\
3\end{array}$ & 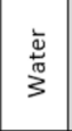 & 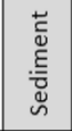 & 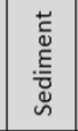 & 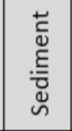 & 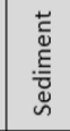 \\
\hline \multirow{5}{*}{ 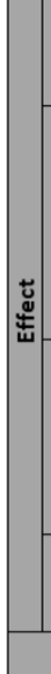 } & әd $\Lambda_{7}$ גәғе $M$ & $\begin{array}{l}\frac{5}{\bar{\omega}} \\
\frac{\omega}{4}\end{array}$ & $\begin{array}{l}\frac{c}{\tilde{Q}} \\
\frac{0}{L}\end{array}$ & 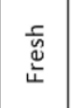 & 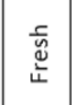 & $\begin{array}{l}\frac{5}{\omega} \\
\stackrel{\omega}{4}\end{array}$ & $\begin{array}{l}\frac{5}{\bar{y}} \\
\stackrel{w}{4}\end{array}$ & $\begin{array}{l}\frac{5}{\bar{\omega}} \\
\frac{\omega}{4}\end{array}$ & 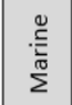 & 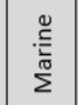 & 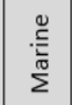 & 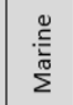 \\
\hline & qu!̣odpuヨ & 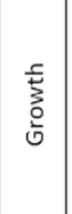 & 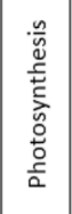 & 吝 & 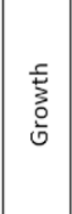 & 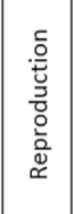 & 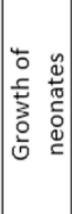 & 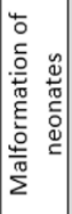 & $\begin{array}{l}\frac{1}{5} \\
\frac{.00}{00} \\
3 \\
3\end{array}$ & 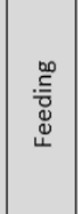 & 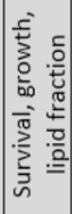 & 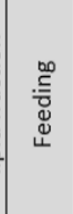 \\
\hline & sə!̣ads & 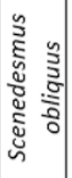 & 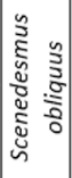 & 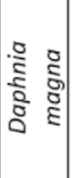 & 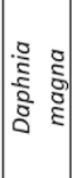 & $\mid \begin{array}{ll}0 & 8 \\
0 & 0 \\
0 & 0 \\
0 & 0 \\
0 & 8 \\
0\end{array}$ & $\begin{array}{ll}0 \\
0 \\
2 & 8 \\
\frac{2}{2} & 0 \\
0 & 8 \\
0 & 8\end{array}$ & 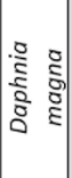 & 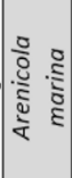 & 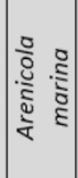 & 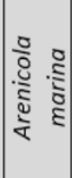 & 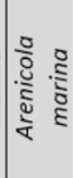 \\
\hline & aunseaW & 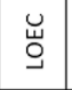 & 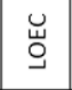 & 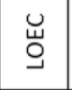 & 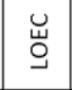 & 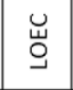 & 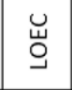 & 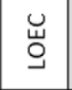 & 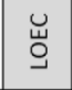 & 岀 & $\begin{array}{l}u \\
\stackrel{u}{u} \\
z\end{array}$ & 亗 \\
\hline & дәздеपગ & $m$ & $m$ & m & $m$ & m & $m$ & $m$ & $\sigma$ & $\sigma$ & 0 & 6 \\
\hline
\end{tabular}




\subsubsection{Review of the literature on effect thresholds}

The scientific literature was searched for data on effect levels in order to ascertain how adverse effects of micro- and nanoplastic are distributed among species, ecosystems, exposure media and plastic particles with varying characteristics. The overview presented here is based on 174 published effect levels from 69 different studies, including the effect levels presented in the previous chapters of this thesis (Table 8.5) as well as many effect levels summarised by Lusher ${ }^{296}$ and Connors et al..$^{294}$ and supplemented with effect thresholds published until July 2017. These threshold data are summarised in Table 8.6 for each exposure medium, size category, ecosystem and threshold value.

What has to be considered an effect of plastic on an organism is still being debated in the field of plastic debris research. In some studies, for instance, plastic ingestion or trophic transfer, that is, secondary ingestion via a plasticcontaining lower trophic organism ${ }^{364-367}$ has been defined as an effect in itself, whereas the effect actually arises from the physiological consequences of the ingestion, such as gut obstruction and the consequent growth reduction, or an adverse effect may in fact even be absent. For this reason, plastic ingestion as an endpoint of effect is excluded here and only the following endpoints are considered: survival, feeding, growth, weight loss, reproduction, moulting, malformation, uptake in tissue beyond the gastrointestinal tract, behaviour, photosynthesis, oxidative stress, enzyme activity, inflammation, gene expression and nutrient cycling. These endpoints can all be assumed to affect population size, given time, eventually leading to a change of community composition and possible of ecological functioning. Only those studies were included that did not report the inclusion of associated chemicals at relevant effect concentrations. Effects of plastic on bioaccumulation of chemical substances are discussed separately further on in this chapter. This is in line with the suggestion by Koelmans et al. ${ }^{293}$ to deal with the additional hazard of bioaccumulation of chemical substances separately following existing risk assessment methods.

The effect thresholds derived from the literature were partly $\mathrm{EC}_{50}$ (Effect concentration at which $50 \%$ of the exposed organisms is affected) values, partly LOEC (Lowest observed effect concentration) values and partly NOEC (No observed effect concentration) values for organisms that were exposed to micro- or nanoplastic via water, food or sediment. As threshold concentrations are provided in varying units in the literature, the following conversions were used to express all data on the basis of weight per litre of water or $\mathrm{kg}$ of DW 
sediment or food: particle numbers were converted into mass data for spherical particles and fibres using the formulas for sphere and cylinder volume, respectively. ${ }^{294}$ For same-diameter but irregular particles, half the volume of a sphere was assumed. If a range of particle sizes was used, the average radius was used in the equations. If no polymer density was provided, the polymer densities given by Andrady ${ }^{8}$ were used, and when exposure included a mixture of polymer types, the average polymer density was calculated. A sediment density of $1.8 \mathrm{~kg} / \mathrm{L}^{51}$ and a porewater fraction of $20 \%$ were used where needed. $^{321}$

\subsubsection{Construction of species sensitivity distributions (SSDs) as a tool to explore effect data}

Effects of chemical stressors are often reported for individual species. This, however, does not offer insights into the consequences of the respective stressors at community level. To increase the relevance of the effect data for this community level, an approach has been developed that combines effect data for individual species in a species sensitivity distribution (SSD). SSDs are log-linear regressions through measures of effect to determine the affected fraction of species at a given concentration. ${ }^{368}$ SSDs are used in the environmental risk assessment of substances. SSDs can be used to estimate the concentration at which $5 \%$ of the species in a community is affected, which is referred to as the 'Hazardous Concentration for $5 \%$ of the Species' $\left(\mathrm{HC}_{5}\right)$. Using the literature data, preliminary SSDs for the stressors micro- and nanoplastic were generated with the SSD generator from the US-EPA. ${ }^{369}$

These SSDs are presented as provisional, since they involve several uncertainties. Ideally, SSDs use the effect threshold values of one single endpoint (one type of harm) for $\geq 10$ different species, with environmental variables kept constant. ${ }^{370}$ Consequently, the observed SSD only expresses the variability of the species sensitivities and the experimental variability. Such data is not yet available for plastic as a stressor. Plastic as a stressor has unique features, which means that an SSD for microplastic is fundamentally different from single substance-single endpoint SSDs. First, microplastic or "plastic debris' is a mixture of different sizes and types of particles, which implies that the observed distribution of the stress response reflects this variability. Second, the different types and sizes of particles trigger responses through different modes of action (different types of harm), which implies that the observed distribution of the stress response reflects this variability too. Previous studies have combined data from different endpoints to overcome the lack of available data. ${ }^{338,371}$ Here, a pragmatic criterion for combining different 
Synthesis

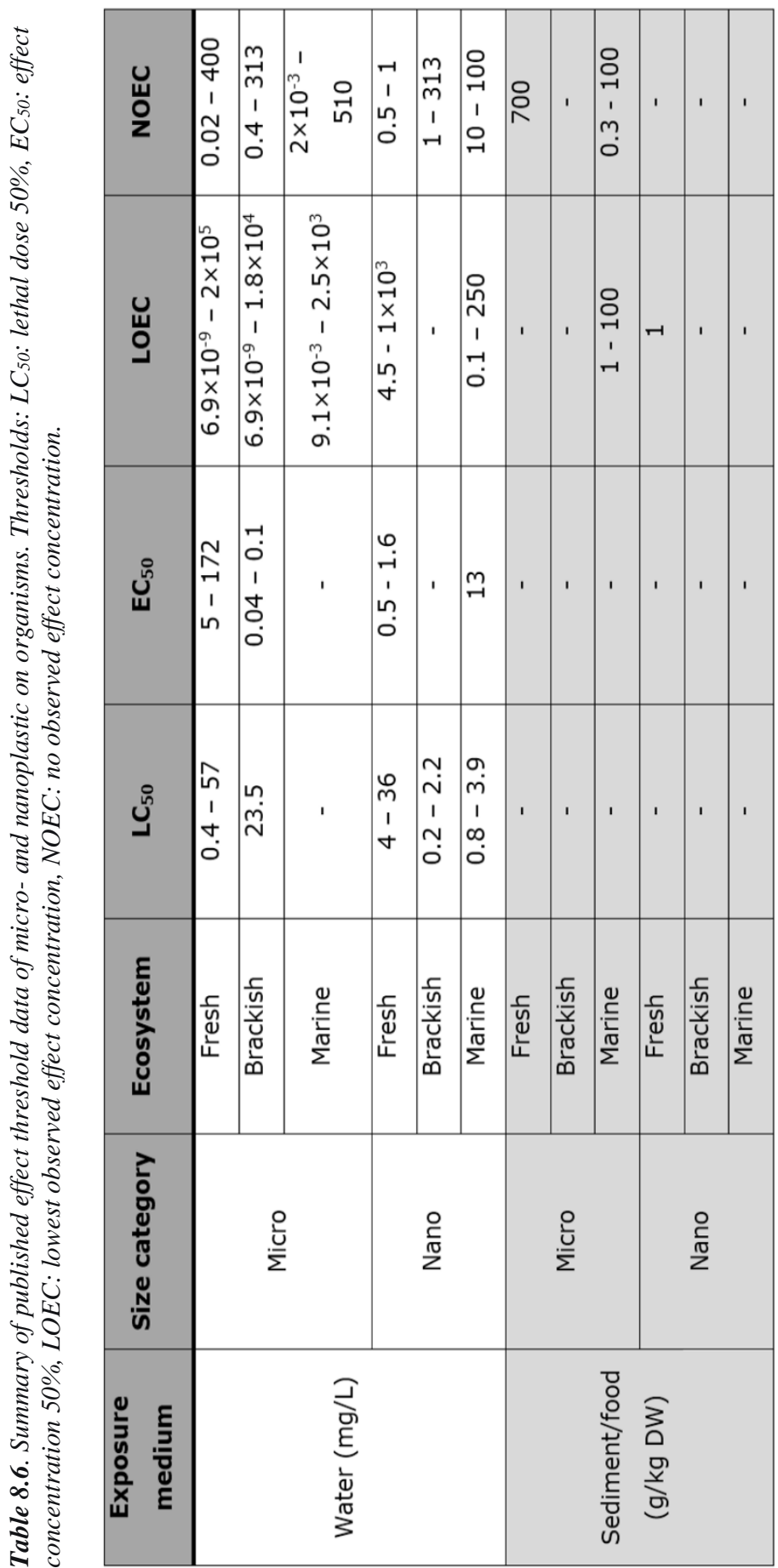


endpoints was used, by combining those endpoints that all imply harm at the population level of a species. Hence, from the large set of thresholds summarised in the previous section, we here combine the endpoints of survival, reproduction and growth, the latter including any reduction in weight, length or hampering of moulting (while a subsequent effect of these endpoints on reproduction can be expected too) to construct SSDs, as they are all population-threatening. Both acute and chronic $\mathrm{LC}_{50}, \mathrm{EC}_{50}$ and LOEC values were used, with exposure durations varying from minutes to months. The comparability of these data was improved by using extrapolation factors from Diepens et al. ${ }^{370}$ to infer chronic LOEC values for these different effect thresholds. The ranges of extrapolation factors used for $\leq 21$-day $\mathrm{LC}_{50}, \mathrm{EC}_{50}$ and LOECs values were 10-30, 5-15 and 3-10, respectively. ${ }^{370}$ When exposure duration was $\leq 5 \mathrm{~d}$, the higher ends of these ranges were used (extrapolation factors of 30, 15 and 10), while for exposure durations $>5 \mathrm{~d}$ but $<5 \mathrm{~d}$, the intermediate values of these ranges of extrapolation factors were used $(20,10$, 6.5 ), and when exposure duration was $\geq 15 \mathrm{~d}$ but $<21 \mathrm{~d}$, the lower ends of these ranges were used $(10,5,3)$. For 21-day $\mathrm{LC}_{50}$ and $\mathrm{EC}_{50}$ values, an extrapolation factor of 5 was used to derive the chronic LOEC. ${ }^{370}$ Since data included those for several organisms that inhabit a salinity range from fresh to brackish, and since no mechanism is known or expected for an effect of salinity on the physical adverse outcome pathways related to microplastic, effect thresholds for marine, estuarine and freshwater species were combined. This combination of taxa from different habitats and ecosystem types is strictly for calculation purposes, and does not imply that they are supposed to share the same habitat. A similar approach of combining data for freshwater and marine invertebrate species has been used for pesticide risk assessment. ${ }^{370,372}$ In conclusion, the tentative SSDs for plastic debris presented here reflect the combined variability of species sensitivity, properties of the stressor and effect mechanisms, as a function of the dosage. The extrapolated chronic effect thresholds used to construct the SSDs can be found in the Appendix Tables A8.1 and A8.2.

The separately constructed SSDs for organisms exposed to micro- and nanoplastic via the water phase (expressed as plastic mass per volume) are shown in Figure 8.9. Of the species studied, the one that seems the most sensitive to exposure to microplastic via the water phase is the rotifer Brachionus koreanus $(0.5-6 \mu \mathrm{m}$ spherical PS particles), and the least sensitive the amphipod Gammarus fossarum $(32-250 \mu \mathrm{m}$ irregular PMMA and PHB particles) (Table A8.1). For nanoplastic, the most sensitive is the 
copepod Tigriopus japonicus (50 $\mathrm{nm}$ spherical PS particles) and the least sensitive the algae Scenedesmus obliquus (70 nm spherical PS particles), the latter threshold being derived in Chapter 3 (Table A8.2). Relatively high sensitivities of the juveniles and of reproduction endpoints were observed for microplastic (Table A8.1) compared to the growth and survival endpoints. Effects on growth might be due to an overall decreased nutritional value of the food as it becomes diluted with plastic. ${ }^{49,51,53,373}$ Several studies suggest that such an effect, however, would not necessarily be unique for plastic but could similarly be caused by natural (e.g., mineral) particles. ${ }^{358}$ On the other hand,
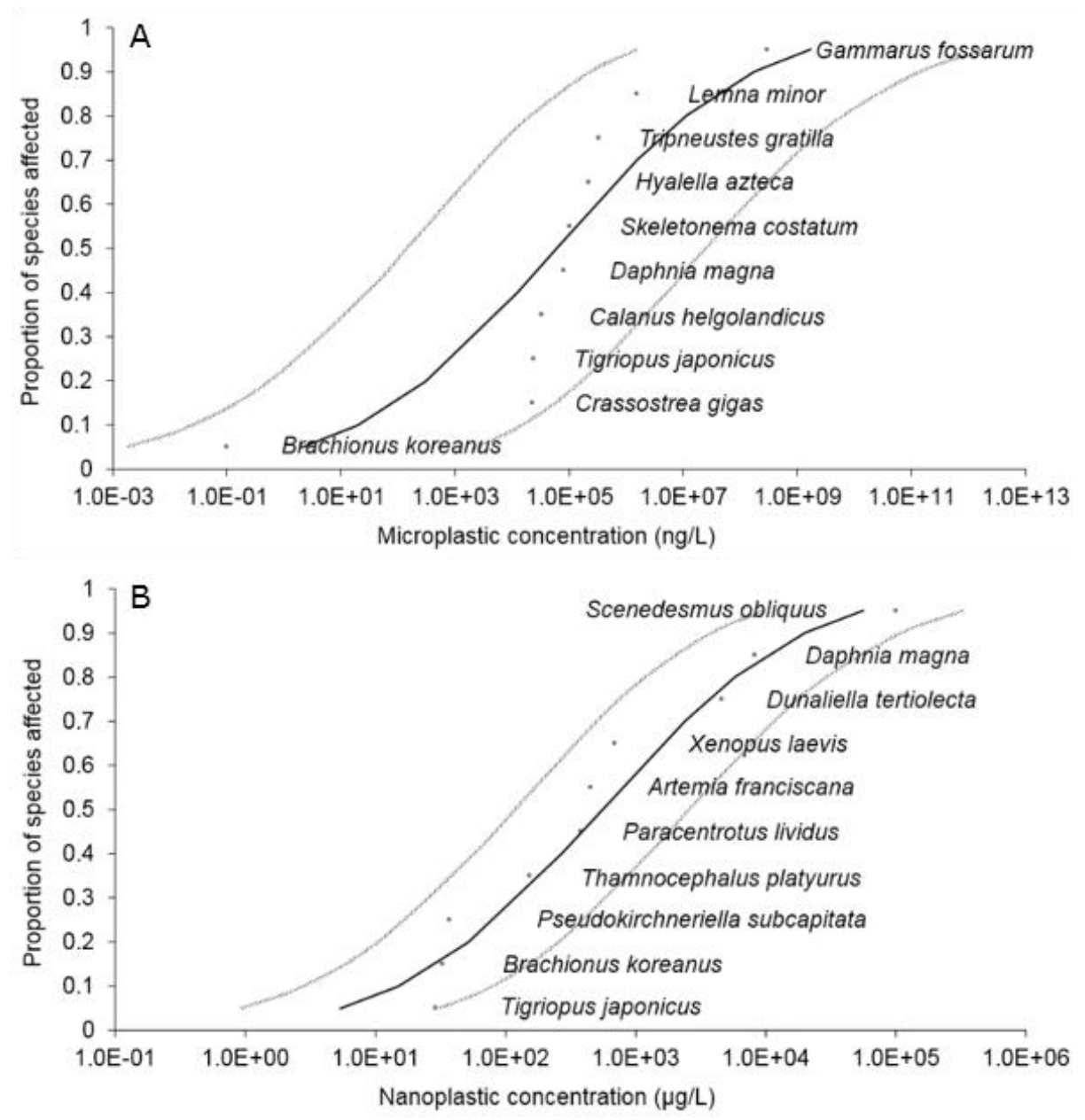

Figure 8.9. Species sensitivity distributions of organisms from the marine, estuarine and freshwater environments exposed to microplastic (Panel A) or nanoplastic (Panel B) via the water phase. Effect thresholds represent chronic LOECs. 
the study exposing G. fossarum showed that effects of particles were not found when natural silica particles instead of plastic particles were used. ${ }^{374}$ From these SSDs, a hazardous concentration $\left(\mathrm{HC}_{5}\right)$ of $2.0 \mathrm{ng} / \mathrm{L}\left(\mathrm{R}^{2}: 0.78,95 \%\right.$ confidence interval $\left.(95 \% \mathrm{CI}): 1.8 \times 10^{-3}-2.2 \times 10^{3} \mathrm{ng} / \mathrm{L}\right)$ were derived here for microplastic. The here derived $\mathrm{HC}_{5}$ for nanoplastic is $5.4 \mu \mathrm{g} / \mathrm{L}\left(\mathrm{R}^{2}: 0.93\right.$, $95 \% \mathrm{CI} 0.93-31 \mu \mathrm{g} / \mathrm{L}$ ), which is over three thousand times higher than that for microplastic. Confidence intervals, however, are wide and overlapping, implying a large degree of uncertainty. In the SSD for microplastic (Figure 8.9A), the effect data for the species Brachionus koreanus seem to be outlying. Removal of this data point would result in an $\mathrm{HC}_{5}$ of $9.2 \times 10^{2} \mathrm{ng} / \mathrm{L}(3.3-1.0$ $\left.\times 10^{4} \mathrm{ng} / \mathrm{L}\right)$. However, this removal does not improve the $\mathrm{R}^{2}(0.78)$, and as four effect thresholds for the same species underlie the data point for Brachionus koreanus in Figure 8.9A, the likelihood of it being an outlier is not very high. Nevertheless, this underlines the uncertainty involved. When using an SSD approach to derive $\mathrm{HC}_{5}$ values, an assessment factor (AF) of 5 has been applied by Van Cauwenberghe to obtain a predicted no effect concentration (PNEC). ${ }^{338}$ In this section an example is provided of how such a concentration could be estimated, and referred to as a preliminary safe standard (PSS). This results in PSS concentrations of $0.4 \mathrm{ng}$ microplastic/L and $1.1 \mu \mathrm{g}$ nanoplastic/L water (Table 8.7).

For exposure via food or sediment, insufficient chronic LOEC data was available to construct SSDs. For the effects of microplastic on survival, growth or reproduction, two LOEC values had been derived before. A LOEC of 12 $\mathrm{g} / \mathrm{kg}$ food for survival of fish was published by Mazurais et al. ${ }^{375}$ and a LOEC of $74 \mathrm{~g} / \mathrm{kg} \mathrm{DW}$ in sediment for the growth of lugworms was reported in Chapter 4 of this thesis. When only a single or a few effect thresholds are available, an AF of 1000 has been used before to derive PNEC values based on data for the most sensitive endpoint available. ${ }^{338}$ For microplastic, in addition to effect thresholds for survival, growth and reproduction, effect thresholds could also be obtained from the literature for a variety of other, partly more sensitive, endpoints (Table A8.4). The most sensitive endpoint is translocation to tissue beyond the gastrointestinal tract, though it can be debated whether this has to be considered an effect on biological functions. Another aspect to keep in mind while interpreting translocation to tissues beyond the gastrointestinal tract when working with high concentrations ${ }^{366}$ is that apparent translocation might be due to cutting during dissection of tissue. For these reasons, we chose not to use this endpoint here and work with the second most sensitive endpoint, which is oxidative stress and liver damage in 
fish at $0.1 \mathrm{~g} / \mathrm{kg}$ DW food. The resulting PSS calculated from this effect threshold is $0.1 \mathrm{mg} / \mathrm{kg}$ food (Table 8.7). For nanoplastic, the LOEC of $1 \mathrm{~g} / \mathrm{kg}$ food for the growth of fish (Table A8.3) provided by Cedervall et al. is the only available effect threshold for exposure via media other than water, so this value was used with an AF of 1000 to derive a preliminary PSS of $1 \mathrm{mg} / \mathrm{kg}$ DW food.

Previous calculations by Van Cauwenberghe for microplastic in the marine environment resulted in a PNEC of 640 particles/L for exposure via water and a PNEC of 540 particles $/ \mathrm{kg}$ WW for exposure via sediment. ${ }^{338}$ Use of the aforementioned conversion factors for particle weight $(5 \mu \mathrm{g} /$ particle $)$ and porewater fraction (20\%) converts these to PNECs of $3.2 \mathrm{mg} / \mathrm{L}$ and $3.4 \mathrm{mg} / \mathrm{kg}$ DW, respectively. The PSSs derived in this synthesis are a factor $10^{-7}$ lower than those for exposure via water, whereas for exposure via sediment or food, the suggested safe concentrations are reasonably within the same range (here a factor 34 lower than the PNEC reported by Van Cauwenberghe et al. ${ }^{338}$ ). That the PSS values derived here are lower than the PNECs reported by Van Cauwenberghe is because more recently available effect thresholds were used in this chapter and all thresholds were scaled to chronic LOEC values using extrapolation factors, leading to relatively conservative estimates. Following Van Cauwenberghe et al. ${ }^{338}$ it should be emphasised that because of the limited availability of suitable threshold data and large confidence intervals, the derived safe values (PSS) are very preliminary and thus should be used with caution.

Table 8.7. Preliminary safe standard (PSS) values for exposure to micro- and nanoplastic via different compartments in different ecosystems. $\mathrm{HC}_{5}$ : hazardous concentration for $5 \%$ of the species, LOEC: lowest observed effect concentration, AF: assessment factor, 95\% CI: 95\% confidence interval.

\begin{tabular}{|c|c|c|c|c|c|c|}
\hline $\begin{array}{c}\text { Size } \\
\text { category }\end{array}$ & Ecosystem & $\begin{array}{c}\text { Exposure } \\
\text { medium }\end{array}$ & HC $_{5}$ & LOEC & AF & PSS \\
\hline \multirow{2}{*}{$\begin{array}{c}\text { Micro } \\
\text { plastic }\end{array}$} & $\begin{array}{c}\text { Aquatic } \\
\text { environment }\end{array}$ & Water & $2.0 \mathrm{ng} / \mathrm{L}$ & & 5 & $\begin{array}{c}0.4 \mathrm{ng} / \mathrm{L} \\
(95 \% \mathrm{Cl}: 3.6 \times \\
\left.10^{-4}-4.5 \times 10^{2}\right)\end{array}$ \\
\cline { 3 - 7 } & Food/Sediment & & $\begin{array}{c}0.1 \mathrm{~g} / \mathrm{kg} \\
\mathrm{DW}\end{array}$ & 1000 & $0.1 \mathrm{mg} / \mathrm{kg} \mathrm{DW}$ \\
\hline \multirow{2}{*}{$\begin{array}{c}\text { Nano } \\
\text { plastic }\end{array}$} & $\begin{array}{c}\text { Aquatic } \\
\text { environment }\end{array}$ & Water & $5.4 \mu \mathrm{g} / \mathrm{L}$ & & 5 & $\begin{array}{c}1.1 \mu \mathrm{\mu g} / \mathrm{L} \\
\left(\begin{array}{c}9 \% \mathrm{Cl}: 0.19- \\
6.2)\end{array}\right.\end{array}$ \\
\cline { 3 - 7 } & & Food & $\begin{array}{c}1 \mathrm{~g} / \mathrm{kg} \\
\mathrm{DW}\end{array}$ & 1000 & $1 \mathrm{mg} / \mathrm{kg} \mathrm{DW}$ \\
\hline
\end{tabular}




\subsubsection{Effect thresholds of plastic particles with varying characteristics}

In addition to the variability in species sensitivity and effect mechanisms, the data underlying the SSDs reflect that plastic as a stressor is present in a variety of sizes, shapes and types. This is why the SSD was used here as a tool to explore whether a relation could be found between the effect level and the varying characteristics of different plastic particles (SSDs not shown). However, these SSD analyses revealed no relation between effect level and polymer type, and within each size class (i.e., $>100 \mathrm{~nm}$ for microplastic and $<100 \mathrm{~nm}$ for nanoplastic) no relation with size could be found. This can mean either that no such dependence exists, or that the large variation in tested species, endpoints and scientific quality of the data used made that no dependence could be detected. While nanoplastic is considered the potentially most harmful size class, ${ }^{15}$ the $\mathrm{EC}_{50}$ values of the smallest nanoplastic particles tested (diameter around $50 \mathrm{~nm}$ ) lie within the upper right half of the curve. Imhof and Laforsch did not find effects of a mixture of polymer types on mud snails, but suggest that a link between effect levels and either polymer type or size might exist, based on a comparison with effects found in studies using $\leq 20 \mu \mathrm{m}$ polystyrene beads. ${ }^{376}$ The constructed SSDs showed no clear relation with nanoparticle size or charge either. Several studies have reported effects on growth, survival and embryo toxicity for positively charged nanoplastic, whereas these effects are lacking, or only occur at higher concentrations, when the same particles are used with negatively charged surface groups. ${ }^{377-379}$ When combining data from different studies in one SSD, these differences in effects within studies were obscured by the differences between studies. The effect levels for microplastic included here are largely based on particles with a spherical shape (16 studies); only two studies used fibres and 8 studies used irregularly shaped particles. From that, no difference in effect levels between microplastic particles with different shapes could be distinguished. One might expect a more difficult egestion or severe effects from fibre-shaped particles based on the known effects of asbestos and different phagocytic reactions to particles with this shape. ${ }^{380}$ Indeed, one study found effects on the assimilation efficiency for fibres, and not for spherical particles. ${ }^{374}$ Yet, the effect data for the fibres of both studies were in the upper right part of the SSD curve, which presents the data for the least sensitive cases. This might be due to the micrometre size and might thus be different for nanoplastic. However, all effect levels reported for nanoplastic thus far relate to spherical particles (Table A8.2). 
Another particle characteristic that varies among studies is whether pristine particles are used or particles that had weathered in an environmental or laboratory setting. The latter is done for different purposes, to actively promote biofilm growth on the particles, give the particles a natural flavouring or make them more environmentally relevant in a general sense. ${ }^{49,358,381}$ In this SSD analysis, no distinction was made between these different ways of preparing the particles, because there was a large variation in the extent to which these preparation methods were described in the original articles in the first place. However, the inclusion of a biofilm has been found to affect the ingestion and egestion efficiency in organisms. ${ }^{358,379}$ Where egestion became reduced due to ingestion of plastic particles with biofilms, a reduced feeding rate was also found. ${ }^{379}$ This implies that although biofilm formation is fast and might (even when not specifically quantified) have been present in studies that claimed to use pristine particles, outcomes of effect studies with pristine particles might be more unfavourable for organisms under environmentally realistic conditions.

The final variable particle characteristic that needs to be mentioned here is the concentration of chemicals within the plastic particles used to derive effect thresholds. The effect thresholds found might apply to the effects of plastic particles themselves or to chemicals transported by these particles, or to the combined effects of multiple stressors, as many studies assessing effects of plastic particles do not exclude that the particles they used contained additives or other chemicals. Further discussion of chemical transport by plastic particles is provided below in section 8.3.5.

\subsubsection{Role of plastic in bioaccumulation of chemicals}

Chapters 4-6 of this thesis showed that the effect of microplastic ingestion on the bioaccumulation of omnipresent hydrophobic chemicals, also referred to as persistent organic pollutants (POPs), is restricted to a twofold increase or decrease in tissue of lugworms. ${ }^{51,245,373}$ Whether an actual increase or decrease of bioaccumulation is found depends on the polymer type and chemical characteristics, as well as complex counteracting mechanisms of contribution to chemical uptake through plastic versus food, and whether or not there is chemical equilibrium. ${ }^{246,352,373}$ In short, this is demonstrated by the fact that the POP concentrations in the surrounding water (or porewater for sedimentdwelling organisms) fully explained the observed bioaccumulation in bioassays, which is the main finding reported in Chapter 6. ${ }^{373}$ 
Although the role of microplastic ingestion in the bioaccumulation of POPs by organisms has been suggested to be minor for most aquatic habitats in this thesis and related publications, there has been considerable debate on this. ${ }^{240,244,248,352,373,382,383}$ The hypothesis that microplastic affects bioaccumulation has dominated a large part of the microplastic research during the past decade, and both those who do and those who do not think that microplastic increases the uptake of POPs find proof in experimental data. ${ }^{51,236,238,239,244,373,384}$ The contrasting views can be explained by taking a closer look at the precise hypotheses that underlie the different studies. Studies with varying types of polymers and POPs found that the more amorphous, low-density polymer types (i.e. PE) have the highest affinity for POPs and, just as in other absorbing pools such as lipids and organic matter, the most hydrophobic POPs will be most attracted to plastic. Apart from differences in polymer types and chemicals, it is the use of different species and in particular different exposure scenarios in different studies which results in different conclusions. ${ }^{238,239,244,364}$ Microplastic ingestion is likely to increase bioaccumulation in organisms when these organisms are relatively clean at the start of exposure and are fed with microplastic loaded with POPs. Microplastic is less likely to increase bioaccumulation in organisms fed with microplastic when the POP concentrations in the organisms are already closer to - or at chemical equilibrium with - the surrounding environmental media like water and diet components. In hotspot locations, where plastic makes up a relatively large fraction of the diet and the POP concentration gradient allows chemical transfer to the organism, plastic might however significantly increase exposure to chemicals. ${ }^{385,386}$

As for nanoplastic particles, there are two reasons why they might deliver a more substantial contribution to bioaccumulation of POPs in organisms than microplastic particles. The first reason is a much higher (1-2 orders of magnitude) affinity of POPs to these particles, compared to microplastic. ${ }^{75,387}$ The second reason is that nanoplastic might be able to reach other tissues than microplastic. ${ }^{15}$ The small size of nanoparticles allows them to enter cells via endocytosis, penetrate tissues, move directly from the digestive tract to the circulatory system, and cross the blood-brain barrier. ${ }^{29,388,389}$ Biofilm formation has been found to decrease sorption to nanoplastic, whereas sorption was enhanced in a marine as opposed to a freshwater setting. ${ }^{75}$ This suggests that the presence of nanoplastic may potentially increase the uptake of POPs from the environment in organisms, the extent of which depends on the specific environment. 


\subsection{Risk characterisation for nano- and microplastic particle effects}

A comparison of plastic particle effect thresholds with exposure concentrations reveals to what extent organisms may be at risk (Figure 8.10). As exposure concentrations of micro- and nanoplastic were given in particles per volume in Table 8.1, the comparison with chronic LOEC values is made here using the unit of particles/L. It might be preferable to work with particle mass per medium volume or mass, as in Figure 8.9. However, environmental fate studies (section 8.2.1) often do not provide detailed information on particle characteristics from which a conversion factor from mass to particle numbers or vice versa could be derived (a general particle mass of 5 $\mu \mathrm{g} /$ particle could be used instead, but this is a rough estimate), whereas the effect threshold studies from the literature (section 8.3.2) often do include this information. Therefore, less uncertainty is introduced when the effect threshold dataset is converted to particle concentrations, rather than converting the environmental concentrations dataset to mass concentrations. Hence, exposure and effect levels are compared here as particle concentrations. A further advantage is that, additional to the SSDs using mass concentrations (Figure 8.9), the same data is here presented as SSDs using particle concentrations (Figure 8.10).

Risks of microplastic in water. For organisms exposed to microplastic in water, the $\mathrm{HC}_{5}$ derived from the SSD in Figure 8.10 is 113 particles/L $\left(\mathrm{R}^{2}\right.$ : $0.96,95 \%$ CI $13-1000$ particles/L). This $\mathrm{HC}_{5}$ for microplastic is of the same order as the worldwide HLRR of microplastic concentrations in-near shore surface water (Figure 8.10A). Worldwide HLRR in freshwater up to and including the year 2016 are three orders of magnitude lower, and those in open ocean surface water almost five orders of magnitude lower, than this $\mathrm{HC}_{5}$. Taking into account that amounts of microplastic are underestimated by up to a factor of 30 when based on surface sampling, ${ }^{297}$ microplastic concentrations might present a risk to $10-20 \%$ of the species at hotspot locations in nearshore regions (95\% CI 5-30\%).

Risks of nanoplastic in water. For organisms exposed to nanoplastic in water, the $\mathrm{HC}_{5}$ is $5.97 \times 10^{10}$ particles/L $\left(\mathrm{R}^{2}: 0.96,95 \% \mathrm{CI}(1.6-22) \times 10^{10}\right.$ particles/L). Effect threshold concentrations for nanoplastic expressed in particles/L are generally seven orders of magnitude higher than those for microplastic. Since measured concentrations of nanoplastic in the environment are lacking, no direct comparison with environmental nanoplastic concentrations can be made. However, environmental 
concentrations of nanoplastic potentially becoming 14 orders of magnitude higher than those of microplastic (section 8.2.4) in the future, due to fragmentation of larger plastic particles, would mean that environmental nanoplastic concentrations could exceed the effect thresholds over time. Within a timeframe of several hundreds of years, ${ }^{338}$ nanoplastic concentrations would then be within the range of effect thresholds included in Figure 8.10B.

Risks of microplastic in sediment or food. For aquatic organisms exposed to microplastic via the media food and sediment, only two chronic LOEC values could be derived when limiting the selection of data to the endpoints survival, growth and reproduction $\left(2.2 \times 10^{5}\right.$ and $4.1 \times 10^{9}$ particles $\left./ \mathrm{kg}\right)$. Effect threshold values for other endpoints are available in the literature (Table A8.4), these endpoints are not by definition threatening populations, although they might lead to community changes. The worldwide HLRR of microplastic concentrations in freshwater sediment and beach sediment are within the range of effect thresholds for these not by definition population threatening endpoints (Table A8.4) but microplastic concentrations in freshwater, subtidal, beach and seafloor sediment are below the two chronic LOEC values for population threatening effects.

In conclusion, based on the preliminary SSDs and worst case worldwide HLRR exposure estimates, the exceedance of hazardous microplastic concentrations for the most sensitive species currently occurs in hotspot locations of near-shore surface waters. Van Cauwenberghe (2016) predicted that only sediment-dwelling organisms would be at risk of exposure to microplastic concentrations exceeding the effect thresholds. ${ }^{338}$ The difference between her prediction and the findings in the present chapter is due to the (recent) availability of more effect thresholds and the use of extrapolation factors to scale these thresholds consistently to chronic LOECs. The present risk assessment is based on comparing effect threshold values from separate bioassays and HLRR values. However, it is strongly advisable to address and quantify the present uncertainties using probabilistic risk assessment methods. 

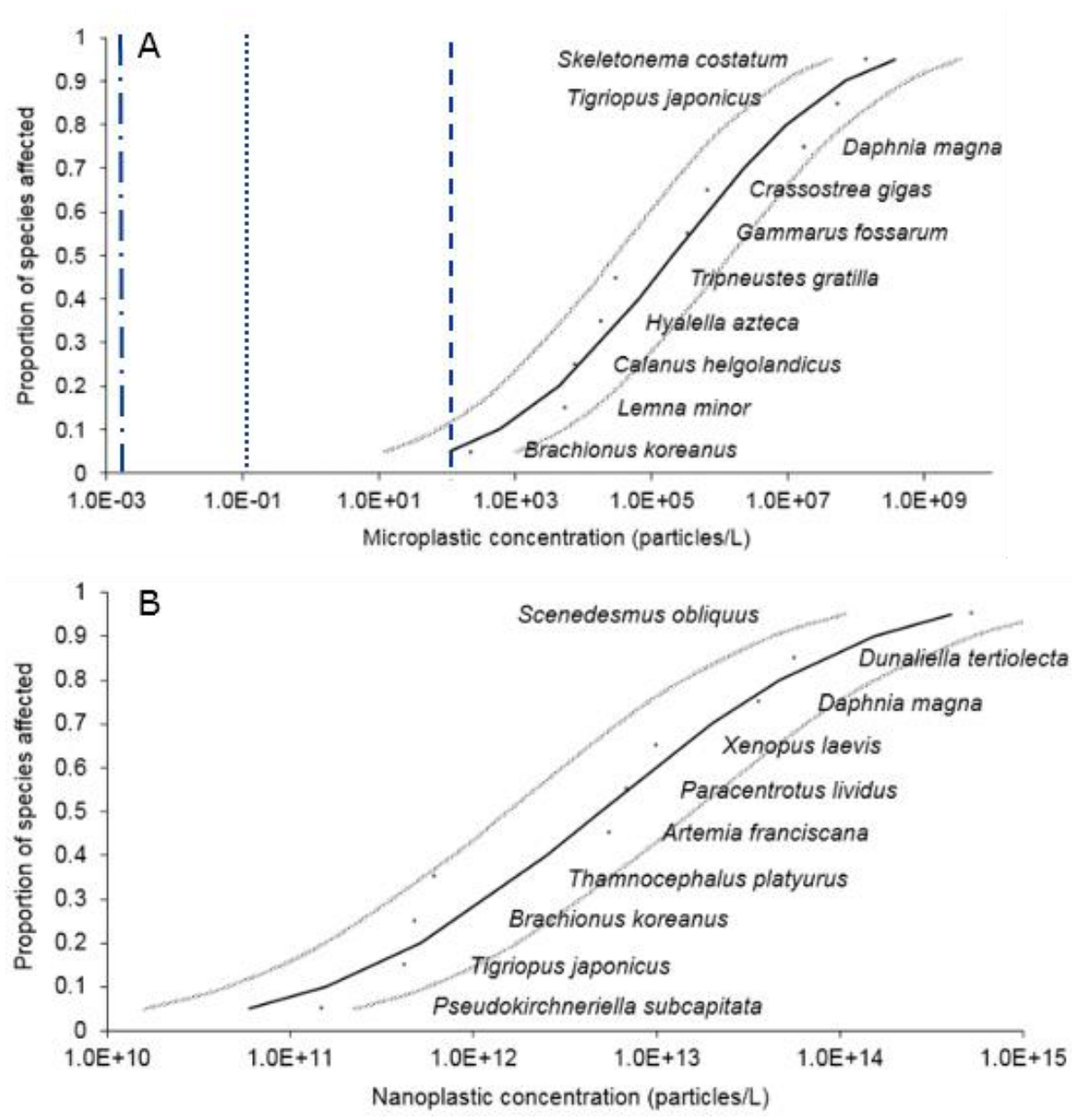

Figure 8.10. Risk characterisation for microplastic and nanoplastic. Separate panels are provided for exposure to microplastic via water (Panel A), and to nanoplastic via water (Panel $B)$. Solid black curves represent SSDs with plastic concentrations expressed in particles per volume. Grey curves represent the 95\% confidence intervals. SSDs are based on (Panel A); chronic LOEC concentration data (particles/L) for microplastic and the endpoints survival, growth and reproduction (Table A8.1, as in Figure 8.9A) and on (Panel B); LOEC concentration data (particles/L) for nanoplastic and the endpoints survival, growth and reproduction (Table A8.2, as in Figure 8.9B). Vertical lines indicate measured environmental concentrations as worldwide highest limits of reported ranges (HLRR) (Table 8.1) for exposure via water (Panel A); microplastic concentrations measured in freshwater surface water (blue dotted line, near-shore surface water (blue dashed line) and open ocean surface water (blue dot-dashed line). For nanoplastic (Panel B) no vertical lines are drawn because no HLRR data are available yet. 


\subsection{Outlook}

Hotspots, underestimated environmental concentrations ${ }^{297,384}$ and ongoing degradation of macro- and microplastic into nanoplastic result in exceedance of effect thresholds, causing risks of organisms being exposed to plastic particles at specific locations. As regards microplastic, current scientific methods are fairly well able to assess their occurrence, effects and hazards. Previous detection of microplastic and other plastic debris was often based on visual appearance, but this is increasingly being replaced by techniques like FTIR and Raman spectroscopy. These techniques are accessible to more and more research groups, so that confirmation of the occurrence in different media and different organisms with these techniques will continue for several more years. The level of public interest in the subject of microplastic pollution is expected to peak around 2022, as has been forecast based on the history of attention focused on other contaminants of emerging concern (CECs, i.e., harmful environmental agents whose identities, occurrences, effects and hazards are not yet sufficiently understood). ${ }^{390}$ Some of the currently assumed effects of microplastic can be considered less harmful than anticipated before. An example is the often limited effect of microplastic on bioaccumulation of other contaminants in organisms. This view appears to receive growing recognition, ${ }^{51,240,241,243-246,352,373,391-393}$ which might constitute a paradigm shift. On the other hand, the work reported on in this thesis provisionally shows that particle effect thresholds inferred from scientific literature data are exceeded, in particular in near-shore surface water hotspot locations and, although very limited population threating effect threshold data are available, possibly also in freshwater sediment and beach sediment. The replaceability of microplastic by more natural materials, the public interest in microplastic pollution, the improvement of WWTPs ${ }^{330}$ and bans on the use of microplastic in several countries make it unlikely that the development, marketing, disposal and consumption of products containing microplastic will increase. However, the degradation of macroplastic that is already present in the environment and the ongoing disposal of new macroplastic by our consumer society might lead to new, higher, levels of microplastic pollution, with consequences for species and possible renewed political and scientific attention. Further development of $\operatorname{SSDs}^{293}$ based on additional and higher quality effect threshold data will further improve insight in the risk of exposure to plastic. Separating the risk of particle effects from that of chemical transfer effects, and considering the latter within a more complete environmental setting including all relevant chemical transfer pathways, will help to assess the overall risk of exposure to plastic. ${ }^{293,392}$ The generation of knowledge about the occurrence and effects of 
nanoplastic has been different from that for microplastic. The level of interest in nanomaterials in general was expected to peak in $2016,{ }^{390}$ but nanoplastic is a specific case. Although these particles are often bracketed together with microplastic, our current technical ability to determine their occurrence, fate and effects is far less highly developed. Breakthroughs in detection techniques for nanoplastic in environmental media, as well as methods to assess their fate and effects at the level of tissues or organisms, will lead to enhanced knowledge about nanoplastic, but this will come later than for microplastic. For other contaminants of emerging concern, attention by policy makers and the development of new regulations have been shown to peak a few years after the peak in scientific attention. ${ }^{390}$ Therefore, within about a decade, policy regulations might be introduced for microplastic and could be under development for nanoplastic too. Further assessment of the risk of micro- and nanoplastic and further introduction of regulations on the use of plastic will diminish the risk of exposure and will help frame the public concern and the scientific debate relative to those regarding environmental concerns other than plastic. 


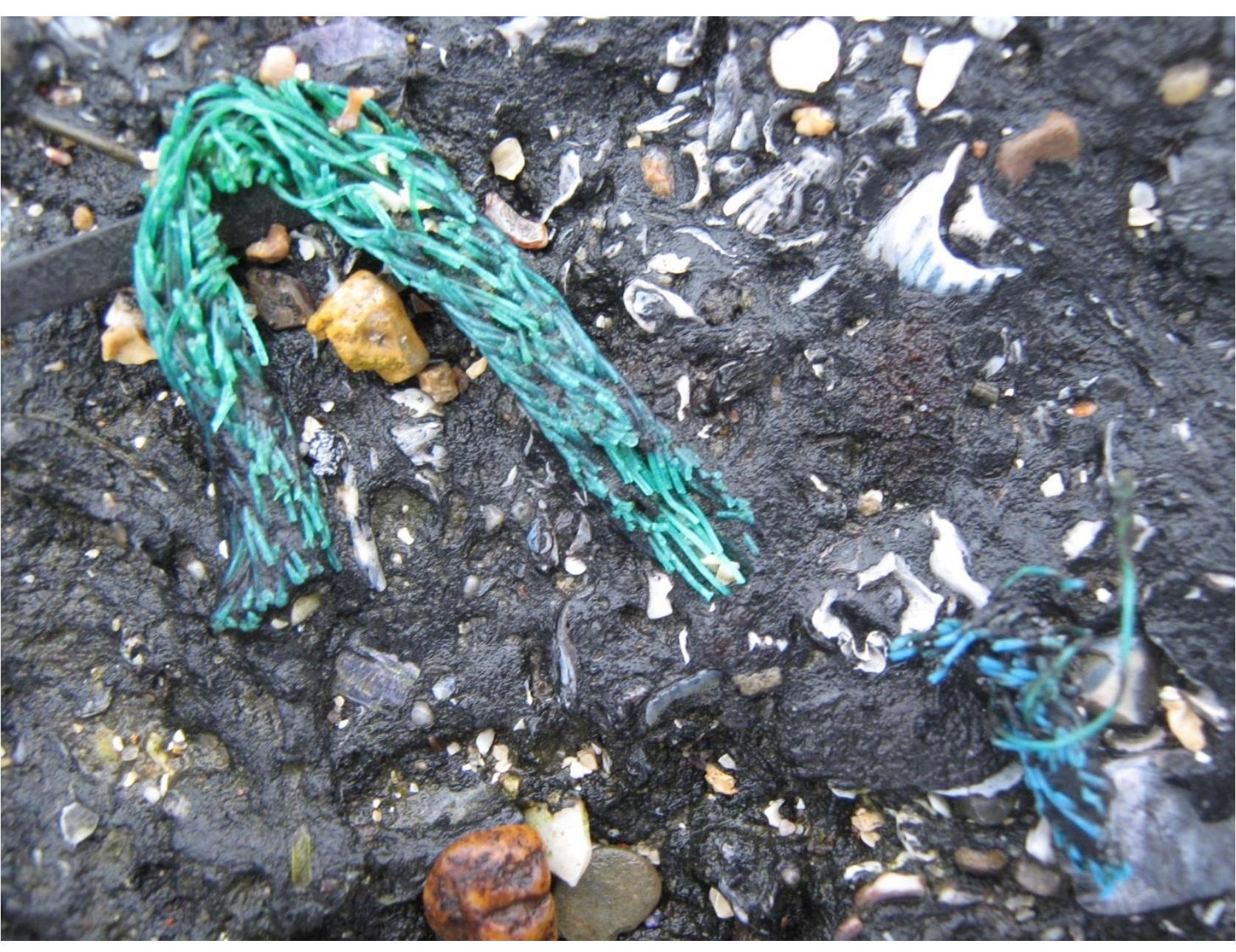




\section{Appendix to Chapter 2}

1. Figure $\mathrm{A} 2.1-\mathrm{A} 2.7$

2. Table A2.1-A2.4

3. NanoDUFLOW Model description, with parameter values (Table A2.5)

4. Calculation of the attachment efficiency ( $\left.\alpha_{\text {het }}\right)$ between nanoplastic, microplastic and clays from experimental data, with $\alpha$ values (Table A2.6) 
Figures
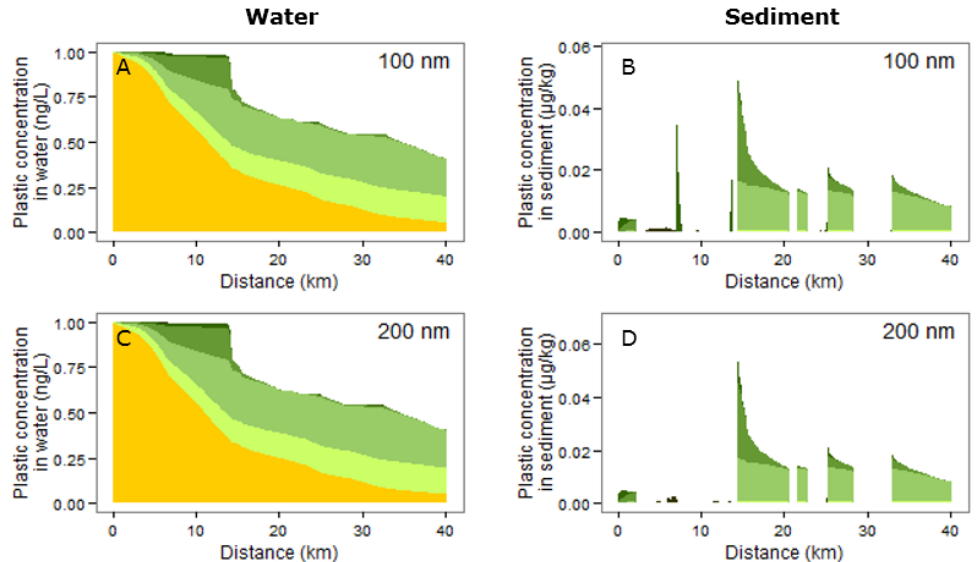

Hetero-

aggregates

$\mathrm{SS}_{5} \mathrm{Pl}_{1-5}$ $\mathrm{SS}_{4} \mathrm{Pl}_{1-5}$ $\mathrm{SS}_{3} \mathrm{Pl}_{1}$ $\mathrm{SS}_{2} \mathrm{Pl}_{1-5}$ $\mathrm{SS}_{1} \mathrm{Pl}_{1-5}$
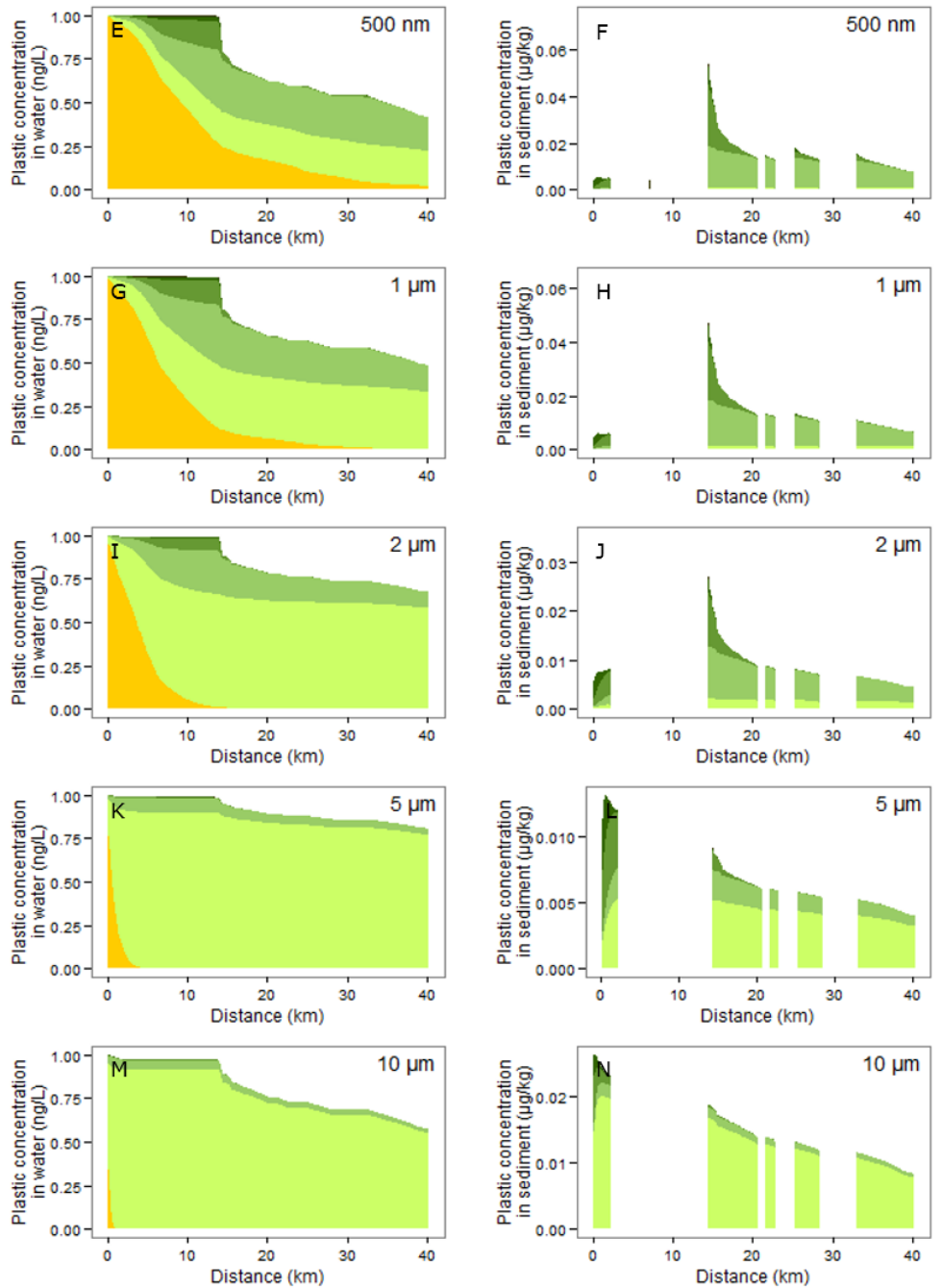

Singular particles $\mathrm{Pl}_{1}$ 
Appendix Chapter 2
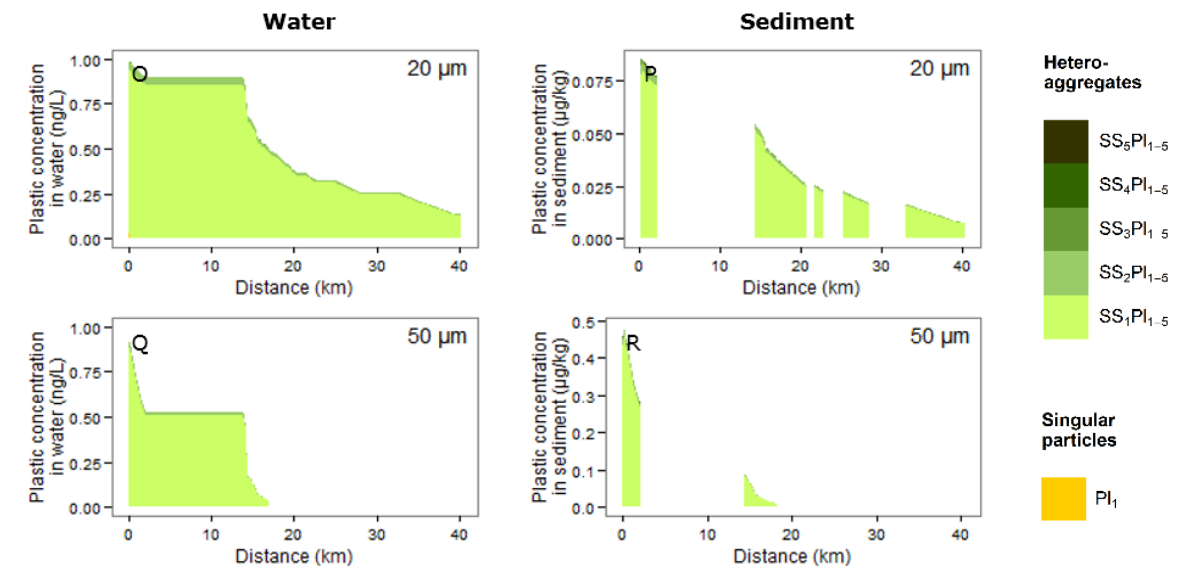

$\mathrm{SS}_{1} \mathrm{Pl}_{1-5}$
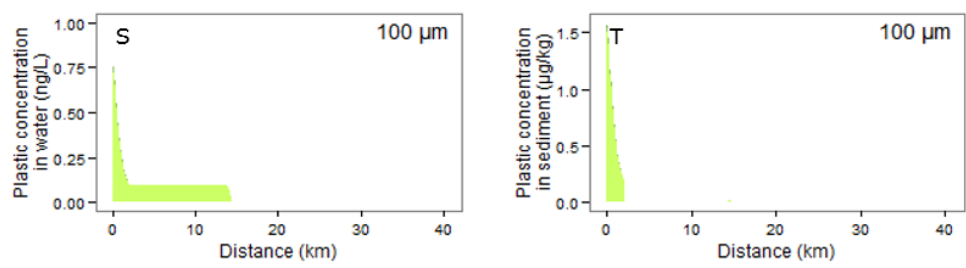

Singular
particles

particles

$\mathrm{Pl}_{1}$
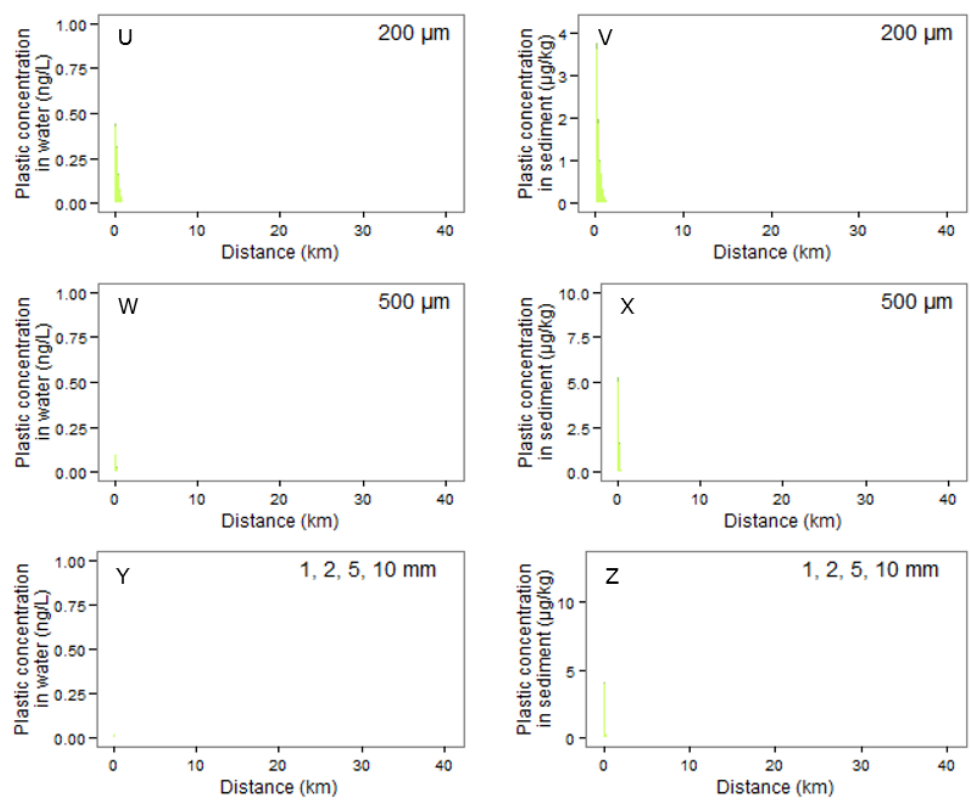

Figure A2.1. Description see Figure A2.4. Scenarios with average polymer density (1040 $\left.\mathrm{kg} / \mathrm{m}^{3}\right)$. 
Figures
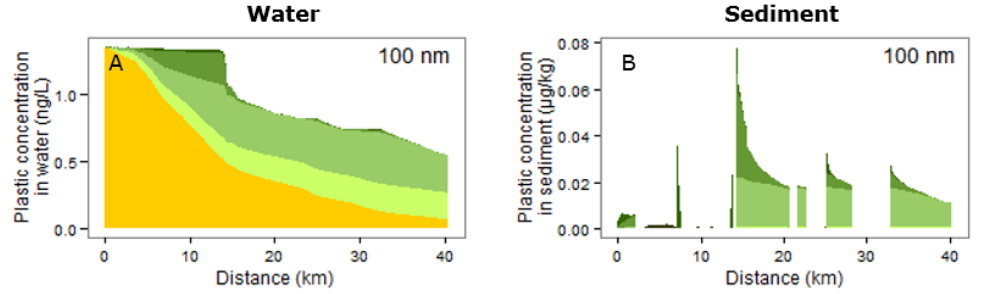

Hetero

aggregates
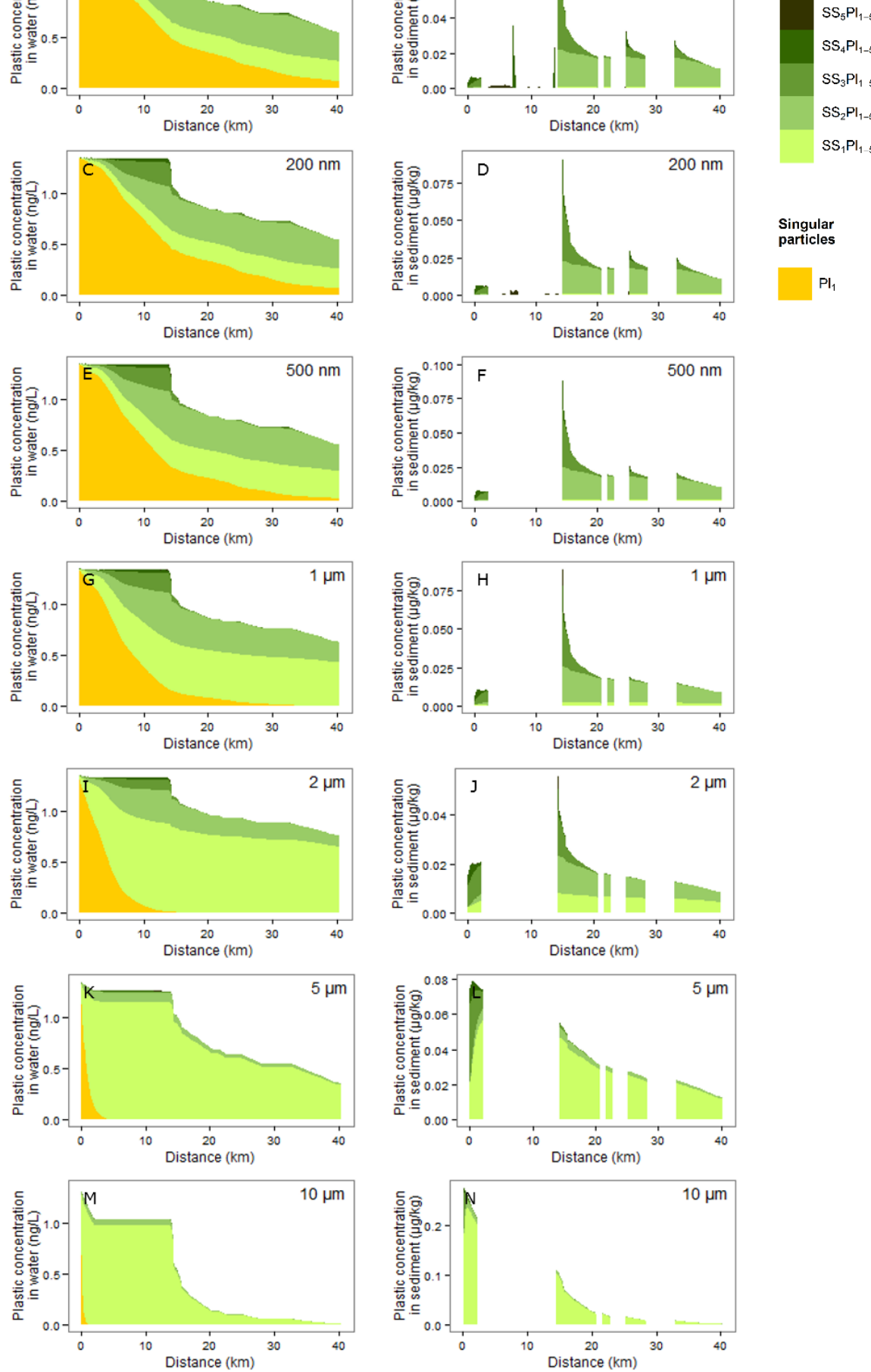

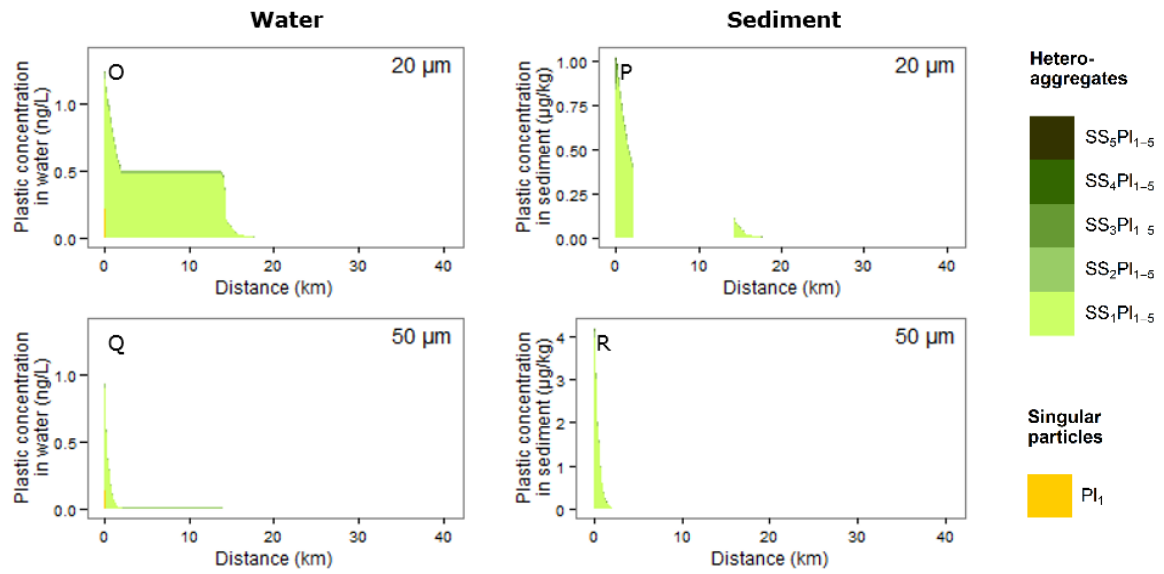

$\mathrm{SS}_{1} \mathrm{Pl}_{1-5}$
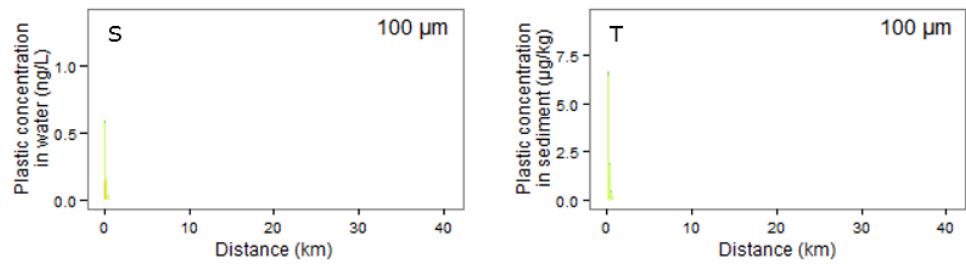

Singular

particles
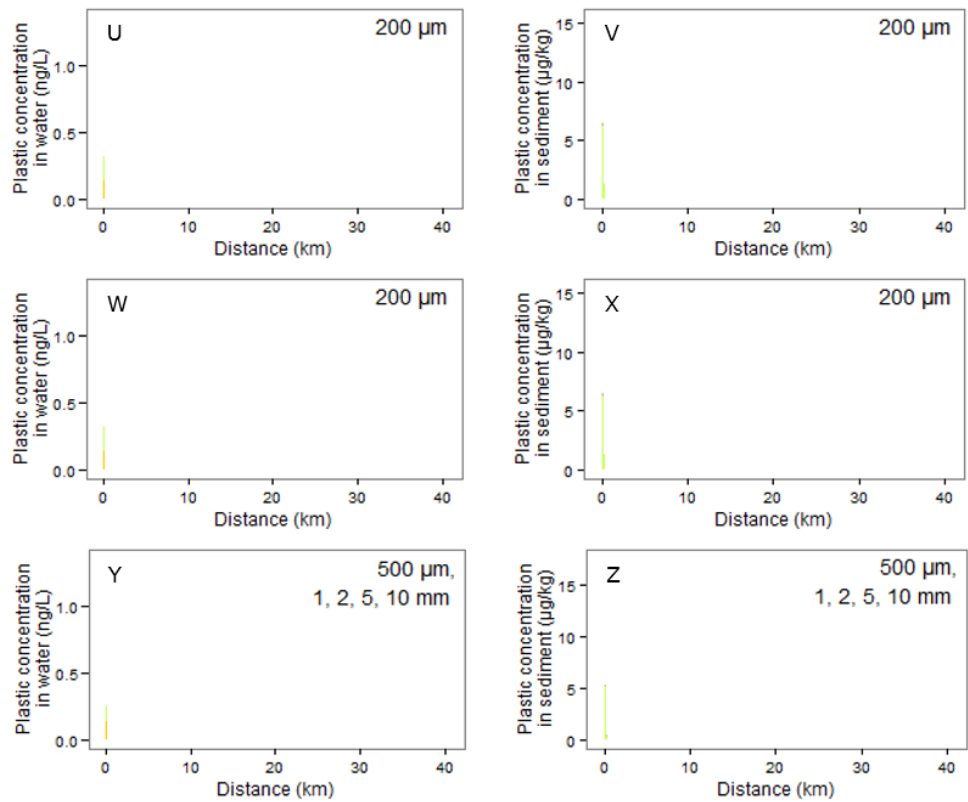

Figure A2.2. Description see Figure A2.4. Scenarios with high polymer density $\left(1400 \mathrm{~kg} / \mathrm{m}^{3}\right)$. 
Figures
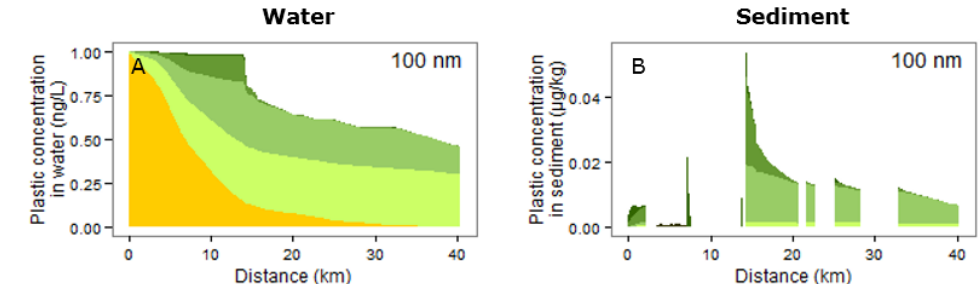

Hetero-

aggregates
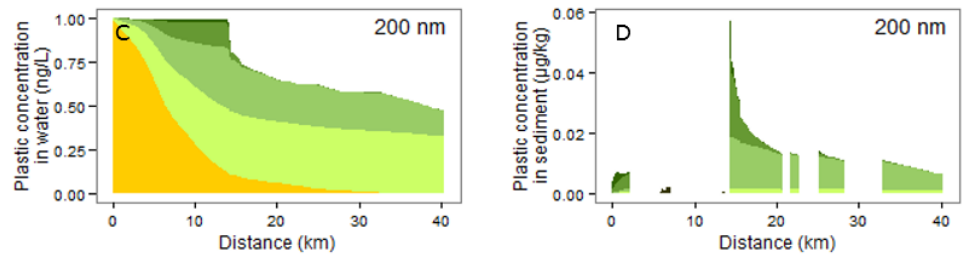

$\mathrm{SS}_{5} \mathrm{Pl}_{1-5}$ $\mathrm{SS}_{4} \mathrm{Pl}_{1-5}$ $\mathrm{SS}_{3} \mathrm{Pl}_{1-5}$ $\mathrm{SS}_{2} \mathrm{Pl}_{1-5}$ $\mathrm{SS}_{1} \mathrm{PI}_{1-5}$
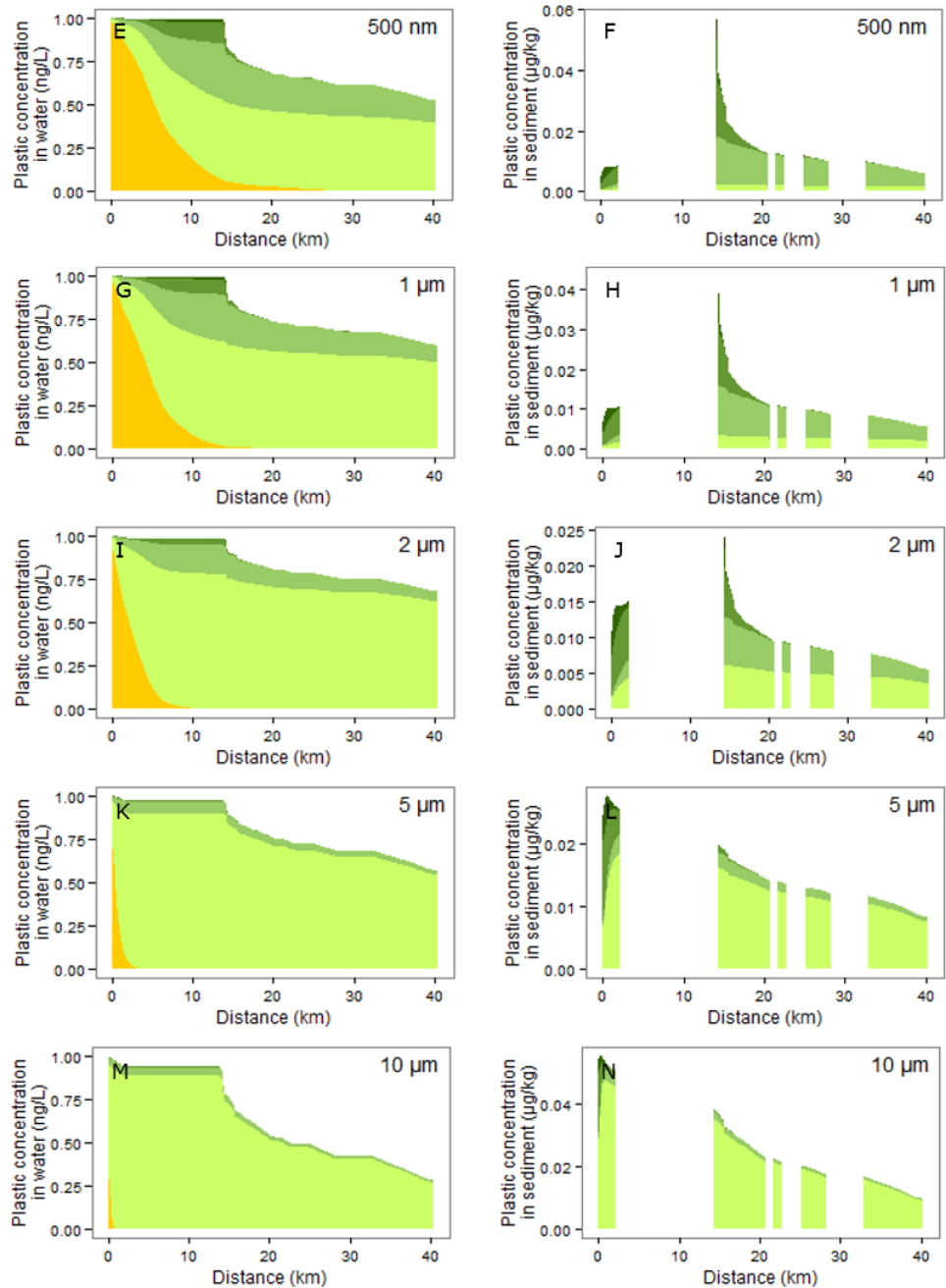

Singular

particles

$\mathrm{PI}_{1}$ 
Appendix Chapter 2
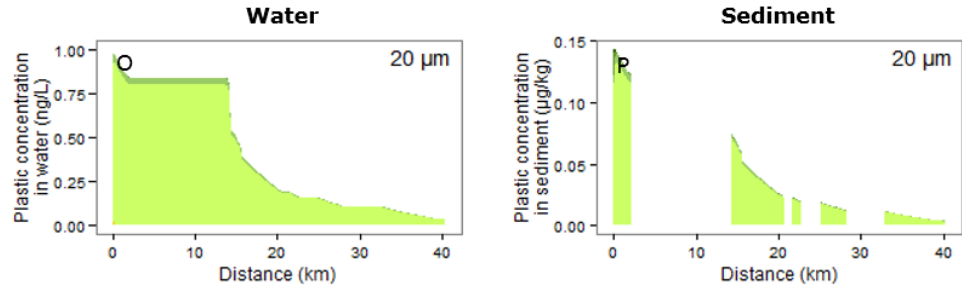

Hetero-

aggregates
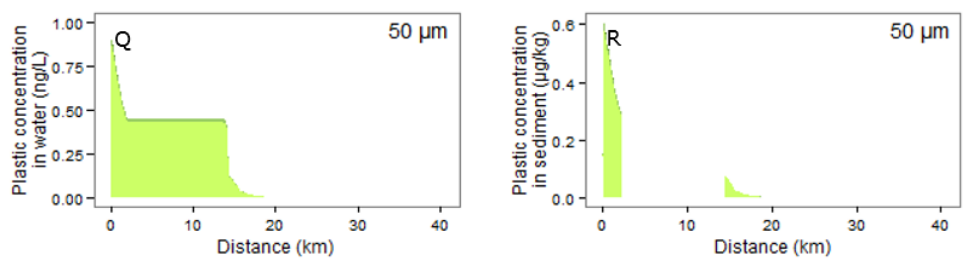

$\mathrm{SS}_{5} \mathrm{PI}_{1-5}$ $\mathrm{SS}_{4} \mathrm{Pl}_{1-5}$ $\mathrm{SS}_{3} \mathrm{PI}_{1-5}$ $\mathrm{SS}_{2} \mathrm{PI}_{1-5}$ $\mathrm{SS}_{1} \mathrm{PI}_{1-5}$
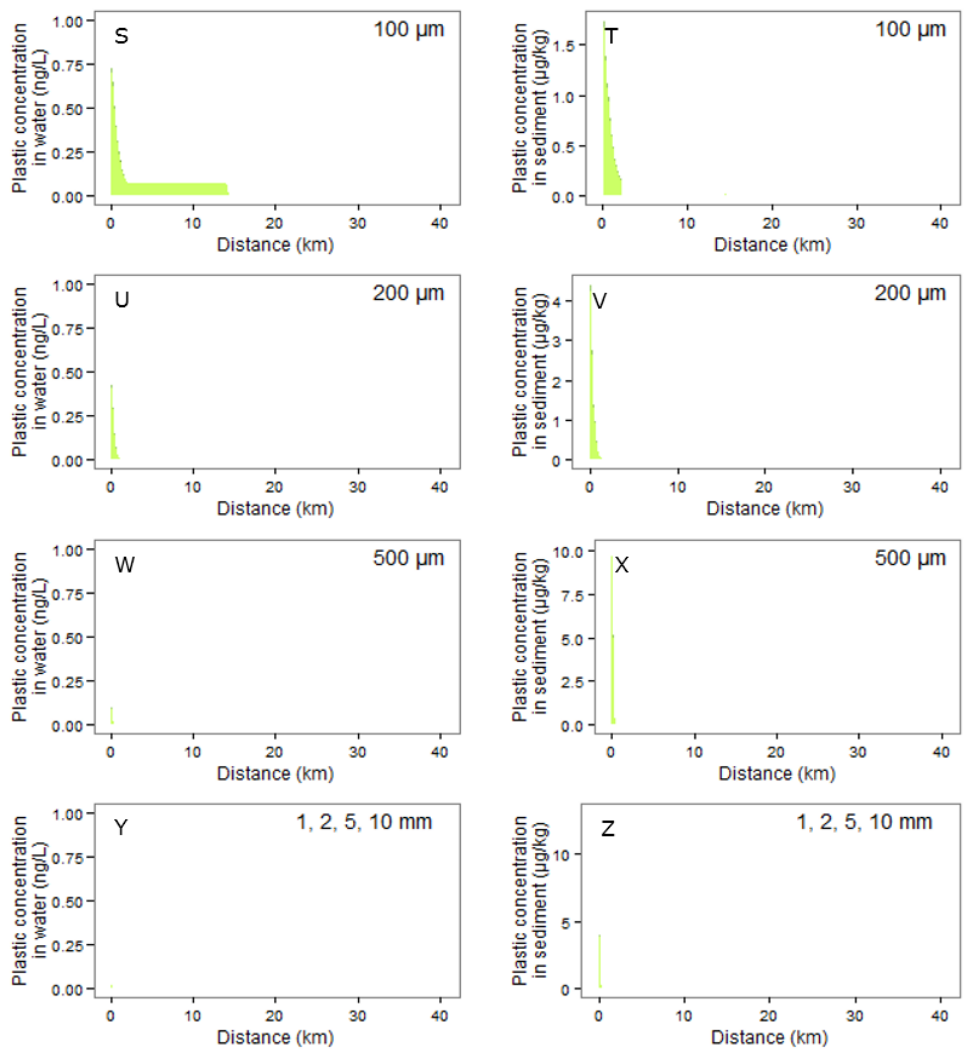

Singular particles $\mathrm{Pl}_{1}$ 
Figures
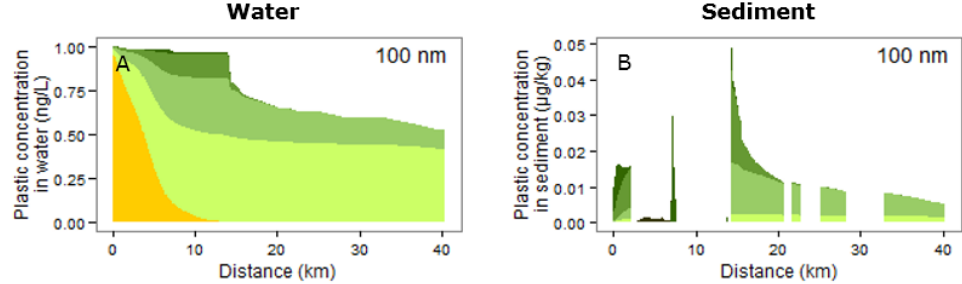

aggregates
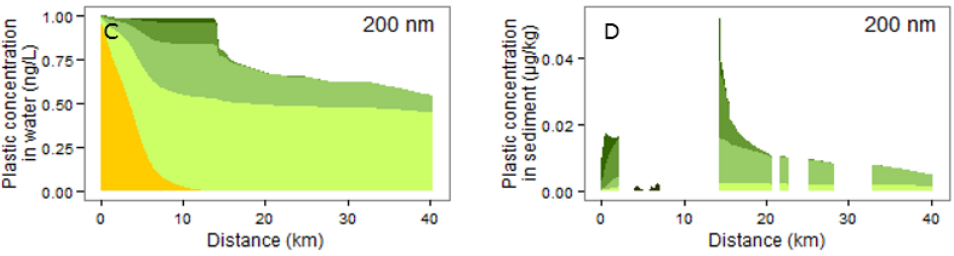

$\mathrm{SS}_{5} \mathrm{Pl}_{1-5}$
$\mathrm{SS}_{4} \mathrm{Pl}_{1-5}$
$\mathrm{SS}_{3} \mathrm{Pl}_{1-5}$
$\mathrm{SS}_{2} \mathrm{Pl}_{1-5}$
$\mathrm{SS}_{1} \mathrm{Pl}_{1-5}$
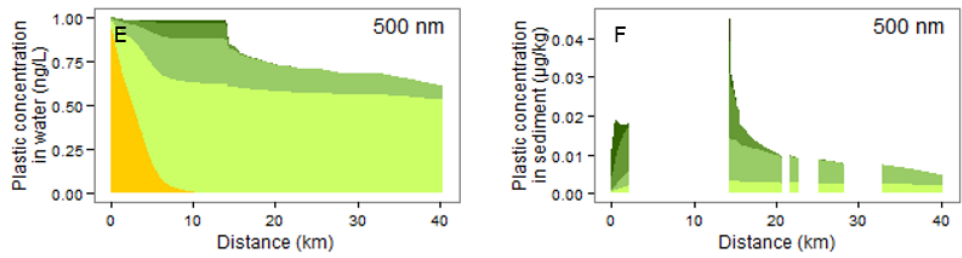

ingular

particles
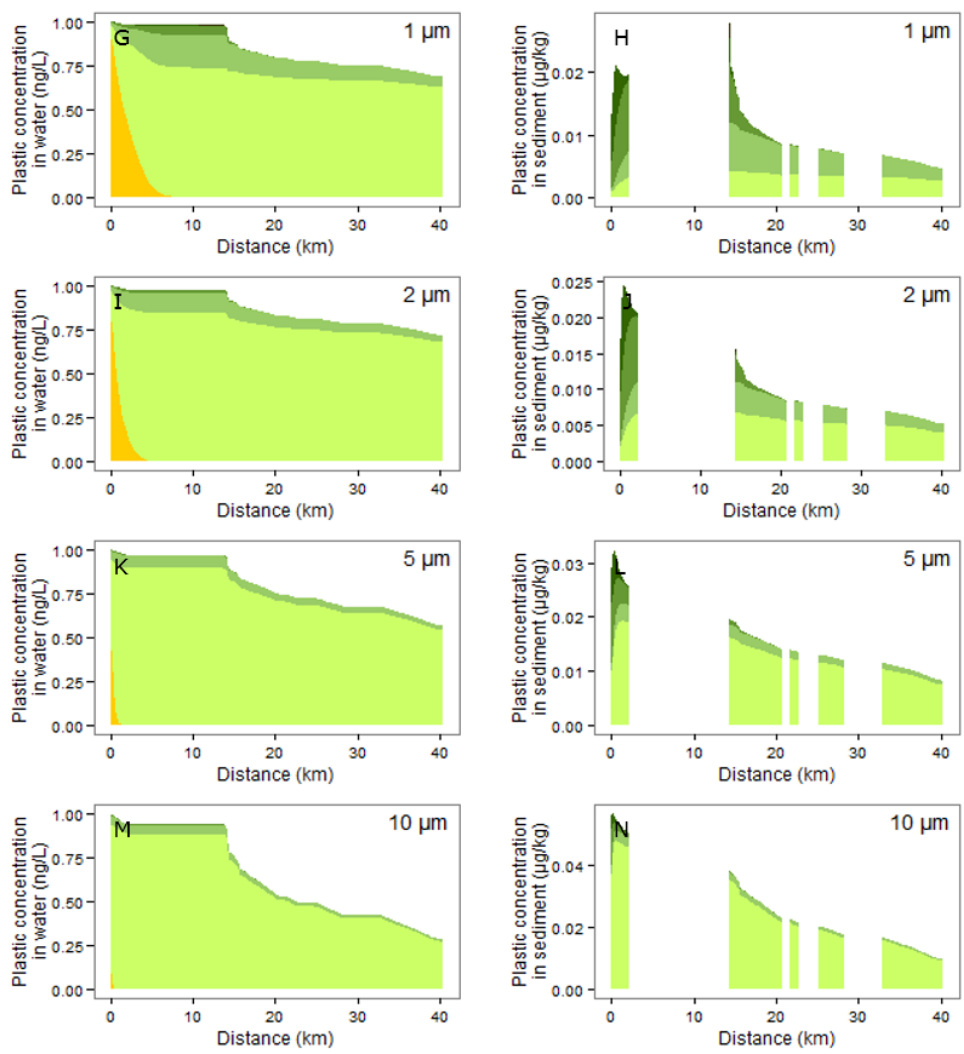

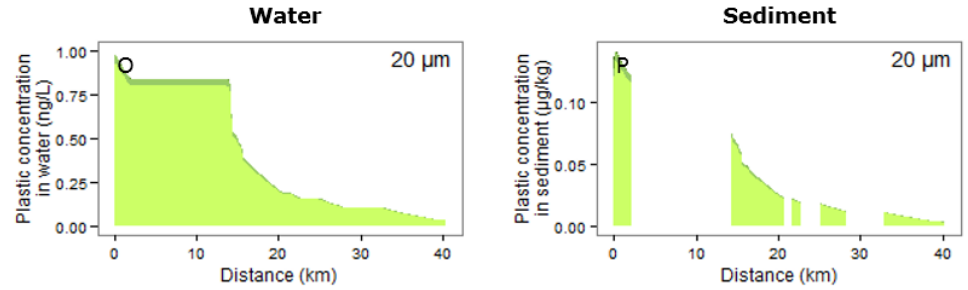

Hetero-

aggregates
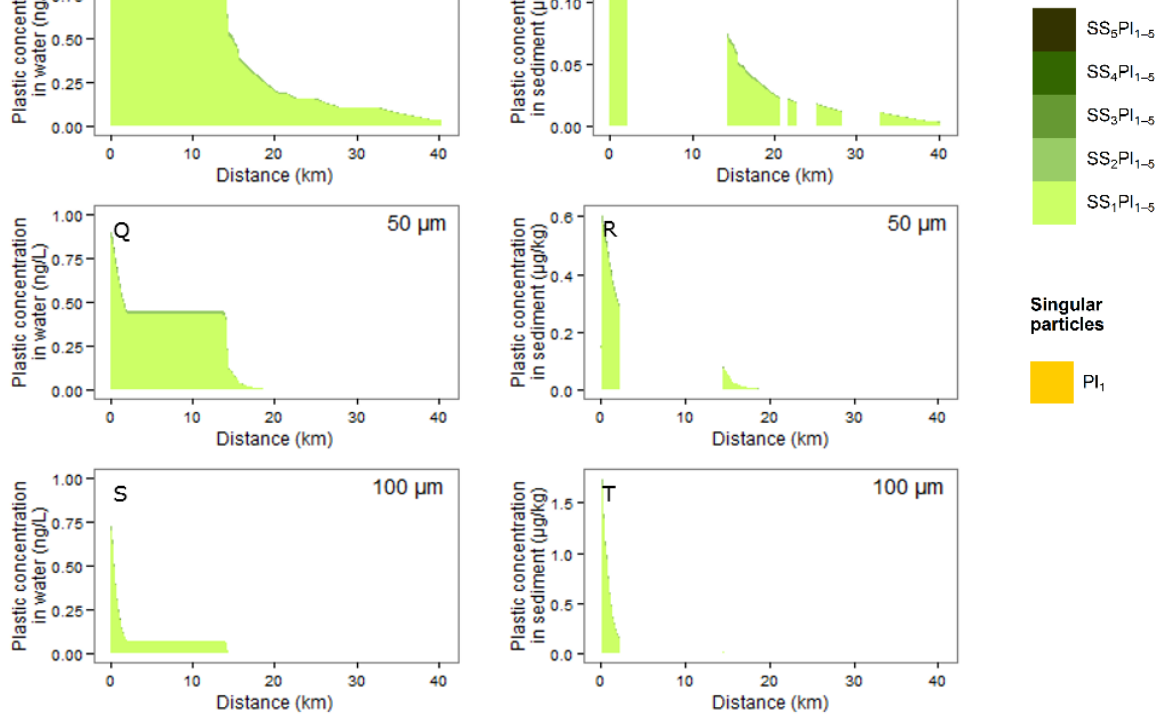

Singular particles
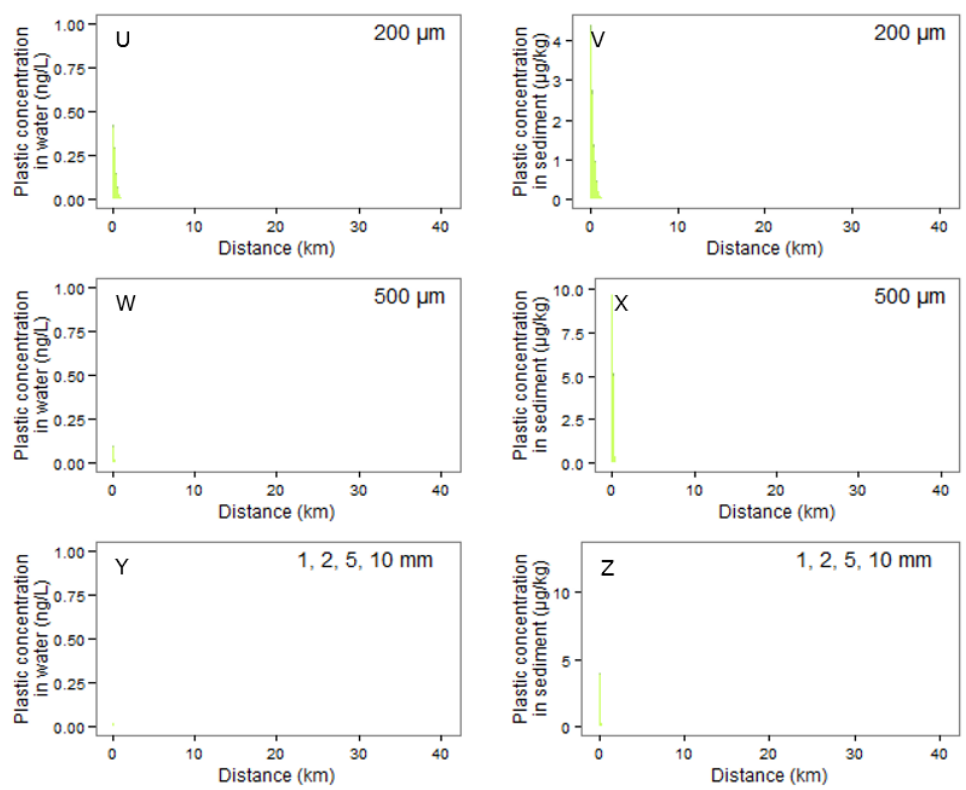

Figure A2.4. Scenarios with biofilm, $\alpha=0.03$. Figure A2.1-4. Spatial distribution of plastic over a $40 \mathrm{~km}$ river stretch. Plastic concentrations are given for the water column (left-sided panels) and for sediment (right-sided panels). From top to bottom, concentrations are given with increasing initial plastic particle sizes ranging from $100 \mathrm{~nm}$ to $10 \mathrm{~mm}$. These concentrations are reached after nine days of plastic input into the river, representing steady- 


\section{Figures}

state concentrations for the water column and intermediate state for the sediment. Different sections along the river are characterised by either net sedimentation or net resuspension, sections with net resuspension show no accumulation of plastic in the sediment. The simulations used an 'average plastic' density of $1040 \mathrm{~kg} / \mathrm{m}^{3}$ (Fig. A2.1), which is similar to that of polystyrene.The upper curves in the panels indicate the total concentration of microplastic, whereas the coloured areas indicate how plastic particles in singular form $\left(\mathrm{Pl}_{1}\right)$ and plastic in heteroaggregates $\left(S S_{1-5} P l_{1-5}\right)$ with suspended solids of different size classes $\left(S S_{1-5}\right)$, contribute to the total concentration. Homoaggregate $\left(\mathrm{Pl}_{2-5}\right)$ concentrations were negligible and therefore not visible in the figure. Heteroaggregate concentrations are plotted as sum for $S S_{x} P l_{1-5}$, though are mainly composed of $S_{x} P l_{1}$. Additionally shown here are simulations with a plastic density of $1400 \mathrm{~kg} / \mathrm{m}^{3}$ (Fig. A2.2), which is similar to that of the polymer types PET and PVC. Figures A3-4 show scenarios with a biofilm on the plastic particles, with a default attachment efficiency of 0.01 (Fig. A2.3) and elevated attachement efficiency of 0.03 (Fig. A2.4).
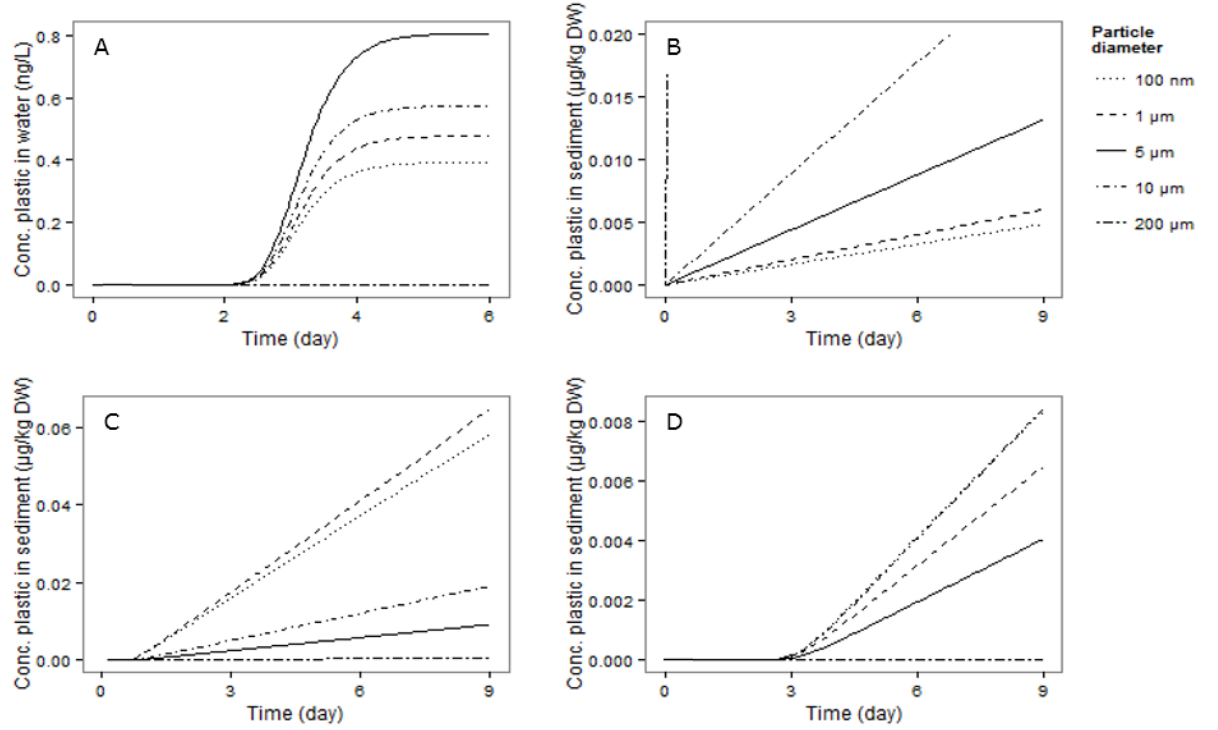

Fig. A2.5. Concentration of plastic as a function of time, in water at the end of the $40 \mathrm{~km}$ river system $(A)$ and in sediment at the distance within $1 \mathrm{~km}$ from the input source where plastic concentrations are highest ( $B$, for the 5 plotted particle sizes respectively 538, 638, 638, 340 and $49 \mathrm{~m}$ downstream the input source), at the main sedimentation area $14.4 \mathrm{~km}$ downstream $(C)$ and at the end of the $40 \mathrm{~km}$ river system $(D)$. In the water compartment, steady-state concentrations are reached in 5 days. In the sediment compartment, steady-state is not reached within the simulated period of 9 days. There is a linear increase of plastic concentrations in the sediment (Table A2.3) within $1 \mathrm{~km}$ from the input source. After a time lag of $1(C)$ or $3(D)$ days a linear increase of plastic concentrations in the sediment starts at the main sedimentation area and $40 \mathrm{~km}$ downstream respectively. For bigger particles (i.e. $\geq 500 \mu \mathrm{m}$, Table A2.4) rapid sedimentation resulted in high concentrations at the start of the river and in low concentrations further downstream. 

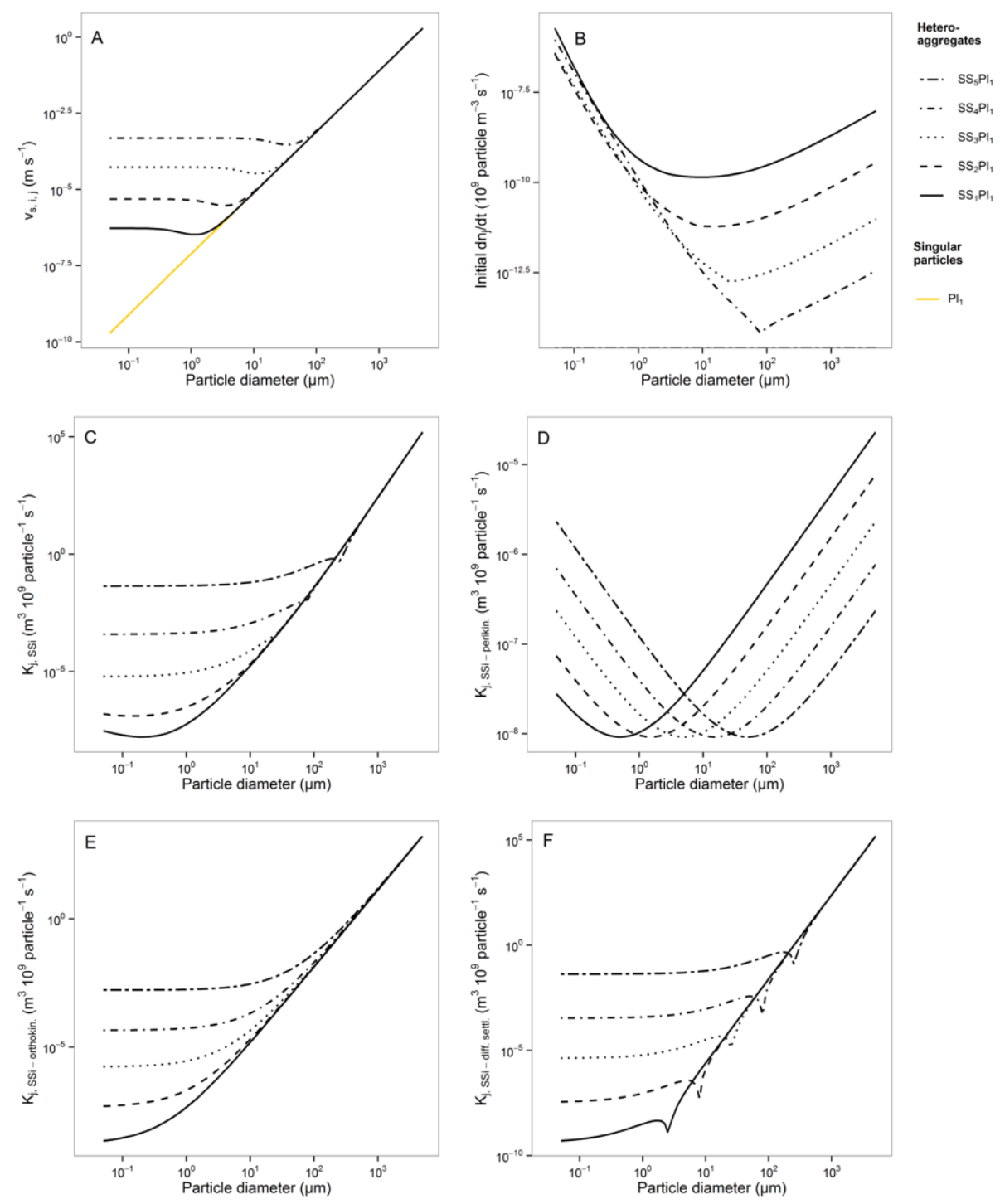

Figure A2.6. Subprocess rates for plastic particles with a size of $100 \mathrm{~nm}$ to $10 \mathrm{~mm}$ at a plastic input concentration of $1 \mathrm{ng} / \mathrm{L}$, and a default 'average plastic' density $\left(1040 \mathrm{~kg} / \mathrm{m}^{3}\right)$ and attachment efficiency (0.01). Panel A: Sedimentation rate of heteroaggregates and singular particles. Panel B: initial heteroaggregation rate of singular plastic particles $\left(\mathrm{Pl}_{1}\right)$ with suspended solids of different size classes $\left(S S_{1-5}\right)$ available in the water phase of the river system. Panel C: Collision frequency as part of heteroaggregation, of singular plastic particles $\left(P_{1}\right)$ with suspended solids of different size classes $\left(S S_{1-5}\right)$ available in the water phase of the river system. Three aggregation regimes ${ }^{77}$ affect the overall collision frequency and are calculated here separately. Panel D: perikinetic aggregation, Panel E: orthokinetic aggregation, Panel F: and differential settling. Dips in panel F occur where the sedimentation rate of $P l$ approaches that of SS, reducing the absolute difference (Eq. A2.5, last term) to zero. 
Figures
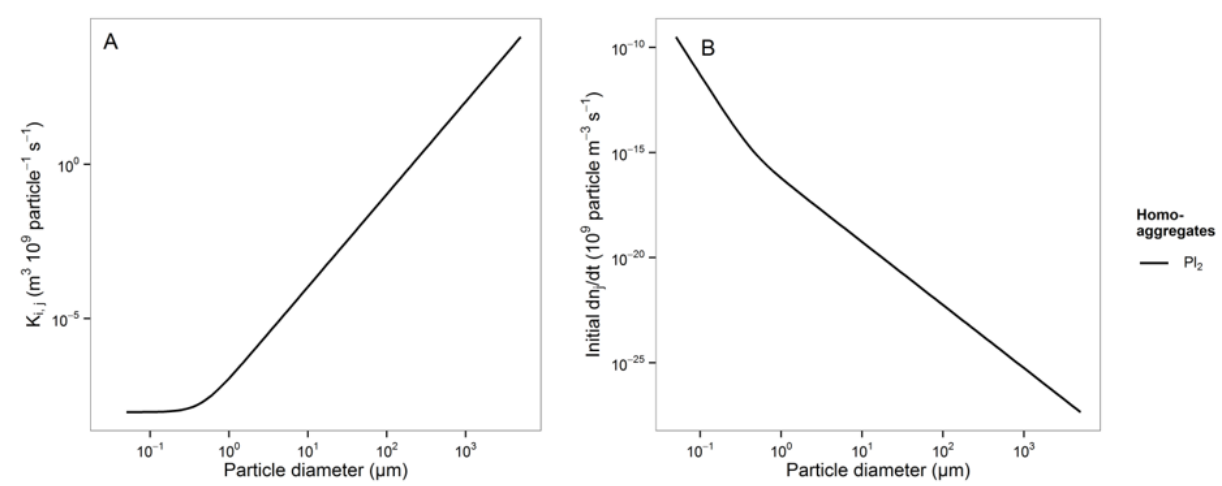

Figure A2.7. Collision frequency as part of homoaggregation (Panel A) and its corresponding initial homoaggregation rate (Panel B) of singular plastic particles $\left(P l_{1}\right)$, with a size of $100 \mathrm{~nm}$ to $10 \mathrm{~mm}$ at a plastic input concentration of $1 \mathrm{ng} / \mathrm{L}$ and a default density $\left(1040 \mathrm{~kg} / \mathrm{m}^{3}\right)$ and attachment efficiency (0.01). The relatively low homoaggregation rate compared to the heteroaggregation rate (Fig. A2.6B) makes that exclusion of homoaggregation does not affect overall retention. 
Table A2.1. Characteristics of the spatially heterogeneous Dommel model (after Quik et al., 2015). ${ }^{71}$

\begin{tabular}{|l|l|}
\hline Length & $40.3 \mathrm{~km}$ \\
\hline Discharge & $1 \mathrm{~m}^{3} \mathrm{~s}^{-1}$ \\
\hline $\begin{array}{l}\text { Min - max (Avg.) } \\
\text { flow rate }\end{array}$ & $\begin{array}{l}0.00155-0.373 \\
(0.199) \mathrm{m} \mathrm{s}^{-1}\end{array}$ \\
\hline Min - max width & $8-288 \mathrm{~m}$ \\
\hline Min - max depth & $0.4-3.6 \mathrm{~m}$ \\
\hline Calc. sections & 477 \\
\hline Avg. section length & $87.7 \mathrm{~m}$ \\
\hline
\end{tabular}

Table A2.2. Average microplastic concentrations in freshwater. Where originally numbers / area were given a sampling depth of $0.1 \mathrm{~m}$ was assumed and where numbers per volume were given a mass per particle of $5 \mu \mathrm{g}$ was used. ${ }^{105}$

\begin{tabular}{|l|c|c|c|c|}
\hline Location & $\begin{array}{c}\text { particle } \\
\mathbf{k m}^{-2}\end{array}$ & $\begin{array}{c}\text { particle } \\
\mathbf{L}^{-\mathbf{1}}\end{array}$ & $\mathbf{n g ~ \mathbf { L } ^ { - 1 }}$ & Reference \\
\hline US, five great lakes & $4.3 \mathrm{E}+04$ & $4.3 \mathrm{E}-04$ & 2.2 & 57 \\
\hline $\begin{array}{l}\text { Switzerland, lake } \\
\text { Geneva }\end{array}$ & $4.8 \mathrm{E}+04$ & $4.8 \mathrm{E}-04$ & 2.4 & 38 \\
\hline Austria, Danube & & $3.2 \mathrm{E}-04$ & 1.6 & 58 \\
\hline $\begin{array}{l}\text { Netherlands, Maas and } \\
\text { Waal catchment area }\end{array}$ & & $7.9 \mathrm{E}-05$ & $4.0 \mathrm{E}-01$ & 101 \\
\hline
\end{tabular}


Tables

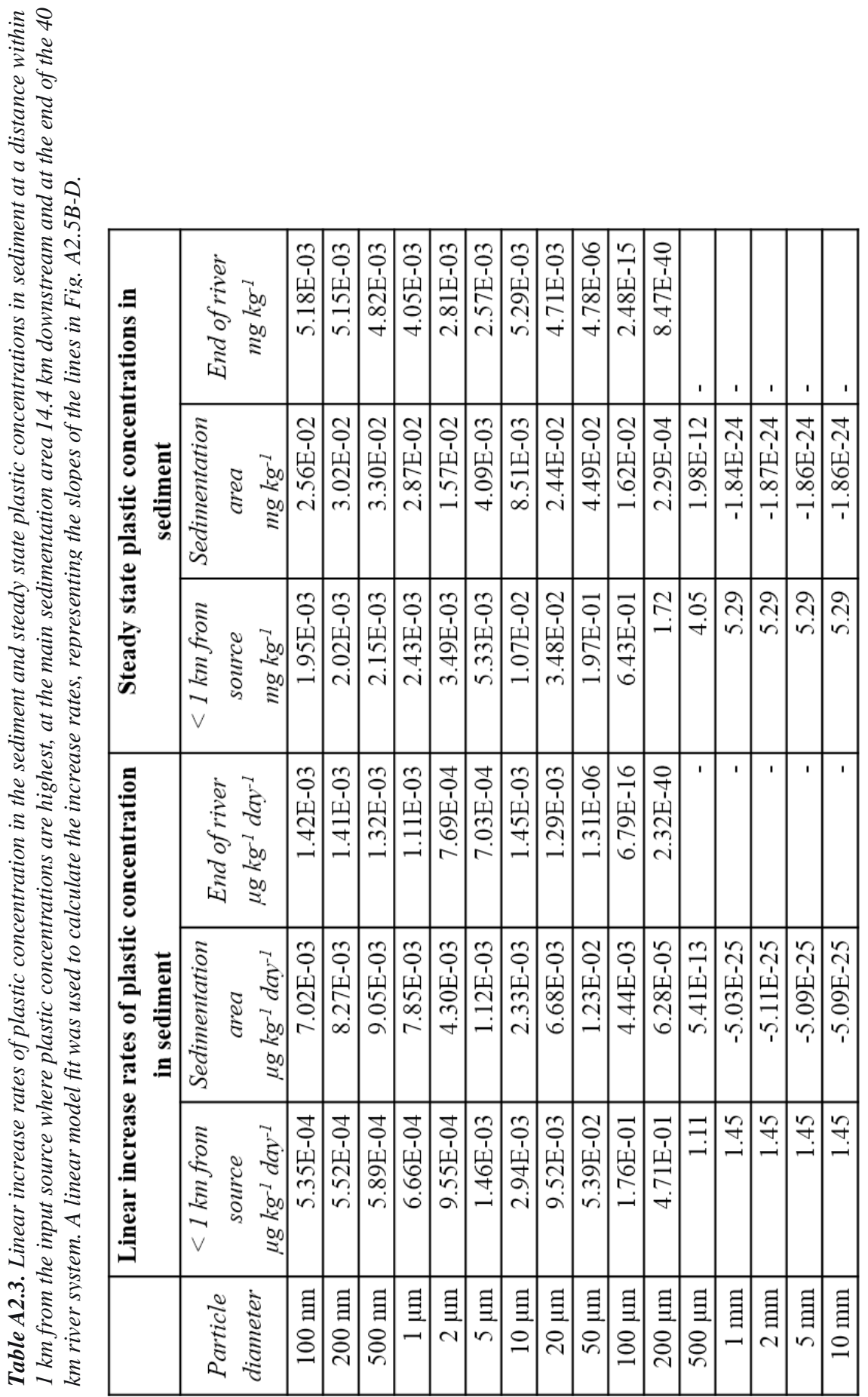



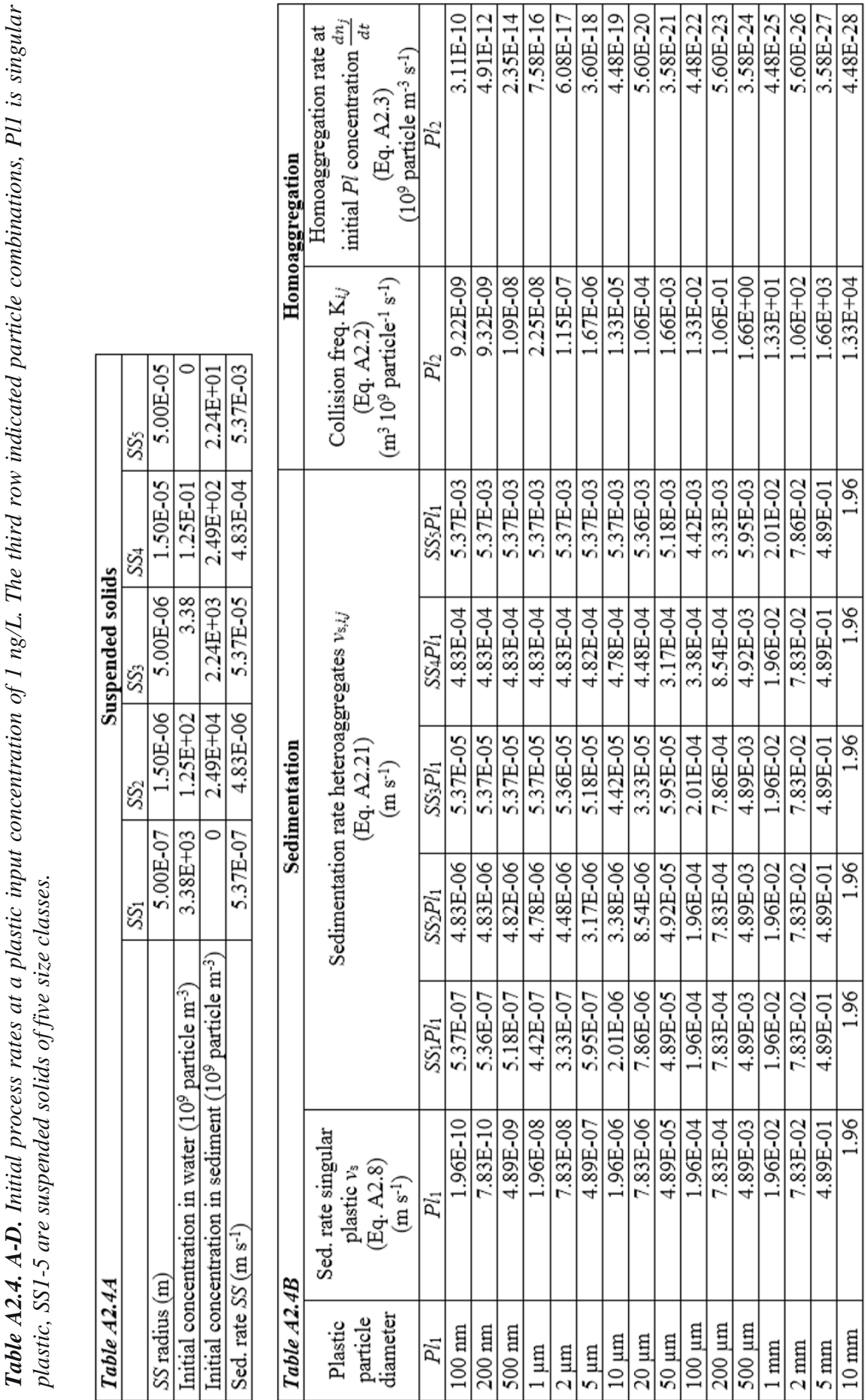
Tables

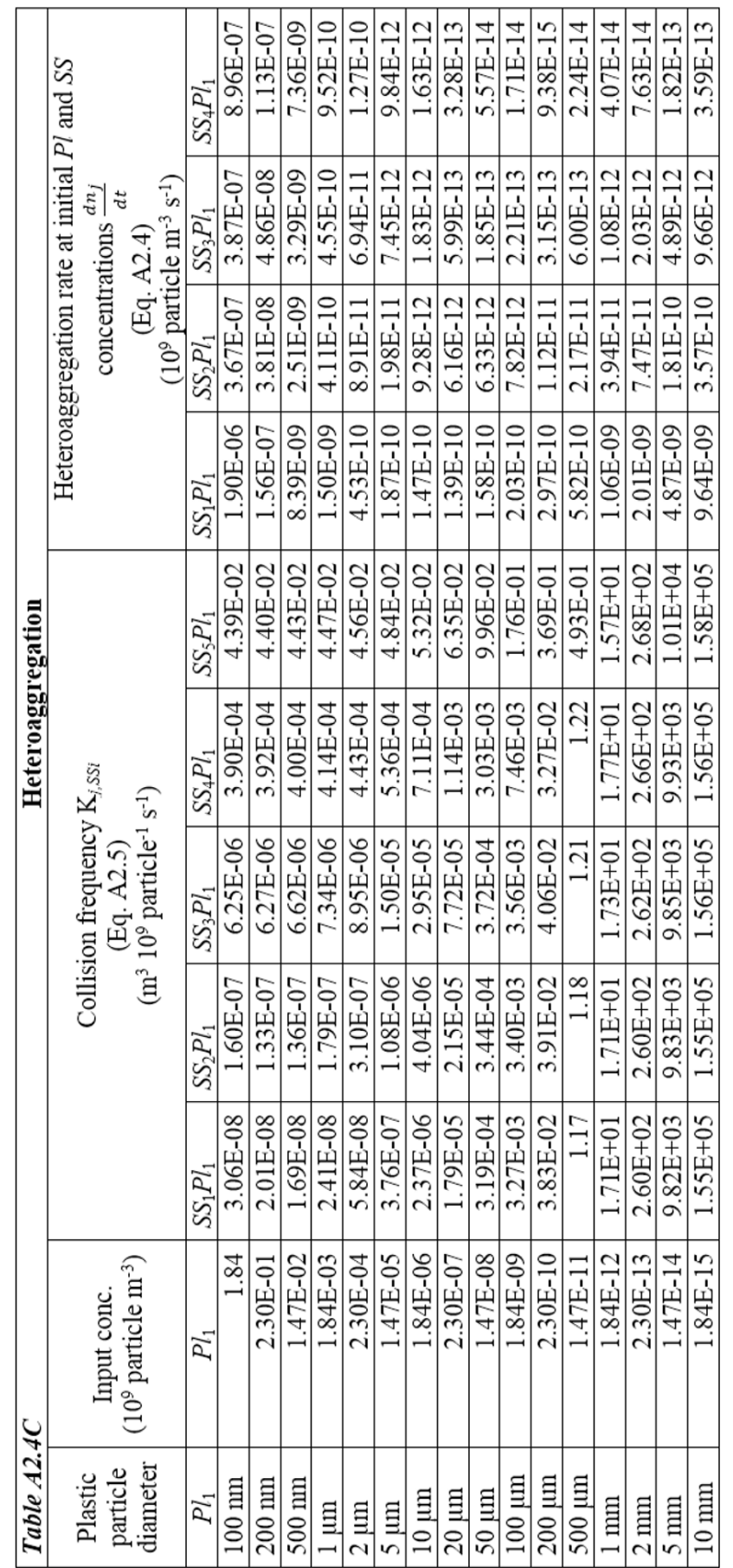




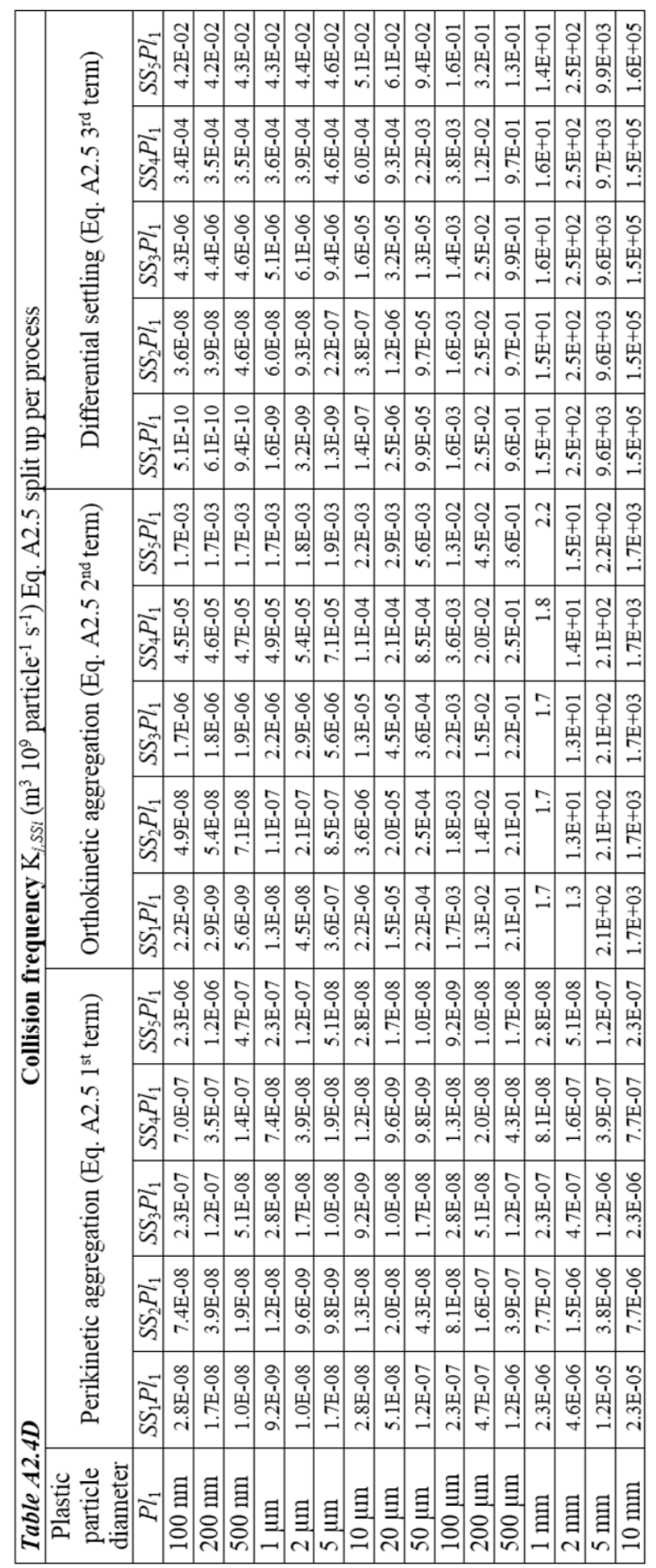




\section{NanoDUFLOW Model description}

Adjusted from Quik et al. (2015). An extended overview of process descriptions for NanoDUFLOW is provided below, followed by parameter values in Table A2.5.

\section{Transformation processes}

\section{Homoaggregation}

Homoaggregation is the process where plastic particles interact with each other to form aggregates. The aggregation rate constant is the product of the frequency of collisions between plastic particles $(K)$ and the attachment efficiency $(\alpha)$. Homoaggregation can be quantitatively described by the Von Smoluchowski equation, ${ }^{394}$ as recommended before for modeling the fate of nano particles in natural waters: ${ }^{71,82,395,396}$

$\frac{d n_{j}}{d t}=\frac{1}{2} \sum_{i=1}^{i=j-1} \alpha_{i, j-1} K_{i, j-i} n_{i} n_{j-i}-n_{j} \sum_{i=1}^{i=\infty} \alpha_{i, j} K_{i, j} n_{i}$

$n_{\mathrm{j}} \quad$ Particle number concentration of size class $j\left[10^{9}\right.$ particle $\left.\mathrm{m}^{-3}\right]$ in giga particles $\left(10^{9}\right)$.

$t \quad$ Time [s]

$\alpha_{i, j} \quad$ Attachment efficiency of particle $i$ with j [-]

$K_{i, j} \quad$ Collision frequency of particle $i$ with $\mathrm{j}\left[\mathrm{m}^{3} 10^{9}\right.$ particle $\left.^{-1} \mathrm{~s}^{-1}\right]$

The collision frequency $\left(K_{i, j}\right)$ is given by:

$$
\begin{aligned}
& K_{i, j}=\left(\frac{2 k_{b} T}{3 \mu} \frac{\left(a_{i}+a_{j}\right)^{2}}{a_{i} a_{j}}+\frac{4}{3} G\left(a_{i}+a_{j}\right)^{3}+\left(\frac{2 \pi g}{9 \mu}\right)\left(\rho_{p}-\right.\right. \\
& \left.\left.\rho_{w}\right)\left(a_{i}+a_{j}\right)\left(a_{i}-a_{j}\right)\right) 10^{9}
\end{aligned}
$$

$k_{\mathrm{b}} \quad$ Boltzman constant $\left[\mathrm{m}^{2} \mathrm{~kg} \mathrm{~s}^{-2} \mathrm{~K}^{-1}\right]$

$T \quad$ Temperature [K]

$\mu \quad$ Viscosity $\left[\mathrm{kg} \mathrm{s}^{-1} \mathrm{~m}^{-1}\right]$

a Particle radius [m]

$G \quad$ Shear rate $\left[\mathrm{s}^{-1}\right]$ (calculated from DUFLOW flow rate, see Table A2.5, Eq. A2.20)

$g \quad$ Gravitation acceleration $\left[\mathrm{m} \mathrm{s}^{-2}\right]$ 
$\rho_{\mathrm{p}} \quad$ Density of the particle $\left[\mathrm{kg} \mathrm{m}^{-3}\right]$

$\rho_{\mathrm{w}} \quad$ Density of the suspending medium $\left[\mathrm{kg} \mathrm{m}^{-3}\right]$

Homoaggregation is implemented in DUFLOW by using five size classes of plastic particles, which grow from one class to the next and are corrected for the difference in mass of the two size classes (Eq. A2.3).

This leads to the following simplification of Eq. A1:

$\frac{d n_{j}}{d t}=-\frac{1}{2} \alpha_{h o m} K_{j, j} n_{j} n_{j}+\frac{1}{2} \alpha_{h o m} K_{i, i} n_{i} n_{i} \frac{\rho_{i} a_{i}^{3}}{\rho_{j} a_{j}^{3}} \quad$ with $i=j-1$

Where the second term in Eq. A3 is zero for $j=1$.

This simplification implies that plastic removal due to interactions of homoaggregates with primary plastic particles and plastic homoaggregates from other size classes is assumed to be negligible, which is based on the extremely fast initial removal of these primary plastic particles due to homoand heteroaggregation. In a previous study we used a full Smoluchowski model (i.e. Eq. A2.1) and showed that sedimentation due to homoaggregation is negligible at low particle concentrations (e.g. $10 \mu \mathrm{g} \mathrm{L}^{-1} \mathrm{CeO}_{2} \mathrm{ENPs}$ ). ${ }^{82} \mathrm{In}$ order to test if this is also true for the present simplified homoaggregation implementation (Eq. A2.3), Quik et al. (2015) performed two different model simulations: (1) with $\alpha_{\mathrm{hom}}=0$ and (2) with $\alpha_{\mathrm{hom}}=1$ (i.e. minimizing and maximizing the role of homoaggregation, respectively) for the default modeled plastic concentration. This resulted in no discernible difference in the concentration profile, which implies that the simplification in Eq. A3 does not affect the model scenarios calculations in the present chapter and thus that the assumption is valid.

\section{Heteroaggregation}

Heteroaggregation is the process where plastic particles interact with natural suspended solids (SS) to form an aggregate. The quantitative description of heteroaggregation is based on the same principles as homoaggregation, where the rate of change in heteroaggregate concentrations is made up by the attachment efficiency combined with the collision frequency. For plastic particles this is given by: ${ }^{71,72,80,82,395}$

$\frac{d n_{j}}{d t}=-\alpha_{h e t} n_{j} \sum_{1}^{i} K_{j, S S i} n_{S S i}$

Where $n$ is the number of size classes of $S S$ and $K_{j, S S j}$ given by: ${ }^{396}$ 


$$
\begin{aligned}
& K_{j, S S i}=\left(\frac{2 k_{b} T}{3 \mu} \frac{\left(a_{j}+a_{S S i}\right)^{2}}{a_{j} a_{S S i}}+\frac{4}{3} G\left(a_{j}+a_{S S i}\right)^{3}+\pi\left(a_{j}+a_{S S i}\right)^{2} \mid v_{S, j}-\right. \\
& \left.v_{S, S S i} \mid\right) 10^{9}
\end{aligned}
$$

where in the term for orthokinetic aggregation $G$ is calculated from the flow rate (calculated by the DUFLOW hydrological model), thus providing a direct link between river morphometry, hydrology and aggregation behaviour (river morphometry $\rightarrow$ flow velocity $\rightarrow$ shear $\rightarrow$ orthokinetic aggregation $\rightarrow$ collision frequency $\rightarrow$ heteroaggregation rate), see Table A2.5, Eq. A2.20.

\section{Surface modification}

The surfaces of pristine plastic particles are supposed to be modified by natural organic matter such as humic acid, fulvic acid or biofilm growth. Particles may be repelled from each other by an electric charge on the particle surface, i.e. electrostatic repulsion. Aggregation can also be limited by a physical barrier formed by large organic molecules, which is referred to as steric hindrance. In NanoDUFLOW these issues are implicitly accounted for by assigning a conditional attachment efficiency $(\alpha)$, which scales between 0 and $1 .^{71}$

Formation of a biofilm on plastic particles was included as an extra scenario. Implementation involved adjustment of total radius and overall density of the plastic particle as a result of the addition of a biofilm layer with a thickness of $0.4 \mu \mathrm{m}$, with a density of $1250 \mathrm{~kg} / \mathrm{m}^{3}$ on the particle. Attachment efficiencies for plastic particles with a biofilm were simultaneously changed by a factor 1$3 .^{70,87}$

Following Praetorius et $a l^{72}$ and De Klein et al. ${ }^{84}$ our scenario calculations used an attachment efficiency for homoaggregation and for heteroaggregation ( $\alpha_{\text {hom }}$ and $\alpha_{\text {het }}$ ) that was constant over the flow distance.

\section{Degradation}

To take into account processes that transform the plastic particles, such as physical weathering, a degradation term is introduced using a first order removal rate, $k_{\text {deg }}$.

$\frac{d n_{j}}{d t}=-k_{d e g} n_{j}$

$k_{\text {deg }} \quad$ Degradation rate constant 


\section{General}

Most of these transformation processes take place in the water phase, though degradation takes place in the sediment as well. Furthermore, heteroaggregation also takes place in the sediment when singular plastic particles or homoaggregates settle to the sediment. Plastic particles that have entered the sediment are assumed not to remain as non-attached singular plastic particle or plastic homoaggregates in the sediment phase. Because of the very high collision frequency between plastic particles and SSs in the sediment, they can be assumed to be converted to heteroaggregates. This is opposing to the water phase, where the collision frequency is more limited.

\section{Transport processes}

\section{Sedimentation}

Sedimentation is the gravitational transport of plastic particles or SSs from the water column to the sediment. Separate sedimentation rates are calculated for each plastic particle size, homoaggregate class, SS class and heteroaggregate class. The sedimentation rate is calculated using Stokes law. ${ }^{397}$ with the assumption that the particles are on average spherical. ${ }^{396}$

$\frac{\mathrm{dn}_{\mathrm{j}}}{\mathrm{dt}}=-\frac{\mathrm{v}_{\mathrm{s}}}{\mathrm{d}} \mathrm{n}_{\mathrm{j}}$

d Sedimentation length [m]

$v_{\mathrm{s}} \quad$ Sedimentation rate $\left[\mathrm{m} \mathrm{s}^{-1}\right]$

The sedimentation rate $\left(v_{\mathrm{s}}\right)$ can be calculated with:

$\mathrm{v}_{\mathrm{s}}=\frac{2 \mathrm{a}_{\mathrm{j}}^{2}\left(\rho_{\mathrm{p}}-\rho_{\mathrm{w}}\right) \mathrm{g}}{9 \mu}$

Sedimentation rates for plastic-SS heteroaggregates are calculated based on the size $\left(a_{\mathrm{j}}\right)$ and density $(\rho)$ of the $S S$ and plastic in the heteroaggregate.

The density of biofouled plastic particles is calculated according to Table A2.5, Eq. A2.19.

\section{Sediment transport}

Lateral transport of sediment is modeled as resuspension - sedimentation and horizontal sediment transport within the water column. ${ }^{398,399}$ 


\section{Burial to deeper sediment layers}

When there is net sedimentation, burial from the mixed sediment top layer is modeled as a first order loss process. ${ }^{102}$ The top sediment layer of $10 \mathrm{~cm}$ is assumed to be available for resuspension. Burial converts this layer to more compact deeper sediment layers, which results in burial of the incorporated plastic-SS heteroaggregates to these deeper sediment layers.

Sediment burial is quantified using first order kinetics:

$\frac{\mathrm{dn}_{\mathrm{j}}}{\mathrm{dt}}=-\mathrm{k}_{\mathrm{bur}} \mathrm{n}_{\mathrm{j}}$

Resuspension

Resuspension is described using a critical shear stress level below which resuspension does not occur according to the equations of Krone and Partheniades, applying for the suspended load transport. ${ }^{398}$ When the critical shear stress $\left(\tau_{\text {crit }}\right)$ is exceeded, a resuspension flux $\left(R_{j}\right)$ is calculated based on the ratio between the actual and the critical shear stress and a resuspension rate constant.

$\mathrm{R}_{\mathrm{j}}=\mathrm{R}_{\mathrm{jmax}}\left(\frac{\tau}{\tau_{\text {crit }}}-1\right)$

Where $\tau=\rho_{\mathrm{w}}\left(\frac{\mathrm{g}^{0.5} \mathrm{v}_{\mathrm{w}}}{\text { Chezy }}\right)^{2}$

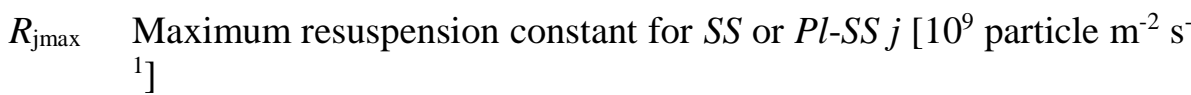
$v_{\mathrm{w}} \quad$ Flow rate of water obtained from DUFLOW $\left[\mathrm{m} \mathrm{s}^{-1}\right]$

Chezy Chezycoefficient $\left[\mathrm{m}^{0.5} \mathrm{~s}^{-1}\right]$

Advection

Advection is implemented using DUFLOW Modeling Studio (v3.8.7) which is a software package for one-dimensional unsteady flow in open-channel systems. $^{212,400}$ Water levels and flow rates are determined by solving the St. Venant equations of continuity and momentum with the Preissmann scheme, using initial and boundary conditions such as an incoming flow at the upstream part of the model and a fixed downstream water level. DUFLOW calculates discharge, water level and mean velocity for each section and for each time step. Chemical transport is modeled by solving the advection-diffusion equation simultaneously with the hydrological equations for all network sections. 


\section{State variables}

The state of $i$ classes of plastic homoaggregates, $j$ classes of $S S$ and all $i \times j$ combinations of $\mathrm{Pl}$-SS heteroaggregates are accounted for using the above mentioned processes. This is done in the water and sediment compartment with $i=5$ and $j=5$.

\section{Rate equations}

This results in the following overall mass balance equation for plastic particles in water:

$$
\begin{aligned}
& \frac{d n_{i}}{d t}=n_{i}\left(-\alpha_{\text {het }} \sum_{1}^{j}\left(K_{i, j} n_{j}\right)-\frac{v_{s, i}}{\mathrm{~d}}-k_{d e g, i}-\frac{1}{2} \alpha_{\text {hom }} K_{i, i} n_{i}\right)+ \\
& \frac{1}{2} \alpha_{\text {hom }} K_{i-1, i-1} n_{i-1} n_{i-1} \frac{\rho_{i-1} a_{i-1}^{3}}{\rho_{i} a_{i}^{3}}
\end{aligned}
$$

The mass balance equation for suspended solids in water reads:

$\frac{d n_{j}}{d t}=n_{j}\left(-\alpha_{\text {het }} \sum_{1}^{i}\left(K_{i, j} n_{i}\right)-\frac{v_{s, j}}{\mathrm{~d}}\right)+\frac{R_{j}}{\mathrm{~d}}$

And for heteroaggregates in water:

$\frac{d n_{i, j}}{d t}=-n_{i, j}\left(\frac{v_{s, i j}}{\mathrm{~d}}+k_{\text {deg, } i}\right)+\alpha_{h e t} K_{i, j} n_{i} n_{j}+\frac{R_{i, j}}{\mathrm{~d}}$

Plastic particles which are removed from the water phase due to direct sedimentation are accounted for, but are assumed to be transformed to heteroaggregates instantaneously upon arrival in the sediment.

$\frac{d n_{s, i}}{d t}=n_{i} v_{s, i}$

The mass balance for the settled (formerly suspended) solids present in the sediment

$\frac{d n_{s, j}}{d t}=n_{j} v_{s, j}-n_{s j} k_{b u r, j}-F_{P l, S S j} \sum_{i}\left(v_{s, i} n_{i}\right)-R_{j}$

Where $F_{P l, S S j}$ is:

$F_{P l, S S j}=\frac{n_{j}}{\sum_{1}^{j} n_{j}}$

The mass balance for heteroaggregates present in sediment is:

$$
\frac{d n_{s, i, j}}{d t}=n_{i, j} v_{s, i, j}+n_{i} v_{s, i} F_{P l, S S j}-n_{s, i, j}\left(k_{d e g, P l j}+k_{b u r, P l j}\right)-R_{i, j}
$$




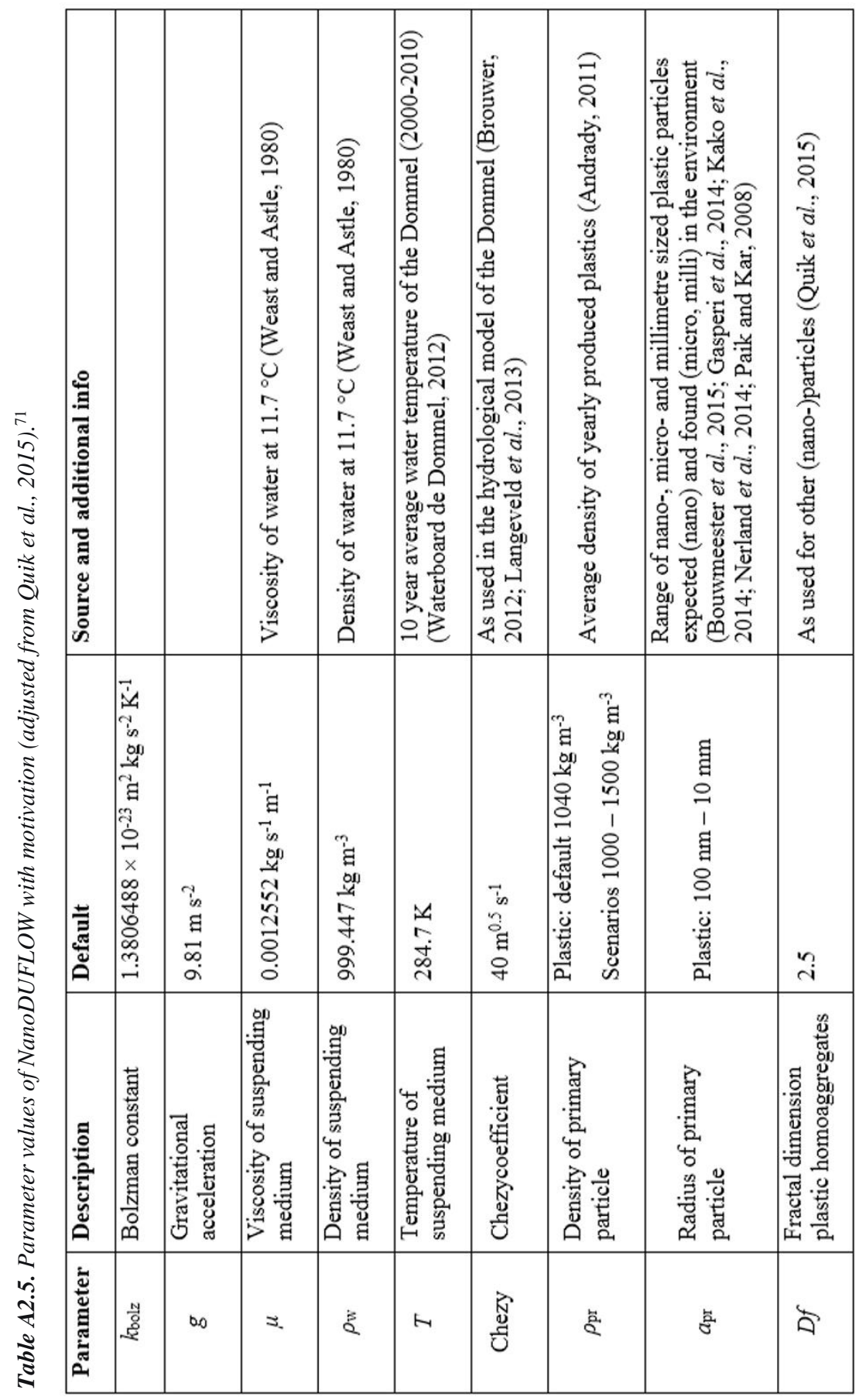


Appendix Chapter 2

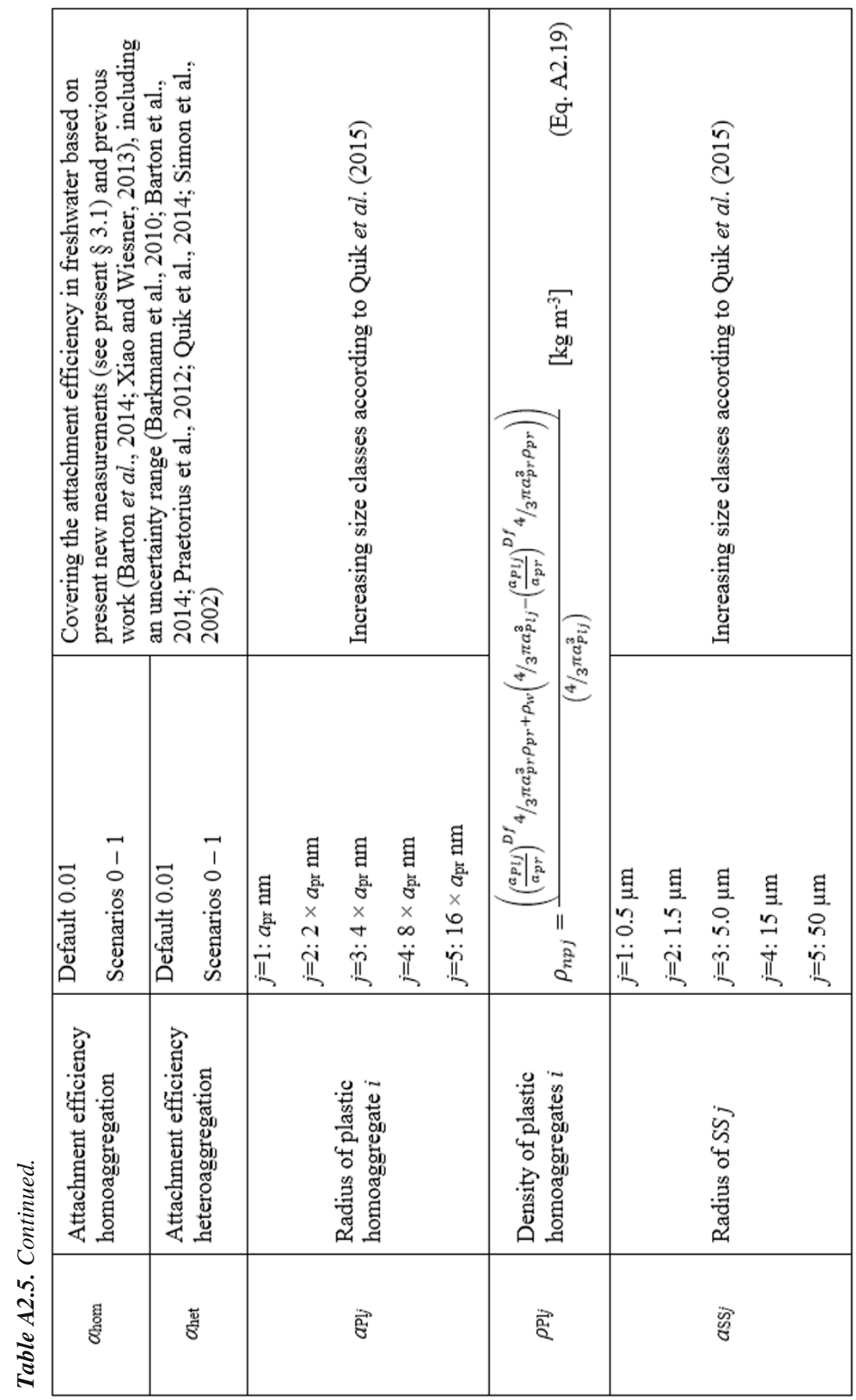


NanoDUFLOW model description

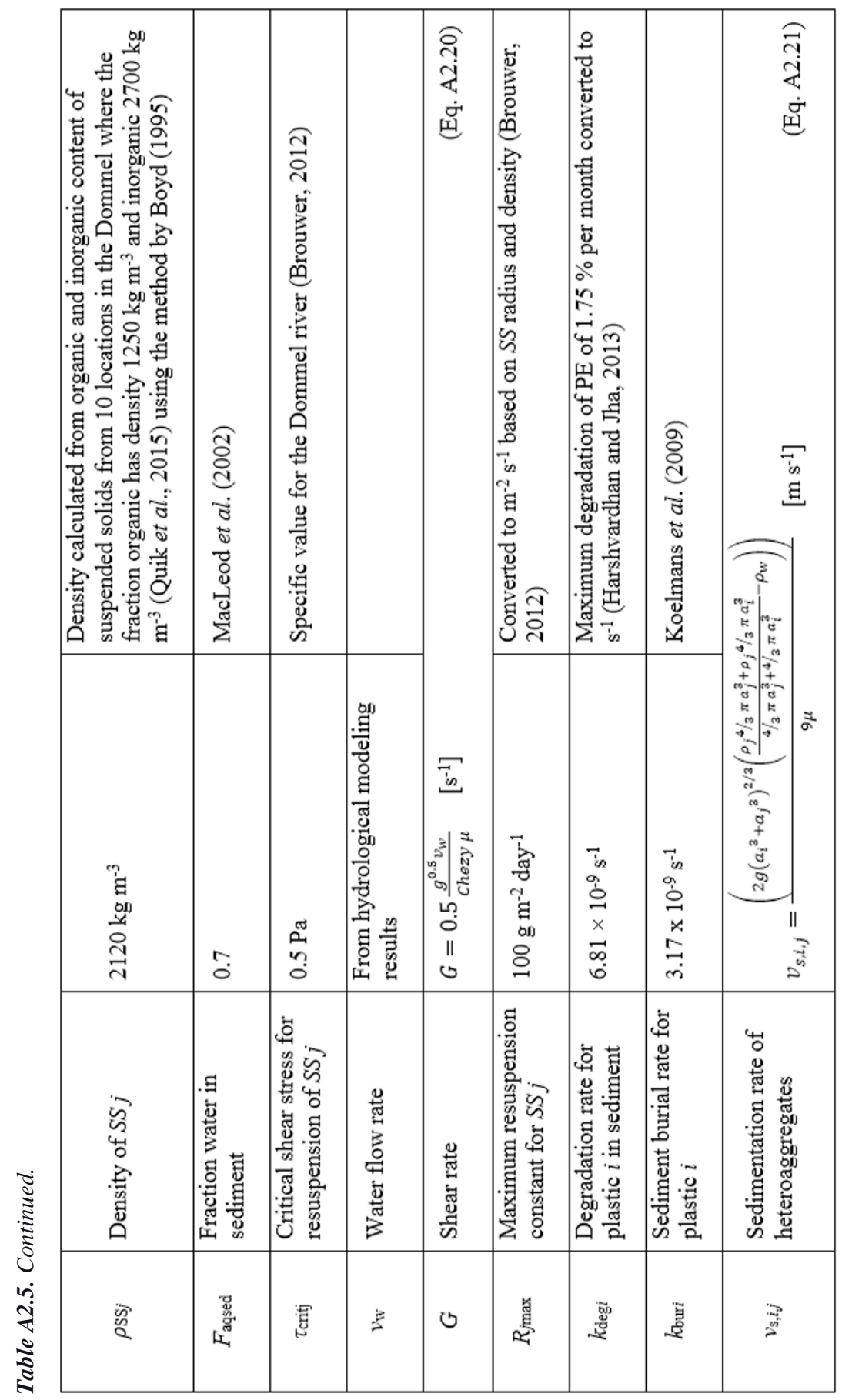




\section{Calculation of the attachment efficiency ( $\left.\alpha_{\text {het }}\right)$ between nanoplastic, microplastic and clays from experimental data}

\section{Summary of aggregation-sedimentation experiment}

Heteroaggregation was studied in three experiments for two plastic particle sizes and two clay types representing suspended solids. The aggregationsedimentation experiments of plastic with suspended solids were performed in 0.9L glass columns (diameter $5 \mathrm{~cm}$, height $43 \mathrm{~cm}$ ) with lake water (Wageningen, Droevendaalsesteeg) filtered through $0.7 \mu \mathrm{m}$ (Whatman, GF/F). The suspended solids kaolin clay (Fluka 60609) or bentonite clay (Sigma 285234) were dispersed at a concentration of $5 \mathrm{mg} / \mathrm{L}$, which is a representative value for rivers with low discharge. Either $70 \mathrm{~nm}$ or $1050 \mathrm{~nm}$ polystyrene (Chapter 3) ( $^{49,75}$ was mixed with the lake water at a concentration of $50 \mathrm{mg} / \mathrm{L}$. Supernatants were sampled after settling times of 20 and 40 minutes, 1, 4 and 6 hours and 1 - 3 days, and measured by Dynamic Light Scattering (DLS) and by UV-VIS spectrometry. Values for $\alpha_{\text {het }}$ were calculated following the method of Barton et al. ${ }^{74}$ using the data obtained by both detection methods.

\section{Calculation of attachment efficiency}

DLS (number concentrations) and spectrophotometry (absorption ) data were transferred to particle concentrations in the supernatants of the sedimentation columns with calibration lines $\left(R^{2}>0.93 ; R^{2}>0.98\right)$. From these the removal from the water phase relative to the initial concentrations was calculated. The distribution coefficient $y$ after settling time $t$ was calculated by:

$y_{t}=r_{t} \frac{c_{0}}{c_{t} \times C_{B}}$

with $c_{0}$ and $c_{\mathrm{t}}$ the plastic particle concentrations at the start of the experiment and after the settling time respectively, $C_{B}$ the mass concentration of background particles (clay) and $r_{\mathrm{t}}$ the removal from the water column. ${ }^{74}$

$$
r_{t}=\frac{c_{0}-c_{t}}{c_{0}}
$$

Barton subsequently uses the following relation between the distribution coefficient over time and attachment efficiency: 


$$
y_{\mathrm{t}}=\frac{1}{c_{\mathrm{B}}}\left(\frac{1}{\left(e^{-\left(\alpha K_{j, S S j} B+k_{\mathrm{B}}\right) t}+\frac{k_{\mathrm{B}}}{\left(\alpha K_{j, S S j} B+k_{\mathrm{B}}\right)}\left(1-e^{-\left(\alpha K_{j, S S j} B+k_{\mathrm{B}}\right) t}\right)\right)}-1\right)
$$

with $K_{j, S S j}$ the collision rate constant, $B$ the particle number concentration of background particles and $k_{\mathrm{B}}$ the breakup constant. ${ }^{74}$ To relate distribution coefficients to attachment efficiency during early time periods, Barton assumes a negligible role of breakup of heteroaggregates, resulting in a simplified equation:

$$
\ln \left(y_{t} C_{B}+1\right)=\alpha K_{j, S S j} B t
$$

In our experiment increased removal from the water phase was observed until a settling time of 1 hour, after which break up of heteroaggregates might have started to play a growing role. We therefore used the particle concentrations in the supernatant after 40 minutes settling time and the simplified relation between $y_{\mathrm{t}}$ and the attachment efficiency $\alpha$ (Eq. A2.25) to determine the experimental alpha values given in table A2.6. $K_{j, S S j}$, was calculated by Eq. A2.5 for water at room temperature $\left(20^{\circ} \mathrm{C}\right)$ with a flow rate according to Table A2.1 and theoretical sedimentation rates by Eq. A2.8. 


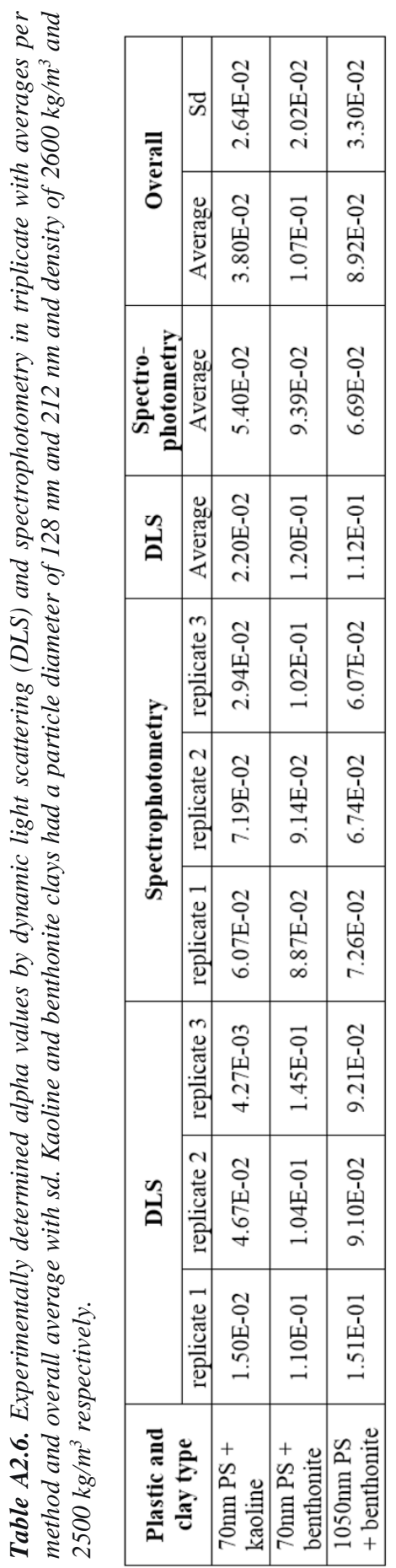




\section{Appendix to Chapter 3}

1. Details on the nano-PS concentrations in the bioassays

2. Pilot bioassay to assess sodium dodecyl sulphate (SDS) toxicity thresholds for Scenedesmus obliquus

3. Nile Red availability in the bioassays

4. Figure A3.1. TEM image of nano-PS 


\section{Details on the nano-PS concentrations in the bioassays}

\section{Scenedesmus obliquus bioassay}

Scenedesmus obliquus were exposed to $44 \times 10-1100 \mathrm{mg} / \mathrm{L}$ nano-PS, specifically being $4.4 \times 10^{1}, 9.9 \times 10^{1}, 2.2 \times 10^{2}, 4.9 \times 10^{2}$ and $1.1 \times 10^{3} \mathrm{mg}$ nanoPS/L.

\section{Daphnia magna bioassay}

Pristine exposures were applied at ten microplastic concentrations in the range of $0.22-150 \mathrm{mg}$ nano-PS/L, specifically being $2.2 \times 10^{-1}, 4.4 \times 10^{-1}, 8.8 \times 10^{-1}$, $1.8,3.5,7.0,1.4 \times 10^{1}, 3.2 \times 10^{1}, 7.0 \times 10^{1}, 1.5 \times 10^{2} \mathrm{mg}$ nano-PS/L. Pristinekairomone exposures were at $8.8 \times 10^{-1}$ and $1.8 \mathrm{mg}$ nano-PS/L. The aged treatment contained $3.2 \times 10^{1}$ nano-PS/L and the aged-filtered treatment was pre-treated with $3.2 \times 10^{1}$ nano-PS/L, after which it was filtered as stated in the main chapter.

\section{Pilot bioassay to assess sodium dodecyl sulphate (SDS) toxicity thresholds for Scenedesmus obliquus}

\section{Methods}

The test algae Scenedesmus obliquus SAG276/3a was obtained from the culture collection of SAG (Göttingen, Germany). It was grown in a $1 \mathrm{~L}$ chemostat on a modified WC medium in a temperature-controlled chamber at $20^{\circ} \mathrm{C}$ under continuous light $\left(100 \mu \mathrm{mol} / \mathrm{m}^{2} / \mathrm{s}\right)$. Algae were collected from the chemostat and used in several assays run in $100 \mathrm{ml}$ Erlenmeyer flasks containing $50 \mathrm{ml}$ of medium. Flasks were incubated in a climate controlled chamber at $20{ }^{\circ} \mathrm{C}$ under continuous cool-fluorescent light at $175 \mu \mathrm{mol} / \mathrm{m}^{2} / \mathrm{s}$ for $48 \mathrm{~h}$ on a rotating shaking table $(80 \mathrm{rpm})$. Algae were exposed to sodium dodecyl sulphate (SDS, Merck, Amsterdam, The Netherlands, 113760) at a $\log$ increase concentration range of $0.001-10 \mathrm{mg} / \mathrm{L}$. A control without SDS was included and all treatments were performed in triplicate. Test dispersions were diluted 200×, after which cell densities and size distributions were determined by Coulter Multisizer II (Coulter Electronics, Luton, UK, capillary $100 \mu \mathrm{m}$ orifice width). Growth rates $(\mu)$ of $S$. obliquus were predicted from the increase in biovolume $(\mathrm{V})$ with the formula $\mu=\left(\ln \left(\mathrm{V}_{\text {end }}\right)-\ln \left(\mathrm{V}_{\text {start }}\right) / d t\right.$, with $t$ being the time in hours.

\section{Results}

Growth rates were within a range of 1.3-1.5 day $^{-1}$, and did not significantly differ among treatments (1-way ANOVA, $p$-value $=0.087$ ). This implies SDS did not cause effects at least up to a concentration of $10 \mathrm{mg} / \mathrm{L}$, which is 200 
times higher than the maximum concentrations that occurred in the assays reported in the main chapter.

\section{Nile Red availability in the bioassays}

Fluorescent dyes have been used before in living systems, without effects reported. ${ }^{11,117,120,140,141,388,401,402}$ In the nano-PS used in this bioassay, the fluorescent hydrophobic dye Nile Red was included in the polymer matrix for visualisation purposes. The nano-PS contained $0.01 \%$ on mass basis of Nile Red. Due to its hydrophobicity, Nile Red becomes practically completely incorporated in the polymer matrix during synthesis of the nano-PS. During the bioassay, leaching of Nile Red from the nano-PS is negligible. As the glass transition temperature of PS is $\sim 100{ }^{\circ} \mathrm{C},{ }^{144}$ PS is a glassy polymer with extremely low intrapolymer diffusivities at the temperatures in the bioassay of respectively $20^{\circ} \mathrm{C}$ and $21^{\circ} \mathrm{C}$. However, as Wu et al. ${ }^{142}$ report an effect of Nile Red on the endpoint chlorophyll fluorescence of the alga Botryococcus braunii at a total concentration of $1 \mathrm{mg} / \mathrm{L}$, we calculated the maximum exposure to Nile Red in our bioassays. Even if all Nile Red would have been released from the nano-PS, the maximum total concentrations in respectively our algae and Daphnia bioassays would have been a factor $9-64$ below the concentration reported by $\mathrm{Wu}$ et al. The previous calculation is worst case, assuming all Nile Red is in the water, which is not realistic. A more realistic maximum Nile Red exposure concentration for our bioassays can be estimated assuming release of Nile Red from the nano-PS only until thermodynamic partitioning equilibrium between nano-PS, water and algae has been established (equilibrium partitioning theory).

\section{Combination of:}

- the mass balance equation: $C_{W}=M_{t o t} /\left(V_{W}+k_{\text {nano-Ps }} \times M_{\text {nano-PS }}+\right.$ $\mathrm{k}_{\mathrm{algae}} \times \mathrm{M}_{\text {algae }}$, Chapter 5), ${ }^{128}$ in which $\mathrm{C}_{\mathrm{W}}$ is the aqueous Nile Red concentration in $\mathrm{mg} / \mathrm{L}, \mathrm{M}_{\text {tot }}$ the total Nile Red mass in $\mathrm{mg}, \mathrm{V}_{\mathrm{W}}$ the water volume in the bioassay in $\mathrm{L}, \mathrm{M}_{\text {nano-PS }}$ and $\mathrm{M}_{\text {algae }}$ the masses of respectively nano-PS and algae in the bioassay in $\mathrm{kg}$ and $\mathrm{k}_{\text {nano-PS }}$ and $\mathrm{k}_{\text {algae }}$ the partitioning coefficients of Nile Red to respectively nano-PS and algae in $\mathrm{L} / \mathrm{kg}$;

- the partitioning relationships and coefficients $\log \mathrm{K}_{\mathrm{OW}}=5,{ }^{141,403} \mathrm{k}_{\text {nano-PS }}=$ 6.5 derived from the relationship between $\mathrm{K}_{\mathrm{Ow}}$ and $\mathrm{K}_{\mathrm{d}}$ of the nano-PS that was used in our bioassay ${ }^{404}$ and $\mathrm{k}_{\mathrm{alg}} \approx \mathrm{K}_{\mathrm{Ow}} * \mathrm{f}_{\text {lip }}$, with $\mathrm{f}_{\text {lip }}$ being the lipid fraction of the algae of $0.075 ; ;^{405}$

- Nile Red, nano-PS and algal masses at the effect thresholds in the bioassays, 
yields an aqueous equilibrium concentration of $3.1 \times 10^{-5}$ and $3.2 \times 10^{-5} \mathrm{mg} / \mathrm{L}$ in the Daphnia and the algae bioassay, respectively. These concentrations are a factor $1.5 \times 10^{4}$ below the toxic aqueous concentration of $0.47 \mathrm{mg} / \mathrm{L}$, which we calculated from the total concentration reported by $\mathrm{Wu}$ et al. ${ }^{142}$ based on partitioning between water and algae. Even taking uncertainty into account, by calculating the aqueous concentration with an initial total amount of Nile Red a hundred times as high as in our bioassay, the aqueous concentration would still be below $3.2 \times 10^{-5} \mathrm{mg} / \mathrm{L}$ due to the high hydrophobicity of Nile Red. We therefore argue that the observed toxicity in our bioassay cannot be explained by the Nile Red concentration. Furthermore, as mentioned in the main manuscript, a radical increase in malformation occurrence was observed in the aged treatment, in contrast to the pristine treatment, which implies that these malformations were not due to any initially present cocontaminant like styrene, SDS or Nile Red.

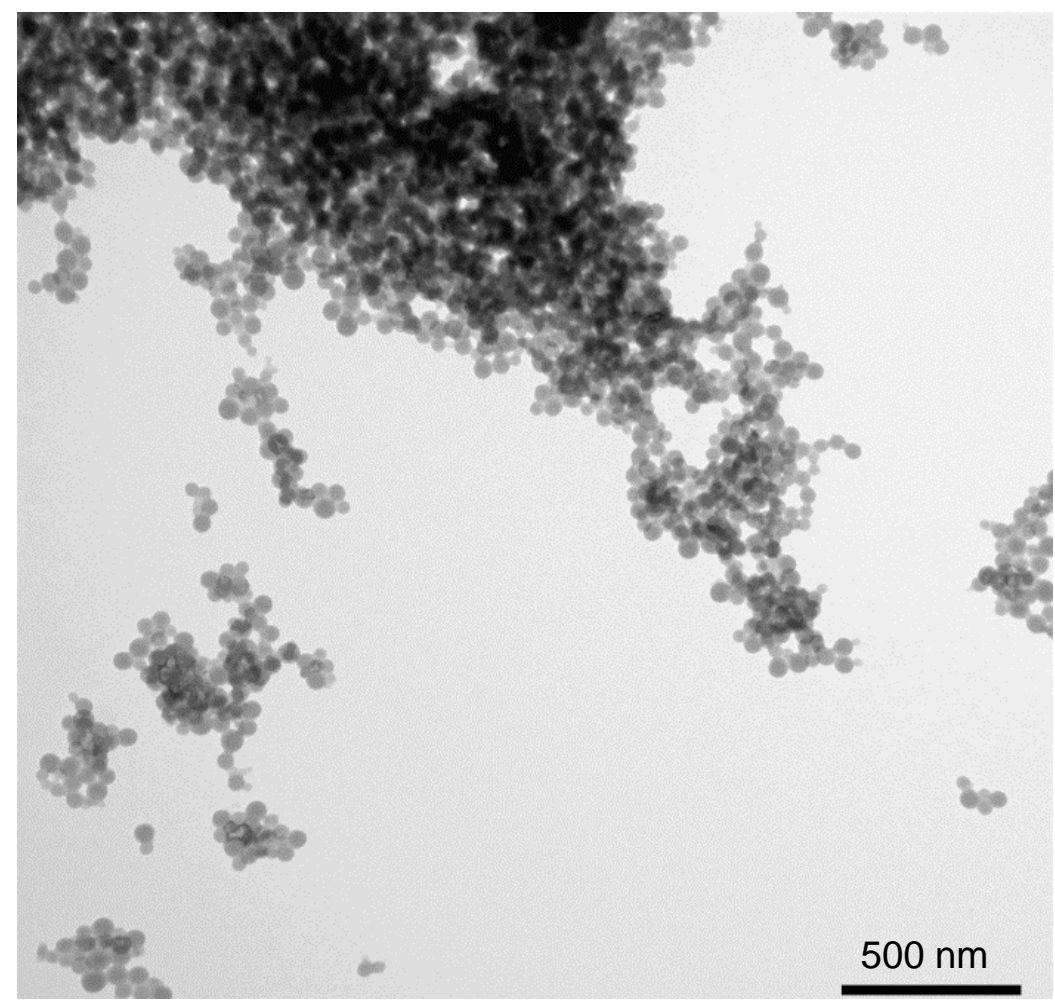

Figure A3.1. The TEM image from Velzeboer et al. 2014 confirms the nominal size of $\sim 70 \mathrm{~nm}$ of the primary nano-PS in freshwater. ${ }^{404}$ 


\section{Appendix to Chapter 4}

1. Pilot experiment

2. Tables A4.1-A4.7

3. Figures A4.1-A4.7 


\section{Pilot experiment: Methods, results and discussion}

In this work two experiments were performed, a pilot experiment and the main bioassay. The pilot experiment followed previously published procedures and is addressed here. The bioassay is described in the main chapter. The overall discussions in the main chapter cover the outcomes of both experiments.

\section{Materials and Methods}

Prior to the pilot experiment, the test organisms cleared their guts in clean sea water overnight and were randomly assigned to the test beakers, such that each beaker contained a group of 5 Arenicola marina individuals with a known weight. The average group weight was $26.5 \mathrm{~g}$ and the variation (SD) among groups was 5.2 g. For the pilot experiment, we used closed $2 \mathrm{~L}$ glass test beakers with a diameter of $19 \mathrm{~cm}$ and a height of $9 \mathrm{~cm}$. Due to the use of small test beakers, the water characteristics were variable over time. Additional to the plastic effect, the impact of these water quality variables on survival, activity and weight could be established. The test beakers contained $\pm 2.2 \mathrm{~kg}$ sediment (WW, $5 \mathrm{~cm}$ thick layer) and $\pm 0.7 \mathrm{~L}$ sea water (water layer of $2.5 \mathrm{~cm}$ ). The lower two-third of the sediment did not contain plastic. The upper onethird of the sediment $(1.7 \mathrm{~cm}, 0.72 \mathrm{~kg})$ contained the polystyrene microplastic (PS), because the lugworm feeds on the upper sediment layer. ${ }^{189}$ Effects of PS were assessed by exposing $A$. marina to a range of PS concentrations: 0, 1, 3, 10,30 and $100 \mathrm{~g}$ PS/L in PCB contaminated sediment. These concentrations agree to $0,0.074,0.22,0.74,2.2$ and $7.4 \%$ DW PS in the sediment. Mixing of the sediment occurred during four weeks prior to the pilot experiment. The systems stabilised during one day, before addition of the lugworms. All treatments were performed in quadruplicate and randomly assigned to the test beakers. We applied aeration and refreshed the water twice a week. The dissolved oxygen saturation, temperature, $\mathrm{pH}$, salinity, $\mathrm{NH}_{4}{ }^{+}$and $\mathrm{NO}_{2}{ }^{-}$ averaged $7.9 \mathrm{mg} / \mathrm{L}$ (79 \% saturation), $13.4{ }^{\circ} \mathrm{C}, 8.0,31 \%$, $6.4 \mathrm{mg} / \mathrm{L}$ and 0.07 $\mathrm{mg} / \mathrm{L}$ respectively. Analysis of the water quality variables and the endpoints were done as in the bioassay, described in the main chapter.

\section{Results and Discussion}

Effects of water quality variables. The use of small test systems showed the sensitivity of the organisms to variable water conditions. There were significant effects of the water quality variables on the endpoints (Fig. A4.2). We found a positive relation between the average amount of days that an organism survived in the experiment and average oxygen concentration 
(Regression, $\mathrm{p}=0.002$ ). Furthermore, a positive relation between average activity and average oxygen concentration $(\mathrm{p}=0.012)$ was detected. Our findings of a significant negative effect of low oxygen concentrations on the activity are in accordance with Cadée ${ }^{180}$ who mentions that feeding might stop at low oxygen levels in the overlying water. High mortality might have been an indirect effect, initiated by starvation as a result of the negative effect of oxygen deficiency on the feeding activity of A. marina. This would imply that the duration of the deficiency was crucial, which is in agreement with our observations that high mortality started after one and a half week of exposure. However, literature shows that A. marina is assumed to be tolerant to oxygen deficiency. ${ }^{406}$ In this pilot experiment, the lowest measured concentrations were on the first day $2.5 \mathrm{mg} / \mathrm{L}$ and remained for the rest of the experiment above $5.5 \mathrm{mg} / \mathrm{L}$, while A. marina can tolerate oxygen concentrations as low as 3.2 - $4.1 \mathrm{mg} / \mathrm{L}$ that occur during ebb. ${ }^{406}$ Additionally, A. marina survived concentrations as low as tenths or even hundredths of $\mathrm{mg} / \mathrm{L}$ in a laboratory experiment and calculations imply that A. marina can survive 71 minutes without external oxygen supply. ${ }^{406}$ For wet, dry and AFD weight (WW, DW, AFDW) loss, a significant negative relation with salinity was discovered $(\mathrm{p}=0.031, \mathrm{p}=0.050, \mathrm{p}=0.002)$, which agrees to previous reports. ${ }^{407}$ For AFDW loss, a significant positive relation with $\mathrm{pH}$ was determined $(\mathrm{p}=0.031)$. As far as we know, this has not been quantified before and might only count for the observed limited $\mathrm{pH}$ range (Appendix Table A4.1). We found no relation between the endpoints and temperature, $\mathrm{NH}_{4}{ }^{+}$and $\mathrm{NO}_{2}{ }^{-}$concentration. Furthermore, there was no significant spatial pattern in water quality variables and endpoints. By using the significantly influential water quality variables as covariables, the relation between the investigated endpoints and the treatment did not change. Nevertheless, we conclude that a set up in which water quality variables can be maintained constant is required in order to detect effects of PS. This was implemented in the bioassay described in the main text.

Effects of microplastic on fitness and performance of A. marina. Survival. The total mortality was $48.3 \%$ in the pilot experiment, which is much higher than the average annual mortality of $22 \%$ observed in the Dutch Wadden Sea. ${ }^{194}$ Irrespective of the plastic concentration (ANOVA, p=0.457) (Fig. A4.3A), survival was low. Also, no relation between the treatments and the amount of days that an organism survived was revealed $(\mathrm{p}=0.460)$. The mortality rate did not significantly differ between the treatments (ANOVA, $\mathrm{p}=0.561$ ) (Fig. $\mathrm{A} 4.6 \mathrm{~A})$, with $\mathrm{K}_{\text {mort }}=-\ln (\mathrm{B} / \mathrm{B} 0) / t$ with $\mathrm{B}=$ survival $\mathrm{n}$ at time $t, \mathrm{~B} 0=$ survival $\mathrm{n}$ at start of the experiment and $t$ is time in days. ${ }^{408}$ It was ascertained that the 
contamination with PCBs did not result in PCB toxicity or plastic avoidance behaviour by A. marina, because of the use of low PCB concentrations (Appendix Table 4.2). The measured PCB concentrations were 350 times lower than toxicity thresholds. ${ }^{183}$

Ingestion of plastic. The organisms that survived the entire 28 days exposure period and were allowed to clear their guts had no plastic in their system, even those being exposed to the highest plastic concentrations. While in some of the organisms that died during the experiment, plastic was encountered after dissection. The difference in the amount of internal plastic particles between organisms that did or did not survive the exposure period was significant (Mann-Whitney $\mathrm{U}$ test, $\mathrm{p}=3.21 \times 10^{-10}$ ). This supports the supposition that $A$. marina ingested PS particles of $\geq 400 \mu \mathrm{m}$ but that these particles did not accumulate in this organism.

Gut content. The material that was egested during gut clearance overnight contained plastic particles. Here, no differentiation is made between internally detected plastic (in worms that died during the experiment) and plastic egested during gut clearance (by worms that survived the experiment), i.e. both are called gut content. By doing so, a negative relation between the amount of plastic particles in the gut content and survival was identified (Mann-Whitney $\mathrm{U}$ test, $\mathrm{p}=9.34 \times 10^{-7}$ ) (Fig. A4.4B). Linear regression showed that the amount of plastic particles in the gut content increased with the plastic concentration to which A. marina was exposed (1-sided P-value $=0.023$ ). Because of the nonnormality of the data, we further investigated this relation with the KruskalWallis test, which gave significant differences between treatments $\left(p=2.48 \times 10^{-4}\right)$. As a post hoc test, pair-wise comparisons of the treatments were done with the Mann-Whitney $\mathrm{U}$ test, which revealed three significantly different treatment classes ( 1 -sided $\mathrm{p}=0.004, \mathrm{p}=3.94 \times 10^{-5}, \mathrm{p}=0.025$ respectively). Fig. A4.4A shows that the amount of plastic particles in the gut content increased significantly over the following grouped treatments: low (treatment 0 and $0.074 \%$ ), middle (treatment 0.22 and $0.74 \%$ ), high (treatment 2.2 and $7.4 \%$ ). The findings imply a positive relation between environmental plastic concentration and ingestion of plastic. To see if the amount of plastic particles in the gut content was proportional to the exposure plastic concentrations, the gut volume was calculated. The average faeces production of $2.4 \mathrm{ml} /$ day during the winter and a defecation time of 20 minutes from Cadée ${ }^{180}$ were used to calculate a gut volume of $3.33 \times 10^{-5} \mathrm{~L}$. The weight of our used plastic particles ranged from $3.5 \times 10^{-8}-1.2 \times 10^{-6} \mathrm{~g}$ (radius of 0.2 $0.65 \mathrm{~mm}$, density of $\left.1.05 \mathrm{~g} / \mathrm{L}^{(8)}\right)$. By using these numbers, the calculated gut 
concentrations ranged in the $0.22 \%$ treatment up to $3.9 \times 10^{-4}-1.3 \times 10^{-2} \%$ (1 - 3 orders of magnitude lower than the exposure concentration), in the $0.74 \%$ treatment up to $7.8 \times 10^{-5}-2.7 \times 10^{-3} \%$ ( $2-4$ orders of magnitude lower), in the 2.2 and $7.4 \%$ treatments up to $7.8 \times 10^{-4}-2.7 \times 10^{-2} \%$ ( 2 - 4 orders of magnitude lower).

Activity. The activity averaged 0.43 heap/individual/day (SD 0.41). Average activity did not significantly differ between the treatments (ANOVA, $\mathrm{p}=0.708$ ) (Fig. A4.1B), also not by considering the activity in the first one, two or three weeks only. This was investigated because it could be speculated that in a later stage of the experiment, treatment effects become overwhelmed by captivity disadvantages, but this was not the case. The activity in the control treatment peaked after two weeks exposure, but was not significantly higher than the activity in the other treatments (Fig. A4.3A). The lack of fit between treatment and activity in the pilot experiment might be explained by the impact of the water quality variables. The oxygen concentration had a significant impact on the activity. To compare the two experiments, we included the activity between the second and ninth day only and excluded the treatments that were not executed in both the pilot experiment and the bioassay $(0.22 \%$ and $2.2 \%$ from the pilot experiment and treatment Oesterput from the bioassay). The calculated activity was 0.27 heap/individual/day in the pilot experiment and 0.42 heap/individual/day in the bioassay, which is a significant difference (Two samples t-test, $\mathrm{p}=2.24 \times 10^{-4}$ ) (Appendix Fig. A4.5A). This is interpreted as worms having a better condition in the bioassay.

Weight loss. Weight loss was observed in all but one group. The mean WW loss was $1.33 \mathrm{~g} /$ individual (25.1\%, SD 0.98), the mean DW loss was 0.31 g/individual (36.1 \%, SD 0.12) and the mean AFDW loss was 0.25 g/individual (36.5\%, SD 0.098). There was no significant relation between plastic concentration and absolute WW/DW/AFDW loss (Regression, $\mathrm{p}=0.810, \mathrm{p}=0.823, \mathrm{p}=0.265$ respectively) (Fig. A4.3C), also not by taking the relative instead of the absolute loss. Some of the worms emerged from the sediment several days before the end of the experiment. Because this was assumed to be a sign of a weak condition, we investigated whether there was a relation between weight loss and the position in the test beaker (in or above the sediment), which was not the case (Two samples $t$ test, $p=0.541, p=0.518$, $\mathrm{p}=0.693$ ) (Fig. A4.6B). The results did not change when worms in and above the sediment were analysed separately. Similar to the activity analysis, an effect of plastic on weight loss could be invisible in this pilot experiment because it might not have been the main stressor in this experiment. It could 
be that the weight losses were highly impacted by the variability of the salinity, which is in accordance with the findings of Spaargaren and Weber, ${ }^{407}$ and the $\mathrm{pH}$. Mortality of heavy (adult) worms might, next to individual weight loss, explain the observed mean weight loss in the pilot experiment, but the latter one is expected to play a major role. ${ }^{192}$ To compare the two experiments, the treatments 0.22 and $2.2 \%$ from the pilot experiment and treatment Oesterput from the bioassay were excluded from analysis. The DW and AFDW loss in the bioassay turned out to be significantly lower than in the pilot experiment (Two samples $t$ test, $\mathrm{p}=3.74 \times 10^{-6}, \mathrm{p}=8.24 \times 10^{-9}$ ) (Appendix Fig. A4.7B). The WW losses did not significantly differ between the two bioassays $(p=0.267)$. The organisms in the bioassay did not preliminary clear their guts, while the organisms in the pilot experiment did. As a result, the start weight in the bioassay was overestimated and the difference in weight loss compared to the pilot experiment even larger than noted.

Table A4.1. Water quality variables in the pilot experiment and the bioassay.

\begin{tabular}{|l|c|c|c|c|}
\hline \multirow{2}{*}{$\begin{array}{l}\text { Water quality } \\
\text { variables }\end{array}$} & \multicolumn{2}{|c|}{ Pilot experiment } & \multicolumn{2}{c|}{ Bioassay } \\
\cline { 2 - 5 } & Mean & Range & Mean & Range \\
\hline Oxygen $(\mathrm{mg} / \mathrm{L})$ & 7.87 & $2.50-10.51$ & 10.07 & $9.66-11.15$ \\
\hline Oxygen $(\%)$ & 78.9 & $29.5-98.7$ & 94.2 & $91.7-103.2$ \\
\hline Temperature $\left({ }^{\circ} \mathrm{C}\right)$ & 13.4 & $11.2-15.1$ & 12.3 & $11.2-13.5$ \\
\hline $\mathrm{pH}$ & 8.03 & $7.61-8.30$ & 8.16 & $8.07-8.25$ \\
\hline Salinity (\%) & 30.9 & $23.4-33.3$ & 32.1 & $31.7-33.0$ \\
\hline $\mathrm{NH}_{4}^{+}(\mathrm{mg} / \mathrm{L})$ & 6.4 & $2-10$ & 0.2 & $0-1$ \\
\hline $\mathrm{NO}_{2}{ }^{-}(\mathrm{mg} / \mathrm{L})$ & 0.07 & $0.0-0.6$ & 0.03 & $0.0-0.2$ \\
\hline
\end{tabular}

Table A4.2. Concentrations of most abundant PCBs and sum of all PCBs ( $\triangle P C B s)$ in the contaminated sediment $(\mu \mathrm{g} / \mathrm{kg} D W)$ (mixture of Diemen and Oesterput sediment).

\begin{tabular}{|c|c|c|c|}
\hline $\begin{array}{l}\text { PCB } \\
\text { congener }\end{array}$ & $\begin{array}{c}\text { Concentration } \\
(\mu \mathrm{g} / \mathrm{kg} \mathrm{DW})\end{array}$ & $\begin{array}{l}\text { PCB } \\
\text { congener }\end{array}$ & $\begin{array}{c}\text { Concentration } \\
(\mu \mathrm{g} / \mathrm{kg} D W)\end{array}$ \\
\hline PCB 28 & 0.11 & PCB 149 & 0.76 \\
\hline PCB 52 & 0.25 & PCB 153 & 0.93 \\
\hline PCB 101 & 0.62 & PCB 170 & 0.28 \\
\hline PCB 118 & 0.33 & PCB 180 & 0.56 \\
\hline PCB 138 & 0.75 & $\Sigma$ PCBs & 5.28 \\
\hline
\end{tabular}


Pilot experiment

Table A4.3. Statistics PCB analysis. ${ }^{a}$ )

\begin{tabular}{|c|c|c|c|c|}
\hline $\begin{array}{c}\text { PCB } \\
\text { congener }\end{array}$ & $\log K_{O W}{ }^{b)}$ & $\begin{array}{l}\text { ANOVA }^{c)} \\
\text { p-value }\end{array}$ & $\#$ values $>$ dl $^{\mathrm{d})}$ & $\begin{array}{c}\text { Treatment } \\
\text { with all } \\
\left.\text { values }<\mathrm{dl}^{\mathrm{e}}\right)\end{array}$ \\
\hline$\overline{\text { PCB } 18}$ & 5.43 & $7.095 \times 10^{-7}$ & 23 & - \\
\hline PCB 20 & 5.58 & $1.287 \times 10^{-5}$ & 21 & Oesterput \\
\hline PCB 28 & 5.58 & $8.133 \times 10^{-8}$ & 25 & - \\
\hline PCB 29 & 5.58 & 0.236 & 20 & $0 \%$ \\
\hline PCB 31 & 5.58 & $8.663 \times 10^{-8}$ & 27 & - \\
\hline PCB 44 & 6.02 & $1.456 \times 10^{-8}$ & 27 & - \\
\hline PCB 52 & 6.02 & $1.504 \times 10^{-8}$ & 27 & - \\
\hline PCB 101 & 6.42 & $6.097 \times 10^{-8}$ & 27 & - \\
\hline PCB 105 & 6.51 & $4.840 \times 10^{-9}$ & 27 & - \\
\hline PCB 118 & 6.51 & $5.064 \times 10^{-8}$ & 27 & - \\
\hline PCB 138 & 6.82 & $7.485 \times 10^{-7}$ & 27 & - \\
\hline PCB 149 & 6.66 & $3.096 \times 10^{-8}$ & 27 & - \\
\hline PCB 153 & 6.82 & $6.324 \times 10^{-7}$ & 27 & - \\
\hline PCB 155 & 6.50 & $3.818 \times 10^{-5}$ & 24 & - \\
\hline PCB 170 & 7.21 & $3.326 \times 10^{-7}$ & 27 & - \\
\hline PCB 180 & 7.21 & $7.120 \times 10^{-7}$ & 27 & - \\
\hline PCB 194 & 7.61 & 0.540 & 8 & $\begin{array}{c}\text { Start, } \\
\text { Oesterput, } \\
0 \%\end{array}$ \\
\hline PCB 204 & 7.39 & 0.583 & 12 & $\begin{array}{l}\text { Sediment, } \\
\text { Start, } \\
\text { Oesterput }\end{array}$ \\
\hline PCB 209 & 8.27 & 0.001 & 12 & $\begin{array}{c}\text { Start, } \\
\text { Oesterput, } \\
0 \%\end{array}$ \\
\hline$\Sigma$ PCBs & & $5.112 \times 10^{-8}$ & 27 & - \\
\hline
\end{tabular}

a) Analysis of differences between treatments.

b) From Van Noort et al. ${ }^{232}$

.c) Appearance of differences between treatments, investigated with ANOVA.

d) Total amount of values: 27.

${ }^{e)}$ In some cases, the outcomes of all quadruplicates within a treatment were below the detection limit. 
Table A4.4. SumPCB ( $\triangle P C B)$ concentrations in the various treatments of the bioassay.

\begin{tabular}{|l|c|c|c|}
\hline & $\begin{array}{l}\text { Mean } \\
(\mu \mathrm{g} / \mathrm{kg})\end{array}$ & SD & \%SD \\
\hline Sediment & 1.84 & 0.22 & 11.7 \\
\hline Non-exp. lugworms & 2.43 & 0.22 & 9.3 \\
\hline Treatment C & 2.40 & 0.61 & 25.5 \\
\hline Treatment 0 \% & 7.00 & 1.35 & 19.2 \\
\hline Treatment 0.074 \% & 9.01 & 1.76 & 19.5 \\
\hline Treatment 0.74 \% & 8.54 & 1.48 & 17.4 \\
\hline Treatment 7.4 \% & 8.31 & 2.17 & 26.1 \\
\hline
\end{tabular}

Table A4.5. BSAFs. ${ }^{a)}$

\begin{tabular}{|l|c|c|c|c|}
\hline PS Treatment & $\mathbf{0} \%$ & $\mathbf{0 . 0 7 4} \%$ & $\mathbf{0 . 7 4} \%$ & $\mathbf{7 . 4} \%$ \\
\hline PCB congener: & & & & \\
\hline PCB 18 & 14.80 & 22.69 & 18.30 & 20.15 \\
\hline PCB 20 & 12.33 & 14.06 & 18.39 & 17.94 \\
\hline PCB 28 & 35.29 & 40.21 & 26.56 & 46.14 \\
\hline PCB 29 & - & 0.40 & 3.08 & 6.89 \\
\hline PCB 31 & 21.03 & 26.64 & 22.47 & 27.04 \\
\hline PCB 44 & 31.52 & 31.92 & 39.26 & 34.75 \\
\hline PCB 52 & 31.35 & 39.40 & 35.92 & 40.45 \\
\hline PCB 101 & 29.59 & 32.48 & 31.73 & 32.71 \\
\hline PCB 105 & 10.60 & 19.36 & 18.91 & 13.90 \\
\hline PCB 118 & 27.52 & 29.01 & 29.80 & 29.95 \\
\hline PCB 138 & 33.86 & 36.20 & 33.79 & 37.27 \\
\hline PCB 149 & 22.75 & 24.73 & 24.93 & 24.87 \\
\hline PCB 153 & 27.23 & 29.46 & 29.91 & 30.45 \\
\hline PCB 155 & 82.31 & 106.72 & 97.10 & 100.57 \\
\hline PCB 170 & 11.35 & 12.61 & 12.48 & 11.80 \\
\hline PCB 180 & 10.29 & 10.53 & 10.56 & 10.96 \\
\hline PCB 194 & - & 20.72 & 10.51 & 10.41 \\
\hline PCB 204 & - & - & - & - \\
\hline PCB 209 & - & 2.60 & 2.47 & 4.23 \\
\hline SPCBs & 20.47 & 23.40 & 22.84 & 24.48 \\
\hline
\end{tabular}

a) Concentration in the organism $(\mu \mathrm{g} / \mathrm{kg}) /$ concentration in sediment $(\mu \mathrm{g} / \mathrm{kg})$, both on a $D W$ basis. 
Tables

Table A4.6. Tissue concentration ratios (Contaminated sediment exposed / clean sediment exposed).

\begin{tabular}{|l|c|c|c|c|}
\hline PS Treatment & $\mathbf{0} \%$ & $\mathbf{0 . 0 7 4} \%$ & $\mathbf{0 . 7 4} \%$ & $\mathbf{7 . 4} \%$ \\
\hline PCB congener: & & & & \\
\hline PCB 18 & 4.32 & 7.49 & 7.98 & 5.80 \\
\hline PCB 20 & - & - & - & - \\
\hline PCB 28 & 4.58 & 5.80 & 3.71 & 5.93 \\
\hline PCB 29 & - & 0.16 & 0.81 & 1.70 \\
\hline PCB 31 & 5.33 & 7.47 & 6.28 & 6.81 \\
\hline PCB 44 & 3.71 & 4.23 & 5.07 & 4.08 \\
\hline PCB 52 & 8.07 & 11.46 & 10.14 & 10.41 \\
\hline PCB 101 & 4.28 & 5.30 & 5.02 & 4.70 \\
\hline PCB 105 & 3.77 & 7.75 & 7.36 & 4.89 \\
\hline PCB 118 & 2.40 & 2.86 & 2.85 & 2.59 \\
\hline PCB 138 & 2.13 & 2.56 & 2.32 & 2.32 \\
\hline PCB 149 & 3.35 & 4.10 & 4.01 & 3.64 \\
\hline PCB 153 & 2.11 & 2.58 & 2.53 & 2.34 \\
\hline PCB 155 & 2.26 & 3.30 & 2.90 & 2.72 \\
\hline PCB 170 & 3.51 & 4.37 & 4.22 & 3.61 \\
\hline PCB 180 & 5.38 & 6.19 & 6.02 & 5.68 \\
\hline PCB 194 & - & - & - & - \\
\hline PCB 204 & - & - & - & - \\
\hline PCB 209 & - & - & - & - \\
\hline PCB & 2.92 & 3.76 & 3.56 & 3.47 \\
\hline
\end{tabular}


Table A4.7. Tissue concentration ratios (Contaminated sediment with PS / Contaminated sediment without PS).

\begin{tabular}{|l|c|c|c|}
\hline PS Treatment & $\mathbf{0 . 0 7 4} \%$ & $\mathbf{0 . 7 4} \%$ & $\mathbf{7 . 4} \%$ \\
\hline PCB congener: & & & \\
\hline PCB 18 & 1.73 & 1.85 & 1.34 \\
\hline PCB 20 & 1.29 & 1.63 & 1.43 \\
\hline PCB 28 & 1.27 & 0.81 & 1.29 \\
\hline PCB 29 & - & - & - \\
\hline PCB 31 & 1.40 & 1.18 & 1.28 \\
\hline PCB 44 & 1.14 & 1.36 & 1.10 \\
\hline PCB 52 & 1.42 & 1.26 & 1.29 \\
\hline PCB 101 & 1.24 & 1.17 & 1.10 \\
\hline PCB 105 & 2.06 & 1.95 & 1.30 \\
\hline PCB 118 & 1.19 & 1.19 & 1.08 \\
\hline PCB 138 & 1.20 & 1.09 & 1.09 \\
\hline PCB 149 & 1.22 & 1.20 & 1.08 \\
\hline PCB 153 & 1.22 & 1.20 & 1.11 \\
\hline PCB 155 & 1.46 & 1.28 & 1.20 \\
\hline PCB 170 & 1.25 & 1.20 & 1.03 \\
\hline PCB 180 & 1.15 & 1.12 & 1.06 \\
\hline PCB 194 & - & - & - \\
\hline PCB 204 & 3.64 & 1.24 & 1.46 \\
\hline PCB 209 & - & - & - \\
\hline SPCB & 1.29 & 1.22 & 1.19 \\
\hline
\end{tabular}


Figures

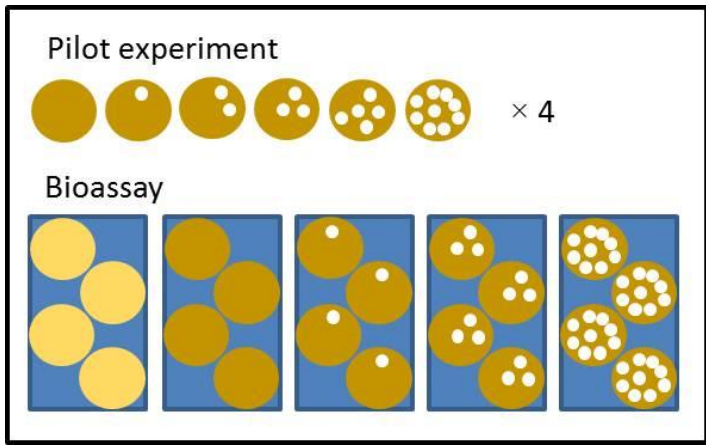

Figure A4.1. Schematic presentation of the experimental set up. The pilot experiment consisted of six treatments in $2 L$ test beakers. The bioassay used five treatments of which one in a PCB clean environment and four in a PCB contaminated environment and was carried out in $2 L$ test beakers in large aquaria. The amount of white dots visually indicates differences in plastic concentration.
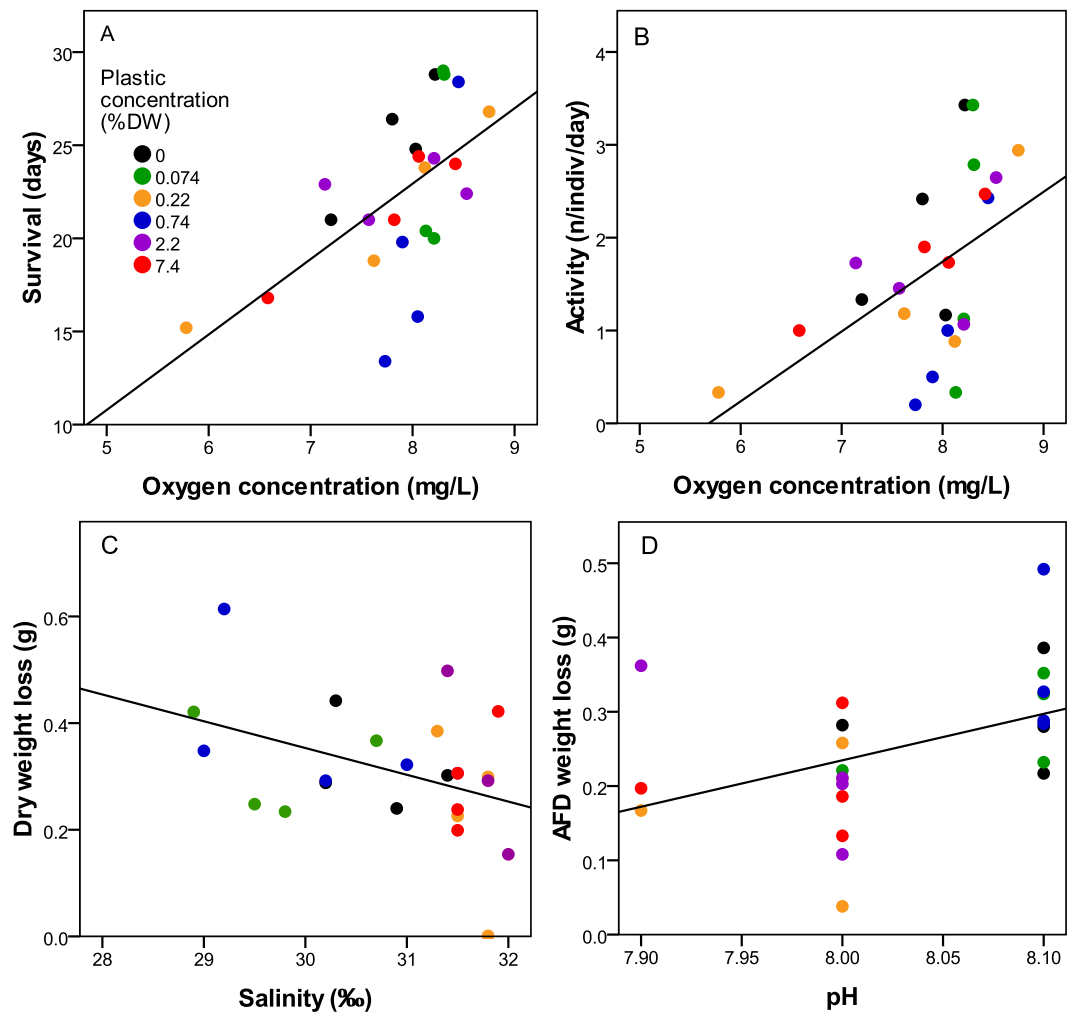

Figure A4.2. Pilot experiment; Influence of water quality variables. A. Relation between oxygen concentration and amount of days of survival. B. Relation between oxygen concentration and mean activity. C. Relation between salinity and DW loss. D. Relation between $p H$ and AFDW loss. 
Appendix Chapter 4
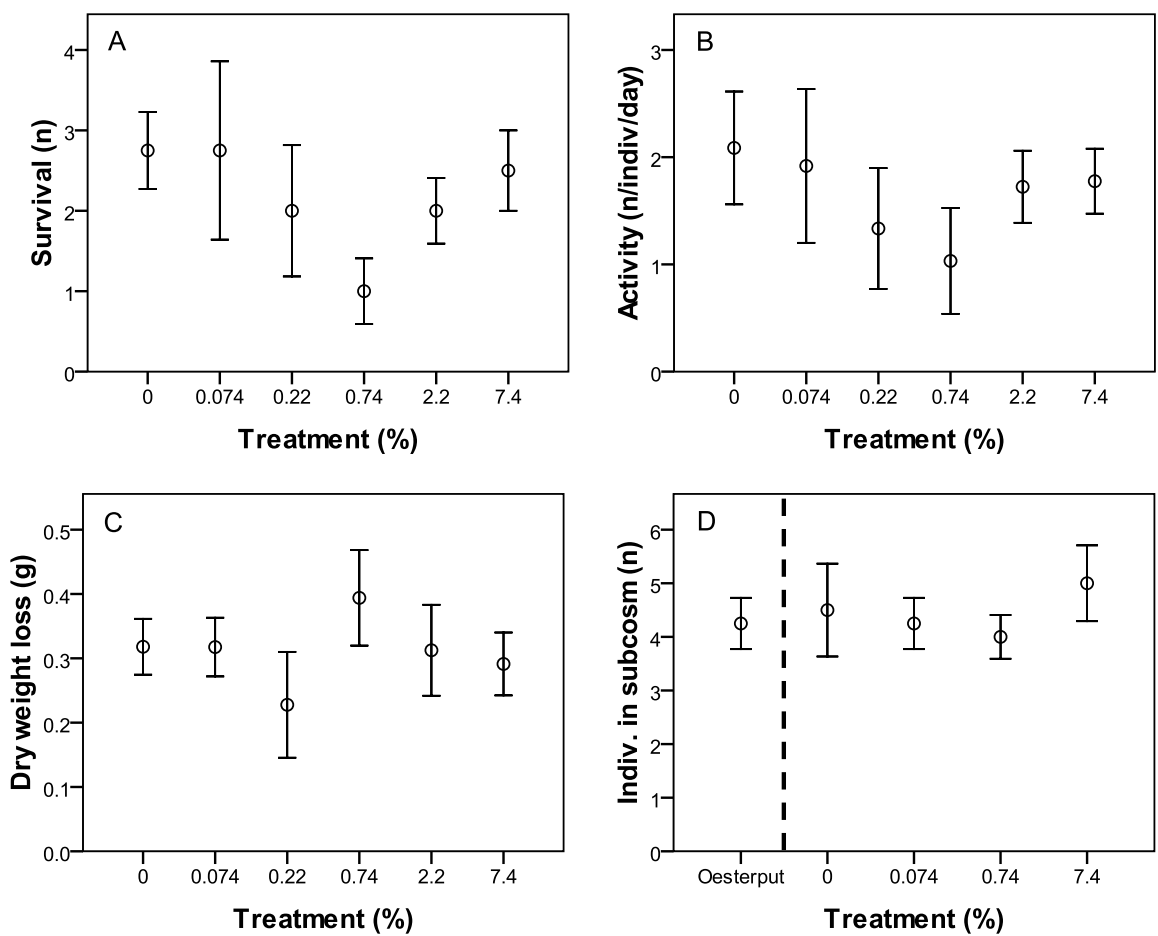

Figure A4.3. A. Pilot experiment; Difference in total amount of organisms that survived between treatments. B. Pilot experiment; Difference in activity between treatments. C. Pilot experiment; Difference in DW loss between treatments. D. Bioassay; Difference in the amount of organisms that remained in their test beaker between treatments. The bars indicate mean \pm standard error (SE). 
Figures
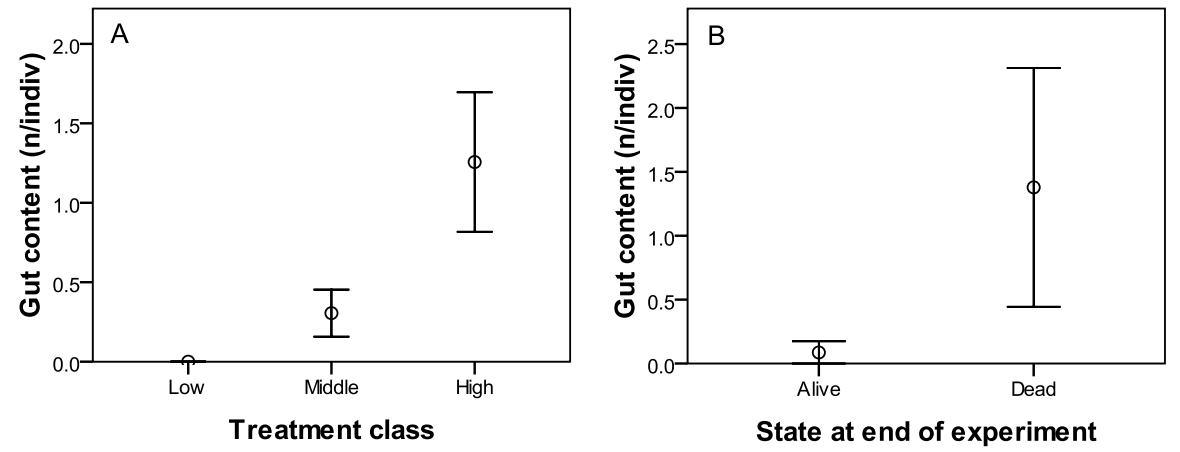

Figure A4.4. Pilot experiment. A. Difference in amount of plastic particles in gut content between exposure plastic concentrations. Expressed in treatment classes (Low $=0$ and 0.074 $\%$. Middle $=0.22$ and $0.74 \%$. High $=2.2$ and $7.4 \%$ ). B. Amount of plastic particles in the gut content of organisms that died during the experiment. compared to the organisms that survived. The bars indicate mean \pm standard error $(S E)$.
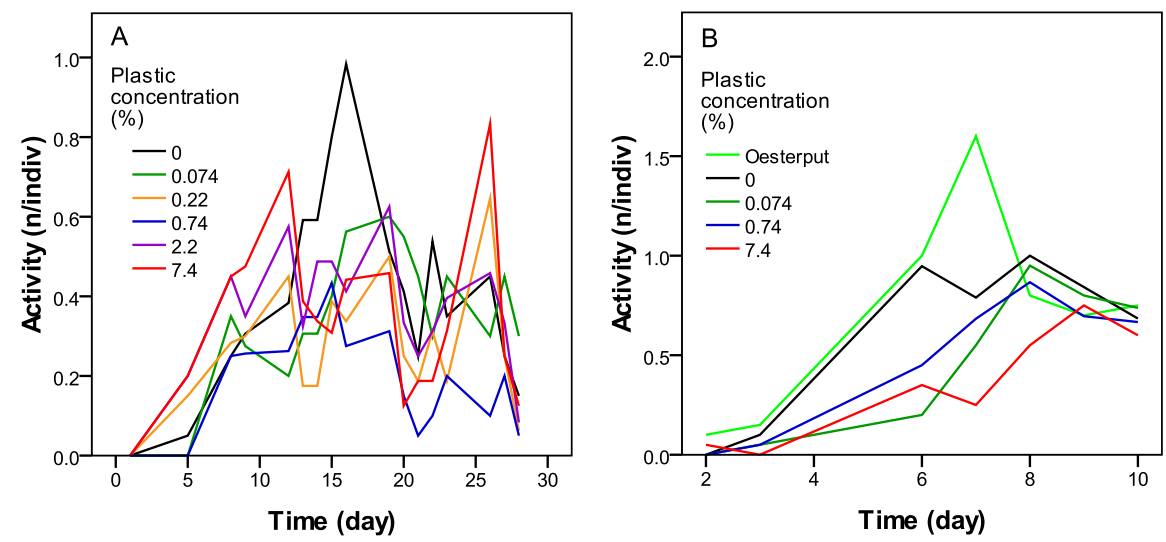

Figure A4.5. Variation of the activity over time. A. Pilot experiment. B. Bioassay. 
Appendix Chapter 4
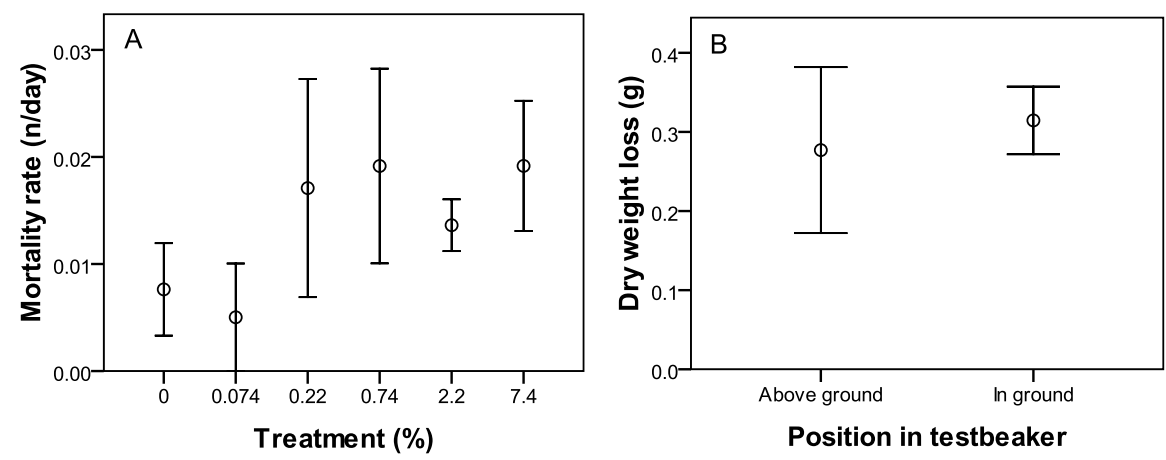

Figure A4.6. Pilot experiment. A. Difference in mortality rate between treatments. B. Difference in DW loss between worms that were in and above the sediment at the end of the experiment. The bars indicate mean $\pm S E$.
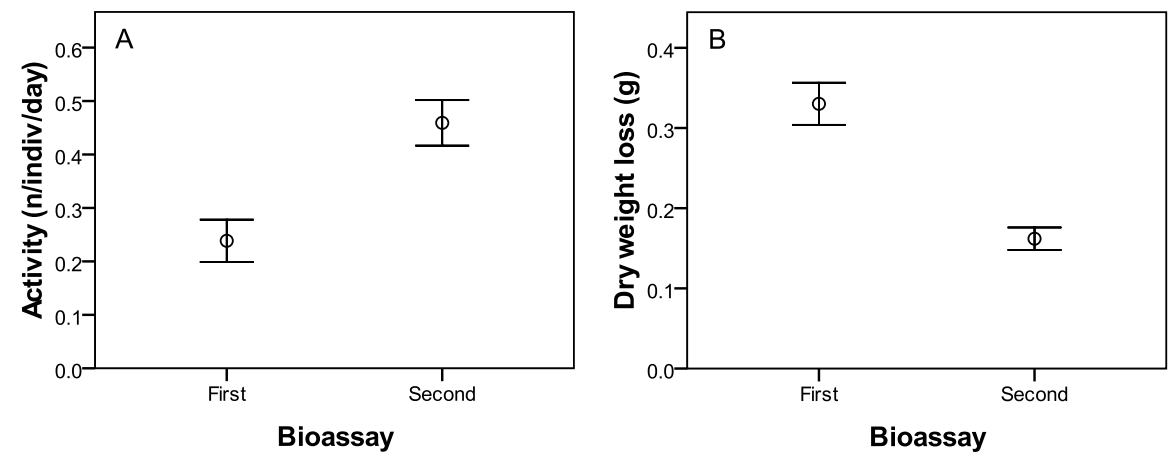

Figure A4.7. A. Activity of A. marina in the pilot experiment compared to the bioassay. B. DW loss of A. marina in the pilot experiment compared to the bioassay. The bars indicate mean \pm SE. 


\section{Appendix to Chapter 5}

1. Derivation of the microplastic absorption efficiency equation (Equation 5.4)

2. Mass transfer from microplastic to the gut fluid in A. Marina. Evaluation of the rate limiting process

3. Tables A5.1-A5.5

4. Figures $\mathrm{A} 4.1-\mathrm{A} 4.7$ 


\section{Derivation of the microplastic absorption efficiency equation (Equation 5.4)}

For calculation of the absorbed concentration $C_{P L R, t}(\mu \mathrm{g} / \mathrm{kg})$, we approximate the exchange of POPs between plastic and lipids as a first order reversible process $C_{P L, t} \leftrightarrow C_{L, t}(\mu \mathrm{g} / \mathrm{kg})$ with forward and backward rate constants $k_{l}$ and $k_{2}\left(\mathrm{~d}^{-1}\right)$.

$\frac{d C_{P L, t}}{d t}=k_{2} C_{L, t}-k_{1} C_{P L, t}$

In Equation (i), $C_{P L, t}$ is the concentration in the plastic during gut passage, $C_{L, t}$ is the concentration in the biota lipids and $t$ is gut passage time (d). This means that $t=0$ at the moment of ingestion of the plastic particle, and $t$ is equal to the total gut retention time $(t=\mathrm{GRT})$ at the moment of egestion of the plastic particle. Exchange of POPs between plastic and biota lipids takes place during $0<t<G R T$. It is assumed that during this exchange process as condensed in Equation i, the total mass of the POP $\left(Q_{\text {total }}\right)$ in the plastic and the biota lipids is constant. This results in the following mass balance equation:

$M_{P L} C_{P L, t}+M_{L} C_{L, t}=M_{P L} C_{P L}^{I n g}+M_{L} C_{L}^{I n g}=Q_{\text {total }}$

in which $M_{P L}$ and $M_{L}(\mathrm{~kg})$ are the masses of plastic and biota lipids present in the organism and $C_{P L}^{I n g}$ and $C_{L}^{I n g}(\mu \mathrm{g} / \mathrm{kg})$ are the concentrations of the POP in the ingested plastic particle and the biota lipids at the moment of ingestion (i.e. at $t=0$ ), respectively. Equation (ii) defines that at any time between $t=0$ and $t=G R T$, the sum of the time dependent masses of POP in plastic and biota lipids, equates to the initial masses in these phases at the moment of microplastic ingestion $(t=0)$. Equation (i) expresses the change of the concentration in the plastic as a function of time. However, to calculate the absorption from the plastic, we need the concentration that has been removed (i.e. absorbed) from the plastic as a result of the concentration gradient between plastic and lipids. This is calculated as follows. The removed concentration $\mathrm{C}_{\mathrm{PLR}, \mathrm{t}}$ during gut passage is equal to the initial concentration $(t=0)$ in the plastic at ingestion $\left(C_{P L}^{I n g}\right)$, minus the actual concentration during gut passage:

$$
C_{P L R, t}=C_{P L}^{I n g}-C_{P L, t} \text { or } \quad C_{P L, t}=C_{P L}^{I n g}-C_{P L R, t}
$$

This Equation (iii) shows how the actual concentration in the plastic $C_{P L, t}$, can be rewritten as the (constant) concentration at ingestion, minus the concentration that has been absorbed from the plastic. The absorbed quantity 
of POP from the plastic leads to an increase in the quantity and concentration of POP in the biota lipids over time. This increase can be calculated using the mass balance equation (ii) and elimination of $C_{P L, t}$ using Equation (iii):

$M_{P L}\left(C_{P L}^{I n g}-C_{P L R, t}\right)+M_{L} C_{L, t}=M_{P L} C_{P L}^{I n g}+M_{L} C_{L}^{I n g}$

which after rearrangement gives:

$C_{L, t}=C_{L}^{I n g}+\frac{M_{P L}}{M_{L}} C_{P L R, t}$

Using Equations (iii) and (iv), $C_{P L, t}$ and $C_{L, t}$ in Equation (i) can be eliminated, which after rearrangement expresses the exchange process in terms of the absorbed concentration only, instead of the concentrations in the separate phases:

$\frac{d C_{P L R, t}}{d t}=k_{1}\left(C_{P L}^{I n g}-C_{P L R, t}\right)-k_{2}\left(C_{L}^{I n g}+\frac{M_{P L}}{M_{L}} C_{P L R, t}\right)$

Equation (v) is an ordinary linear differential equation of the form $\mathrm{dx} / \mathrm{dt}=\mathrm{a} *(\mathrm{~b}-$ $\mathrm{x})-\mathrm{c}^{*}\left(\mathrm{~d}+\mathrm{e}^{*} \mathrm{x}\right)$, which can be analytically solved to yield:

$C_{P L R, G R T}=\frac{k_{1} C_{P L}^{I n g}-k_{2} C_{L}^{I n g}}{k_{1}+\frac{M_{P L}}{M_{L}} k_{2}}\left(1-e^{-\left(k_{1}+\frac{M_{P L}}{M_{L}} k_{2}\right) G R T}\right)$

in which time $t$ is replaced by the gut retention time GRT. Equation (vi) expresses the concentration absorbed from the plastic during gut passage, as a function of kinetic constants, masses of plastic and biota lipids, initial concentrations in plastic and biota lipids and gut retention time. Note that in Equations (i-v), time $t$ does not relate to total bioaccumulation time (by default simulated for $28 \mathrm{~d}$ ), but to gut passage time only (about $0.03 \mathrm{~d}$ ). This serves the calculation of the absorbed concentration i.e. Equation (vi), which then is recalculated for each time step in the $28 \mathrm{~d}$ bioaccumulation simulation, according to Equation 5.1 in the main manuscript. The value of $C_{P L}^{I n g}$ is assumed constant during the simulation, due to the excess of sediment and plastic compared to biota lipids. However, the value of $C_{L}^{I n g}$ changes over time and equates to $C_{B, t} / f_{\text {lip }}$ in Equation $1\left(C_{B, t} / f_{\text {lip }} C_{L}^{\text {Ing }}\right)$, where the subscript 't' now refers to $28 \mathrm{~d}$ simulation time. Similarly, $C_{P L R}$ and $G R T$ also change during simulation time and therefore are written as $C_{P L R, t}$ and $G R T, t$ :

$C_{P L R, t}=\frac{k_{1} C_{P L}^{I n g}-k_{2} C_{L, t}^{I n g}}{k_{1}+\frac{M_{P L}}{M_{L}} k_{2}}\left(1-e^{-\left(k_{1}+\frac{M_{P L}}{M_{L}} k_{2}\right) G R T, t}\right)$ 
Equation (vii) is Equation 4 in the main manuscript. From Equation (A5.vii / 4) the absorption efficiency can be calculated as $a_{P L, t}=C_{P L R, t} / C_{P L}^{I n g}$ (Eq. 5.5 in main manuscript). Note that $a_{P L, t}$ is defined only for absorption, i.e., the concentrations in $C_{P L R, t} / C_{P L}^{I n g}$ both higher than zero.

\section{Mass transfer from microplastic to the gut fluid in $A$. Marina. Evaluation of the rate limiting process.}

The release of polychlorobiphenyls from plastic particles to the gut fluid is determined by diffusion in the polymer matrix and diffusion across a stagnant boundary layer surrounding the plastic particle. In this chapter it is shown that the first-mentioned polymer diffusion is the slowest step and therefore is rate limiting for the overall exchange process. This section provides a quantitative theoretical analysis of the rates for the two processes in order to support this approach. This is done by calculating the effective first order kinetic constant $\left(\mathrm{k}_{1}\right.$ in main chapter Eq. 2) for each of the rate limitations. Here, the rate constants for polymer (P) diffusion and for boundary layer (BL) diffusion will be referred to as $\mathrm{k}_{\mathrm{P}}$ and $\mathrm{k}_{\mathrm{BL}}$, respectively. It will be shown that expected values for $k_{P}\left(d^{-1}\right)$ are orders of magnitude smaller than those for $k_{B L}\left(d^{-1}\right)$, which supports our assumption that polymer diffusion is rate limiting.

\section{Polymer diffusion}

If the desorption rate is limited by diffusion through a homogeneous polymeric sphere, the release of PCBs can be describe by Fick's law of diffusion in radial coordinates: ${ }^{21,223,227}$

$\frac{d C}{d t}=D_{p}\left(\frac{d^{2} C}{d x^{2}}+\frac{2}{x} \frac{d C}{d x}\right)$

where $\mathrm{C}$ is the $\mathrm{PCB}$ concentration in the plastic, $t$ is time, $x$ is the distance from the centre of the sphere with radius $r$, and $0<x<r$. Wu and Gschwend ${ }^{223}$ and Schwarzenbach et al. ${ }^{227}$ provide a linear approximation of the radial diffusion model, according to which a first order desorption rate constant for polymer diffusion from a spherical particle can be expressed as:

$k_{D} \cong 23 \frac{D_{P}}{r^{2}}$

This equation allows estimation of $k_{D}$ for PCBs if polymer diffusivities $D_{p}$ and particle radius $r$ are known. Polymer diffusivities $\mathrm{D}_{\mathrm{p}}$ for diffusion of PCBs in polystyrene (PS) and polyethylene (PE) were taken from Pascall et al. ${ }^{188}$ and 
Rusina et al. ${ }^{234}$ respectively. Particle radius $r$ for our scenario studies were 50 $\mathrm{nm}, 0.2 \mathrm{~mm}$ and $0.65 \mathrm{~mm}$ respectively. The resultant $\mathrm{k}_{\mathrm{P}}$ values for PCBs are presented in Table A5.2.

Diffusion across the stagnant boundary layer

If transfer through the stagnant film surrounding the microplastic particle is rate limiting, the release of PCBs can be described by: $:^{227,409}$

$\frac{d C}{d t}=-\frac{K_{L} \times A}{V \times K_{P L}^{G U T}} C=-k_{B} C$

Where $K_{L}$ is the mass-transfer coefficient $\left(\mathrm{m} \times \mathrm{d}^{-1}\right)$, $A$ is the spherical particle surface $\left(A=4 \pi r^{2} ; m^{2}\right)$, $V$ is plastic sphere volume $\left(V=4 / 3 \pi r^{3} ; m^{3}\right)$ and $K_{P L}$ GUT is the apparent plastic-water partition coefficient in the gut. $\mathrm{K}_{\mathrm{PL}}{ }^{\mathrm{GUT}}$ is the plastic-water partition coefficient for pure water, corrected for the solubility increase due to digestive surfactants, proteins, food hydrolysates such as membrane fragments and other colloidal or dissolved organic matter: ${ }^{228,410-413}$

$K_{P L}^{G U T}=\frac{K_{P L}}{1+K_{D O M}[D O M]}$

in which $[\mathrm{DOM}]$ is the concentration of DOM in the gut $(\mathrm{kg} / \mathrm{L}), \mathrm{K}_{\mathrm{DOM}}(\mathrm{L} / \mathrm{kg})$ is a DOM water partition coefficient and $\mathrm{K}_{\mathrm{PL}}(\mathrm{L} / \mathrm{kg})$ is a plastic-water partition coefficient. For transport across a planar boundary layer (BL), usually a linear concentration gradient is assumed, so that $\mathrm{K}_{\mathrm{L}}$ can be approximated by $\mathrm{D}_{\mathrm{M}} / \delta$, where $\mathrm{D}_{\mathrm{M}}$ is PCB diffusivity in water and $\delta$ is the thickness of the BL. ${ }^{227}$ However, for a spherical particle, the BL also will be spherical. The spherical boundary increases the concentration gradient across the boundary, leading to an increase of $\mathrm{K}_{\mathrm{L}}$ by a factor $(1+\delta / \mathrm{r})$ (Schwarzenbach et al. ${ }^{227} \mathrm{p} 874$, eq. 1968):

$K_{L}^{\text {Spherical particle }}=K_{L}\left(1+\frac{\delta}{r}\right)$

Consequently, the increase is larger for smaller microparticles. Besides molecular diffusion of dissolved PCBs across the BL, in an organism's gut diffusion of PCBs that are associated with organic molecules (DOM) will contribute to the flux across the BL. ${ }^{229,257,261,414}$ The extent of association of PCBs with DOM can be quantified through a traditional DOM water partition coefficient $\mathrm{K}_{\mathrm{DOM}}(\mathrm{L} / \mathrm{kg})$. The (labile) PCB-DOM complexes will have a lower aqueous diffusivity than the freely dissolved $\mathrm{PCBs}^{229,257}$ which has to be accounted for. The overall mass transfer coefficient including DOM facilitated transport can be expressed as: $:^{229,261}$ 
$K_{L}^{\text {DOM inclusive }}=K_{L}+K_{L}^{\text {DOM }} K_{\text {DOM }}[D O M]$

in which $\mathrm{K}_{\mathrm{L}}{ }^{\mathrm{DOM}}$ is the mass transfer coefficient of the PCB-DOM complexes $(\mathrm{m} / \mathrm{d})$ and $[\mathrm{DOM}]$ is the concentration of DOM in the gut $(\mathrm{kg} / \mathrm{L})$. Consequently, the transport facilitation by DOM will be more important for more hydrophobic PCBs. Accounting for both mechanisms and because the relative increase due to the spherical boundary is identical for diffusion of freely as well as DOM-associated PCBs, $\mathrm{k}_{\mathrm{B}}$ in Eq. 3 becomes:

$k_{B}=\frac{3\left(1+\frac{\delta}{r}\right)\left(K_{L}+K_{L}^{D O M} K_{D O M}[D O M]\right)\left(1+K_{D O M}[D O M]\right)}{r K_{P L}}$

Values for $\mathrm{K}_{\mathrm{L}}{ }^{\text {DOM }}$ were taken as $0.02 \times \mathrm{K}_{\mathrm{L}}$, based on data provided by Ter Laak et al. ${ }^{229}$ showing a more or less constant ratio of $0.02 \pm 0.01$ between stagnant boundary layer diffusivities for DOM-bound and freely dissolved PCBs. A very similar value of $\mathrm{K}_{\mathrm{L}}{ }^{\mathrm{DOM}} / \mathrm{K}_{\mathrm{L}}=0.025$ was recently reported by Kupryianchyk et $a l .^{261}$

To assess the solubility increase in the gut, the term $K_{D O M}[D O M]$ in Eq. A5.4 and A5.7 needs to be estimated. $\mathrm{K}_{\mathrm{DOM}}$ values can be approximated as $0.06 \times \mathrm{K}_{\mathrm{Ow}},{ }^{410,415}$ with $\mathrm{K}_{\mathrm{Ow}}$ the octanol-water partition coefficient. The DOM concentration in the gut relates to a mixture of solubilised sediment organic matter, surfactants and proteins present in the gut. ${ }^{228,257,412,413}$ Mayer et al. ${ }^{257}$ report a value of $[\mathrm{DOM}]=15.5 \times 10^{-3} \mathrm{~kg} / \mathrm{L}$ for the polychaete Neris virens, which is of the same class as A. Marina. Alternatively, we may assume that the modeled PCB absorption efficiency from sediment organic matter $\left(a_{S E D}=0.015\right)$ matches the actual fraction of organic matter solubilised at the end of the gut. For the entire gut, this would yield a DOM concentration $(\mathrm{kg} / \mathrm{L})$ of:

$$
\begin{aligned}
& {[D O M]=\frac{0.5 \times V_{G U T} \sigma_{S E D} f_{O M} a_{S E D}}{V_{G U T}}=0.5 \times 1.8 \times 0.0173 \times 0.015} \\
& =2.3 \times 10^{-3} \mathrm{~kg} / \mathrm{L}
\end{aligned}
$$

In Eq. A5.8, the factor 0.5 accounts for averaging the solubilisation over the gut $\left(\mathrm{a}_{\mathrm{SED}}=0\right.$ at start and $\mathrm{a}_{\mathrm{SED}}=0.015$ at the end of the gut), $\mathrm{V}_{\mathrm{GUT}}$ is gut volume (calculated through Eq. 5.9 in the main chapter) and $\sigma_{\mathrm{SED}}$ is the density $(\mathrm{kg} / \mathrm{L})$ of the sediment. Based on these data, the solubility increase $K_{D O M}[D O M]$ in Eq. A5.4 and A5.7, would equate to $0.06 K_{O W}[D O M]$, which yields a solubility increase ranging from $0.14 \times 10^{-3} K_{O W}$ to $1 \times 10^{-3} K_{O W}$ based on this range of DOM values of 2.3 to $15.5 \mathrm{~g} / \mathrm{L}$. Interestingly, Voparil et al. ${ }^{228}$ 
reported a solubility increase in A. Marina gut fluids of $\sim 10,000$ for dibenzo(a,h)-anthracene (Figure 2 in Voparil et al. ${ }^{228}$ ). With $\mathrm{K}_{\mathrm{OW}}=10^{7.11}$ for dibenzo(a,h)-anthracene this translates into a solubility increase of $0.78 \times 10^{-}$ ${ }^{3} \times \mathrm{K}_{\mathrm{Ow}}$, which agrees well to the range of $0.14 \times 10^{-3} \mathrm{~K}_{\mathrm{Ow}}$ to $1 \times 10^{-3} \mathrm{~K}_{\mathrm{OW}}$ mentioned above. Therefore, in the calculation of $\mathrm{k}_{\mathrm{B}}$ (Eq. A5.7) this latter intermediate value is used.

Values for $\mathrm{K}_{\mathrm{PL}}(\mathrm{L} / \mathrm{kg})$ were taken from Pascall et al. ${ }^{188}$ and Smedes et al. ${ }^{216}$ for partitioning of PCBs to PS and PE, respectively.

For the thickness of the undisturbed BL $(\delta)$ in surface water, values of $50-$ $250 \mu \mathrm{m}$ are often used. ${ }^{227}$ In the gut, however, direct sediment-plastic particle contact and peristaltic contractions of the gut will drastically increase transport rates across the UBL, because the UBL would no longer be 'undisturbed'. Direct contact and particle collisions will drastically reduce the effective thickness of the BL, if not, completely eliminate the BL. This accelerating effect of direct contact has been shown by Mayer et al. ${ }^{257}$ and by Smedes et $a l .{ }^{224}$ They conclude that direct particle contact ${ }^{257}$ and/or turbulence in dense sediment suspensions ${ }^{224}$ disrupt the water UBL and decrease the diffusion distance resulting in a faster transport of compounds. If the BL thickness $(\delta)$ decreases, the factor increase in $\mathrm{K}_{\mathrm{L}}$ (i.e. $\mathrm{D}_{\mathrm{M}} / \delta$ ) is higher than the factor decrease due to the transport enhancement due to the spherical boundary (Eq. A5.5). Therefore, the net effect is a further increase in mass transfer. Although $\delta$ probably is much smaller than the range for surface water, we use a conservative estimate of $50 \mu \mathrm{m}$ in the calculations.

Particle radius $r$ for our scenario studies were $50 \mathrm{~nm}, 0.2 \mathrm{~mm}$ and $0.65 \mathrm{~mm}$ respectively. The resultant $\mathrm{k}_{\mathrm{P}}$ values for PCBs are presented in Table A5.3.

\section{Determination of the rate limiting step}

Apparent first order constants for the release of PCBs from plastic particles were calculated based on a radial polymer diffusion mechanism ( $\mathrm{k}_{\mathrm{D}}$, Eq. A5.2) and a boundary layer resistance mechanism ( $\mathrm{k}_{\mathrm{B}}$, Eq. A5.7). This was done for PS and for PE particles of $100 \mathrm{~nm}, 0.4 \mathrm{~mm}$ and $1.3 \mathrm{~mm}$.

For PS particles with a diameter of $100 \mathrm{~nm}$ to $1.3 \mathrm{~mm}$, BL limited mass transfer is calculated to be 4 to 9 orders of magnitude faster than polymer diffusion (Table A5.2 ; Figure A5.1A), dependent on particle size and $\operatorname{LogK}_{\text {ow }}$ of the PCB. Even with a low DOM estimate of $2.3 \mathrm{~g} / \mathrm{L}$ and a higher BL thickness $\delta$ of $100 \mu \mathrm{m}, \mathrm{BL}$ mass transfer is still 3 to 7 orders of magnitude faster. Completely neglecting DOM transport facilitation still shows 1 to 2.5 
orders of magnitude higher BL mass transfer rates. Consequently, we conclude that we can safely assume that PS polymer diffusion (Eq. A5.2) is rate limiting for the PS scenarios, and therefore can be used to estimate the rate of PCB release from PS in the gut of A. Marina.

Similarly, for PE particles, BL limited mass transfer is calculated to be 0.4 to 4 orders of magnitude faster than PE polymer diffusion (Table A5.3, Figure A5.1B), dependent on particle size and $\log K_{o w}$ of the PCB. Although for the less hydrophobic PCBs the difference is not large, for PCB52 and higher, polymer diffusion is calculated to be at least a factor of 20 slower than BL limited mass transfer, which also confirms the validity of our assumption for the PE scenarios. The slower BL mass transfer for PE compared to PS can be explained from the higher $\mathrm{K}_{\mathrm{PL}}{ }^{\mathrm{GUT}}$ for $\mathrm{PE}$, which reduces the release rate (Eq. A5.3). Consequently, in contrast to PS, if DOM associated transport would not contribute to the total flux through the BL, polymer diffusion would not be rate limiting. However, the role of DOM facilitated transport in aqueous media and specifically gut fluids is well-established as explained above. $229,257,261,414$ 
Tables

Table A5.1. Rate constants.

Uptake from water

Hendriks et al. (2001):

$k_{\text {derm }}=\left(\frac{w^{-\kappa}}{\rho_{H_{2} O, 0}+\frac{\rho_{C H 2}}{K_{O W}}+\frac{1}{\gamma_{0}}}\right) \times \frac{1}{D W} \times 10^{-3}$

Which translates to (Hendriks et al., 2001, Fig. 3, Annelida):

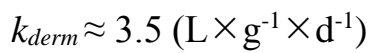

\section{Loss rate}

Following Janssen et al. ${ }^{218}$ and Hendriks et al. ${ }^{221}$ elimination, growth dilution and egestion were modeled using a lumped loss rate constant. The rate constant was approximated as (Fig. 5, 'Annelida', in Hendriks et al. ${ }^{221}$ ):

$k_{\text {loss }} \approx-0.75 \times \log K_{O W}+3.65$ and $4<\log K_{O W}<8$

Used factors, with standard values for benthic invertebrates

\begin{tabular}{|l|l|}
\hline $\mathrm{DW}$ & lugworm dry weight fraction \\
\hline $\mathrm{k}_{\mathrm{derm}}$ & dermal absorption rate constant from water $\left(\mathrm{L} \times \mathrm{g}^{-1} \times \mathrm{d}^{-1}\right)$ \\
\hline $\mathrm{k}_{\text {loss }}$ & loss rate into water $\left(\mathrm{d}^{-1}\right)$ \\
\hline$\gamma_{0}$ & water absorption-excretion coefficient $\left(\mathrm{kg}^{\mathrm{K}} \times \mathrm{d}^{-1} ; 200\right)$ \\
\hline $\mathrm{K}_{\mathrm{OW}}$ & octanol-water partition ratio $(-)$ \\
\hline$\kappa$ & rate exponent $(-; 0.25)$ \\
\hline$\rho_{\mathrm{H} 2 \mathrm{O}, \mathrm{j}}$ & water layer diffusion resistance $\left(\mathrm{d} \times \mathrm{kg}^{-\mathrm{k}} ; 2.8 \times 10^{-3}\right.$ if $\mathrm{j}=0 ;$ \\
& $1.1 \times 10^{-5}$ if $\left.\mathrm{j}=1\right)^{221}$ \\
\hline$\rho_{\mathrm{CH} 2}$ & lipid layer permeation resistance $\left(\mathrm{d} \times \mathrm{kg}^{-\mathrm{k}} ; 68\right)$ \\
\hline $\mathrm{w}$ & species wet weight $(\mathrm{g})$ \\
\hline
\end{tabular}


Table A5.2. Calculated apparent first order release constants from polystyrene nano- and microplastic particles assuming rate limitation in the boundary layer $\left(k_{B}\right)$ and assuming rate limitation by polymer diffusion $\left(k_{D}\right)$.

\begin{tabular}{|c|c|c|c|c|c|c|c|c|c|}
\hline \multirow[t]{2}{*}{ PCB: } & \multicolumn{3}{|c|}{$\begin{array}{c}\text { Boundary resistance }^{a)} \\
\qquad k_{B}\left(d^{-1}\right)\end{array}$} & \multicolumn{3}{|c|}{$\begin{array}{l}\text { Diffusive resistance }^{b)} \\
\qquad k_{D}\left(d^{-1}\right)\end{array}$} & \multicolumn{3}{|c|}{$\log \left(\mathbf{k}_{B} / \mathbf{k}_{D}\right)^{c)}$} \\
\hline & $100 \mathrm{~nm}$ & $0,4 \mathrm{~mm}$ & $1,3 \mathrm{~mm}$ & $100 \mathrm{~nm}$ & $0,4 \mathrm{~mm}$ & $1,3 \mathrm{~mm}$ & $100 \mathrm{~nm}$ & $0,4 \mathrm{~mm}$ & $1,3 \mathrm{~mm}$ \\
\hline РCB31 & $3,57 E+11$ & $1,11 E+05$ & $2,95 E+04$ & $3,34 \mathrm{E}+07$ & $2,09 E+00$ & $1,98 \mathrm{E}-01$ & 4,03 & 4,73 & 5,17 \\
\hline РCB47 & $1,79 \mathrm{E}+12$ & $5,58 E+05$ & $1,48 \mathrm{E}+05$ & $8,74 E+06$ & $5,46 \mathrm{E}-01$ & $5,17 \mathrm{E}-02$ & 5,31 & 6,01 & 6,46 \\
\hline PCB103 & $4,90 E+12$ & $1,53 E+06$ & $4,06 E+05$ & $7,15 E+06$ & 4,47E-01 & 4,23E-02 & 5,84 & 6,53 & 6,98 \\
\hline PCB128 & $1,91 E+13$ & $5,98 E+06$ & $1,58 \mathrm{E}+06$ & $6,36 \mathrm{E}+06$ & 3,97E-01 & $3,76 \mathrm{E}-02$ & 6,48 & 7,18 & 7,62 \\
\hline PCB171 & $1,96 \mathrm{E}+14$ & $6,11 E+07$ & $1,62 E+07$ & $8,74 E+06$ & $5,46 \mathrm{E}-01$ & $5,17 \mathrm{E}-02$ & 7,35 & 8,05 & 8,50 \\
\hline PCB200 & $7,73 E+14$ & $2,41 E+08$ & $6,40 E+07$ & $5,56 \mathrm{E}+06$ & $3,48 \mathrm{E}-01$ & $3,29 \mathrm{E}-02$ & 8,14 & 8,84 & 9,29 \\
\hline
\end{tabular}

a) Calculated using Eq 2 with PS polymer diffusivities.

b) Calculated using Eq 7 with PS partition coefficients.

${ }^{c)} \log$ of the dimensionless ratio $k_{B} / k_{D}$. If $\log \left(k_{B} / k_{D}\right)>0$ polymer diffusion is rate limiting.

Table A5.3. Calculated apparent first order release constants from polyethylene nano- and microplastic particles assuming rate limitation in the boundary layer $\left(k_{B}\right)$ and assuming rate limitation by polymer diffusion $\left(k_{D}\right)$.

\begin{tabular}{|c|c|c|c|c|c|c|c|c|c|}
\hline \multirow[t]{2}{*}{ PCB: } & \multicolumn{3}{|c|}{$\begin{array}{c}\text { Boundary resistance }^{a)} \\
k_{B}(d-1)\end{array}$} & \multicolumn{3}{|c|}{$\begin{array}{l}\left.\text { Diffusive resistance }^{b}\right) \\
\qquad k_{D}(d-1)\end{array}$} & \multicolumn{3}{|c|}{$\log \left(k_{B} / k_{D}\right)^{c)}$} \\
\hline & $100 \mathrm{~nm}$ & $1 \mu \mathrm{m}$ & $1 \mathrm{~mm}$ & $100 \mathrm{~nm}$ & $1 \mu \mathrm{m}$ & $1 \mathrm{~mm}$ & $100 \mathrm{~nm}$ & $1 \mu \mathrm{m}$ & $1 \mathrm{~mm}$ \\
\hline РCB28 & $6,02 E+08$ & $6,07 E+06$ & $6,61 E+01$ & $2,46 E+08$ & $2,46 E+06$ & $2,46 E+00$ & 0,39 & 0,39 & 1,43 \\
\hline РCB35 & $7,70 E+08$ & $7,77 E+06$ & $8,46 E+01$ & $1,37 E+08$ & $1,37 E+06$ & $1,37 \mathrm{E}+00$ & 0,75 & 0,75 & 1,79 \\
\hline PCB52 & $7,59 \mathrm{E}+08$ & $7,66 \mathrm{E}+06$ & $8,35 E+01$ & $1,05 E+08$ & $1,05 E+06$ & $1,05 E+00$ & 0,86 & 0,86 & 1,90 \\
\hline РСB72 & $1,59 E+09$ & $1,60 E+07$ & $1,75 E+02$ & $8,53 E+07$ & $8,53 E+05$ & $8,53 E-01$ & 1,27 & 1,27 & 2,31 \\
\hline РСB77 & $1,90 E+09$ & $1,92 E+07$ & $2,09 E+02$ & $7,67 E+07$ & $7,67 E+05$ & 7,67E-01 & 1,40 & 1,40 & 2,44 \\
\hline PCB101 & $1,89 \mathrm{E}+09$ & $1,91 E+07$ & $2,08 \mathrm{E}+02$ & $6,92 E+07$ & $6,92 \mathrm{E}+05$ & $6,92 \mathrm{E}-01$ & 1,44 & 1,44 & 2,48 \\
\hline PCB118 & $3,68 \mathrm{E}+09$ & $3,71 E+07$ & $4,04 E+02$ & $7,08 \mathrm{E}+07$ & $7,08 \mathrm{E}+05$ & $7,08 \mathrm{E}-01$ & 1,72 & 1,72 & 2,76 \\
\hline PCB126 & $4,86 E+09$ & $4,91 E+07$ & $5,34 \mathrm{E}+02$ & $4,34 \mathrm{E}+07$ & $4,34 E+05$ & 4,34E-01 & 2,05 & 2,05 & 3,09 \\
\hline PCB153 & $4,94 \mathrm{E}+09$ & $4,98 \mathrm{E}+07$ & $5,42 E+02$ & $4,17 E+07$ & $4,17 E+05$ & 4,17E-01 & 2,07 & 2,08 & 3,11 \\
\hline PCB180 & $1,08 \mathrm{E}+10$ & $1,09 E+08$ & $1,19 E+03$ & $2,14 E+07$ & $2,14 E+05$ & $2,14 \mathrm{E}-01$ & 2,70 & 2,71 & 3,75 \\
\hline РCB203 & $1,81 \mathrm{E}+10$ & $1,82 E+08$ & $1,99 E+03$ & $1,92 E+07$ & $1,92 E+05$ & 1,92E-01 & 2,97 & 2,98 & 4,01 \\
\hline
\end{tabular}

a) Calculated using Eq. A5.2 with PE polymer diffusivities.

b) Calculated using Eq. A5.7 with PE partition coefficients.

c) Log of the dimensionless ratio $k_{B} / k_{D}$. If $\log \left(k_{B} / k_{D}\right)>0$ polymer diffusion is rate limiting. 


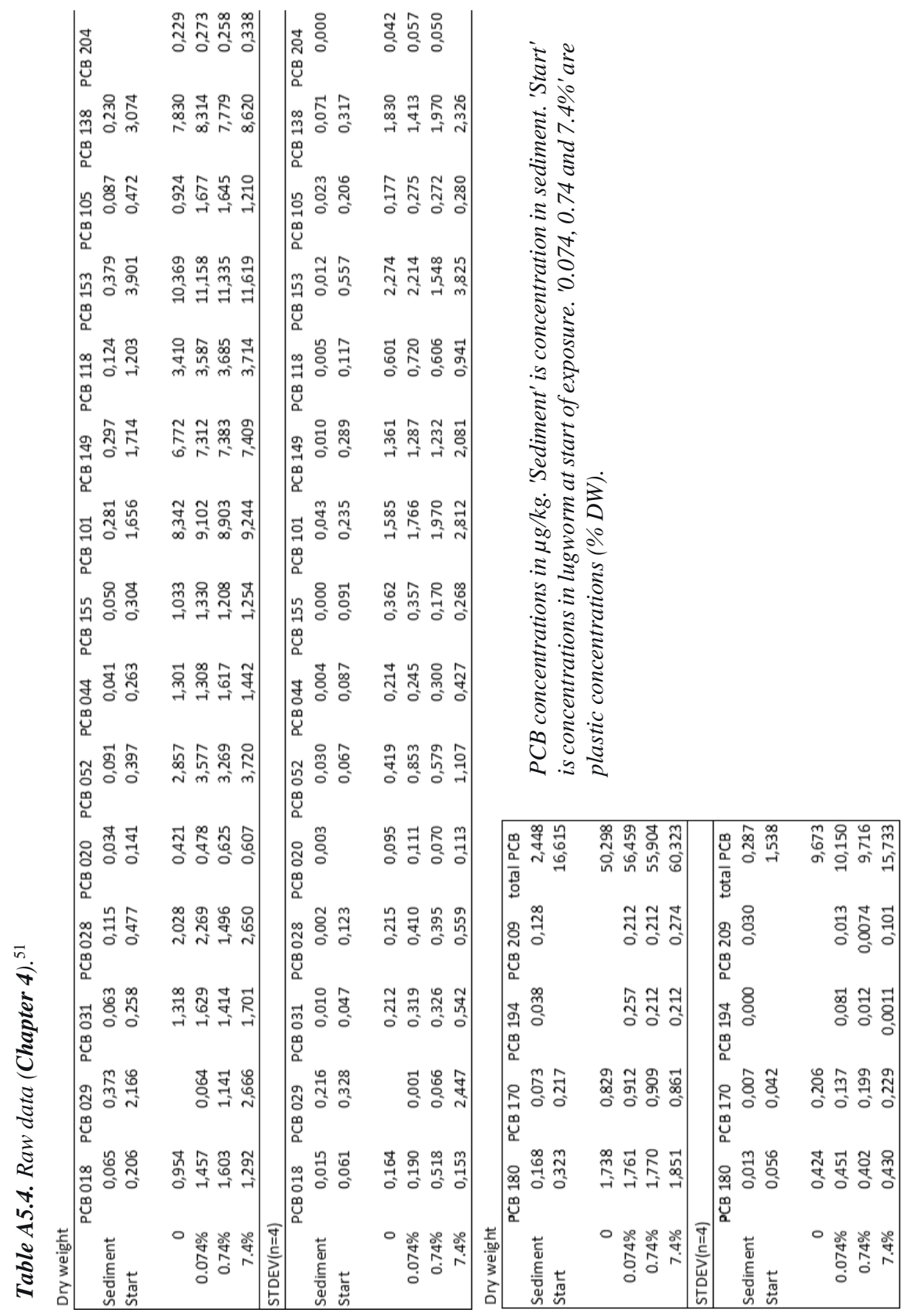


Table A5.5. Parameter ranges and optimized values. Literature citations relate to references in the main chapter. ${ }^{a)}$

\begin{tabular}{|c|c|}
\hline Parameter & Calculation - Source \\
\hline$\delta$ & $\begin{array}{l}\text { Gut content's density }\left(\mathrm{g} / \mathrm{mm}^{3}\right) \text { calculated as weighted } \\
\text { average of sediment and plastic densities: } \\
\delta=\delta_{\mathrm{SED}} S_{\mathrm{SED}}+\delta_{\mathrm{PL}} S_{\mathrm{PL}} \text {. Sediment density calculated from OM } \\
\left(1.0 \mathrm{~g} / \mathrm{cm}^{3}\right) \text { and mineral density }\left(2.65 \mathrm{~g} / \mathrm{cm}^{3}\right) \text { and } \mathrm{OM} \\
\text { weight fraction }\left(\mathrm{f}_{\mathrm{OM}}\right) \text { based on measured loss on ignition). } \\
\text { PS density } 1.05 \mathrm{~g} / \mathrm{cm}^{3} .\end{array}$ \\
\hline asED & $\begin{array}{l}\text { Absorption efficiency from sediment (-) from Hendriks et } \\
a l .{ }^{221}\end{array}$ \\
\hline$a_{S E D, L}$ & Absorption efficiency per mm worm length $\left(\mathrm{mm}^{-1}\right)$; $\mathrm{a}_{\mathrm{SED}} / \mathrm{L}_{\mathrm{t}}$ \\
\hline $\mathrm{aPL}$ & $\begin{array}{l}\text { Absorption efficiency from plastic (-). Time variable, } \\
\text { calculated with Equation A5.5. }\end{array}$ \\
\hline $\mathrm{C}_{\mathrm{B}, \mathrm{t}}$ & $\begin{array}{l}\text { PCB Concentration in biota }(\mu \mathrm{g} / \mathrm{g} \text { DW). Measured by } \\
\text { Besseling } \text { et al. }(\text { Chapter } 4)^{51}(t=0, t=28 \mathrm{~d}) \text {. Modeled with } \\
\text { Equation A5.1. }\end{array}$ \\
\hline $\mathrm{C}_{\mathrm{L}, \mathrm{t}}$ & $\begin{array}{l}\text { Lipid normalised concentration in biota }(\mu \mathrm{g} / \mathrm{g} \mathrm{DW}) . \mathrm{C}_{\mathrm{L}}^{0} \text { is } \\
\text { the concentration at } t=0 . \mathrm{C}_{\mathrm{L}, \mathrm{t}}^{\text {Ing }} \text { is the concentration at the } \\
\text { time of plastic ingestion. At any time, } \mathrm{C}_{\mathrm{L}, \mathrm{t}}=\mathrm{C}_{\mathrm{B}, \mathrm{t}} / \mathrm{f}_{\text {lip }}\end{array}$ \\
\hline $\mathrm{C}_{\mathrm{PL}}$ & $\begin{array}{l}\text { Concentration in plastic (outside worm and at time of } \\
\text { ingestion by worm) }(\mu \mathrm{g} / \mathrm{g} \text { ). At any time calculated using } \\
\text { literature equilibrium partition coefficients. PS: } \operatorname{LogK}_{\mathrm{PL}} \approx \\
\text { 3. }{ }^{188} \mathrm{PE}: \mathrm{LogK}_{\mathrm{PL}}=1.18 \mathrm{LogK}_{\mathrm{OW}}-1.26 .{ }^{216,217}\end{array}$ \\
\hline $\mathrm{C}_{\text {PLR }, \mathrm{t}}$ & $\begin{array}{l}\text { Concentration in plastic that has been absorbed by the } \\
\text { worm, referenced to plastic mass }(\mu \mathrm{g} / \mathrm{g}) \text {. Calculated from } \\
\text { Equation A5.4. }\end{array}$ \\
\hline $\mathrm{C}_{\mathrm{SED}}$ & $\begin{array}{l}\text { Concentration in sediment ( } \mu \mathrm{g} / \mathrm{g} \text { sediment). Measured } \\
\text { using ASE solvent extraction and GC-ECD detection. }\end{array}$ \\
\hline $\mathrm{C}_{\mathrm{w}}$ & $\begin{array}{l}\text { Concentration in water }(\mu \mathrm{g} / \mathrm{L}) \text {. Calculated using Equation } \\
\text { A5.6. }\end{array}$ \\
\hline
\end{tabular}


Tables

Table A5.5. Continued.

\begin{tabular}{|c|c|}
\hline $\mathrm{D}$ & $\begin{array}{l}\text { POP polymer diffusivity }\left(\mathrm{m}^{2} / \mathrm{d}\right) \text {. Values for PCBs from } \\
\text { refs. }{ }^{188,234}\end{array}$ \\
\hline $\mathrm{f}_{\text {lip }}$ & $\begin{array}{l}\text { Lipid fraction (-) of A. Marina. Site-specific literature } \\
\text { values from ref. }\end{array}$ \\
\hline$f_{O C}$ & Sediment organic carbon fraction (-). Estimated as $\mathrm{f}_{\mathrm{OM}} / 2.5$ \\
\hline$f_{O M}$ & $\begin{array}{l}\text { Sediment organic matter fraction (-). Measured as loss on } \\
\text { ignition }\left(3 \mathrm{~h}, 550^{\circ} \mathrm{C}\right) \text {. }\end{array}$ \\
\hline $\mathrm{GRT}_{\mathrm{t}}$ & Gut residence time (d). Calculated using Equation A5.9. \\
\hline $\mathrm{IR}_{\mathrm{t}}$ & $\begin{array}{l}\text { Ingestion rate (g/g DW per day). Calculated using Equation } \\
\text { A5.7, which is based on a regression of ingestion rates, } \\
\text { weights and sediment organic matter contents specifically } \\
\text { derived for deposit feeders and detritivores. }{ }^{204}\end{array}$ \\
\hline $\mathrm{k}_{1}$ & $\begin{array}{l}\text { Apparent first order rate constant for plastic to lipid } \\
\text { transport }\left(\mathrm{d}^{-1}\right) \text {. Assuming polymer diffusion is the rate } \\
\text { limiting step in the bioavailability of plastic bound PCBs, } \\
\mathrm{k}_{1} \text { is estimated using a half-life based on a radial diffusion } \\
\text { model; } \mathrm{k}_{1} \approx 23 \mathrm{D} / \mathrm{r}_{\mathrm{P}}^{2} \text {.(227) }\end{array}$ \\
\hline $\mathrm{k}_{2}$ & $\begin{array}{l}\text { Apparent first order rate constant for lipid to plastic } \\
\text { transport }\left(\mathrm{d}^{-1}\right) \text {, estimated as } \mathrm{k}_{1} / \mathrm{K}_{\text {PLIP. }}\end{array}$ \\
\hline $\mathrm{k}_{\mathrm{derm}}$ & $\begin{array}{l}\text { Rate constant for uptake from water }\left(\mathrm{L} / \mathrm{g} \mathrm{DW} \mathrm{d}^{-1}\right) \text {. See } \\
\text { Table A5.2. }\end{array}$ \\
\hline $\mathrm{k}_{\text {loss }}$ & Loss rate constant $\left(\mathrm{g} / \mathrm{g} \mathrm{DW} \mathrm{d}^{-1}\right)$. See Table A5.2. \\
\hline $\mathrm{K}_{\mathrm{OC}}$ & $\begin{array}{l}\text { Sediment organic carbon - water partition coefficient (- } \\
\text { ).Taken from Seth et al. }{ }^{225} \text { for all scenarios. }\end{array}$ \\
\hline $\mathrm{K}_{\mathrm{OW}}$ & $\begin{array}{l}\text { Octanol water partition coefficient (-). From Van Noort et } \\
\text { al. }{ }^{232}\end{array}$ \\
\hline $\mathrm{K}_{\mathrm{P}}$ & $\begin{array}{l}\text { Sediment-water partition coefficient (-). Calculated as } \\
\mathrm{Kp}=\mathrm{f}_{\mathrm{OC}} \mathrm{K}_{\mathrm{OC}} \text {. }\end{array}$ \\
\hline
\end{tabular}


Table A5.5. Continued.

\begin{tabular}{|c|c|}
\hline $\mathrm{K}_{\text {PLIP }}$ & $\begin{array}{l}\text { Lipid - plastic equilibrium partition coefficient; ratio } \\
\text { between the lipid water partition coefficient }\left(\mathrm{K}_{\mathrm{LIP}}\right) \text { and } \\
\text { plastic - water partition coefficient }\left(\mathrm{K}_{\mathrm{PL}}\right) . \mathrm{K}_{\mathrm{LIP}} \text { is } \\
\text { approximated as } \mathrm{K}_{\mathrm{LIP}}=1000 * \mathrm{k} \text {-derm/(flip*k-loss). }\end{array}$ \\
\hline $\mathrm{L}_{\mathrm{t}}$ & Length of the worm $(\mathrm{mm})$. Measured. \\
\hline $\mathrm{M}_{\mathrm{PS}}$ & Mass of plastic in bioassay system (kg DW) \\
\hline $\mathrm{M}_{\mathrm{SED}}$ & Mass of sediment in bioassay system (kg DW) \\
\hline Q & $\begin{array}{l}\text { Volumetric flow rate ingested particles }\left(\mathrm{mm}^{3} / \mathrm{d}\right) \text {, calculated } \\
\text { according to Equation A5.9. }\end{array}$ \\
\hline $\mathrm{r}_{\mathrm{P}}$ & $\begin{array}{l}\text { Radius of plastic particle }(\mathrm{m}) \text {. According to materials used } \\
\text { in the bioassays (Chapter } 4)^{51} \text { or present in the other } \\
\text { scenario studies. }\end{array}$ \\
\hline $\mathrm{r}_{\mathrm{t}}$ & Radius of the worm (mm). Measured (Chapter 4). \\
\hline $\mathrm{S}_{\mathrm{SED}}$ & $\begin{array}{l}\text { Mass fraction of sediment particles ingested (-). Inferred } \\
\text { from the physical mixture of sediment and plastic particles, } \\
\text { assuming non-specific feeding. }\end{array}$ \\
\hline $\mathrm{S}_{\mathrm{PL}}$ & $\begin{array}{l}\text { Mass fraction of plastic particles ingested (-). Inferred from } \\
\text { the physical mixture of sediment and plastic particles, } \\
\text { assuming non-specific feeding. }\end{array}$ \\
\hline $\mathrm{V}_{\text {gut }}$ & $\begin{array}{l}\text { Lugworm gut volume }\left(\mathrm{mm}^{3}\right) \text {, calculated according to } \\
\text { Equation A5.9. }\end{array}$ \\
\hline $\mathrm{W}_{\mathrm{t}}$ & $\begin{array}{l}\text { Dry weight of organism }(\mathrm{g}) \text { in bioassay. Inferred from wet } \\
\text { weight measurements and dry weight - wet weight (DW) } \\
\text { ratios measured for subsample of worms. }\end{array}$ \\
\hline
\end{tabular}


Figures

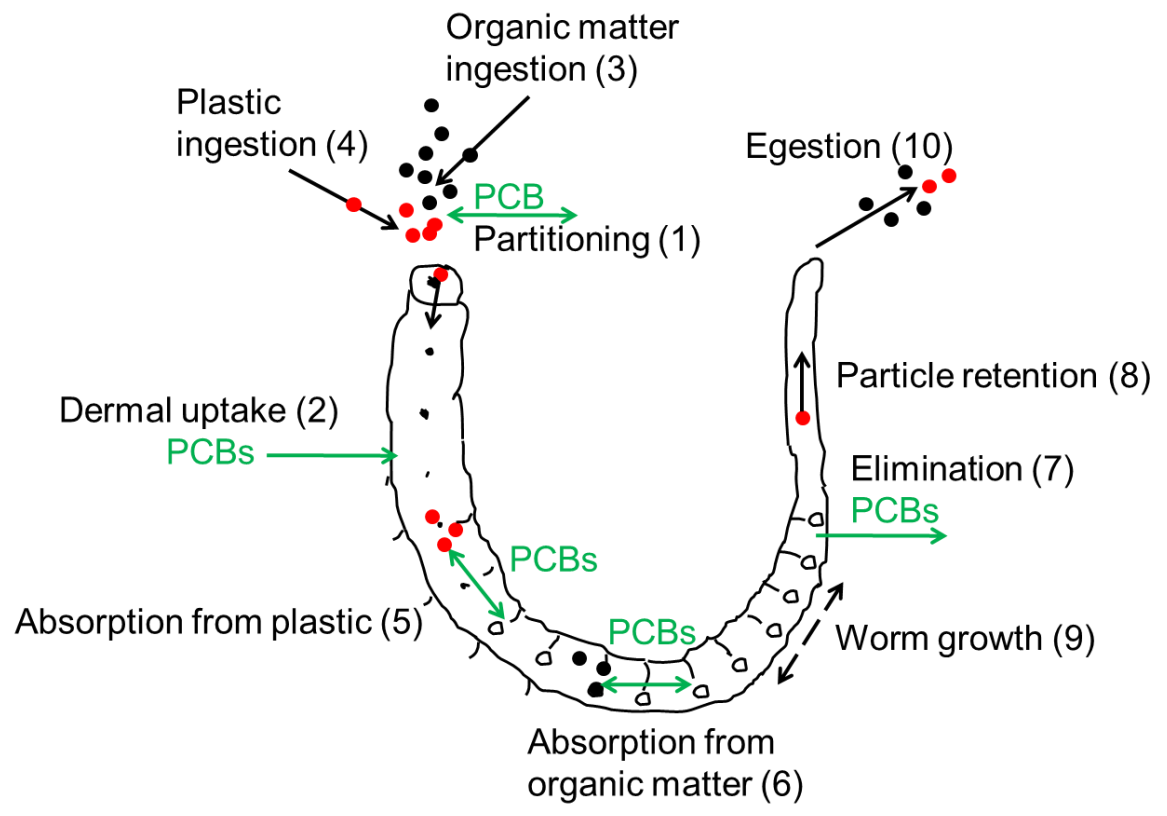

Figure A1. Schematic representation of modeled processes. Adapted from Besseling et al. ${ }^{51}$

1. Partitioning between plastic sediment and water

2. Dermal uptake

3. Organic matter ingestion

4. Plastic ingestion

5. Absorption from plastic

6. Absorption from organic matter

7. Elimination

8. Particle retention

9. Worm growth

10. Particle egestion (sediment and plastic) 

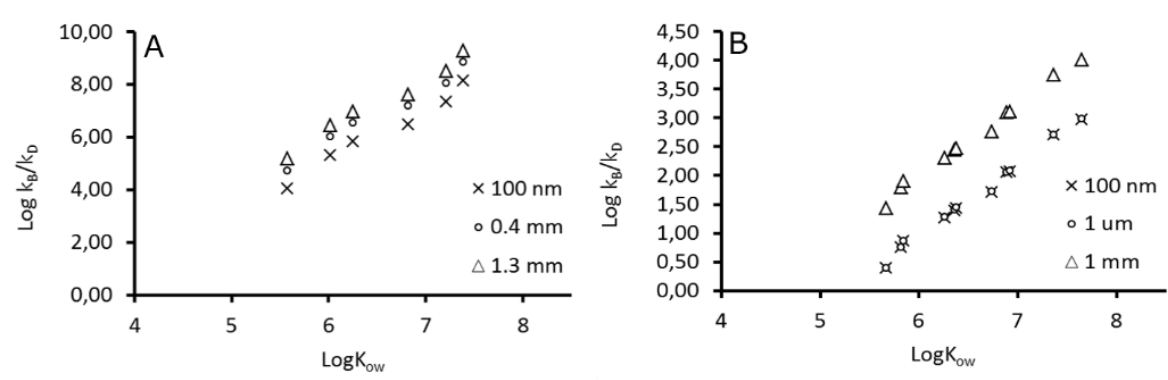

Figure A5.2. Log of the dimensionless ratio $k_{B} / k_{D}$ for polystyrene $(A)$ and polyethylene $(B)$ microparticles with a diameter of $100 \mathrm{~nm}, 0.4 \mathrm{~mm}$ and $1.3 \mathrm{~mm}$. If $\log \left(k_{B} / k_{D}\right)>0$, polymer diffusion is rate limiting. The ratio increases with $\log K_{\text {ow }}$ because DOM facilitated transport through the stagnant boundary layer increases with LogK $K_{\text {ow. }}$. Polystyrene values are higher than for polyethylene because the partition coefficient for polystyrene is lower (Eq. A5.3).
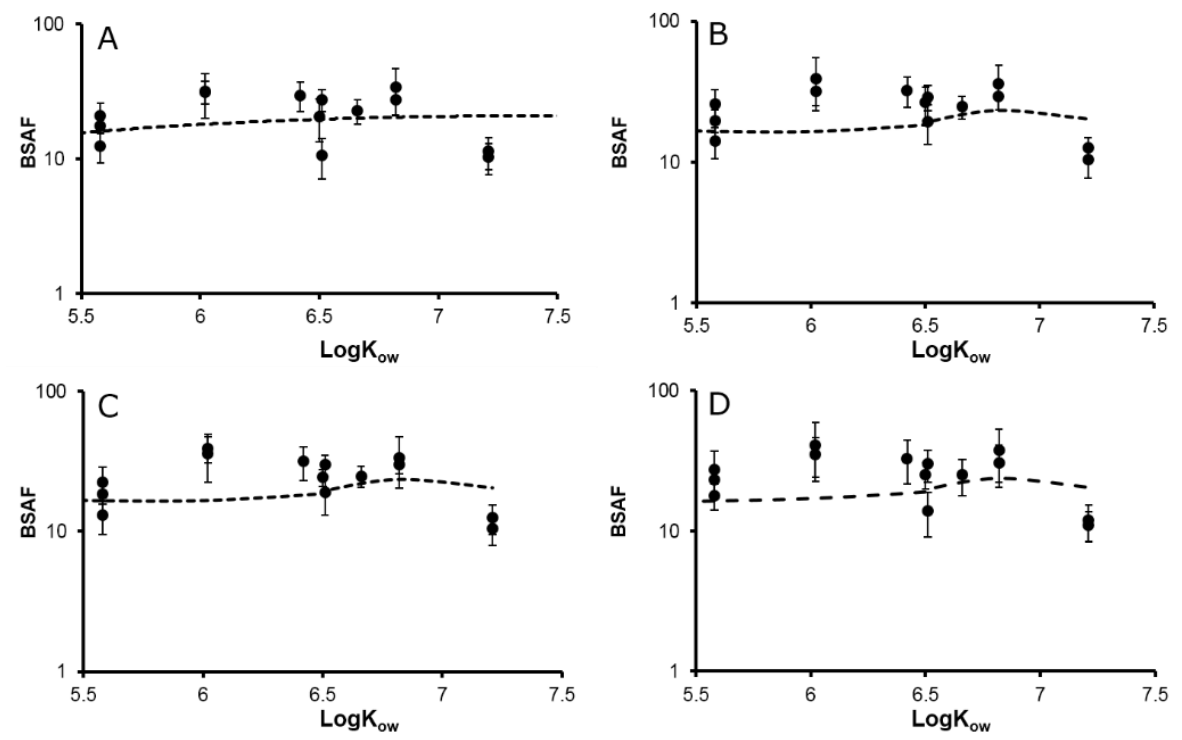

Figure A5.3. Measured ( $\bullet$ ) and modeled (---) PCB BSAF values for A. Marina in sediment with $0 \%(A), 0.074 \%(B), 0.74 \%(C)$ and $7.4 \%(D)$ polystyrene microplastic, calculated from data of Chapter $4 .^{51}$ 
Figures
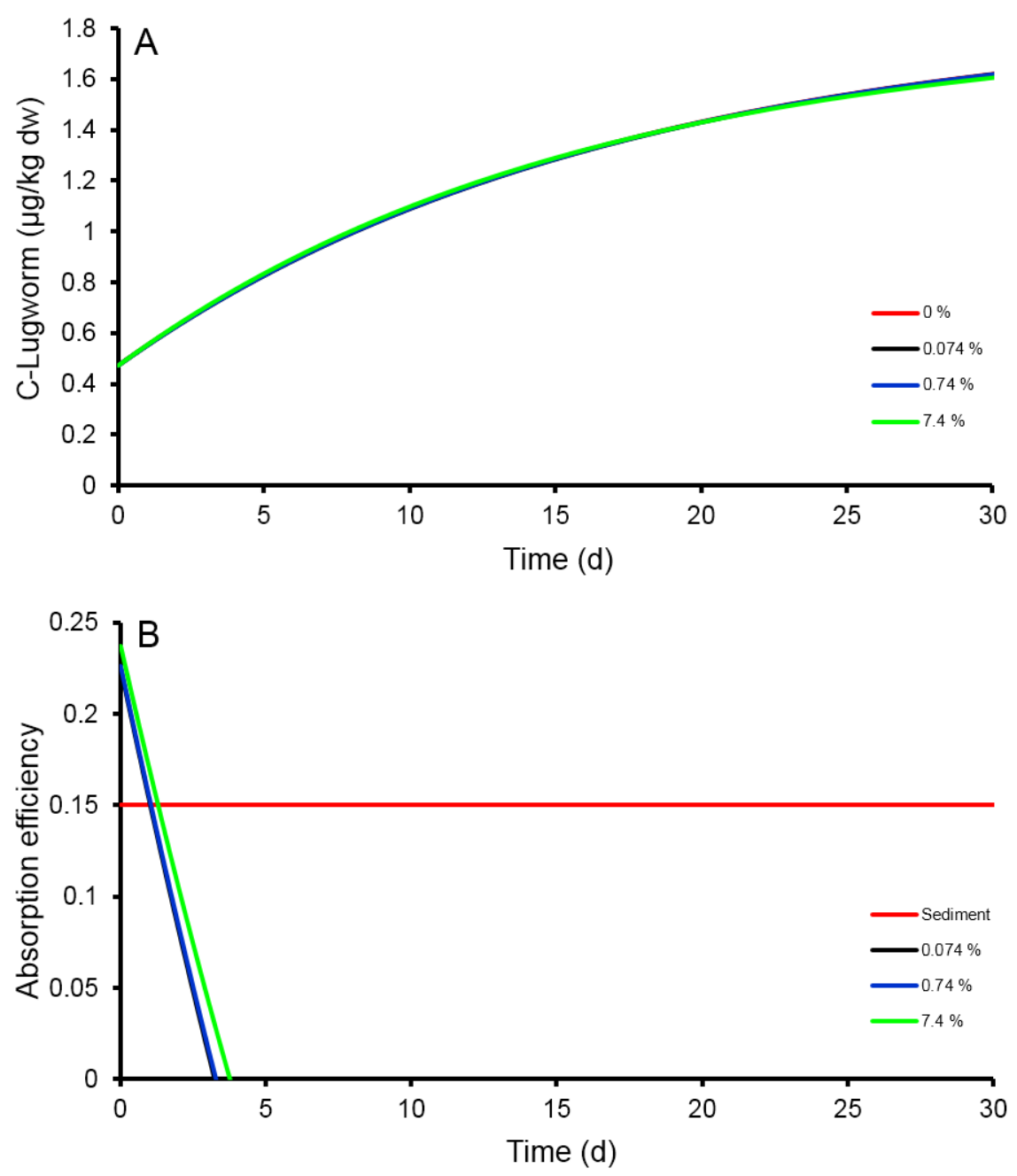

Figure A5.4: Simulated PCB105 concentration as a function of time in closed bioassay systems with polystyrene (A), and absorption efficiencies from sediment and polystyrene (B), for three polystyrene concentrations. Default exchange rate constant $k_{l}$ is $10 d^{-1}$. 


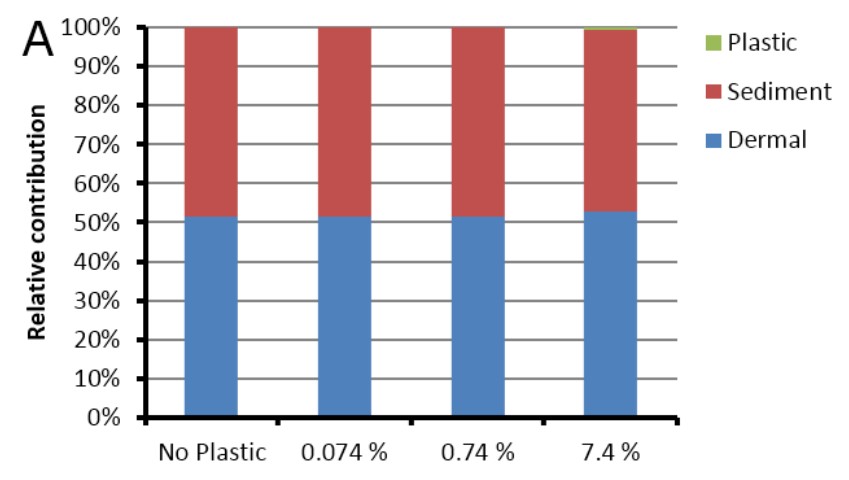

Percentage of plastic

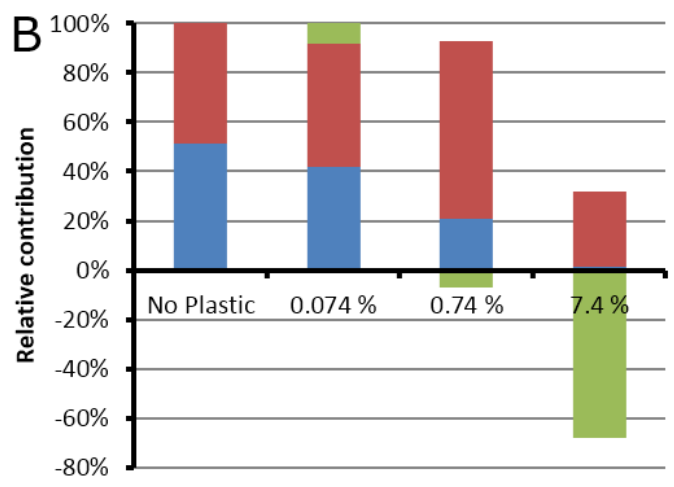

- Plastic

- Sediment

- Dermal

Percentage of plastic

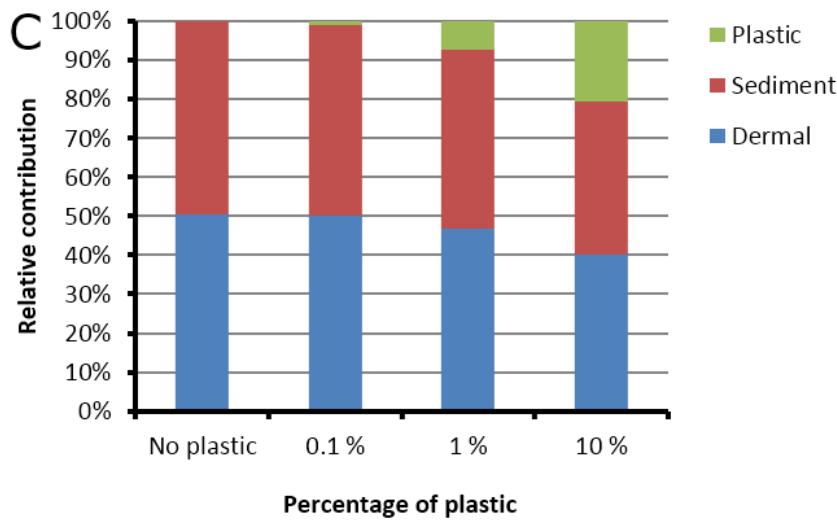

Figure A5.5. Relative contribution to total uptake of PCB105 by the exposure pathways plastic, sediment and water (dermal uptake) as a function of plastic concentration in the sediment, for polystyrene in closed bioassay systems (A), polyethylene in closed bioassay systems (B), and polyethylene in open marine systems $(C)$, at a default exchange rate constant $k_{1}$ of $10 d^{-1}$. Negative values for plastic relate to depuration of the worm by plastic ('cleaning'). 
Figures

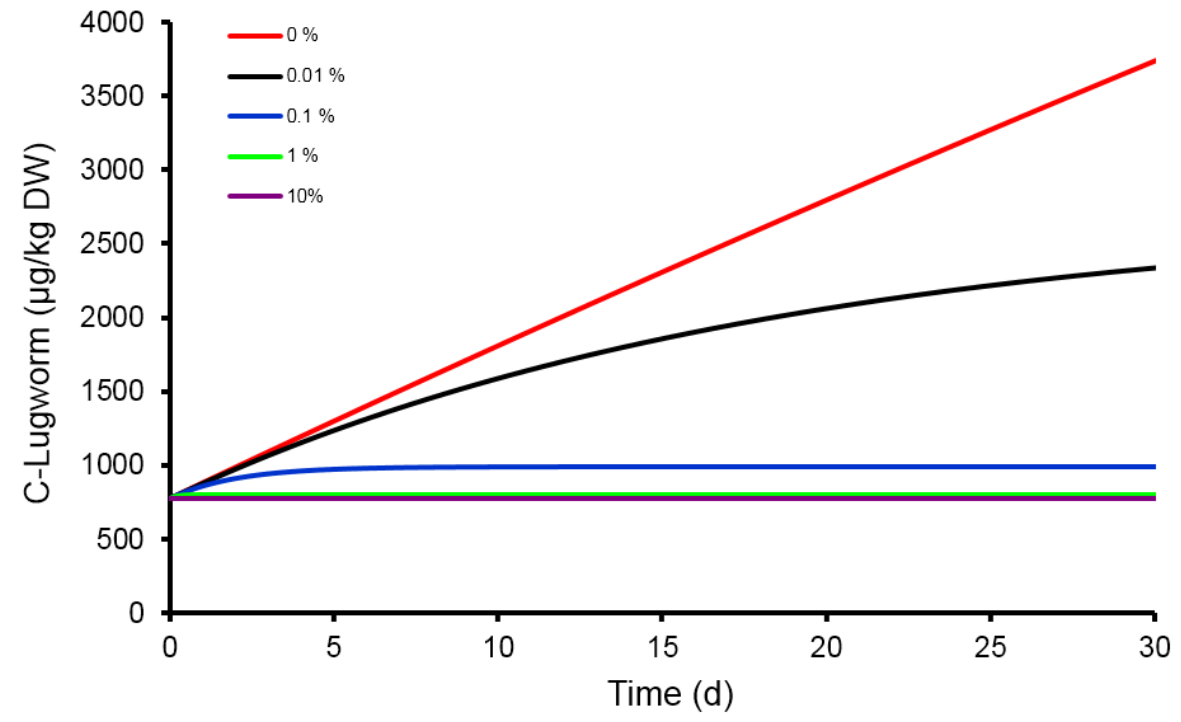

Figure A5.6. Simulated PCB105 concentration as a function of time for an open marine system with polyethylene for three polyethylene concentrations. Default exchange rate constant $k_{1}$ is $10 d^{-1}$. 

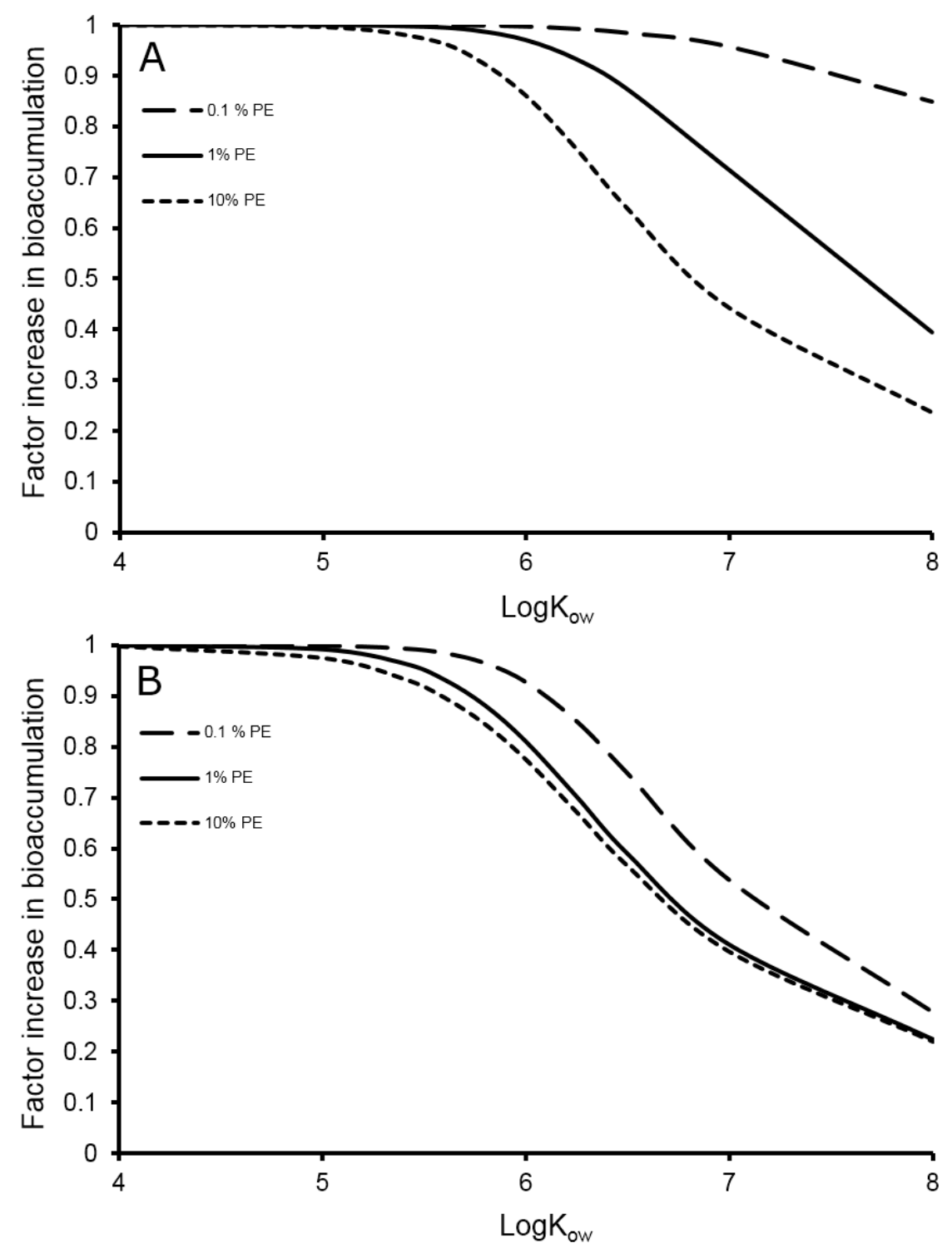

Figure A5.7. Steady-state bioaccumulation in the lugworm in sediment with plastic, relative to a scenario without plastic for open marine systems, for 0.1, 1 and $10 \%$ polyethylene. Simulations are for microplastic particles with diameter $\sim 1 \mathrm{~mm}\left(k_{1}=1 d^{-1}\right)$ (panel A), and for submicron-nanoplastic particles with diameter $0.1-1 \mu \mathrm{m}, k_{1}>100 d^{-1}$ (panel B). 


\section{Appendix to Chapter 6}

1. Materials and methods

2. Calculation of $\mathrm{C}_{P W} \mathrm{t=28}, \mathrm{K}_{\mathrm{OM}}, \mathrm{K}_{\mathrm{PE}}, \mathrm{C}_{\mathrm{PE}}, \mathrm{C}_{\mathrm{OM}}, \mathrm{BSAF}, \mathrm{BPAF}$ and $\mathrm{BAF}$ (including Eq. A6.1-A6.9)

3. Biodynamic model for leaching of chemicals from plastic (including Eq. A10-17 and Table A6.4)

4. Biodynamic model for leaching of chemicals from plastic (including Eq. A6.10-A6.17 and Table A6.4)

5. Table A6.1-A6.3

6. Figure A6.1-A6.8 


\section{Materials and methods}

Materials. Polyethylene (PE, green fluorescent UVPMS-BG, spherical, diameter $10-180 \mu \mathrm{m}$, density $0.94 \mathrm{~kg} / \mathrm{L}$ ) was purchased from Cospheric, Santa Barbara, USA. PE polymer identity was confirmed by FTIR (ThermoFisher, iN10 MX). For microscope images and particle size distributions of the PE the reader is referred to the publication by Velzeboer et al. ${ }^{75}$ Polyoxymethylene sheets (POM, $76 \mu \mathrm{m}$ thickness) from CS Hyde Co (Lake Villa, IL, US) were used as passive samplers as in earlier studies. ${ }^{75,249,253}$ PCB congeners 28, 31, 44, 52, 101, 118, 138, 153, 170 and 180 were obtained from Dr. Ehrenstorfer GmbH (Augsburg, Germany). Acetone and n-hexane (picograde) were obtained from Promochem (Wesel, Germany), diatomaceous earth from Dionex (Camberly, UK) and isooctane from Acros (Geel, Belgium). Silicia gel 63 - 200 mesh was obtained from Merck KGaA (Darmstadt, Germany) and activated overnight at $180{ }^{\circ} \mathrm{C}$. Aluminium oxide super was obtained from ICN Biomedicals (Eschwege, Germany) and deactivated with 10 mass $\%$ Barnstead ${ }^{\mathrm{TM}}$ nanopure water. Before use, copper powder, $99.7 \%$, from Merck KGaA (Darmstadt, Germany) was Soxhletextracted with hexane for $4 \mathrm{~h}$.

Sediment sampling and pre-treatment. The sediment was sampled from the Eastern Scheldt (Location 'Oesterput', the Netherlands, Chapter 4) ${ }^{51,185}$ and had an average density of $1.8 \mathrm{~kg} / \mathrm{L}$ (wet weight; WW) and organic matter $(\mathrm{OM})$ content of $1.15 \% \mathrm{DW}$. It was sieved in order to remove objects $>2 \mathrm{~mm}$ before usage. The sediment was divided in four portions, one for each treatment. PE was added to the sediment, accomplishing plastic concentrations of $0,0.05$ and $0.5 \% \mathrm{DW}$, which are within and above the range found in the marine environment, respectively (Chapter 4). ${ }^{39,51,165,254}$ Subsequently, the sediment-plastic mixture was spiked with the PCB congeners. After addition of PE and PCBs, the sediment was mixed for six weeks. Mixing was performed on a roller apparatus (Willemsen Proefinstallaties, Spijk, the Netherlands). During the last four weeks of mixing, three POM passive samplers $(\approx 0.3 \mathrm{~g} \text { each })^{75,172,249,253}$ were added to each PE-sediment mixture for determination of porewater PCB concentrations. Six and four weeks have been shown sufficient to reach chemical equilibrium between sediment porewater, and 10-180 $\mu \mathrm{m}$ PE particles and POM passive samplers, respectively. ${ }^{75,172,173,216,223,224,249,255-258}$ The mass ratio of OM to POM was 300 to 1 , which implies that passive sampling occurred under negligible chemical depletion conditions, ${ }^{172,249}$ that is, POM extracted less than $1.6 \%$ of each PCB congener present in de sediment. 
Test organisms. A. marina were collected by a professional bait collector (Lugworm wholesale business Rotgans, Hippolytushoef, the Netherlands) in the southern Wadden Sea. The lugworms contained background concentrations of PCBs, representing Dutch estuarine conditions (Fig. 6.2, A6.8, left markers). The lugworms were acclimatized at experimental temperature and the 'digging-in' speed of the organisms in clean sediment was tested, to select fit, fast digging organisms. The organisms were pooled randomly. The $\mathrm{n}=5$ pool weights averaged $18.2 \pm 1.4 \mathrm{~g}$. Three pools were directly stored at $-18{ }^{\circ} \mathrm{C}$ for determination of initial PCB concentrations.

Maintenance. Three times a week, dissolved oxygen (DO) saturation, temperature, $\mathrm{pH}$ and salinity were measured with a HACH HQd Field Case. Reagent kits from Aquamerck (Darmstadt, Germany) were used to monitor $\mathrm{NH}_{4}{ }^{+}$and $\mathrm{NO}_{2}{ }^{-}$(kit range $0.5-10 \mathrm{mg} / \mathrm{L}$ and $0.025-0.5 \mathrm{mg} / \mathrm{L}$ respectively). Averages were DO $9.7 \mathrm{mg} / \mathrm{L}$ ( $95 \%$ saturation), temperature $14.2{ }^{\circ} \mathrm{C}, \mathrm{pH} 8.1$, salinity $33.8 \%, \mathrm{NH}_{4}{ }^{+} 0.7 \mathrm{mg} / \mathrm{L}$ and $\mathrm{NO}_{2}{ }^{-} 0.05 \mathrm{mg} / \mathrm{L}$. Evaporation was compensated by adding demineralised water. The overlying water was continuously aerated and about $30 \mathrm{~L}$ was refreshed with Eastern Scheldt water three times a week, after the water quality measurements.

\section{Calculation of $\mathrm{C}_{P W} \mathrm{t}=28, \mathrm{~K}_{\mathrm{OM}}, \mathrm{K}_{\mathrm{PE}}, \mathrm{C}_{\mathrm{PE}}, \mathrm{C}_{\mathrm{OM}}$, BSAF, BPAF and BAF}

Concentrations of PCBs on polyoxymethylene passive samplers $\left(\mathrm{C}_{\mathrm{POM}}\right)$ were used to determine porewater concentrations of $\mathrm{PCBs}\left(\mathrm{C}_{\mathrm{PW}, \mathrm{t}=0}\right)$, at the start of the experiment. This was done by using the partitioning coefficients to POM in Table A2 (K $\left.\mathrm{K}_{\mathrm{POM}}\right)$ from Hawthorne et al. ${ }^{249}$ and Eq. A1:

$$
C_{P W, t=0}=\frac{C_{P O M}}{K_{P O M}}
$$

From the concentrations in the sediment and the porewater in the $0 \% \mathrm{PE}$ treatments at the start of the experiment, respectively $\mathrm{C}_{\mathrm{SED}, \text { total }}$ and $\mathrm{C}_{\mathrm{PW}, \mathrm{t}=0}$, the partitioning coefficient to $\mathrm{OM}\left(\mathrm{K}_{\mathrm{OM}}\right)$ could be calculated by using Eq. A6.2:

$K_{O M}=\frac{C_{S E D, t o t a l}}{C_{P W}} \times \frac{1}{f_{O M}}$

in which $\mathrm{f}_{\mathrm{OM}}$ is the fraction $\mathrm{OM}$ in the $0 \% \mathrm{PE}$ treatments at $t=0$. 
Similarly, from the concentrations in the sediment and the porewater in the treatments with $\mathrm{PE}$ at the start of the experiment, respectively $\mathrm{C}_{\mathrm{SED} \text {,toal }}$ and $\mathrm{C}_{\mathrm{PW}, \mathrm{t}=0}$ the partitioning coefficient to $\mathrm{PE}\left(\mathrm{K}_{\mathrm{PE}}\right)$ could be calculated by using Eq. A6.4:

$K_{P E}=\left(\frac{C_{S E D \text { total }}}{C_{P W, t=0}}-f_{O M} \times K_{O M}\right) \times \frac{1}{f_{P E}}$

in which $\mathrm{f}_{\mathrm{OM}}$ is the fraction $\mathrm{OM}$ in the treatments with $\mathrm{PE}$ at $t=0, \mathrm{~K}_{\mathrm{OM}}$ is the partitioning coefficient to $\mathrm{OM}$ as calculated in Eq. A6.2 and $\mathrm{f}_{\mathrm{PE}}$ is the fraction $\mathrm{PE}$ in the sediment of the treatments with PE.

The above equations show how from measured concentrations in sediment and porewater at $t=0 \mathrm{~d}$, equilibrium partition coefficients for $\mathrm{OM}\left(\mathrm{K}_{\mathrm{OM}}\right)$ and $\mathrm{PE}$ $\left(\mathrm{K}_{\mathrm{PE}}\right)$ can be derived. Now we use the reversed calculation at $t=28 \mathrm{~d}$, that is, porewater concentrations after $28 \mathrm{~d}\left(\mathrm{C}_{\mathrm{PW}, \mathrm{t}=28}\right)$ are calculated using the measured concentration in sediment after $28 \mathrm{~d}\left(\mathrm{C}_{\mathrm{SED}, \text { total }}\right)$ and the aforementioned values for $\mathrm{K}_{\mathrm{OM}}$ and $\mathrm{K}_{\mathrm{PE}}$. This assumes that these partition coefficients remain constant during $28 \mathrm{~d}$ of exposure. The calculation is done following Eq. A6.4:

$C_{P W, t=28}=\frac{C_{S E D, t o t a l}}{\left(f_{O M} \times K_{O M}+f_{P E} \times K_{P E}\right)}$

in which $\mathrm{C}_{\mathrm{SED} \text {,total }}$ is the concentration of PCBs in the total sediment mixture, including organic matter $(\mathrm{OM})$ and polyethylene (PE) at $t=28 \mathrm{~d}$. To verify whether porewater concentrations were sufficiently constant during the $28 \mathrm{~d}$ assay, we compare $\mathrm{C}_{\mathrm{PW}, \mathrm{t}=28}$ (Eq. A4, Fig. A6.3) with $\mathrm{C}_{\mathrm{PW}, \mathrm{t}=0}$ (Eq. A6.1, Fig. 6.1). The constant partitioning between sediment organic matter, PE and sediment porewater over $28 \mathrm{~d}$ was confirmed by the excellent agreement between $\mathrm{C}_{\mathrm{PW}, \mathrm{t}=0}$ and $\mathrm{C}_{\mathrm{PW}, \mathrm{t}=28}$ as illustrated in Fig. A6.4.

The PCB concentration on the PE, $\mathrm{C}_{\mathrm{PE}}$ can be calculated at $t=0$ and $t=28$ with Eq. A6.5:

$C_{P E}=C_{P W} \times K_{P E}$

with $\mathrm{C}_{\mathrm{PW}}$ being the concentration of PCBs in the porewater at either $t=0$ or $t=28$ (Eq. A6.1 or A6.4) and $\mathrm{K}_{\mathrm{PE}}$ the partitioning coefficient to PE (Eq. A3). Similarly, the PCB concentration on the OM, $\mathrm{C}_{\mathrm{OM}}$ can be calculated with Eq. A6.6: 
Biodynamic model

$C_{O M}=C_{P W} \times K_{O M}$

with $\mathrm{C}_{\mathrm{PW}}$ being the concentration of PCBs in the porewater at either $t=0$ or $t=28$ (Eq. A6.1 or A6.4) and $\mathrm{K}_{\mathrm{OM}}$ the partitioning coefficient to OM (Eq. A6.2).

After the 28 days exposure assay, PCB concentrations in the tissue of the lugworms were determined and normalised on the lipid concentration in the tissue $\left(\mathrm{C}_{\text {lip }}\right)$. Biota to sediment accumulation factors (BSAFs) normalised on lipids and sediment OM were subsequently calculated by using Eq. A6.7:

$B S A F_{\text {lip }, O M}=\frac{C_{\text {lip }}}{C_{O M}}$

in which $\mathrm{C}_{\mathrm{OM}}$ is the PCB concentration in $\mathrm{OM}$ of the sediment, calculated before by Eq. A6.6. Likewise, the new metric; biota plastic accumulation factor (BPAF), was calculated by using Eq. A6.8:

$B P A F_{\text {lip }, P E}=\frac{C_{\text {lip }}}{C_{P E}}$

with $\mathrm{C}_{\mathrm{PE}}$ being the PCB concentration on PE, calculated by Eq. A6.5. Correspondingly, bioaccumulation factors were calculated from the PCB concentration in the lipids, $\mathrm{C}_{\text {lip }}$, and in the porewater, $\mathrm{C}_{\mathrm{PW}}$, with Eq. A6.9:

$B A F=\frac{C_{\text {lip }}}{C_{P W}}$

Biodynamic model for leaching of chemicals from plastic The model description below follows the description provided in the Appendix of Chapter $5 .{ }^{129}$

Koelmans et al. (Chapter 5) $)^{128,129}$ modeled bioaccumulation of hydrophobic chemicals $\left(\mathrm{dC}_{\mathrm{B}, \mathrm{t}} / \mathrm{dt} ; \mu \mathrm{g} \times \mathrm{g}^{-1} \mathrm{~d}^{-1}\right)$ from an environment containing plastic as a mass balance of uptake and loss processes: 
$\frac{\mathrm{dC}_{\mathrm{B}, \mathrm{t}}}{\mathrm{dt}}=\mathrm{k}_{\mathrm{derm}} \mathrm{C}_{\mathrm{PW}}+\mathrm{IR}\left(\mathrm{S}_{\mathrm{FOOD}} \mathrm{a}_{\mathrm{FOOD}} \mathrm{C}_{\mathrm{FOOD}}+\mathrm{S}_{\mathrm{PL}} \mathrm{C}_{\mathrm{PLR}, \mathrm{t}}\right)-\mathrm{k}_{\text {loss }} \mathrm{C}_{\mathrm{B}, \mathrm{t}}$

The first term in Eq. A6.10 quantifies dermal (including gills) uptake from ambient water. The second term quantifies uptake from ingested food and exchange with plastic particles. The third term quantifies overall loss due to elimination and egestion. The first and third term are parameterised following traditional approaches with $\mathrm{C}_{\mathrm{PW}}(\mu \mathrm{g} / \mathrm{L})$ being the concentration in the ambient

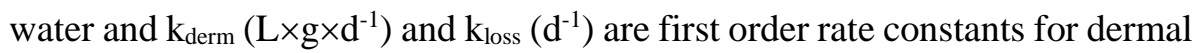
uptake and overall loss through elimination and egestion. Following Hendriks et al. ${ }^{221} \mathrm{k}_{\text {loss }}$ is a minimum value, excluding possible biotransformation. In the

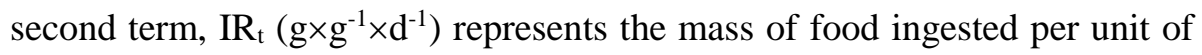
time and organism dry weight, $\mathrm{a}_{\mathrm{FOOD}}$ is the absorption efficiency from food, $\mathrm{S}_{\mathrm{FOOD}}$ and $\mathrm{S}_{\mathrm{PL}}$ are the mass fractions of food and plastic in ingested material respectively $\left(S_{\mathrm{FOOD}}+\mathrm{S}_{\mathrm{PL}}=1\right)$ and $\mathrm{C}_{\mathrm{FOOD}}$ is the chemical concentration in food. The product $a_{F O O D} \times \mathrm{C}_{\mathrm{FOOD}}$ quantifies the contaminant concentration that is transferred from food, i.e. prey species, to the organism during gut passage. Note that for species like fish, weight usually is expressed as wet weight $(\mathrm{WW})$, in which case $\mathrm{IR}_{\mathrm{t}}$ also is based on wet weight. The transferred concentration from plastic during gut passage (GP), $\mathrm{C}_{\mathrm{PLR}, \mathrm{t}},(\mu \mathrm{g} / \mathrm{g})$ is dynamically modeled using (see Chapter $5^{128}$ for detailed derivation):

$C_{P L R, t}=\frac{k_{1} C_{P L}-k_{2} C_{L, t}}{k_{1}+\frac{M_{P L}}{M_{L}} k_{2}}\left(1-e^{-\left(k_{1}+\frac{M_{P L}}{M_{L}} k_{2}\right) G R T}\right)$

In which $\mathrm{k}_{1}$ and $\mathrm{k}_{2}\left(\mathrm{~d}^{-1}\right)$ are forward and backward first order rate constants describing the transport between plastic and biota lipids, GRT is gut residence time (d), $C_{P L}$ and $C_{L, t}(\mu \mathrm{g} / g)$ are the chemical concentrations in the ingested plastic particle and the biota lipids at the moment of ingestion (i.e. $C_{L, t}=$ $C_{B, t} / f_{\text {lip }}, \mu \mathrm{g} / \mathrm{g}$ ), and $\mathrm{M}_{\mathrm{PL}}$ and $\mathrm{M}_{\mathrm{L}}$ are the mass of plastic and lipids in the organism respectively (g). Eq. A6.11 can be rewritten as: ${ }^{129}$

$\mathrm{C}_{\mathrm{PLR}, \mathrm{t}}=\mathrm{A}_{\mathrm{PL}} \mathrm{k}_{1} C_{P L}-\mathrm{A}_{\mathrm{PL}} \mathrm{k}_{2} C_{L, t}$

in which

$A_{P L}=\frac{1-e^{-\left(k_{1}+\frac{M_{P L}}{M_{L}} k_{2}\right) G R T_{t}}}{k_{1}+\frac{M_{P L}}{M_{L}} k_{2}}$ 
If GRT is constant, also $A_{P L}$ is constant over time. Combination of equations A6.10, A6.12 and A6.13 and using $C_{L, t}=C_{B, t} / f_{\text {lip }}$, yields the mass balance equation for bioaccumulation: ${ }^{129}$

$$
\begin{aligned}
& \frac{d C_{B, t}}{d t}=k_{d e r m} C_{W}+I R \times S_{F O O D} a_{F O O D} C_{F O O D}+I R \times S_{P L} A_{P L} k_{1} C_{P L} \\
& -\left(I R \times S_{P L} A_{P L} k_{2} / f_{\text {lip }}+k_{\text {loss }}\right) C_{B, t}
\end{aligned}
$$

for which the following steady-state solution (body burden at steady-state, $\mathrm{C}_{\mathrm{B}}^{\mathrm{SS}}$ ) can be calculated:

$$
C_{B}^{S S}=\frac{k_{d e r m} C_{W}+I R\left(S_{F O O D} a_{F O O D} C_{F O O D}+S_{P L} k_{1} C_{P L} A_{P L}\right)}{I R S_{P L} k_{2} A_{P L} / f_{l i p}+k_{l o s s}}
$$

The steady-state concentration thus reflects the balance between rates for dermal uptake, uptake by food and uptake by plastic ('carrier') all in the numerator, versus 'cleaning' by plastic ingestion and chemical loss, which are covered by the denominator. The analytical solution to Eq. A6.14 is (Koelmans et al.): $:^{129}$

$$
C_{B, t}=\left(C_{B, t=0}-C_{B}^{S S}\right) \times\left(e^{-\left(I R S_{P L} k_{2} A_{P L} / f_{\text {lip }}+k_{\text {loss }}\right) t}\right)+C_{B}^{S S}
$$

The time required to reach $95 \%$ of steady-state ( $\left.\mathrm{tss}_{\mathrm{ss}}\right)$ can be approximated as three times the time constant of the system $\left(1-\mathrm{e}^{-3}\right)$ :

$$
t_{S S}=3 /\left(I R \frac{S_{P L} k_{2} A_{P L}}{f_{\text {lip }}}+k_{\text {loss }}\right)
$$

We modeled bioaccumulation at $28 \mathrm{~d}$ using the analytical solution of Eq. A6.10 and measured values for $\mathrm{C}_{\mathrm{PW}}, \mathrm{C}_{\mathrm{SED}}, \mathrm{C}_{\mathrm{PL}} \mathrm{S}_{\mathrm{SED}}$ and $\mathrm{S}_{\mathrm{PL}}$. The relative share of an uptake pathway (either term 1, 2 or 3 in Eq. A6.10) was quantified as the ratio of the magnitude of that term, and that of the sum of all three uptake terms.

\section{Parameters}

Parameters and variables for the experimental treatments $0 \%$ PE B, $0.05 \%$ PE and $0.5 \% \mathrm{PE}$, were taken from the experimental data and literature and are provided in Table A6.4. 
Table A6.1. PCB concentration added to the sediment of the different treatments at start of the bioassay. PCB concentrations are on a dry weight $(D W)$ basis. The $0 \%$ PE A treatment has similar background quantities spiked for all ten congeners. The 0\% PE B treatment has elevated concentrations of five of the ten congeners spiked to the sediment. These five congeners are also extra spiked to the $0.05 \% \mathrm{DW} P E$ treatment and $0.5 \% \mathrm{DW} P E$ treatment, to compensate for the anticipated chemical dilution by the PE, thus representing the infinite source scenario.

\begin{tabular}{|l|l|c|c|c|c|}
\hline \multirow{2}{*}{$\begin{array}{l}\text { PCB } \\
\begin{array}{l}\text { Congener } \\
\boldsymbol{\mu g / k g ~ D W ~}\end{array}\end{array}$} & Scenario & $\mathbf{4}$ Treatment \\
\cline { 3 - 6 } & & $\begin{array}{c}\mathbf{0 \%} \\
\text { PE A }\end{array}$ & $\begin{array}{c}\mathbf{0 \%} \\
\text { PE B }\end{array}$ & $\begin{array}{c}\mathbf{0 . 0 5 \%} \\
\text { PE }\end{array}$ & $\begin{array}{c}\mathbf{0 . 5 \%} \\
\text { PE }\end{array}$ \\
\hline PCB 28 & Chemical dilution & 0.92 & 3.87 & 1.31 & 3.83 \\
\hline PCB 52 & Chemical dilution & 0.97 & 4.07 & 1.38 & 4.03 \\
\hline PCB 101 & Chemical dilution & 0.92 & 4.90 & 1.41 & 4.86 \\
\hline PCB 153 & Chemical dilution & 0.80 & 4.76 & 1.28 & 4.71 \\
\hline PCB 180 & Chemical dilution & 0.93 & 5.65 & 1.50 & 5.61 \\
\hline PCB 31 & Infinite source & 0.92 & 0.97 & 1.01 & 0.96 \\
\hline PCB 44 & Infinite source & 0.94 & 0.99 & 1.03 & 0.98 \\
\hline PCB 118 & Infinite source & 0.94 & 0.98 & 1.02 & 0.98 \\
\hline PCB 138 & Infinite source & 0.94 & 0.99 & 1.03 & 0.98 \\
\hline PCB 170 & Infinite source & 0.94 & 0.98 & 1.02 & 0.97 \\
\hline
\end{tabular}

Table A6.2. Partitioning coefficients used for calculations and comparisons. ${ }^{75,217,225,232,249}$

\begin{tabular}{|l|l|l|l|l|l|}
\hline $\begin{array}{l}\text { PCB } \\
\text { congener }\end{array}$ & $\begin{array}{l}\text { Log } \\
\text { Kow }^{(232)}\end{array}$ & $\begin{array}{l}\text { Log } \\
\mathbf{K}_{\text {POM }} \\
\mathbf{L / k g} \mathbf{g}^{(24)}\end{array}$ & $\begin{array}{l}\mathbf{L o g} \\
\mathbf{K}_{\mathbf{O M}} \\
\mathbf{L / k g}\end{array}$ & $\begin{array}{l}\mathbf{L o g} \mathbf{K}_{\mathbf{P E}} \\
\mathbf{L} / \mathbf{k g}^{(225)}\end{array}$ & $\begin{array}{l}\mathbf{L o g} \mathbf{K}_{\mathbf{P E}} \\
\mathbf{L / k g} \mathbf{k g}^{(75)}\end{array}$ \\
\hline PCB 28 & 5.58 & 5.68 & 4.99 & 5.85 & 6.23 \\
\hline PCB 31 & 5.58 & 5.51 & 4.96 & 5.81 & 6.05 \\
\hline PCB 44 & 6.02 & 5.65 & 5.01 & 5.87 & 6.03 \\
\hline PCB 52 & 6.02 & 5.65 & 5.07 & 5.94 & 6.04 \\
\hline PCB 101 & 6.42 & 5.90 & 5.56 & 6.50 & 6.55 \\
\hline PCB 118 & 6.51 & 6.32 & 5.85 & 6.83 & 6.30 \\
\hline PCB 138 & 6.82 & 6.50 & 6.01 & 7.01 & 6.74 \\
\hline PCB 153 & 6.82 & 6.64 & 6.08 & 7.09 & 7.59 \\
\hline PCB 170 & 7.21 & 6.54 & 6.36 & 7.41 & 8.03 \\
\hline PCB 180 & 7.21 & 6.67 & 6.49 & 7.56 & 7.94 \\
\hline
\end{tabular}


Tables

Table A6.3: Relative importance (\%) of the various PCB uptake pathways for A. marina at a realistic $(0.05 \%)$ and a high $(0.5 \%) P E$ concentration in marine sediment. ${ }^{a}{ }^{\text {) }}$

\begin{tabular}{|c|c|c|c|c|c|c|c|c|c|c|}
\hline & \multicolumn{5}{|c|}{ IS Scenario PCBs } & \multicolumn{5}{|c|}{ CD Scenario PCBs } \\
\hline & 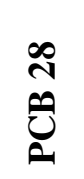 & $\begin{array}{l}\hat{0} \\
\text { in } \\
\tilde{0}\end{array}$ & $\underset{\theta}{\theta}$ & $\stackrel{\infty}{n}$ & 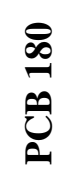 & $\begin{array}{l}\infty \\
0 \\
0\end{array}$ & \begin{tabular}{l}
$\nexists$ \\
\multirow{2}{*}{}
\end{tabular} & $\underset{\sim}{=}$ & $\underset{n}{\infty}$ & $\frac{P}{0}$ \\
\hline & \multicolumn{10}{|c|}{ Environmentally realistic dose of $0.05 \%$ PE } \\
\hline $\begin{array}{l}\text { Uptake } \\
\text { from } \\
\text { water }\end{array}$ & 96.2 & n.a. & 77.7 & 32.6 & 16.6 & 95.3 & 89.4 & 56.1 & 34.4 & 18.9 \\
\hline $\begin{array}{l}\text { Sediment } \\
\text { ingestion }\end{array}$ & 3.6 & n.a. & 21.6 & 64.4 & 80.7 & 4.6 & 10.3 & 40.7 & 63.5 & 78.5 \\
\hline $\begin{array}{l}\text { Plastic } \\
\text { ingestion }\end{array}$ & 0.1 & n.a. & 0.7 & 3.0 & 2.8 & 0.1 & 0.3 & 3.2 & 2.2 & 2.6 \\
\hline & \multicolumn{10}{|c|}{ High dose of $0.5 \% \mathrm{PE}$} \\
\hline $\begin{array}{l}\text { Uptake } \\
\text { from } \\
\text { water }\end{array}$ & 95.8 & n.a. & 75.5 & 26.2 & 10.7 & 95.6 & 89.5 & 49.7 & 28.4 & 12.9 \\
\hline $\begin{array}{l}\text { Sediment } \\
\text { ingestion }\end{array}$ & 1.9 & n.a. & 11.1 & 27.4 & 27.7 & 2.4 & 5.4 & 19.1 & 27.8 & 28.2 \\
\hline $\begin{array}{l}\text { Plastic } \\
\text { ingestion }\end{array}$ & 2.3 & n.a. & 13.4 & 46.4 & 61.6 & 2.0 & 5.1 & 31.2 & 43.8 & 58.9 \\
\hline
\end{tabular}

a) Based on evaluation of the first three terms in Eq. 6.1 of the main manuscript. The model used variable values from the actual experiments, established default parameters for uptake from water and sediment (Chapter 5), ${ }^{128,129,221,245}$ and optimized parameters for ingestion rate (IR) and plastic-gut fluid exchange coefficient $k_{l}$.

b) Omitted due to detection problems. 


\begin{tabular}{|c|c|c|c|c|c|c|c|c|c|c|c|c|c|c|c|c|c|c|c|c|c|c|c|c|c|}
\hline 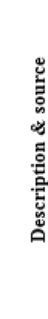 & & & & 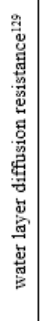 & 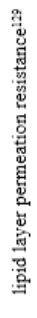 & 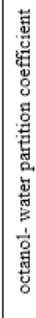 & 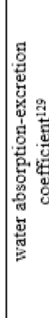 & 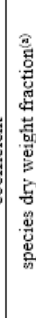 & 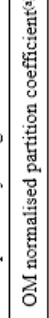 & 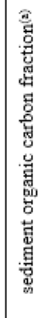 & 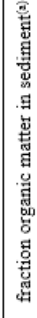 & 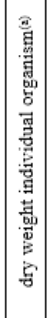 & 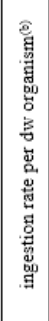 & 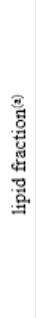 & 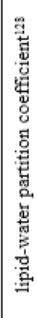 & 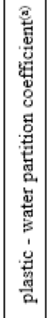 & 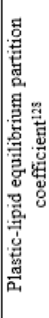 & 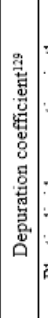 & 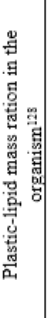 & 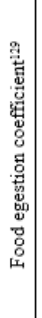 & 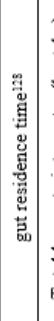 & 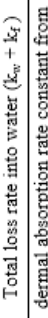 & & 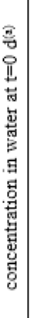 & \\
\hline 吾 & & 만 & & 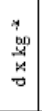 & $\frac{\frac{p 0}{x}}{x}$ & ' & 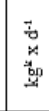 & & & & ' & on & 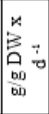 & & ' & & & & on & & 0 & & 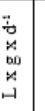 & 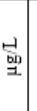 & 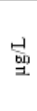 \\
\hline & 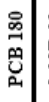 & 总 & สู่ & $\begin{array}{l}\infty \\
\tilde{0} \\
\dot{0}\end{array}$ & $\mathscr{0}$ & $\vec{r}$ & ః्रे & $\stackrel{t}{0}$ & 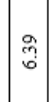 & 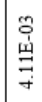 & $\stackrel{\circ}{\circ}$ & 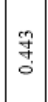 & \begin{tabular}{|l|l}
$\infty$ \\
$\vdots$ \\
$\alpha$ \\
$\alpha$
\end{tabular} & $\begin{array}{l}\tilde{\partial} \\
0 \\
0\end{array}$ & 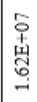 & & 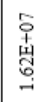 & 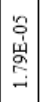 & & $\begin{array}{l}\vec{J} \\
\stackrel{\overrightarrow{0}}{0} \\
0\end{array}$ & $\mid$\begin{tabular}{l}
2 \\
\hdashline \\
0 \\
0
\end{tabular} & $\begin{array}{l}\vec{J} \\
\overrightarrow{0} \\
0\end{array}$ & $\frac{a}{c}$ & 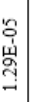 & \\
\hline & 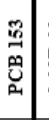 & 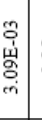 & ત્રે & $\begin{array}{l}0 \\
0 \\
0 \\
0\end{array}$ & $\mathscr{0}$ & \begin{tabular}{|c}
0 \\
0 \\
0
\end{tabular} & ષ્ণి & $\stackrel{\Xi}{0}$ & o. & 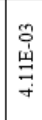 & 응 & 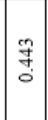 & \begin{tabular}{|l}
$\infty$ \\
$\vdots$ \\
$\alpha$ \\
$\alpha$
\end{tabular} & $\begin{array}{l}\tilde{0} \\
\dot{0}\end{array}$ & 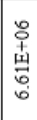 & & $\begin{array}{l}0 \\
0 \\
1 \\
\text { 怘 } \\
0 \\
0\end{array}$ & $\begin{array}{l}\text { 总 } \\
\text { 岁 } \\
\text { 子 }\end{array}$ & & $\begin{array}{l}\mathcal{T} \\
\stackrel{7}{0} \\
0\end{array}$ & 㽬 & 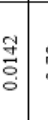 & 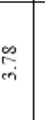 & 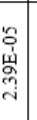 & \\
\hline 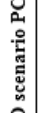 & $\begin{array}{l}\overrightarrow{0} \\
\overrightarrow{0} \\
\text { : } \\
\text { | }\end{array}$ & 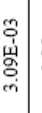 & ત્વ & $\begin{array}{l}0 \\
0 \\
0 \\
0\end{array}$ & $\mathscr{0}$ & f7 & ః् & $\stackrel{\vec{J}}{0}$ & $\overrightarrow{i n}$ & 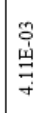 & $\stackrel{\circ}{\circ}$ & 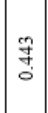 & \begin{tabular}{|c|}
$\infty$ \\
$\vdots$ \\
$\alpha$ \\
$\alpha$
\end{tabular} & $\begin{array}{l}\stackrel{m}{0} \\
\stackrel{0}{0}\end{array}$ & 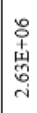 & & 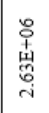 & 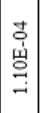 & & \begin{tabular}{l}
7 \\
\multirow{0}{0}{} \\
0
\end{tabular} & $\mid \begin{array}{l}2 \\
- \\
0 \\
0\end{array}$ & 管 & $\begin{array}{l}\infty \\
\text { co } \\
\text { in }\end{array}$ & 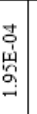 & - \\
\hline & 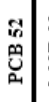 & 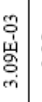 & 'ָ & $\begin{array}{l}\infty \\
0 \\
0 \\
0\end{array}$ & $\mathscr{0}$ & o. & ¿্ণ & $\stackrel{ \pm}{0}$ & $\begin{array}{c}\vec{\Phi} \\
\stackrel{+}{+}\end{array}$ & 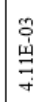 & $\stackrel{\circ}{\circ}$ & 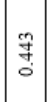 & \begin{tabular}{|l|}
$\infty$ \\
$\vdots$ \\
$\alpha$ \\
$\alpha$
\end{tabular} & $\begin{array}{l}0 \\
0 \\
0\end{array}$ & 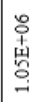 & & 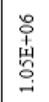 & 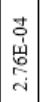 & & $\begin{array}{l}\frac{T}{7} \\
\stackrel{0}{0} \\
0\end{array}$ & 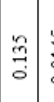 & '高 & $\begin{array}{l}\stackrel{\circ}{c} \\
\text { id }\end{array}$ & 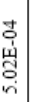 & $n$ \\
\hline & 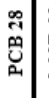 & 总 & สુ & $\begin{array}{l}0 \\
0 \\
0 \\
0\end{array}$ & $\mathscr{0}$ & مֶ. & ڤ્ণ & $\frac{\mathrm{J}}{0}$ & 7 & 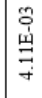 & $\stackrel{\circ}{\circ}$ & $\begin{array}{l}\text { 导 } \\
\text { o }\end{array}$ & $\begin{array}{c}\infty \\
\stackrel{\infty}{2} \\
\alpha\end{array}$ & $\begin{array}{l}\tilde{c} \\
\stackrel{0}{0}\end{array}$ & $\begin{array}{l}\text { 省 } \\
\text { 岁 } \\
\text { or } \\
\text { ris }\end{array}$ & $\begin{array}{l} \\
\text { 䓵 } \\
\text { 惫 }\end{array}$ & 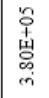 & 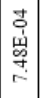 & $\begin{array}{l}\text { 䕒 } \\
\text {. }\end{array}$ & $\begin{array}{l}\mathcal{T} \\
0 \\
0 \\
0\end{array}$ & $\mid$\begin{tabular}{l}
2 \\
\hdashline \\
0 \\
0
\end{tabular} & 웡 & i & 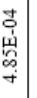 & n \\
\hline & 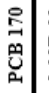 & 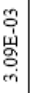 & "ું & $\begin{array}{l}0 \\
0 \\
0 \\
0\end{array}$ & $\mathscr{0}$ & $\vec{\Xi}$ & ठ્సి & $\stackrel{ \pm}{0}$ & ชే & 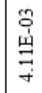 & $\stackrel{\circ}{\circ}$ & 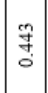 & \begin{tabular}{|l|}
$\infty$ \\
$\vdots$ \\
$\alpha$ \\
$\alpha$
\end{tabular} & $\begin{array}{l}0 \\
\stackrel{0}{0} \\
0\end{array}$ & 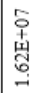 & 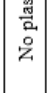 & 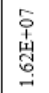 & 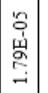 & 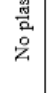 & $\begin{array}{l}\vec{J} \\
\overrightarrow{0} \\
0\end{array}$ & $\begin{array}{l}2 \\
\stackrel{2}{a} \\
0\end{array}$ & 壱 & $\stackrel{\text { aे }}{c}$ & 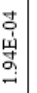 & $\vec{i}$ \\
\hline & 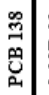 & 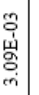 & 'ָ & $\begin{array}{l}0 \\
\stackrel{0}{0} \\
0\end{array}$ & $\mathscr{8}$ & שֶ & હે & $\stackrel{ \pm}{\circ}$ & $\begin{array}{l}\circ \\
\stackrel{2}{\circ}\end{array}$ & 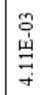 & $\stackrel{\circ}{\circ}$ & \begin{tabular}{l}
0 \\
\multirow{J}{*}{} \\
0 \\
0
\end{tabular} & $\begin{array}{c}\infty \\
\stackrel{\infty}{\sigma} \\
\alpha\end{array}$ & $\begin{array}{l}0 \\
\stackrel{0}{0} \\
0\end{array}$ & $\begin{array}{l}0 \\
0 \\
+4 \\
1 \\
0 \\
0 \\
0\end{array}$ & & 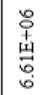 & 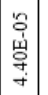 & & $\begin{array}{l}\mathcal{T} \\
\stackrel{7}{0} \\
0\end{array}$ & $\mid \begin{array}{l}2 \\
9 \\
0 \\
0\end{array}$ & ج & $\begin{array}{l}\infty \\
i \\
i j\end{array}$ & 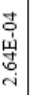 & ส่ \\
\hline 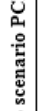 & 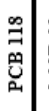 & 愛 & ત્a & $\begin{array}{l}\infty \\
\stackrel{0}{0} \\
0\end{array}$ & $\mathscr{0}$ & है & ठ્તે & $\stackrel{ \pm}{\circ}$ & 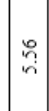 & 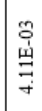 & O롱 & 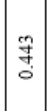 & \begin{tabular}{c}
$\infty$ \\
\multirow{2}{\alpha}{} \\
$\sigma$
\end{tabular} & $\begin{array}{l}0 \\
\stackrel{0}{0} \\
0\end{array}$ & $\begin{array}{l}\text { 号 } \\
\text { 荘 } \\
\text { y } \\
\text { r }\end{array}$ & & 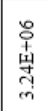 & 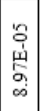 & & $\begin{array}{l}\frac{7}{7} \\
0 \\
0\end{array}$ & $\mid$\begin{tabular}{l}
2 \\
\hdashline \\
0 \\
0
\end{tabular} & 童 & 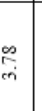 & 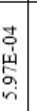 & c) \\
\hline & 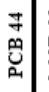 & 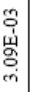 & "ָે & 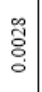 & $\mathscr{0}$ & b & હ્సి & $\frac{ \pm}{0}$ & $\begin{array}{l}\circ \\
\stackrel{\leftarrow}{+}\end{array}$ & 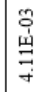 & $\stackrel{\circ}{\circ}$ & $\begin{array}{l}\text { 等 } \\
0\end{array}$ & 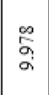 & $\begin{array}{l}0 \\
\stackrel{0}{0} \\
0\end{array}$ & 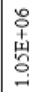 & & 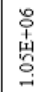 & 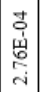 & & $\begin{array}{l}\mathcal{T} \\
\stackrel{T}{0} \\
0\end{array}$ & $\mid \begin{array}{l}2 \\
9 \\
0 \\
0\end{array}$ & '管 & : & 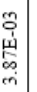 & 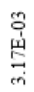 \\
\hline & 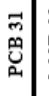 & 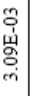 & 'ָे & $\begin{array}{l}0 \\
0 \\
0 \\
0\end{array}$ & $\mathscr{\circ}$ & ". & ఫે & $\frac{7}{0}$ & $\hat{7}$ & 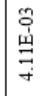 & 응 & $\begin{array}{l}\frac{9}{f} \\
0 \\
0\end{array}$ & $\begin{array}{c}\infty \\
\vdots \\
\vdots \\
\alpha\end{array}$ & $\frac{\tilde{a}}{0} \dot{0}$ & 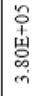 & & \begin{tabular}{|c} 
ò \\
c. \\
岁 \\
c
\end{tabular} & $\begin{array}{l}\text { 志 } \\
\text { 岁 } \\
\text { g } \\
\sim\end{array}$ & & 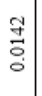 & $\mid \begin{array}{l}2 \\
-2 \\
0 \\
0\end{array}$ & 咅 & i⿱ & 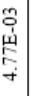 & $\begin{array}{l}\text { 岁 } \\
\text { 㟋 } \\
\stackrel{4}{+}\end{array}$ \\
\hline & & B & ${ }^{2}$ & gี & 要 & 童 & $\because$ & 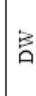 & $\begin{array}{l}0 \\
0 \\
0 \\
0 \\
0 \\
\vdots\end{array}$ & $\ddot{\leftrightarrow}$ & 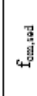 & $\nabla$ & $\underline{\mu}$ & $4^{9}$ & 4 & $\mid \begin{array}{c}2 \\
8 \\
0 \\
0 \\
0\end{array}$ & 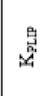 & 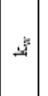 & $\begin{array}{l}\frac{g}{3} \\
\frac{a}{2}\end{array}$ & 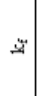 & 骂 & 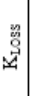 & 3 & d & 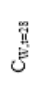 \\
\hline
\end{tabular}


Tables

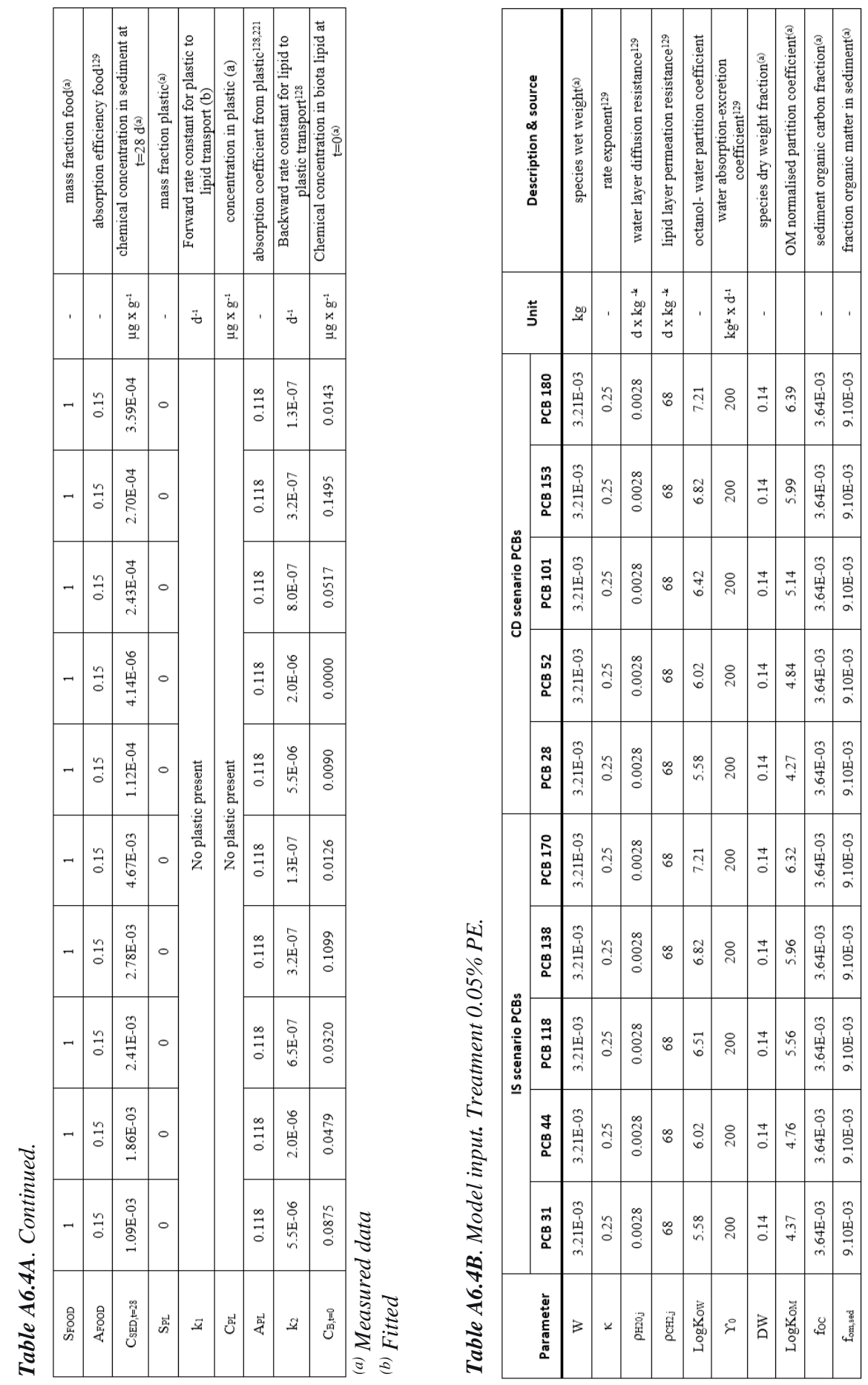




\begin{tabular}{|c|c|c|c|c|c|c|c|c|c|c|c|c|c|c|c|c|c|c|c|c|c|c|c|c|}
\hline 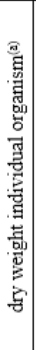 & 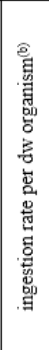 & 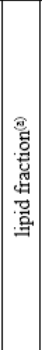 & 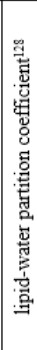 & 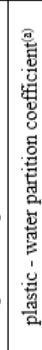 & 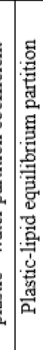 & : & 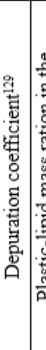 & 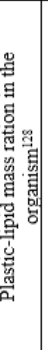 & 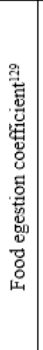 & 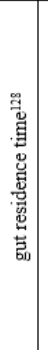 & 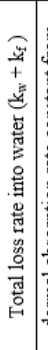 & 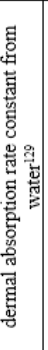 & 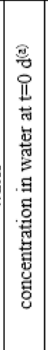 & 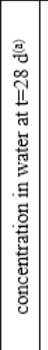 & 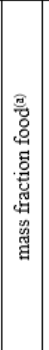 & 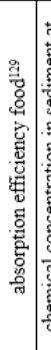 & 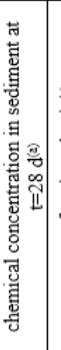 & 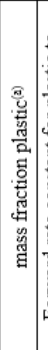 & 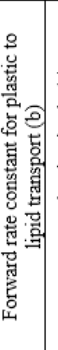 & 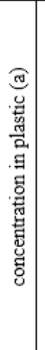 & 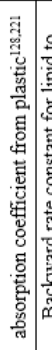 & 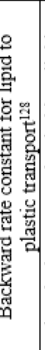 & 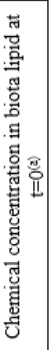 & \\
\hline on & 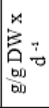 & & & ' & & & & Do & & 0 & & 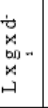 & 总 & $\mid \begin{array}{l}\mathrm{b} \\
\mathrm{ob}\end{array}$ & & ' & $\begin{array}{l}\vec{b} \\
\vec{b} \\
x \\
o p \\
z\end{array}$ & & $\because 0$ & $\begin{array}{l}b_{0} \\
x \\
x \\
z \\
z\end{array}$ & & $\ddot{0}$ & $\begin{array}{l}\vec{b}_{0} \\
x \\
b \\
a \\
z\end{array}$ & \\
\hline $\begin{array}{l}0 \\
\vdots \\
0 \\
0\end{array}$ & \begin{tabular}{|l}
$\vec{n}$ \\
$i n$
\end{tabular} & $\mid \begin{array}{l}0 \\
0 \\
0 \\
0\end{array}$ & 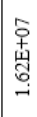 & लै & 8 & 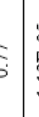 & , & $\begin{array}{l}\infty \\
\stackrel{0}{0} \\
0\end{array}$ & 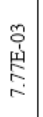 & 嘼 & 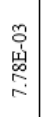 & $\stackrel{n}{n}$ & 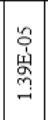 & \begin{tabular}{|c|} 
\\
ù \\
岁 \\
- \\
\end{tabular} & 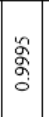 & $\stackrel{2}{\circ}$ & 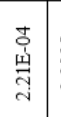 & 管 & 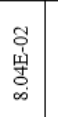 & $\vec{a}$ & $\begin{array}{l}\stackrel{2}{m} \\
\stackrel{m}{0}\end{array}$ & $\begin{array}{l}\overrightarrow{0} \\
\text { 岕 } \\
\stackrel{-}{*}\end{array}$ & 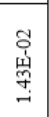 & \\
\hline $\begin{array}{l}0 \\
+ \\
0 \\
0\end{array}$ & \begin{tabular}{|l}
$\vec{n}$ \\
$i n$
\end{tabular} & $\mid \begin{array}{l}0 \\
\vdots \\
0 \\
0\end{array}$ & 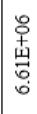 & : & {$\left[\begin{array}{c}\infty \\
0 \\
0\end{array}\right.$} & 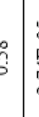 & 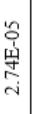 & $\begin{array}{l}\infty \\
\stackrel{0}{0} \\
0\end{array}$ & 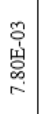 & $\stackrel{\substack{m \\
0}}{0}$ & 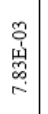 & $\stackrel{n}{\stackrel{n}{n}}$ & 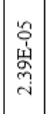 & 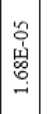 & \begin{tabular}{|l|} 
\\
$\alpha$ \\
$\alpha$ \\
$o$ \\
\end{tabular} & $\stackrel{?}{0}$ & 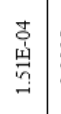 & 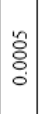 & 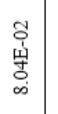 & $\underset{\sigma}{\sigma}$ & $\begin{array}{l}\stackrel{2}{9} \\
0\end{array}$ & $\begin{array}{l}\overrightarrow{0} \\
\stackrel{\leftrightarrow}{+} \\
\underset{-}{+}\end{array}$ & $\begin{array}{l}\overrightarrow{0} \\
\text { 㟋 } \\
-\end{array}$ & \\
\hline $\begin{array}{l}0 \\
+ \\
0 \\
0\end{array}$ & $\begin{array}{l}\overrightarrow{3} \\
\text { in }\end{array}$ & $\mid \begin{array}{l}0 \\
\grave{0} \\
0 \\
0\end{array}$ & 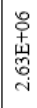 & 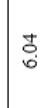 & $\stackrel{\infty}{\sigma}$ & $\stackrel{\infty}{n}$ & 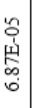 & $\stackrel{\infty}{0}$ & 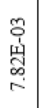 & $\stackrel{\substack{m \\
0}}{0}$ & 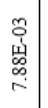 & $\underset{\sim}{\stackrel{t}{i}}$ & 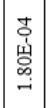 & 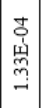 & 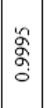 & $\stackrel{2}{0}$ & 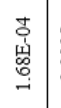 & ì & 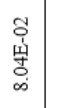 & 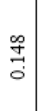 & $\stackrel{\stackrel{m}{9}}{0}$ & 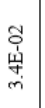 & 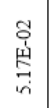 & \\
\hline $\begin{array}{l}0 \\
\vdots \\
0 \\
0\end{array}$ & \begin{tabular}{|l}
$\vec{n}$ \\
in
\end{tabular} & $\mid \begin{array}{l}0 \\
0 \\
0 \\
0\end{array}$ & 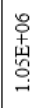 & $\begin{array}{c}8 \\
\text { in } \\
\text { in }\end{array}$ & 5 & 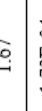 & 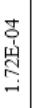 & $\begin{array}{l}\infty \\
\stackrel{0}{0} \\
0\end{array}$ & 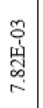 & 孚 & 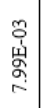 & $\underset{\sim}{\stackrel{N}{m}}$ & 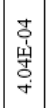 & 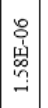 & 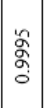 & $\stackrel{2}{0}$ & $\begin{array}{l}0 \\
0 \\
\dot{1} \\
\text { 足 } \\
-\end{array}$ & 管 & 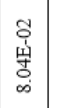 & $\overrightarrow{0}$ & $\begin{array}{l}m \\
m \\
0 \\
0\end{array}$ & $\begin{array}{c}\underset{S}{ } \\
\text { 㟧 } \\
+\end{array}$ & $\begin{array}{l}8 \\
+ \\
\text { 岁 } \\
\text { : }\end{array}$ & \\
\hline $\begin{array}{l}0 \\
+ \\
0 \\
0\end{array}$ & $\begin{array}{l}\vec{n} \\
\text { in }\end{array}$ & $\begin{array}{l}0 \\
0 \\
0 \\
0\end{array}$ & 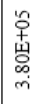 & 7ี & 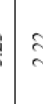 & N & 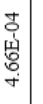 & $\stackrel{\infty}{\circ}$ & 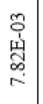 & 嘼 & 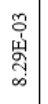 & $\begin{array}{l}\overrightarrow{0} \\
\dot{m}\end{array}$ & \begin{tabular}{|l|}
\multirow{0}{0}{} \\
㟯 \\
子
\end{tabular} & 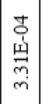 & \begin{tabular}{|l|}
$\alpha$ \\
o. \\
o \\
\end{tabular} & 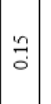 & 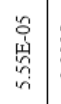 & 管 & 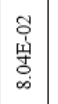 & 产 & $\mid \begin{array}{l}m \\
\tilde{a} \\
0\end{array}$ & 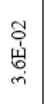 & $\begin{array}{l}\text { ô } \\
\text { 岕 } \\
\text { o } \\
\infty\end{array}$ & 10 \\
\hline 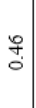 & \begin{tabular}{|l}
$\vec{n}$ \\
$i n$
\end{tabular} & $\mid \begin{array}{l}0 \\
0 \\
0 \\
0\end{array}$ & 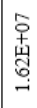 & 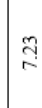 & $1 \%$ & ही & 苍 & $\begin{array}{l}\infty \\
\stackrel{0}{0} \\
0\end{array}$ & 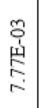 & $\begin{array}{l}m \\
m \\
0 \\
0\end{array}$ & 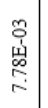 & $\stackrel{n}{\stackrel{n}{n}}$ & 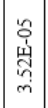 & 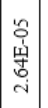 & 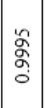 & $\stackrel{2}{0}$ & 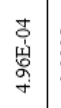 & 然 & $\begin{array}{l}\text { Oे } \\
\text { 㝘 } \\
\infty\end{array}$ & $\begin{array}{l}0 \\
\text { 品 } \\
0\end{array}$ & $\begin{array}{l}2 \\
9 \\
0 \\
0\end{array}$ & 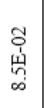 & $\begin{array}{l}\text { S } \\
\text { 莕 } \\
\text { - }\end{array}$ & 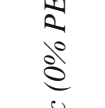 \\
\hline $\begin{array}{l}0 \\
\vdots \\
0 \\
0\end{array}$ & 空 & $\mid \begin{array}{l}0 \\
0 \\
0 \\
0\end{array}$ & $\begin{array}{l}0 \\
0 \\
+ \\
4 \\
0 \\
0 \\
0 \\
0\end{array}$ & \&̊요 & J & t. & 总 & $\begin{array}{l}\infty \\
\stackrel{0}{0} \\
0\end{array}$ & 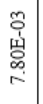 & 嘼 & 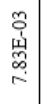 & $\stackrel{n}{\stackrel{n}{n}}$ & 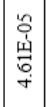 & 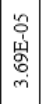 & 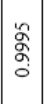 & $\frac{2}{0}$ & 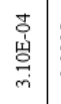 & 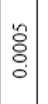 & 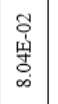 & $\vec{a}$ & 急 & 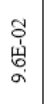 & $\begin{array}{l}\overrightarrow{0} \\
\text { 峉 } \\
\stackrel{-}{-}\end{array}$ & $\begin{array}{l}\frac{v}{3} \\
\frac{3}{3} \\
0\end{array}$ \\
\hline 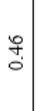 & $\begin{array}{l}\vec{n} \\
\text { in }\end{array}$ & $\mid \begin{array}{l}0 \\
0 \\
0 \\
0\end{array}$ & 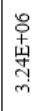 & : & $\frac{1}{4}$ & f. & 资 & $\begin{array}{l}\infty \\
\stackrel{0}{0} \\
0\end{array}$ & 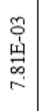 & 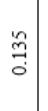 & 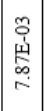 & $\underset{\sim}{\stackrel{D}{D}}$ & 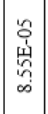 & 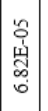 & \begin{tabular}{|l|}
\multirow{2}{\alpha}{} \\
$\mathrm{o}$ \\
$\mathrm{o}$
\end{tabular} & $\frac{1}{0}$ & 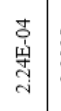 & . & 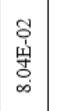 & 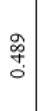 & $\stackrel{\leftrightarrow}{\stackrel{m}{0}}$ & 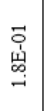 & $\begin{array}{l}\text { S } \\
\text { 岕 } \\
\text { ल }\end{array}$ & ฐ \\
\hline 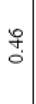 & 鿖 & 㝘 & 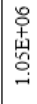 & $\begin{array}{l}\text { : } \\
\text { in }\end{array}$ & 7 & J & 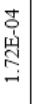 & $\begin{array}{l}\infty \\
\stackrel{0}{0}\end{array}$ & 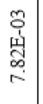 & 畋 & 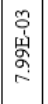 & $\underset{\sim}{\stackrel{\sim}{*}}$ & 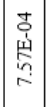 & 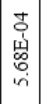 & 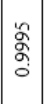 & $\frac{1}{0}$ & 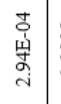 & iू & $\begin{array}{l}\text { Oे } \\
\text { 㟯 } \\
\infty\end{array}$ & $\begin{array}{l}\text { 志 } \\
\text { c }\end{array}$ & $\mid$\begin{tabular}{c}
$m$ \\
\hdashline \\
0 \\
0
\end{tabular} & 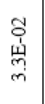 & 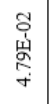 & o \\
\hline $\begin{array}{l}0 \\
\vdots \\
0 \\
0\end{array}$ & \begin{tabular}{|l}
$\vec{n}$ \\
in
\end{tabular} & \begin{tabular}{|l|}
0 \\
0 \\
0 \\
0
\end{tabular} & 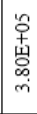 & הี & ભొ & $\frac{8}{8}$ & $\begin{array}{l}\text { 草 } \\
\text { 岁 } \\
\vdots\end{array}$ & $\begin{array}{l}\infty \\
\stackrel{0}{0} \\
0\end{array}$ & 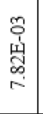 & $\begin{array}{l}m \\
m \\
0\end{array}$ & 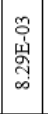 & $\begin{array}{l}\hat{0} \\
\dot{n}\end{array}$ & 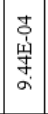 & 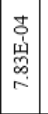 & 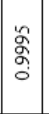 & $\stackrel{2}{0}$ & 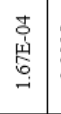 & 管 & 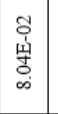 & ఫి & $\stackrel{\leftrightarrow}{\stackrel{m}{0}}$ & 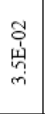 & 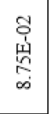 & 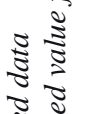 \\
\hline$\dot{\xi}$ & 电 & 4 & $\frac{9}{4}$ & 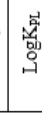 & 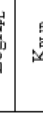 & 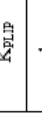 & $\stackrel{4}{*}$ & 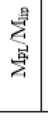 & $\ddot{\Xi}$ & 递 & 解 & $\begin{array}{l}0.9 \\
-4\end{array}$ & 竞 & 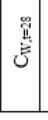 & \begin{tabular}{|c}
0 \\
0 \\
0 \\
0 \\
0
\end{tabular} & \begin{tabular}{|c|} 
\\
妾 \\
\end{tabular} & 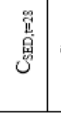 & 尚 & $\vec{A}$ & 今心 & 草 & $\approx$ & 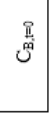 & 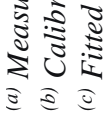 \\
\hline
\end{tabular}


Tables

\begin{tabular}{|c|c|c|c|c|c|c|c|c|c|c|c|c|c|c|c|c|c|c|c|c|c|c|c|c|c|c|}
\hline 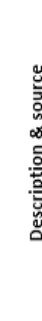 & & 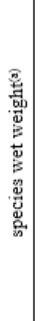 & 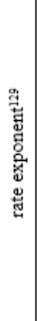 & 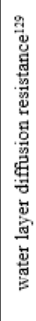 & 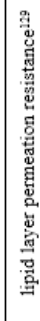 & 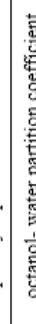 & 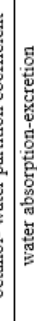 & . & 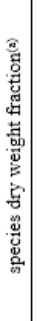 & 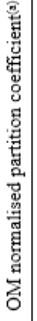 & 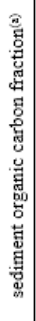 & 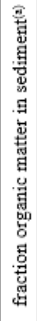 & 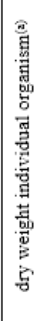 & 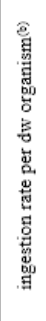 & & 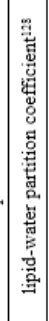 & 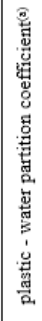 & 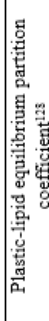 & 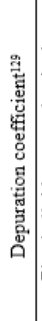 & 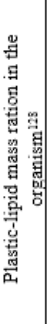 & 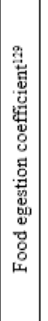 & 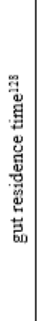 & 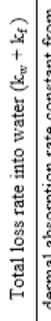 & 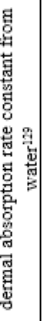 & 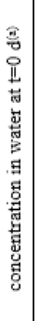 & 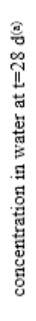 \\
\hline 壱 & & $\stackrel{\text { Do }}{-1}$ & & 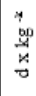 & 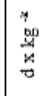 & & 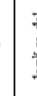 & : & & & & ' & on & 0 & & & ' & & & on & & 0 & & 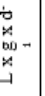 & 架 & 点 \\
\hline & $\begin{array}{c}0 \\
0 \\
0 \\
0 \\
0 \\
0\end{array}$ & 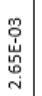 & 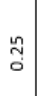 & 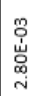 & 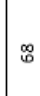 & $\bar{\beth}$ & 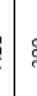 & S. & गे & 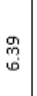 & 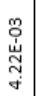 & $\begin{array}{l}\text { no } \\
\text { : } \\
0 \\
0\end{array}$ & $\mid \begin{array}{c}\infty \\
0 \\
0 \\
0\end{array}$ & $\begin{array}{c}\tilde{N} \\
\tilde{m}\end{array}$ & : & 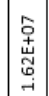 & 点 & ปี & 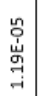 & $\begin{array}{l}\stackrel{9}{7} \\
0\end{array}$ & $\left|\begin{array}{l}0 \\
0 \\
\dot{u} \\
\omega \\
\sigma \\
\sigma\end{array}\right|$ & $\begin{array}{l}\stackrel{\sim}{9} \\
\stackrel{9}{0}\end{array}$ & 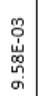 & 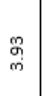 & 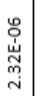 & 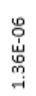 \\
\hline & 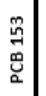 & 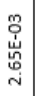 & 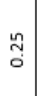 & 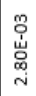 & ๕ & $\begin{array}{c}\infty \\
\infty \\
0 \\
0\end{array}$ & 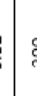 & S. & 茕 & 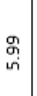 & 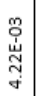 & $\begin{array}{l}\text { no } \\
0 \\
0 \\
0 \\
0\end{array}$ & $\begin{array}{c}\infty \\
0 \\
0\end{array}$ & 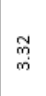 & ○̊ & $\mid \begin{array}{l}0 \\
0 \\
4 \\
0 \\
0 \\
0\end{array}$ & 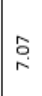 & 㔛 & 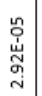 & $\begin{array}{l}0 \\
7 \\
0 \\
0\end{array}$ & 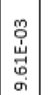 & 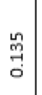 & 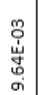 & 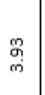 & 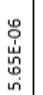 & 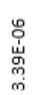 \\
\hline & 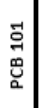 & 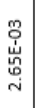 & $\stackrel{\mathscr{n}}{\stackrel{2}{0}}$ & 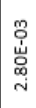 & ஜ & ปี & 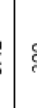 & 5 & वे & 吉 & 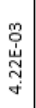 & 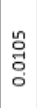 & $\begin{array}{c}\infty \\
0 \\
0 \\
0\end{array}$ & $\begin{array}{c}\tilde{N} \\
\text { m. }\end{array}$ & :̊ & 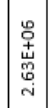 & \begin{tabular}{|l}
0 \\
0 \\
0
\end{tabular} & స̃ & 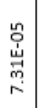 & $\begin{array}{l}0 \\
\stackrel{7}{0} \\
0\end{array}$ & $\mid$\begin{tabular}{c|}
0 \\
0 \\
$\dot{u}$ \\
0 \\
$o$ \\
$o$ \\
$o$
\end{tabular} & 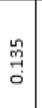 & $\begin{array}{c}0 \\
\stackrel{0}{0} \\
\dot{⿱} \\
\vdots \\
\sigma\end{array}$ & $\underset{\text { స్ }}{\text { J్ }}$ & 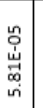 & 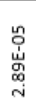 \\
\hline & 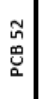 & 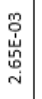 & $\begin{array}{c}\mathfrak{n} \\
0 \\
0\end{array}$ & 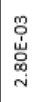 & $\stackrel{\infty}{\circledR}$ & వి & 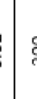 & 8 & 离 & 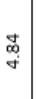 & 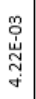 & 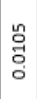 & $\begin{array}{c}\infty \\
0 \\
0 \\
0\end{array}$ & 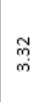 & o̊ & 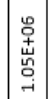 & $\begin{array}{l}n \\
\hat{n} \\
\text { in }\end{array}$ & 怘 & 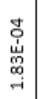 & \begin{tabular}{l}
$\circ$ \\
\multirow{0}{0}{} \\
\end{tabular} & $\left|\begin{array}{c}0 \\
0 \\
\dot{u} \\
0 \\
\sigma \\
\sigma \\
\sigma\end{array}\right|$ & 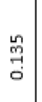 & 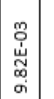 & 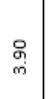 & 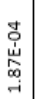 & 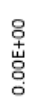 \\
\hline & $\begin{array}{l}\stackrel{\infty}{2} \\
\stackrel{0}{0} \\
0\end{array}$ & 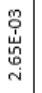 & $\begin{array}{c}\stackrel{n}{:} \\
\text { D }\end{array}$ & 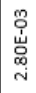 & 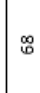 & "ె & 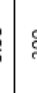 & 5 & 齐 & $\begin{array}{c}\hat{\mathfrak{g}} \\
\text {. }\end{array}$ & 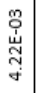 & $\begin{array}{l}\text { 哭 } \\
0 \\
0 \\
0\end{array}$ & 离 & 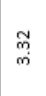 & $\begin{array}{l}\tilde{O} \\
\text { ơ }\end{array}$ & 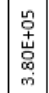 & 俩 & $\stackrel{\text { I }}{\mathrm{N}}$ & 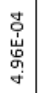 & \begin{tabular}{l}
0 \\
\multirow{3}{0}{} \\
0
\end{tabular} & $\mid \begin{array}{c}0 \\
0 \\
\dot{u} \\
0 \\
\sigma \\
\sigma \\
\end{array}$ & 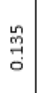 & 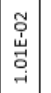 & 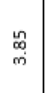 & 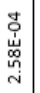 & 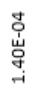 \\
\hline \multirow{5}{*}{ 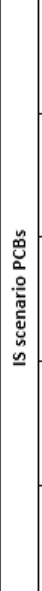 } & $\begin{array}{l}\stackrel{2}{7} \\
\stackrel{8}{0} \\
0\end{array}$ & 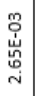 & $\begin{array}{c}\mathfrak{n} \\
\vdots \\
0\end{array}$ & 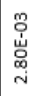 & $\stackrel{\infty}{\infty}$ & న్ & 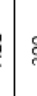 & S. & वै. & $\begin{array}{c}\widetilde{N} \\
0 \\
0\end{array}$ & 惫 & 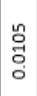 & $\begin{array}{c}\infty \\
0 \\
0 \\
0\end{array}$ & $\begin{array}{l}\tilde{N} \\
\tilde{m}\end{array}$ & o̊ & 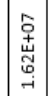 & $\underset{\text { g }}{\stackrel{g}{r}}$ & 员 & 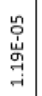 & $\begin{array}{l}0 \\
7 \\
0\end{array}$ & $\mid \begin{array}{l}0 \\
0 \\
u \\
\omega \\
\sigma \\
\sigma\end{array}$ & 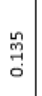 & 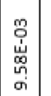 & 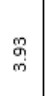 & 筩 & 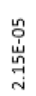 \\
\hline & $\begin{array}{l}\stackrel{\infty}{0} \\
\stackrel{7}{7} \\
\stackrel{8}{0} \\
0\end{array}$ & 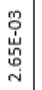 & $\stackrel{\mathscr{n}}{\underset{D}{0}}$ & $\begin{array}{l}\text { 总 } \\
\text { 峞 }\end{array}$ & 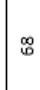 & 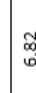 & 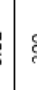 & 8 & 売 & 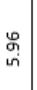 & 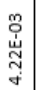 & 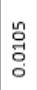 & $\mid \begin{array}{c}\infty \\
0 \\
0\end{array}$ & 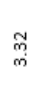 & o̊ & 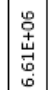 & 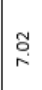 & 壳 & 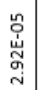 & $\begin{array}{l}\stackrel{0}{7} \\
\stackrel{7}{0}\end{array}$ & 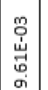 & 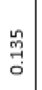 & 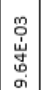 & $\begin{array}{c}m \\
m \\
m\end{array}$ & 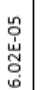 & 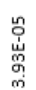 \\
\hline & 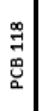 & 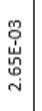 & 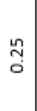 & 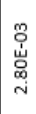 & $\stackrel{\infty}{๕}$ & $\begin{array}{l}\vec{n} \\
0\end{array}$ & & S. & 总 & 号 & 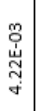 & $\begin{array}{l}\text { no } \\
\text { : } \\
0 \\
0\end{array}$ & $\begin{array}{c}\infty \\
0 \\
0 \\
0\end{array}$ & $\underset{\tilde{m}}{\tilde{m}}$ & ס̊ & 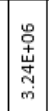 & \begin{tabular}{|l|l}
$\tilde{O}$ \\
0 \\
0
\end{tabular} & 合 & 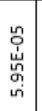 & $\begin{array}{l}0 \\
\stackrel{7}{0} \\
0\end{array}$ & \begin{tabular}{|c|} 
\\
0 \\
$\dot{u}$ \\
0 \\
$o$ \\
$\sigma$ \\
$\sigma$
\end{tabular} & $\begin{array}{l}\text { 品 } \\
\stackrel{0}{0}\end{array}$ & $\begin{array}{l}0 \\
0 \\
\dot{u} \\
0 \\
0 \\
\sigma \\
\sigma\end{array}$ & 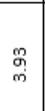 & 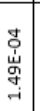 & 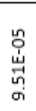 \\
\hline & 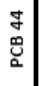 & 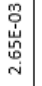 & $\stackrel{\mathscr{n}}{\tilde{O}}$ & 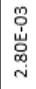 & œ & శ్రి & 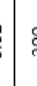 & S. & ने & 号 & 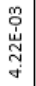 & $\begin{array}{l}\text { : } \\
\stackrel{0}{0} \\
0 \\
0\end{array}$ & $\mid \begin{array}{c}\infty \\
0 \\
0\end{array}$ & 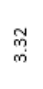 & ס̊ & 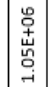 & 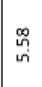 & A & 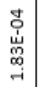 & $\begin{array}{l}\circ \\
\stackrel{7}{0} \\
0\end{array}$ & $\mid \begin{array}{c}0 \\
0 \\
\dot{u} \\
\vdots \\
\sigma \\
\sigma\end{array}$ & $\begin{array}{c}\stackrel{\sim}{m} \\
\stackrel{9}{0} \\
0\end{array}$ & 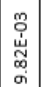 & 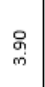 & 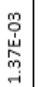 & 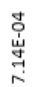 \\
\hline & $\begin{array}{l}\overrightarrow{1} \\
0 \\
0 \\
0\end{array}$ & 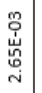 & 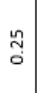 & 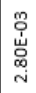 & $\stackrel{\infty}{\circledR}$ & "n & & : & 离 & \begin{tabular}{c}
$\hat{m}$ \\
\multirow{r}{*}{}
\end{tabular} & 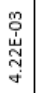 & $\begin{array}{l}\text { 管 } \\
0 \\
0\end{array}$ & $\mid \begin{array}{c}\infty \\
0 \\
0 \\
0\end{array}$ & 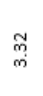 & סे & 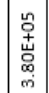 & 売 & $\stackrel{\text { L̊ }}{\text { ì }}$ & 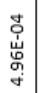 & \begin{tabular}{l}
0 \\
\multirow{7}{0}{} \\
0
\end{tabular} & $\mid \begin{array}{c}0 \\
0 \\
\dot{u} \\
\vdots \\
0 \\
\sigma \\
\sigma\end{array}$ & 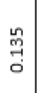 & 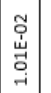 & 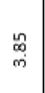 & 总 & 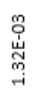 \\
\hline & 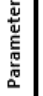 & $B$ & $*$ & 명 & 高 & 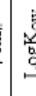 & & 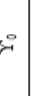 & 合 & 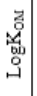 & 8 & 4 & g & 복 & $4^{4}$ & $: \frac{2}{4}$ & 密 & 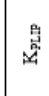 & 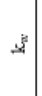 & 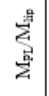 & $\because$ & 品 & 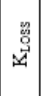 & $\stackrel{g}{9}$ & 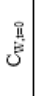 & है \\
\hline
\end{tabular}




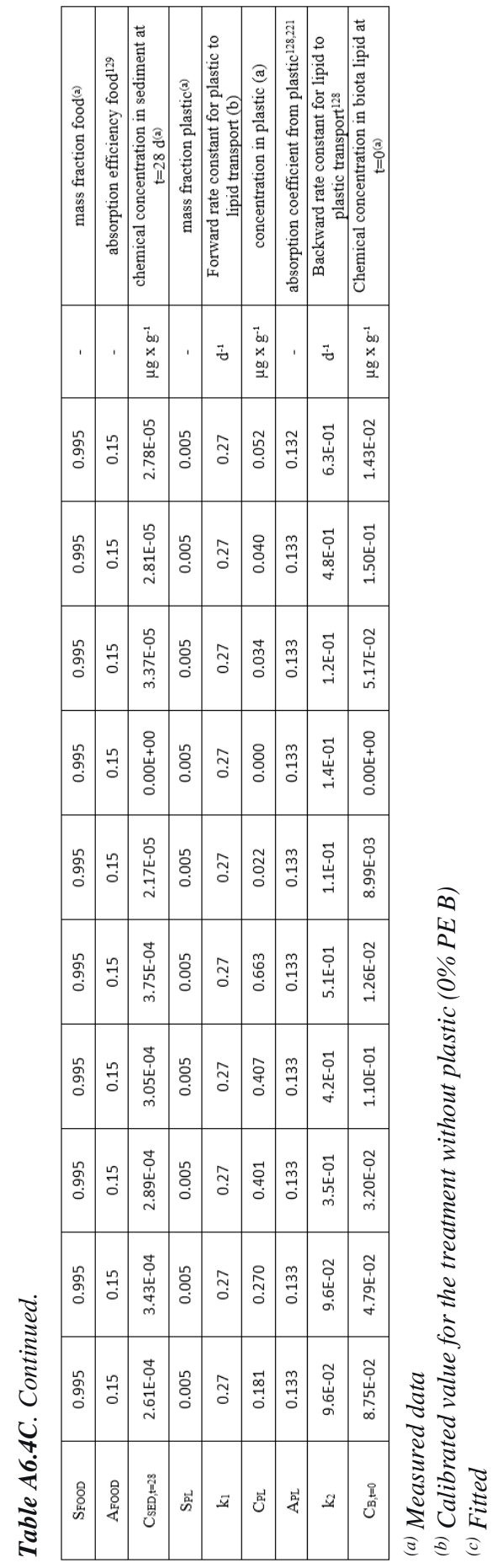


Figures

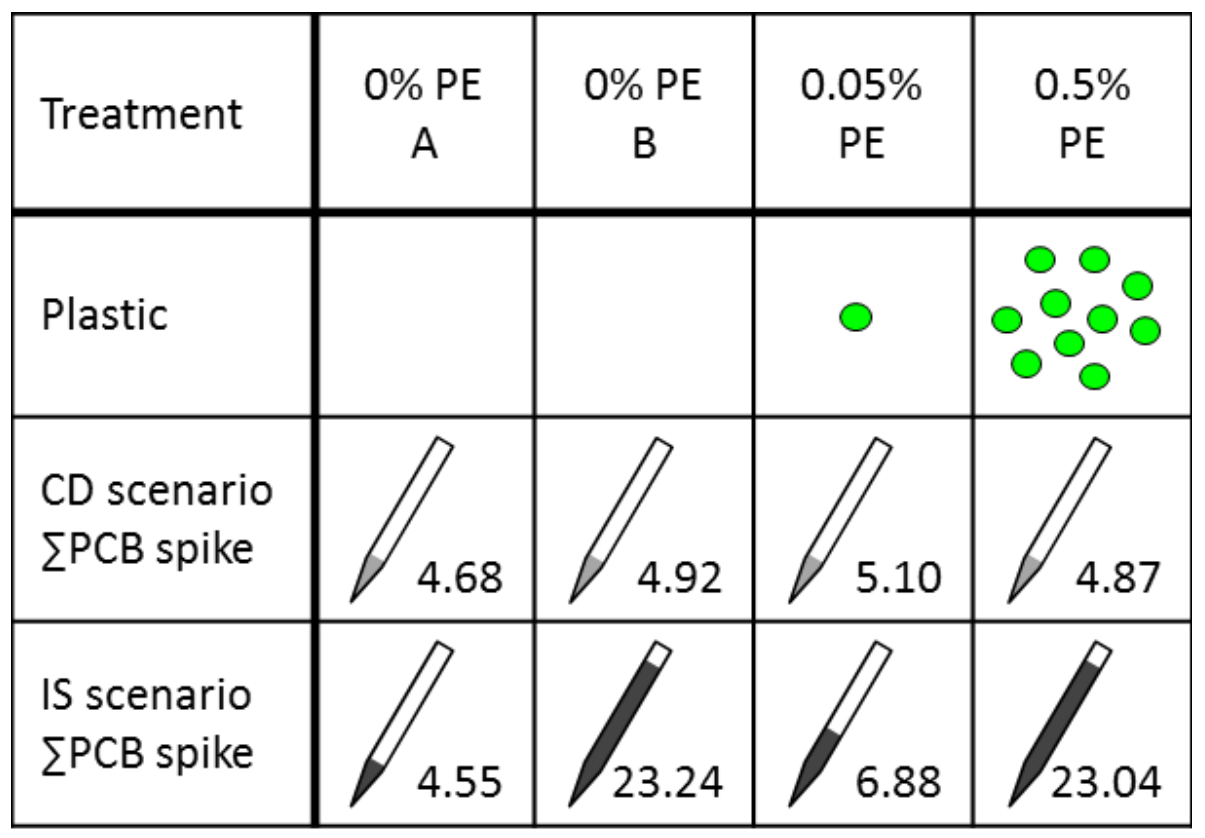

Figure S1. Schematization of the four treatments. $\Sigma P C B$ concentrations are the sum of 5 congeners representing the respective scenario, in $\mu \mathrm{g} / \mathrm{kg} D W$. Concentrations per congener can be found in Table A6.1. $0 \%$ PE A: no plastic and a similar concentration of the PCB congeners representing the $C D$ and $I S$ scenario. $0 \%$ PE B: no plastic, a low concentration of $P C B$ congeners representing the $C D$ scenario $(P C B$ 28, 52, 101, 153 and 180) and a higher concentration of $P C B$ congeners representing the IS scenario $(P C B 31,44,118,138$ and 170). $0.05 \%$ PE: $0.05 \%$ polyethylene, a low concentration of $P C B$ congeners representing the $C D$ scenario and a higher concentration of $P C B$ congeners representing the IS scenario to compensate for dilution by the $0.05 \%$ PE. $0.5 \%$ PE: $0.5 \%$ polyethylene, a low concentration of $P C B$ congeners representing the $C D$ scenario and a higher concentration of $P C B$ congeners representing the IS scenario to compensate dilution by the $0.5 \%$ PE. The extra spike in the $0.5 \%$ PE IS scenario is higher than in the 0.05\% PE IS scenario to compensate for the higher anticipated dilution effect at $0.5 \%$ PE compared to $0.05 \%$ PE. 

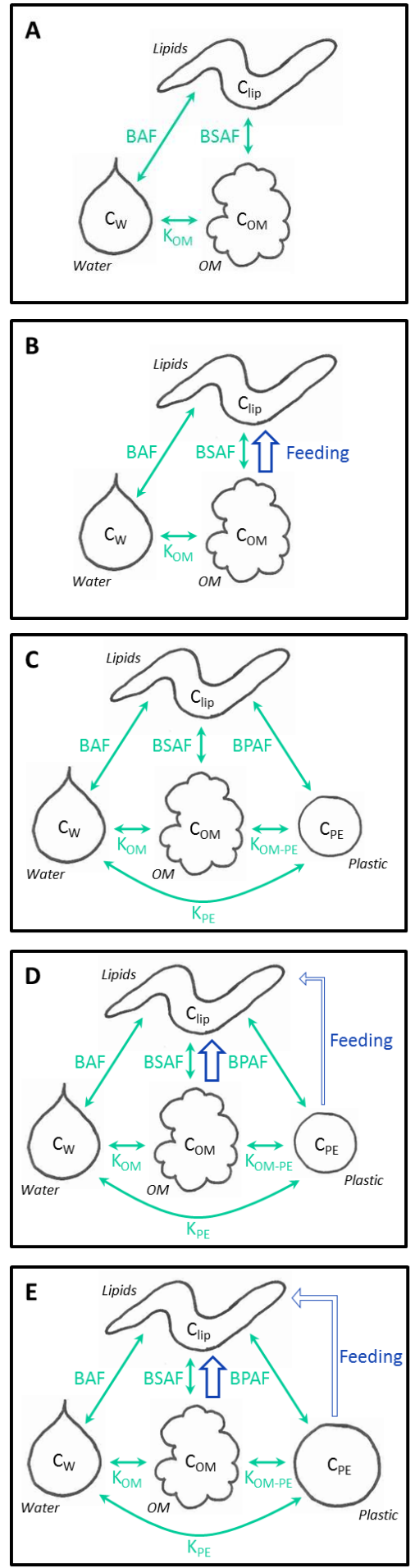

Figure A6.2. Schematic visualization of the compartments and metrics used in the study design. The main transport pathways of $P C B$ s are indicated with arrows. $C_{l i p}, C_{P W}, C_{O M}$, and $C_{P E}$ are the $P C B$ concentration in respectively the lugworm lipids, the porewater, the organic matter (OM) and the plastic (PE) in the sediment. Ком, Ком-PE, and KРE are the equilibrium partitioning coefficients between porewater, OM and PE. The biota to sediment accumulation factor is calculated as: BSAF = $C_{\text {lip }} /$ Сом. The bioaccumulation factor is calculated as: $B A F=C_{\text {lip }} / C_{P W}$. The biota to plastic accumulation factor is calculated as $B P A F=C_{\text {lip }} / C_{P E}$. Chemical transfer according to equilibrium partitioning theory $(E P T)$ and including realistic feeding in the treatments are shown. The relative importance of feeding pathways in Panel $B, D$ and $E$ is indicated with the thickness of the blue arrows. Panel A - EPT 0\% PE: Hypothetical equilibrium partitioning between the compartments lipids, porewater and OM. Panel B-0\% PE: Partitioning as in Panel A, but know with realistic feeding included. Panel C - EPT 0.05\% PE: Hypothetical equilibrium partitioning between the compartments of Panel A, but now including $0.05 \%$ plastic. As the addition of plastic is the introduction of an extra hydrophobic sorption domain, this causes transport of PCBs from the porewater, OM and lipids towards the plastic. The resulting dilution of $P C B$ concentrations in the compartments other than plastic, is referred to as the 'chemical dilution' (CD) scenario. In the 'infinite source' (IS) scenario, extra PCBs are spiked to overcome this dilution effect and thus represent oceanic conditions with excess $P C B$ availability from surrounding sediment. Panel D-0.05\% PE: Partitioning as in Panel $B$, but now with realistic feeding included. Feeding on OM leads to higher than equilibrium steady state $P C B$ concentrations in lipids, leading to BSAF and BPAF values higher than 1-2. Panel E-0.5\% PE: Feeding inclusive steady state partitioning as in Panel $C$, but now with a higher PE concentration $(0.5 \%)$. Feeding on OM can lead to higher than equilibrium steady state $P C B$ concentrations in lipids, leading to BSAF and $B P A F$ values higher than 1-2. 
Figures
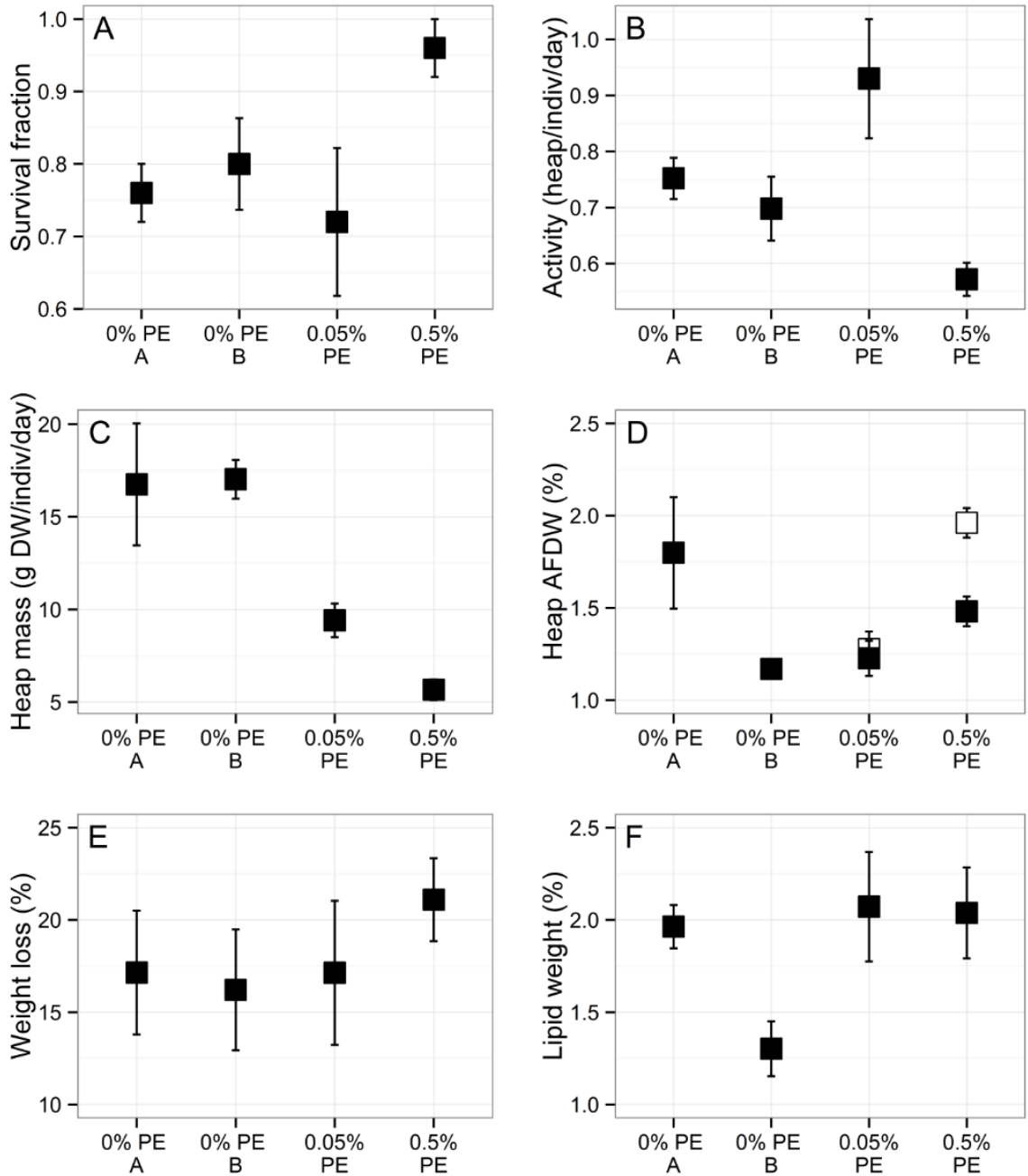

Figure A6.3. Physiological endpoints. Panel A: Average fraction of lugworms surviving the 28 day exposure assay. Panel B: Average feeding activity during the 28 day exposure assay in number of faeces heaps produced per organism per day. Panel C: Average total mass of faeces heaps produced on day 28 of the exposure assay per surviving individual. Panel D: Average $A F D W$ in the faeces heaps produced on day 28 of the exposure assay. White markers: percentage of all material $(O M+P E)$ that burned at $600{ }^{\circ} \mathrm{C}$. Black markers: percentage of $O M$ estimated as AFDW minus the nominal percentage of PE. Panel E: Average percentage wet weight loss of lugworms during the 28 days exposure assay. Panel F: Average lipid weight as percentage of the lugworm $D W$ after the 28 day exposure assay. 

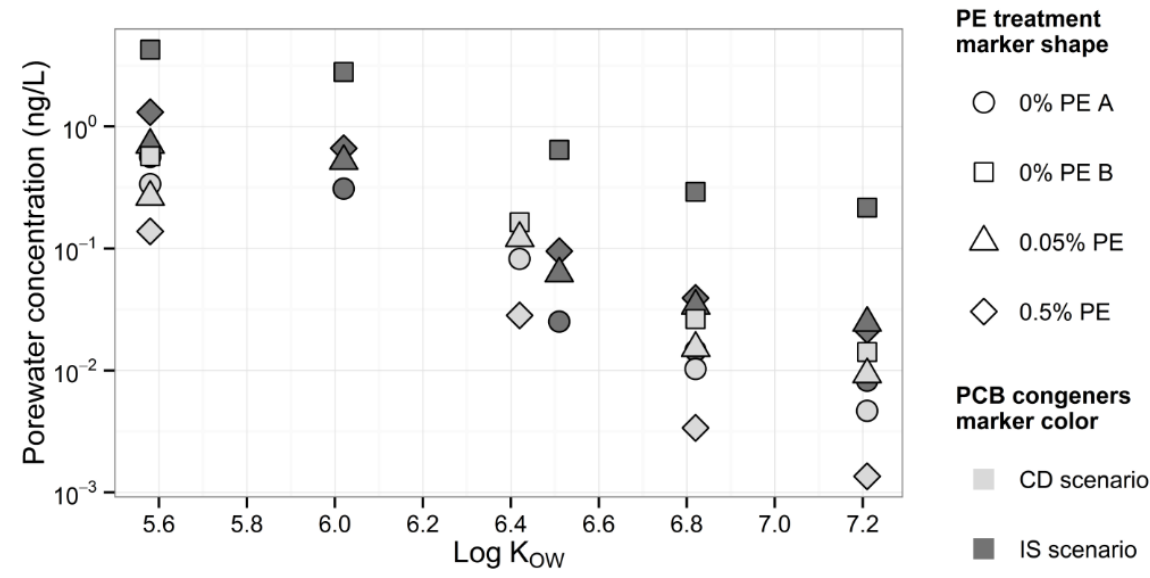

Figure A6.4. $C_{P W}$ values $\pm S E 0.009-0.179$ (not shown) at $t=28$ days, calculated with Eq. A6.2.

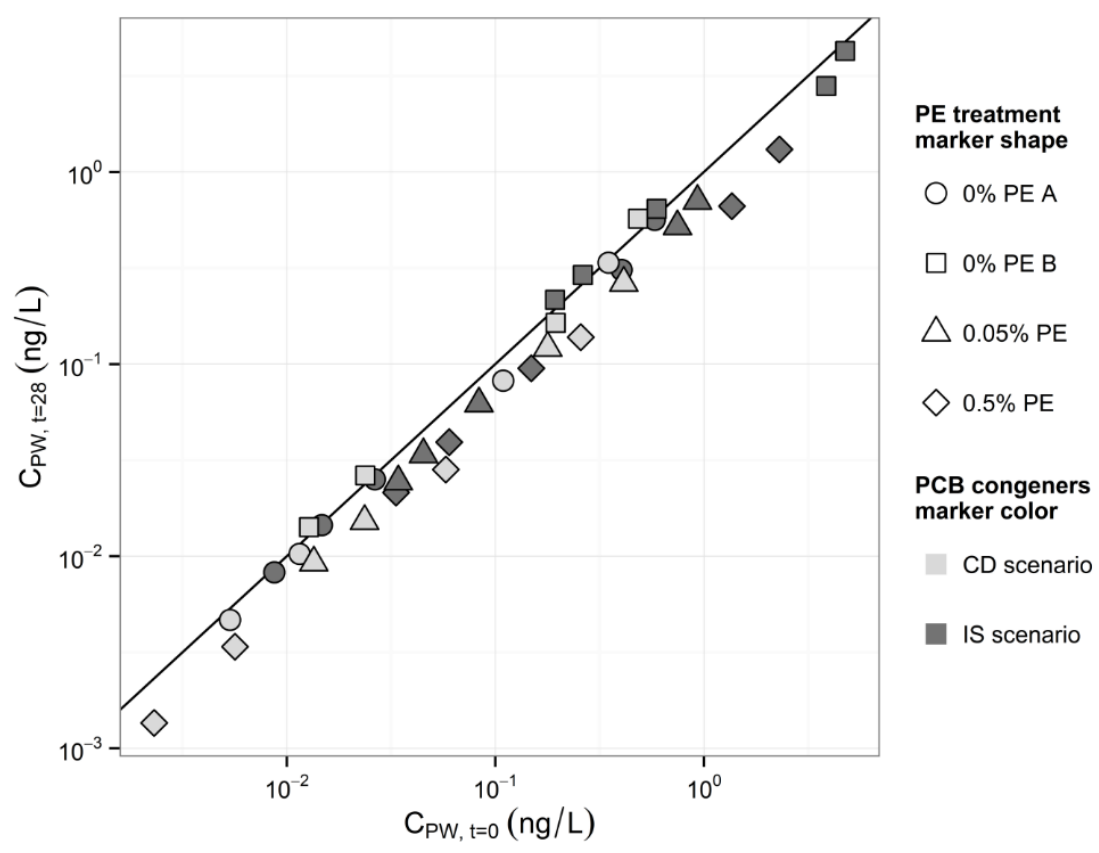

Figure A6.5. Agreement of $C_{P W, t=28}$ with $C_{P W, t=0}$ (1:1 line drawn for comparison) over four orders of magnitude, used to support the assumption of constant aqueous exposure during the 28 d exposure assay. 
Figures

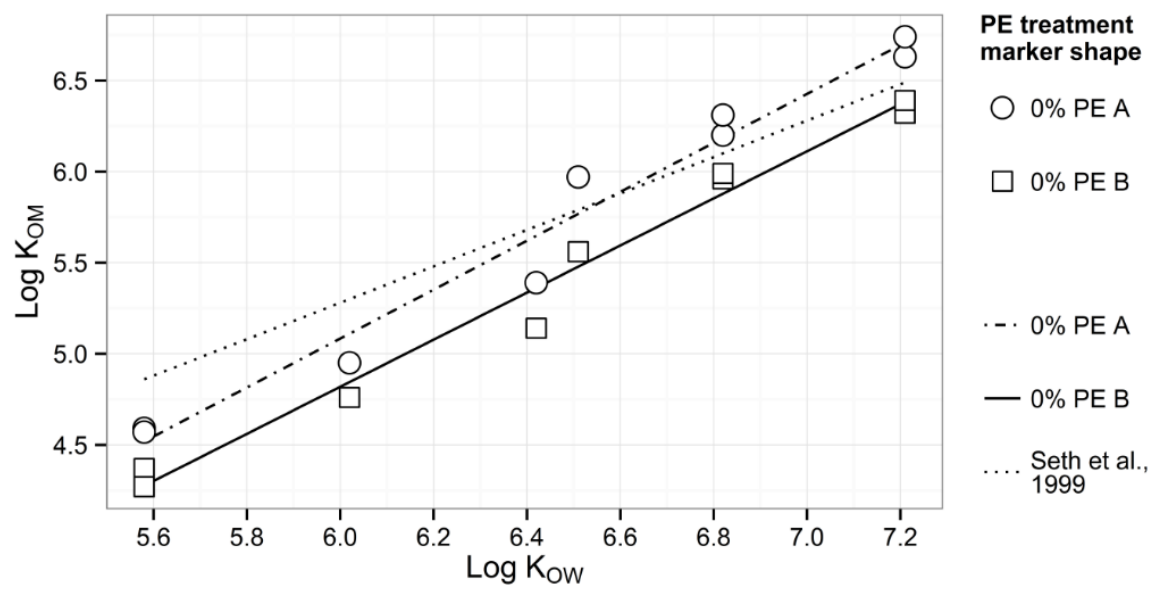

Figure A6.6. Sorption of PCB congeners to organic matter ( $\left.\log K_{O M}\right)$ in sediment of the $0 \%$ $P E A$ (Regression line: $\log K_{O M}=1.34 \times \log K_{O W}-2.98, R^{2}=0.97$ ) and the $0 \% P E B$ treatment with elevated PCB concentration (Regression line: $\log K_{O M}=1.29 \times \log K_{O W}-2.94, R^{2}=0.98$ ) as a function of their hydrophobicity (Log Kow). For comparison KOM values according to the formula by Seth et al. are given. ${ }^{225}$
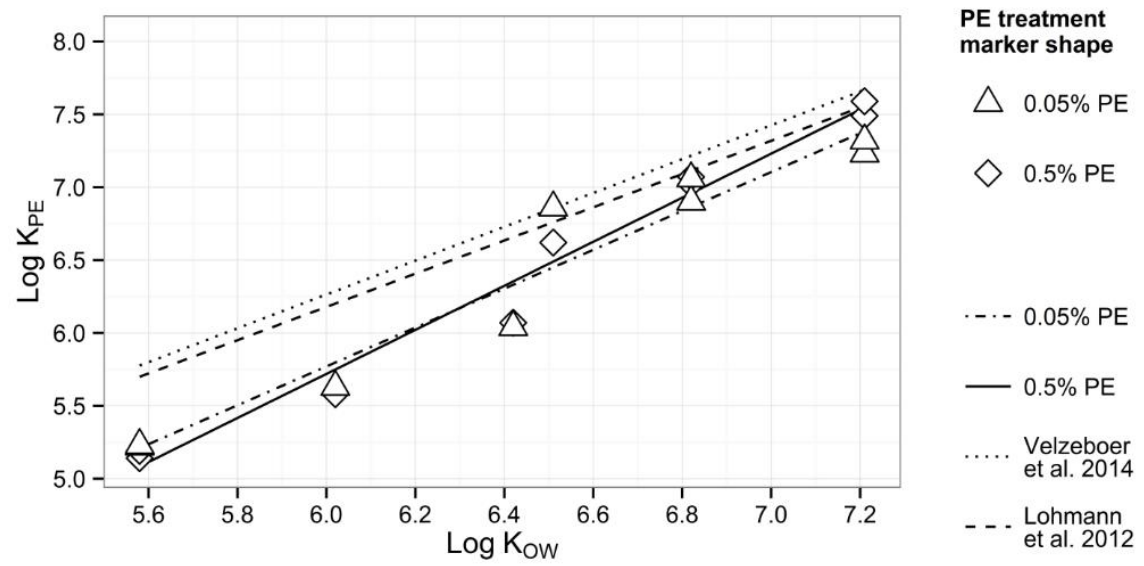

Figure A6.7. Sorption of $P C B$ congeners to polyethylene ( $\left.\log K_{P E}\right)$ as a function of $P C B$ hydrophobicity $\left(\log K_{O W}\right)^{232}$. Regression line $0.05 \%$ PE treatment: $\log K_{P E}=1.33 \times \log K_{O W}$ $-2.23, R^{2}=0.94$. Regression line $0.5 \%$ PE treatment: $\log K_{P E}=1.51 \times \operatorname{LogKow}-3.34, R^{2}=$ 0.98 . 

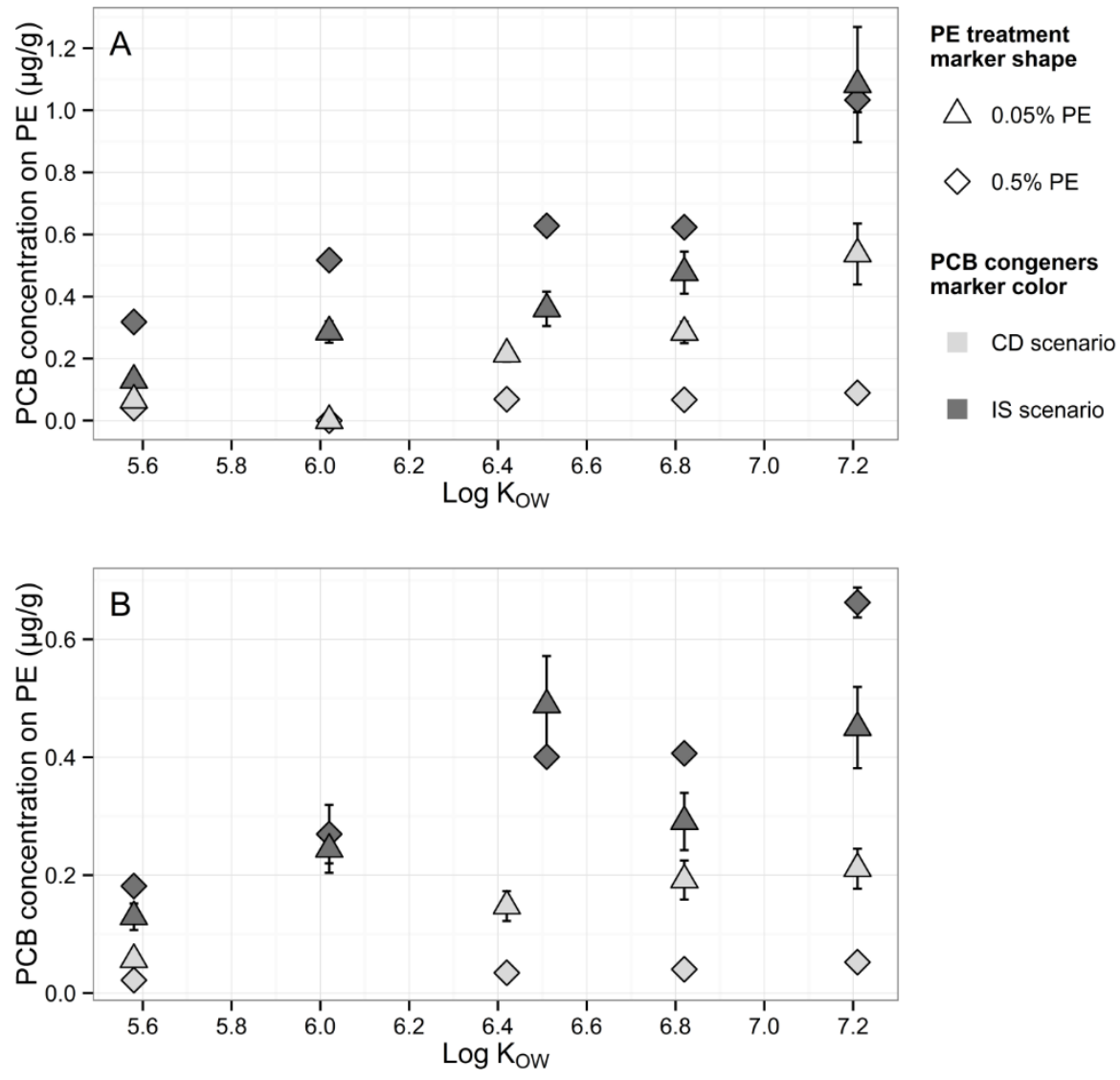

Figure A6.8. Average PCB concentration on PE in the sediment-PE mixture. Panel A: at $t=0$. Panel B: at $t=28$ days. At $t=0$ these PCB concentrations on the PE were calculated from the concentration in the porewater, determined using triplicate POM passive samplers that equilibrated with the sediment mixture, KPOM and KPE. At $t=28 d$ the PCB concentrations on the $P E \pm S E$ were calculated from the concentration in the sediment, which was for each treatment in quintuplicate, $K_{O M}$ and $K_{P E}$ by Eq. A6.2-5. Where error bars are invisible they are small and thus lie behind the markers. 
Figures
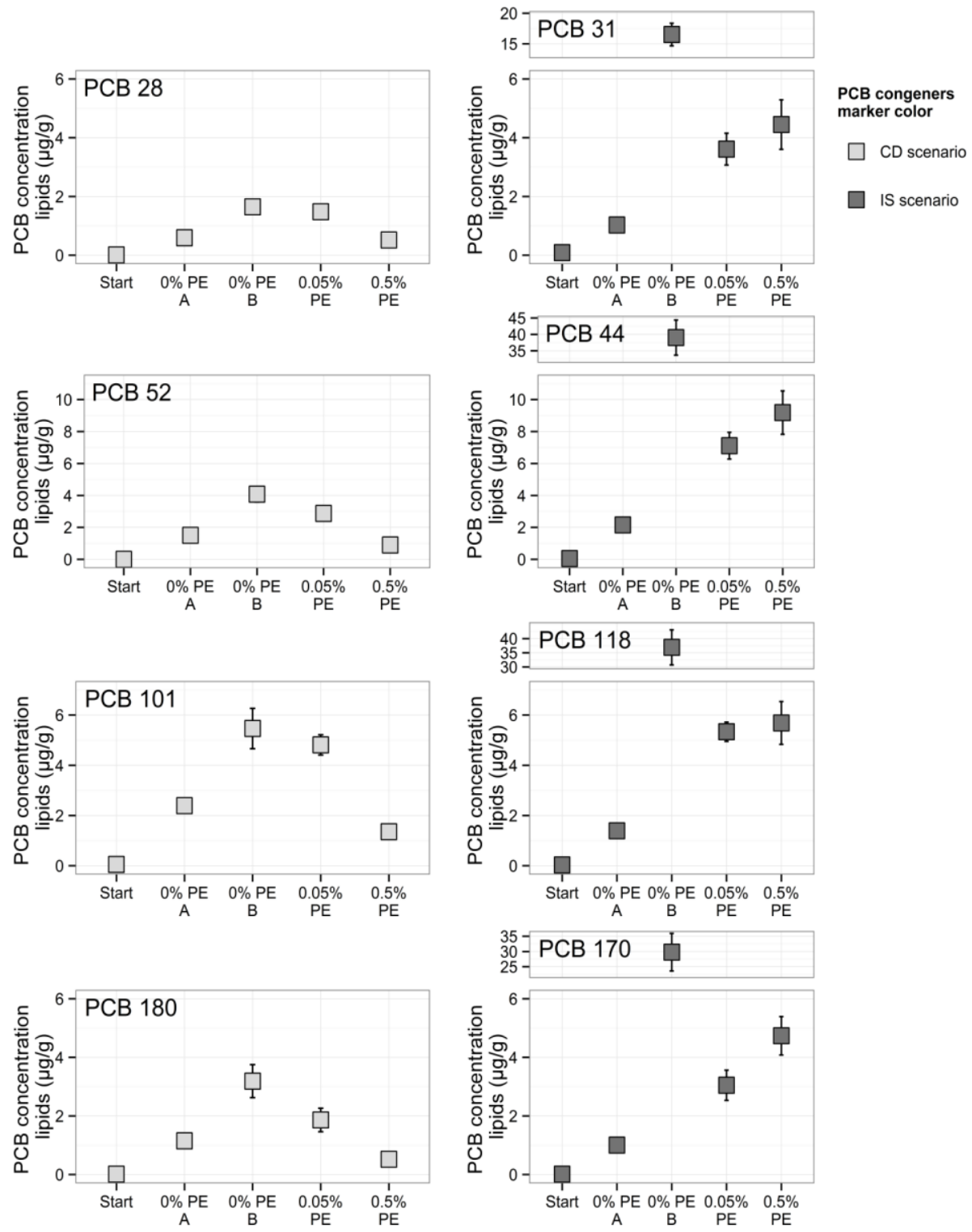

Figure A6.9. Average PCB concentrations $\pm S E$ in lugworms (lipid normalised) after exposure to the different treatments and their background PCB concentrations before start of the exposure assay. Left panels: $P C B$ congener spiked equally in all treatments representing the $C D$ (chemical dilution) scenario. Right panels: $P C B$ congener extra spiked in the treatments with PE and the 0\% PE B treatment to correct for the dilution mechanism representing the IS (infinite source) scenario. Where error bars are invisible they are smaller than the markers. 


\section{Appendix to Chapter 7}

1. Figures $A 7.1-A 7.2$ 
Figures

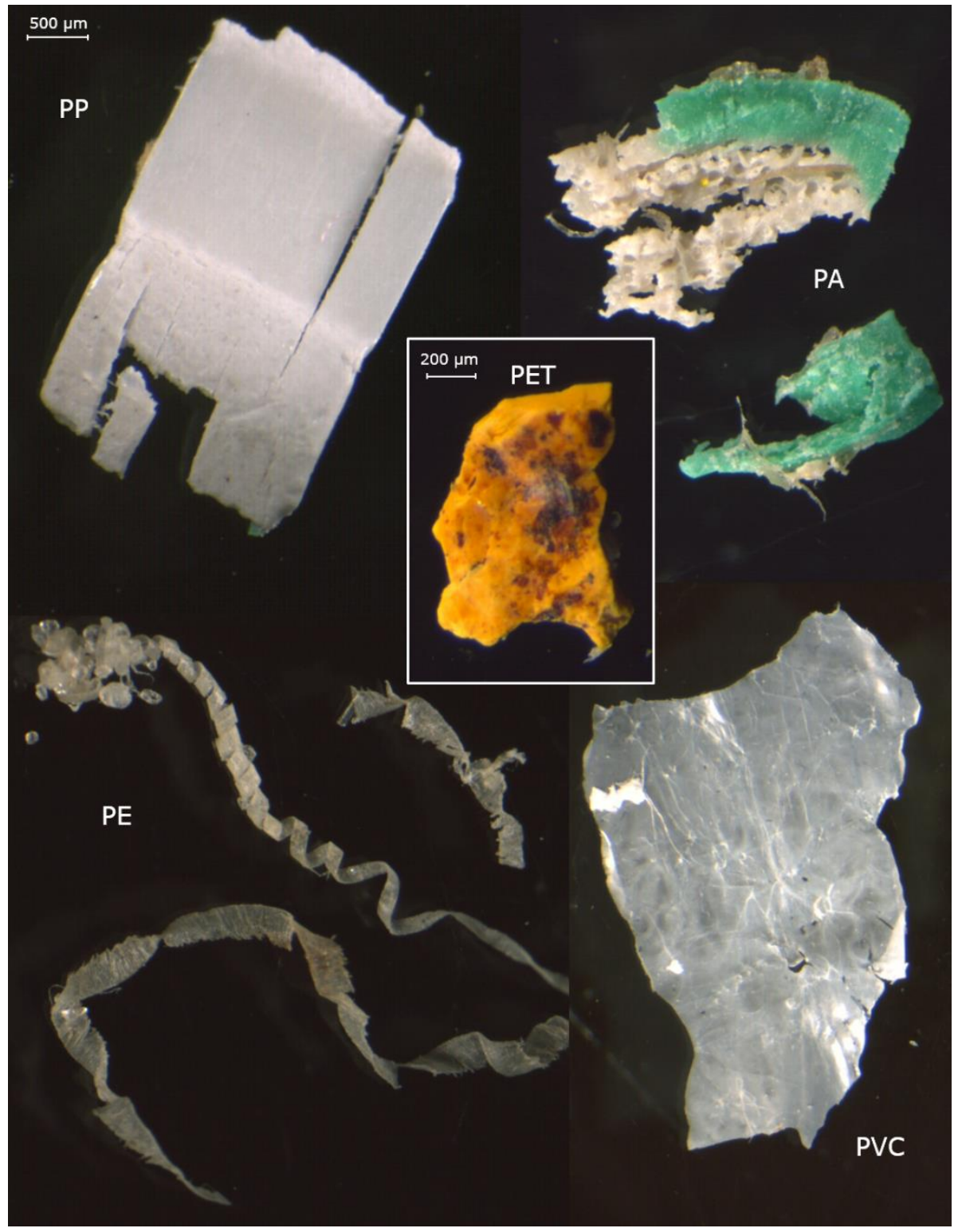

Figure A7.1. Plastic fragments and sheets encountered in the intestines of a humpback whale, of the polymer types polypropylene $\left(P P, R^{2}=0.82\right)$, nylon $\left(P A, R^{2}=0.73\right)$, polyethylene $(P E$, $\left.R^{2}=0.88\right)$, polyvinylchloride $\left(P V C, R^{2}=0.82\right)$ and polyethylene terephthalate $\left(P E T, R^{2}=\right.$ $0.82)$. 
Appendix to Chapter 7

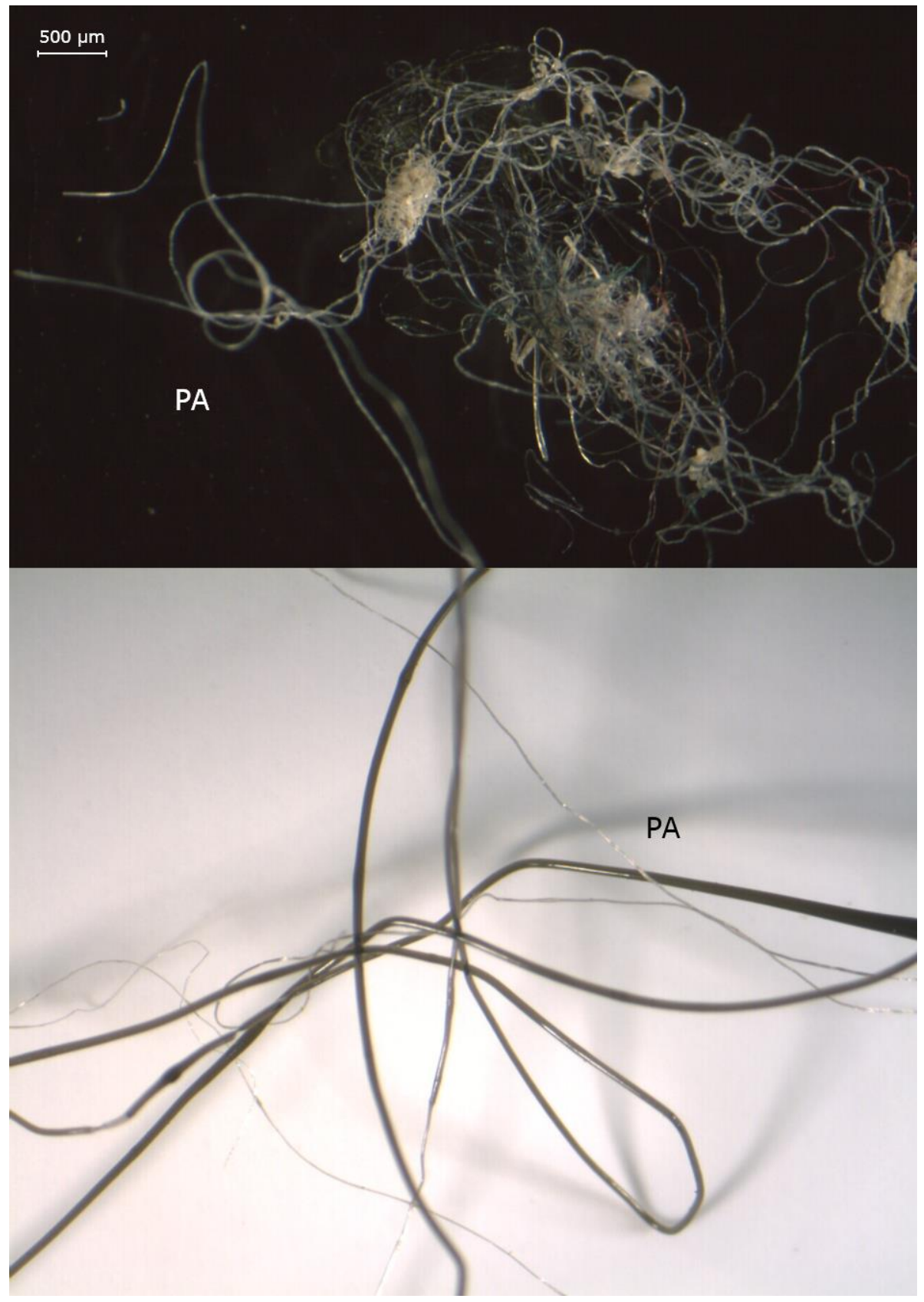

Figure A7.2. Plastic threads encountered in the intestines of a humpback whale, of the polymer type nylon $\left(P A, R^{2}=0.78,0.96\right)$. 
Appendix to Chapter 8

1. Tables A8.1-A8.4 


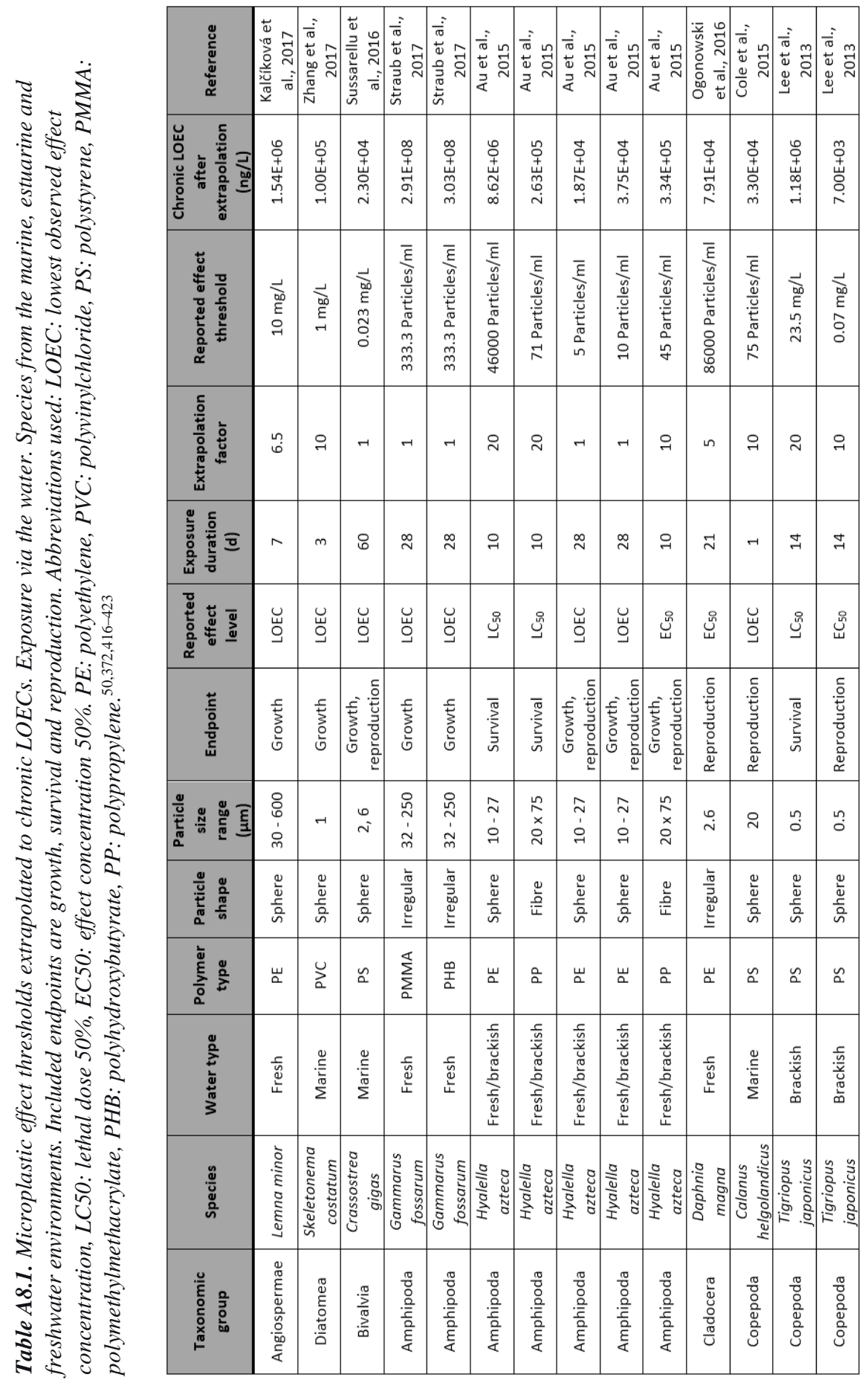


Tables

\begin{tabular}{|c|c|c|c|c|c|c|c|c|c|c|c|c|c|c|}
\hline 离 & 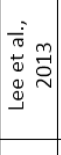 & 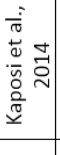 & 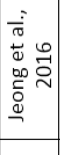 & 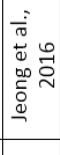 & 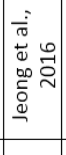 & 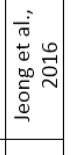 & \multirow{20}{*}{ 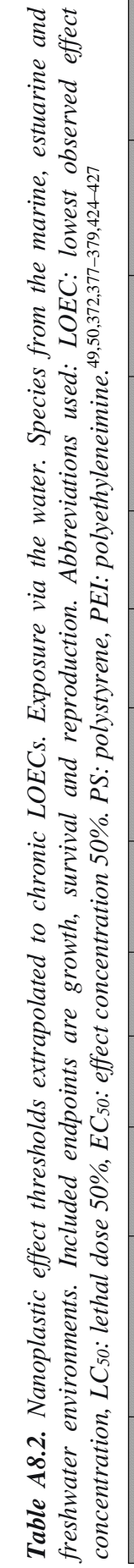 } & 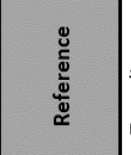 & 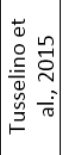 & 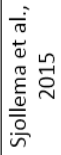 & 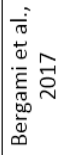 & 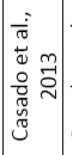 & $\begin{array}{l}\frac{\dot{0}}{\overline{0}} \\
\stackrel{0}{0} \\
0 \\
0 \\
0 \\
0 \\
\tilde{c} \\
\tilde{c}\end{array}$ & 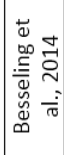 \\
\hline 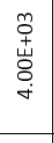 & 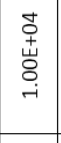 & $\begin{array}{l}\stackrel{\sim}{+} \\
\stackrel{+}{\sim} \\
\underset{\sim}{\sim} \\
m\end{array}$ & 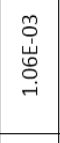 & $\begin{array}{l}\stackrel{\overrightarrow{+}}{山} \\
\stackrel{\vec{J}}{i}\end{array}$ & $\begin{array}{l}\infty \\
\infty \\
-i\end{array}$ & 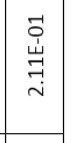 & & 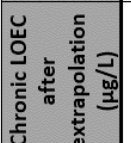 & 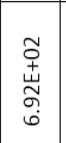 & 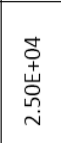 & $\begin{array}{l}\text { Oे } \\
\text { 岃 } \\
\text { 岁 } \\
\infty\end{array}$ & $\begin{array}{l}\overrightarrow{0} \\
\stackrel{+}{+} \\
\infty \\
\infty \\
m\end{array}$ & 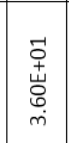 & $\begin{array}{l}\stackrel{u}{0} \\
+ \\
\stackrel{4}{\circ} \\
\stackrel{r}{r}\end{array}$ \\
\hline \multirow[t]{2}{*}{ 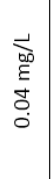 } & \multirow[t]{2}{*}{ 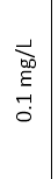 } & \multirow{2}{*}{ 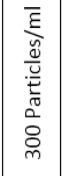 } & \multirow{2}{*}{ 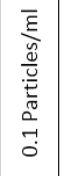 } & \multirow{2}{*}{ 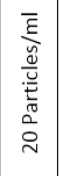 } & \multirow{2}{*}{ 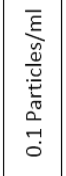 } & \multirow{2}{*}{ 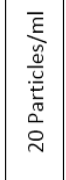 } & & 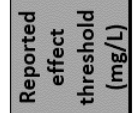 & & 只 & t. & : & นี & ○ి \\
\hline & & & & & & & & & \multirow[b]{2}{*}{ นึ } & \multirow[b]{2}{*}{ 우 } & \multirow[b]{2}{*}{$\stackrel{\Omega}{\sim}$} & \multirow[b]{2}{*}{$\stackrel{\sim}{\sim}$} & \multirow[b]{2}{*}{$\stackrel{\sim}{\sim}$} & \multirow[b]{2}{*}{ 우 } \\
\hline \multirow[t]{2}{*}{ ㄱ } & \multirow[t]{2}{*}{$\rightarrow$} & \multirow[t]{2}{*}{9} & \multirow[t]{2}{*}{ นn } & \multirow[t]{2}{*}{ ڤึ } & \multirow[t]{2}{*}{ 资 } & \multirow[t]{2}{*}{$\stackrel{\text { นn }}{6}$} & & 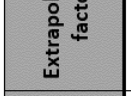 & & & & & & \\
\hline & & & & & & & & 홀 & r & $m$ & $m$ & $m$ & $m$ & $m$ \\
\hline \multirow{2}{*}{ 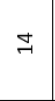 } & \multirow{2}{*}{$\underset{ন}{J}$} & \multirow{2}{*}{ in } & \multirow{2}{*}{$\approx$} & \multirow{2}{*}{ ㄱ. } & $\simeq$ & $\simeq$ & & & & & & & & \\
\hline & & & & & & & & 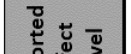 & 嵌 & 岀 & 号 & 品 & 品 & 岀 \\
\hline 总 & in & u & 㟧 & 㟧 & $\vec{u}$ & ü & & & 与 & ב & & & & \\
\hline & & & & & & & & $=$ & $\overrightarrow{0}=\mid$ & $F$ & & & & \\
\hline 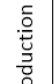 & $\begin{array}{l}\frac{\overline{0}}{\overline{\underline{E}}} \\
\frac{\mathrm{y}}{\bar{z}}\end{array}$ & 章 & 旁 & 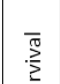 & 訔 & 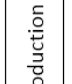 & & 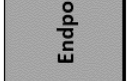 & $\mid$ & 竜 & 豙 & 竞 & 竞 & $\begin{array}{l}3 \\
0 \\
0 \\
0\end{array}$ \\
\hline 产 & $\begin{array}{c}\bar{a} \\
\propto \\
\propto\end{array}$ & & & & & 兗 & & 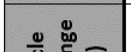 & & & & & & \\
\hline 0 & n̊. & $\begin{array}{l}\text { r } \\
f \\
0\end{array}$ & $\stackrel{n}{0}$ & $\stackrel{n}{0}$ & 0 & $\stackrel{n}{o}$ & & 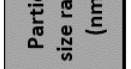 & in & in & ڤ̊ & น & 임 & 尺 \\
\hline 岕 & $\frac{0}{0}$ & 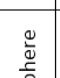 & 这 & $\frac{0}{0}$ & 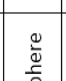 & 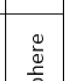 & & 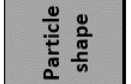 & 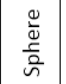 & $\begin{array}{l}\frac{0}{0} \\
\frac{c}{2} \\
\frac{0}{n}\end{array}$ & $\begin{array}{l}\frac{0}{0} \\
\frac{0}{0} \\
\text { in }\end{array}$ & $\frac{0}{\frac{0}{0}}$ & $\frac{0}{\frac{0}{0}}$ & $\begin{array}{l}\frac{0}{0} \\
\frac{c}{0} \\
\frac{0}{n}\end{array}$ \\
\hline in & के & के & जे & 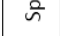 & के & iे & & & & & & & & \\
\hline$\check{\alpha}$ & 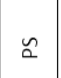 & wa & $\simeq$ & $\check{a}$ & $\simeq$ & $\simeq$ & & 竞 & $\tilde{a}$ & 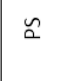 & $\check{a}$ & $\begin{array}{l}\text { 퐁 } \\
\bar{\Sigma}\end{array}$ & $\begin{array}{l}\overrightarrow{\underline{\alpha}} \\
\bar{\alpha}\end{array}$ & $\bumpeq$ \\
\hline 喜 & 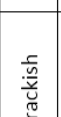 & 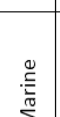 & $\begin{array}{l}\frac{5}{5} \\
\frac{5}{2} \\
\frac{\pi}{2} \\
\frac{2}{2}\end{array}$ & 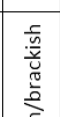 & 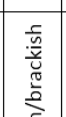 & 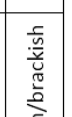 & & 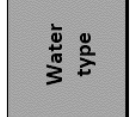 & 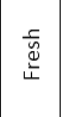 & 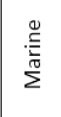 & 竞 & 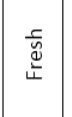 & $\begin{array}{l}\frac{5}{\bar{w}} \\
\frac{\tilde{L}}{4}\end{array}$ & $\begin{array}{l}\frac{5}{\bar{w}} \\
\text { 这 }\end{array}$ \\
\hline$\overline{\tilde{\omega}}$ & $\infty$ & & 铥 & $\begin{array}{l}\text { 离 } \\
\text { 这 }\end{array}$ & $\begin{array}{l}\bar{s} \\
\text { 产 } \\
\end{array}$ & $\begin{array}{l}\overline{\bar{s}} \\
\overline{\underline{4}} \\
\end{array}$ & & & $\frac{\omega}{3}$ & & & $\stackrel{0}{2}$ & & \\
\hline 离. & 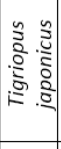 & 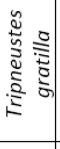 & 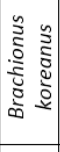 & 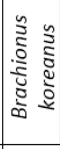 & 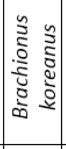 & 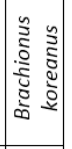 & & ڤँّ & 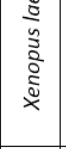 & 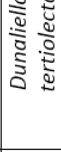 & 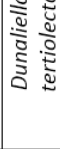 & 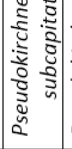 & 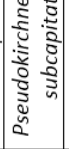 & 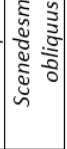 \\
\hline $\begin{array}{l}\text { 융 } \\
\text { 잉 } \\
\text { 잉 }\end{array}$ & \begin{tabular}{|l}
$\frac{\pi}{0}$ \\
0 \\
00 \\
0 \\
0
\end{tabular} & 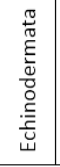 & 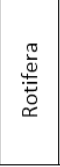 & 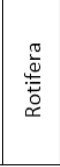 & 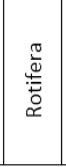 & 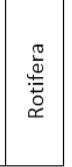 & & 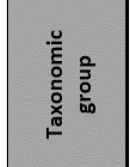 & $\frac{\cdot \frac{0}{0}}{\frac{2}{\frac{2}{2}}}$ & 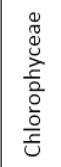 & 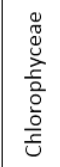 & 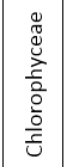 & 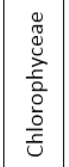 & 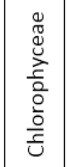 \\
\hline
\end{tabular}




\begin{tabular}{|c|c|c|c|c|c|c|c|c|c|c|c|c|c|c|c|c|c|}
\hline 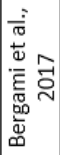 & 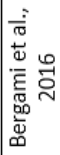 & 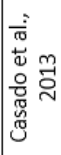 & 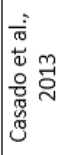 & 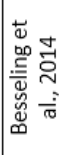 & 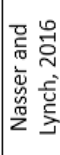 & 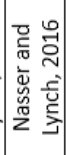 & 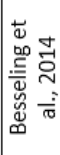 & 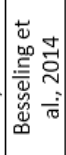 & 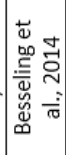 & 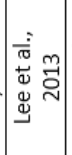 & 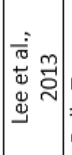 & 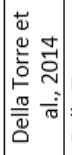 & 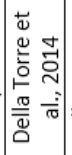 & 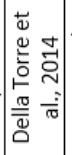 & 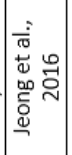 & 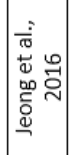 & 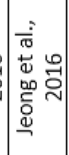 \\
\hline $\begin{array}{l}\vec{c} \\
\stackrel{+}{+} \\
\stackrel{4}{\leftrightarrow} \\
+ \\
+\end{array}$ & 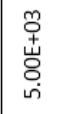 & $\begin{array}{l}\stackrel{\sim}{+} \\
\stackrel{+}{\sim} \\
\stackrel{-}{-}\end{array}$ & 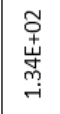 & 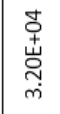 & 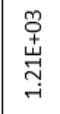 & 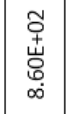 & 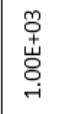 & 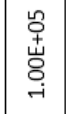 & 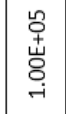 & 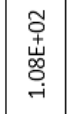 & 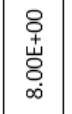 & 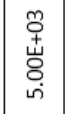 & 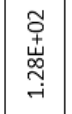 & 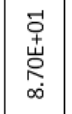 & 㕝 & 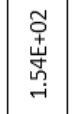 & 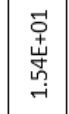 \\
\hline 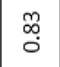 & in & กี & $\stackrel{\substack{\leftrightarrow \\
\leftarrow}}{0}$ & $\tilde{m}$ & 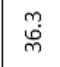 & $\begin{array}{l}\stackrel{\infty}{\sim} \\
\stackrel{\sim}{~}\end{array}$ & $\rightarrow$ & 우 & 우 & $\stackrel{\mathscr{\sim}}{\sim}$ & $\begin{array}{l}\text { 웅 } \\
\text {. }\end{array}$ & 요 & 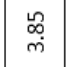 & $\overrightarrow{\mathrm{i}}$ & -1. & 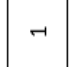 & ન્' \\
\hline
\end{tabular}

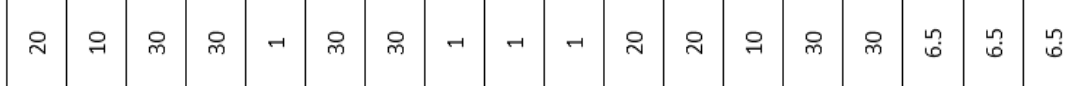

\begin{tabular}{|c|c|c|c|c|c|c|c|c|c|c|c|c|c|c|c|c|c|}
\hline$\underset{ন}{J}$ & $\sim$ & $\rightarrow$ & $\rightarrow$ & $\vec{N}$ & $\rightarrow$ & $\rightarrow$ & $\vec{N}$ & $\vec{N}$ & $\vec{\lambda}$ & A & $\underset{-}{J}$ & $\sim$ & $\rightarrow$ & $\sim$ & $\approx$ & $\approx$ & $\approx$ \\
\hline 品 & 亗 & 总 & 㔽 & 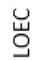 & 吢 & 号 & 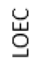 & 岀 & 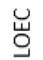 & 号 & 品 & 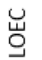 & 品 & త্త & 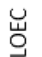 & 岀 & 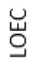 \\
\hline
\end{tabular}

\begin{tabular}{|c|c|c|c|c|c|c|c|c|c|c|c|c|c|c|c|c|c|}
\hline & & & & & & & & & & & & & & & & & \\
\hline 丞 & 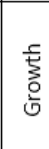 & 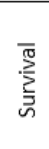 & 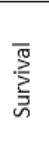 & 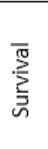 & 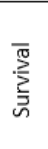 & 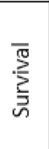 & 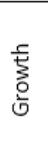 & 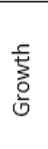 & 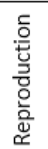 & 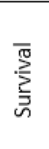 & 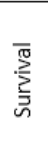 & 㞼 & 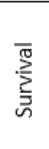 & 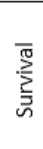 & $\begin{array}{l}\frac{5}{5} \\
\frac{0}{0} \\
0\end{array}$ & 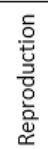 & 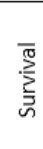 \\
\hline
\end{tabular}

\begin{tabular}{|c|c|c|c|c|c|c|c|c|c|c|c|c|c|c|c|c|c|}
\hline 吊 & in & 出 & 욱 & 尺 & $\mid \begin{array}{ll}1 & 0 \\
n & 0 \\
\infty & \vdots \\
\infty & \infty\end{array}$ & 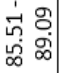 & $\curvearrowright$ & 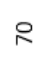 & 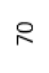 & 요 & 요 & 우 & 으 & 요 & 요 & 요 & 으 \\
\hline $\begin{array}{l}\frac{0}{\bar{\nu}} \\
\frac{\bar{\nu}}{n}\end{array}$ & $\begin{array}{l}\frac{0}{\overline{0}} \\
\frac{\overline{0}}{\bar{n}}\end{array}$ & 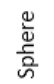 & $\begin{array}{l}\frac{\alpha}{0} \\
\frac{\bar{c}}{2} \\
\frac{0}{n}\end{array}$ & 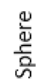 & $\begin{array}{l}\frac{0}{\overline{0}} \\
\frac{\overline{0}}{0}\end{array}$ & $\begin{array}{l}\frac{0}{0} \\
\frac{\bar{c}}{0} \\
\bar{n}\end{array}$ & 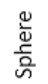 & $\begin{array}{l}\frac{0}{0} \\
\frac{0}{0} \\
\frac{0}{n}\end{array}$ & 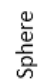 & $\begin{array}{l}\frac{0}{0} \\
\frac{0}{0} \\
\bar{c}\end{array}$ & $\frac{0}{\frac{0}{0}}$ & $\begin{array}{l}\frac{0}{0} \\
\frac{\bar{c}}{0} \\
\bar{n}\end{array}$ & $\begin{array}{l}\frac{0}{0} \\
\frac{\bar{c}}{2}\end{array}$ & 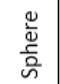 & 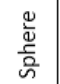 & $\begin{array}{l}\frac{0}{0} \\
\frac{\bar{c}}{0} \\
\bar{n}\end{array}$ & $\begin{array}{c}\frac{0}{0} \\
\frac{\bar{c}}{0} \\
\bar{n}\end{array}$ \\
\hline$\approx$ & 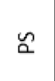 & $\begin{array}{l}\overline{\underline{a}} \\
\bar{a}\end{array}$ & $\begin{array}{l}\overline{\underline{a}} \\
\bar{a}\end{array}$ & $\check{a}$ & $\approx$ & $\approx$ & $\check{a}$ & $\approx$ & $\cong$ & $\cong$ & $\check{a}$ & 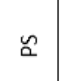 & $\approx$ & $\approx$ & $\check{\alpha}$ & 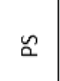 & $\check{a}$ \\
\hline 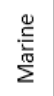 & 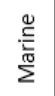 & $\begin{array}{l}\text { 离 } \\
\text { 这 }\end{array}$ & 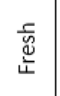 & 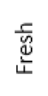 & 系 & 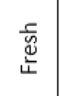 & 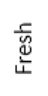 & 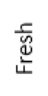 & $\begin{array}{l}\frac{5}{\tilde{W}} \\
\text { W. }\end{array}$ & 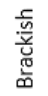 & 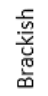 & 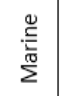 & 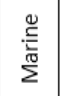 & 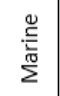 & 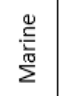 & 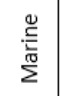 & 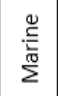 \\
\hline 宽 & 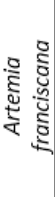 & 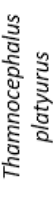 & 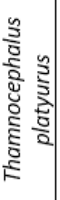 & 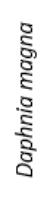 & 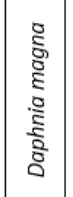 & 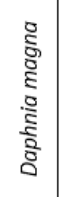 & 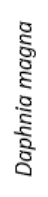 & 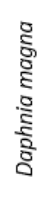 & 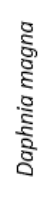 & 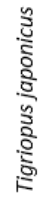 & 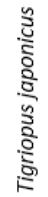 & 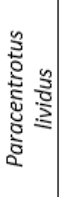 & 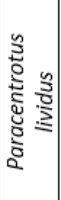 & 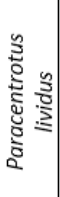 & 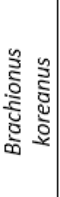 & 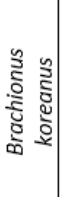 & 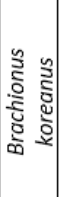 \\
\hline 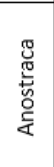 & 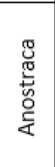 & 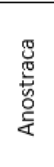 & 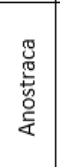 & $\begin{array}{l}\frac{\pi}{0} \\
\frac{0}{0} \\
\frac{\pi}{\pi} \\
\frac{\pi}{0}\end{array}$ & $\begin{array}{l}\frac{\pi}{0} \\
\frac{0}{0} \\
\frac{\pi}{0} \\
\frac{\pi}{0}\end{array}$ & $\begin{array}{l}\frac{\pi}{0} \\
\frac{0}{0} \\
\frac{\pi}{0} \\
\frac{\pi}{0}\end{array}$ & $\begin{array}{l}\frac{\pi}{0} \\
\frac{0}{0} \\
\frac{0}{\pi} \\
\frac{\pi}{0}\end{array}$ & 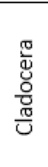 & $\begin{array}{l}\frac{\pi}{0} \\
\frac{0}{0} \\
\frac{\pi}{0} \\
\frac{\pi}{U}\end{array}$ & $\begin{array}{l}\text { 융 } \\
\text { 웅 } \\
\text { 웅 }\end{array}$ & $\begin{array}{l}\frac{\pi}{0} \\
0 \\
00 \\
0 \\
0\end{array}$ & 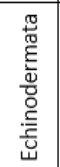 & 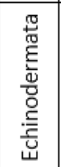 & 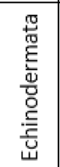 & 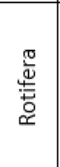 & 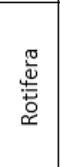 & 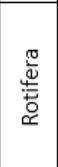 \\
\hline
\end{tabular}


Tables

\begin{tabular}{|c|c|c|c|c|c|c|c|c|c|c|}
\hline 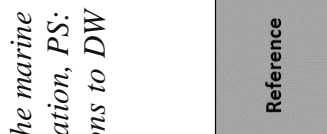 & 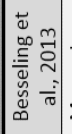 & 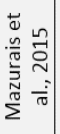 & 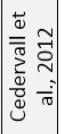 & 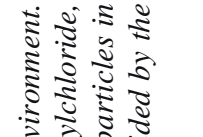 & 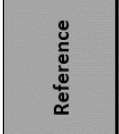 & 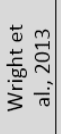 & 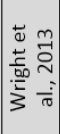 & 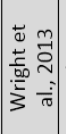 & 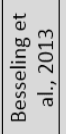 & 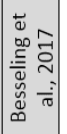 \\
\hline 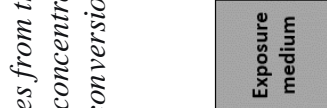 & 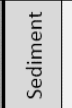 & : & 훈 & 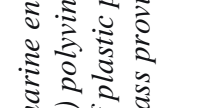 & 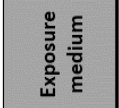 & 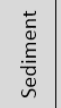 & 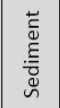 & 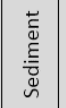 & 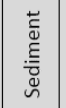 & 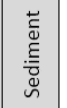 \\
\hline 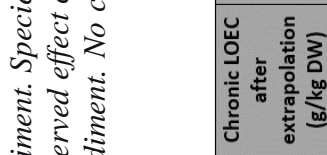 & I & $\approx$ & $\rightarrow$ & 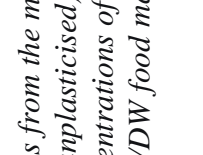 & 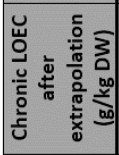 & $\stackrel{\sim}{6}$ & $\stackrel{\sim}{\underset{\sim}{\sim}}$ & $\stackrel{n}{\mathfrak{G}}$ & न̆ & $\stackrel{n}{0}$ \\
\hline 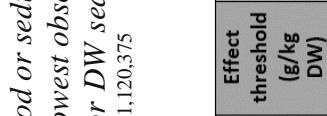 & ন & $\approx$ & -1 & 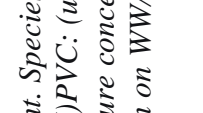 & 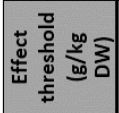 & $\stackrel{n}{\mathfrak{G}}$ & $\underset{\sim}{\underset{7}{7}}$ & $\stackrel{n}{G}$ & $\frac{0}{4}$ & $\stackrel{n}{o}$ \\
\hline 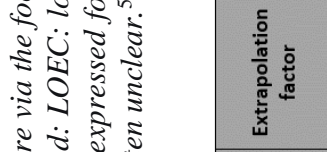 & -1 & -1 & $\rightarrow$ & 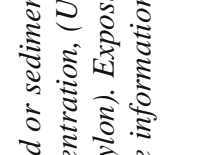 & 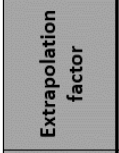 & 우 & $\rightarrow$ & $r$ & ్ֶ & $r$ \\
\hline 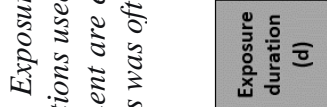 & $\stackrel{\infty}{\sim}$ & $\stackrel{m}{\infty}$ & $\stackrel{m}{\circ}$ & 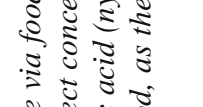 & 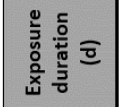 & $\sim$ & $\stackrel{\infty}{\sim}$ & $\stackrel{\infty}{\sim}$ & ㄱ. & $\stackrel{\infty}{\sim}$ \\
\hline 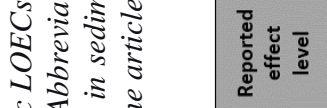 & 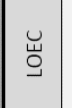 & 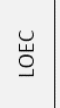 & 岂 & 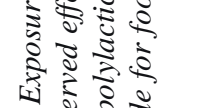 & 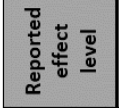 & 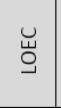 & 岀 & 亗 & 岃 & 亗 \\
\hline 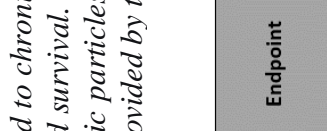 & 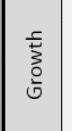 & $\stackrel{\bar{m}}{\sum_{3}^{n}}$ & 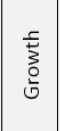 & 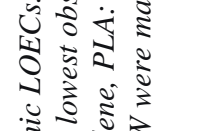 & 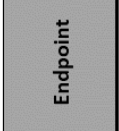 & 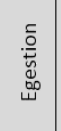 & 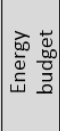 & 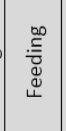 & 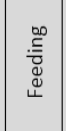 & 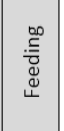 \\
\hline 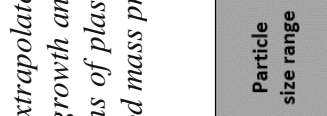 & 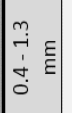 & 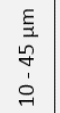 & 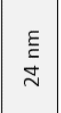 & 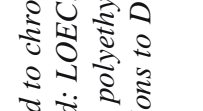 & 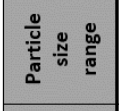 & 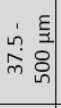 & 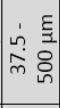 & 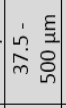 & 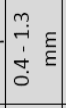 & $\begin{array}{c}\infty \\
0 \\
-1 \\
\vdots \\
\vdots \\
1\end{array}$ \\
\hline 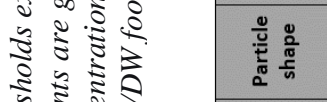 & $\begin{array}{l}\frac{0}{0} \\
\frac{\pi}{0} \\
\text { in }\end{array}$ & $\begin{array}{c}\frac{0}{0} \\
\frac{c}{n} \\
\text { ñ }\end{array}$ & $\begin{array}{l}\frac{0}{\overline{0}} \\
\frac{0}{2} \\
\bar{n}\end{array}$ & 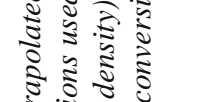 & 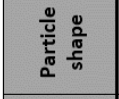 & 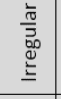 & 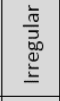 & 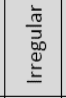 & 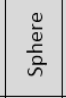 & 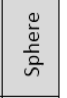 \\
\hline غุ̀ & $\simeq$ & 똠 & $\check{\Sigma}$ & 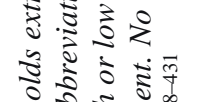 & 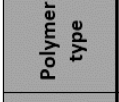 & 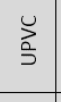 & $\begin{array}{l}\text { ב̀ } \\
\text { a }\end{array}$ & בั้ & $\simeq$ & $\begin{array}{l}\text { 峑 } \\
\text { 呈 }\end{array}$ \\
\hline 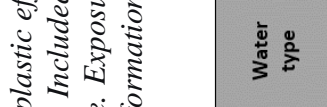 & 竞 & 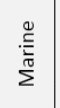 & 离 & 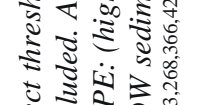 & 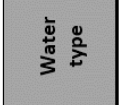 & 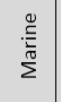 & 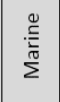 & 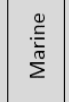 & 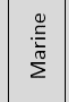 & 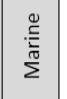 \\
\hline 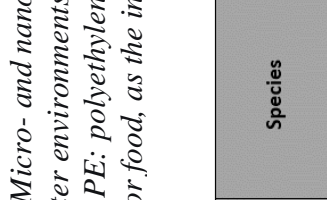 & 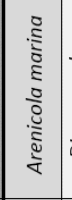 & 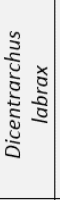 & 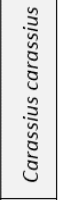 & 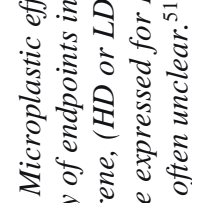 & 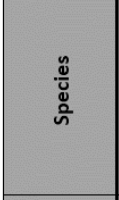 & 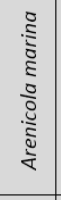 & 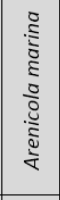 & 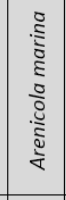 & 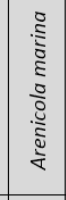 & 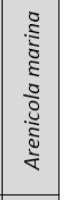 \\
\hline 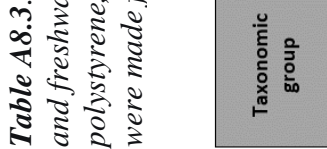 & $\frac{\frac{\pi}{0}}{\frac{0}{0}}$ & $\begin{array}{l}\frac{\pi}{0} \\
\frac{\pi}{0} \\
\frac{0}{0} \\
\frac{\delta}{U}\end{array}$ & 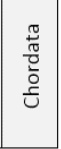 & 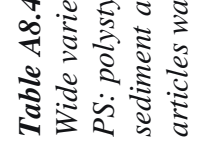 & 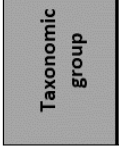 & \begin{tabular}{|l}
$\frac{\pi}{0}$ \\
$\frac{0}{0}$ \\
$\frac{5}{5}$ \\
$\frac{5}{4}$
\end{tabular} & $\frac{\frac{\pi}{0}}{\frac{0}{0}}$ & $\begin{array}{l}\frac{\pi}{0} \\
\frac{0}{0} \\
\frac{0}{5} \\
\frac{5}{4}\end{array}$ & $\begin{array}{l}\frac{\pi}{\frac{\pi}{0}} \\
\frac{\sum_{0}^{2}}{4} \\
\frac{5}{4}\end{array}$ & $\begin{array}{l}\frac{\pi}{0} \\
\frac{0}{0} \\
\frac{5}{4} \\
\frac{5}{4}\end{array}$ \\
\hline
\end{tabular}




\section{Appendix to Chapter 8}

\begin{tabular}{|c|c|c|c|c|c|c|c|c|c|c|c|}
\hline 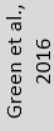 & 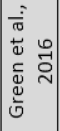 & 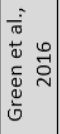 & 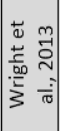 & 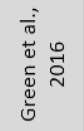 & 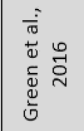 & 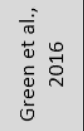 & 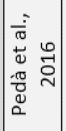 & 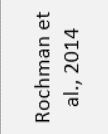 & 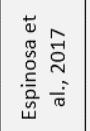 & 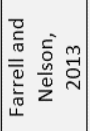 & 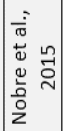 \\
\hline 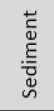 & 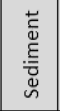 & 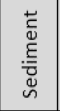 & 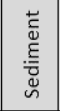 & 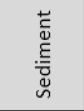 & 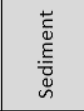 & 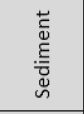 & 웅 & 임 & 임 & 현 & 형 \\
\hline$\stackrel{\Perp}{\sim}$ & $\stackrel{\sim}{\sim}$ & $\stackrel{\mathscr{N}}{\sim}$ & 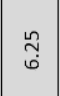 & 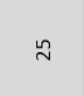 & 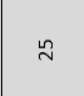 & 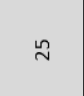 & $ન$ & : & 5 & 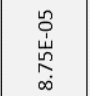 & 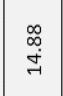 \\
\hline$\stackrel{\sim}{\sim}$ & $\stackrel{\mathscr{N}}{0}$ & $\stackrel{\mathscr{N}}{*}$ & 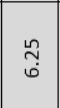 & $\stackrel{\Perp}{\sim}$ & $\stackrel{\sim}{\sim}$ & $\stackrel{\sim}{ }$ & $ન$ & ঃ্ণ & - & 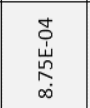 & 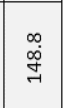 \\
\hline$\rightarrow$ & $\rightarrow$ & -1 & $\rightarrow$ & -1 & - & - & - & - & - & 악 & 우 \\
\hline$\vec{m}$ & $\vec{m}$ & $\vec{m}$ & $\stackrel{\infty}{\sim}$ & $\vec{m}$ & $\vec{m}$ & $\vec{m}$ & ८ & ৪ & 이 & $\frac{5}{-1}$ & - \\
\hline 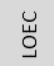 & 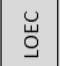 & 岕 & 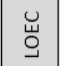 & 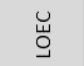 & 亗 & 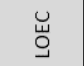 & 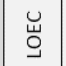 & 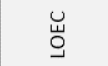 & 亗 & 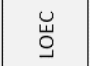 & 兽 \\
\hline 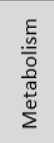 & 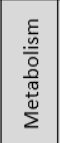 & 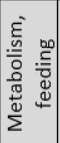 & 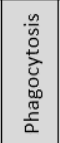 & 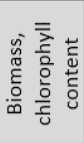 & 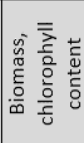 & 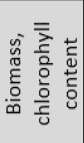 & 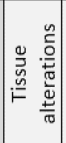 & 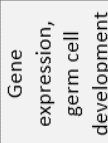 & 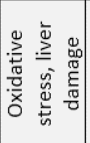 & 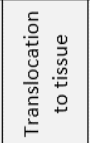 & 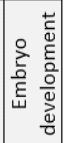 \\
\hline 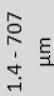 & 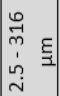 & 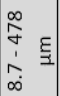 & 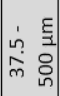 & $\begin{array}{l}\hat{O} \\
\dot{j} \\
\dot{j} \\
\dot{T}\end{array}$ & $\begin{array}{l}\stackrel{0}{1} \\
\stackrel{-1}{0} \xi \\
\stackrel{n}{N}\end{array}$ & 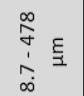 & 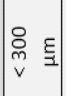 & 品 & 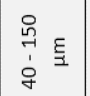 & $\begin{array}{l}\xi \\
\xi \\
n \\
0\end{array}$ & $\begin{array}{l}\varepsilon \\
\varepsilon \\
\stackrel{\varepsilon}{n} \\
\vee\end{array}$ \\
\hline 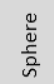 & 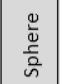 & $\begin{array}{l}\frac{0}{0} \\
\frac{0}{0} \\
\text { in }\end{array}$ & 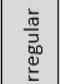 & $\begin{array}{l}\frac{0}{0} \\
\frac{0}{0}\end{array}$ & 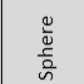 & 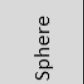 & 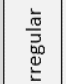 & 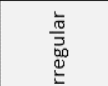 & & 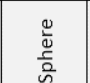 & $\frac{\overrightarrow{\mathrm{w}}}{\overline{\bar{\sigma}}}$ \\
\hline$\$$ & $\begin{array}{l}\text { 䚯 } \\
\text { 呈 }\end{array}$ & ¿ & \ั) & 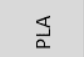 & $\begin{array}{l}\text { 山्̀ } \\
\text { ⿳亠口冋 }\end{array}$ & ૫a & ¿ & щu & ૫্ & $\approx$ & $\underline{a}$ \\
\hline 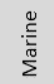 & 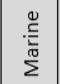 & 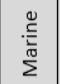 & 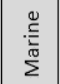 & 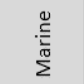 & 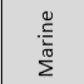 & 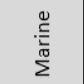 & 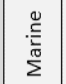 & 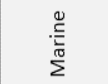 & 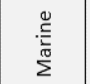 & 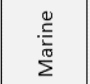 & 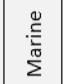 \\
\hline 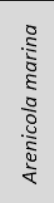 & 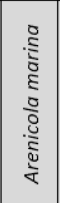 & 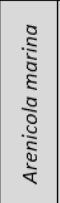 & 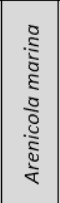 & 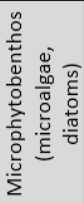 & 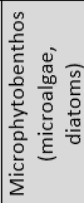 & 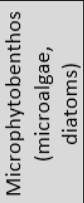 & 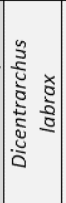 & 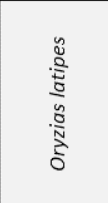 & 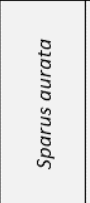 & 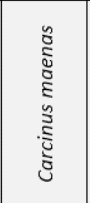 & 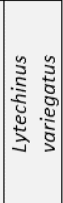 \\
\hline $\begin{array}{l}\frac{\pi}{\frac{0}{0}} \\
\frac{0}{0} \\
\frac{5}{4}\end{array}$ & $\begin{array}{l}\frac{\pi}{0} \\
\frac{0}{0} \\
\frac{0}{2} \\
\frac{5}{4}\end{array}$ & $\begin{array}{l}\frac{\pi}{0} \\
\frac{0}{0} \\
\frac{0}{5} \\
\frac{5}{4}\end{array}$ & $\begin{array}{l}\frac{\pi}{0} \\
\frac{0}{0} \\
\frac{0}{5} \\
\frac{5}{4}\end{array}$ & 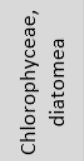 & 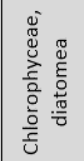 & 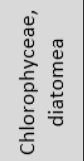 & 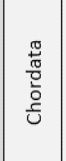 & $\begin{array}{l}\frac{\pi}{0} \\
\frac{\pi}{0} \\
\text { o } \\
\text { d }\end{array}$ & 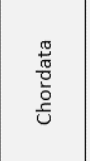 & 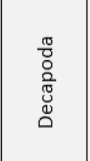 & 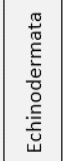 \\
\hline
\end{tabular}




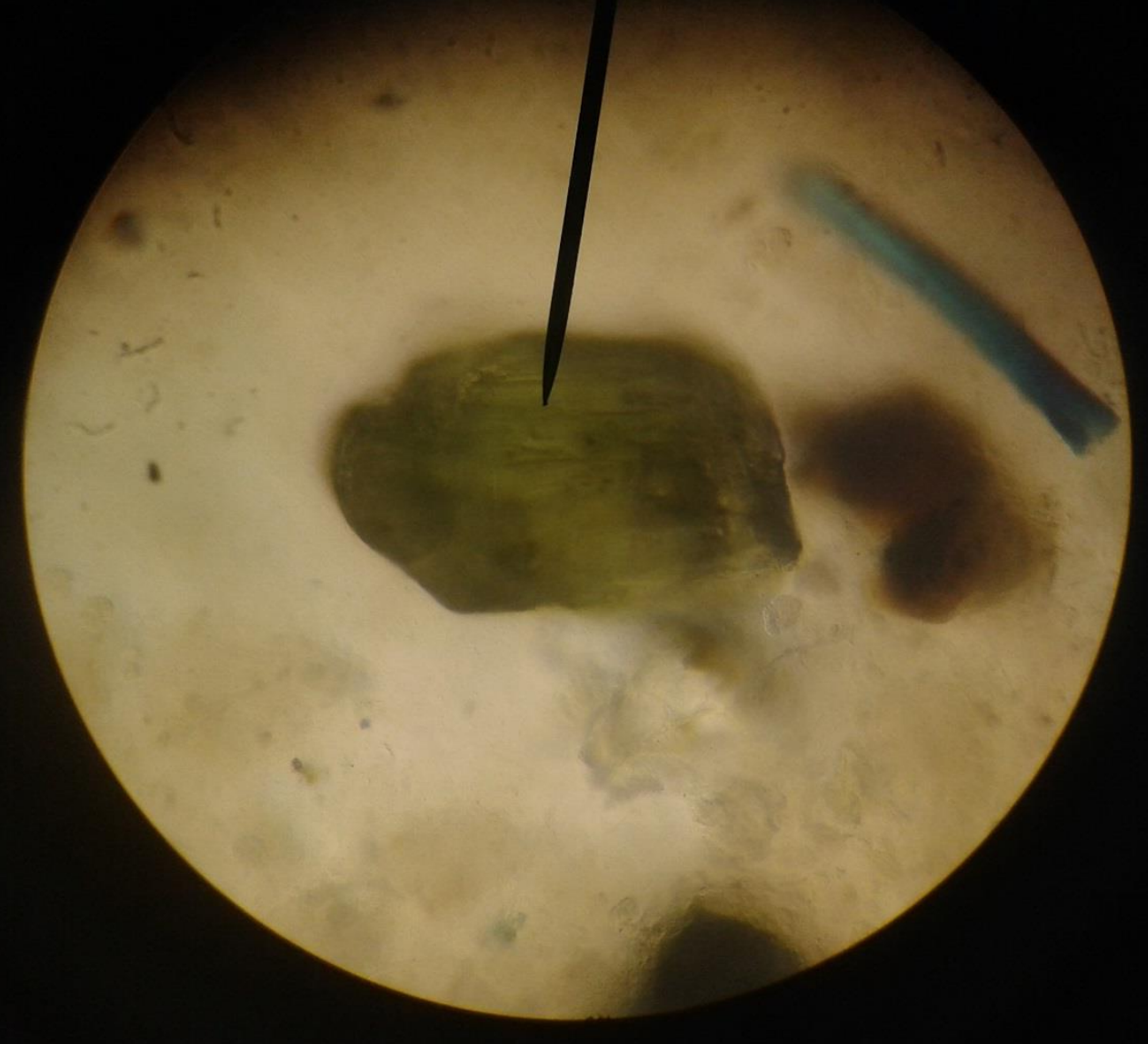




\section{References}

(1) Andrady, A. L.; Neal, M. a Applications and societal benefits of plastics. Philos. Trans. R. Soc. Lond. B. Biol. Sci. 2009, 364, 1977-1984.

(2) PlasticsEurope Plastics - the Facts; 2016.

(3) Azzarello, M.; Van Vleet, E. Marine birds and plastic pollution. Mar. Ecol. Prog. Ser. 1987, 37, 295-303.

(4) Jambeck, J. R.; Geyer, R.; Wilcox, C.; Siegler, T. R.; Perryman, M.; Andrady, A. L.; Narayan, R.; Law, K. L. Plastic waste inputs from land into the ocean. Science 2015, 347, 768-771.

(5) Galgani, F.; Hanke, G.; Werner, S.; De Vrees, L. Marine litter within the European marine strategy framework directive. ICES J. Mar. Sci. 2013, 70, 1055-1064.

(6) Wong, C. S.; Green, D. R.; Cretney, W. J. Quantitative tar and plastic waste distributions in the Pacific Ocean. Nature 1974, 247, 30-32.

(7) Shaw, D. G.; Mapes, G. A. Surface circulation and the distribution of pelagic tar and plastic. Mar. Pollut. Bull. 1979, 10, 160-162.

(8) Andrady, A. L. Microplastics in the marine environment. Mar. Pollut. Bull. 2011, 62, 1596-1605.

(9) Moore, C. J. Synthetic polymers in the marine environment: A rapidly increasing, long-term threat. Environ. Res. 2008, 108, 131-139.

(10) Carpenter, E. J.; Anderson, S. T.; Harvey, G. R.; Miklas, H. P.; Peck, B. B. Polystyrene spherules in coastal water. Science (80-. ). 1972, 178, 749750.

(11) Cole, M.; Lindeque, P.; Halsband, C.; Galloway, T. S. Microplastics as contaminants in the marine environment: A review. Mar. Pollut. Bull. 2011, 62, 2588-2597.

(12) Fendall, L. S.; Sewell, M. a Contributing to marine pollution by washing your face: microplastics in facial cleansers. Mar. Pollut. Bull. 2009, 58, 1225-1228.

(13) Browne, M. A.; Crump, P.; Niven, S. J.; Teuten, E.; Tonkin, A.; Galloway, T.; Thompson, R. Accumulation of microplastic on shorelines woldwide: sources and sinks. Environ. Sci. Technol. 2011, 45, 9175-9179.

(14) Zubris, K. A. V; Richards, B. K Synthetic fibers as an indicator of land application of sludge. Environ. Pollut. 2005, 138, 201-211.

(15) Koelmans, A. A.; Besseling, E.; Shim, W. J. Nanoplastics in the aquatic environment. Critical review. In Marine anthropogenic litter; Bergmann, M.; Gutow, L.; Klages, M., Eds.; Springer Cham Heidelberg: New York Dordrecht, London, 2015, 325-340.

(16) Wagner, M.; Scherer, C.; AlvarezMuñoz, D.; Brennholt, N.; Bourrain, X.; Buchinger, S.; Fries, E.; Grosbois, C.; Klasmeier, J.; Marti, T.; RodriguezMozaz, S.; Urbatzka, R.; Vethaak, A D.; Winther-Nielsen, M.; Reifferscheid, G. Microplastics in freshwater ecosystems: what we know and what we need to know. Environ. Sci. Eur. 2014, 26, 1-9.

(17) Klaine, S. J.; Koelmans, A. a; Horne, N.; Carley, S.; Handy, R. D.; Kapustka L.; Nowack, B.; von der Kammer, F. Paradigms to assess the environmental impact of manufactured nanomaterials. Environ. Toxicol. Chem. 2012, 31, 314.

(18) Stephens, B.; Azimi, P.; El Orch, Z.; Ramos, T. Ultrafine particle emissions from desktop 3D printers. Atmos. Environ. 2013, 79, 334-339.

(19) Gouin, T.; Roche, N.; Lohmann, R.; Hodges, G. A thermodynamic approach for assessing the environmental exposure of chemicals absorbed to microplastic. Environ. Sci. Technol. 2011, 45, 1466-1472. 
(20) Teuten, E. L.; Rowland, S. J.; Galloway, T. S.; Thompson, R. C. Potential for plastics to transport hydrophobic contaminants. Environ. Sci. Technol. 2007, 41, 7759-7764.

(21) Teuten, E. L.; Saquing, J. M.; Knappe, D. R. U.; Barlaz, M. a; Jonsson, S.; Björn, A.; Rowland, S. J.; Thompson, R. C.; Galloway, T. S.; Yamashita, R.; Ochi, D.; Watanuki, Y.; Moore, C.; Viet, P. H.; Tana, T. S.; Prudente, M.; Boonyatumanond, R.; Zakaria, M. P.; Akkhavong, K.; Ogata, Y.; Hirai, H.; Iwasa, S.; Mizukawa, K.; Hagino, Y.; Imamura, A.; Saha, M.; Takada, H. Transport and release of chemicals from plastics to the environment and to wildlife. Philos. Trans. R. Soc. Lond. B. Biol. Sci. 2009, 364, 2027-2045.
Thompson, R. C.; Olsen, Y.; Mitchell, R. P.; Davis, A.; Rowland, S. J.; John, A. W. G.; McGonigle, D.; Russell, A. E. Lost at sea: where is all the plastic? Science 2004, 304, 838.

Gregory, M. R. Environmental implications of plastic debris in marine settings--entanglement, ingestion, smothering, hangers-on, hitch-hiking and alien invasions. Philos. Trans. R. Soc. Lond. B. Biol. Sci. 2009, 364 , 2013-2025

Pierce, K. E.; Harris, R. J.; Larned, L. S.; Pokras, M. A. Obstruction and starvation associated with plastic ingestion in a Northern Gannet Morus bassanus and a Greater Shearwater Puffinus gravis. Mar. Ornithol. 2004, 32, 187-189.

Van Franeker, J. A.; Blaize, C.; Danielsen, J.; Fairclough, K.; Gollan, J.; Guse, N.; Hansen, P.-L.; Heubeck, M.; Jensen, J.-K.; Le Guillou, G.; Olsen, B.; Olsen, K.; Pedersen, J.; Stienen, E.; Turner, D. Monitoring plastic ingestion by the northern fulmar Fulmarus glacialis in the North Sea. Environ. Pollut. 2011, 159, 2609-2615.

Moore, C. J.; Moore, S. L.; Leecaster, M. K.; Weisberg, S. B. A comparison of plastic and plankton in the North Pacific Central Gyre. Mar. Pollut. Bull. 2001, 42, 1297-1300.

Zarfl, C.; Fleet, D.; Fries, E.; Galgani, F.; Gerdts, G.; Hanke, G.; Matthies, M. Microplastics in oceans. Mar. Pollut.
Bull. 2011, 62, 1589-1591.

(28) Bhattacharya, P.; Lin, S.; Turner, J. P.; Ke, P. C. Physical adsorption of charged plastic nanoparticles affects algal photosynthesis. J. Phys. Chem. C. 2010 , 114, 16556-16561.

(29) Browne, M. a; Dissanayake, A.; Galloway, T. S.; Lowe, D. M.; Thompson, R. C. Ingested microscopic plastic translocates to the circulatory system of the mussel, Mytilus edulis (L). Environ. Sci. Technol. 2008, 42, 5026-5031.

(30) Koehler, A.; Marx, U.; Broeg, K.; Bahns, S.; Bressling, J. Effects of nanoparticles in Mytilus edulis gills and hepatopancreas - a new threat to marine life? Mar. Environ. Res. 2008, 66, 12 14.

(31) Auffan, M.; Rose, J.; Bottero, J.-Y.; Lowry, G. V; Jolivet, J.-P.; Wiesner, M. R. Towards a definition of inorganic nanoparticles from an environmental, health and safety perspective. Nat. Nanotechnol. 2009, 4, 634-641.

(32) Van Franeker, J. A. Fulmar litter EcoQO Monitoring in the Netherlands 1979-2008 - in relation to EU Directive 2000/59/EC on port reception facilities. 2010.

(33) De Gruijter, C. Occurrence and effects of plastic in North Sea fish species. MSc. Thesis Wageningen University. 2011.

(34) Murray, F.; Cowie, P. R. Plastic contamination in the decapod crustacean Nephrops norvegicus (Linnaeus, 1758). Mar. Pollut. Bull. 2011, 62, 1207-1217.

(35) Possatto, F. E.; Barletta, M.; Costa, M. F.; do Sul, J. a I.; Dantas, D. V Plastic debris ingestion by marine catfish: an unexpected fisheries impact. Mar. Pollut. Bull. 2011, 62, 1098-1102.

(36) Lindborg, V. a; Ledbetter, J. F.; Walat, J. M.; Moffett, C. Plastic consumption and diet of Glaucous-winged Gulls (Larus glaucescens). Mar. Pollut. Bull. 2012, 64, 2351-2356.

Hirai, H.; Takada, H.; Ogata, Y.; Yamashita, R.; Mizukawa, K.; Saha, M.; Kwan, C.; Moore, C.; Gray, H.; Laursen, D.; Zettler, E. R.; Farrington, J. W.; Reddy, C. M.; Peacock, E. E.; 
Ward, M. W. Organic micropollutants in marine plastics debris from the open ocean and remote and urban beaches. Mar. Pollut. Bull. 2011, 62, 1683-1692.

Faure, F.; Corbaz, M.; Baecher, H.; Felippe, L. Pollution due to plastics and microplastics in Lake Geneva and in the Mediterranean Sea. Arch. Des Sci. 2012, 65, 157-164.

(39) Claessens, M.; De Meester, S.; Van Landuyt, L.; De Clerck, K.; Janssen, C. R. Occurrence and distribution of microplastics in marine sediments along the Belgian coast. Mar. Pollut. Bull. 2011, 62, 2199-2204.

(40) Shimeta, J.; Koehl, M. a. R Mechanisms of particle selection by tentaculate suspension feeders during encounter, retention, and handling. $J$. Exp. Mar. Bio. Ecol. 1997, 209, 47-73.

(41) Wright, S. L.; Thompson, R. C.; Galloway, T. S. The physical impacts of microplastics on marine organisms: A review. Environ. Pollut. 2013, 178, 483-492.

(42) Collignon, A.; Hecq, J.-H.; Galgani, F.; Voisin, P.; Collard, F.; Goffart, A. Neustonic microplastic and zooplankton in the North Western Mediterranean Sea. Mar. Pollut. Bull. 2012, 64, 861-864.

(43) Dantas, D. V; Barletta, M.; da Costa, M F. The seasonal and spatial patterns of ingestion of polyfilament nylon fragments by estuarine drums (Sciaenidae). Environ. Sci. Pollut. Res. Int. 2012, 19, 600-606.

(44) Denuncio, P.; Bastida, R.; Dassis, M.; Giardino, G.; Gerpe, M.; Rodríguez, D. Plastic ingestion in Franciscana dolphins, Pontoporia blainvillei (Gervais and d'Orbigny, 1844), from Argentina. Mar. Pollut. Bull. 2011, 62, 1836-1841.

(45) Davison, P.; Asch, R. Plastic ingestion by mesopelagic fishes in the North Pacific Subtropical Gyre. Mar. Ecol. Prog. Ser. 2011, 432, 173-180.

(46) Boerger, C. M.; Lattin, G. L.; Moore, S. L.; Moore, C. J. Plastic ingestion by planktivorous fishes in the North Pacific Central Gyre. Mar. Pollut. Bull. 2010, 60, 2275-2278.

Secchi, E. R.; Zarzur, S. Plastic debris ingested by a Blainville's beaked whale, Mesoplodon densirostris, washed ashore in Brazil. Aquat. Mamm. 1999, 25, 21-24.

(48) European Environmental Agency State of Europe's seas; Copenhagen, Denmark, 2015, 1-218.

(49) Besseling, E.; Wang, B.; Lürling, M.; Koelmans, A. A. Nanoplastic affects growth of S. obliquus and reproduction of D. magna. Environ. Sci. Technol. 2014, 48, 12336-12343.

(50) Lee, K.; Shim, W. J.; Kwon, O. Y.; Kang, J. Size-dependent effects of micro polystyrene particles in the marine copepod Tigriopus japonicus. Environ. Sci. Technol. 2013, 47, 1127811283.

(51) Besseling, E.; Wegner, A.; Foekema, E. M.; Van den Heuvel-Greve, M. J.; Koelmans, A. A. Effects of microplastic on fitness and PCB bioaccumulation by the lugworm Arenicola marina (L.). Environ. Sci. Technol. 2013, 47, 593 600.

(52) Wegner, A.; Besseling, E.; Foekema, E. M.; Kamermans, P.; Koelmans, A. A Effects of nanopolystyrene on the feeding behavior of the blue mussel (Mytilus edulis L.). Environ. Toxicol. Chem. 2012, 31, 2490-2497.

Wright, S. L.; Rowe, D.; Thompson, R. C.; Galloway, T. S. Microplastic ingestion decreases energy reserves in marine worms. Curr. Biol. 2013, 23, 1031-1033.

(54) Kühn, S.; Bravo Rebolledo, E. L.; Van Franeker, J. A. Deleterious effects of litter on marine life. In Marine anthropogenic litter; Bergmann, M.; Gutow, L.; Klages, M., Eds.; Springer International Publishing: New York, Dordrecht, London. 2015, 75-116.

Barnes, D. K. A.; Galgani, F.; Thompson, R. C.; Barlaz, M. Accumulation and fragmentation of plastic debris in global environments. Philos. Trans. R. Soc. B. 2009, 364, 1985-1998.

(56) Browne, M. A.; Galloway, T. S.; Thompson, R. C. Spatial patterns of plastic debris along estuarine shorelines. Environ. Sci. Technol. 2010, 44, 3404-3409. 
Eriksen, M.; Mason, S.; Wilson, S.; Box, C.; Zellers, A.; Edwards, W.; Farley, H.; Amato, S. Microplastic pollution in the surface waters of the Laurentian Great Lakes. Mar. Pollut. Bull. 2013, 77, 177-182.

(58) Lechner, A.; Keckeis, H.; LumesbergerLoisl, F.; Zens, B.; Krusch, R.; Tritthart, M.; Glas, M.; Schludermann, E. The Danube so colourful: A potpourri of plastic litter outnumbers fish larvae in Europe's second largest river. Environ. Pollut. 2014, 188, 177-181.

(59) Dubaish, F.; Liebezeit, G. Suspended microplastics and black carbon particles in the Jade system, Southern North Sea. Water, Air, Soil Pollut. 2013, 224, 1352.

(60) Klein, S.; Worch, E.; Knepper, T. P. Occurrence and spatial distribution of microplastics in river shore sediments of the Rhine-Main area in Germany. Environ. Sci. Technol. 2015, 49, 6070-6076.

(61) Eerkes-Medrano, D.; Thompson, R. C.; Aldridge, D. C. Microplastics in freshwater systems: a review of the emerging threats, identification of knowledge gaps and prioritisation of research needs. Water Res. 2015, 75, 63-82.

(62) Zbyszewski, M.; Corcoran, P. L.; Hockin, A. Comparison of the distribution and degradation of plastic debris along shorelines of the Great Lakes, North America. J. Great Lakes Res. 2014, 40, 288-299.

(63) Hidalgo-Ruz, V.; Gutow, L.; Thompson, R. C.; Thiel, M. Microplastics in the marine environment: a review of the methods used for identification and quantification. Environ. Sci. Technol. 2012, 46, 3060-3075.

(64) Löder, M. G. J.; Kuczera, M.; Mintenig, S.; Lorenz, C.; Gunnar, G. Focal plane array detector-based micro-Fouriertransform infrared imaging for the analysis of microplastics in environmental samples. Environ. Chem. 2015, 12, 563-581.

(65) Song, Y. K.; Hong, S. H.; Jang, M.; Han, G. M.; Rani, M.; Lee, J.; Shim, W. J. A comparison of microscopic and spectroscopic identification methods for analysis of microplastics in environmental samples. Mar. Pollut. Bull. 2015, 93, 202-209.

(66) Lambert, S.; Wagner, M Characterisation of nanoplastics during the degradation of polystyrene. Chemosphere 2016, 145, 265-268.

(67) Gigault, J.; Pedrono, B.; Maxit, B.; Ter Halle, A. Marine plastic litter: the unanalyzed nano-fraction. Environ. Sci. Nano 2016, 3, 346-350.

(68) Gottschalk, F.; Sun, T.; Nowack, B. Environmental concentrations of engineered nanomaterials: Review of modeling and analytical studies. Environ. Pollut. 2013, 181, 287-300.

(69) Cózar, A.; Echevarría, F.; GonzálezGordillo, J. I.; Irigoien, X.; Ubeda, B.; Hernández-León, S.; Palma, A. T.; Navarro, S.; García-de-Lomas, J.; Ruiz, A.; Fernández-de-Puelles, M. L.; Duarte, C. M. Plastic debris in the open ocean. PNAS 2014, 111, 10239-10244.

(70) Quik, J. T. K.; Vonk, J. A.; Hansen, S. F.; Baun, A.; Meent, D. van de How to assess exposure of aquatic organisms to manufactured nanoparticles? Environ Int. 2011, 37, 1068-1077.

(71) Quik, J. T. K.; Klein, J. J. M. de; Koelmans, A. A. Spatially explicit fate modelling of nanomaterials in natural waters. Water Res. 2015, 80, 200-208.

(72) Praetorius, A.; Scheringer, M.; Hungerbühler, K. Development of environmental fate models for engineered nanoparticles - A case study of $\mathrm{TiO} 2$ nanoparticles in the Rhine River. Environ. Sci. Technol. 2012, 46, 6705-6713.

(73) Labille, J.; Harns, C.; Bottero, J.-Y.; Brant, J. Heteroaggregation of titanium dioxide nanoparticles with natural clay colloids. Environ. Sci. Technol. 2015, 49, 6608-6616.

(74) Barton, L. E.; Therezien, M.; Auffan, M.; Bottero, J.-Y.; Wiesner, M. R. Theory and methodology for determining nanoparticle affinity for heteroaggregation in environmental matrices using batch measurements. Environ. Eng. Sci. 2014, 31, 1-7.

(75) Velzeboer, I.; Kwadijk, C.; Koelmans, A. A. Strong sorption of PCBs to nanoplastics, microplastics, carbon nanotubes and fullerenes. Environ. Sci. 
Technol. 2014, 48, 4869-4876.

Quik, J. T. K.; Velzeboer, I.; Wouterse, M.; Koelmans, A. A.; Meent, D. van de Heteroaggregation and sedimentation rates for nanomaterials in natural waters. Water Res. 2014, 48, 269-279.

(77) Farley, K. J.; Morel, F. M. Role of coagulation in the kinetics of sedimentation. Environ. Sci. Technol. 1986, 20, 187-195.

(78) Atteia, O. Evolution of size distributions of natural particles during aggregation: Modelling versus field results. Colloids Surfaces A Physicochem. Eng. Asp. 1998, 139, 171-188.

(79) Burd, A. B.; Jackson, G. a. Particle aggregation. Ann. Rev. Mar. Sci. 2009. 1, 65-90.

(80) Meesters, J. A. J.; Koelmans, A. A.; Quik, J. T. K.; Hendriks, A. J.; van de Meent, D. Multimedia modeling of engineered nanoparticles with SimpleBox4nano: model definition and evaluation. Environ. Sci. Technol. 2014, 48, 5726-5736.

(81) Sani-Kast, N.; Scheringer, M.; Slomberg, D.; Labille, J.; Praetorius, A.; Ollivier, P.; Hungerbühler, K. Addressing the complexity of water chemistry in environmental fate modeling for engineered nanoparticles. Sci. Total Environ. 2015, 535, 150-159.

Quik, J. T. K.; Van de Meent, D.; Koelmans, A. A. Simplifying modeling of nanoparticle aggregationsedimentation behavior in environmental systems: A theoretical analysis. Water Res. 2014, 62, 193-201.

(83) Lenz, R.; Enders, K.; Gissel, T. Microplastic exposure studies should be environmentally realistic. Proc. Natl. Acad. Sci. U. S. A. 2016, 113, 2-3.

(84) De Klein, J. J. M.; Quik, J. T. K.; Bauerlein, P.; Koelmans, A. A. Towards validation of the NanoDUFLOW nanoparticle fate model for the river Dommel, the Netherlands. Environ. Sci. Nano. 2016, 3, 434-441.

(85) Langeveld, J.; Nopens, I.; Schilperoort, R.; Benedetti, L.; De Klein, J.; Amerlinck, Y.; Weijers, S. On data requirements for calibration of integrated models for urban water systems. Water Sci. Technol. 2013, 68, 728-736.

(86) R Core Team R: A language and environment for statistical computing In; R Foundation for Statistical Computing, Vienna, Austria. URL https://www.r-project.org/, 2015.

(87) Xiao, Y.; Wiesner, M. R. Transport and retention of selected engineered nanoparticles by porous media in the presence of a biofilm. Environ. Toxicol. Chem. 2013, 47, 2246-2253.

Therezien, M.; Thill, A.; Wiesner, M. R. Importance of heterogeneous aggregation for NP fate in natural and engineered systems. Sci. Total Environ. 2014, 485-486, 309-318.

Barkmann, W.; Schäfer-Neth, C.; Balzer, W. Modelling aggregate formation and sedimentation of organic and mineral particles. J. Mar. Syst. 2010, 82, 81-95.

(90) Simon, M.; Grossart, H.; Schweitzer, B.; Ploug, H. Microbial ecology of organic aggregates in aquatic ecosystems. Aquat. Microb. Ecol. 2002, $28,175-211$.

(91) Dris, R.; Gasperi, J.; Rocher, V.; Saad, M.; Renault, N.; Tassin, B. Microplastic contamination in an urban area: A case study in Greater Paris. Environ. Chem. 2015, 12, 592-599.

(92) Dris, R.; Gasperi, J.; Saad, M.; Mirande, C.; Tassin, B. Synthetic fibers in atmospheric fallout: A source of microplastics in the environment? Mar. Pollut. Bull. 2015, 104, 290-293.

(93) Kiørboe, T.; Tang, K.; Grossart, H.; Ploug, H. Dynamics of microbial communities on marine snow aggregates: Colonization, growth, detachment, and grazing mortality of attached bacteria. Appl. Environ. Microbiol. 2003, 69, 3036-3047.

(94) Lobelle, D.; Cunliffe, M. Early microbial biofilm formation on marine plastic debris. Mar. Pollut. Bull. 2011, 62, 197-200

(95) Ye, S.; Andrady, A. L. Fouling of floating plastic debris under Biscayne Bay exposure conditions. Mar. Pollut. Bull. 1991, 22, 608-613. 
(96) Zardus, J. D.; Nedved, B. T.; Huang, Y.; Tran, C.; Hadfield, M. G. Microbial biofilms facilitate adhesion in biofouling invertebrates. Biol. Bull. 2008, 214, 91-98.

(97) McCormick, A.; Hoellein, T. J.; Mason, S. A.; Schluep, J.; Kelly, J. J. Microplastic is an abundant and distinct microbial habitat in an urban river. 2014, 48, 11863-11871.

(98) Pernthaler, J.; Sattler, B.; Simek, K.; Schwarzenbacher, A.; Psenner, R. Topdown effects on the size-biomass distribution of a freshwater bacterioplankton community. Aquat. Microb. Ecol. 1996, 10, 255-263.

(99) Avnimelech, Y.; Ritvo, G.; Meijer, L. E.; Kochba, M. Water content, organic carbon and dry bulk density in flooded sediments. Aquac. Eng. 2001, 25, 2533.

(100) Godin, M.; Bryan, A. K.; Burg, T. P.; Babcock, K.; Manalis, S. R. Measuring the mass, density, and size of particles and cells using a suspended microchannel resonator. Appl. Phys. Lett. 2007, 91, 123121.

(101) Besseling, E.; Foekema, E. M.; Koelmans, A. A. Preliminary investigation of microplastic in the management area of water board rivierenland (In Dutch). 2014, 1-18.

(102) Koelmans, A. A.; Nowack, B.; Wiesner, M. R. Comparison of manufactured and black carbon nanoparticle concentrations in aquatic sediments. Environ. Pollut. 2009, 157, 1110-1116.

(103) Harshvardhan, K.; Jha, B. Biodegradation of low-density polyethylene by marine bacteria from pelagic waters, Arabian Sea, India. Mar. Pollut. Bull. 2013, 77, 100-106.

(104) Praetorius, A.; Labille, J.; Scheringer, M.; Thill, A.; Hungerbu, K.; Bottero, J.Y. Heteroaggregation of titanium dioxide nanoparticles with model natural colloids under environmentally relevant conditions. Environ. Sci. Technol. 2014, 48, 10690-10698.

(105) Van Cauwenberghe, L.; Claessens, M.; Vandegehuchte, M. B.; Mees, J.; Janssen, C. R. Assessment of marine debris on the Belgian Continental Shelf. Mar. Pollut. Bull. 2013, 73, 161-169.
(106) Kukulka, T.; Proskurowski, G.; MorétFerguson, S.; Meyer, D. W.; Law, K. L. The effect of wind mixing on the vertical distribution of buoyant plastic debris. Geophys. Res. Lett. 2012, 39, 16.

(107) Song, Y. K.; Hong, S. H.; Jang, M.; Kang, J.-H.; Kwon, O. Y.; Han, G. M.; Shim, W. J. Large accumulation of micro-sized synthetic polymer particles in the sea surface microlayer. Environ. Sci. Technol. 2014, 48, 9014-9021.

(108) Sundt, P.; Schulze, P.-E.; Syversen, F. Sources of microplastic-pollution to the marine environment; Norwegian Environmental Agency. Mepex 1032. 2014.

(109) Gouin, T.; Avalos, J.; Brunning, I.; Brzuska, K.; Graaf, J. De; Kaumanns, J.; Koning, T.; Meyberg, M.; Rettinger, K.; Schlatter, H.; Thomas, J.; Van Welie, R.; Wolf, T. Use of micro-plastic beads in cosmetic products in Europe and their estimated emissions to the North Sea environment. SOFW J. 2015 , 141, 40-46.

(110) Cornelissen, G.; Gustafsson, Ö.; Bucheli, T. D.; Jonker, T. O.; Koelmans, A. A.; Van Noort, P. C. M. Extensive sorption of organic compounds to black carbon, coal, and kerogen in sediments and soils: mechanisms and consequences for distribution, bioaccumulation, and biodegradation. Environ. Sci. Technol. 2005, 39, 6881-6895.

(111) Corcoran, P. L.; Norris, T.; Ceccanese, T.; Walzak, M. ; Helm, P. A.; Marvin, C. H. Hidden plastics of Lake Ontario, Canada and their potential preservation in the sediment record. Environ. Pollut 2015, 204, 17-25

(112) Rech, S.; Macaya-Caquilpán, V.; Pantoja, J. F.; Rivadeneira, M. M.; Jofre Madariaga, D.; Thiel, M. Rivers as a source of marine litter - A study from the SE Pacific. Mar. Pollut. Bull. 2014, $82,66-75$.

(113) Eriksen, M.; Mason, S.; Wilson, S.; Box, C.; Zellers, A.; Edwards, W.; Farley, H.; Amato, S. Microplastic pollution in the surface waters of the Laurentian Great Lakes. Mar. Pollut. Bull. 2013, 77, 177-182.

(114) Free, C. M.; Jensen, O. P.; Mason, S. A.; 
Eriksen, M.; Williamson, N. J.; Boldgiv, B. High-levels of microplastic pollution in a large, remote, mountain lake. Mar. Pollut. Bull. 2014, 85, 156163.

(115) Imhof, H. K.; Ivleva, N. P.; Schmid, J.; Niessner, R.; Laforsch, C. Contamination of beach sediments of a subalpine lake with microplastic particles. Curr. Biol. 2013, 23, 867868.

(116) Moore, M. N. Do nanoparticles present ecotoxicological risks for the health of the aquatic environment? Environ. Int. 2006, 32, 967-976.

(117) Rothen-Rutishauser, B.; Mühlfeld, C.; Blank, F.; Musso, C.; Gehr, P. Translocation of particles and inflammatory responses after exposure to fine particles and nanoparticles in an epithelial airway model. Part. Fibre Toxicol. 2007, 4, 1-9.

(118) Brown, D. M.; Wilson, M. R.; MacNee, W.; Stone, V.; Donaldson, K. Sizedependent proinflammatory effects of ultrafine polystyrene particles: A role for surface area and oxidative stress in the enhanced activity of ultrafines. Toxicol. Appl. Pharmacol. 2001, 175, 191-199.

(119) Oberdörster, G.; Ferin, J.; Lehnert, B. E Correlation between particle size, in vivo particle persistence, and lung injury. Environ. Health Perspect. 1994 102, 173-179.

(120) Cedervall, T.; Hansson, L.-A.; Lard, M.; Frohm, B.; Linse, S. Food chain transport of nanoparticles affects behaviour and fat metabolism in fish. PLoS One. 2012, 7, e32254

(121) Sivan, A. New perspectives in plastic biodegradation. Curr. Opin. Biotechnol. 2011, 22, 422-426.

(122) Shim, W.; A, S.; Hong, S.; Jang, M.; Han, G. Producing fragmented microand nano-sized expanded polystyrene particles with an accelerated mechanical abrasion experiment. In Abstract Book 24nd Annual Meeting SETAC. 2014.

(123) Laforsch, C.; Imhof, H. K.; Ivleva, N. P. Beyond the ocean: plastic particles in limnetic ecosystems. In Abstract Book 24nd Annual Meeting SETAC. 2014.
(124) Browne, M. A.; Niven, S. J.; Galloway, T. S.; Rowland, S. J.; Thompson, R. C. Microplastic moves pollutants and additives to worms, reducing functions linked to health and biodiversity. Curr. Biol. 2013, 23, 2388-2392.

(125) Cole, M.; Lindeque, P.; Fileman, E.; Halsband, C.; Goodhead, R.; Moger, J.; Galloway, T. S. Microplastic ingestion by zooplankton. Environ. Sci. Technol. 2013, 47, 6646-6655.

(126) Navarro, E.; Baun, A.; Behra, R.; Hartmann, N. B.; Filser, J.; Miao, A.-J.; Quigg, A.; Santschi, P. H.; Sigg, L. Environmental behavior and ecotoxicity of engineered nanoparticles to algae, plants, and fungi. Ecotoxicology. 2008, 17, 372-386.

(127) Nowack, B.; Bucheli, T. D. Occurrence, behavior and effects of nanoparticles in the environment. Environ. Pollut. 2007 $150,5-22$.

(128) Koelmans, A. A.; Besseling, E. Wegner, A.; Foekema, E. M. Plastic as a carrier of POPs to aquatic organisms. A model analysis. Environ. Sci. Technol. 2013, 47, 7812-7820.

(129) Koelmans, A. A.; Besseling, E.; Foekema, E. M. Leaching of plastic additives to marine organisms. Environ. Pollut. 2014, 187, 49-54.

(130) Hanazato, T.; Dodson, S. I. Synergistic effects of low oxygen concentration, predator kairomone, and a pesticide on the cladoceran Daphnia pulex. Limnol. Oceanogr. 1995, 40, 700-709.

(131) Riessen, H. P. Predator-induced life history shifts in Daphnia: a synthesis of studies using meta-analysis. Can. J. Fish. Aquat. Sci. 1999, 56, 2487-2494.

(132) Lürling, M. Infodisruption: pollutants interfering with the natural chemical information conveyance in aquatic systems. In Brönmark, C., Hansson, LA. (eds.) Chemical ecology in aquatic systems, Oxford University Press. 2012 250-271.

(133) Lürling, M.; Beekman, W. Palmelloids formation in Chlamydomonas reinhardtii: defence against rotifer predators? Ann. Limnol. - Int. J. Limnol. 2006, 42, 65-72.

(134) Lürling, M.; Roessink, I. On the way to cyanobacterial blooms: Impact of the 
herbicide metribuzin on the competition between a green alga (Scenedesmus) and a cyanobacterium (Microcystis). Chemosphere. 2006, 65, 618-626.

(135) Lürling, M.; Faassen, E. J.; Van Eenennaam, J. S. Effects of the cyanobacterial neurotoxin $\beta-\mathrm{N}$ methylamino-L-alanine (BMAA) on the survival, mobility and reproduction of Daphnia magna. J. Plankton Res. 2011, 33, 333-342.

(136) Lu, S.; Qu, R.; Forcada, J. Preparation of magnetic polymeric composite nanoparticles by seeded emulsion polymerization. Mater. Lett. 2009, 63, 770-772.

(137) Lürling, M.; Beekman, W. Extractable substances (anionic surfactants) from membrane filters induce morphological changes in the green alga Scenedesmus obliquus (Chlorophyceae). Environ. Toxicol. Chem. 2002, 21, 1213-1218.

(138) Radix, P.; Léonard, M.; Papantoniou, C.; Roman, G.; Saouter, E.; GallottiSchmitt, S.; Thiébaud, H.; Vasseur, P. Comparison of Brachionus calyciflorus 2-d and microtox chronic 22-h tests with Daphnia magna 21- d test for the chronic toxicity assessment of chemicals. Environ. Toxicol. Chem. 1999, 18, 2178-2185.

(139) Alexander, M. Environmental fate and effects of styrene. Crit. Rev. Environ. Sci. Technol. 1997, 27, 383-410.

(140) Greenspan, P.; Mayer, E. P.; Fowler, S. D. Nile red: A selective fluorescent stain for intracellular lipid droplets. $J$. Cell Biol. 1985, 100, 965-973.

(141) Küchler, S.; Abdel-Mottaleb, M.; Lamprecht, A.; Radowski, M. R.; Haag, R.; Schäfer-Korting, M. Influence of nanocarrier type and size on skin delivery of hydrophilic agents. Int. J. Pharm. 2009, 377, 169-172.

(142) Wu, H.; Volponi, J. V; Oliver, A. E.; Parikh, A. N.; Simmons, B. A. Supporting information - In vivo lipidomics using single-cell raman spectroscopy. PNAS. 2011, 108, 3809 3814.

(143) Fotopoulou, K. N.; Karapanagioti, H. K Surface properties of beached plastic pellets. Mar. Environ. Res. 2012, 81, $70-77$.
(144) Rieger, J. The glass transition temperature of polystyrene - Results of a round robin test. J. Therm. Anal. 1996, 46, 965-972.

(145) Mallick, N.; Mohn, F. H. Use of chlorophyll fluorescence in metal-stress research: a case study with the green microalga Scenedesmus. Ecotoxicol. Environ. Saf. 2003, 55, 64-69.

(146) Moed, J. R.; Hallegraeff, G. M. Some problems in the estimation of chlorophyll-a and phaeopigments from pre- and post-acidification spectrophotometric measurements. Int. Rev. der gesamten Hydrobiol. und Hydrogr. 1978, 63, 787-800.

(147) OECD Test No. 202: Daphnia sp. acute immobilisation test in OECD guidelines for the testing of chemicals. OECD Publishing. 2004.

(148) OECD Test No. 211: Daphnia magna reproduction test in OECD guidelines for the testing of chemicals. OECD Publishing. 2008.

(149) Reede, T. Effects of neonate size and food concentration on the life history responses of a clone of the hybrid Daphnia hyalina $\mathrm{x}$ galeata to fish kairomones. Freshw. Biol. 1997, 37, 389-396.

(150) Weber, A.; Declerck, S. Phenotypic plasticity of Daphnia life history traits in response to predator kairomones: genetic variability and evolutionary potential. Hydrobiologia. 1997, 360, 89-99.

(151) Lürling, M. Effects of microcystin-free and microcystin-containing strains of the cyanobacterium Microcystis aeruginosa on growth of the grazer Daphnia magna. Environ. Toxicol. 2003, 18, 202-210

(152) Lürling, M.; Beekman, W. Growth of Daphnia magna males and females fed with the cyanobacterium Microcystis aeruginosa and the green alga Scenedesmus obliquus in different proportions. Acta Hydrochim. Hydrobiol. 2006, 34, 375-382.

(153) International Organization for Standardization International standard, water quality - Freshwater algal growth inhibition test with unicellular green algae. ISO guideline, Switzerland. 
2004.

(154) Senger, H.; Fleischhacker, P. H Adaptation of the photosynthetic apparatus of Scenedesmus obliquus to strong and weak light conditions. Physiol. Plantarium. 1978, 43, 35-42.

(155) Saido, K.; Koizumi, K.; Sato, H.; Ogawa, N.; Kwon, B. G.; Chung, S.-Y.; Kusui, T.; Nishimura, M.; Kodera, Y. New analytical method for the determination of styrene oligomers formed from polystyrene decomposition and its application at the coastlines of the North-West Pacific Ocean. Sci. Total Environ. 2014, 473 474, 490-495.

(156) Hanazato, T. Pesticide effects on freshwater zooplankton: an ecological perspective. Environ. Pollut. 2001, 112, $1-10$.

(157) Kast-Hutcheson, K.; Rider, C.; LeBlanc, G. A. The fungicide propiconazole interferes with embryonic devolopment of the crustacean Daphnia magna. Environ. Toxicol. Chem. 2001, 20, 502-509.

(158) Flaherty, C. M.; Dodson, S. I. Effects of pharmaceuticals on Daphnia survival, growth, and reproduction. Chemosphere 2005, 61, 200-207.

(159) Khangarot, B. S.; Das, S. Toxicity of mercury on in vitro development of parthenogenetic eggs of a freshwater cladoceran Daphnia carinata. J. Hazard. Mater. 2009, 161, 68-73.

(160) Dao, T. S.; Do-Hong, L.-C.; Wiegand, C. Chronic effects of cyanobacterial toxins on Daphnia magna and their offspring. Toxicon. 2010, 55, 1244 1254.

(161) Connell, M. J. O.; Boul, P.; Ericson, L. M.; Hu, C.; Wang, Y.; Haroz, E.; Kuper, C.; Tour, J.; Ausman, K. D.; Smalley, R. E. Reversible water-solubilization of single-walled carbon nanotubes by polymer wrapping. Chem. Phys. Lett. 2001, 342, 265-271.

(162) Lundqvist, M.; Stigler, J.; Elia, G.; Lynch, I.; Cedervall, T.; Dawson, K. A. Nanoparticle size and surface properties determine the protein corona with possible implications for biological impacts. Proc. Natl. Acad. Sci. U. S. A. 2008, 105, 14265-14270.
(163)

Rossi, G.; Barnoud, J.; Monticelli, L. Polystyrene nanoparticles perturb lipid membranes. J. Phys. Chem. Lett. 2014, 5, 241-246.

(164) Van Cauwenberghe, L.; Vanreusel, A.; Mees, J.; Janssen, C. R. Microplastic pollution in deep-sea sediments Environ. Pollut. 2013, 182, 495-499.

(165) Reddy, M. S.; Adimurthy, S.; Ramachandraiah, G. Description of the small plastics fragments in marine sediments along the Alang-Sosiya shipbreaking yard, India. Estuar. Coast. Shelf Sci. 2006, 68, 656-660.

(166) Stolzenbach, K. D.; Newman, K. A.; Wong, C. S. Aggregation of fine particles at the sediment-water interface. J. Geophys. Res. 1992, 97, 17889-17898.

(167) Wiench, K.; Wohlleben, W.; Hisgen, V.; Radke, K.; Salinas, E.; Zok, S.; Landsiedel, R. Acute and chronic effects of nano- and non-nano-scale $\mathrm{TiO}(2)$ and $\mathrm{ZnO}$ particles on mobility and reproduction of the freshwater invertebrate Daphnia magna Chemosphere. 2009, 76, 1356-1365.

(168) Webb, H. K.; Crawford, R. J.; Sawabe, T.; Ivanova, E. P. Poly(ethylene terephthalate) Polymer Surfaces as a Substrate for Bacterial Attachment and Biofilm Formation. Microbes Environ. 2009, 24, 39-42.

(169) Paik, P.; Kar, K. K. Kinetics of thermal degradation and estimation of lifetime for polypropylene particles: Effects of particle size. Polym. Degrad. Stab. 2008, 93, 24-35.

(170) Foekema, E. M.; De Gruijter, C.; Mergia, M. T.; Kotterman, M.; Van Franeker, J. A.; Klok, C.; Murk, A. J.; Koelmans, A. A. Inventory of the presence of plastics in the digestive tract of North Sea fishes. In Abstract Book SETAC Europe 21st Annual Meeting. 2011.

(171) Mato, Y.; Isobe, T.; Takada, H.; Kanehiro, H.; Ohtake, C.; Kaminuma, T. Plastic resin pellets as a transport medium for toxic chemicals in the marine environment. Environ. Sci. Technol. 2001, 35, 318-324.

(172) Jonker, M. T.; Koelmans, A. A. Polyoxymethylene solid phase 
extraction as a partitioning method for hydrophobic organic chemicals in sediment and soot. Environ. Sci. Technol. 2001, 35, 3742-3748.

(173) Hale, S. E.; Martin, T. J.; Goss, K.-U.; Arp, H. P. H.; Werner, D. Partitioning of organochlorine pesticides from water to polyethylene passive samplers. Environ. Pollut. 2010, 158, 2511-2517.

(174) Ogata, Y.; Takada, H.; Mizukawa, K.; Hirai, H.; Iwasa, S.; Endo, S.; Mato, Y.; Saha, M.; Okuda, K.; Nakashima, A.; Murakami, M.; Zurcher, N.; Booyatumanondo, R.; Zakaria, M. P.; Dung, L. Q.; Gordon, M.; Miguez, C.; Suzuki, S.; Moore, C.; Karapanagioti, H. K.; Weerts, S.; McClurg, T.; Burres, E.; Smith, W.; Van Velkenburg, M.; Lang, J. S.; Lang, R. C.; Laursen, D.; Danner, B.; Stewardson, N.; Thompson, R. C. International pellet watch: global monitoring of persistent organic pollutants (POPs) in coastal waters. 1. Initial phase data on PCBs, DDTs, and HCHs. Mar. Pollut. Bull. 2009, 58, 1437-1446.

(175) Rios, L. M.; Moore, C.; Jones, P. R. Persistent organic pollutants carried by synthetic polymers in the ocean environment. Mar. Pollut. Bull. 2007, 54, 1230-1237.

(176) Kershaw, P.; Katsuhiko, S.; Lee, S.; Samseth, J.; Woodring, D. Plastic debris in the ocean. In UNEP Yearbook Emerging Issues in our Global Environment; United Nations Environment Programm. 2011.

(177) Ryan, P. G.; Connell, A. D.; Gardner, B. D. Plastic ingestion and PCBs in seabirds: Is there a relationship? Mar. Pollut. Bull. 1988, 19, 174-176.

(178) Yamashita, R.; Takada, H.; Fukuwaka, M.; Watanuki, Y. Physical and chemical effects of ingested plastic debris on short-tailed shearwaters, Puffinus tenuirostris, in the North Pacific Ocean. Mar. Pollut. Bull. 2011, 62, 2845-9.

(179) Kaag, N. H. B. M.; Foekema, E. M.; Bowmer, C. T. A new approach for testing contaminated marine sediments: fertilization success of lugworms following parental exposure. J. Aquat. Ecosyst. Heal. 1994, 3, 177-184.

(180) Cadée, G. C. Sediment reworking by
Arenicol marina on tidal flats in the Dutch Wadden Sea. Netherlands J. Sea Res. 1976, 10, 440-460.

(181)

Van Cauwenberghe, L.; Vandegehuchte, M. B.; Claessens, M.; Janssen, C. R. Occurence of microplastics in mussels (Mytilus edulis) and lugworms (Arenicola marina) collected along the BelgianFrench-Dutch coast. In Abstract Book 6th SETAC World Congress / SETAC Europe 22nd Annual Meeting. 2012, 59.

(182) Zarfl, C.; Matthies, M. Are marine plastic particles transport vectors for organic pollutants to the Arctic? Mar. Pollut. Bull. 2010, 60, 1810-1814.

(183) MacDonald, D. D.; Dipinto, L. M.; Field, J.; Ingersoll, C. G.; Long, E. R.; Swartz, R. C. Development and evaluation of consensus-based sediment effect concentrations for polychlorinated biphenyls. Environ. Toxicol. Chem. 2000, 19, 1403-1413.

(184) Thain, J.; Bifield, S.; Science, A.; Avenue, R.; Kingdom, U. ICES Techniques in Marine Environmental Sciences, No. 29 - Biological effects of contaminants: Sediment bioassay using the polychaete Arenicola marina; International council for the exploration of the sea: Copenhagen, Denmark, 2001, 29.

(185) Brils, J. M.; Huwer, S. L.; Kater, B. J.; Schout, P. G.; Harmsen, J.; Delvigne, G a L.; Scholten, M. C. T. Oil effect in freshly spiked marine sediment on Vibrio fischeri, Corophium volutator, and Echinocardium cordatum. Environ. Toxicol. Chem. 2002, 21, 2242-2251.

(186) Moermond, C. T. A.; Roessink, I.; Jonker, M. T. O.; Meijer, T.; Koelmans, A. A. Impact of polychlorinated biphenyl and polycyclic aromatic hydrocarbon sequestration in sediment on bioaccumulation in aquatic food webs. Environ. Toxicol. Chem. 2007, 26, 607-615.

(187) Ten Hulscher, T. E. M.; Vrind, B. a.; van den Heuvel, H.; van der Velde, L. E.; van Noort, P. C. M.; Beurskens, J. E. M.; Govers, H. a. J. Triphasic desorption of highly resistant chlorobenzenes, polychlorinated biphenyls, and polycyclic aromatic hydrocarbons in field contaminated 
sediment. Environ. Sci. Technol. 1999 , 33, 126-132.

(188) Pascall, M. A.; Zabik, M. E.; Zabik, M J.; Hernandez, R. J. Uptake of polychlorinated biphenyls (PCBs) from an aqueous medium by polyethylene, polyvinyl chloride, and polystyrene films. J. Agric. Food Chem. 2005, 53, 164-169.

(189) Retraubun, a. S. W.; Dawson, M.; Evans, S. M. The role of the burrow funnel in feeding processes in the lugworm Arenicola marina (L.). J. Exp. Mar. Bio. Ecol. 1996, 202, 107-118.

(190) Kaag, N. H. B. M.; Foekema, E. M.; Scholten, M. c. T.; Van Straalen, N. M. Comparison of contaminant accumulation in three species of marine invertebrates with different feeding habits. Environ. Toxicol. Chem. 1997, $16,837-842$.

(191) Allen, Y. T.; Thain, J. E.; Haworth, S.; Barry, J. Development and application of long-term sublethal whole sediment tests with Arenicola marina and Corophium volutator using Ivermectin as the test compound. Environ. Pollut. 2007, 146, 92-99.

(192) Beukema, J. J. Seasonal changes in the biomass of the macro-benthos of a tidal flat area in the Dutch Wadden Sea. Netherlands J. Sea Res. 1974, 107, 94 107.

(193) Roessink, I.; Moermond, C. T. A.; Gillissen, F.; Koelmans, A. A. Impacts of manipulated regime shifts in shallow lake model ecosystems on the fate of hydrophobic organic compounds. Water Res. 2010, 44, 6153-6163.

(194) Beukema, J. J.; De Vlas, J. Population parameters of the lugworm, Arenicola marina, living on tidal flats in the Dutch Wadden Sea. Neth. J. Sea Res. 1979, 13 , 331-353.

(195) Casado-Martínez, M. C.; Branco, V.; Vale, C.; Ferreira, a M.; Delvalls, T. a Is Arenicola marina a suitable test organism to evaluate the bioaccumulation potential of $\mathrm{Hg}$, PAHs and PCBs from dredged sediments? Chemosphere. 2008, 70, 1756-1765.

(196) Di Toro, D. M.; Mahony, J. D.; Gonzalez, A. M. Particle oxidation model of synthetic FeS and sediment acid-volatile sulfide. Environ. Toxicol Chem. 2008, 15, 2156-2167.

Selck, H.; Drouillard, K.; Eisenreich, K.; Koelmans, A. a; Palmqvist, A.; Ruus, A.; Salvito, D.; Schultz, I.; Stewart, R.; Weisbrod, A.; van den Brink, N. W.; van den Heuvel-Greve, M. Explaining differences between bioaccumulation measurements in laboratory and field data through use of a probabilistic modeling approach. Integr. Environ. Assess. Manag. 2012, $8,42-63$.

(198) Pettit, T. N.; Grant, G. S.; Causy Whittow, G. Ingestion of plastics by Laysan Albatross. The Auk. 2012, 98, 839-841.

(199) Ryan, P. G. Effects of ingested plastic on seabird feeding: evidence from chickens. Mar. Pollut. Bull. 1988, 19 125-128.

(200) Spear, L. B.; Ainley, D. G.; Ribi, A Incidence of plastic in seabirds from the Tropical Pacific, 1984-91: Relation with distribution of species, sex, age, season, year and body weight. Mar Environ. Res. 1995, 40, 123-146.

(201) Colabuono, F. I.; Barquete, V.; Domingues, B. S.; Montone, R. C Plastic ingestion by Procellariiformes in Southern Brazil. Mar. Pollut. Bull. 2009, 58, 93-96.

(202) De Wilde, P. A. W. J.; Berghuis, E. M. Laboratory experiments on growth of juvenile lugworms, Arenicola marina Netherlands J. Sea Res. 1979, 13, 487502 .

(203) Arthur, C.; Baker, J.; Bamford, H. Proceedings of the international research workshop on the occurrence, effects, and fate of microplastic marine debris. In International research workshop on the occurrence, effects, and fate of microplastic marine debris University of Washington Tacoma, Tacoma, WA, USA, September 9-112008. 2009, 1-49.

(204) Cammen, L. M. Ingestion rate: An empirical model for aquatic deposit feeders and detritivores. Oecologia. 1980, 44, 303-310.

Jonker, M. T. O.; Suijkerbuijk, M. P. W.; Schmitt, H.; Sinnige, T. L. Ecotoxicological effects of activated 


\section{References}

carbon addition to sediments. Environ. Sci. Technol. 2009, 43, 5959-5966.

(206) Gschwend, P. M.; MacFarlane, J. K.; Reible, D. D.; Lu, X.; Hawthorne, S. B.; Nakles, D. V; Thompson, T. Comparison of polymeric samplers for accurately assessing PCBs in pore waters. Environ. Toxicol. Chem. 2011, 30, 1288-1296.

(207) Derraik, J. G. B. The pollution of the marine environment by plastic debris: a review. Mar. Pollut. Bull. 2002, 44, 842-852.

(208) Pruter, A. T. Sources, quantities and distribution of persistent plastics in the marine-environment. Mar. Pollut. Bull. 1987, 18, 305-310.

(209) Eriksson, C.; Burton, H. Origins and biological accumulation of small plastic particles in fur seals from Macquarie Island. AMBIO A J. Hum. Environ. 2003, 32, 380 .

(210) Law, K. L.; Moret-Ferguson, S.; Maximenko, N. A.; Proskurowski, G.; Peackock, E. E.; Hafner, J.; Reddy, C. M. Plastic accumulation in the North Atlantic subtropical gyre. Science. 2010, 329, 1185-1189.

(211) Goldstein, M. C.; Rosenberg, M.; Cheng, L. Increased oceanic microplastic debris enhances oviposition in an endemic pelagic insect. Biol. Lett. 2012, 8, 817-820.

(212) Aalderink, R. H.; Zoeteman, A.; Jovin, R. Effect of input uncertainties upon scenario predictions for the river Vecht. Water Sci. Technol. 1996, 33, 107-118.

(213) Foekema, E. M.; De Gruijter, C.; Mergia, M. T.; Murk, A. J.; Van Franeker, J. A.; Koelmans, A. A. Plastic in North Sea fish. Environ. Sci. Technol. 2013, 47, 8818-8824.

(214) Endo, S.; Takizawa, R.; Okuda, K.; Takada, H.; Chiba, K.; Kanehiro, H.; Ogi, H.; Yamashita, R.; Date, T. Concentration of polychlorinated biphenyls (PCBs) in beached resin pellets: variability among individual particles and regional differences. Mar. Pollut. Bull. 2005, 50, 1103-1114.

(215) Rochman, C. M.; Hoh, E.; Hentschel, B T.; Kaye, S. Long-term field measurement of sorption of organic contaminants to five types of plastic pellets: Implications for plastic marine debris. Environ. Sci. Technol. 2013, 47, 1646-1654.

(216) Smedes, F.; Geertsma, R. W.; Van Der Zande, T.; Booij, K. Polymer-water partition coefficients of hydrophobic compounds for passive sampling: Application of cosolvent models for validation. Environ. Sci. Technol. 2009, 43, 7047-7054.

(217) Lohmann, R. Critical review of lowdensity polyethylene's partitioning and diffusion coefficients for trace organic contaminants and implications for its use as a passive sampler. Environ. Sci. Technol. 2012, 46, 606-618.

(218) Janssen, E. M.-L.; Croteau, M.-N.; Luoma, S. N.; Luthy, R. G. Measurement and modeling of polychlorinated biphenyl bioaccumulation from sediment for the marine polychaete Neanthes arenaceodentata and response to sorbent amendment. Environ. Sci. Technol. 2010, 44, 2857-2863.

(219) Hauck, M.; Hendriks, H. W. M.; Huijbregts, M. A. J.; Ragas, A. M. J.; Van de Meent, D.; Hendriks, A. J. Parameter uncertainty in modeling bioaccumulation factors of fish Environ. Toxicol. 2011, 30, 403-412.

(220) Thomann, R. V.; Connolly, J. P.; Parkerton, T. F. An equilibrium model of organic chemical accumulation in aquatic food webs with sediment interaction. Environ. Toxicol. Chem. 1992, 11, 615-629.

(221) Hendiks, A. J.; Van der Linde, A.; Cornelissen, G.; Sijm, D. T. H. M. The power of size. 1. Rate constants and equilibrium ratios for accumulation of organic substances related to octanolwater partition ratio and species weight Environ. Toxicol. Chem. 2001, 20 1399-1420.

(222) Brezonik, P. L. Chemical kinetics and process dynamics in aquatic systems CRC Press: Boca Raton, Florida, 1994.

(223) Wu, S. C.; Gschwend, P. M. Sorption kinetics of hydrophobic organic compounds to natural sediments and soils. Environ. Sci. Technol. 1986, 20, 717-725

(224) Smedes, F.; van Vliet, L. A.; Booij, K. 
Multi-ratio equilibrium passive sampling method to estimate accessible and pore water concentrations of polycyclic aromatic hydrocarbons and polychlorinated biphenyls in sediment. Environ. Sci. Technol. 2013, 47, 510 517.

(225) Seth, R.; Mackay, D.; Muncke, J. Estimating the organic carbon partition coefficient and its variability for hydrophobic chemicals. Environ. Sci. Technol. 1999, 33, 2390-2394.

(226) Rusina, T. P.; Smedes, F.; Klanova, J.; Booij, K.; Holoubek, I. Polymer selection for passive sampling: A comparison of critical properties. Chemosphere. 2007, 68, 1344-1351.

(227) Schwarzenbach R.P., Gschwend, P. M.; Imboden, D. M. Environmental organic chemistry; 2nd edition; WileyInterscience. 2003.

(228) Voparil, I. M.; Burgess, R. M.; Mayer, L. M.; Tien, T.; Cantwell, M. G.; Ryba, S. A. Digestive bioavailability to a deposit feeder (Arenicola marina) of polycyclic aromatic hydrocarbons associated with anthropogenic particles. Environ. Toxicol. Chem. 2004, 23, 2618-2626.

(229) Ter Laak, T. L.; Van Eijkeren, J. C. H.; Busser, F. J. M.; Van Leeuwen, H.P. Hermens, J. L. M. Facilitated transport of polychlorinated biphenyls and polybrominated diphenyl ethers by dissolved organic matter. Environ. Sci. Technol. 2009, 43, 1379-1385.

(230) Bakir, A. A.; Wright, S. W.; Rowland, S. J.; Galloway, T. S.; Thompson, R. C. Sorption and desorption of persistent organic pollutants from microplastics in the marine environment. In Abstract Book SETAC 6th World Congress / SETAC Europe 22nd Annual Meeting. 2012, p. 58

(231) Chen, C.; Liu, T. Fill the gap: Developing management strategies to control garbage pollution from fishing vessels. Mar. policy. 2013, 40, 34-40.

(232) Van Noort, P. C. M.; Haftka, J. J. H.; Parsons, J. R. Updated Abraham solvation parameters for polychlorinated biphenyls. Environ. Sci. Technol. 2010, 44, 7037-7042.

(233) Hauck, M.; Hendriks, A. J.; Huijbregts,
M. A. J.; Koelmans, A. A.; Van den Heuvel-Greve, M. J.; Moermond, C. T. A.; Veltman, K.; Vethaak, A. D. Including sorption to black carbon in modelling bioaccumulation of polycyclic aromatic hydrocarbons: Uncertainty analysis and comparison with field data. Environ. Sci. Technol. 2007, 41, 2738-2744.

(234) Rusina, R. P.; Smedes, F.; Klanova, J. Diffusion coefficients of polychlorinated biphenyls and polycyclic hydrocarbons in polydimethylsiloxane and low-density polyetylene polymers. J. Appl. Polym. Sci. 2010, 116, 1803-1810.

Avio, C. G.; Gorbi, S.; Milan, M.; Benedetti, M.; Fattorini, D.; d'Errico, G.; Pauletto, M.; Bargelloni, L.; Regoli, F. Pollutants bioavailability and toxicological risk from microplastics to marine mussels. Environ. Pollut. 2015 198, 211-222.

(236) Chua, E. M.; Shimeta, J.; Nugegoda, D.; Morrison, P. D.; Clarke, B. O Assimilation of polybrominated diphenyl ethers from microplastics by the marine amphipod, allorchestes compressa. Environ. Sci. Technol. 2014, 48, 8127-8134.

(237) Oliveira, M.; Ribeiro, A.; Hylland, K.; Guilhermino, L. Single and combined effects of microplastics and pyrene on juveniles $(0+$ group) of the common goby Pomatoschistus microps (Teleostei, Gobiidae). Ecol. Indic. 2013, 34, 641-647.

(238) Wardrop, P.; Shimeta, J.; Nugegoda, D.; Morrison, P. D.; Miranda, A.; Tang, M.; Clarke, B. O. Chemical pollutants sorbed to ingested microbeads from personal care products accumulate in fish. Environ. Sci. Technol. 2016, 50, 4037-4044.

(239) Rochman, C. M.; Hoh, E.; Kurobe, T.; Teh, S. J. Ingested plastic transfers hazardous chemicals to fish and induces hepatic stress. Sci. Rep. 2013, 3, 3263.

(240) Herzke, D.; Anker-Nilssen, T.; Nøst, T. H.; Götsch, A.; Christensen-Dalsgaard S.; Langset, M.; Fangel, K.; Koelmans, A. A. Negligible impact of ingested microplastics on tissue concentrations of persistent organic pollutants in northern fulmars off coastal Norway. 


\section{References}

Environ. Sci. Technol. 2016, 50, 1924 1933.

(241) Beckingham, B.; Ghosh, U. Differential bioavailability of polychlorinated biphenyls associated with environmental particles: Microplastic in comparison to wood, coal and biochar. Environ. Pollut. 2017, 220, 150-158.

(242) Paul-Pont, I.; Lacroix, C.; González Fernández, C.; Hégaret, H.; Lambert, C.; Le Goïc, N.; Frère, L.; Cassone, A. L.; Sussarellu, R.; Fabioux, C.; Guyomarch, J.; Albentosa, M.; Huvet, A.; Soudant, P. Exposure of marine mussels Mytilus spp. to polystyrene microplastics: Toxicity and influence on fluoranthene bioaccumulation. Environ. Pollut. 2016, 216, 724-737.

(243) GESAMP Sources, fate and effects of microplastics in the marine environment: a global assessment (Kershaw, P. J., ed.). (IMO/FAO/ UNESCO - IOC/UNIDO/WMO/IAEA/ UN/UNEP/UNDP Joint Group of Experts on the Scientific Aspects of Marine Environmental Protection). 2015, 90, 1-96.

(244) Koelmans, A. A.; Bakir, A.; Burton, G. A.; Janssen, C. R. Microplastic as a vector for chemicals in the aquatic environment: critical review and modelsupported reinterpretation of empirical studies. Environ. Sci. Technol. 2016, 50, 3315-3326.

(245) Bakir, A.; O’Connor, I. A.; Rowland, S J.; Hendriks, A. J.; Thompson, R. C. Relative importance of microplastics as a pathway for the transfer of hydrophobic organic chemicals to marine life. Environ. Pollut. 2016, 219 , 56-65.

(246) Koelmans, A. A. Modeling the role of microplastics in bioaccumulation of organic chemicals to marine aquatic organisms. A critical review. In Marine Anthropogenic Litter; Bergmann, M.; Gutow, L.; Klages, M., Eds.; Springer International Publishing. 2015, 309 324.

(247) Rummel, C. D.; Adolfsson-Erici, M.; Jahnke, A.; MacLeod, M. No measurable "cleaning" of polychlorinated biphenyls from Rainbow Trout in a 9 week depuration study with dietary exposure to $40 \%$ polyethylene microspheres. Environ. Sci. Process Impacts. 2016, 18, 788-795.

(248) Endo, S.; Koelmans, A. A. Sorption of Hydrophobic Organic Compounds to Plastics in the Marine Environment: Equilibrium. In Hazardous Chemicals Associated with Plastics in the Marine Environment, Hdb Env Chem; Takada, H.; Karapanagioti, H. K., Eds.; Springer International Publishing Switzerland. 2016, 41-53.

(249) Hawthorne, S. B.; Miller, D. J.; Grabanski, C. B. Measuring low picogram per liter concentrations of freely dissolved polychlorinated biphenyls in polyoxymethylene. Anal. Chem. 2009, 81, 9472-9480.

(250) Van Cauwenberghe, L.; Devriese, L.; Galgani, F.; Robbens, J.; Janssen, C. R. Microplastics in sediments: A review of techniques, occurrence and effects. Mar. Environ. Res. 2015, 111, 5-17.

(251) Van Cauwenberghe, L.; Vanreusel, A.; Mees, J.; Janssen, C. R. Microplastic pollution in deep-sea sediments. Environ. Pollut. 2013, 182, 495-499.

Weisbrod, A.V.; Woodburn, K.B.; Koelmans, A.A.; Parkerton, T.F.; McElroy, A.E.; Borga, K. Evaluation of bioaccumulation using in vivo laboratory and field studies. Integr. Environ. Assess. Manag. 2009, 5, 598623 .

(253) Kupryianchyk, D.; Reichman, E. P.; Rakowska, M. I.; Peeters, E. T. H. M.; Grotenhuis, J. T. C.; Koelmans, A. A. Ecotoxicological effects of activated carbon amendments on macroinvertebrates in nonpolluted and polluted sediments. Environ. Sci. Technol. 2011, 45, 8567-8574.

(254) Lopez Lozano, R.; Mouat, J. Marine litter in the North-East Atlantic Region. 2009.

(255) Adams, R. G.; Lohmann, R.; Fernandez, L. A.; MacFarlane, J. K.; Gschwend, P. M. Polyethylene devices: Passive samplers for measuring dissolved hydrophobic organic compounds in aquatic environments. Environ. Sci. Technol. 2007, 41, 1317-1323.

(256) Van der Heijden, S. A.; Jonker, M. T. O PAH bioavailability in field sediments: Comparing different methods for 
predicting in situ bioaccumulation. Environ. Sci. Technol. 2009, 43, 3757 3763.

(257) Mayer, P.; Fernquist, M. M.; Christensen, P. S.; Karlson, U.; Trapp, S. Enhanced diffusion of polycyclic aromatic hydrocarbons in artificial and natural aqueous solutions. Environ. Sci. Technol. 2007, 41, 6148-6155.

(258) Ghosh, U.; Kane Driscoll, S.; Burgess, R. M.; Jonker, M. T. O.; Reible, D.; Gobas, F.; Choi, Y.; Apitz, S. E.; Maruya, K. A.; Gala, W. R.; Mortimer, M.; Beegan, C. Passive sampling methods for contaminated sediments: practical guidance for selection, calibration, and implementation. Integr. Environ. Assess. Manag. 2014, 10, 210 223.

(259) Bligh, E. G.; Dyer, W. J. A rapid method of total lipid extraction and purification. Can. J. Biochem. Physiol. 1959, 37, 911-917.

(260) Kupryianchyk, D.; Rakowska, M. I.; Grotenhuis, J. T. C.; Koelmans, A. A. In situ sorption of hydrophobic organic compounds to sediment amended with activated carbon. Environ. Pollut. 2012 , 161, 23-29.

(261) Kupryianchyk, D.; Noori, A.; Rakowska, M. I.; Grotenhuis, J. T. C.; Koelmans, A. A. Bioturbation and dissolved organic matter enhance contaminant fluxes from sediment treated with powdered and granular activated carbon. Environ. Sci. Technol. 2013, 47, 5092-5100.

(262) Rakowska, M. I.; Kupryianchyk, D.; Grotenhuis, T.; Rijnaarts, H. H. M.; Koelmans, A. A. Extraction of sediment-associated polycyclic aromatic hydrocarbons with granular activated carbon. Environ. Toxicol. Chem. 2013, 32, 304-311.

(263) Rakowska, M. I.; Kupryianchyk, D.; Koelmans, A. A.; Grotenhuis, T.; Rijnaarts, H. H. M. Equilibrium and kinetic modeling of contaminant immobilization by activated carbon amended to sediments in the field. Water Res. 2014, 67, 96-104.

(264) Diepens, N. J.; Van Den Heuvel-Greve, M. J.; Koelmans, A. A. Modeling of bioaccumulation in marine benthic invertebrates using a multispecies experimental approach. Environ. Sci. Technol. 2015, 49, 13575-13585.

(265) Sidney, L. A.; Diepens, N. J.; Guo, X.; Koelmans, A. A. Trait-based modelling of bioaccumulation by freshwater benthic invertebrates. Aquat. Toxicol. 2016, 176, 88-96.

(266) Morrison, H. A.; Gobas, F. A. P. ; Lazar, R.; Haffner, G. D. Development and verification of a bioaccumulation model for organic contaminants in benthic invertebrates. Environ. Sci. Technol. 1996, 30, 3377-3384.

(267) Jonker, M. T. O.; Van der Heijden, S. A Bioconcentration factor hydrophobicity cutoff: An artificial phenomenon reconstructed. Environ. Sci. Technol. 2007, 41, 7363-7369.

(268) Rochman, C. M.; Kurobe, T.; Flores, I.; Teh, S. J. Early warning signs of endocrine disruption in adult fish from the ingestion of polyethylene with and without sorbed chemical pollutants from the marine environment. Sci. Total Environ. 2014, 493C, 656-661.

(269) De Witte, B.; Devriese, L.; Bekaert, K.; Hoffman, S.; Vandermeersch, G.; Cooreman, K.; Robbens, J. Quality assessment of the blue mussel (Mytilus edulis): Comparison between commercial and wild types. Mar. Pollut. Bull. 2014, 85, 146-155.

(270) Van Cauwenberghe, L.; Janssen, C. R Microplastics in bivalves cultured for human consumption. Environ. Pollut. 2014, 193, 65-70.

(271) Nemoto, T. Marine food chains Feeding pattern of baleen whales in the ocean. In; Ocean Research Institute, University of Tokyo, Tokyo. 1970, 241-252.

(272) Deméré, T. A. Humpback whale Megaptera novaeangliae. In D.E. Wilson \& R.A. Mittelmeier (eds.) Handbook of the mammals of the world. Vol. 4: Sea mammals; Lynx Edicions: Barcelona. 2014, 297-299.

(273) Fossi, M. C.; Coppola, D.; Baini, M.; Giannetti, M.; Guerranti, C.; Marsili, L.; Panti, C.; de Sabata, E.; Clò, S. Large filter feeding marine organisms as indicators of microplastic in the pelagic environment: the case studies of the Mediterranean basking shark 


\section{References}

(Cetorhinus maximus) and fin whale (Balaenoptera physalus). Mar. Environ. Res. 2014, 100, 17-24.

(274) Fossi, M. C.; Panti, C.; Guerranti, C.; Coppola, D.; Giannetti, M.; Marsili, L.; Minutoli, R. Are baleen whales exposed to the threat of microplastics? A case study of the Mediterranean fin whale (Balaenoptera physalus). Mar. Pollut. Bull. 2012, 64, 2374-2379.

(275) Galgani, F.; Claro, F.; Depledge, M.; Fossi, C. Monitoring the impact of litter in large vertebrates in the Mediterranean Sea within the European marine strategy framework directive (MSFD): constraints, specificities and recommendations. Mar. Environ. Res. 2014, 100, 3-9.

(276) Simmonds, M. P. Cetaceans and marine debris: The great unknown. J. Mar. Biol. 2012, 2012, 1-8.

(277) Baulch, S.; Perry, C. Evaluating the impacts of marine debris on cetaceans. Mar. Pollut. Bull. 2014, 80, 210-221.

(278) Kühn, S.; Bravo Rebolledo, E. L.; Van Franeker, J. A. Deleterious effects of litter on marine life. In Marine Anthropogenic Litter; Bergmann, M.; Gutow, L.; Klages, M., Eds.; Springer International Publishing. 2015, 75-116.

(279) Lusher, A. Microplastics in the marine environment: distribution, interactions and effects. In Marine Anthropogenic Litter; Bergmann, M.; Gutow, L.; Klages, M., Eds.; Springer International Publishing. 2015, 245-307.

(280) De Stephanis, R.; Giménez, J.; Carpinelli, E.; Gutierrez-Exposito, C.; Cañadas, A. As main meal for sperm whales: Plastics debris. Mar. Pollut. Bull. 2013, 69, 206-214.

(281) Di Beneditto, A. P. M.; Awabdi, D. R How marine debris ingestion differs among megafauna species in a tropical coastal area. Mar. Pollut. Bull. 2014, $88,86-90$.

(282) Simmonds, M. P. Cetaceans and Marine Debris: The Great Unknown. J. Mar. Biol. 2012, 2012, 1-8.

(283) Johnson, J. H.; Wolman, A. A. The humpback whale, Megaptera novaeangliae. Mar. Fish. Rev. 1984, 46, 30-37.
(284) Bravo Rebolledo, E. L.; Van Franeker, J. a; Jansen, O. E.; Brasseur, S. M. J. M. Plastic ingestion by harbour seals (Phoca vitulina) in the Netherlands. Mar. Pollut. Bull. 2013, 67, 200-202.

(285) Van Franeker, J. A.; Blaize, C. Danielsen, J.; Fairclough, K.; Gollan, J.; Guse, N.; Hansen, P. L.; Heubeck, M.; Jensen, J.-K.; Le Guillou, G.; Olsen, B.; Olsen, K. O.; Pedersen, J.; Stienen, E. W. M.; Turner, D. M. Monitoring plastic ingestion by the northern fulmar Fulmarus glacialis in the North Sea. Environ. Pollut. 2011, 159, 2609-2615.

(286) Gonzalez-Contreras, P.; Weijma, J.; Van der Weijden, R.; Buisman, C. J. N. Biogenic scorodite crystallization by Acidianus sulfidivorans for arsenic removal. Environ. Sci. Technol. 2010 , 44, 675-680.

(287) Ng, K. L.; Obbard, J. P. Prevalence of microplastics in Singapore's coastal marine environment. Mar. Pollut. Bull. 2006, 52, 761-767.

(288) Thompson, R. C.; Olsen, Y.; Mitchell, R. P.; Davis, A.; Rowland, S. J.; John, A. W. G.; McGonigle, D.; Russell, A. E. Supporting information - Lost at sea: Where is all the plastic? Science. 2004, 304,838 .

(289) Fries, E.; Dekiff, J. H.; Willmeyer, J.; Nuelle, M.-T.; Ebert, M.; Remy, D. Identification of polymer types and additives in marine microplastic particles using pyrolysis-GC/MS and scanning electron microscopy. Environ. Sci. Process. Impacts. 2013, 15, 1949 1956.

(290) Slijper, E. J. Whales; Hutchinson: London, 1979

(291) Kastelein, R. A.; Neurohr, B.; Nieuwstraten, S. H.; Wiepkema, P. R. Food consumption and body measurements of Amazon river dolphins (Inia geoffrensis). Aquat. Mamm. 1999, 25, 173-182.

(292) Lusher, A. L.; Burke, A.; O’Connor, I.; Officer, R. Microplastic pollution in the Northeast Atlantic Ocean: Validated and opportunistic sampling. Mar. Pollut. Bull. 2014, 88, 325-333.

Koelmans, A. A.; Besseling, E.; Foekema, E. M.; Kooi, M.; Mintenig, S.; Ossendorp, B. C.; Redondo- 
Hasselerharm, P. E.; Verschoor, A.; Van Wezel, A. P.; Scheffer, M. Risk of plastic debris: unravelling fact, opinion, perception and belief. Environ. Sci. Technol. 2017, 51, 11513-11519.

(294) Connors, K. A.; Dyer, S. D.; Belanger, S. E. Advancing the quality of environmental microplastic research. Environ. Toxicol. Chem. 2017, 36, 1697-1703.

(295) Koelmans, A. A.; Diepens, N. J.; Velzeboer, I.; Besseling, E.; Quik, J. T. K.; van de Meent, D. Guidance for the prognostic risk assessment of nanomaterials in aquatic ecosystems. Sci. Total Environ. 2015, 535, 141-149.

(296) Lusher, A. Microplastics in the marine environment: distribution, interactions and effects. In Marine anthropogenic litter; Bergmann, M.; Gutow, L.; Klages, M., Eds.; Springer International Publishing. 2015, 245-307.

(297) Kooi, M.; Reisser, J.; Slat, B.; Ferrari, F. F.; Schmid, M. S.; Cunsolo, S.; Brambini, R.; Noble, K.; Sirks, L.-A.; Linders, T. E. W.; Schoeneich-Argent, R. I.; Koelmans, A. A. The effect of particle properties on the depth profile of buoyant plastics in the ocean. Sci. Rep. 2016, 6, 33882.

(298) Besseling, E.; Quik, J. T. K.; Sun, M.; Koelmans, A. A. Fate of nano- and microplastic in freshwater systems: a modeling study. Environ. Pollut. 2017, 220, 540-548.

(299) Kooi, M.; Nes, E. H.; Scheffer, M.; Koelmans, A. A. Ups and downs in the ocean: Effects of biofouling on vertical transport of microplastics. Environ. Sci. Technol. 2017, 51, 7963-7971.

(300) Gewert, B.; Plassmann, M. M.; MacLeod, M. Pathways for degradation of plastic polymers floating in the marine environment. Environ. Sci. Process. Impacts. 2015, 17, 1513-1521.

(301) Van Sebille, E.; England, M. H.; Froyland, G. Origin, dynamics and evolution of ocean garbage patches from observed surface drifters. Environ. Res. Lett. 2012, 7, 1-6.

(302) Isobe, A.; Kubo, K.; Tamura, Y.; Kako, S.; Nakashima, E.; Fujii, N. Selective transport of microplastics and mesoplastics by drifting in coastal waters. Mar. Pollut. Bull. 2014, 89 324-330.

(303) Wilcox, C.; Heathcote, G.; Goldberg, J.; Gunn, R.; Peel, D.; Hardesty, B. D Understanding the Sources and Effects of Abandoned, Lost, and Discarded Fishing Gear on Marine Turtles in Northern Australia. Conserv. Biol. 2014, 29, 198-206.

(304) Barnes, D. K. A.; Milner, P. Drifting plastic and its consequences for sessile organism dispersal in the Atlantic Ocean. Mar. Biol. 2004, 146, 815-825.

(305) Katsanevakis, S.; Amato, E.; Birkun, A.; Fleet, D.; Franeker, J. A. Van; Hanke, G.; Janssen, C.; Maes, T.; Mouat, J.; Oosterbaan, L.; Poitou, I.; Thompson, R. Proposing methodological standards for monitoring marine litter, in order to achieve good environmental status in the framework of the marine strategy framework directive (MSFD). 2008

(306) Kataoka, T.; Hinata, H. Evaluation of beach cleanup effects using linear system analysis. Mar. Pollut. Bull. 2015, 91, 73-81.

(307) Kako, S.; Isobe, A.; Kataoka, T.; Hinata, H. A decadal prediction of the quantity of plastic marine debris littered on beaches of the East Asian marginal seas. Mar. Pollut. Bull. 2014, 81, 174184.

(308) Kataoka, T.; Hinata, H.; Kato, S. Analysis of a beach as a time-invariant linear input/output system of marine litter. Mar. Pollut. Bull. 2013, 77, 266273.

(309) Foekema, E.; Hoornsman, G.; Sonneveld, C.; Arenoe, F. Microplastics in the management area of waterboard rivierenland (In Dutch). IMARES Wageningen UR. 2015, 1-22.

(310) Besseling, E.; Foekema, E. M.; De Hoon, K.; Wegner, A.; Gylstra, R.; Koelmans, A. A. Occurrence of microplastic in the river Rhine and Meuse delta. Poster TU057. In Abstract Book SETAC Annual Meeting; Barcelona, Spain. 2015.

Hermsen, E.; Pompe, R.; Besseling, E.; Koelmans, A. A. Detection of low numbers of microplastics in North Sea fish using strict quality assurance 
criteria. Mar. Pollut. Bull. 2017, 122, 253-258.

(312) Besseling, E.; Foekema, E. M.; Van Franeker, J. A.; Leopold, M. F.; Kühn, S.; Bravo Rebolledo, E. L.; Heße, E.; Mielke, L.; IJzer, J.; Kamminga, P.; Koelmans, A. A. Microplastic in a macro filter feeder: Humpback whale Megaptera novaeangliae. Mar. Pollut. Bull. 2015, 95, 248-252.

(313) Leslie, H. A.; Brandsma, S. H.; van Velzen, M. J. M.; Vethaak, A. D. Microplastics en route: Field measurements in the Dutch river delta and Amsterdam canals, wastewater treatment plants, North Sea sediments and biota. Environ. Int 2017, 101, 133 142.

(314) Kooi, M.; Besseling, E.; Kroeze, C.; Wezel, A. P. Van; Koelmans, A. A. Modeling the fate and transport of plastic debris in fresh waters. Review and guidance. In Freshwater microplastics; Wagner, M.; Lamber, S., Eds.; Springer International Publishing. 2017, 125-152.

(315) Azimi, P.; Zhao, D.; Pouzet, C.; Crain, N. E.; Stephens, B. Emissions of ultrafine particles and volatile organic compounds from commercially available desktop three-dimensional printers with multiple filaments. Environ. Sci. Technol. 2016, 50, 1260 1268.

(316) Fischer, M.; Scholz-Böttcher, B. M. Simultaneous Trace Identification and Quantification of Common Types of Microplastics in Environmental Samples by Pyrolysis-Gas Chromatography-Mass Spectrometry. Environ. Sci. Technol. 2017, 51, 50525060 .

(317) Hernandez, L. M.; Yousefi, N.; Tufenkji, N. Are There Nanoplastics in Your Personal Care Products? Environ. Sci. Technol. Lett. 2017, 4, 280-285.

(318) Zhang, H.; Kuo, Y.-Y.; Gerecke, A. C.; Wang, J. Co-release of hexabromocyclododecane (HBCD) and Nano- and microparticles from thermal cutting of polystyrene foams. Environ. Sci. Technol. 2012, 46, 10990-10996.

(319) Song, Y. K.; Hong, S. H.; Jang, M.; Han, G. M.; Jung, S. W.; Shim, W. J. Combined Effects of UV Exposure
Duration and Mechanical Abrasion on Microplastic Fragmentation by Polymer Type. Environ. Sci. Technol. 2017, 51, 4368-4376.

(320) Besseling, E.; Wang, B.; Lürling, M.; Koelmans, A. A. Correction to: Nanoplastic affects growth of $\mathrm{S}$ obliquus and reproduction of D. magna Environ. Sci. Technol. 2014, 48, 1233612343.

(321) Claessens, M.; Van Cauwenberghe, L.; Vandegehuchte, M. B.; Janssen, C. R. New techniques for the detection of microplastics in sediments and field collected organisms. Mar. Pollut. Bull. 2013, 70, 227-233.

(322) Kühn, S.; Van Werven, B.; Van Oyen, A.; Meijboom, A.; Bravo Rebolledo, E. L.; Van Franeker, J. A. The use of potassium hydroxide $(\mathrm{KOH})$ solution as a suitable approach to isolate plastics ingested by marine organisms. Mar. Pollut. Bull. 2017, 115, 86-90.

(323) Lusher, A. L.; Welden, N. A.; Sobral, P.; Cole, M. Sampling, isolating and identifying microplastics ingested by fish and invertebrates. Anal. Methods. 2017, 9, 1346-1360.

(324) Rocha-Santos, T.; Duarte, A. C. A critical overview of the analytica approaches to the occurrence, the fate and the behavior of microplastics in the environment. TrAC Trends Anal. Chem. 2014, 65, 47-53.

(325) Torre, M.; Digka, N.; Anastasopoulou, A.; Tsangaris, C.; Mytilineou, C. Anthropogenic microfibres pollution in marine biota. A new and simple methodology to minimize airborne contamination. Mar. Pollut. Bull. 2016 113, 55-61.

(326) Dümichen, E.; Barthel, A.-K.; Braun, U.; Bannick, C. G.; Brand, K.; Jekel, M.; Senz, R. Analysis of polyethylene microplastics in environmental samples, using a thermal decomposition method. Water Res. 2015, 85, 451-457.

(327) Löder, M. G. J.; Gerdts, G. Methodology used for the detection and identification of microplastics - a critical appraisal. In Marine anthropogenic litter; Bergmann, M.; Gutow, L.; Klages, M., Eds.; Springer International Publishing. 2015, 201227. 
(328) Twiss, M. R. Standardized methods are required to assess and manage microplastic contamination of the Great Lakes system. J. Great Lakes Res. 2016, 42, 921-925.

(329) Nizzetto, L.; Bussi, G.; Futter, M. N.; Butterfield, D.; Whitehead, P. G. A theoretical assessment of microplastic transport in river catchments and their retention by soils and river sediments. Environ. Sci. Process. Impacts. 2016, 18, 1050-1059.

(330) Siegfried, M.; Koelmans, A. A.; Besseling, E.; Kroeze, C. Export of microplastics from land to sea. A global modelling approach. Water Res. 2017, 15, 249-257.

(331) Sherman, P.; van Sebille, E. Modeling marine surface microplastic transport to assess optimal removal locations. Environ. Res. Lett. 2016, 11, 14006.

(332) Long, M.; Moriceau, B.; Gallinari, M.; Lambert, C.; Huvet, A.; Raffray, J.; Soudant, P. Interactions between microplastics and phytoplankton aggregates: Impact on their respective fates. Mar. Chem. 2015, 175, 39-46.

(333) Carson, H. S.; Nerheim, M. S.; Carroll, K. a; Eriksen, M. The plastic-associated microorganisms of the North Pacific Gyre. Mar. Pollut. Bull. 2013, 75, 126132.

(334) Artham, T.; Sudhakar, M.; Venkatesan, R.; Madhavan Nair, C.; Murty, K. V. G. K.; Doble, M. Biofouling and stability of synthetic polymers in sea water. Int Biodeterior. Biodegradation. 2009, 63, 884-890.

(335) Lebreton, L. C. M.; van der Zwet, J.; Damsteeg, J.-W.; Slat, B.; Andrady, A.; Reisser, J. River plastic emissions to the world's oceans. Nat. Commun. 2017, 8 , 15611.

(336) Van Wezel, A.; Caris, I.; Kools, S. A. E Release of primary microplastics from consumer products to wastewater in the Netherlands. Environ. Toxicol. Chem. 2016, 35, 1627-1631.

(337) Sherrington, C.; Darrah, C.; Hann, S.; Cordle, M.; Cole, G. Study to support the development of measures to combat a range of marine litter sources. Report for European Commission DG Environment. 2016.
(338) Van Cauwenberghe, L. Occurrence, effects and risks of marine microplastics. Thesis submitted in fulfilment of the requirements for the degree of Doctor (PhD) in Applied Biological Sciences. 2016.

(339) Koelmans, A. A.; Kooi, M.; LavenderLaw, K.; Van Sebille, E. All is not lost: Deriving a top-down mass budget of plastic at sea. Environ. Res. Lett. 2017.

(340) Kooi, M.; Besseling, E.; Kroeze, C.; Van Wezel, A. P.; Koelmans, A. A. Modeling the fate and transport of plastic debris in freshwaters: review and guidance. Poster WE138. In Abstract Book SETAC Annual Meeting; Brussels, Belgium. 2017.

(341) Williams, J.; Hindell, J. S.; Swearer, S. E.; Jenkins, G. P. Influence of freshwater flows on the distribution of eggs and larvae of black bream Acanthopagrus butcheri within a drought-affected estuary. J. Fish Biol. 2012, 80, 2281-2301.

(342) Lima, A. R. A.; Costa, M. F.; Barletta, M. Distribution patterns of microplastics within the plankton of a tropical estuary. Environ. Res. 2014, 132, 146-155.

(343) Obbard, R. W.; Sadri, S.; Wong, Y. Q.; Khitun, A. a.; Baker, I.; Thompson, R. C. Global warming releases microplastic legacy frozen in Arctic Sea ice. Earth's Futur. 2014, 2, 315-320.

(344) Ballent, A.; Pando, S.; Purser, A.; Juliano, M. F.; Thomsen, L. Modelled transport of benthic marine microplastic pollution in the Nazaré Canyon. Biogeosciences. 2013, 10, 7957-7970.

(345) Mathalon, A.; Hill, P. Microplastic fibers in the intertidal ecosystem surrounding Halifax Harbor, Nova Scotia. Mar. Pollut. Bull. 2014, 81, 6979.

(346) Maximenko, N.; Hafner, J.; Niiler, P. Pathways of marine debris derived from trajectories of Lagrangian drifters. Mar. Pollut. Bull. 2012, 65, 51-62.

(347) Wilcox, C.; Van Sebille, E.; Hardesty, B. D. Threat of plastic pollution to seabirds is global, pervasive, and increasing. Proc. Natl. Acad. Sci. 2015 , 112, 11899-11904

(348) Wilcox, C.; Hardesty, B. D.; Sharples, 
R.; Griffin, D. A.; Lawson, T. J.; Gunn, R. Ghostnet impacts on globally threatened turtles, a spatial risk analysis for northern Australia. Conserv. Lett. 2013, 6, 247-254.

(349) Schuyler, Q.; Hardesty, B. D.; Wilcox, C.; Townsend, K. Global Analysis of Anthropogenic Debris Ingestion by Sea Turtles. Conserv. Biol. 2014, 28, 129 139.

(350) Besseling, E.; Foekema, E. M.; Ren, Y.; Van den Heuvel-Greve, M. J.; Koelmans, A. A. Feedbacks between bioturbation and microplastic at the sediment-water interface. Poster TU065. In Abstract Book SETAC Annual Meeting; Barcelona, Spain. 2015.

(351) Paredes, I.; Koelmans, A. A.; Besseling, E. Interactions of microplastics with freshwater mussels (Anodonta anatina). Poster. In Abstract Book SETAC Annual Meeting; Barcelona, Spain. 2015.

(352) Koelmans, A. A.; Besseling, E.; Wegner, A.; Foekema, E. M. Plastic as a carrier of POPs to aquatic organisms: A model analysis. Environ. Sci. Technol. 2013, 47, 7812-7820.

(353) Diepens, N. J.; Koelmans, A. A. Food web accumulation of microplastics and associated contaminants. In prep.

(354) Huntley, M. E.; Barthel, K.-G.; Star, J. L. Particle rejection by Calanus pacificus: discrimination between similarly sized particles. Mar. Biol. 1983, 74, 151-160.

(355) Brillant, M. G. S.; MacDonald, B. A. Postingestive selection in the sea scallop, Placopecten magllanicus (Gmelin): the role of particle size and density. J. Exp. Mar. Bio. Ecol. 2000, 253, 211-227.

(356) Ayukai, T. Discriminate feeding of the calanoid copepod Acartia clausi. Mar. Biol. 1987, 94, 579-587.

(357) Donaghay, P. L.; Small, L. F. Food selection capabilities of the estuarine copepod Acartia clausi. Mar. Biol. 1979, 52, 137-146.

(358) Vroom, R. J. E.; Koelmans, A. A.; Besseling, E.; Halsband, C. Aging of microplastics promotes their ingestion by marine zooplankton. Environ. Pollut. 2017, 231, 987-996.
(359) Wilson, D. S. Food size selection among copepods. Ecology. 1973, 54, 909-914.

(360) González Carman, V.; Acha, E. M.; Maxwell, S. M.; Albareda, D.; Campagna, C.; Mianzan, H. Young green turtles, Chelonia mydas, exposed to plastic in a frontal area of the SW Atlantic. Mar. Pollut. Bull. 2014, 78, 56-62.

(361) Taylor, M. L.; Gwinnett, C.; Robinson, L. F.; Woodall, L. C. Plastic microfibre ingestion by deep-sea organisms. Sci. Rep. 2016, 6, 33997.

(362) Jabeen, K.; Su, L.; Li, J.; Yang, D.; Tong, C.; Mu, J.; Shi, H. Microplastics and mesoplastics in fish from coastal and fresh waters of China. Environ. Pollut. 2017, 221, 141-149.

(363) Santos, R. G.; Andrades, R.; Boldrini, M. A.; Martins, A. S. Debris ingestion by juvenile marine turtles: An underestimated problem. Mar. Pollut. Bull. 2015, 93, 37-43.

(364) Au, S. Y.; Lee, C. M.; Weinstein, J. E.; van den Hurk, P.; Klaine, S. J. Trophic transfer of microplastics in aquatic ecosystems: Identifying critical research needs. Integr. Environ. Assess. Manag. 2017, 13, 505-509.

(365) Setälä, O.; Fleming-Lehtinen, V.; Lehtiniemi, M. Ingestion and transfer of microplastics in the planktonic food web. Environ. Pollut. 2013, 185C, 7783.

(366) Farrell, P.; Nelson, K. Trophic level transfer of microplastic: Mytilus edulis (L.) to Carcinus maenas (L.). Environ. Pollut. 2013, 177, 1-3.

(367) Tosetto, L.; Williamson, J. E.; Brown, C. Trophic transfer of microplastics does not affect fish personality. Anim. Behav. 2017, 123, 159-167.

(368) Posthuma, L.; Suter II, G. W.; Traas, T. P. Species sensitivity distributions in ecotoxicology; Lewis publishers. 2002.

(369) EPA (United States Environmental Protection Agency) Species sensitivity distribution generator. 2016.

(370) Diepens, N. J.; Koelmans, A. A.; Baveco, H.; Van den Brink, P. J.; Van den Heuvel-Greve, M. J.; Brock, T. C. M. Prospective environmental risk 
assessment for sediment-bound organic chemicals: a proposal for tiered effect assessement. In Reviews of environmental contamination and toxicology. 2016, 239, 1-77.

(371) Hermsen, E. Microplastics in the marine environment: how much is too much? BSc. Thesis Wageningen University. 2015.

(372) Jeong, C. B.; Won, E. J.; Kang, H. M.; Lee, M. C.; Hwang, D. S.; Hwang, U. K.; Zhou, B.; Souissi, S.; Lee, S. J.; Lee, J. S. Microplastic size-dependent toxicity, oxidative stress induction, and p-JNK and p-p38 activation in the monogonont rotifer (Brachionus koreanus). Environ. Sci. Technol. 2016, 50, 8849-8857.

(373) Besseling, E.; Foekema, E. M.; van den Heuvel-Greve, M. J.; Koelmans, A. A. The effect of microplastic on chemical uptake by the lugworm Arenicola marina (L.) under environmentally relevant conditions. Environ. Sci. Technol. 2017, 51, 8795-8804.

(374) Blarer, P.; Burkhardt-Holm, P. Microplastics affect assimilation efficiency in the freshwater amphipod Gammarus fossarum. Environ. Sci. Pollut. Res. 2016, 23, 23522-23532.

(375) Mazurais, D.; Ernande, B.; Quazuguel, P.; Severe, A.; Huelvan, C.; Madec, L.; Mouchel, O.; Soudant, P.; Robbens, J.; Huvet, A.; Zambonino-Infante, J. Evaluation of the impact of polyethylene microbeads ingestion in European sea bass (Dicentrarchus labrax) larvae. Mar. Environ. Res. 2015 , $112,78-85$.

(376) Imhof, H. K.; Laforsch, C. Hazardous or not - Are adult and juvenile individuals of Potamopyrgus antipodarum affected by non-buoyant microplastic particles? Environ. Pollut. 2016, 218, 383-391.

(377) Bergami, E.; Pugnalini, S.; Vannuccini, M. L.; Manfra, L.; Faleri, C.; Savorelli, F.; Dawson, K. A.; Corsi, I. Long-term toxicity of surface-charged polystyrene nanoplastics to marine planktonic species Dunaliella tertiolecta and Artemia franciscana. Aquat. Toxicol. 2017, 189, 159-169.

(378) Della Torre, C.; Bergami, E.; Salvati, A.; Faleri, C.; Cirino, P.; Dawson, K. A.; Corsi, I. Accumulation and embryo- toxicity of polystyrene nanoparticles at early stage of development of sea urchin embryos Paracentrotus lividus. Environ. Sci. Technol. 2014, 48, 12302 12311.

(379) Nasser, F.; Lynch, I. Secreted protein eco-corona mediates uptake and impacts of polystyrene nanoparticles on Daphnia magna. J. Proteomics. 2016, 137, 45-51.

(380) Sharma, G.; Valenta, D. T.; Altman, Y.; Harvey, S.; Xie, H.; Mitragotri, S.; Smith, J. W. Polymer particle shape independently influences binding and internalization by macrophages. $J$. Control. Release. 2010, 147, 408-412.

(381) DeMott, W. R. Discrimination between particles algae and artificial particles by freshater and marine copepods. Limnology. 1988, 33, 397-408.

(382) Lohmann, R. Microplastics are not important for the cycling and bioaccumulation of organic pollutants in the oceans - but should microplastics be considered POPs themselves? Integr. Environ. Assess. Manag. 2017, 13, 460 465 .

(383) Ziccardi, L. M.; Edgington, A.; Hentz, K.; Kulacki, K. J.; Kane Driscoll, S. Microplastics as vectors for bioaccumulation of hydrophobic organic chemicals in the marine environment: A state-of-the-science review. Environ. Toxicol. Chem. 2016 , 35, 1667-1676.

(384) Hartmann, N. B.; Rist, S.; Bodin, J.; Jensen, L. H. S.; Schmidt, S. N.; Mayer, P.; Meibom, A.; Baun, A. Microplastics as vectors for environmental contaminants: Exploring sorption, desorption, and transfer to biota. Integr. Environ. Assess. Manag. 2017, 13, 488493.

(385) Jang, M.; Shim, W. J.; Han, G. M.; Rani, M.; Song, Y. K.; Hong, S. H. Styrofoam debris as a source of hazardous additives for marine organisms. Environ. Sci. Technol. 2016, 50, 4951-4960.

(386) Chen, Q.; Reisser, J.; Cunsolo, S.; Kwadijk, C.; Kotterman, M.; Proietti, M.; Slat, B.; Ferrari, F.; Schwarz, A.; Levivier, A.; Yin, D.; Hollert, H.; Koelmans, A. A. Persistent organic pollutants in plastics within the Great 
Pacific Garbage Patch. Environ. Sci. Technol. 2018, 52, 446-456.

(387) Liu, L.; Fokkink, R.; Koelmans, A. A Sorption of polycyclic aromatic hydrocarbons to polystyrene nanoplastic. Environ. Toxicol. Chem. 2015, 35, 1650-1655.

(388) Kashiwada, S. Distribution of nanoparticles in the see-through medaka (Oryzias latipes). Environ. Health Perspect. 2006, 114, 16971702.

(389) Koziara, J. M.; Lockman, P. R.; Allen, D. D.; Mumper, R. J. In Situ Blood Brain Barrier Transport of Nanoparticles. Pharm. Res. 2003, 20, 1772-1778.

(390) Halden, R. U. Epistemology of contaminants of emerging concern and literature meta-analysis. J. Hazard. Mater. 2015, 282, 2-9.

(391) Gassel, M.; Harwani, S.; Park, J.-S.; Jahn, A. Detection of nonylphenol and persistent organic pollutants in fish from the North Pacific Central Gyre. Mar. Pollut. Bull. 2013, 73, 231-242.

(392) EPA (United States Environmental Protection Agency) State of the Science White Paper. A Summary of Literature on the Chemical Toxicity of Plastics Pollution to Aquatic Life and AquaticDependent Wildlife. 2016, 50.

(393) Devriese, L. I.; De Witte, B.; Vethaak, A. D.; Hostens, K.; Leslie, H. A. Bioaccumulation of PCBs from microplastics in Norway lobster (Nephrops norvegicus): An experimental study. Chemosphere. 2017, 186, 10-16.

(394) Von Smoluchowski, M. Versuch einer mathematischen Theorie der Koagulationskinetik kolloider Lösungen. Zeitschrift fuer Phys. Chemie 1917, 92, 129-168.

(395) Arvidsson, R.; Molander, S.; Sandén, B. A.; Hassellöv, M. Challenges in exposure modeling of nanoparticles in aquatic environments. Hum. Ecol. Risk Assess. 2011, 17, 245-262.

(396) Lyklema, J. Particulate colloids, volume IV. In Fundamentals of interface and colloid science; Lyklema, J., Ed.; Elsevier Academic Press: Amsterdam. 2005.
Stokes, G. G. On the effect of the internal friction of fluids on the motion of pendulums; Transactions of the Cambridge Philosophical Society IX, 8. 1850.

(398) Blom, G.; Aalderink, R. H. Calibration of three resuspension/sedimentation models. Water Sci. Technol. 1998, 37 41-49.

(399) Brouwer, L. Obtaining a clear view: Sediment dynamics of river de Dommel and the possible consequences of suspended solids on the underwater light climate; Wageningen University, Wageningen. 2012

(400) Clemmens, A.; Holly, F. J.; Schuurmans, W. Description and evaluation of program: Duflow. J. Irrig Drain. Eng. 1993, 119, 724-734.

(401) Rao, J. P.; Geckeler, K. E. Polymer nanoparticles: Preparation techniques and size-control parameters. Prog. Polym. Sci. 2011, 36, 887-913.

(402) Rosenkranz, P.; Chaudhry, Q.; Stone, V.; Fernandes, T. F. A comparison of nanoparticle and fine particle uptake by Daphnia magna. Environ. Toxicol. Chem. 2009, 28, 2142-2149.

(403) Wang, J. D.; Douville, N. J.; Takayama, S.; ElSayed, M. Quantitative analysis of molecular absorption into PDMS microfluidic channels. Ann. Biomed. Eng. 2012, 40, 1862-1873.

(404) Velzeboer, I.; Kwadijk, C.; Koelmans, A. A. Supporting information - Strong sorption of PCBs to nanoplastics, microplastics, carbon nanotubes and fullerenes. Environ. Sci. Technol. 2014, 48, 4869-4876.

(405) Lürling, M.; De Lange, H. J.; Van Donk, E. Changes in food quality of the green alga Scenedesmus induced by Daphnia infochemicals: biochemical composition and morphology. Freshw. Biol. 1997, 38, 619-628.

(406) Alyakrinskaya, I. O. Some ecological features of the lugworm Arenicola marina L. (Annelida, Polychaeta) and its morphological and biochemical adaptations to burrowing. Biol. Bull. 2003, 30, 411-418.

(407) Spaargaren, D. H.; Weber, R. E Osmotic responses in the coelomic fluid of Arenicola marina subjected to 
salinity change. Netherlands J. Sea Res. 1979, 13, 547-561.

(408) Gourley, S. A.; Kuang, Y. A stage structured predator-prey model and its dependence on maturation delay and death rate. Math. Biol. 2004, 200, 188 200.

(409) Koelmans, A. A.; Jonker, M. T. O. Effects of black carbon on bioturbationinduced benthic fluxes of polychlorinated biphenyls. Chemosphere. 2011, 84, 1150-1157.

(410) Koelmans, A. A. Correction of experimental sorption coefficients using DOC measurements and apparent solubility enhancements. Environ. Toxicol. Chem. 1995, 14, 2015-2016.

(411) Ahrens, M. J.; Hertz, J.; Lamoureux, E. M.; Lopez, G. R.; McElroy, A. E.; Brownawell, B. J. The role of digestive surfactants in determining bioavailability of sediment-bound hydrophobic organic contaminants to two deposit-feeding polychaetes. Mar. Ecol. Prog. Ser. 2001, 212, 145-157.

(412) Voparil, I.; Mayer, L. M. Dissolution of sedimentary polycyclic hydrocarbons (PAHs) into the lugworm's (Arenicola marina) digestive fluids. Environ. Sci. Technol. 2000, 34, 1221-1228.

(413) Weston, D. P.; Millward, R. N.; Mayer, L. M.; Voparil, I.; Lotufo, G. R. Sediment extraction using depositfeeder gut fluids: A potential rapid tool for assessing bioaccumulation potential of sediment- associated contaminants. ERDC/EL T R-02-18, U.S. Army Engineer Research and Development Center, Vicksburg, MS. 2002.

(414) Kramer, N. I.; Van Eijkeren, J. C. H.; Hermens, J. L. M. Influence of albumin on sorption kinetics in solid phase microextraction: Consequences for chemical analyses and uptake processes. Anal. Chem. 2007, 79, 69416948.

(415) Burkhard, L. P. Estimating dissolved organic carbon partition coefficients for nonionic organic chemicals. Environ. Sci. Technol. 2000, 34, 4663-4668.

(416) Kalčíková, G.; Žgajnar Gotvajn, A.; Kladnik, A.; Jemec, A. Impact of polyethylene microbeads on the floating freshwater plant duckweed Lemna minor. Environ. Pollut. 2017, 230 1108-1115.

(417) Zhang, C.; Chen, X.; Wang, J.; Tan, L. Toxic effects of microplastic on marine microalgae Skeletonema costatum: Interactions between microplastic and algae. Environ. Pollut. 2017, 220, 1282-1288.

(418) Sussarellu, R.; Suquet, M.; Thomas, Y.; Lambert, C.; Fabioux, C.; Pernet, M. E. J.; Le Goïc, N.; Quillien, V.; Mingant, C.; Epelboin, Y.; Corporeau, C.; Guyomarch, J.; Robbens, J.; Paul-Pont, I.; Soudant, P.; Huvet, A. Oyster reproduction is affected by exposure to polystyrene microplastics. Proc. Natl. Acad. Sci. 2016, 113, 2430-2435.

(419) Straub, S.; Hirsch, P. E.; BurkhardtHolm, P. Biodegradable and PetroleumBased Microplastics Do Not Differ in Their Ingestion and Excretion but in Their Biological Effects in a Freshwater Invertebrate Gammarus fossarum. Int. J. Environ. Res. Public Health. 2017, 14,774 .

(420) Ogonowski, M.; Schür, C.; Jarsén, Å. Gorokhova, E. The effects of natural and anthropogenic microparticles on individual fitness in daphnia magna. PLoS One. 2016, 11, 1-20.

(421) Au, S. Y.; Bruce, T. F.; Bridges, W. C.; Klaine, S. J. Responses of Hyalella azteca to acute and chronic microplastic exposures. Environ. Toxicol. Chem. 2015, 34, 2564-2572.

(422) Cole, M.; Lindeque, P.; Fileman, E.; Halsband, C.; Galloway, T. S. The impact of polystyrene microplastics on feeding, function and fecundity in the marine copepod Calanus helgolandicus Environ. Sci. Technol. 2015, 49, 11301137.

(423) Kaposi, K. L.; Mos, B.; Kelaher, B. P.; Dworjanyn, S. A. Ingestion of microplastic has limited impact on a marine larva. Environ. Sci. Technol. 2014, 48, 1638-1645.

(424) Tussellino, M.; Ronca, R.; Formiggini, F.; Marco, N. De; Fusco, S.; Netti, P. A.; Carotenuto, R. Polystyrene nanoparticles affect Xenopus laevis development. J. Nanoparticle Res. 2015, 17, 70 .

(425) Sjollema, S. B.; Redondo- 


\section{References}

Hasselerharm, P.; Leslie, H. A.; Kraak, M. H. S.; Vethaak, A. D. Do plastic particles affect microalgal photosynthesis and growth? Aquat. Toxicol. 2015, 170, 259-261.

(426) Casado, M.; Macken, A.; Byrne, H. J. Ecotoxicological assessment of silica and polystyrene nanoparticles assessed by a multitrophic test battery. Environ. Int. 2013, 51, 97-105.

(427) Bergami, E.; Bocci, E.; Vannuccini, M L.; Monopoli, M.; Salvati, A.; Dawson, K. A.; Corsi, I. Nano-sized polystyrene affects feeding, behavior and physiology of brine shrimp Artemia franciscana larvae. Ecotoxicol. Environ. Saf. 2016, 123, 18-25.

(428) Green, D. S. Effects of microplastics on European flat oysters, Ostrea edulis and their associated benthic communities. Environ. Pollut. 2016, 216, 95-103.

(429) Pedà, C.; Caccamo, L.; Fossi, M. C.;
Gai, F.; Andaloro, F.; Genovese, L.; Perdichizzi, A.; Romeo, T.; Maricchiolo, G. Intestinal alterations in European sea bass Dicentrarchus labrax (Linnaeus, 1758) exposed to microplastics: Preliminary results. Environ. Pollut. 2016, 212, 251-256.

(430) Espinosa, C.; Cuesta, A.; Esteban, M. Á. Effects of dietary polyvinylchloride microparticles on general health, immune status and expression of several genes related to stress in gilthead seabream (Sparus aurata L.). Fish Shellfish Immunol. 2017, 68, 251-259.

(431) Nobre, C. R.; Santana, M. F. M.; Maluf, A.; Cortez, F. S.; Cesar, A.; Pereira, C. D. S.; Turra, A. Assessment of microplastic toxicity to embryonic development of the sea urchin Lytechinus variegatus (Echinodermata: Echinoidea). Mar. Pollut. Bull. 2015, 92, 99-104. 
References 


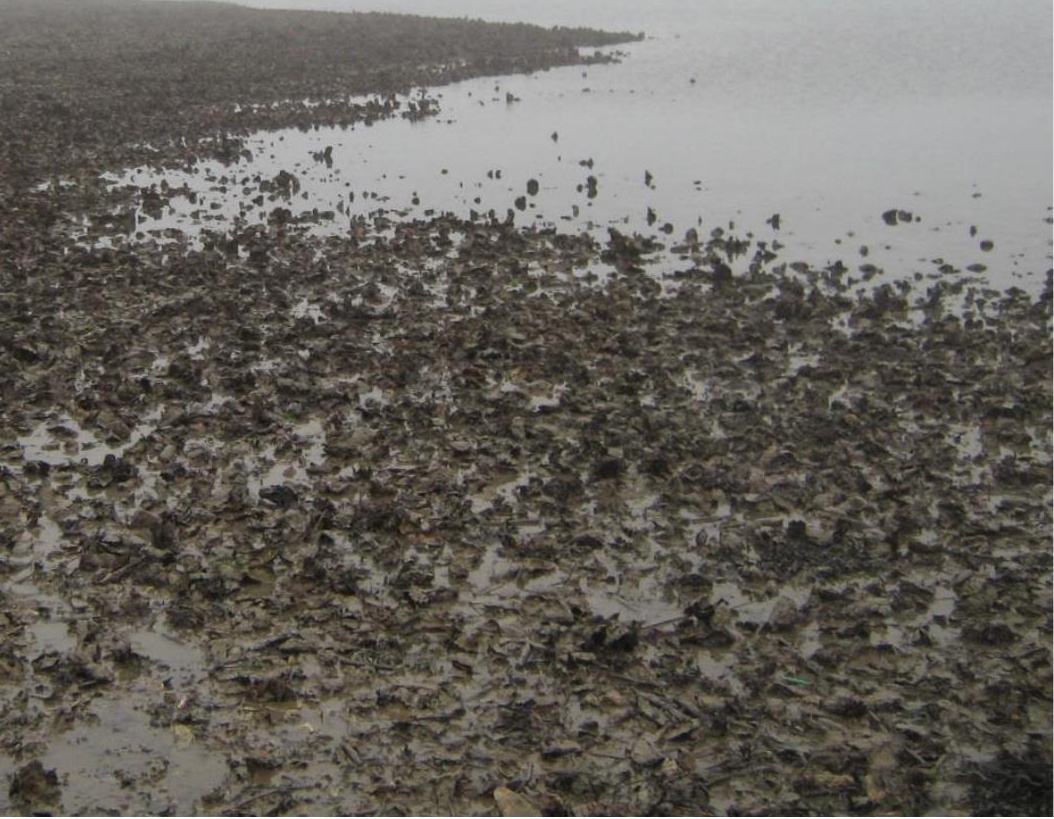




\section{Summary}

Although plastic has unquestionable benefits to modern society, its current use leads to pollution of the environment. Via direct disposal and degradation of larger plastic items, microplastic $(\leq 5 \mathrm{~mm})$ and nanoplastic $(\leq 100 \mathrm{~nm})$ particles reach the environment. Apart from these broad size ranges, micro- and nanoplastic exist with a variety of other characteristics of which part is material specific, such as polymer type, and others change in the environment, such as presence of a biofilm and sorbed hydrophobic chemicals. Potential negative effects include ecological harm to species due to particle effects of plastic and effects of plastic on the transfer of chemicals between organisms and the environment. The particle characteristics as well as many environmentally relevant processes affect the fate of micro- and nanoplastic particles, the exposure, effects and consecutive risk to organisms. This thesis addresses a great number of these characteristics and processes in depth in six separate chapters that each provide innovative concepts for a full-fledge risk assessment from the riverine towards the marine environment (Chapter 2-7) and combines these with other fate and effect studies from the literature to derive a provisional quantitative analysis of the ecological risk of micro- and nanoplastic in the aquatic environment (Chapter 8).

Riverine transport is considered an important pathway for microplastic to the marine environment. In Chapter 2, for the first time, fate and transport of nano- and microplastic in freshwater systems are modeled. Scenario studies are presented on the fate and transport of nano- to millimetre sized spherical particles $(100 \mathrm{~nm}-10 \mathrm{~mm})$ with a spatiotemporally resolved hydrological model. The processes included in the model are: advective transport, homoand heteroaggregation, sedimentation-resuspension, polymer degradation, presence of biofilm and burial. The model is parameterised with the use of literature data and additionally the attachment efficiency for heteroaggregation, is determined experimentally. The attachment efficiency ranges from 0.004 to 0.2 for $70 \mathrm{~nm}$ and $1050 \mathrm{~nm}$ polystyrene particles aggregating in natural freshwater with kaolin or bentonite clay particles. Model scenario calculations reveal that effects of polymer density (1000 $1500 \mathrm{~kg} / \mathrm{m}^{3}$ ) and biofilm formation on the predicted fate of the particles are not large. This is explained by the fact that variations in polymer density and 
biofilm formation are overwhelmed by excess mass of suspended solids that form heteroaggregates with microplastic. The size of the plastic particles has a huge effect on the modeled fate, the retention of plastic particles within the river stretch and the positioning of accumulation hotspots in the sediment. Retention is lowest (18-25\%) for intermediate sized particles of about $5 \mu \mathrm{m}$, which suggests that the smaller submicron as well as larger micro- and millimetre sized plastic particles are favourably retained. These results indicate that river hydrodynamics affect riverine microplastic size distributions with profound implications for emissions to the marine environment.

Plastic particles with a size in the nanoscale might, like other nanoparticles, affect organisms via particular size related effects mechanisms. Chapter 3 addresses the effects of $70 \mathrm{~nm}$ polystyrene nanoplastic on organisms in the freshwater environment. Effects of nanoplastic on the growth and photosynthesis of the green alga Scenedesmus obliquus and the growth, survival, neonate production and malformations of the water flea Daphnia magna are assessed. Population growth and chlorophyll concentrations of the algae become reduced during exposure to nanoplastic. Reduced body size and severe alterations in reproduction are observed in exposed water flea. Numbers and body size of newborn water flea are lower and the number of neonate malformations rises up to $68 \%$ of the newborn water flea. These effects of nanoplastic are observed at exposure concentrations from $0.22 \mathrm{mg} / \mathrm{L}$ onwards. Malformations occur at exposure concentrations from $30 \mathrm{mg} / \mathrm{L}$ onwards. These results appear to be novel in showing that direct physiological changes in algae and water flea populations may occur due to exposure to nanoplastic.

It has been speculated that microplastic causes negative physiological effects and increased bioaccumulation of hydrophobic, contaminants in organisms. Chapter 4 presents the first controlled bioassay on effects of microplastic on benthic organisms including transfer of hydrophobic chemicals. The physiological endpoints survival, feeding activity and bodyweight as well as uptake of microplastic particles and transfer of hydrophobic contaminants, are assessed in bioassays with the marine lugworm Arenicola marina exposed to microplastic. Microplastic is pre-equilibrated in natively contaminated sediment before the start of the exposure. Uptake of plastic particles and weight loss by the lugworms increases with exposure to increasing concentrations of microplastic. Furthermore, a reduction in feeding activity is observed at a microplastic concentration of $7.4 \%$ sediment dry weight. A low 
microplastic dose of $0.074 \%$ dry weight sediment increases bioaccumulation of hydrophobic contaminants by a factor $1.1-3.6$, an effect that is significant for the sum of the 19 used hydrophobic contaminants and for several individual contaminants. At higher plastic doses, bioaccumulation decreases compared to the low dose, but this is only significant for one individual contaminant. This decreasing effect on bioaccumulation with increasing microplastic concentration might be explained by dilution of overall concentrations of hydrophobic contaminants as a result of the introduction of a large absorbing pool, namely the microplastic. Microplastic thus shows to have statistically significant effects on organisms' fitness and bioaccumulation of hydrophobic contaminants, although the magnitude of the effects is not high. The effect on bioaccumulation is explained from physical impacts of the microplastic rather than from the microplastic acting as a carrier of chemicals to the organism.

Complex counteracting mechanisms play a role in the transfer of hydrophobic chemicals from plastic to organisms and vice versa. In Chapter 5 a conceptual model is developed and analyzed that simulates the effects of plastic on bioaccumulation of hydrophobic contaminants. The model involves the processes dilution of exposure concentration by sorption of hydrophobic contaminants to plastic ('dilution'), increased bioaccumulation by ingestion of plastic containing hydrophobic contaminants ('carrier'), and decreased bioaccumulation by ingestion of clean plastic ('cleaning'). Parameterization of the model is based on the lugworm Arenicola marina and evaluated against bioaccumulation data from the bioassay in Chapter 4. Further scenarios include a different microplastic polymer type, nanoplastic, and open marine systems. The model analysis demonstrates that plastic polymer types with low affinity for hydrophobic contaminants, as for example the polystyrene in Chapter 4, have a marginal decreasing effect on bioaccumulation in organisms, directed by dilution. For stronger sorbing polymers such as polyethylene, the dilution as well as the carrier and cleaning mechanism are more important in driving bioaccumulation. In closed laboratory bioassay systems, 'dilution' and 'cleaning' dominate, leading to decreased bioaccumulation. In open marine systems a decrease in bioaccumulation of hydrophobic contaminants as a result of exposure to plastic is predicted as well, due to the cleaning mechanism counteracting biomagnification.

Chapter $\mathbf{4}$ and $\mathbf{5}$ indicate the importance of including all relevant processes and transport pathways in assessing the effect of microplastic on bioaccumulation of hydrophobic contaminants in organisms. In Chapter 6 the 
effect of the strong sorbing polymer polyethylene microplastic on uptake of hydrophobic contaminants by the lugworm Arenicola marina is assessed in a bioassay, quantifying uptake fluxes from all natural exposure pathways. Concentrations of hydrophobic contaminants in sediment, biota lipids $\left(\mathrm{C}_{\text {lip }}\right)$ and porewater are measured with passive samplers to derive lipid-normalised bioaccumulation $\mathrm{C}_{\mathrm{lip}}$, the Biota - sediment accumulation factor (BSAF), the Bioaccumulation factor (BAF) and a new metric: the Biota - plastic accumulation factor (BPAF). Small effects of exposure to plastic are detected, suggesting either slightly increased or decreased bioaccumulation. However, the differences decrease in magnitude dependent on the used bioaccumulation metric in the order: $\mathrm{C}_{\text {lip }}>\mathrm{BSAF}>\mathrm{BPAF}>\mathrm{BAF}$, and are non-significant for $\mathrm{BAF}$. That $\mathrm{BAF}$, i.e. normalization of $\mathrm{C}_{\text {lip }}$ on porewater concentration, merely removes all effects of exposure to plastic on bioaccumulation, shows that polyethylene does not act as a measurable vector of hydrophobic contaminants. Biodynamic model analysis confirms that the ingestion of polyethylene, although being one of the polymers potentially affecting bioaccumulation most, contributes marginally to bioaccumulation. Chapter 6 therewith empirically confirms the model-based predictions in Chapter 5 that under environmentally realistic conditions the relevance of microplastic for bioaccumulation is limited. Furthermore, Chapter 6 illustrates the importance of assessing exposure to hydrophobic contaminants through all media in microplastic bioaccumulation studies, for instance by using elaborate passive sampling technologies.

Marine filter feeders are thought to be exposed to microplastic because of their selection of small particles as food source. In Chapter 7 the occurrence of microplastic in the major marine filter feeding species Megaptera novaeangliae, a baleen whale, is assessed. Macroplastic had been found in baleen whales before and as these organisms feed by filtering small particles from large water volumes, they potentially collect microplastic particles too. Chapter 7 presents the first study confirming the presence of microplastic in intestines of a baleen whale. Potential synthetic polymer particles from the intestinal content are selected based on density and appearance, and analysed by Fourier transform infrared (FTIR) spectroscopy. Several polymer types (polyethylene, polypropylene, polyvinylchloride, polyethylene terephthalate, nylon) are found, in varying particle shapes: sheets, fragments and threads with a size of $1 \mathrm{~mm}$ to $17 \mathrm{~cm}$. This diversity in polymer types and particle shapes, can be interpreted as a representation of the varying characteristics of marine plastic and the unselective way of ingestion by filter feeding baleen 
whales. A basic model is developed to estimate plastic concentrations in the water column of the region a sampled organism inhabited, based on daily filtered water volume, gut passage time and plastic particle numbers detected in the organism. The model outcomes correspond with average microplastic concentrations in the literature measured in surface water from the same oceanic region the sampled whale inhabited. This illustrates the usefulness of the model for estimations of internal plastic concentrations in filter feeding organisms from regions with known plastic concentrations in the water, and, the other way around, the potential utility of gut contents of filter feeding species by representing average environmental plastic concentrations.

Combining knowledge on fate, exposure concentrations and effect thresholds and risk characterisation leads to insight in the ecological risk of a pollutant. In Chapter $\mathbf{8}$ such a quantitative risk assessment is performed for micro- and nanoplastic. The remaining uncertainties are described. Highest limits of reported ranges of microplastic concentrations are standardized per habitat type and global region, and calculated to be highest in beach sediment, freshwater sediment and near shore surface water. Based on microplastic concentrations and degradation mechanisms, potential future nanoplastic number concentrations are estimated to become up to 14 orders of magnitude higher than microplastic concentrations. Species sensitivity distributions are constructed for micro- and nanoplastic separately. Effect thresholds for aquatic organisms with potential population level consequences from the literature are standardized and combined in these species sensitivity distributions. From these species sensitivity distributions, provisional safe standards are derived. For micro- and nanoplastic in water these are $0.4 \mathrm{ng} / \mathrm{L}$ (microplastic) and $1.1 \mu \mathrm{g} / \mathrm{L}$ (nanoplastic) respectively. In food for aquatic organisms or in sediment these are $0.1 \mathrm{mg} / \mathrm{kg}$ (microplastic) and $1 \mathrm{mg} / \mathrm{kg}$ (nanoplastic) dry weight. A comparison of exposure concentrations with the hazardous concentration for $5 \%$ of the species $\left(\mathrm{HC}_{5}\right)$, derived from the species sensitivity distributions, indicates that microplastic concentrations in the water are below effect thresholds for organisms in most locations but on some near shore hotspot locations might be hazardous for up to 10-20\% of the species. Microplastic concentrations at the higher ends of worldwide reported ranges in both freshwater sediment and beach sediment are within the limited set of currently available microplastic particle effect thresholds for organisms inhabiting sediment. Whereas previous research indicated a risk of microplastic for sediment dwelling organisms only, the use of new effect threshold data and extrapolation factors to scale thresholds consistently to 
chronic effect concentrations leads to the characterisation of a risk for organisms inhabiting the water column too. For both micro- and nanoplastic, current predictions on the development of environmental concentrations suggest that more species will be at risk in the future. The transfer of hydrophobic contaminants by microplastic seems to have a negligible contribution to the overall risk of exposure to microplastic. Exceptions to this are (a) hotspot locations, where a large fraction of an organisms' diet consists of microplastic (yet still only if the chemical concentration gradient allows transfer of contaminants towards the organism) and (b) nanoplastic particles, where the risk might be considerable due to strong binding of hydrophobic contaminants and possible translocation to tissues beyond the intestines. Several uncertainties and data gaps remain, such as the environmental concentrations of nanoplastic, importance of food selection strategies for exposure and general or species dependent effect mechanisms. Additionally, the material plastic as a pollutant comes with different polymer types, shapes and sizes of particles, with concomitant variations in fate and effects. However, the combination of novel information and concepts provided in this thesis together with an extensive literature review of fate, exposure and effect data made a tentative yet quantitative risk assessment for micro- and nanoplastic possible. 
Summary 


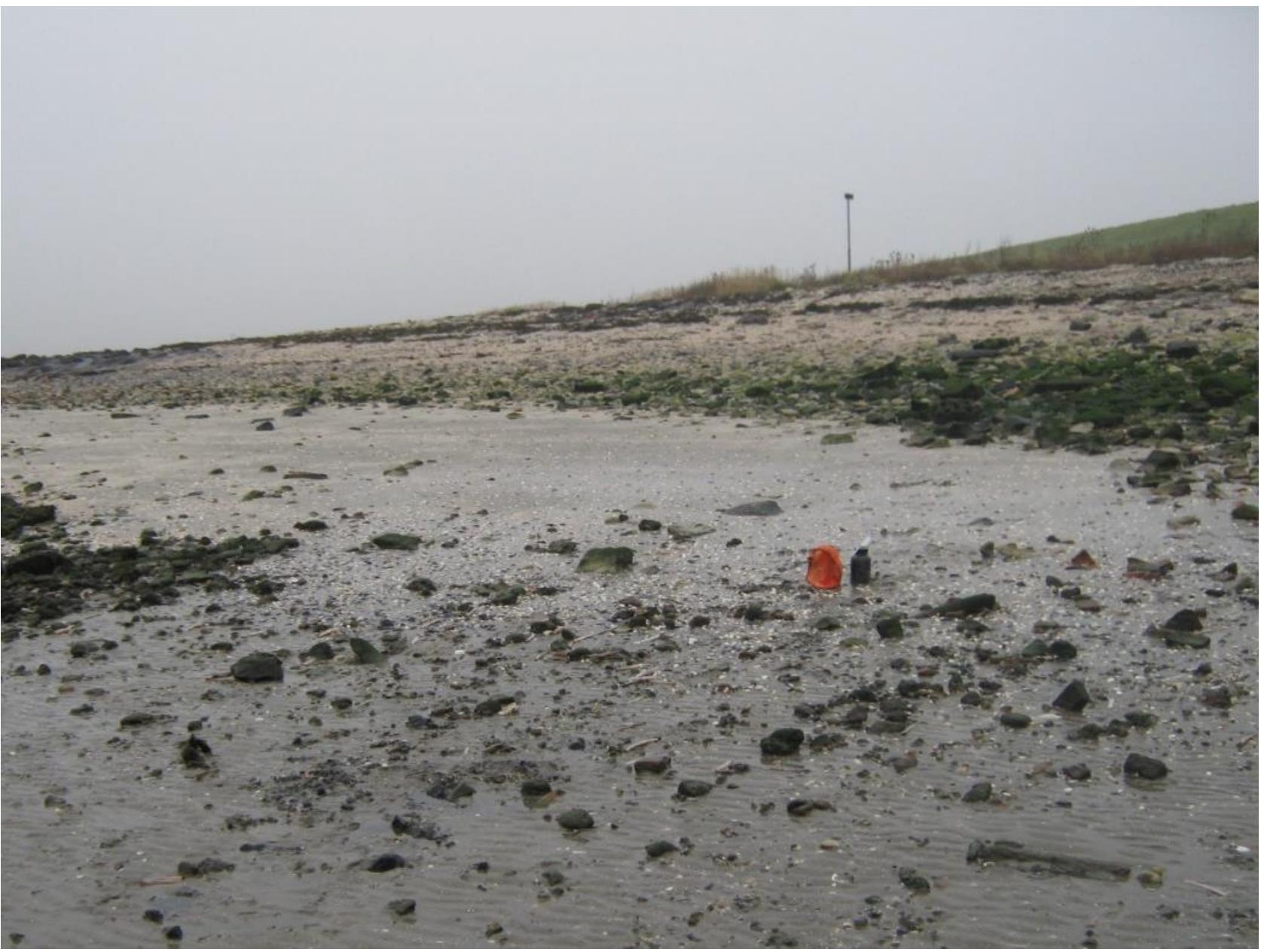




\section{Samenvatting}

Hoewel plastic onmiskenbare voordelen biedt voor de moderne maatschappij, leidt het huidige gebruik tot vervuiling van het milieu. Door rechtstreekse emissie en degradatie van grotere plastic items komen microplastic $(\leq 5 \mathrm{~mm})$ en nanoplastic $(\leq 100 \mathrm{~nm})$ deeltjes in het milieu terecht. Behalve deze ruime ranges van deeltjes groottes, hebben micro- en nanoplastic meer variërende eigenschappen waarvan sommige materiaal specifiek zijn, zoals polymeertype. Andere eigenschappen kunnen veranderen in het milieu, zoals de aanwezigheid van een biofilm en geabsorbeerde hydrofobe chemicaliën. Potentiële negatieve ecologische effecten worden ofwel veroorzaakt door de fysieke aanwezigheid van plastic deeltjes zelf, ofwel door de effecten van plastic op de overdracht van chemicaliën tussen organismen en het milieu. Zowel de eigenschappen van de deeltjes als vele milieurelevante processen beïnvloeden de verspreiding van micro- en nanoplastic deeltjes, de blootstelling, de effecten en het risico voor organismen. Dit proefschrift behandelt een groot aantal van deze eigenschappen en processen in detail, in zes hoofdstukken. Elk van deze hoofdstukken levert innovatieve concepten voor het maken van een volledige risico inschatting van het rivierenlandschap tot het marine milieu (Hoofdstuk 2-7). Vervolgens worden deze hoofdstukken gecombineerd met andere verspreidings- en effectstudies uit de literatuur, om te komen tot een voorlopige kwantitatieve analyse van het ecologische risico van micro- en nanoplastic in het aquatische milieu (Hoofdstuk 8).

Rivieren worden gezien als belangrijke transportroute van microplastic naar het mariene milieu. In Hoofdstuk 2 wordt voor de eerste keer de verspreiding en het transport van nano- en microplastic in zoetwatersystemen gemodelleerd. Scenario studies van de verspreiding en het transport van nanotot millimeter grote bolvormige deeltjes $(100 \mathrm{~nm}-10 \mathrm{~mm})$ worden gepresenteerd door gebruik van een in ruimte en tijd expliciet model. De in het model opgenomen processen zijn: transport door stroming, aggregatie met plastic en andere deeltjes, sedimentatie, opwerveling, afbraak, aanwezigheid van een biofilm en opslag in de waterbodem. De modelparameters zijn vastgesteld op basis van gegevens uit de literatuur en daarnaast is experimenteel de aggregatie efficiëntie van nano- en microplastic met deeltjes in het water, zoals kleideeltjes, bepaald. Deze aggregatie efficiëntie varieerde 
van 0.004 tot 0.2 voor $70 \mathrm{~nm}$ en $1050 \mathrm{~nm}$ polystyreen deeltjes aan kleideeltjes in natuurlijk zoetwater. Scenarioberekeningen met het model laten zien dat de invloed van de polymeerdichtheid $\left(1000-1500 \mathrm{~kg} / \mathrm{m}^{3}\right)$ en van de aanwezigheid van een biofilm betrekkelijk weinig invloed hebben op de verspreiding van de plastic deeltjes. Dit wordt verklaard door het feit dat variaties in polymeerdichtheid en biofilmformatie worden overschaduwd door het effect van de overmaat aan in de waterkolom zwevende deeltjes die aggregeren met microplastic. De grootte van de plastic deeltjes heeft een enorm effect op de gemodelleerde verspreiding, de retentie van plastic deeltjes in de rivierloop en de locatie waar ophoping in het sediment plaatsvindt. Retentie is het laagst (18-25\%) voor deeltjes van een middelmatige grootte rond de $5 \mu \mathrm{m}$. Dit suggereert dat zowel de kleinere sub-micrometer deeltjes als de grotere micro- en millimeter plastic deeltjes vooral worden vastgelegd in rivierlopen. Deze resultaten laten zien dat de hydrodynamiek in rivieren de deeltjesgrootteverdeling van plastic verandert, met gevolgen voor de emissie naar zee.

Plastic deeltjes van nanometer grootte beïnvloeden mogelijk, net als andere nanodeeltjes, organismen via specifieke grootte-gerelateerde effect mechanismen. Hoofdstuk 3 gaat in op de effecten van $70 \mathrm{~nm}$ polystyreen nanoplastic op organismen in het zoetwater milieu. Effecten van nanoplastic op de groei en fotosynthese van de groene alg Scenedesmus obliquus en de groei, overleving, voortplanting en misvormingen van de watervlo Daphnia magna worden behandeld. Populatiegroei en chlorofyl concentraties van de algen zijn lager tijdens blootstelling aan nanoplastic. Blootgestelde watervlooien zijn kleiner en verschillende veranderingen worden geobserveerd in hun nakomelingen. Aantallen en grootte van pasgeboren watervlooien zijn kleiner en het aantal misvormingen stijgt naar $68 \%$ van de individuen. Deze effecten van nanoplastic worden gezien bij blootstellingsconcentraties vanaf $0.22 \mathrm{mg} / \mathrm{L}$. Misvormingen van jonge watervlooien treden op bij blootstellingsconcentraties vanaf $30 \mathrm{mg} / \mathrm{L}$. Nieuw aan deze resultaten is dat ze laten zien dat directe fysiologische veranderingen in algen en watervlopopulaties kunnen optreden door blootstelling aan nanoplastic.

Er wordt over gespeculeerd dat microplastic negatieve fysiologische effecten en verhoging van bioaccumulatie van hydrofobe verontreinigende stoffen in organismen kan veroorzaken. Hoofdstuk 4 beschrijft het eerste onder gecontroleerde omstandigheden uitgevoerde experiment met benthische organismen blootgesteld aan microplastic waarbij de overdracht van hydrofobe contaminanten wordt geanalyseerd. Zowel de fysiologische 
parameters overleving, foerageeractiviteit en gewicht als de opname van microplastic deeltjes en de opname van hydrofobe chemicaliën zijn onderzocht in een experiment waarin de mariene wadpier Arenicola marina is blootgesteld aan microplastic. Voorafgaand aan de blootstelling is de concentratie contaminanten in microplastic deeltjes in evenwicht gebracht met die in het sediment. De wadpieren nemen meer plastic deeltjes op en verliezen meer gewicht bij blootstelling aan een toenemende concentratie microplastic. Verder is een vermindering van de activiteit geobserveerd bij een microplastic concentratie van $7.4 \%$ van het sediment (droog gewicht). Een lage microplastic dosering van $0.074 \%$ van het sediment verhoogt de bioaccumulatie van hydrofobe contaminanten met een factor 1.1 tot 3.6. Dit effect is significant voor de concentratie van de 19 gebruikte contaminanten samen en voor een aantal individuele contaminanten. Bij hogere plastic doseringen is de bioaccumulatie van hydrofobe contaminanten lager dan bij lage dosering, maar dit is alleen significant voor één individuele contaminant. Dit verminderde effect op bioaccumulatie met toenemende plastic concentratie kan worden verklaard door verdunning van de totale hoeveelheid hydrofobe chemicaliën als gevolg van de toevoeging van een grote hoeveelheid chemicaliën-absorberend materiaal, namelijk de microplastic deeltjes. Microplastic heeft dus statistisch significante effecten op de gezondheid van organismen en op de bioaccumulatie van hydrofobe contaminanten, al is de omvang van het effect op bioaccumulatie niet groot. Het effect op de bioaccumulatie wordt eerder verklaard door de fysieke aanwezigheid van het plastic dan door het voorheen vermeende optreden als drager van contaminanten naar organismen toe.

Complexe tegengestelde mechanismen spelen een rol in het transport van chemicaliën van plastic naar organismen en omgekeerd. In Hoofdstuk 5 is een model ontwikkeld en geanalyseerd dat het effect van plastic op de bioaccumulatie van hydrofobe contaminanten simuleert. Het model bevat de processen verdunning van de blootstellingsconcentratie door opname van hydrofobe contaminanten door plastic ('verdunning'), toenemende bioaccumulatie door ingestie van plastic dat hydrofobe contaminanten bevat ('drager'), en verminderde bioaccumulatie door ingestie van schoon plastic ('reiniging'). Parameterisatie van het model is gebaseerd op de wadpier Arenicola marina en geëvalueerd met de bioaccumulatie gegevens van het experiment uit Hoofdstuk 4. Overige scenario's bevatten: een ander microplastic polymeer type, nanoplastic, en open marine systemen. De model analyse laat zien dat plastic polymeer typen met een lage affiniteit voor 
hydrofobe contaminanten, zoals bijvoorbeeld het polystyreen gebruikt in Hoofdstuk 4, een marginale vermindering van de bioaccumulatie in organismen veroorzaken, gestuurd door verdunning. Bij sterker absorberende polymeer typen zoals polyethyleen, zijn zowel verdunning als het drager- en reinigingsmechanisme meer bepalend voor de mate van bioaccumulatie. In laboratoriumexperimenten met gesloten systemen, leidt het domineren van de mechanismen 'verdunning' en 'reiniging' tot verminderde bioaccumulatie in organismen. Voor open mariene systemen wordt ook een vermindering van de bioaccumulatie van hydrofobe contaminanten door blootstelling aan plastic voorspeld, doordat het reinigingsmechanisme een tegengestelde werking heeft ten opzichte van biomagnificatie.

Hoofdstuk 4 en 5 geven het belang aan van het meenemen van alle relevante processen en transport routes in het beoordelen van het effect van microplastic op de bioaccumulatie van hydrofobe contaminanten in organismen. In Hoofdstuk $\mathbf{6}$ is het effect van het sterk absorberende polymeertype polyethyleen microplastic op de opname van hydrofobe contaminanten in de wadpier Arenicola marina onderzocht, in een laboratorium experiment waarin de opnamefluxen vanuit alle natuurlijke blootstellingsroutes zijn gekwantificeerd. Concentraties van hydrofobe contaminanten in sediment, wadpier lipiden $\left(\mathrm{C}_{\mathrm{lip}}\right)$ en poriewater zijn gemeten met 'passive samplers' om de lipiden-genormaliseerde bioaccumulatie $\mathrm{C}_{\text {lip }}$, de Biota-Sediment Accumulatie Factor (BSAF), de Bioaccumulatie Factor (BAF) en een nieuwe parameter: de Biota-Plastic Accumulatie Factor (BPAF) te bepalen. Kleine effecten van de blootstelling aan plastic zijn gedetecteerd, soms duidend op een kleine toename, en soms op een afname van de bioaccumulatie. De grootte van de gedetecteerde verschillen in bioaccumulatie neemt echter afhankelijk van de gebruikte bioaccumulatie parameter af, in de volgorde: $\mathrm{C}_{\text {lip }}>\mathrm{BSAF}>\mathrm{BPAF}>\mathrm{BAF}$, om uiteindelijk niet meer significant te zijn voor de $\mathrm{BAF}$. Dat de BAF, oftewel $\mathrm{C}_{\text {lip }}$ genormaliseerd op de poriewaterconcentratie in het sediment, alle effecten van blootstelling aan plastic op bioaccumulatie wegneemt, laat zien dat polyethyleen niet als meetbare vector voor hydrofobe contaminanten fungeert. Biodynamische model analyse bevestigt dat ingestie van polyethyleen, hoewel dit een van de polymeertypen is met het grootste potentiële effect op de bioaccumulatie, nauwelijks bijdraagt aan de bioaccumulatie. Hiermee bevestigt Hoofdstuk 6 empirisch de modelvoorspellingen uit Hoofdstuk 5, dat wil zeggen: dat onder milieu realistische condities de relevantie van microplastic voor bioaccumulatie beperkt is. Verder illustreert Hoofdstuk 6 het belang van het meenemen van blootstelling 
aan contaminanten via alle media in microplastic bioaccumulatie studies, bijvoorbeeld middels gebruik van 'passive sampling' technieken.

Mariene filterfeeders worden naar verwachting blootgesteld aan microplastic doordat ze kleine deeltjes selecteren als voedsel. In Hoofdstuk $\mathbf{7}$ is het voorkomen van microplastic in de grote mariene filterfeeder Megaptera novaeangliae, een baleinwalvis, onderzocht. Macroplastic was voorheen al gevonden in baleinwalvissen en omdat deze dieren zich voeden door kleine deeltjes uit grote watervolumes te filteren, verzamelen ze mogelijk ook microplastic deeltjes. Hoofdstuk 7 presenteert de eerste studie die het voorkomen van microplastic in de ingewanden van een baleinwalvis bevestigd. Potentiële synthetische polymeer deeltjes zijn geselecteerd uit de inhoud van de ingewanden op basis van dichtheid en uiterlijk, en geanalyseerd met Fourier-Transform-Infraroodspectroscopie (FTIR). Verschillende polymeertypen (polyethyleen, polypropyleen, polyvinylchloride, polyethyleentereftalaat, nylon) zijn gevonden, in wisselende deeltjesvorm: folie, brok en draad met een grootte van $1 \mathrm{~mm}$ tot $17 \mathrm{~cm}$. Deze diversiteit aan polymeertypen en deeltjesvormen kan worden geïnterpreteerd als een afspiegeling van de variërende kenmerken van marien plastic en de nietselectieve manier van ingestie door filterende baleinwalvissen. Een voorlopig model is ontwikkeld voor het inschatten van plastic concentraties in de waterkolom van de regio waar een bemonsterd dier vandaan komt, gebaseerd op het dagelijks gefilterde watervolume, de verblijftijd van voedsel in de ingewanden en het in het dier gedetecteerde aantal plastic deeltjes. De modeluitkomsten komen overeen met de gemiddelde in de literatuur vermeldde microplastic concentratie in oppervlaktewater in de oceaanregio waar de bemonsterde walvis vandaan komt. Dit illustreert de bruikbaarheid van het model voor inschatting van interne plastic concentraties in filterende organismen uit regio's waarvan de plastic concentraties in het water bekend zijn en, andersom, de potentiële bruikbaarheid van de inhoud van ingewanden van filterende diersoorten voor het vertegenwoordigen van gemiddelde plastic concentraties in het milieu.

Het combineren van kennis over verspreiding, blootstellingsconcentraties en effectdrempels en risicokarakterisering leidt tot inzicht in het ecologische risico van een verontreiniging. In Hoofdstuk 8 is zo'n risicobeoordeling uitgevoerd voor micro- en nanoplastic. De resterende onzekerheden zijn beschreven. De bovengrenzen van gerapporteerde microplastic concentraties zijn gestandaardiseerd per habitat type en mondiale regio. Vergelijking geeft aan dat zij het hoogst te zijn in strandzand, zoetwater sediment en 
oppervlaktewater dicht bij de kust. Gebaseerd op microplastic concentraties en afbraakmechanismen zijn potentiële toekomstige nanoplastic concentraties in deeltjes aantallen ingeschat tot 14 grootteorders hoger te worden dan microplastic concentraties. 'Species sensitivity distributions' (SSDs) zijn geconstrueerd voor micro- en nanoplastic afzonderlijk. Uit de literatuur zijn voor aquatische organismen effectdrempels met potentiële consequenties op het populatieniveau gestandaardiseerd en gecombineerd in deze SSDs. Uit de SSDs zijn voorlopige veilige plastic concentraties afgeleid. Voor micro- en nanoplastic in water zijn dit respectievelijk $0.4 \mathrm{ng} / \mathrm{L}$ (microplastic) en $1.1 \mu \mathrm{g} / \mathrm{L}$ (nanoplastic). In voedsel van aquatische organismen en in sediment zijn dit $0.1 \mathrm{mg} / \mathrm{kg}$ (microplastic) en $1 \mathrm{mg} / \mathrm{kg}$ (nanoplastic) droog gewicht. Een vergelijking van blootstellingsconcentraties met de schadelijke concentratie voor 5\% van de soorten organismen $\left(\mathrm{HC}_{5}\right)$, afgeleid uit de SSDs, wijst uit dat microplastic concentraties in de waterkolom op de meeste locaties onder de effectdrempel voor organismen liggen maar op sommige hotspot locaties nabij de kust gevaarlijk kunnen zijn voor 10-20\% van de soorten. De bovengrenzen van wereldwijd gerapporteerde microplastic concentraties in zowel zoetwater sediment als strandzand vallen binnen de beperkte set momenteel beschikbare effectdrempels van microplastic deeltjes voor sedimentbewonende organismen. Voorgaand onderzoek duidde op een risico van microplastic voor alleen sedimentbewonende organismen. Het gebruik van nieuwe effectdrempel data en het gebruik van extrapolatie factoren voor het consistent schalen van effectdrempels naar chronische effectconcentraties leidt ertoe dat ook een risico voor organismen in de waterkolom kenbaar wordt in dit proefschrift. Voor zowel micro- als nanoplastic suggereren actuele voorspellingen van de ontwikkeling van milieuconcentraties dat er in de toekomst voor meer soorten een risico zal zijn. Het transport van hydrofobe contaminanten door microplastic lijkt een verwaarloosbare bijdrage te leveren aan het totale risico door blootstelling aan microplastic. Uitzonderingen hierop zijn (a) hotspot locaties, waar een groot aandeel van het dieet van een organisme bestaat uit plastic (hoewel alleen als de chemische concentratiegradiënt transport naar het organisme toe mogelijk maakt) en (b) nanoplastic deeltjes, waarvoor het risico aanzienlijk kan zijn door de sterke binding van hydrofobe contaminanten en mogelijk translocatie naar weefsels buiten de ingewanden. Meerdere onzekerheden en datalacunes resteren, zoals de nanoplastic concentraties in het milieu, het belang van voedselselectie strategieën voor de blootstelling en generieke of soortafhankelijke effectmechanismen. Bovendien komt het materiaal plastic als vervuiling voor in een verscheidenheid aan polymeertypen, deeltjesvormen en -groottes, met 
bijbehorende variaties in verspreiding en effecten. Desondanks maakt de combinatie van nieuwe informatie en concepten in dit proefschrift samen met een uitgebreide literatuurevaluatie van verspreiding, blootstelling en effect data, een voorlopige kwantitatieve risicobeoordeling voor micro- en nanoplastic mogelijk. 


\section{Acknowledgements}

First of all, I'd like to thank Anna for starting all of this together. Thanks for your good company and delicious baking's, during our stay-over weekends taking care of our first experiments with micro- and nanoplastic in Den Helder. Thank you Frits for your ever sound laboratory instructions and for ordering a package of 'suspiciously white powder' every now and then. Thanks to all colleagues at IMARES (nowadays WMR) in Den Helder, Yerseke, IJmuiden and Texel and our neighbours at NIOZ Yerseke for your warm welcome along the coast. Thanks to all colleagues and students at our group AEW in Wageningen for the great atmosphere, the good talks, thinking along in science and teaching together. Thank you Rudi for trusting your course to me. Thank you John for all the support and the good times teaching together, in particular on Texel. Thank you Joris for you kind introduction in the modeling work you started. Thank you Martine, Pauline, Ilona, Michiel, Suze and Jan Andries for our joined work on plastic. Yu, Bo, Sanne, Muzhi, Kim, Lijing, Fani, Lyke, Dianneke, Renske and Enya thanks for your curiosity in microand nanoplastic research during your theses and internships. Svenja, Merel and Paula thanks for exchanging thoughts on our plastic theses and for continuing with all the open ends. A very special thanks to Edwin and Bart. Thank you Edwin for the good times in Den Helder, the long phone conversations, your guidance and in particular your ever critical approach. Most of all, thank you Bart for all that without you would never have been there. Thanks for our joint interest in a quantitative approach, for all that I learned, for your sharp-mindedness, the many advises, specific and helpful commentaries and the good company. Thank you Sil for getting me through the steep learning curve of the program R, exchanging thoughts on our theses, but most of all thank you and Tijl for the very warm homecomings. Thanks to all friends and family for bearing with us while Sil and me were spending years on accomplishing this. Thanks in particular for babysitting and for your interest in our thesis topics, or patient nodding. And, a very special thanks to my parents for raising me with a love of nature and for the opportunity to go study in Wageningen. 


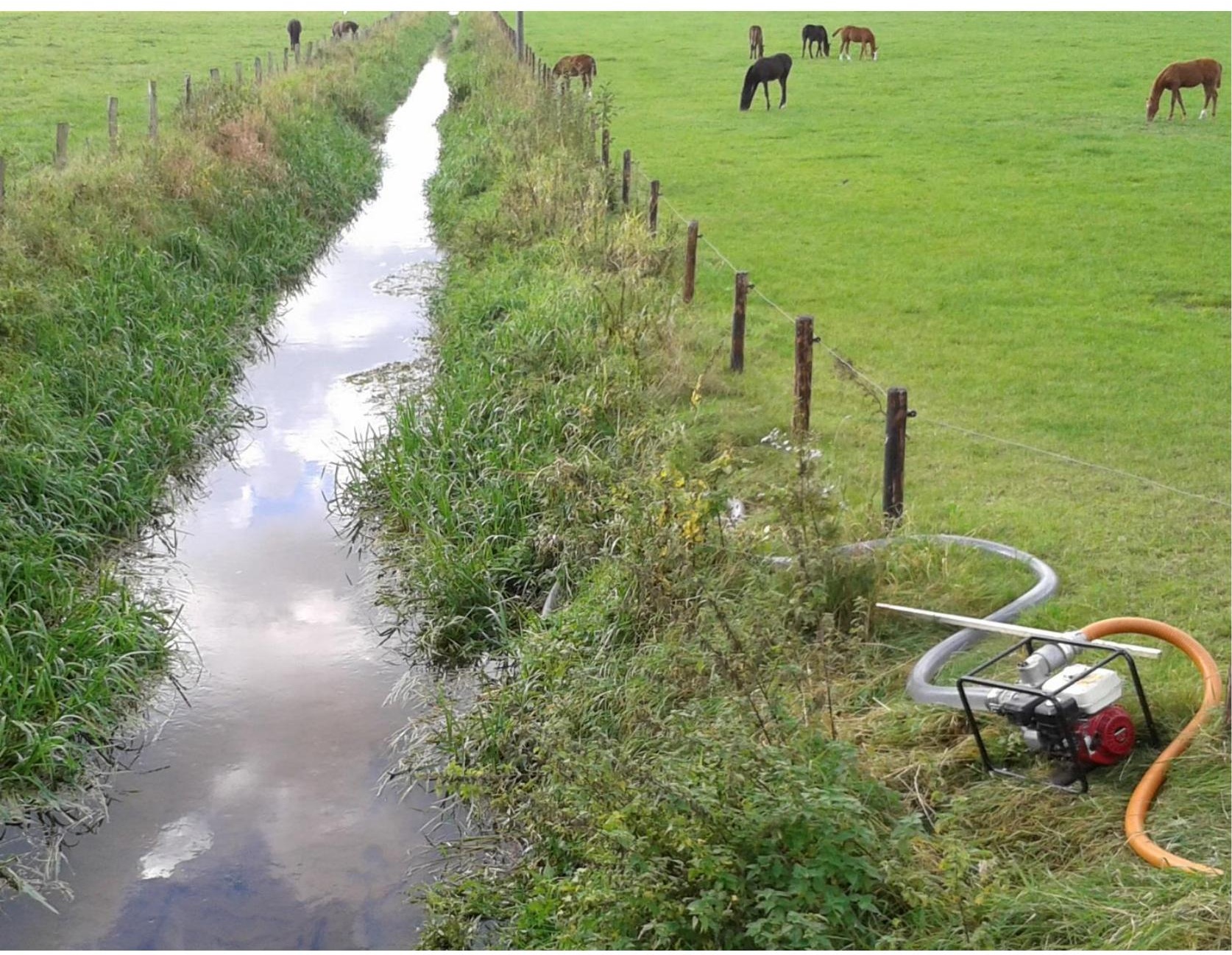




\section{Curriculum vitae}

Als kind al was Ellen verwonderd over de ondergrond, het water en alles wat er op en in leeft. Zodra de eerste stapjes werden gezet had haar moeder de schone taak zonder steel geplukte bloemen op vaas te zetten. Ze werd door haar ouders aangestoken met het enthousiasme voor de tuin en plantte al jong bloemen en groenten, maar ook eikels en kastanjes. In de winter groef zij in de moestuin van haar ouders op de hoge enkgrond van Vorden kuilen om het grondwater te vinden. De verwachtte ondergrondse rivier bleef uit maar dat de metersdiepe kuil toch langzaam vol sijpelde met water, was des te mysterieus. De kuil ging op een paar bodemschatten, scherven van oud servies, na weer dicht om in het voorjaar op een andere plek opnieuw gegraven te worden. Eerst als kleine vijver, met een vuilniszak als bodembedekking. In de zomer namen de mooie dikke kikkers daar genoegen mee, maar voor de herfst moest er een vijver komen voldoende diep om de geliefde amfibieën de vorst te laten overleven. Die kwam er, op een onbestemd stuk grond tussen de schuur en een paar hoge coniferen. Misschien iets te vaak naar smaak van de kikkers werden zij onder de flappen van het vijverfolie weggepakt en geteld. De omgeving werd afgestruind en gele lis en dotterbloem naar de vijver gehaald. Het welig tieren van planten en dieren maakte dat haar ouders de vijver op een plek beter in het zicht, in de bloementuin wilden hebben. Die kwam er, en is er nog steeds met al z'n padden en salamanders. Het was daar dat het niet willen groeien van waterviolier en niet willen bloeien van zwanenbloem deden realiseren hoe afhankelijk al dat leven van omgevingsfactoren is.

Ook buiten de eigen tuin werden groene kikkers gevangen en boomkikkers gezocht met haar vader bij kasteel De Wildenborch en in het bos waar ze op scouting zat. Eerst zelf en later als leidster liet zij kinderen de natuur ontdekken, en in het bijzonder het deel dat je met een schepnet tegenkomt. In de vijvers en het rabattenbos bij een vriendinnetje werden de eerste indrukwekkende kamsalamanders gevangen en daarop volgden er nog vele tijdens tellingen met reptielen- en amfibieën vereniging RAVON. Als bijbaantje onderhield ze de bloemen- en moestuinen van tuinliefhebbers op leeftijd. Op school ging haar interesse uit naar de bèta kant, waarna ze naar de universiteit van Wageningen ging om te studeren. Eerst landschapsinrichting en -planning, later milieuwetenschappen met als specialisatie, het kon 
eigenlijk niet missen, aquatische ecologie en waterkwaliteit. In Wageningen ontmoette ze haar vriend, een geboren bioloog waarmee ze een volkstuin begon, de excursies van zijn studie tijdens vakanties nog eens overdeed en nu een zoon van twee heeft wiens eerste woorden 'bij' en 'vogel' waren. Ze liep stage bij Waterschap Zuiderzeeland waar ze in afstemming met verschillende partijen de optimalisatie van het gebruik van fosfor, een eindige grondstof, afstemde. Vanuit haar afstudeeronderzoek over de effecten van micro- en nanoplastic rolde ze door in haar promotieonderzoek bij de leerstoelgroep aquatische ecologie en waterkwaliteit in Wageningen en onderzoeksinstituut IMARES. Dat er relatief weinig bekend was over dit nieuwe onderwerp maakte dat er zowel vanuit beleid als wetenschappelijk veel interesse was voor het onderzoek en de groep zich in korte tijd ontwikkelde tot een internationale autoriteit op het gebied van verspreiding en effecten van micro- en nanoplastic. Naast haar promotieonderzoek werd Ellen docent bij de leerstoelgroep waar ze met veel plezier les gaf in een aantal aquatische ecologie en waterkwaliteitsvakken en afstudeeronderzoeken begeleidde. Het willen opdoen van meer beleidservaring en werken dichter bij de praktijk maakt dat ze weer terug is in de Achterhoek waar ze bij Waterschap Rijn en IJssel werkt aan het samengaan van een prachtig landschap, een verscheidenheid aan functies en een goede waterkwaliteit. 


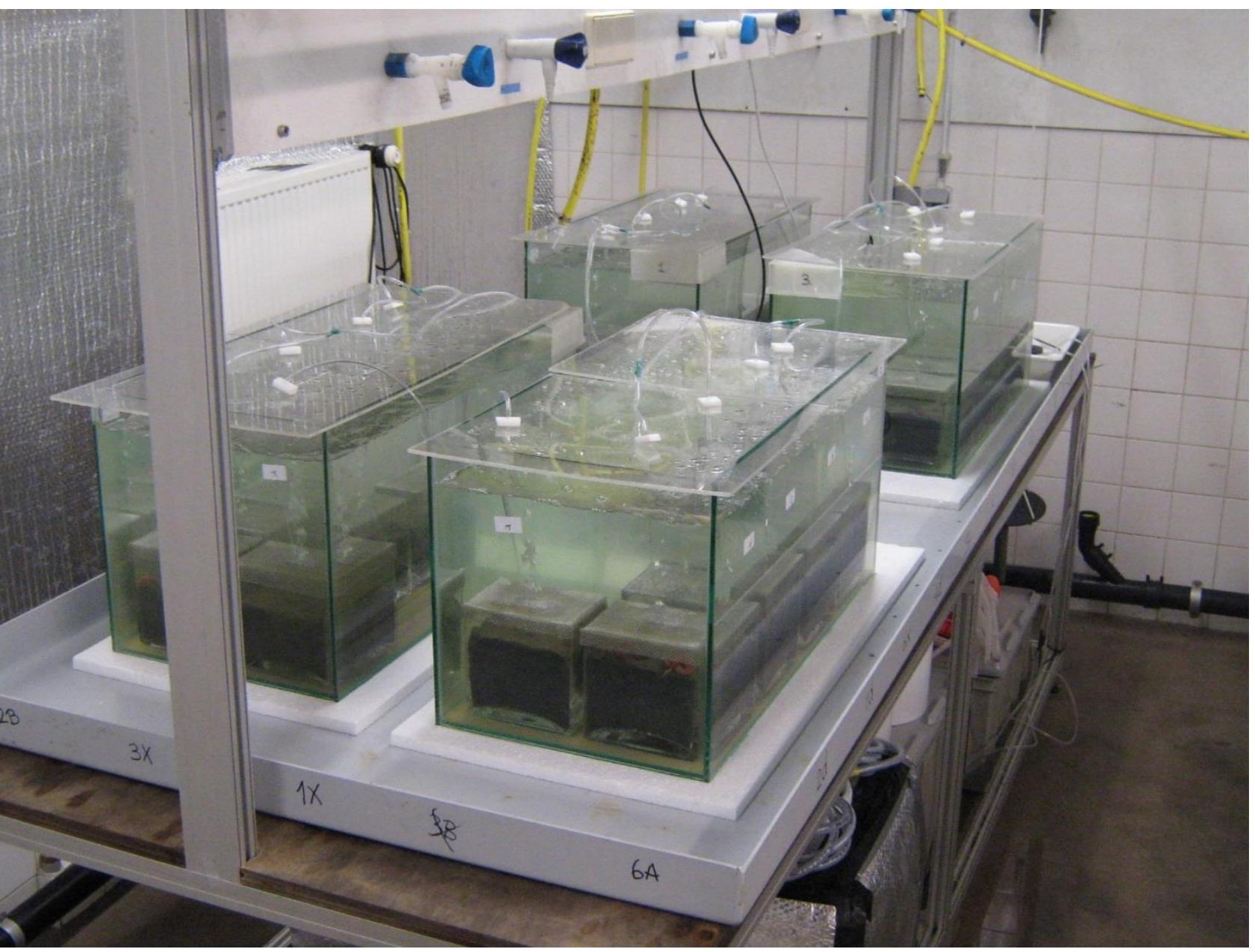




\section{Scientific publications}

Wegner, A., Besseling, E., Foekema, E.M., Kamermans, P., Koelmans, A.A., 2012. Effects of nanopolystyrene on the feeding behavior of the blue mussel (Mytilus edulis L.). Environ. Toxicol. Chem. 31, 2490-2497.

Besseling, E., Wegner, A., Foekema, E.M., Van den Heuvel-Greve, M.J., Koelmans, A.A., 2013. Effects of microplastic on fitness and PCB bioaccumulation by the lugworm Arenicola marina (L.). Environ. Sci. Technol. 47, 593-600.

Koelmans, A.A., Besseling, E., Wegner, A., Foekema, E.M., 2013. Plastic as a carrier of POPs to aquatic organisms: A model analysis. Environ. Sci. Technol. 47, 7812-7820.

Besseling, E., Wang, B., Lürling, M., Koelmans, A.A., 2014. Nanoplastic affects growth of $\mathrm{S}$. obliquus and reproduction of D. magna. Environ. Sci. Technol. 48, 12336-12343.

Koelmans, A.A., Besseling, E., Foekema, E.M., 2014. Leaching of plastic additives to marine organisms. Environ. Pollut. 187, 49-54.

Besseling, E., Foekema, E.M., Van Franeker, J.A., Leopold, M.F., Kühn, S., Bravo Rebolledo, E.L., Heße, E., Mielke, L., IJzer, J., Kamminga, P., Koelmans, A.A., 2015. Microplastic in a macro filter feeder: Humpback whale Megaptera novaeangliae. Mar. Pollut. Bull. 95, 248-252.

Huerta Lwanga, E., Gertsen, H., Gooren, H., Peters, P., Salánki, T., Van Der Ploeg, M., Besseling, E., Koelmans, A.A., Geissen, V., 2016. Microplastics in the Terrestrial Ecosystem: Implications for Lumbricus terrestris (Oligochaeta, Lumbricidae). Environ. Sci. Technol. 50, 26852691.

Koelmans, A.A., Besseling, E., Shim, W.J., 2015. Nanoplastics in the aquatic environment. Critical review, in: Bergmann, M., Gutow, L., Klages, M. (Eds.), Marine Anthropogenic Litter. Springer Cham Heidelberg, New York, Dordrecht, London, pp. 325-340. 
Besseling, E., Foekema, E.M., van den Heuvel-Greve, M.J., Koelmans, A.A., 2017. The effect of microplastic on chemical uptake by the lugworm Arenicola marina (L.) under environmentally relevant conditions. Environ. Sci. Technol. 51, 8795-8804.

Besseling, E., Quik, J.T.K., Sun, M., Koelmans, A.A., 2017. Fate of nano- and microplastic in freshwater systems: a modeling study. Environ. Pollut. $220,540-548$.

Hermsen, E., Pompe, R., Besseling, E., Koelmans, A.A., 2017. Detection of low numbers of microplastics in North Sea fish using strict quality assurance criteria. Mar. Pollut. Bull. 122, 253-258.

Huerta Lwanga, E., Gertsen, H., Gooren, H., Peters, P., Salánki, T., van der Ploeg, M., Besseling, E., Koelmans, A.A., Geissen, V., 2017. Incorporation of microplastics from litter into burrows of Lumbricus terrestris. Environ. Pollut. 220, 523-531.

Koelmans, A.A., Besseling, E., Foekema, E.M., Kooi, M., Mintenig, S., Ossendorp, B.C., Redondo-Hasselerharm, P.E., Verschoor, A., Van Wezel, A.P., Scheffer, M., 2017. Risk of plastic debris: unravelling fact, opinion, perception and belief. Environ. Sci. Technol. 51, 11513-11519.

Kooi, M., Besseling, E., Kroeze, C., Wezel, A.P. Van, Koelmans, A.A., 2017. Modeling the fate and transport of plastic debris in fresh waters. Review and guidance, in: Wagner, M., Lamber, S. (Eds.), Freshwater Microplastics. Springer International Publishing, pp. 125-152.

Siegfried, M., Koelmans, A.A., Besseling, E., Kroeze, C., 2017. Export of microplastics from land to sea. A global modelling approach. Water Res. $15,249-257$.

Vroom, R.J.E., Koelmans, A.A., Besseling, E., Halsband, C., 2017. Aging of microplastics promotes their ingestion by marine zooplankton. Environ. Pollut. 231, 987-996. 


\section{SENSE}

Netherlands Research School for the

Socio-Economic and Natural Sciences of the Environment

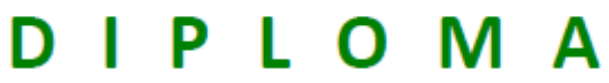

For specialised PhD training

The Netherlands Research School for the Socio-Economic and Natural Sciences of the Environment

(SENSE) declares that

\section{Ellen Besseling}

born on 26 February 1989 in Vorden, The Netherlands

has successfully fulfilled all requirements of the

Educational Programme of SENSE.

Wageningen, 16 April 2018

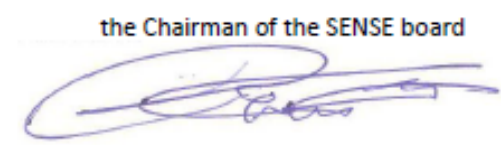

Prof. dr. Huub Rijnaarts

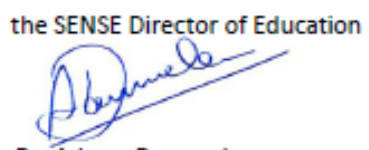

Dr. Ad van Dommelen

The SENSE Research School hes been accredited by the Royal Netherlands Academy of Arts and Sciences (KNAW)

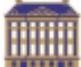

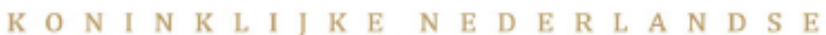
A K A D E M I E V A 


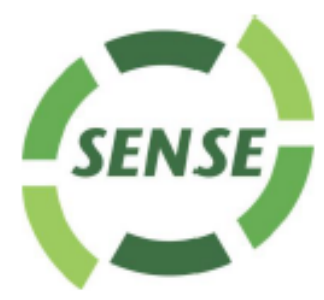

The SENSE Research School declares that Ms Ellen Besseling has successfully fulfilled all requirements of the Educational PhD Programme of SENSE with a work load of $43 \mathrm{EC}$, including the following activities:

\section{SENSE PhD Courses}

- Research in context activity: 'Developing and teaching several times a new part of a course: Marine plastic pollution (theme 6) of the course Water Quality (AEW-31306)' (2014-2017)

\section{Other PhD and Advanced MSc Courses}

- Biochemical modelling in aquatic environments: using $\mathrm{R}$ as a simulation environment, Netherlands Institute for Sea Research (NIOZ) (2012)

- Effective behaviour in your professional surroundings, Wageningen University (2013)

- Voice matters-Voice and presentation skills training, Wageningen University (2013)

- Teaching and supervising thesis students, Wageningen University (2013)

- Techniques for writing and presenting a scientific paper, Wageningen University (2014)

- Brain based teaching, Wageningen University (2014)

- Lecturing, Wageningen University (2016)

\section{Management and Didactic Skills Training}

- Supervising eight MSc students (2013-2016)

- Supervising MBO student (2014)

- Supervising two BSc students (2015)

\section{Oral Presentations}

- Nanoplastic affects growth of S. obliquus and reproduction of D. magna. Workshop MICRO: Fate and Impact of Microplastics in Marine Ecosystems, 13-15 January 2014, Brest, France, and SETAC Europe 24th Annual Meeting, 11-15 May 2015, Basel, Switzerland

- Modeling the fote of nanoplastic and microplastic in rivers. SETAC North America 35th Annual Meeting, 11-13 November 2014, Vancouver, Canada, and ISES, 12 October 2016, Utrecht, The Netherlands

- Relative importance of $P C B$ uptake from sediment and microplastic by the lugworm $A$. marina (L.). SETAC Europe 25th Annual Meeting, 3-7 May 2015, Barcelona, Spain

\section{Societal impact}

- Interview with several newspapers and websites Volkskrant (2012), Plastic tides (2014), Het Parool (2015), Vakblad $\mathrm{H}_{2} \mathrm{O}$ (2015), TV station PO News (2012) and two radio stations Vroege Vogels (2014) and Deutschland Funk (2014)

- Initiated and organized stakeholder participation with several Waterboards to develop a combined project plan (2015)

SENSE Coordinator PhD Education 

Financial support from the Aquatic Ecology and Water Quality Management Group of Wageningen University for staffing, materials and printing this thesis, and of Wageningen Marine Research (formerly IMARES) for providing facilities in Yerseke and Den Helder, is gratefully acknowledged.

Cover \& Layout: Ellen Besseling

Printed by: Digiforce || ProefschriftMaken 